The clinical effectiveness and costeffectiveness of different surveillance mammography regimens after the treatment for primary breast cancer: systematic reviews, registry database analyses and economic evaluation

C Robertson, SK Arcot Ragupathy,

C Boachie, JM Dixon, C Fraser, R Hernández, S Heys, W Jack, GR Kerr, G Lawrence, G MacLennan, A Maxwell, J McGregor, G Mowatt, S Pinder, L Ternent, RE Thomas, L Vale, R Wilson, S Zhu and FJ Gilbert

Health Technology Assessment NIHR HTA programme www.hta.ac.uk 


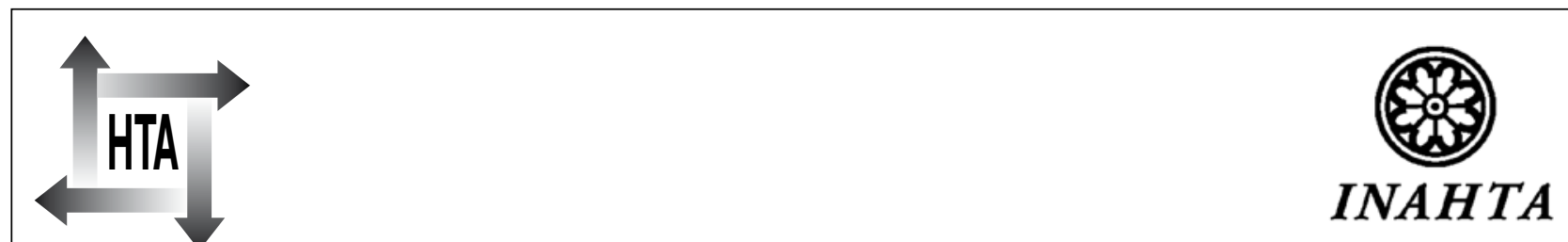

\section{How to obtain copies of this and other HTA programme reports}

An electronic version of this title, in Adobe Acrobat format, is available for downloading free of charge for personal use from the HTA website (www.hta.ac.uk). A fully searchable DVD is also available (see below).

Printed copies of HTA journal series issues cost $£ 20$ each (post and packing free in the UK) to both public and private sector purchasers from our despatch agents.

Non-UK purchasers will have to pay a small fee for post and packing. For European countries the cost is $£ 2$ per issue and for the rest of the world £3 per issue.

How to order:

- fax (with credit card details)

- post (with credit card details or cheque)

- phone during office hours (credit card only).

Additionally the HTA website allows you to either print out your order or download a blank order form.

\section{Contact details are as follows:}

Synergie UK (HTA Department)

Digital House, The Loddon Centre

Wade Road

Basingstoke

Hants RG24 8QW

\section{Email: orders@hta.ac.uk}

Tel: 08458124000 - ask for 'HTA Payment Services'

(out-of-hours answer-phone service)

Fax: 08458124001 - put 'HTA Order' on the fax header

\section{Payment methods}

Paying by cheque

If you pay by cheque, the cheque must be in pounds sterling, made payable to University of Southampton and drawn on a bank with a UK address.

Paying by credit card

You can order using your credit card by phone, fax or post.

\section{Subscriptions}

NHS libraries can subscribe free of charge. Public libraries can subscribe at a reduced cost of $£ 100$ for each volume (normally comprising 40-50 titles). The commercial subscription rate is $£ 400$ per volume (addresses within the UK) and $£ 600$ per volume (addresses outside the UK). Please see our website for details. Subscriptions can be purchased only for the current or forthcoming volume.

\section{How do I get a copy of HTA on DVD?}

Please use the form on the HTA website (www.hta.ac.uk/htacd/index.shtml). HTA on DVD is currently free of charge worldwide.

The website also provides information about the HTA programme and lists the membership of the various committees. 


\title{
The clinical effectiveness and cost- effectiveness of different surveillance mammography regimens after the treatment for primary breast cancer: systematic reviews, registry database analyses and economic evaluation
}

\author{
C Robertson, ${ }^{1 *}$ SK Arcot Ragupathy, ${ }^{2}$ C Boachie, ${ }^{1}$ \\ JM Dixon, ${ }^{3}$ C Fraser,${ }^{1}$ R Hernández, ${ }^{1,4} \mathrm{~S}$ Heys, ${ }^{5}$ W Jack, ${ }^{3}$ \\ GR Kerr, ${ }^{6}$ G Lawrence, ${ }^{7}$ G MacLennan, ${ }^{1}$ A Maxwell, ${ }^{8}$ \\ J McGregor, ${ }^{9}$ G Mowatt, ${ }^{1}$ S Pinder, ${ }^{10}$ L Ternent,,${ }^{1,3}$ \\ RE Thomas, ${ }^{1} \mathrm{~L}$ Vale, ${ }^{1,3} \mathrm{R}$ Wilson, ${ }^{11} \mathrm{~S}$ Zhu ${ }^{1}$ and \\ FJ Gilbert ${ }^{12}$
}

${ }^{1}$ Health Services Research Unit, University of Aberdeen, Aberdeen, UK ${ }^{2}$ Radiology Department, Aberdeen Royal Infirmary, NHS Grampian, Aberdeen, UK ${ }^{3}$ Edinburgh Breast Unit, Edinburgh Cancer Centre, Western General Hospital, Edinburgh, UK

${ }^{4}$ Health Economics Research Unit, University of Aberdeen, Aberdeen, UK ${ }^{5}$ Division of Applied Medicine, School of Medicine and Dentistry, University of Aberdeen, and Aberdeen Royal Infirmary, NHS Grampian, Aberdeen, UK ${ }^{6}$ Department of Clinical Oncology, Edinburgh Cancer Centre, Western General Hospital, Edinburgh, UK

${ }^{7}$ West Midlands Cancer Intelligence Unit, Birmingham Women's NHS Foundation Trust, Birmingham, UK ${ }^{8}$ Breast Unit, Royal Bolton Hospital, Bolton, UK ${ }^{9}$ Breast Cancer Care, London, UK

${ }^{10}$ Research Oncology, Kings College, London, UK

${ }^{11}$ Department of Clinical Radiology, The Royal Marsden, Sutton, UK

${ }^{12}$ Aberdeen Biomedical Imaging Centre, University of Aberdeen, and Aberdeen Royal Infirmary, NHS Grampian, Aberdeen, UK 
Declared competing interests of authors: none

Published September 2011

DOI: 10.3310/hta15340

This report should be referenced as follows:

Robertson C, Arcot Ragupathy SK, Boachie C, Dixon JM, Fraser C, Hernández RS, et al. The clinical effectiveness and cost-effectiveness of different surveillance mammography regimens after the treatment for primary breast cancer: systematic reviews, registry database analyses and economic evaluation. Health Technol Assess 2011;15(34).

Health Technology Assessment is indexed and abstracted in Index Medicus/MEDLINE, Excerpta Medica/EMBASE, Science Citation Index Expanded (SciSearch ${ }^{\circledR}$ ) and Current Contents ${ }^{\circledast} /$ Clinical Medicine. 

The Health Technology Assessment (HTA) programme, part of the National Institute for Health Research (NIHR), was set up in 1993. It produces high-quality research information on the effectiveness, costs and broader impact of health technologies for those who use, manage and provide care in the NHS. 'Health technologies' are broadly defined as all interventions used to promote health, prevent and treat disease, and improve rehabilitation and long-term care.

The research findings from the HTA programme directly influence decision-making bodies such as the National Institute for Health and Clinical Excellence (NICE) and the National Screening Committee (NSC). HTA findings also help to improve the quality of clinical practice in the NHS indirectly in that they form a key component of the 'National Knowledge Service'.

The HTA programme is needs led in that it fills gaps in the evidence needed by the NHS. There are three routes to the start of projects.

First is the commissioned route. Suggestions for research are actively sought from people working in the NHS, from the public and consumer groups and from professional bodies such as royal colleges and NHS trusts. These suggestions are carefully prioritised by panels of independent experts (including NHS service users). The HTA programme then commissions the research by competitive tender.

Second, the HTA programme provides grants for clinical trials for researchers who identify research questions. These are assessed for importance to patients and the NHS, and scientific rigour.

Third, through its Technology Assessment Report (TAR) call-off contract, the HTA programme commissions bespoke reports, principally for NICE, but also for other policy-makers. TARs bring together evidence on the value of specific technologies.

Some HTA research projects, including TARs, may take only months, others need several years. They can cost from as little as $£ 40,000$ to over $£ 1$ million, and may involve synthesising existing evidence, undertaking a trial, or other research collecting new data to answer a research problem.

The final reports from HTA projects are peer reviewed by a number of independent expert referees before publication in the widely read journal series Health Technology Assessment.

Criteria for inclusion in the HTA journal series

Reports are published in the HTA journal series if (1) they have resulted from work for the HTA programme, and (2) they are of a sufficiently high scientific quality as assessed by the referees and editors.

Reviews in Health Technology Assessment are termed 'systematic' when the account of the search, appraisal and synthesis methods (to minimise biases and random errors) would, in theory, permit the replication of the review by others.

The research reported in this issue of the journal was commissioned by the HTA programme as project number 07/47/01. The contractual start date was in June 2008. The draft report began editorial review in February 2010 and was accepted for publication in September 2010. As the funder, by devising a commissioning brief, the HTA programme specified the research question and study design. The authors have been wholly responsible for all data collection, analysis and interpretation, and for writing up their work. The HTA editors and publisher have tried to ensure the accuracy of the authors' report and would like to thank the referees for their constructive comments on the draft document. However, they do not accept liability for damages or losses arising from material published in this report.

The views expressed in this publication are those of the authors and not necessarily those of the HTA programme or the Department of Health.

Editor-in-Chief:

Series Editors:

Professor Tom Walley CBE

Dr Martin Ashton-Key, Professor Aileen Clarke, Dr Tom Marshall, Professor John Powell, Dr Rob Riemsma and Professor Ken Stein

Associate Editor:

Dr Peter Davidson

Editorial Contact: edit@southampton.ac.uk

ISSN 1366-5278 (Print)

ISSN 2046-4924 (Online)

ISSN 2046-4932 (DVD)

(C) Queen's Printer and Controller of HMSO 2011. This work was produced by Robertson et al. under the terms of a commissioning contract issued by the Secretary of State for Health.

This journal is a member of and subscribes to the principles of the Committee on Publication Ethics (COPE) (http://www. publicationethics.org/).

This journal may be freely reproduced for the purposes of private research and study and may be included in professional journals provided that suitable acknowledgement is made and the reproduction is not associated with any form of advertising. Applications for commercial reproduction should be addressed to: NETSCC, Health Technology Assessment, Alpha House, University of Southampton Science Park, Southampton SO16 7NS, UK.

Published by Prepress Projects Ltd, Perth, Scotland (www.prepress-projects.co.uk), on behalf of NETSCC, HTA.

Printed on acid-free paper in the UK by the Charlesworth Group. 


\title{
Abstract
}

\section{The clinical effectiveness and cost-effectiveness of different surveillance mammography regimens after the treatment for primary breast cancer: systematic reviews, registry database analyses and economic evaluation}

\author{
C Robertson, ${ }^{1 *}$ SK Arcot Ragupathy, ${ }^{2} \mathrm{C}$ Boachie, ${ }^{1} \mathrm{JM}$ Dixon, ${ }^{3}$ C Fraser, ${ }^{1}$ \\ R Hernández, ${ }^{1,4}$ S Heys, ${ }^{5}$ W Jack, ${ }^{3}$ GR Kerr, ${ }^{6}$ G Lawrence, ${ }^{7}$ G MacLennan, ${ }^{1}$ \\ A Maxwell, ${ }^{8} \mathrm{~J}$ McGregor, ${ }^{9} \mathrm{G}$ Mowatt, ${ }^{1}$ S Pinder, ${ }^{10} \mathrm{~L}$ Ternent, ${ }^{1,3} \mathrm{RE}$ Thomas, ${ }^{1}$ \\ L Vale, ${ }^{1,3} \mathrm{R}$ Wilson, ${ }^{11} \mathrm{~S} Z$ Zhu ${ }^{1}$ and FJ Gillbert' ${ }^{12}$
}

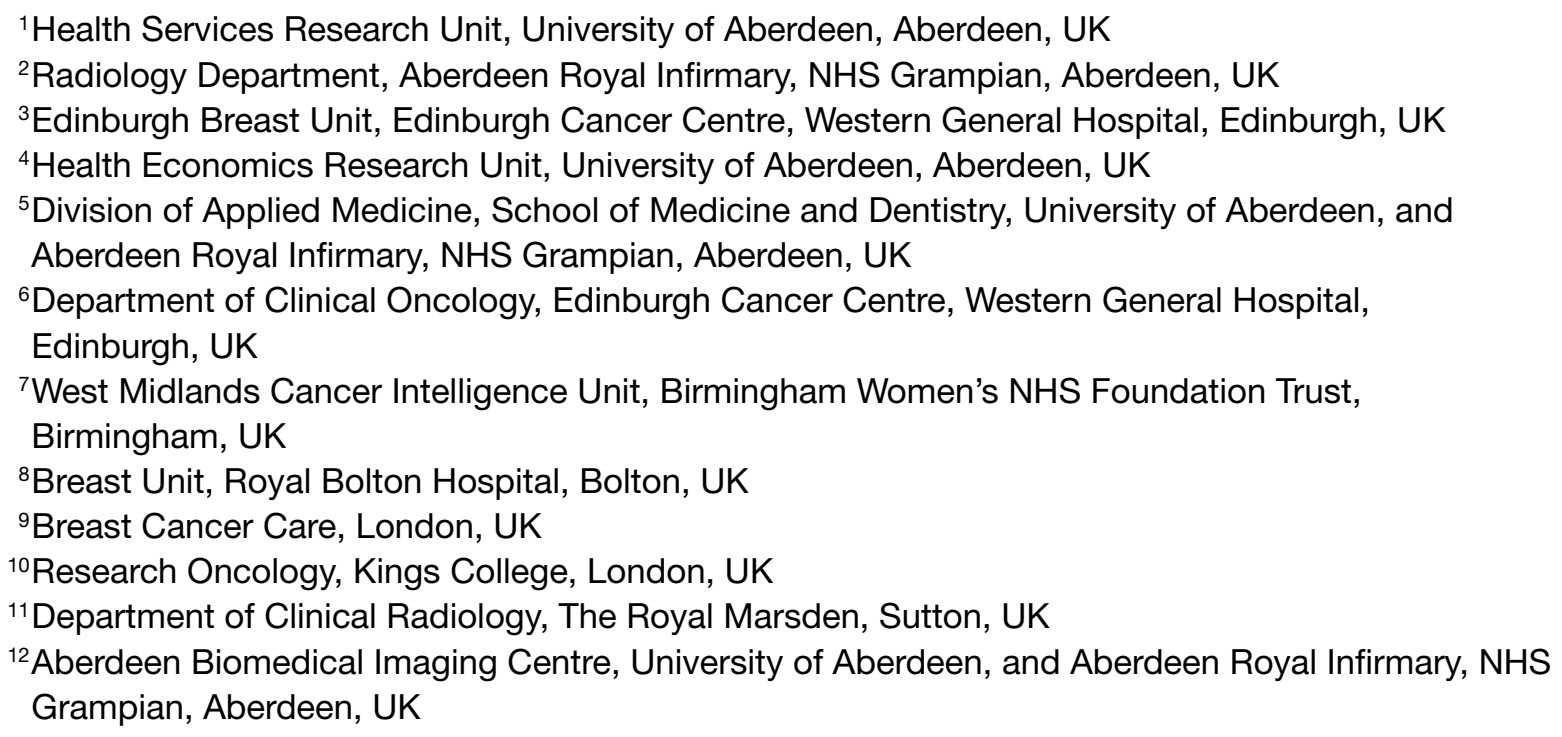

${ }^{*}$ Corresponding author

Background: Following primary breast cancer treatment, the early detection of ipsilateral breast tumour recurrence (IBTR) or ipsilateral secondary cancer in the treated breast and detection of new primary cancers in the contralateral breast is beneficial for survival. Surveillance mammography is used to detect these cancers, but the optimal frequency of surveillance and the length of follow-up are unclear.

Objectives: To identify feasible management strategies for surveillance and follow-up of women after treatment for primary breast cancer in a UK setting, and to determine the effectiveness and cost-effectiveness of differing regimens.

Methods: A survey of UK breast surgeons and radiologists to identify current surveillance mammography regimens and inform feasible alternatives; two discrete systematic reviews of evidence published from 1990 to mid 2009 to determine (i) the clinical effectiveness and cost-effectiveness of differing surveillance mammography regimens for patient health outcomes and (ii) the test performance of surveillance mammography in the detection of IBTR and metachronous contralateral breast cancer (MCBC); statistical analysis of individual patient data (West Midlands Cancer Intelligence Unit Breast Cancer Registry and 
Edinburgh data sets); and economic modelling using the systematic reviews results, existing data sets, and focused searches for specific data analysis to determine the effectiveness and cost-utility of differing surveillance regimens.

Results: The majority of survey respondents initiate surveillance mammography 12 months after breast-conserving surgery (BCS) $(87 \%)$ or mastectomy $(79 \%)$. Annual surveillance mammography was most commonly reported for women after BCS or after mastectomy ( $72 \%$ and $53 \%$, respectively). Most (74\%) discharge women from surveillance mammography, most frequently 10 years after surgery. The majority $(82 \%)$ discharge from clinical follow-up, most frequently at 5 years. Combining initiation, frequency and duration of surveillance mammography resulted in 54 differing surveillance regimens for women after BCS and 56 for women following mastectomy. The eight studies included in the clinical effectiveness systematic review suggest surveillance mammography offers a survival benefit compared with a surveillance regimen that does not include surveillance mammography. Nine studies were included in the test performance systematic review. For routine IBTR detection, surveillance mammography sensitivity ranged from $64 \%$ to $67 \%$ and specificity ranged from $85 \%$ to $97 \%$. For magnetic resonance imaging (MRI), sensitivity ranged from $86 \%$ to $100 \%$ and specificity was $93 \%$. For non-routine IBTR detection, sensitivity and specificity for surveillance mammography ranged from $50 \%$ to $83 \%$ and from $57 \%$ to $75 \%$, respectively, and for MRI from $93 \%$ to $100 \%$ and from $88 \%$ to $96 \%$, respectively. For routine MCBC detection, one study reported sensitivity of $67 \%$ and specificity of $50 \%$ for both surveillance mammography and MRI, although this was a highly select population. Data set analysis showed that IBTR has an adverse effect on survival. Furthermore, women experiencing a second tumour measuring $>20 \mathrm{~mm}$ in diameter were at a significantly greater risk of death than those with no recurrence or those whose tumour was $<10 \mathrm{~mm}$ in diameter. In the base-case analysis, the strategy with the highest net benefit, and most likely to be considered cost-effective, was surveillance mammography alone, provided every 12 months at a societal willingness to pay for a quality-adjusted lifeyear of either $£ 20,000$ or $£ 30,000$. The incremental cost-effectiveness ratio for surveillance mammography alone every 12 months compared with no surveillance was $£ 4727$.

Limitations: Few studies met the review inclusion criteria and none of the studies was a randomised controlled trial. The limited and variable nature of the data available precluded any quantitative analysis. There was no useable evidence contained in the Breast Cancer Registry database to assess the effectiveness of surveillance mammography directly. The results of the economic model should be considered exploratory and interpreted with caution given the paucity of data available to inform the economic model.

Conclusions: Surveillance is likely to improve survival and patients should gain maximum benefit through optimal use of resources, with those women with a greater likelihood of developing IBTR or MCBC being offered more comprehensive and more frequent surveillance. Further evidence is required to make a robust and informed judgement on the effectiveness of surveillance mammography and follow-up. The utility of national data sets could be improved and there is a need for high-quality, direct head-to-head studies comparing the diagnostic accuracy of tests used in the surveillance population. Funding: The National Institute for Health Research Health Technology Assessment programme. 


\section{Contents}

List of abbreviations $\quad$ xi

$\begin{array}{lc}\text { Executive summary } & \text { xiii }\end{array}$

1. Background 1

Description of health problem 1

Aetiology, pathology and prognosis 1

Epidemiology 2

Incidence and prevalence 2

Impact of health problem 3

Current service provision 3

Description of technology 5

2. Definition of the decision problem 11

Decision problem 11

$\begin{array}{ll}\text { Aim of the review } & 17\end{array}$

Structure of the remainder of the report 18

3. Identifying feasible UK management strategies for surveillance mammography and follow-up of women after treatment for primary breast cancer 19

Overview 19

Aim 19

Methods for the survey $\quad 20$

Results of the survey 21

Care pathway development $\quad 25$

Discussion $\quad 27$

4. Systematic review of clinical effectiveness of surveillance mammography after treatment for primary breast cancer $\quad 29$

Overview 29

Methods $\quad 29$

Results 32

Summary 40

5. Systematic review of test performance of surveillance mammography and comparator tests in detecting IBTR/МСВC 41

Overview 41

Methods $\quad 41$

Results 44

Summary

6. Analysis of Breast Cancer Registry database 55

Statement of overall aim and methods $\quad 55$

The WMCIU breast cancer primary tumour cohorts $\quad 55$

WMCIU second tumour cohorts $\quad 66$

$\begin{array}{ll}\text { Edinburgh breast cancer data set } & 73\end{array}$

$\begin{array}{ll}\text { Summary } & 74\end{array}$ 
7. Economic evaluation $\quad 75$

$\begin{array}{lr}\text { Introduction } & 75\end{array}$

Principles of economic evaluation $\quad 75$

$\begin{array}{ll}\text { Methods } & 76\end{array}$

Results

Summary 111

8. Discussion 113

Statement of overall aim and methods 113

Summary of main findings 113

$\begin{array}{ll}\text { Strengths and limitations } & 117\end{array}$

9. Conclusions 123

$\begin{array}{ll}\text { Summary of findings } & 123\end{array}$

Implications for the NHS and patients 123

$\begin{array}{ll}\text { Suggested research priorities } & 124\end{array}$

$\begin{array}{lr}\text { Acknowledgements } & 127\end{array}$

$\begin{array}{lr}\text { References } & 129\end{array}$

Appendix 1 Membership and individual area of expertise of the project Advisory Group

Appendix 2 Questionnaire content: a survey of UK surveillance mammography practice after treatment for primary breast cancer

Appendix 3 E-mail invitation to participate in a survey of UK surveillance mammography practice after treatment for primary breast cancer

Appendix 4 Reminder e-mail invitation to participate in a survey of UK surveillance mammography practice after treatment for primary breast cancer

Appendix 5 Number and percentage of respondents completing questions in the survey of UK surveillance mammography practice after treatment for primary breast cancer

Appendix 6 List of NHS trusts of respondents to the survey of UK surveillance mammography practice after the treatment for primary breast cancer

Appendix 7 Description of mammography surveillance regimens for women following breast-conserving surgery

Appendix 8 Description of mammography surveillance regimens for women following mastectomy

Appendix 9 List of questions used to help define alternative care pathways

Appendix 10 Search strategies: systematic review of clinical effectiveness of surveillance mammography 
Appendix 11 Reports of included studies: systematic review of clinical effectiveness of surveillance mammography

Appendix 12 Excluded studies: systematic review of clinical effectiveness of surveillance mammography

Appendix 13 Data extraction form for the systematic review of clinical effectiveness of surveillance mammography

Appendix 14 Quality assessment tool used in the systematic review of clinical effectiveness of surveillance mammography

Appendix 15 Characteristics of the studies included in the systematic review of clinical effectiveness of surveillance mammography

Appendix 16 Quality assessment of individual studies included in the systematic review of clinical effectiveness of surveillance mammography

Appendix 17 Search strategies: systematic review of test performance of surveillance mammography and comparator tests in detecting IBTR/MCBC

Appendix 18 Reports of included studies: systematic review of test performance of surveillance mammography and comparator tests in detecting IBTR/MCBC

Appendix 19 Reports of excluded studies: systematic review of test performance of surveillance mammography and comparator tests in detecting IBTR/MCBC

Appendix 20 Data extraction form for the systematic review of test performance of surveillance mammography and comparator tests in detecting IBTR/MCBC

Appendix 21 Quality assessment tool used in the systematic review of test performance of surveillance mammography and comparator tests in detecting IBTR/MCBC

Appendix 22 Characteristics of the included studies for the systematic review of test performance of surveillance mammography and comparator tests in detecting IBTR/MCBC

Appendix 23 Quality assessment of individual studies included in the systematic review of test performance of surveillance mammography and comparator tests in detecting IBTR/MCBC

Appendix 24 Histology of cancers detected and not detected (true-positives and false-negatives): systematic review of test performance of surveillance mammography and comparator tests in detecting IBTR/MCBC

Appendix 25 Univariate Cox regression models for the WMCIU cohorts

Appendix 26 Univariate Cox regression models for the Edinburgh cohort

Appendix 27 Example of detailed outline of economic model structure 
Appendix 28 Review of management guidelines for breast cancer

Appendix 29 Estimates of the different types of cancer that exist

Appendix 30 Summary of data from Adjuvant! Online used - estimated 10-year survival

Appendix 31 All-cause mortality rates

Appendix 32 Summary of findings of the structured review of doubling times 


\title{
List of abbreviations
}

\author{
ABS at BASO Association of Breast Surgeons at the British Association of Surgical Oncology \\ BCS breast-conserving surgery \\ BCT breast-conserving therapy \\ CE clinical examination \\ CI confidence interval \\ CT computerised tomography \\ ER oestrogen receptor \\ DOR diagnostic odds ratio \\ EBCTCG Early Breast Cancer Trialists' Collaborative Group \\ FFDM full-field digital mammography \\ GP general practitioner \\ HER2 human epidermal growth factor receptor 2 \\ HR hazard ratio \\ HRT hormone replacement therapy \\ HSRU Health Services Research Unit \\ IBTR ipsilateral breast tumour recurrence \\ ICER incremental cost-effectiveness ratio \\ MCBC metachronous contralateral breast cancer \\ MRI magnetic resonance imaging \\ NHSBSP NHS Breast Screening Programme \\ NICE National Institute for Health and Clinical Excellence \\ NIHR National Institute for Health Research \\ NR not reported \\ OR odds ratio \\ PSSRU Personal Social Services Research Unit \\ QALY quality-adjusted life-year \\ RCR Royal College of Radiologists \\ RCT randomised controlled trial \\ SD standard deviation \\ SEER Surveillance, Epidemiology, and End Results \\ SIGN Scottish Intercollegiate Guidelines Network \\ US ultrasound \\ WMCIU West Midlands Cancer Intelligence Unit \\ XRM X-ray mammography
}

All abbreviations that have been used in this report are listed here unless the abbreviation is well known (e.g. NHS), or it has been used only once, or it is a non-standard abbreviation used only in figures/tables/appendices, in which case the abbreviation is defined in the figure legend or in the notes at the end of the table. 



\section{Executive summary}

\section{Background}

Survival following breast cancer treatment is increasing. A key question is, therefore, how best to follow up patients after completion of primary treatments. There is considerable debate about the role and optimal organisation of the follow-up of patients following treatment for primary breast cancer. Data indicate that the early detection of ipsilateral breast tumour recurrence or ipsilateral second primary cancer [ipsilateral breast tumour recurrence (IBTR)] in the treated breast and detection of new primary cancers in the contralateral breast [metachronous contralateral breast cancer $(\mathrm{MCBC})]$ is beneficial in terms of survival. This raises the question as to how best to identify ipsilateral local recurrence of disease and new contralateral primary breast cancer at the earliest possible stage. Surveillance mammography is directed towards the detection of IBTR and MCBC. However, the optimal frequency of mammographic surveillance and the length of follow-up are unclear.

\section{Objectives}

1. Identify feasible management strategies for surveillance and follow-up of women after treatment for breast cancer in a UK setting.

2. Determine the effectiveness of differing surveillance and follow-up regimens after treatment for breast cancer.

3. Estimate the costs of differing surveillance and follow-up regimens after treatment for breast cancer.

4. Estimate the cost-effectiveness of differing surveillance and follow-up regimens after treatment for breast cancer.

5. Identify future research needs.

\section{Methods}

The work comprised a survey of UK breast surgeons and radiologists to identify current UK surveillance mammography regimens and inform feasible alternative regimens. In addition, we undertook two discrete systematic reviews to determine the clinical effectiveness of differing surveillance mammography regimens carried out after treatment for primary breast cancer on patient health outcomes and the test accuracy of surveillance mammography in the detection of IBTR and MCBC. Sensitive search strategies of several major bibliographic databases were conducted from 1990 to mid 2009. We undertook statistical analysis of individual patient data [West Midlands Cancer Intelligence Unit (WMCIU) Breast Cancer Registry and Edinburgh data sets] and economic modelling using the systematic reviews results, existing data sets, and focused searches for specific data analysis to determine the effectiveness and cost-utility of differing surveillance regimens. We developed an economic model in the form of a Markov model to represent the alternative surveillance regimens modelled at varying surveillance intervals. Parameter estimates for the Markov model were determined from a survey of existing data sets, a series of systematic reviews, and focused searches for specific data. 


\section{Results}

\section{Survey}

We received responses from $17 \%$ (183/1048) of those surveyed, 64\% were surgeons and 35\% radiologists and were based in 105 NHS trusts across the UK. The majority initiate surveillance mammography 12 months post surgery for women who have had breast-conserving surgery (BCS) $(87 \%)$ and for women who have had a mastectomy (79\%). Annual surveillance mammography was the most commonly reported surveillance mammography frequency for women after breast-conserving surgery (BCS) or after mastectomy ( $72 \%$ and $53 \%$, respectively), with biennial mammography the next most frequently reported ( $12 \%$ and $30 \%$, respectively). Most $(74 \%, 136 / 182)$ discharge women from surveillance mammography and they do this most frequently 10 years after surgery. The majority $(82 \%, 148 / 180)$ do discharge from clinical follow-up and most frequently at 5 years. Just over half $(55 \%, 98 / 179)$ responded that they discharge women to the NHSBSP if eligible. Combining initiation, frequency and duration of surveillance mammography resulted in 54 differing surveillance regimens for women after BCS and 56 for women following mastectomy. The most commonly followed four regimens for women after BCS or after mastectomy are to initiate surveillance mammography 12 months after surgery, conduct annual surveillance mammography with indefinite duration $(12 \%, 19 / 154,7 \%$, 10/136 respectively); or discharge from both clinical and mammographic surveillance at 5 years (14\%, 22/154, 10\%, 13/136 respectively); or 10 years $(12 \%, 18 / 154,11 \%, 15 / 136$ respectively) after surgery or discharge from clinical follow-up at 5 years with continued mammographic surveillance until 10 years $(13 \%, 20 / 154,8 \%, 11 / 136$ respectively). Our findings suggest that, although common patterns in surveillance mammography practice exist, there is considerable variation in the combinations of start, frequency, duration and discharge from surveillance mammography. Our findings reflect the different guidance given by the various professional organisations with an interest in surveillance after breast cancer, in combination with the local protocols of the respondents.

\section{Systematic reviews}

Eight studies, involving 3775 women, were included in the systematic review of clinical effectiveness. Although none of the tests of interest was used for the same purpose (i.e. routine or non-routine surveillance) in all studies, results suggest that the use of surveillance mammography offers a survival benefit compared with a surveillance regimen that does not include surveillance mammography.

Nine studies, involving 3724 women, were included in the systematic review of test performance. For the detection of IBTR in routine surveillance where there was no prior suspicion of recurrence, the highest sensitivity was shown for magnetic resonance imaging (MRI) and combined MRI/clinical examination at $100 \%$, whereas the highest specificity was shown for surveillance mammography alone $(97 \%)$ although this was obtained in a highly select population. Clinical examination alone had the lowest sensitivity (50\%) and surveillance mammography with clinical examination had the lowest specificity (67\%). For the detection of IBTR in patients for whom there was a suspicion of recurrence, sensitivity of tests varied from $50 \%$ (surveillance mammography) to $100 \%$ (MRI) and specificity ranged from $31 \%$ (ultrasound) to $96 \%$ (MRI). In the detection of $\mathrm{MCBC}$ in routine surveillance, sensitivity ranged from $0 \%$ (clinical examination) to $100 \%$ for the combination of surveillance mammography, clinical examination, ultrasound and MRI. Specificity ranged from $50 \%$ for surveillance mammography, MRI or clinical examination to $99 \%$ for the combination of surveillance mammography and ultrasound. Again, the highly selected nature of the population should be borne in mind in the context of these results. 


\section{Analyses of the WMCIU and Edinburgh data sets}

The analyses showed that IBTR has an adverse effect on survival that is independent of known risk factors. Furthermore, in those women experiencing a second tumour (either IBTR or MCBC) the size of this second tumour is important, with those women with tumours of $>20 \mathrm{~mm}$ in diameter being at a significantly greater risk of death than those with no recurrence or those whose tumour was $<10 \mathrm{~mm}$ in diameter.

\section{Economic evaluation}

The results of the economic model should be considered exploratory and interpreted with caution given the paucity of data available to inform the economic model. In the base-case analysis, the strategy with the highest net benefit, and therefore the most likely to be considered cost-effective, was surveillance mammography alone, provided every 12 months at a societal willingness to pay for a quality-adjusted life-year (QALY) of either $£ 20,000$ or $£ 30,000$. The incremental cost-effectiveness ratio for surveillance mammography alone every 12 months compared to no surveillance was $£ 4727$. This result holds for women previously treated for their primary cancer with either breast-conserving surgery or mastectomy or for women who suffer IBTR. The results of the model are sensitive to changes in the incidence of recurrent cancer. When the expected incidence is increased towards the maximum that could be expected (approximately 0.008 per annum) clinical surveillance plus mammography has an incremental cost per QALY of approximately $£ 30,000$. As the surveillance interval and incidence increase regimens that are more costly but more effective (proxied by the MRI plus clinical surveillance) may also have incremental costs per QALY $<£ 30,000$. This suggests that there may be some scope for research into alternative technologies that could be used for surveillance.

We did not conduct probabilistic sensitivity analysis due to statistically imprecise and limited data. We did conduct both one-way and multiway sensitivity analyses, however. Sensitivity analysis included varying the probability of cancer, inflating the risk of death from cancer, inflating the risk of cancer progression in undiagnosed or untreated cancers, varying diagnostic accuracy of the surveillance tests and varying costs and age.

The results of the model were sensitive to incidence and other factors, for example age, tumour characteristics, etc., which might define women with greater or lesser likelihoods of developing an IBTR or MCBC. These results suggest that a more intensive follow-up of women with greater likelihood of IBTR or MCBC may be worthwhile. Conversely, for women with less likelihood of IBTR or MCBC it may be more cost-effective for surveillance to be performed less often (every 2 or 3 years) with mammography alone. As the surveillance interval and incidence increase, strategies that are more costly, but more effective, may also have incremental costs per QALY below typical threshold values.

\section{Limitations}

Despite considerable and rigorous methods adopted for both systematic reviews, we found few studies meeting our inclusion criteria, none of which were a randomised controlled trial (RCT). The limited and variable nature of the data available precluded any quantitative analysis. There was no useable evidence contained in the Breast Cancer Registry database to assess the effectiveness of surveillance mammography directly. As few data were available, the economic results need to be treated cautiously. In particular, a series of simplifying assumptions were made about disease progression and prognosis of recurrent cancers. We took care to err on the side of caution when making these assumptions, however, in order to minimise the possibility of overestimating the value of surveillance. Furthermore, few data relevant to the UK were available on health-state utilities. We assumed that the data used were applicable to the UK and the health states modelled. 


\section{Conclusions}

\section{Implications for service provision}

Surveillance is likely to improve survival, with a strategy of mammography alone, every 12-24 months, appearing to have the highest net benefits. The evidence base on which to recommend any change in current practice is relatively weak, however. Careful consideration should be given to stratification of patients to ensure maximum benefit to ensure optimal use of resources, with those women with a greater likelihood of developing IBTR or MCBC being offered more comprehensive (e.g. mammography and clinical follow-up) and more frequent surveillance (every 12 months). The greatest net benefit for women with the lowest likelihood of IBTR or MCBC is mammography only every 3 years. Although there may be arguments for delivering a varying surveillance regimen this would present challenges and, without provision of information and reassurance, might be a source of unnecessary anxiety for patients.

\section{Suggested research priorities}

- The utility of the national data sets could be improved. In addition to the nationally agreed collection, it would be extremely valuable to record details of mode of detection for IBTR or $\mathrm{MCBC}$; the frequency of the clinical and mammographic surveillance regimens, and how this varies over time; and whether a woman's IBTR or MCBC was detected during routine surveillance or as a result of it causing symptoms for the patient.

- There is a need for high-quality, direct head-to-head studies comparing the diagnostic accuracy of tests used in the surveillance population. Further primary work should also consider whether the use of existing technologies, such as MRI, which may have better performance, could be worthwhile for patients at high risk of IBTR or MCBC. An economic analysis should form part of such work.

- Further economic evaluation modelling should compare differing mixed regimens of clinical follow up, delivered in lower cost settings, combined with surveillance mammography in the long term. This would be important to inform further primary research (e.g. an RCT) which could then focus on regimens that appeared most promising.

- A definitive RCT would be ideal and, although costly, could focus on those women at higher risk of IBTR or MCBC. The interventions considered might include mammography and MRI, for those at the highest risk, or surveillance mammography of 1 year versus a longer time interval, for example 3 years. Such a trial might also compare more sophisticated surveillance regimens, which vary not only in terms of the frequency of mammography but also in terms of the frequency and setting of clinical follow-up. An economic evaluation should form part of any RCT.

\section{Funding}

The National Institute for Health Research Health Technology Assessment programme. 


\section{Chapter 1}

\section{Background}

\section{Description of health problem}

Breast cancer affects one in nine women in the UK. At present, the 5-year survival rate in the $\mathrm{UK}$ is $82 \%$. It is estimated that there are $>550,000$ women who are alive and have been treated for breast cancer in the UK, which represents $2 \%$ of the total female population. ${ }^{1}$ Survival following breast cancer treatment is increasing and a key question is how these patients should be followed up after completion of treatment for primary breast cancer. Surveillance mammography is directed towards the detection of ipsilateral breast tumour recurrence or ipsilateral second primary cancer (IBTR) in the treated breast and detection of new primary cancers in the contralateral breast [metachronous contralateral breast cancer (MCBC)]. In addition, follow-up of patients after treatment for primary breast cancer is aimed at detection of regional recurrence in the chest wall (following mastectomy) and axillae, detection of metastatic disease, and monitoring for side effects of adjuvant treatments and making changes in treatment where appropriate, together with psychological support and treatment as required.

The Early Breast Cancer Trialists' Collaborative Group (EBCTCG) meta-analysis of data from randomised controlled trials (RCTs) demonstrated that IBTR is associated with an adverse effect on survival in patients undergoing a variety of standard treatments for breast cancer. This may take up to 15 years to become evident, however. ${ }^{2-4} \mathrm{Lu}^{5}$ recently conducted a systematic review to determine the impact of early detection of isolated locoregional and contralateral recurrence on survival. The author reported better overall survival for recurrences detected by mammography or in asymptomatic patients, with an absolute reduction in mortality of $17-28 \%$ if all breast cancer recurrences are detected early. Similarly, an analysis of the Surveillance, Epidemiology, and End Results (SEER) database reporting on 174,453 women who had previously been treated for a primary breast cancer demonstrated that, for women with MCBC with stage 0-I disease, there was an $81 \%$ reduction in breast cancer mortality compared with that of women who were identified with more advanced contralateral disease. ${ }^{6}$ Therefore, the data suggest that the early detection of IBTR and of MCBC is beneficial in terms of survival. This raises the question as to how best to identify IBTR and MCBC.

\section{Aetiology, pathology and prognosis}

It is now generally accepted that IBTR has an adverse influence on patient survival. There is, however, no general agreement on the best treatment regimen for those who have experienced an IBTR. ${ }^{7}$ Furthermore, it is not clear whether IBTR itself increases the risk of the development of distant metastases, or whether IBTR and risk of distant disease is merely a reflection of the original aggressiveness of the tumour with its resultant effect on survival.

Younger women (defined as those $<35$ years of age), extensive ductal carcinoma in situ, excision margins involved with tumour and lymphovascular invasion by tumour are well recognised risk factors for IBTR. ${ }^{8}$ Tumour size, grade and nodal involvement are risk factors for both local and distant recurrence. ${ }^{9}$ Of patients experiencing IBTR, $40-50 \%$ will have further recurrence or 


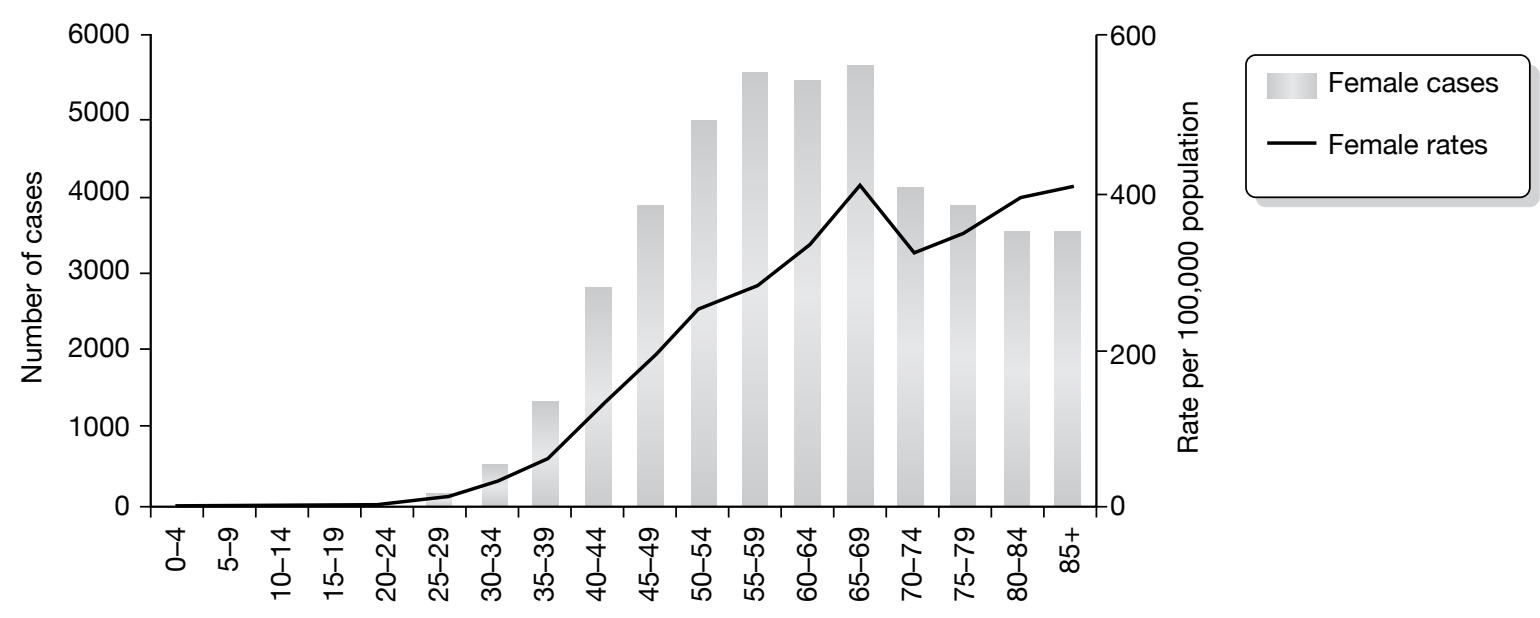

Age at diagnosis (years)

FIGURE 1 Number of new cases and age-specific incidence rates for female breast cancer, UK 2006. Reproduced with permission from Cancer Research UK. ${ }^{14}$

develop distant disease subsequently, particularly if the local recurrence occurs within 2 years of the initial presentation and treatment. ${ }^{10}$

\section{Epidemiology}

Breast cancer is an age-related disease, with $81 \%$ of cases occurring in those over 50 years of age. It is more prevalent in affluent societies, with the highest incidence being in North America and Western/Northern Europe. In those areas and countries, the most deprived socioeconomic groups have a lower incidence compared with the most affluent groups. Women who migrate from countries with a low incidence to those with a high incidence (e.g. Japan to the USA) often increase their risk, suggesting a strong effect for lifestyle and/or environmental factors. Early menarche, late menopause, oral contraceptive use, later age at first pregnancy, lower parity, lack of breastfeeding and use of hormone replacement therapy (HRT) after the age of 50 years are all recognised risk factors for breast cancer development. Following the publication of the Women's Health Initiative trial in $2002^{11}$ there was a marked reduction in the use of HRT, which has resulted in a reduction of breast cancer incidence in the over-50-years age group..$^{12}$ In the UK, approximately $25 \%$ of women in the 45 - to 69 -year age group were using HRT by 2001 but this had fallen by half by 2006. This is estimated to have resulted in a reduction of around 1400 cases of breast cancer in the 50 - to 59 -year age group. ${ }^{13}$

\section{Incidence and prevalence}

There are 45,822 new cases of breast cancer in the UK each year. ${ }^{14}$ Figure 1 details Cancer Research UK incidence rates for women by age for 2006 in the UK. Approximately $20 \%$ of patients will develop a systemic recurrence and die within 5 years. In patients who undergo breast-conserving therapy (BCT), IBTR occurs in $1-2 \%$ of patients each year ${ }^{15}$ and MCBC occurs at a rate of up to $0.8 \%$ each year. ${ }^{16,17}$

Around 550,000 women were living in the UK with a diagnosis of breast cancer in 2008. ${ }^{18}$ 


\section{Impact of health problem}

\section{Significance for patients in terms of ill health}

Ipsilateral breast tumour recurrence and $\mathrm{MCBC}$ have significant adverse effects on the patient. Further treatment is required and this often necessitates mastectomy for the patient who has a previously conserved breast together with the consideration of the use of systemic treatments (chemotherapy, hormone therapy, biological therapy). Disease recurrence has a significant adverse impact psychologically with major implications for the patient, their health and quality of life. There are data to indicate that patients who experience local disease recurrence have a poorer survival than those who do not have local recurrence., ${ }^{2,7}$

\section{Significance for the NHS}

The significance to the NHS in terms of the provision of clinical and mammographic surveillance, and treatment of IBTR and MCBC, is great. Following the treatment of primary breast cancer, patients are followed up with regular clinical examinations and surveillance mammography carried out at intervals as described below. Subsequent investigations are carried out to confirm disease recurrence or to exclude disease in those incorrectly identified as positive by a prior test or examination ('false-positive').

For each annual cohort of approximately 45,000 new cases of breast cancer, 20\% (9000) will have developed metastatic disease and die within 5 years, requiring complex, demanding and costly treatment regimens. Taking a mean age of 70 years for primary breast cancer diagnosis, if $1 \%$ develop IBTR each year, and accounting for death from other causes, then over a 20-year period approximately 6358 patients will require treatment for this with $20 \%$ requiring further treatment for systemic disease.

\section{Current service provision}

Ipsilateral breast tumour recurrence and $\mathrm{MCBC}$ are detected by mammography, clinical breast examination and/or by the presence of symptoms noted by the patient. There are various recommendations in the UK for surveillance of patients with breast cancer ranging from 'no follow-up' to 6-monthly clinical examination (sometimes more frequently) and surveillance mammography (carried out at intervals ranging from 1- to 3-yearly). Follow-up is sometimes stratified by the likelihood of disease recurrence (defined as local and/or distant metastatic disease), based on prognostic factors taken from the patient's initial primary breast cancer. The majority of surveillance is carried out in secondary care (e.g. surgeons, oncologists, breast-care nurses), with patients being discharged to primary care after an interval which varies from 3 years to continued follow-up in secondary care. The uncertainty about best practice and current knowledge about variation in practice is further described below (see Variation in services and/or uncertainty about best practice).

\section{Management of disease}

Women with primary disease are usually treated using a combination of treatment modalities, including surgery, radiotherapy, chemotherapy, hormone therapy and biological therapy (trastuzumab), either alone or in a variety of combinations tailored to the patient and the type and stage of disease. However, most patients undergo surgery initially, which is either mastectomy or BCT, together with axillary surgery (sentinel lymph node biopsy, axillary sample or axillary clearance) and tailored adjuvant therapy afterwards. Patients with large or locally advanced breast cancer may receive either primary (neoadjuvant) chemotherapy or hormone 
therapy prior to undergoing surgery. A small number of patients who are unfit for surgery but are hormone receptor positive may receive endocrine treatment and/or palliative radiotherapy as their sole treatment.

The management of locoregional recurrence following the treatment of primary breast cancer is variable in the UK, as there is an absence of RCTs to guide management decisions. Following breast conservation surgery it has been reported that, in those patients who experience locoregional recurrence, up to $20 \%$ may have distant metastases at the same time. ${ }^{19,20}$ Furthermore, other studies have indicated that patients who experience a locoregional relapse have a reduced 5-year survival. ${ }^{2,3}$ Where there is a recurrence in the chest wall after mastectomy, patients will frequently have systemic recurrence and therefore all patients presenting with recurrent breast cancer should be restaged [investigations may include combinations of haematological investigations, chest radiography, abdominal ultrasound, computerised tomography (CT) scan of chest and abdomen, isotope bone scan] prior to definitive management. ${ }^{21}$

A multidisciplinary approach is required for the management of patients with locoregional recurrence following treatment for primary breast cancer. For patients who have undergone breast conservation surgery, treatment is usually mastectomy (with axillary clearance if not already performed), together with consideration of further systemic therapy (chemotherapy, hormone therapy) to reduce the risk of subsequent metastatic relapse. In patients who have a local chest wall recurrence following mastectomy, local therapy (surgery if possible with radiotherapy afterwards or if surgery is not possible due to the extensive nature of the disease then radiotherapy alone) may be undertaken together with systemic therapy (chemotherapy, hormone therapy) given the high risk of subsequent metastatic relapse in this group of patients.

There are no RCTs on which to base the decision to offer systemic adjuvant therapies. In those patients without detectable systemic metastases, factors taken into account are as for the use of adjuvant therapy, for example tumour size, tumour grade, lymph node status, lymphovascular invasion, hormone receptor and human epidermal growth factor receptor 2 (HER2) status and the time interval from the treatment of the patient's original primary breast cancer.

\section{Variation in services and/or uncertainty about best practice}

There is considerable debate about the optimal organisation of a surveillance service following treatment for primary breast cancer. The number of different guidelines produced by various professional groups and policy-makers reflects this. ${ }^{21-25}$ Previous surveys of breast surgeons, oncologists $^{26}$ and NHS breast-screening units ${ }^{27}$ indicate that there is wide variation in follow-up practice but, in general terms, surveillance includes regular clinical examination, up to 3-5 years, with annual mammography for up to 10 years, or in some units this is carried out indefinitely. The most recent National Institute for Health and Clinical Excellence (NICE) guidance recommends mammography annually for 5 years and then follow-up through the NHS Breast Screening Programme (NHSBSP). ${ }^{24}$ There is concern over whether the 3-yearly interval is sufficient for the group under surveillance, and some believe a stratified approach based on risk of recurrence or contralateral disease is more appropriate. The American Society of Clinical Oncology ${ }^{28}$ recommended that surveillance should include annual mammography but acknowledges that there is a lack of high-level evidence to support decisions about the frequency and timing of mammographic surveillance. These guidelines have usually been based on consensus approaches and literature reviews, and their key recommendations are shown in Table 1.

For women who have had treatment for breast cancer there is, however, general agreement that there is no survival advantage conferred by an intensive surveillance regimen (comprising chest 
TABLE 1 Comparison of guidelines for the surveillance of women who have received treatment for primary breast cancer

\begin{tabular}{|c|c|c|c|}
\hline $\begin{array}{l}\text { Organisation publishing } \\
\text { guidelines }\end{array}$ & Clinical follow-up & Mammography & Additional comments \\
\hline $\mathrm{ABS}$ at $\mathrm{BASO}^{21}$ & $\begin{array}{l}\text { Continue follow-up while on } \\
\text { active treatments; at least } 5 \text { years } \\
\text { recommended }\end{array}$ & $\begin{array}{l}1-2 \text { yearly for up to } 10 \text { years after } \\
\text { the primary diagnosis }\end{array}$ & $\begin{array}{l}\text { High-risk or active treatment then } \\
\text { more frequent and with agreed } \\
\text { local protocols }\end{array}$ \\
\hline $\mathrm{SIGN}^{22}$ & Pragmatic - indefinite & $1-2$ yearly & \\
\hline NICE (Cancer Service) $)^{25}$ & Up to 3 years' follow-up & No recommendation & \\
\hline $\begin{array}{l}\text { NICE (Early and Locally Advanced } \\
\text { Breast Cancer) }{ }^{24}\end{array}$ & $\begin{array}{l}\text { Follow-up to completion of } \\
\text { adjuvant treatment then choose } \\
\text { setting in which follow-up occurs }\end{array}$ & $\begin{array}{l}\text { Yearly for } 5 \text { years then move } \\
\text { to NHSBSP screening - more } \\
\text { frequently than 3-yearly stratified } \\
\text { by risk }\end{array}$ & \\
\hline $\mathrm{RCR}^{23}$ & & $1-2$ yearly & \\
\hline
\end{tabular}

ABS, Association of Breast Surgery; BASO, British Association of Surgical Oncology; RCR, Royal College of Radiologists; SIGN, Scottish Intercollegiate Guidelines Network.

radiography, liver ultrasound, haematological investigations and isotope bone scans) compared with a simpler follow-up schedule of clinical examination and mammographic surveillance. ${ }^{29}$

Given the variation in recommendations, it would be surprising if there were no variation in practice. The results of a survey of practice conducted for this study are described in more detail in Chapter 3.

\section{Current and anticipated costs}

As reported above, there are follow-up regimens for women following treatment of primary breast cancer. Illustrative annual costs of alternative surveillance regimens are presented in Table 2. The calculation of these costs is reported in detail in Chapter 7.

\section{Description of technology}

\section{Surveillance mammography}

Mammography has been in use for $>30$ years and is the standard imaging technique for detection of breast cancer..$^{30}$ In women previously treated for breast cancer, surveillance mammography is useful for early detection of tumour recurrence or for confirming the absence of recurrent cancer. While tumour recurrence may display similar mammographic features to the previous primary breast cancer, ${ }^{31}$ interpretation of the surveillance mammogram is hindered by changes in the breast caused by postoperative scarring and changes to breast density caused by primary treatment modalities. For example, following surgery and/or radiotherapy, detectable abnormalities on mammography include haematoma, scar formation, fat necrosis, skin thickening, increased soft tissue density in the ipsilateral breast and microcalcifications, all of which can be misinterpreted as malignancy. Therefore, surveillance mammography after the treatment for breast cancer is associated with the possibility of false-positive results causing further unnecessary investigations (invasive and non-invasive) and reduction in sensitivity for the detection of IBTR. There may also be an effect on MCBC detection with a lack of a comparator side.

Although published figures vary, it has been estimated that approximately $50 \%$ of IBTRs will be detected by mammography, with the remainder being detected by clinical examination. ${ }^{31-33}$ 
TABLE 2 Illustrative costs of alternative surveillance regimens

\begin{tabular}{lllll}
\hline & $\begin{array}{l}\text { Mammographic } \\
\text { surveillance yearly for } \\
\text { 10 years }\end{array}$ & $\begin{array}{l}\text { Mammographic and } \\
\text { clinical follow-up yearly } \\
\text { for 10 years }\end{array}$ & $\begin{array}{l}\text { Mammographic } \\
\text { surveillance every 2 } \\
\text { years for 10 years }\end{array}$ & $\begin{array}{l}\text { Mammographic and } \\
\text { clinical follow-up every } \\
\text { 2 years for 10 years }\end{array}$ \\
\hline $\begin{array}{l}\text { Cost per woman per screen } \\
(£)\end{array}$ & 55.45 & 141.45 & 55.45 & 141.45 \\
$\begin{array}{l}\text { Cost for first episode of } \\
\text { surveillance for an initial } \\
\text { cohort of 10,000 women }(£)\end{array}$ & 552,204 & $1,408,643$ & 544,259 & $1,388,376$ \\
Cost over 10 years $(£)$ & $5,147,650$ & $13,131,382$ & $2,552,363$ & $6,510,943$ \\
\hline
\end{tabular}

a Assuming that mean age of cohort is 57 years, women face the same risk of death as the general population and that they leave this followup regimen once cancer is detected.

Patients who have had a mastectomy or those who have undergone breast reconstruction following mastectomy do not have mammographic surveillance of that side. Clinical examination alone is the standard method of follow-up to detect IBTR, although mammography is undertaken of the remaining breast. Patients who have undergone mastectomy may find it easier to detect IBTR clinically than those who have undergone breast conservation surgery or reconstruction. Approximately $10 \%$ of breast cancers that can be palpated on clinical examination are not clearly visible on mammography and require the use of additional imaging techniques. Recurrent breast cancers detected by mammography are generally smaller and less aggressive than those found on clinical examination. ${ }^{31,32}$ It is presumed, therefore, that mammography, combined with clinical examination, allows the earliest possible diagnosis of IBTR and also allows surveillance of the contralateral breast for the detection of $\mathrm{MCBC}$. Whether such surveillance regimens reduce mortality remains unclear at present.

Mammography involves low-dose X-ray imaging of the breast to create detailed soft tissue, high-contrast, high-resolution images, which are recorded on photographic film. Mammograms are usually produced by a radiographer and interpreted by a radiologist who is trained in breast imaging. Recent developments have led to an increasing use of solid-state detectors rather than X-ray film, termed digital mammography or full-field digital mammography (FFDM). These convert X-rays into electrical signals. The images produced are displayed on a computer screen but can be printed onto radiographic film that is similar to conventional mammograms. It is possible to manipulate digital images on-screen to enhance visibility of certain areas. Digital mammography is quicker to produce than film mammography, uses lower doses of radiation and digital images require less physical storage space than traditional films. Digital mammography systems are, however, one to four times more costly than film mammography systems ${ }^{34}$ In the screening population, digital mammography has improved performance over film mammography in younger women and in women with dense breasts. Overall, however, the diagnostic accuracy of digital mammography is not significantly greater than that of film mammography.

\section{Other relevant new interventions Imaging tests Ultrasound}

Breast ultrasound is an imaging technique for diagnosing breast cancer. A hand-held transducer produces high-frequency sound waves, which pass through the breast and reflect back or echo from various tissue surfaces to form an image of the internal structures. It is not an invasive technique and does not involve radiation. The transducer is linked to a computer so that images 
can be viewed on a monitor screen. A radiologist or radiographer is responsible for interpreting the ultrasound images generated.

Breast ultrasound has been reported as having a lower sensitivity for detecting breast cancer than mammography. ${ }^{36}$ When biopsies were indicated on the basis of the results of either mammography or ultrasound the positive predictive value was lower for ultrasonography than for mammography. ${ }^{34}$ The use of ultrasound in routine practice is therefore mainly as an adjunct to mammography as a problem-solving tool to investigate an area in the breast which has been designated as 'suspicious' by mammography. Here, ultrasound acts as an additional diagnostic tool in determining whether a lesion identified on mammography or clinical examination requires further invasive investigation, for example in distinguishing between a fluid-filled cyst and a solid mass. Breast ultrasound is also particularly useful in evaluating women whose mammograms are difficult to interpret due to the density of their breasts. ${ }^{37-39}$ Ultrasound is not used as a surveillance technique due to the low sensitivity of detection of breast cancer but can be helpful after mammography in order to differentiate scar tissue from IBTR or MCBC. It can also be used to undertake an ultrasound-guided biopsy of a suspicious area found on mammography. Ultrasound is a low-cost technique and, unlike mammography, does not involve any harm or discomfort to the patient. Test performance is operator dependent and time-consuming, however (approximately 30 minutes per ultrasound), and the results are less consistent than mammography.

\section{Magnetic resonance imaging}

Magnetic resonance imaging (MRI) uses a powerful magnetic field and radio frequencies to produce detailed images of the breast. Breast imaging is undertaken using dedicated breast coils and with the patient lying prone on the examination table. The body coil around the base of the magnet sends radio waves into the breast tissues. The breast coils receive radio waves recording signals from the breast tissue. A computer then processes the signals and generates a series of images, each of which shows a thin slice of the breast, which can be viewed in different planes. An intravenous contrast agent is required to highlight breast cancers within the glandular tissue. The examination is carried out in the second week of the menstrual cycle to reduce background tissue enhancement.

Magnetic resonance imaging is used in women who are at high risk of developing breast cancer as a result of their family history; in distinguishing between scar tissue and recurrent tumour; in women for whom ultrasound and mammography findings are discordant; and in patients with lobular cancer and/or where there is suspected multifocal disease and a higher risk of MCBC. MRI has greater sensitivity than mammography or ultrasound, which makes it a particularly useful imaging modality for detecting small tumours and in women with dense breast tissue. Specificity, however, has been reported as being as low as $37 \%$, as MRI is poor at distinguishing between cancer and benign breast disease. ${ }^{34} \mathrm{MRI}$ is not used as a primary surveillance tool at present.

The magnetic field used in MRI is not harmful, although internal medical devices that contain metal may malfunction or cause problems during an MRI examination. Some women may find the procedure uncomfortable and claustrophobic. There is also a small risk of mild allergic reaction if contrast material is injected. MRI investigations carry a greater financial cost (the NHS Reference $\operatorname{Costs}^{40}$ report a mean cost of $£ 232$ ) than mammography (a mean cost of $£ 45$ for the mammogram alone has been estimated from Breast Screening Programme data) or ultrasound (the NHS Reference Costs ${ }^{40}$ report a mean cost of $£ 56$ ). MRI is also more time consuming to perform (30-40 minutes) than mammography and is not as readily available, making it impractical to perform as part of the outpatient breast clinic. 


\section{Physical examination tests Specialist-led clinical examination}

Up to $10 \%$ of breast cancers are not detected by mammography but can be felt during a clinical breast examination. The clinical examination involves taking a history together with performing a physical examination (visual and palpation) of the breast(s) and lymph-draining areas (axillae, supraclavicular regions) and sometimes abdominal examination. It also provides an opportunity for monitoring treatment side effects (with appropriate therapy given as required) and its outcomes, changing adjuvant therapies, and providing psychological reassurance and treatment, if necessary, for the patient. This is conducted by a trained clinician and/or a nurse practitioner, and may be performed by other appropriately trained health-care professionals. If there are causes for concern then additional investigations can be undertaken, for example mammography, ultrasound or MRI. There is broad agreement for frequent clinical examination, usually at 6-monthly intervals initially and then annually for up to 3-5 years. ${ }^{21,22,25}$

\section{Unstructured primary care follow-up (including primary care follow- up and self-examination)}

Primary care follow-up Clinical follow-up of breast cancer patients is routinely coordinated and conducted in the secondary care outpatient hospital setting. Patients, however, may detect IBTR or MCBC themselves during the interval between clinic visits. There have been suggestions that follow-up could be transferred to the primary care setting with the general practitioner (GP) performing a similar role to the specialist clinician during the clinical examination. ${ }^{29,41}$ If a GP detects a possible IBTR or MCBC, they should refer the patient back to the secondary care breast unit for further investigation. ${ }^{21,24}$ Primary care follow-up also encompasses the role of the GP in assessment and referral of patients presenting in primary care with self-reported symptoms. These patients may be under secondary care supervision and/or may not have been taking part in any follow-up regimen for a variety of reasons. This latter group of women is classified as undergoing unstructured primary care follow-up, as they commonly self present with symptoms in the primary care setting. ${ }^{41}$

Self-examination The breast self-examination is a physical examination performed by the patient to detect any changes in the breast. Special training is given to the patient in palpation techniques and to promote breast self-awareness. Training teaches women how to identify new lumps in the breast or the axilla that may be potentially malignant, and to identify any changes, such as skin rash or nipple discharge, on examining the breast surface. Patients are also taught to recognise additional symptoms, such as bone, chest or abdominal pain, difficulty breathing and persistent coughs or headaches, that may be indicative of recurrent breast cancer. It is recommended that patients should perform a self-examination every month in addition to scheduled imaging and clinical examinations. ${ }^{42}$

\section{Reference standard test}

Histopathological examination is the commonly agreed reference standard for diagnosing IBTR or MCBC, based on tissue obtained from either a biopsy or cytological examination of a fineneedle aspirate. Histopathological examination of tissue is usually undertaken where there is a suspicion of malignancy on a prior surveillance test. In contrast, there is no reference standard for ascertaining the true-negative and false-negative measures of a surveillance test for IBTR or MCBC, although this is usually ascertained by a negative result or a positive test result at subsequent testing after a period of follow-up has elapsed (e.g. 1 year - mammography interval, 2-3 years - MRI interval, etc.). A pragmatic reference standard is therefore the diagnosis of IBTR or MCBC up to 3 years after a surveillance test has been carried out. 


\section{Identification of important subgroups of patients}

It is known that certain groups of patients are at increased risk of IBTR. Those patients tend to be under 50 years of age at the time of diagnosis of their first breast cancer, have tumours classified histologically as being grade 3 cancers, have larger tumours, lymph node involvement and lymphovascular invasion (Table 3 gives an estimate of importance of these factors in IBTR). Pathologists in the UK report this information routinely and much of these data are held by the cancer registries. We focused on these risk factors as it is possible to stratify patients according to these variables and also give some indication on the hazard ratio (HR) of developing IBTR or MCBC when taking these factors into account. These variables have been used to estimate prognosis, as with the Nottingham Prognostic Index, for example, which is based on tumour size, grade and lymph node status. Adjuvant! Online also uses similar criteria to calculate the benefits of different types of treatment for each particular patient.

TABLE 3 Factors associated with ipsilateral breast tumour recurrence ${ }^{8}$

\begin{tabular}{|c|c|c|}
\hline Characteristics associated with primary tumour & 10-year LR rate (\%) & $95 \% \mathrm{Cl}$ \\
\hline \multicolumn{3}{|l|}{ Age at diagnosis (years) } \\
\hline$\leq 35$ & 35 & 17 to 53 \\
\hline $36-40$ & 9 & 2 to 17 \\
\hline $41-50$ & 9 & 7 to 13 \\
\hline $51-60$ & 11 & 7 to 16 \\
\hline$>60$ & 7 & 4 to 11 \\
\hline \multicolumn{3}{|l|}{ Tumour size (pathological) (cm) } \\
\hline$<1.0$ & 4 & 0 to 8 \\
\hline $1.2-2.0$ & 13 & 9 to 16 \\
\hline$\geq 2.1$ & 11 & 7 to 15 \\
\hline \multicolumn{3}{|l|}{ Nodal status (pathological) } \\
\hline Negative & 10 & 7 to 13 \\
\hline Positive & 11 & 7 to 15 \\
\hline \multicolumn{3}{|l|}{ Histological type } \\
\hline Infiltrating ductal & 9 & 7 to 12 \\
\hline Infiltrating ductal with EIC & 21 & 11 to 32 \\
\hline Infiltrating lobular & 10 & 2 to 18 \\
\hline \multicolumn{3}{|l|}{ Histological grade } \\
\hline Low/intermediate & 7 & 5 to 10 \\
\hline High & 15 & 10 to 19 \\
\hline \multicolumn{3}{|l|}{ Vascular invasion } \\
\hline No & 8 & 6 to 11 \\
\hline Yes & 15 & 10 to 20 \\
\hline
\end{tabular}

$\mathrm{Cl}$, confidence interval; EIC, extensive intraductal component; LR, local recurrence. 



\section{Chapter 2}

\section{Definition of the decision problem}

\section{Decision problem}

There is considerable debate about the role and optimal organisation of follow-up of patients after treatment for primary breast cancer. The number of differing guidelines that have been produced by various professional groups and policy-makers reflects this. ${ }^{21-23,25}$ There is broad agreement that surveillance should include frequent clinical examination, initially up to 3-5 years, with mammography starting 6 months after treatment, and then 1- to 2-yearly, for up to 10 years or indefinitely. The American Society of Clinical Oncology ${ }^{28}$ recommended in 2006 that surveillance should include annual mammography, although acknowledging that there is a lack of high level evidence to support decisions about the frequency and timing of mammographic surveillance.

Seven reviews, ${ }^{4,29,41,43-48}$ of varying methodological quality, have summarised the evidence of the effectiveness of differing surveillance regimens after treatment for breast cancer.

\section{Summary of existing systematic reviews}

$\mathrm{Lu}^{5}$ recently conducted a systematic review to determine the impact of early detection of isolated locoregional and contralateral recurrence on survival. Thirteen studies considered routine follow-up (regular mammography and physical examination) or intensive follow-up (with the inclusion of routine additional tests) aimed at early detection of recurrence. The authors defined early detection as detection by mammography instead of physical examination or in asymptomatic patients, as opposed to those presenting with symptoms or detected via physical examination either by clinician or by the patient. The authors reported better overall survival for recurrences detected by mammography or in asymptomatic patients, with an absolute reduction in mortality of $17-28 \%$ if all breast cancer recurrences are detected early. The authors had insufficient data to study the contribution of differing frequency of mammography (e.g. yearly mammography compared with 6-monthly mammography) or to analyse the effect of prognostic factors such as age, tumour stage and surgical treatment. Rojas and colleagues ${ }^{29}$ focused on the effectiveness of different surveillance policies for the detection of distant metastatic disease and concluded that follow-up programmes of regular physical examination and yearly mammography alone are as effective as more intensive approaches involving laboratory and radiological tests in terms of timeliness of detection of tumour recurrences, overall survival and quality of life. In addition, follow-up of patients performed by a trained general primary care practitioner is comparable to that of hospital-based secondary care specialists in terms of detection of tumour recurrence and quality of life. Collins and colleagues ${ }^{44}$ reported that patient survival and quality of life were not affected by intensity of follow-up or location of care, although the authors note that data were not sufficiently homogeneous to integrate statistically. Montgomery and colleagues ${ }^{46}$ systematically reviewed RCT evidence for alternative follow-up methods and concluded that the trials reviewed were not adequately powered to establish the safety of reducing or replacing hospital clinic visits.

Only Grunfeld and colleagues, ${ }^{47}$ Montgomery and colleagues ${ }^{46}$ and Barnsley and colleagues ${ }^{43}$ specifically considered the role of mammography in surveillance, and Barnsley and colleagues ${ }^{43}$ focused solely on surveillance mammography of the reconstructed breast, concluding that 
certain local recurrences are detectable by surveillance mammography but that there is a paucity of evidence.

De Bock and colleagues ${ }^{45}$ conducted a review of 18 uncontrolled, prospective and retrospective non-randomised studies of the effectiveness of routine follow-up visits and tests. The proportion of isolated locoregional recurrences diagnosed during routine visits or routine tests in asymptomatic patients was compared with the proportion of isolated locoregional recurrences in symptomatic patients. The authors were unable to assess whether recurrences, as defined by the study authors, were detected by physical examination or other tests, including mammography, or whether the detection of asymptomatic isolated recurrences had any influence on the potential for cure or quality of life of the patients.

Montgomery and colleagues, ${ }^{4}$ in a meta-analysis of 12 studies (11 non-randomised cohorts and one randomised trial), aimed to determine the relevant contributions of clinical examination, patient self-examination and mammography to the detection of potentially treatable locoregional recurrence and contralateral primary cancers. It was reported that $30-40 \%$ of treatable recurrences, as defined by the study authors, were detected by the patients self-examining. Prior to $2000,15 \%$ of relapses were mammographically detected compared with $46 \%$ detected by routine clinical examination. Post $2000,40 \%$ were mammographically detected and $15 \%$ were detected through routine clinical examination. Patients with ipsilateral recurrence detected by clinical examination appeared to do less well in terms of survival than those detected by selfexamination or mammography. The authors concluded that there was no evidence that clinical examination confers a survival advantage compared with other methods of detection and thus the need for clinical follow-up in detection of relapse is uncertain. The authors suggest that the temporal trend for increased detection by mammography is due to technical improvements in mammography and better quality assurance. Houssami and colleagues ${ }^{48}$ recently reported a similar trend for mammographic detection, although they propose that this effect is largely due to increased uptake of surveillance mammography by women rather than increased sensitivity of mammography.

Grunfeld and colleagues ${ }^{47}$ systematic review to define the effect of routine surveillance mammograms in detecting ipsilateral and contralateral cancer included 15 observational studies (published 1980-99). The 10 studies of ipsilateral recurrence showed that mammography detected the recurrence in between one-quarter and one-half of the women (range 8-50\%), with the remainder being found by the women themselves between follow-up or by a hospital practitioner during clinical examination. The majority of studies did not report outcomes. Where this was reported, the method of detection of ipsilateral tumour did not appear to influence survival, except in the study conducted by Voogd..$^{49}$ Here it was reported that patients had a better 5 -year survival if their tumour recurrence was detected mammographically. The nine studies of MCBC showed similar variation of detection methods. However, only one study reported outcome and this showed there was no difference in survival when comparing mammographic detection of the tumour with other methods. ${ }^{33}$ The authors did not conduct a meta-analysis and concluded that further research is needed to better define the optimum surveillance mammography regimen following breast cancer treatment.

Overall, from these reviews the optimal frequency and duration of surveillance mammography is not clear. Furthermore, more recent information is required on whether there is new evidence concerning the effectiveness of surveillance mammography. This is now extremely important because of the development and use of new and increasingly effective treatments for patients with breast cancer since 1990. These may offer women an improvement in survival if there is an early detection of either IBTR and/or MCBC. A further limitation of all these reviews is that they did not consider the costs and cost-effectiveness of surveillance mammography compared with 
other follow-up regimens despite there being methods for incorporating economic evidence into systematic reviews. ${ }^{50}$ This is important because the evidence to date has not been systematically reviewed to assess whether or not surveillance mammography is cost-effective in the follow-up of patients with breast cancer. As health-care resources are limited, they have to be used effectively for the benefit of society. Using limited resources to provide surveillance mammography will mean that we cannot use those resources to provide some other form of potentially beneficial care. For surveillance mammography to be considered efficient the benefits that it provides must be greater than the benefits we could have obtained from providing other care.

\section{Relevance of existing data to the decision problem}

The introduction of the NHSBSP in the UK in 1988, coupled with advances in the treatment of primary breast cancer around 1990, has led to improvements in overall survival, with the 5-year relative survival rate now 82\% in England and Wales. ${ }^{1}$ Although long-term follow-up would be the most useful to inform the decision problem, technological developments in all aspects of diagnosis, treatment and follow-up of women make those women with the longest follow-up data the less relevant and their outcomes less generalisable to current practice. Therefore, in consultation with expert members of our Advisory Group (see Appendix 1 for details of Advisory Group members) we decided to narrow our population of interest to consider data only for women treated for breast cancer from 1990 onwards. In addition, we decided not to include information from 1990 onwards relating to the Breast Screening Programme. This is because the screening population differs greatly to women who have been diagnosed and treated for breast cancer due to changes in breast density following treatment for primary breast cancer. We therefore felt that it would be inappropriate to use data from breast-screening studies of test performance in the systematic reviews (Chapters 4 and 5) to make assumptions regarding test performance for surveillance of the contralateral breast. We used parameter estimates for MRI test performance in the screening population in the economic evaluation in Chapter 7 of this report; however, it was felt that these would provide an indication of the relative value of a more costly but more effective test.

\section{Key issues}

The key issues to be addressed are:

- Can surveillance mammography improve overall survival for women treated for primary breast cancer?

- Does surveillance mammography improve detection of IBTR and MCBC?

- What is the incremental cost-effectiveness of surveillance mammography?

\section{Current care pathway}

There are a number of different surveillance mammography regimens in place in the UK for women following the treatment of primary breast cancer. In this section, we describe current pathways of care for women who are diagnosed, treated and followed up for breast cancer. In Chapter 3 we describe the potential alternative care pathways that we will attempt to consider and how we derived them.

However, when evaluating different surveillance regimens it is important to understand the sequence of care that a woman might receive after treatment for a primary breast cancer. Consideration can then turn to how different surveillance regimens may alter the care that a woman may receive over time.

\section{Care pathways for current practice}

We developed a care pathway for Aberdeen via discussions with experts in Aberdeen

(Figures 2-4). This care pathway starts with initial presentation and describes the sequence of 


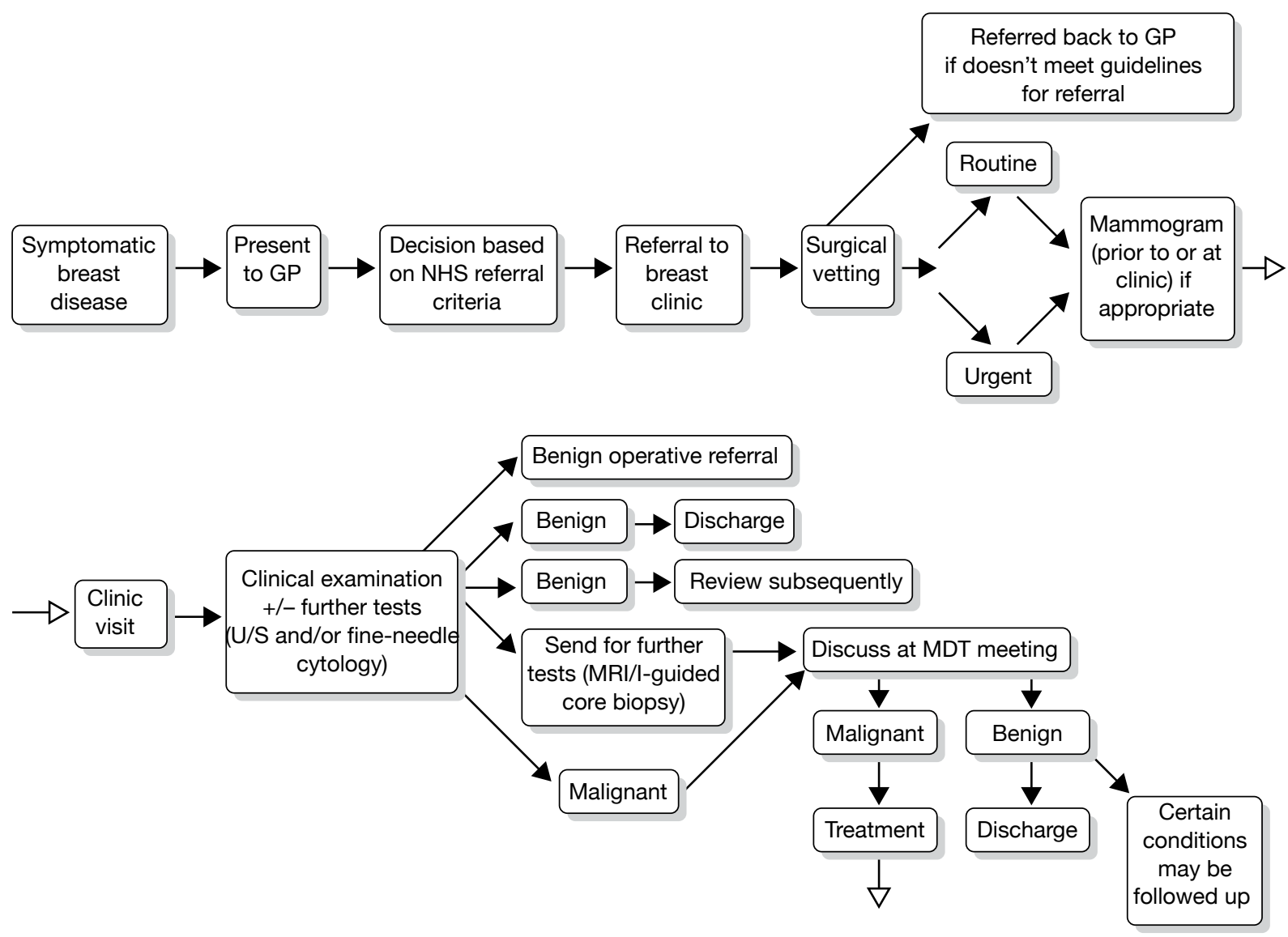

FIGURE 2 Current care pathway: Aberdeen: diagnosis of breast cancer - symptomatic presentation. I-guided core biopsy, image-guided core biopsy; MDT, multidisciplinary team; U/S, ultrasound.

events from diagnosis, through treatment and eventual longer-term follow-up. It is useful to consider the whole sequence of events, but of central importance to this project is how different surveillance regimens will potentially alter this care pathway.

\section{Development of alternative surveillance regimens}

Taking the care pathways above as a basis, we used the data reported in Chapter 3 to identify potentially relevant pathways for alternative surveillance regimens. We also considered whether or not there are any clinically attractive follow-up regimens that might not be used in practice but that we might consider useful to estimate their effectiveness, cost-effectiveness and feasibility in our subsequent modelling exercise. This consideration was partly informed by our discussions at the last Advisory Group Meeting, the literature and the results of our survey reported in Chapter 3.

When considering what surveillance regimens might be relevant, answers to the following questions were sought.

For mammographic surveillance:

- For which women is the issue of mammographic surveillance relevant?

- What mammography surveillance should be used?

- Does it vary between women and if so why would it vary?

- How often is it performed?

- Where does it take place? 


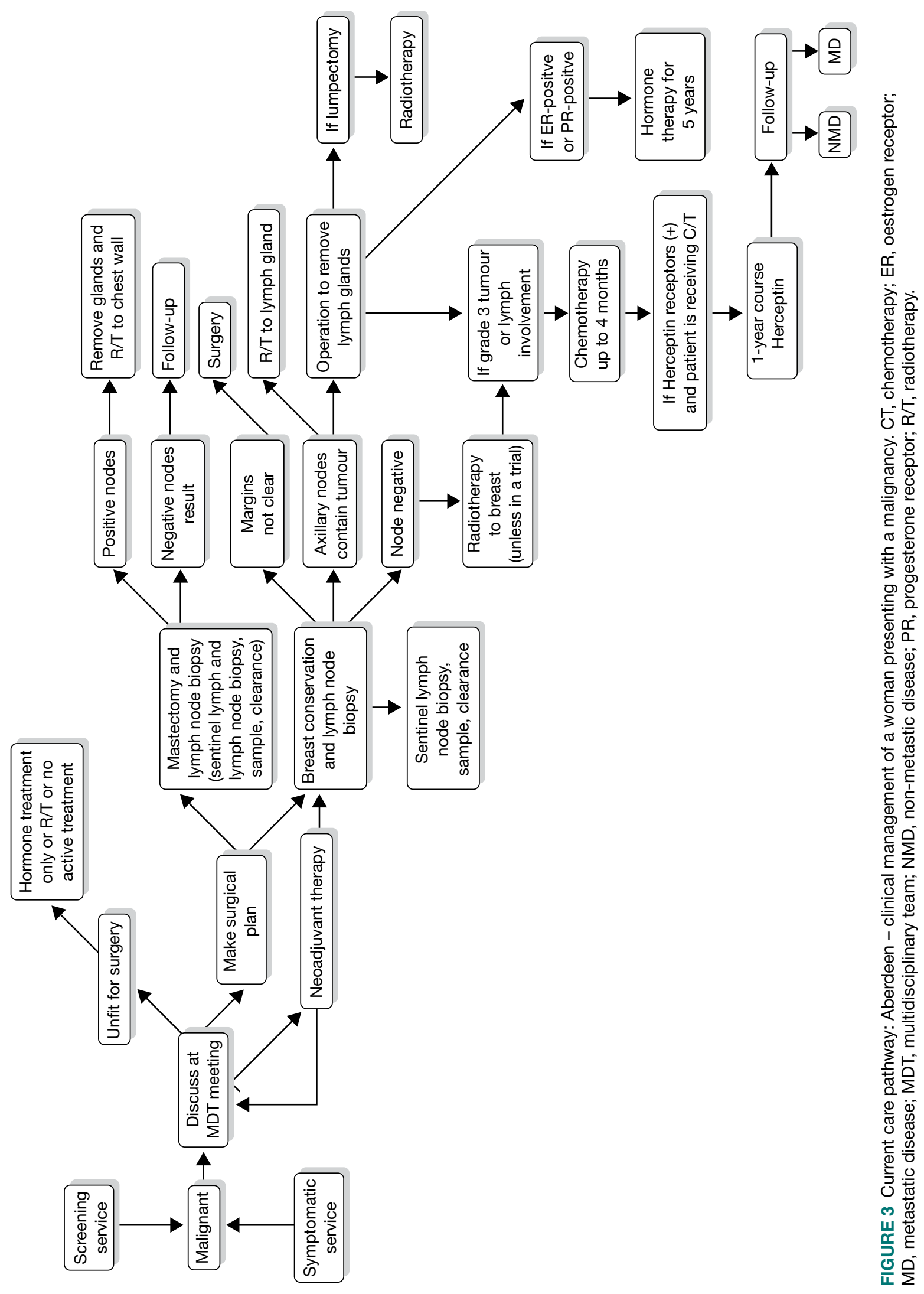




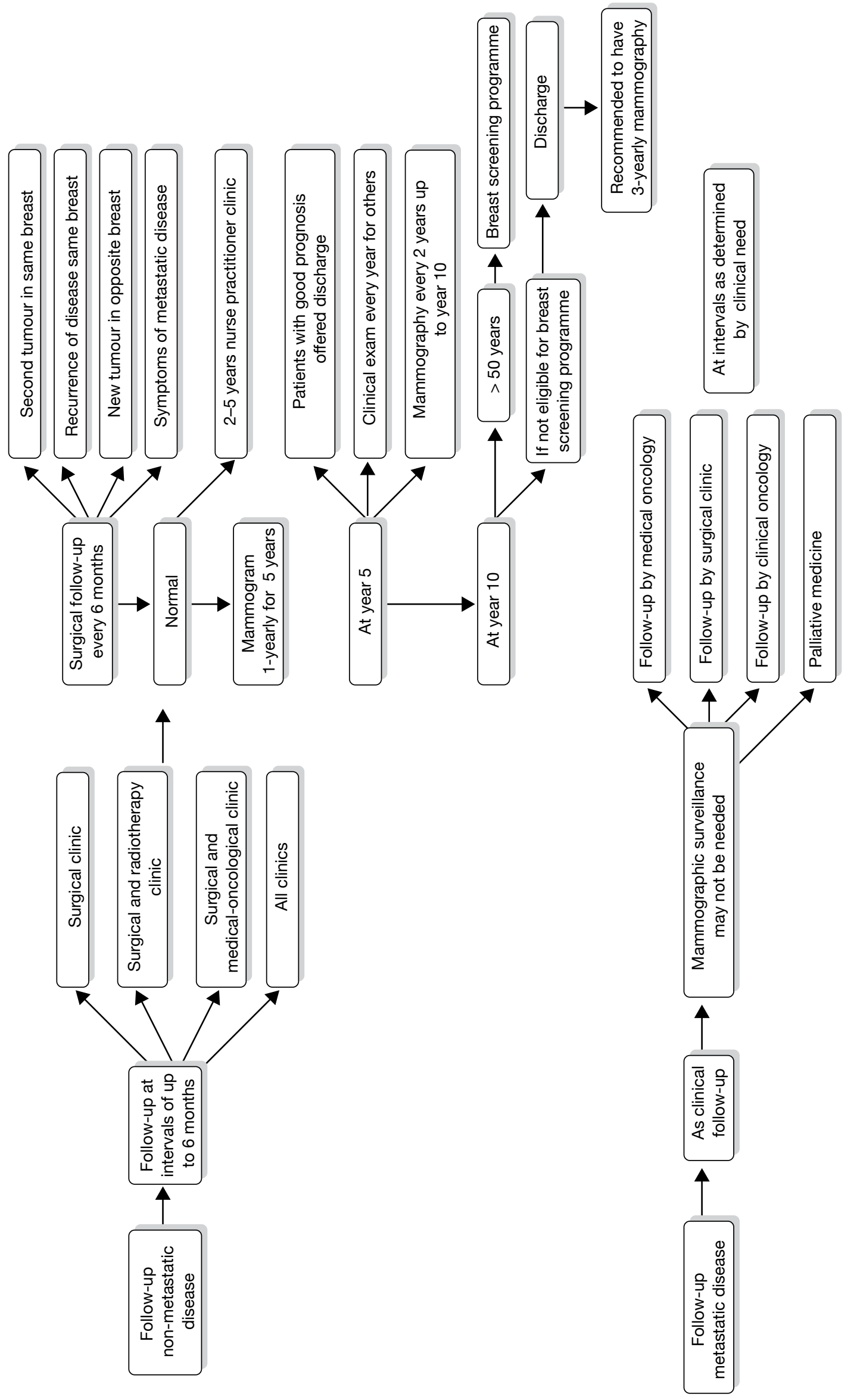

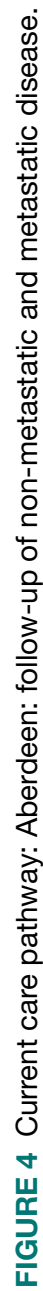


For clinical follow-up:

- What clinical follow-up is used?

- Does it vary between women and if so why?

- Where does it take place?

- How often?

For unstructured primary care follow-up:

- How might a diagnosis be made?

- At what point would these women enter the care pathway described in Figures 2-4?

More specifically, what factors might influence the choices made about mammographic surveillance and clinical follow-up?

Other factors to consider:

- age

- risk factors

- type of primary disease

- type of treatment.

We describe the care pathways developed from this process in Chapter 3. We then used the care pathways to structure data collection in the remainder of the project and to help to define the comparators for the economic evaluation. The structure of the model, which is detailed in Chapter 7, was based upon the current care pathway described above. The structure of the economic model was defined to directly address the aim of the review set out below.

\section{Aim of the review}

To examine the clinical effectiveness and cost-effectiveness of different surveillance mammography regimens after the treatment for primary breast cancer in the UK in primary and secondary care settings.

The aim is addressed through:

1. A survey of UK breast surgeons and radiologists to identify current practice and estimate resource consequences of the varying regimens.

2. Two discrete systematic reviews to determine the clinical effectiveness of differing surveillance mammography regimens after treatment for breast cancer on patient health outcomes and the diagnostic accuracy of surveillance mammography in the detection of IBTR and MCBC.

3. Statistical analysis of individual patient data and and economic modelling using the systematic review results, existing data sets and focused searches for specific data analysis to determine the effectiveness and cost-utility of differing surveillance regimens.

The specific objectives are to:

1. identify feasible management strategies for surveillance and follow-up of women after treatment for breast cancer in a UK context 
2. determine the effectiveness of differing surveillance and follow-up regimens after treatment for breast cancer

3. estimate the costs of differing regimens for surveillance and follow-up regimens after treatment for breast cancer

4. estimate the cost-effectiveness of differing regimens for surveillance and follow-up regimens after treatment for breast cancer

5. identify future research needs.

\section{Structure of the remainder of the report}

The remainder of the report is structured as follows: Chapter 3 describes a survey of current practice and feasible management strategies for surveillance in the UK; Chapter 4 assesses the clinical effectiveness of surveillance mammography, Chapter 5 assesses test performance of surveillance mammography compared with alternative diagnostic tests and combinations of diagnostic tests. Chapter 6 describes the analysis of individual patient data (Breast Cancer Registry database) and Chapter 7 assesses the cost-effectiveness of surveillance mammography. Chapter 8 is a discussion of the findings and Chapter 9 presents the review's conclusions, including implications for NHS service provision and for future research priorities. 


\section{Chapter 3}

\section{Identifying feasible UK management strategies for surveillance mammography and follow-up of women after treatment for primary breast cancer}

\section{Overview}

In the UK, there is considerable debate about the role and optimal organisation of a surveillance service following breast cancer treatment. The number of different guidelines produced by various professional groups and policy-makers reflects this. The guidelines include those of the British Association of Surgical Oncology (BASO) 2009, ${ }^{21}$ the NICE Guidance on Cancer Services $2002,{ }^{25}$ the NICE Early and Locally Advanced Breast Cancer: Full Guideline (CG80) 2009, ${ }^{24}$ the Royal College of Radiologists (RCR) Breast Group 2003, ${ }^{23}$ and the Scottish Intercollegiate Guidelines Network (SIGN) 2005.22 Typically, consensus approaches and literature reviews are the basis for these guidelines. We show the variations in key recommendations in Table 1 (see Chapter 1).

Donnelly and colleagues' survey in $2007^{26}$ of 256 specialists registered to Cancer Trials Units suggested that the majority (84\%) adhered to locally developed protocols for surveillance, and annual mammography was the most common surveillance interval for both invasive and non-invasive disease. Maxwell and colleagues' survey of breast-screening units taking part in the Sloane project ${ }^{27}$ concluded that there was wide variation in follow-up practice after surgery (breast conservation or mastectomy) for non-invasive breast cancer. The most common frequency of clinical follow-up was annual for up to 5 years and, for surveillance mammography, annual up to 10 years after breast conservation, and annual or biennial to 10 years after mastectomy.

The optimal frequency and duration for conducting surveillance mammography is unclear, thus leading to variation in follow-up protocols for breast cancer throughout the UK.

Aim

To describe the variations in current mammographic surveillance and follow-up practice in the $\mathrm{UK}$, in order to inform the development of the care pathways for the economic evaluation.

We addressed this aim via a survey of breast surgeons and radiologists (see Methods for the survey and Results of the survey) and consultation with our expert project Advisory Group. 


\section{Methods for the survey}

\section{Objectives}

- To identify current UK surveillance mammography regimens.

- To inform the feasible alternative surveillance regimens (care pathways) for:

- populating the economic model

- informing the systematic reviews

- providing context for the individual patient data analysis.

- To inform the choice of comparator surveillance regimens (inclusive or not of mammography) for the systematic review components of the wider project.

\section{Population and sample}

Our population was all health-care professionals providing surveillance of women following treatment for primary breast cancer. We chose our sample from this population to reflect those most likely to be currently involved with organising and/or undertaking surveillance mammography and to try to ensure UK-wide information. We sampled from the Association of Breast Surgery (ABS) at the BASO and the RCR Breast Group. Both the ABS and RCR Breast Group (to the best of their knowledge) held current and complete e-mail contacts for their members and these lists formed our sample. We sampled all full members (496) and associate members (73) of the ABS, and ordinary members (447 radiologists) and associate members (32 breast physicians) of the RCR Breast Group. We excluded the retired and overseas members of both organisations.

\section{Data collection, management and analysis Questionnaire development}

We developed a questionnaire for use in a web-based survey (Appendix 2). The development of the data items and questions were informed by recently published similar surveys ${ }^{26,27}$ and with the expert advice of the project Advisory Group. We tested the questionnaire items with members of the project Advisory Group and subsequently piloted the questionnaire with the first 100 members on the ABS and RCR e-mail distribution lists.

\section{Survey invitation and distribution}

We invited the ABS and RCR Breast Group members to participate in the survey via an e-mailbased web link to the online questionnaire sent to them by the ABS and RCR Breast Group membership administrators. All responses to the survey were anonymous. The Health Services Research Unit (HSRU) (University of Aberdeen) Programming Team created a web-based version of the survey questionnaire (see Appendix 2 for content details).

The ABS and RCR Breast Group membership administrators e-mailed the link to the questionnaire through a secure website using the e-mail distribution lists for each college. The website used for sending the questionnaire URL was password protected to prevent unauthorised access. Only persons with authorised login were able to access this website. Once logged on to this site, the administrators added the individual e-mail addresses into a text box for submission. An automated e-mail (see Appendix 3 for content details) was then sent to all submitted e-mail addresses, containing text inviting potential participants to consider participating in the survey and the URL link to the survey questionnaire itself. The administrators were able to view only the e-mail addresses they submitted and were not able to view e-mail addresses entered by any other administrator. 
Once potential participants received the e-mail they could then access the survey questionnaire by clicking the URL hyperlink contained in the e-mail. Participants could then complete and submit the questionnaire. Participants could view their own data only.

We could not target reminders to individuals who had not responded, as participants' responses were anonymous. One general reminder e-mail was sent via the ABS and the RCR Breast Group membership administrators to all potential participants after the initial mailing, asking those members who had not yet completed the survey to consider doing so (see Appendix 4 for content details).

We tested the survey invitation and distribution with members of the project Advisory Group and subsequently piloted with the first 100 members on the ABS and RCR e-mail distribution lists.

\section{Ethics and consent}

We consulted members of the North of Scotland Research Ethics Service to consider whether the survey required review by a Research Ethics Committee (REC). They had access to the aims, objectives and the content of the questionnaire and concluded that as they viewed it as service evaluation it did not require REC review.

The text of the accompanying e-mail and the survey form itself informed potential participants that their responses were anonymous and the information would be held in strictest confidence.

The project team did not have access to the e-mail addresses of individual ABS or RCR Breast Group members, nor did the questionnaire contain individual personal details. Submitted survey data were stored within a secure database on a secure server within HSRU. All researchers who could have access to the data had signed and were required to adhere to a 'Protecting Information Policy' and were expected to adhere to the principles of Good Clinical Practice (GCP).

\section{Data management}

The website used for sending the survey invitation e-mails automatically generated unique, study identifiers (study ID) for each completion of the survey without identifying individual e-mail addresses. Submitted survey data were stored within a secure database on a secure server within HSRU. We cleaned survey data and recoded text fields as required.

\section{Data analysis}

We defined the response rate to the survey as the number of participants who responded divided by the numbers of potential participants on the ABS and RCR Breast Group e-mail distribution lists. We summarised the responses to the survey by type and frequency (\%).

\section{Results of the survey}

We received responses from 17\% (183/1048) of those surveyed (see Appendix 5 for the number and percentage of respondents who completed each question). The majority of respondents (64\%) named their specialty as surgery and $35 \%$ as radiology (Table 4$)$. Fifty per cent of individuals practise in units that annually manage $>300$ women with newly diagnosed breast cancer, with $26 \%$ practising in units managing between 200 and 300 women with breast cancer (Table 4). Respondents were based in 105 NHS trusts across the UK (see Appendix 6 for the list 
TABLE 4 Respondent specialty and unit size

\begin{tabular}{lcc}
\hline & No. (\%) of respondents & \\
\cline { 2 - 3 } Specialty & $\boldsymbol{n}=\mathbf{1 8 3}$ & Percentage of $\boldsymbol{n}$ \\
\hline Surgery & 117 & 63.9 \\
Radiology & 64 & 35.0 \\
Other & 2 & 1.1 \\
No. of women with newly diagnosed breast cancer unit manages annually & \\
$<100$ & 5 & 2.7 \\
$100-199$ & 33 & 18.0 \\
$200-300$ & 48 & 26.2 \\
$>300$ & 92 & 50.3 \\
\hline
\end{tabular}

of NHS trusts) and one in full-time private practice. The number of respondents per trust was just one for 60 trusts (57\%); in 26 trusts (25\%) there were two respondents and in 12 trusts (11\%) there were three respondents; the maximum number of respondents per trust was six, with only seven trusts with four or more respondents per trust.

\section{Initiation, frequency and duration of surveillance mammography}

The large majority of respondents initiate surveillance mammography at 12 months post surgery for women who have had breast-conserving surgery (BCS) $(87 \%)$ and for women who have had a mastectomy (79\%) (Table 5).

Responses ranged from six to 24 months post surgery, with the next most frequent being 24 months (13\%) post mastectomy.

Table 6 shows the respondents standard practice frequencies or intervals of surveillance mammography for women after BCS and after mastectomy. Annual surveillance mammography was the most commonly reported standard frequency of surveillance mammography for women after BCS or after mastectomy ( $72 \%$ and $53 \%$, respectively), with biennial mammography the next most frequently reported ( $12 \%$ and $30 \%$, respectively). The 'other' responses varied but can generally be described as showing a pattern of increasing mammography surveillance intervals with increasing time since surgery, for example surveillance mammography at 1,2,3,5,7 and 10 years.

Fourteen per cent (26/180) of respondents said that they varied their standard surveillance mammography practice (initiation of surveillance mammography post surgery or frequency of mammography) for women who had BCS. They varied their practice according to the surveyprompted criteria of in situ tumours $(n=14)$; size of tumour $(n=5)$; grade of tumour $(n=1)$; lymphovascular invasion $(n=4)$; age $(n=9)$; absence of radiotherapy $(n=3)$; combinations of these $(n=2)$; or other criteria $(n=10)$. Other criteria included 'close margins', comorbidities, family history and genes predisposing to breast cancer.

Similarly, 13\% (23/180) varied their standard surveillance mammography practice (initiation of or frequency) for women who had a mastectomy, by factors such as age, cancer grade and size, comorbidities, family history, genes predisposing to breast cancer or 'high-risk' groups.

In addition, a further 16/183 (9\%) commented in text within the questionnaire that they vary their standard initiation and frequency surveillance practices, trying to take into account factors 
TABLE 5 Initiation of surveillance mammography

\begin{tabular}{|c|c|c|c|c|}
\hline \multirow[b]{3}{*}{ No. of months post surgery } & \multicolumn{2}{|c|}{ After BCS } & \multicolumn{2}{|c|}{ After mastectomy } \\
\hline & \multicolumn{4}{|c|}{ Number and percentage of respondents } \\
\hline & $n=181$ & Percentage of $n$ & $n=159$ & Percentage of $n$ \\
\hline 6 & 7 & 4 & 2 & 1 \\
\hline 8 & 1 & 1 & 0 & 0 \\
\hline 9 & 2 & 1 & 1 & 1 \\
\hline 11 & 3 & 2 & 1 & 1 \\
\hline 12 & 157 & 87 & 125 & 79 \\
\hline 18 & 7 & 4 & 9 & 6 \\
\hline 24 & 4 & 2 & 21 & 13 \\
\hline
\end{tabular}

TABLE 6 Frequency of surveillance mammography

\begin{tabular}{|c|c|c|c|c|}
\hline \multirow{3}{*}{$\begin{array}{l}\text { Frequency of surveillance } \\
\text { mammography }\end{array}$} & \multicolumn{2}{|c|}{ After BCS } & \multicolumn{2}{|c|}{ After mastectomy } \\
\hline & \multicolumn{4}{|c|}{ Number and percentage of respondents } \\
\hline & $n=182$ & Percentage of $n$ & $n=181$ & Percentage of $n$ \\
\hline Annually & 131 & 72 & 96 & 53 \\
\hline Every 18 months & 11 & 6 & 16 & 9 \\
\hline Every 2 years & 22 & 12 & 54 & 30 \\
\hline Every 3 years & 0 & 0 & 5 & 3 \\
\hline Annually to 5 years then every 2 years & 9 & 5 & 6 & 3 \\
\hline Other & 9 & 5 & 4 & 2 \\
\hline
\end{tabular}

such as age, density of breast tissue, comorbidities, family history, genes predisposing to breast cancer or 'high-risk' groups.

When asked about through which service they arrange their surveillance mammography, the majority responded [175/182 (96\%)] that it is through their symptomatic breast service, although seven $(4 \%)$ said through the NHSBSP.

The majority $(75 \%, 136 / 182)$ indicated they discharge women from surveillance mammography and they do this most frequently 10 years after surgery (Table 7$)$. The majority $(82 \%, 148 / 180)$ do discharge from clinical follow-up and most frequently at 5 years (Table 7). Just over half (55\%, 98/179) responded that they discharge women to the NHSBSP (Table 8) if eligible.

However, around 28\% (47/167) of those who discharge from follow-up (clinical and/ or mammographic follow-up) commented that they vary the duration of surveillance mammography and this is influenced by the age of the women $(24 \%)$, or by other factors including family history, genes predisposing to breast cancer, and tumour characteristics.

Combining our respondents' standard initiation, frequency and duration of surveillance mammography resulted in 54 differing surveillance regimens for women after BCS and 56 for women following mastectomy (Appendices 7 and 8, respectively). Fifty-one per cent (79/154) of respondents follow one of four surveillance regimens for women after BCS. The most commonly followed regimens are to initiate surveillance mammography at 12 months after surgery and conduct annual surveillance mammography with indefinite duration (12\%, 19/154); discharge 
TABLE 7 Duration of surveillance mammography: discharge from surveillance mammography and from clinical follow-up

\begin{tabular}{|c|c|c|c|c|}
\hline & \multicolumn{4}{|c|}{ Discharge from: } \\
\hline & \multicolumn{2}{|c|}{ Surveillance mammography } & \multicolumn{2}{|c|}{ Clinical follow-up } \\
\hline & \multicolumn{4}{|c|}{ Number and percentage of respondents } \\
\hline & $n=182$ & Percentage of $n$ & $n=180$ & Percentage of $n$ \\
\hline No & 46 & 25 & 32 & 18 \\
\hline Yes & 136 & 75 & 148 & 82 \\
\hline \multicolumn{5}{|c|}{ How many years post surgery } \\
\hline & $n=121$ & Percentage of $n$ & $n=130$ & Percentage of $n$ \\
\hline 1 & 0 & 0 & 3 & 2 \\
\hline 2 & 0 & 0 & 2 & 1 \\
\hline 3 & 1 & 1 & 9 & 7 \\
\hline 5 & 42 & 35 & 85 & 65 \\
\hline 6 & 3 & 2 & 1 & 1 \\
\hline 8 & 1 & 1 & 1 & 1 \\
\hline 9 & 6 & 5 & 0 & 0 \\
\hline 10 & 67 & 55 & 29 & 22 \\
\hline 11 & 1 & 1 & 0 & 0 \\
\hline
\end{tabular}

TABLE 8 Duration of surveillance mammography: discharge to NHSBSP

\begin{tabular}{lll}
\hline Discharge to NHSBSP & \multicolumn{1}{l}{ Number and percentage of respondents } \\
\hline \multirow{2}{*}{ No } & $n=179$ & Percentage of $n$ \\
Yes & 81 & 45 \\
At age (years) & 98 & \\
& & Percentage of $\boldsymbol{n}$ \\
50 & $n=39$ & 67 \\
70 & 26 & 8 \\
75 & 3 & 2 \\
Or years of follow-up & 1 & \\
& & Percentage of $\boldsymbol{n}$ \\
5 & $n=39$ & 5 \\
9 & 2 & 2 \\
10 & 1 & 2 \\
\hline
\end{tabular}

from both clinical and mammographic surveillance at 5 years $(14 \%, 22 / 154)$ or 10 years $(12 \%, 18 / 154)$ after surgery; or discharge from mammographic surveillance at 10 years and clinical follow-up at 5 years $(13 \%, 20 / 154)$. Similarly, after mastectomy the most commonly followed regimens are to initiate surveillance mammography at 12 months after surgery and conduct annual surveillance mammography, with indefinite duration (7\%, 10/136); or discharge from both clinical and mammographic surveillance at 5 years $(10 \%, 13 / 136)$; or 10 years 
$(11 \%, 15 / 136)$ after surgery; or discharge from clinical follow-up at 5 years with continued mammographic surveillance until 10 years $(8 \%, 11 / 136)$.

\section{Ideal practice}

Twenty-nine per cent (53/180) of respondents suggested that their ideal surveillance mammographic practice differs from their current practice and that this is influenced by the factors listed in Box 1.

The most common ideal frequency of surveillance mammography given was annually for women who had undergone BCS $(80 \%, 85 / 106)$ or mastectomy $(57 \%, 61 / 106)$ (Table 9$)$. These ideal frequencies of surveillance did not differ from their current practice for the majority of respondents, for women after BCS $(80 \%, 84 / 106)$ or for women after mastectomy $(69 \%, 73 / 106)$. However, three respondents suggested that their ideal practice would be to arrange surveillance mammography through the screening units, as they are set up to manage the appointment and recall system.

\section{Care pathway development}

We used the results of our survey, other relevant surveys ${ }^{26,27}$ and advice from the clinical experts involved in this study to identify alternative follow-up regimens. This work formed the basis for the care pathways, which, in turn, provided the basis of the economic model that is described in Chapter 7. The economic model aims to determine the effectiveness and cost-utility of differing surveillance regimens.

BOX 1 Factors that influence why ideal practice differs from current practice

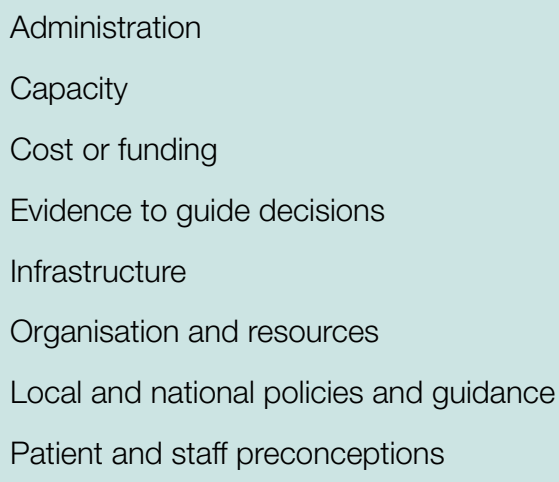

TABLE 9 Ideal frequency of surveillance mammography

\begin{tabular}{|c|c|c|c|c|}
\hline \multirow[b]{3}{*}{ Ideal frequency of surveillance mammography } & \multicolumn{2}{|c|}{ After BCS } & \multicolumn{2}{|c|}{ After mastectomy } \\
\hline & \multicolumn{4}{|c|}{ Number and percentage of respondents } \\
\hline & $n=106$ & Percentage of $n$ & $n=106$ & Percentage of $n$ \\
\hline Annually & 85 & 80 & 61 & 57 \\
\hline Every 18 months & 5 & 5 & 18 & 17 \\
\hline Every 2 years & 9 & 8 & 21 & 20 \\
\hline Every 3 years & 0 & 0 & 0 & 0 \\
\hline Annually to 5 years, then every 2 years & 0 & 0 & 0 & 0 \\
\hline Other & 7 & 7 & 6 & 6 \\
\hline
\end{tabular}




\section{Development of alternative surveillance regimens}

Taking the care pathways described in Table 1 (see Chapter 1) and Figures 1-3 (see Chapter 2) as a basis, we used the results of the surveys to identify if there were any clinically attractive follow-up regimens that might be used in practice or are currently not used in practice. This consideration was partly informed by our discussions during project Advisory Group Meetings (which were informed by the literature and the results of the survey described above).

When considering what surveillance regimens might be relevant, answers to the following questions were sought, which can be briefly summarised as: who would be under surveillance/ follow-up; what technology would be used (e.g. mammography, clinical examination, etc.); where would the surveillance be performed; who would perform the surveillance; and what would be the frequency of surveillance/follow-up (the questions used are described in more detail in Appendix 9).

\section{Surveillance regimens}

Figure 5 describes the potential alternative care pathways developed from this process. For example, individuals can be followed up using surveillance mammography at different intervals, for example once yearly, every 18 months, every 24 months or every 36 months. Alternatively, individuals could present to a GP with a problem, i.e. discover a lump. Individuals who present to their GP with a lump would be given a clinical examination by the GP. Current practice in the economic model is assumed to be once-yearly mammograms.

The alternative surveillance regimens in the economic model vary by screening interval and/ or screening technology. For example, alternative mammographic surveillance regimens to the standard regimen would be for mammography to take place at less or more frequent intervals, for example every 18 months or every 24 months. Although not explicitly noted, one important option to consider as an alternative would be surveillance mammography organised through the NHSBSP.

Alternative primary care regimens would be for an individual to attend a GP surgery and receive a clinical examination followed by a mammogram if there was a suspicious finding on the clinical examination. Other potential surveillance regimens include the use of alternative technologies, i.e. MRI or ultrasound in replacement of mammography. For all regimens other than GP opportunistic finding, individuals are invited to attend screening at different intervals, for example once yearly. An individual can either choose to attend or not attend the screening programme. Given that this is a higher-risk group (women who have previously had breast cancer), and, also for simplicity of modelling, we are assuming that all individuals who are invited for screening do attend. In the intervals in which screening does not occur, we assumed that individuals could still be diagnosed with breast cancer through their GP.

Following further discussion within the project Advisory Group, these options were further reduced to three regimens that we felt broadly represented the most relevant comparators. This decision was also informed by knowledge of the preliminary findings of the research reported in Chapters 4-6. These regimens were: mammographic surveillance with and without clinical follow-up organised either through secondary care or through the screening service (this option embraces regimens 1, 3 and 6 in Figure 5) and the identification of cancer following referral from primary care following the identification of a suspicious lump on self-examination (regimen 7). 


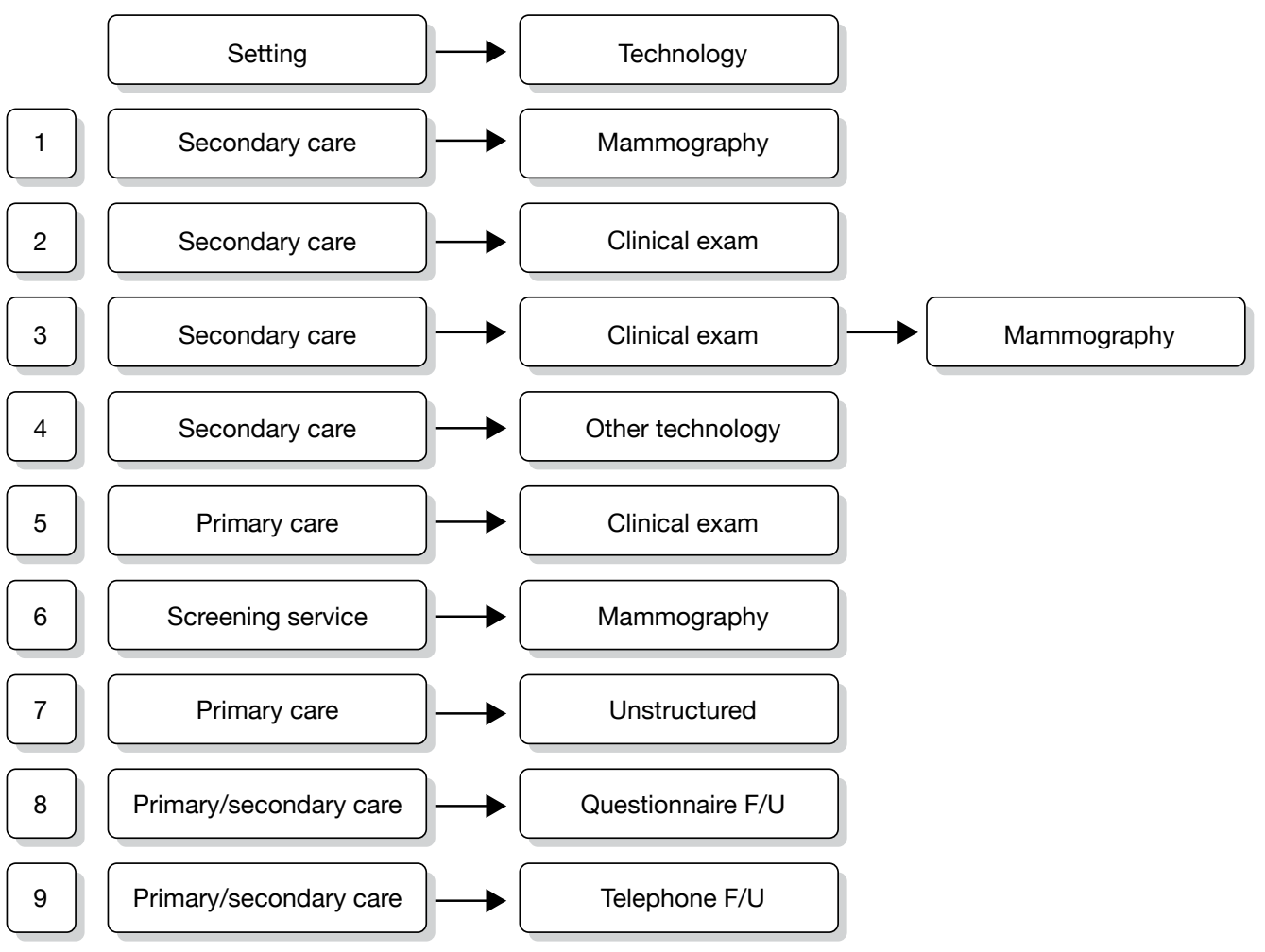

FIGURE 5 Potential alternative care pathways. F/U, follow-up.

\section{Discussion}

The findings of our survey suggest that although common patterns in surveillance mammography practice exist there is considerable variation in the way surveillance is organised.

The most commonly followed regimens for surveillance of women after BCS are to initiate surveillance mammography at 12 months after surgery and conduct annual surveillance mammography with indefinite duration, discharge from both clinical and mammographic surveillance at 5 or 10 years after surgery, or discharge from mammographic surveillance at 10 years and clinical follow-up at 5 years. Similarly, after mastectomy the most commonly followed regimens are to initiate surveillance mammography at 12 months after surgery and conduct annual surveillance mammography, with indefinite duration or discharge from both clinical and mammographic surveillance at 5 or 10 years after surgery or discharge from clinical follow-up at 5 years with continued mammographic surveillance until 10 years. However, respondents varied greatly in the combinations of start, frequency, duration and discharge from surveillance mammography.

Our findings reflect the varying guidance given by the various professional organisations with an interest in surveillance after breast cancer (see Table 1, Chapter 1) in combination with 'local circumstances' of the respondents, and are generally consistent with those of recent surveys addressing similar questions. ${ }^{26,27}$ 
Our response rate was not high in comparison with what might be expected for a postal questionnaire in medicine or in comparison with the recent surveys. ${ }^{26,27}$ We discuss further the implications of this in Chapter 8.

The plethora of different ways of conducting surveillance mammography in practice mean that there are potentially a large number of care pathways that are worthy of further consideration. It would not be possible to consider the merits of each of these options especially when there are additional methods of follow-up that do not include mammography. Therefore, using the results of the survey and expert opinion provided by the project group, a relatively small number of follow-up methods were defined, which differed by the diagnostic technology used. It is possible, however, that this list excludes potentially important pathways. 


\section{Chapter 4}

\section{Systematic review of clinical effectiveness of surveillance mammography after treatment for primary breast cancer}

\section{Overview}

As Chapter 3 illustrated, although there is some similarity between surveillance regimens used in practice there is also considerable variation. It is unclear which of these alternative methods of organising a service represent the most effective and efficient regimen. Previous systematic reviews ${ }^{4,29,43-47}$ have been unable to clarify the optimal frequency and duration of surveillance mammography.

We conducted a systematic review to determine the clinical effectiveness of differing surveillance mammography regimens after treatment for primary breast cancer in detecting IBTR and MCBC. The results of this review were used to inform the economic evaluation described later in this report (Chapter 7).

\section{Methods}

\section{Inclusion and exclusion criteria}

Types of study

Evaluations of any screening or follow-up regimens are prone to lead and length time bias, i.e. detecting tumours early in the course of disease without influencing survival (lead time bias) or detecting slower growing tumours that would not reduce length of life or underdetecting rapidly progressive tumours that have a poorer survival prognosis (length time bias). As RCTs are the optimal method of ameliorating for these biases, we included RCTs of differing surveillance mammography regimens and alternative breast cancer follow-up regimens in this evaluation, i.e. where women are randomly allocated to one surveillance regimen or another. In addition, we included non-randomised comparative studies of differing surveillance mammography regimens and alternative breast cancer follow-up regimens. We also included prospective and retrospective cohort studies of differing surveillance mammography regimens or alternative breast cancer follow-up regimens containing 100 or more participants and where participants were followed up for at least a median time of 5 years.

All studies were required to include participants with a diagnosis of primary breast cancer from 1990 or later. In consultation with expert members of our Advisory Group, we chose 1990 as the start date for our inclusion criteria to avoid the introduction of bias for overall survival outcomes. Improvements in overall survival around 1990 are attributable to the introduction of the NHSBSP in the UK, coupled with advances in the treatment of primary breast cancer and changes to the ways in which patients with breast cancer are managed. The 5-year relative survival rate is now $80 \%$ in England and Wales. ${ }^{1}$ 


\section{Types of participants}

Women previously treated for primary breast cancer without detectable metastatic disease at their initial presentation for treatment.

\section{Types of interventions}

Routine surveillance mammography for detecting IBTR and/or MCBC.

\section{Comparator(s)}

- No surveillance mammography.

- Differences in frequency of surveillance mammography regimens.

- Alternative follow-up regimens, for example, including, but not limited to, breast-care physician-led clinical examination.

\section{Types of outcome measures}

We included studies that reported any of the following outcomes:

- Primary outcome - overall survival. We defined overall survival as the amount of time that a woman remains free from all-cause mortality following primary treatment. We also considered mortality rates to optimise data collection of outcome measures relating to the effectiveness of a surveillance regimen.

- Secondary outcomes - IBTR and/or MCBC event rate. We considered event rates and time to detection of IBTR and/or MCBC. We did not include tumour recurrences outside of the breast, for example regional (axillary) recurrence, or distant metastatic disease, as we would not expect surveillance mammography to detect these types of disease recurrence.

- Quality of life - We considered any measure of health-related quality of life attributable to surveillance mammography or other follow-up regimen.

- Harms of mammography (adverse events) - Undergoing mammography entails exposure to radiation, which carries a small risk of developing cancer. Mammography can also be an uncomfortable procedure that can cause pain in some women. Following mammography, further investigations may be required, which subsequently turn out to be normal.

- Uptake of mammography - We considered data relating to numbers of women attending for planned surveillance mammography.

- Economic data - Reporting of any data on costs to the NHS and patients including resource use for the actual interventions and resource use consequent to implementation of that intervention, which may occur up to the end of the woman's life, were planned. This would have included the costs arising as a consequence of both true-positives and false-negatives and the costs of investigating incidental findings. Estimates of cost-effectiveness would have been taken as they were defined in the study and incremental cost per life-year or qualityadjusted life-year (QALY) were calculated if they could be estimated from the data reported.

In addition to our study eligibility criteria, economic evaluations had to meet the following inclusion criteria:

- compare a surveillance mammography regimen with an alternative follow-up regimen

- relate information on costs to effectiveness or have reported both cost and effectiveness data (in the same or separate study reports) for the reviewer to relate data on costs to effectiveness

- include detailed methods for estimating costs and effects data in the methods section of the study report and report the results in a results section. 


\section{Search methods for identification of studies}

We conducted an extensive electronic search to identify reports of relevant published and ongoing studies, as well as any grey literature. The search strategies were designed to be highly sensitive, including both appropriate subject heading and text word terms to capture the concepts of surveillance mammography or other follow-up strategies and the study designs meeting the inclusion criteria for this review. The searches were restricted to full text papers published from 1990 onwards without language restriction. We searched the following databases for primary studies: MEDLINE, MEDLINE In-Process, EMBASE, BIOSIS, Science Citation Index (SCI), CANCERLIT and Cochrane Central Register of Controlled Trials (CENTRAL). We also searched the Cochrane Database of Systematic Reviews (CDSR), Database of Abstracts of Reviews of Effects (DARE) and the HTA Database for reports of evidence syntheses. Reports of ongoing and recently completed trials were sought from the Current Controlled Trials (CCT), Clinical Trials, WHO International Clinical Trials Registry Platform (ICTRP), NCI Clinical Trials Database, National Research Register (NRR) Archive, and NIHR Portfolio Database. Appendix 10 gives full details of the search strategies used.

In addition, we searched relevant websites, namely those of the National Cancer Institute, National Comprehensive Cancer Network, CancerWEB, Breast Cancer Surveillance Consortium, and the National Library for Health, as well as relevant professional organisations including the American Society of Clinical Oncology, the American Society of Breast Disease, the American College of Radiology, and the European Society for Medical Oncology. We scanned reference lists of all included studies for additional reports.

\section{Data extraction strategy}

One reviewer (from GM, CR, RT and SZ) screened the titles and available abstracts of all reports identified by the search strategy for relevance to the inclusion criteria. One reviewer independently assessed full text copies of all potentially relevant studies to assess them for inclusion (from CB, CR and SZ). An economist reviewer examined reports relating to an economic evaluation or cost analysis.

We conducted a 10\% check of inclusion assessment for all potentially relevant studies (RT). We resolved any disagreements by consensus or arbitration by a third party. A list of the included and excluded studies is given in Appendices 11 and 12, respectively.

One reviewer (from CR and SZ) independently extracted details of study design, participant characteristics, description of the intervention and outcome data (see Appendix 13 for data extraction form). A second reviewer independently validated the data extraction (from CR and SZ). In the event of any uncertainty, a third reviewer advised on and validated the data extraction $(\mathrm{CB})$.

\section{Quality assessment strategy}

We assessed the methodological quality of non-randomised studies using a quality assessment tool (Appendix 14) adapted from the Review Body for Interventional Procedures (ReBIP) checklist for quality assessment of non-randomised studies (comparative studies and case series). We included additional items (questions 18 and 19) to assess whether study authors attempt to correct for lead and length time bias in their analyses. Each of the items was checked as 'yes', 'no' or 'unclear'. Each item was worded so that a rating of 'yes' was the optimal rating of methodological quality, except item 14 regarding differential dropout rate/participants lost 
to follow-up. We planned to use an adapted version of the Cochrane Collaboration's tool for assessing risk of bias ${ }^{51}$ for assessing the methodological quality of individual RCTs. For the quality assessment of any economic evaluations, we planned to use the NHS Economic Evaluation Database Handbook. ${ }^{52}$

\section{Data analysis}

We planned statistical synthesis of results (using meta-analysis) of included studies directly comparing different surveillance mammography regimens or comparing surveillance mammography with an alternative follow-up regimen, for RCTs and non-randomised comparative studies, favouring intention to treat over per-protocol results for our analysis. We planned to derive a pooled HR for time-to-event outcomes (e.g. recurrence and survival). For data on harms of mammography, adverse events and quality of life we planned to use standardised mean difference to combine quality-of-life scores depending on the suitability of study data. We did not plan quantitative synthesis of economic outcomes data.

\section{Results}

\section{Number and type of studies included and excluded}

Figure 6 shows the number of potentially relevant studies identified by the search strategy with details of the number meeting the inclusion criteria and the number that were ineligible by exclusion criteria.

From the literature searches 2849 titles and abstracts were identified, 422 of which were selected for full text assessment. We excluded 414 reports, which did not meet the inclusion criteria for this review, of which we retained 114 reports to assess eligibility for inclusion in the systematic review of test performance (see Chapter 5 of this report). Seven reports were unavailable. We list the bibliographic details of the eight studies that met the inclusion criteria in Appendix 11. We list the bibliographic details of the excluded studies, plus the reasons for exclusion in Appendix 12.

\section{Characteristics of the included studies}

Eight studies met our inclusion criteria. ${ }^{53-60}$ Appendix 15 provides full details of the characteristics of the included studies. Six studies ${ }^{53-58}$ were retrospective cohort studies. Two studies ${ }^{59,60}$ were prospective cohort studies. We did not identify any RCTs or economic evaluation studies meeting our inclusion criteria. Three studies ${ }^{53-55}$ were conducted in the UK, whereas three en-60 $^{58}$ were conducted in the USA. The study by Paszat and colleagues ${ }^{56}$ was conducted in Canada, and the study by Yau and colleagues ${ }^{57}$ was conducted in China (Hong Kong). Table 10 provides a summary of overall characteristics for the included studies. Table 11 provides further details of the characteristics of individual studies.

As we lacked RCT studies directly comparing different surveillance mammography regimens we could not conduct a formal meta-analysis of these studies. In addition, none of the included studies compared surveillance regimens; therefore, it was not possible to undertake meta-analysis assessing surveillance regimens, including investigation of subgroup factors. Consequently, we decided to present a narrative synthesis of results for this review.

Four studies reported data for surveillance mammography only $y^{56,58-60}$ and did not report details of any additional follow-up given to participants. Three studies considered surveillance mammography combined with clinical examination. ${ }^{53-55}$ The study conducted by Yau and colleagues ${ }^{57}$ considered surveillance mammography, combined with clinical and ultrasound examination of the breasts, conducted at the clinician's discretion. Six studies did not include 


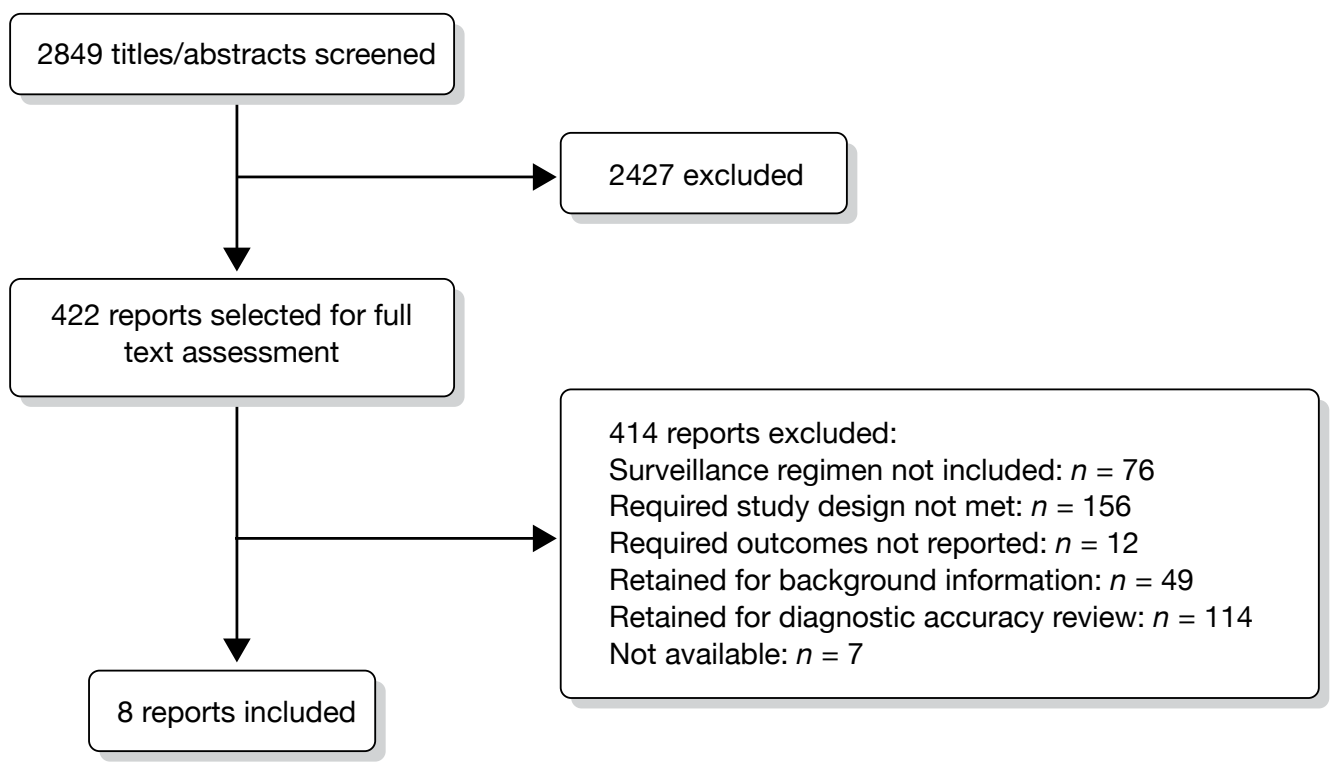

FIGURE 6 Flow chart of the number of potentially relevant reports of identified studies and the number subsequently included and excluded from the effectiveness review.

TABLE 10 Summary of the total number of patients and number of studies by study type, primary surgical treatment and type of surveillance test for the included effectiveness studies

\begin{tabular}{lrl}
\hline Characteristic & No. of patients & No. of studies \\
\hline Patients & 7337 & 8 \\
Enrolled & 3775 & \\
Analysed & & \\
Type of study & 637 & 2 \\
Prospective cohort & 3138 & 6 \\
Retrospective cohort & & \\
Type of primary surgical treatment & 1190 & 5 \\
Mastectomy & 2571 & 8 \\
Breast conserving & 3 & 1 \\
No surgery & 9 & 2 \\
Information missing & 2 & 1 \\
Reported as 'other' & & \\
Type of surveillance test & 2350 & 4 \\
XRM & 918 & 3 \\
Combined XRM, CE & 507 & 1 \\
Combined XRM, CE, US & & \\
\hline
\end{tabular}

CE, clinical examination; US, ultrasound; XRM, X-ray mammography.

a comparator regimen ${ }^{53-55,57,58,60}$ Lash and colleagues ${ }^{59}$ comparatively analysed the number of consecutive years of guideline surveillance (defined as annual history, annual clinical examination and annual surveillance mammography) received by women in their cohort with women who had not received consecutive years of guideline surveillance (i.e. women who 


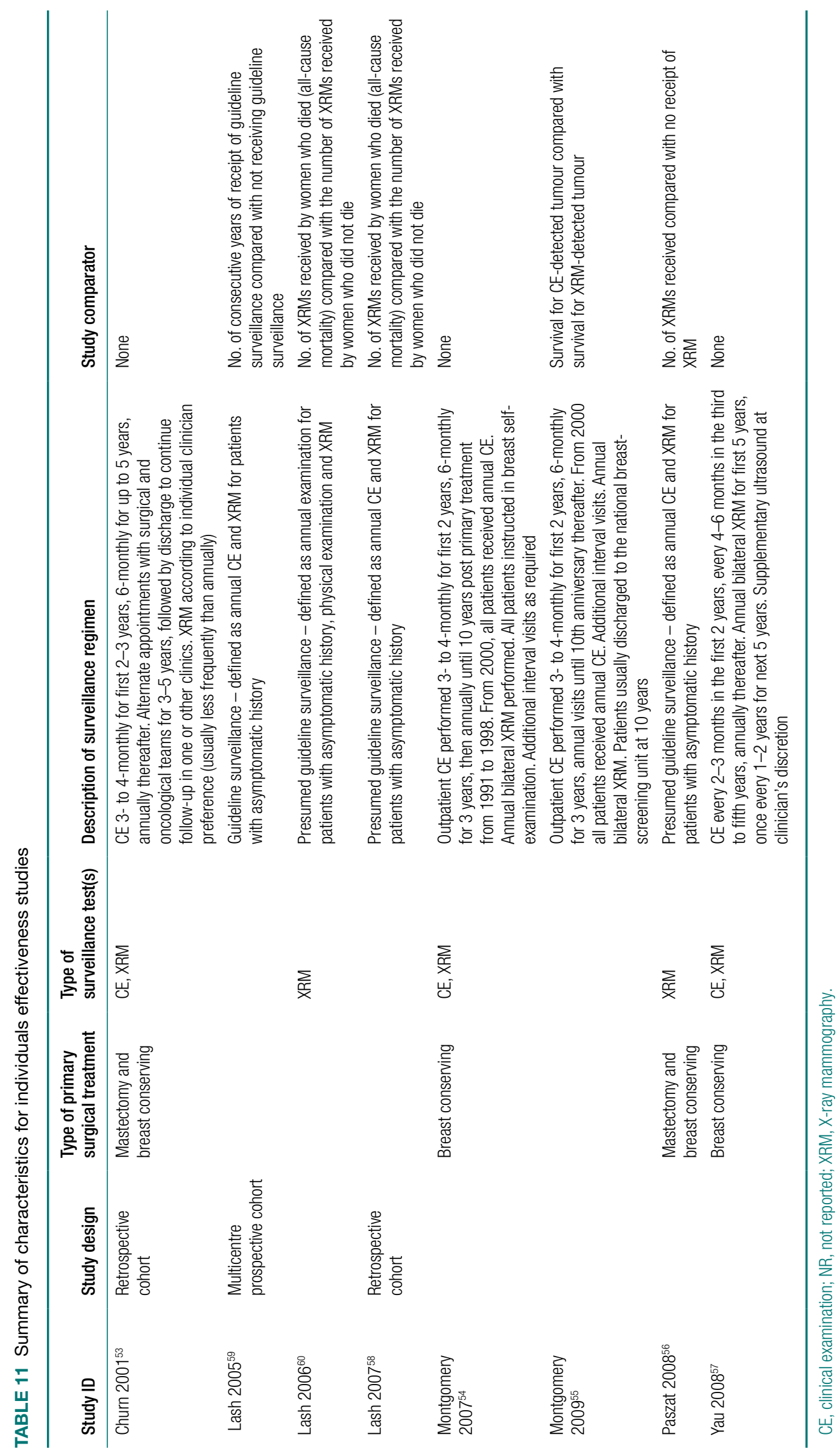


had missed one or more annual surveillance appointment for unspecified reasons). Paszat and

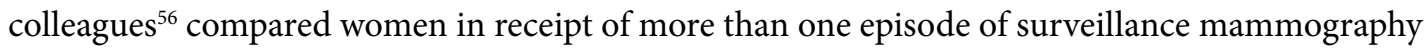
within their cohort with women who did not receive surveillance mammography.

Overall, the eight studies enrolled 7337 patients. After exclusions, due to eligibility or participant dropout, the studies included 3775 patients in their analyses. The studies included 1626 mastectomy patients and 4864 breast conservation surgery patients all treated for primary breast cancer and without detectable metastatic disease. Five studies ${ }^{53,56,58-60}$ reported participant age details, with 444 participants aged $<50$ years and 4168 participants aged 50 years or older. Two studies ${ }^{54,55}$ reported mean ages, 56 and 58 years, and age ranges, $24-91$ and $28-91$ years, respectively. Yau and colleagues ${ }^{57}$ reported a median age of 46 years and range $25-90$ years. The earliest report was published in $2001^{53}$ and the latest in $2009 .{ }^{55}$ The earliest date of primary treatment reported was $1990^{58}$ and the latest was $2003 .{ }^{57}$ Follow-up ranged from 2.4 months to 15 years.

\section{Quality of the included studies}

The results of the quality assessment for the individual studies are shown in Appendix 16. Figure 7 summarises the quality assessment of the included studies.

Four studies ${ }^{56,58-60}$ were considered to include samples that were unrepresentative of those women who we considered eligible for surveillance mammography (i.e. all women treated for primary breast cancer). Three of these studies, conducted by the same lead author, ${ }^{58-60}$ included only women aged over 65 years. It is unclear whether the cohorts of women included in the studies conducted in $2005^{59}$ and by Lash and colleagues ${ }^{58}$ included the same women and hence whether or not the studies had an overlap of patients. This older age group represents only a proportion of what we consider the eligible population. A pragmatic surveillance mammography regimen could include women of all ages, with those over the age of 50 years possibly benefiting from eligibility for inclusion in the national NHSBSP. In addition, we also considered that the sample included in the study conducted by Paszat and colleague ${ }^{56}$ was unrepresentative. In this study, the authors randomly selected two samples from their previously identified population of women treated for

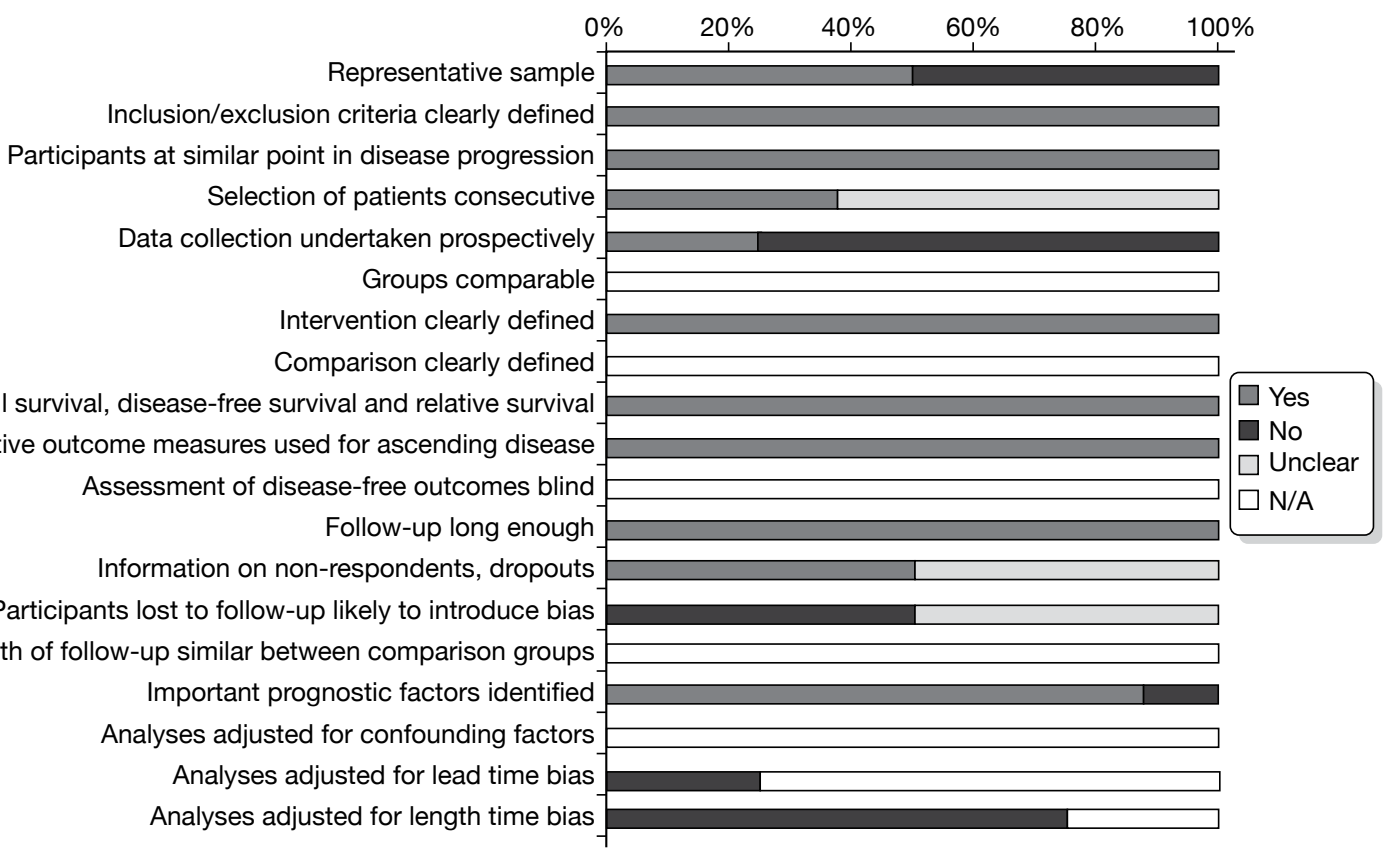

FIGURE 7 Summary of quality assessment of the included effectiveness studies. 
primary breast cancer. The authors drew one random sample from women without any further breast surgery after their initial primary treatment. A larger second sample was drawn from the same group who had undergone further breast surgery 6 or more months after their initial treatment. The second sampling fraction was larger $(0.237$ compared with 0.055$)$ to increase the probability of including women with an episode of IBTR and/or MCBC. We therefore considered that this second group of women with subsequent breast surgery were over-represented in comparison to our study population.

It was unclear for five reports ${ }^{53,55-58}$ whether participants were a consecutively treated series of patients, whereas in three studies ${ }^{54,59,60}$ patient selection was consecutive. All studies clearly described their inclusion/exclusion criteria and the intervention, and avoided disease progression bias by enrolling participants who were all at a similar point in their condition, as opposed to including patients at mixed levels of advancement in their cancer. All studies used objective outcome measures for ascertaining overall and disease-free survival, and mortality and IBTR/ MCBC event rates. All studies included a median follow-up time of at least 5 years, which we considered adequate for detecting important outcome effects. We did not consider lead and length time bias to be applicable to the studies by Montgomery and colleagues ${ }^{55}$ and Yau and colleagues $^{57}$ as neither study reported mortality data. The remaining studies did not adjust for lead or length time bias in their analyses.

Four studies ${ }^{54,55,59,60}$ provided information on non-respondents and dropouts (e.g. incomplete case note data, losses to follow-up, etc.) and all were judged to have avoided attrition bias, defined as bias introduced by high or differential dropout of patients. This information was judged as being unclear in the remaining studies. ${ }^{53,56-58}$ All but one study ${ }^{54}$ identified important prognostic factors for patients' overall survival or mortality.

Two studies ${ }^{59,60}$ undertook prospective data collection, whereas the remainder undertook retrospective data review. ${ }^{53-58}$

\section{Assessment of effectiveness}

Two studies provided data on overall survival, cause-specific survival and the annual hazard rate of ipsilateral locoregional and new contralateral relapse in graph form. ${ }^{54,55}$ Those remaining reports eligible for inclusion in this review reported numbers of overall deaths, ${ }^{53,56,58-60}$ deaths due to breast cancer ${ }^{53,56,58-60}$ and IBTR and/or MCBC events only. ${ }^{53,56-58}$

\section{Overall survival and mortality}

Table 12 summarises the data for mortality as reported by the individual studies. For breast cancer-specific mortality, four studies ${ }^{53,56,59,60}$ did not report whether deaths were due to primary breast cancer, breast cancer recurrence or a second primary cancer. Two studies ${ }^{54,58}$ reported deaths due to recurrent cancer or a second primary cancer.

Churn and colleagues ${ }^{53}$ reported 112 deaths from all causes from an analysis of 612 women.

Lash and colleagues ${ }^{59}$ reported 63 deaths from all causes from an analysis of 303 women. Of these deaths, 27 were due to breast cancer. For all-cause mortality the authors reported a crude conditional odds ratio (OR) of 0.62 [95\% confidence interval (CI) 0.48 to 0.80 ] for the effect of a consecutive year of mammographic surveillance with clinical examination compared with lack of consecutive years of surveillance (i.e. women did not receive surveillance at each consecutive year). The age-adjusted OR was reported as 0.66 (95\% CI 0.51 to 0.86 ). For breast cancer-specific mortality an OR of 0.7 (95\% CI 0.52 to 1.1 ) was reported. For all but breast cancer mortality, the OR was 0.69 (95\% CI 0.48 to 0.99 ). 
TABLE 12 Numbers of deaths due to all causes, breast cancer and IBTR/MCBC, as reported by the included effectiveness studies

\begin{tabular}{|c|c|c|c|c|c|c|c|c|}
\hline \multirow[b]{3}{*}{ Study ID } & \multirow{3}{*}{$\begin{array}{l}\text { No. of patients } \\
\text { studied }\end{array}$} & \multicolumn{6}{|c|}{ No. of deaths due to: } & \multirow[b]{3}{*}{ Follow-up time for whole cohort } \\
\hline & & \multicolumn{2}{|c|}{$\begin{array}{l}\text { All-cause } \\
\text { mortality }\end{array}$} & \multicolumn{2}{|c|}{$\begin{array}{l}\text { Breast cancer- } \\
\text { specific mortality }\end{array}$} & \multicolumn{2}{|c|}{$\begin{array}{l}\text { IBTR/MCBC } \\
\text { specific }\end{array}$} & \\
\hline & & $n / N$ & $\%$ & $n / N$ & $\%$ & $n / N$ & $\%$ & \\
\hline Churn $2001^{53}$ & 612 & $112 / 612$ & 18 & & NR & & NR & 5 years \\
\hline Lash $2005^{59}$ & 303 & 63/303 & 21 & $27 / 303$ & 9 & & NR & $\begin{array}{l}\text { Median } 7.4 \text { years (range } 10 \text { months to } \\
9.5 \text { years) }\end{array}$ \\
\hline Lash $2006^{60}$ & 334 & $32 / 334$ & 10 & $13 / 334$ & 4 & & NR & $5-8$ years \\
\hline Lash $2007^{58}$ & 812 & $391 / 812$ & 48 & $178 / 812$ & 22 & 130 & & 5 years \\
\hline $\begin{array}{l}\text { Montgomery } \\
2007^{54}\end{array}$ & 110 & & NR & & NR & $24 / 110$ & 22 & Median 10 years (range $1.5-15$ years) \\
\hline $\begin{array}{l}\text { Montgomery } \\
2009^{55}\end{array}$ & 196 & & NR & & NR & & NR & $\begin{array}{l}\text { Median } 5.9 \text { years (range } 4 \text { months to } \\
10.5 \text { years) }\end{array}$ \\
\hline Paszat $2008^{56}$ & 901 & $512 / 901$ & 57 & $374 / 901$ & 42 & & NR & 15 years \\
\hline Yau $2008^{57}$ & 507 & & NR & & NR & & $\mathrm{NR}$ & $\begin{array}{l}\text { Median } 5.9 \text { years (range } 0.2-13 \\
\text { years) }\end{array}$ \\
\hline
\end{tabular}

Lash and colleagues ${ }^{60}$ reported 32 deaths from all causes from an analysis of 334 women. Of these deaths, 13 were due to breast cancer. The authors reported a decline in mortality rate with increasing number of surveillance mammograms received ( $p$ for trend $=0.007$ ). The crude OR for an additional surveillance mammogram compared with no surveillance mammography was 0.79 (95\% CI 0.56 to 1.1). After adjusting for age, primary tumour stage and receipt of adjuvant tamoxifen treatment, this figure was reported as 0.77 (95\% CI 0.53 to 1.1). The authors stated that they had insufficient data to analyse the effect of surveillance mammography on breast cancerspecific mortality rates.

Lash and colleagues ${ }^{58}$ reported 391 deaths from all causes and 178 deaths due to breast cancer within the first 5 years of follow-up. Of those women dying from breast cancer, 130 had experienced breast cancer recurrence. Thirteen of these recurrences were local and five of the women received a surveillance mammogram in the preceding year (38\%). In addition, 101 women had a recurrence and did not die from breast cancer. Of these women 24 had a local recurrence, of whom 19 received a surveillance mammogram in the preceding year (79\%).

Montgomery and colleagues ${ }^{54}$ reported a series of 1312 patients who were treated by breast conservation surgery. There were 116 patients with an isolated local recurrence and from this group 110 were available for analysis (attrition due to either incomplete case notes or patients lost to follow-up). In this group of 110 patients, two patients had ipsilateral breast relapse diagnosed incidentally during breast reshaping procedures, both of whom subsequently died. Thirty-five of the remaining 108 women died. Of these 35 deaths, 12 had IBTR recurrence, 11 had ipsilateral axillary recurrence, three had recurrence in both the breast and axilla, eight had MCBC and one patient had bilateral breast (IBTR and MCBC) recurrence.

Paszat and colleagues ${ }^{56}$ reported 512 deaths, of which 374 were breast cancer specific, from an analysed study population of 901 patients. The authors analysed the association between exposure to one or more episodes of surveillance mammography and the risk of death. Adjusting for age, tumour stage, surgery, and adjuvant chemotherapy and adjuvant tamoxifen treatment, the authors reported a HR of 0.28 (95\% CI 0.22 to 0.37 ) for breast cancer-specific death. Of those 
women with IBTR the authors reported an unadjusted HR of 0.36 (95\% CI 0.13 to 1.00 ). The authors reported an unadjusted figure of 0.86 (95\% CI 0.20 to 3.77) for women with MCBC.

The remaining studies ${ }^{55,57}$ did not report survival data.

\section{IBTR and/or MCBC event rate}

Table 13 summarises the data for IBTR and/or MCBC event rates as reported by the individual studies. Two studies did not report details of IBTR and/or MCBC recurrence and are therefore excluded from this table..$^{59,60}$ The remaining six studies ${ }^{53-58}$ did not report raw data time points for when IBTR and/or MCBC events occurred but reported numbers of individual IBTR and/or MCBC events experienced within the individual cohort. We have not included any recurrences occurring outside the breast in Table 13, for example regional (axillary) recurrences or distant metastatic recurrences. Details of these recurrence events are available in Appendix 15 if reported by individual study authors.

Lash and colleagues ${ }^{58}$ reported details for locoregional recurrence. We were able to identify 37 cases of IBTR recurrence in this study but were unable to identify numbers with MCBC only. Montgomery and colleagues ${ }^{55}$ reported one case of $\mathrm{MCBC}$ in the first year following treatment of the primary breast cancer, with a case of IBTR and one case of MCBC within the first 3 years; with a further three IBTR and four MCBC cases occuring 3 years after treatment.

\section{Method of detection}

Five studies ${ }^{53-57}$ reported details of the mode of IBTR/MCBC detection where this was known. The study conducted by Montgomery and colleagues ${ }^{55}$ reports subsequent deaths where known. Table 14 summarises this data. The remaining studies ${ }^{58-60}$ did not report data regarding the method of detection.

We did not find any studies meeting our inclusion criteria that reported data on quality of life, harms of mammography, uptake of mammography or economic data.

TABLE 13 Number of patients diagnosed with IBTR/MCBC as reported by the included effectiveness studies

\begin{tabular}{|c|c|c|c|c|c|c|c|c|}
\hline \multirow[b]{3}{*}{ Study ID } & \multirow{3}{*}{$\begin{array}{l}\text { No. of patients } \\
\text { studied }\end{array}$} & \multicolumn{6}{|c|}{ Nos. of patients diagnosed with: } & \multirow[b]{3}{*}{ Follow-up time for whole cohort } \\
\hline & & \multicolumn{2}{|l|}{ IBTR } & \multicolumn{2}{|l|}{ MCBC } & \multicolumn{2}{|c|}{$\begin{array}{l}\text { Bilateral IBTR } \\
\text { and MCBC }\end{array}$} & \\
\hline & & $n / N$ & $\%$ & $n / N$ & $\%$ & $n / N$ & $\%$ & \\
\hline Churn $2001^{153}$ & 612 & $25 / 505$ & 5 & $9 / 104$ & 9 & 0 & 0 & 5 years \\
\hline Lash $2007^{58}$ & 812 & $37 / 851$ & $4^{a}$ & & NR & & NR & 5 years \\
\hline $\begin{array}{l}\text { Montgomery } \\
2007^{54}\end{array}$ & 110 & $48 / 110$ & 44 & $35 / 110$ & 32 & $2 / 110$ & 2 & Median 10 years (range $1.5-15$ years) \\
\hline $\begin{array}{l}\text { Montgomery } \\
2009^{55}\end{array}$ & 196 & $4 / 196$ & 2 & $6 / 196$ & 3 & 0 & 0 & $\begin{array}{l}\text { Median } 5.9 \text { years (range } 4 \text { months to } \\
10.5 \text { years) }\end{array}$ \\
\hline Paszat $2008^{56}$ & 901 & $84 / 584$ & 14 & $49 / 317$ & 16 & 0 & 0 & 15 years \\
\hline Yau $2008^{57}$ & 507 & $23 / 507$ & 5 & $13 / 507$ & 3 & 0 & 0 & $\begin{array}{l}\text { Median } 5.9 \text { years (range } 0.2-13 \\
\text { years) }\end{array}$ \\
\hline
\end{tabular}

a Overall, 851 BCS patients and 973 mastectomy patients were enrolled in this cohort and 812 patients were analysed. Breakdown of surgery type included in the analysis is not given. 


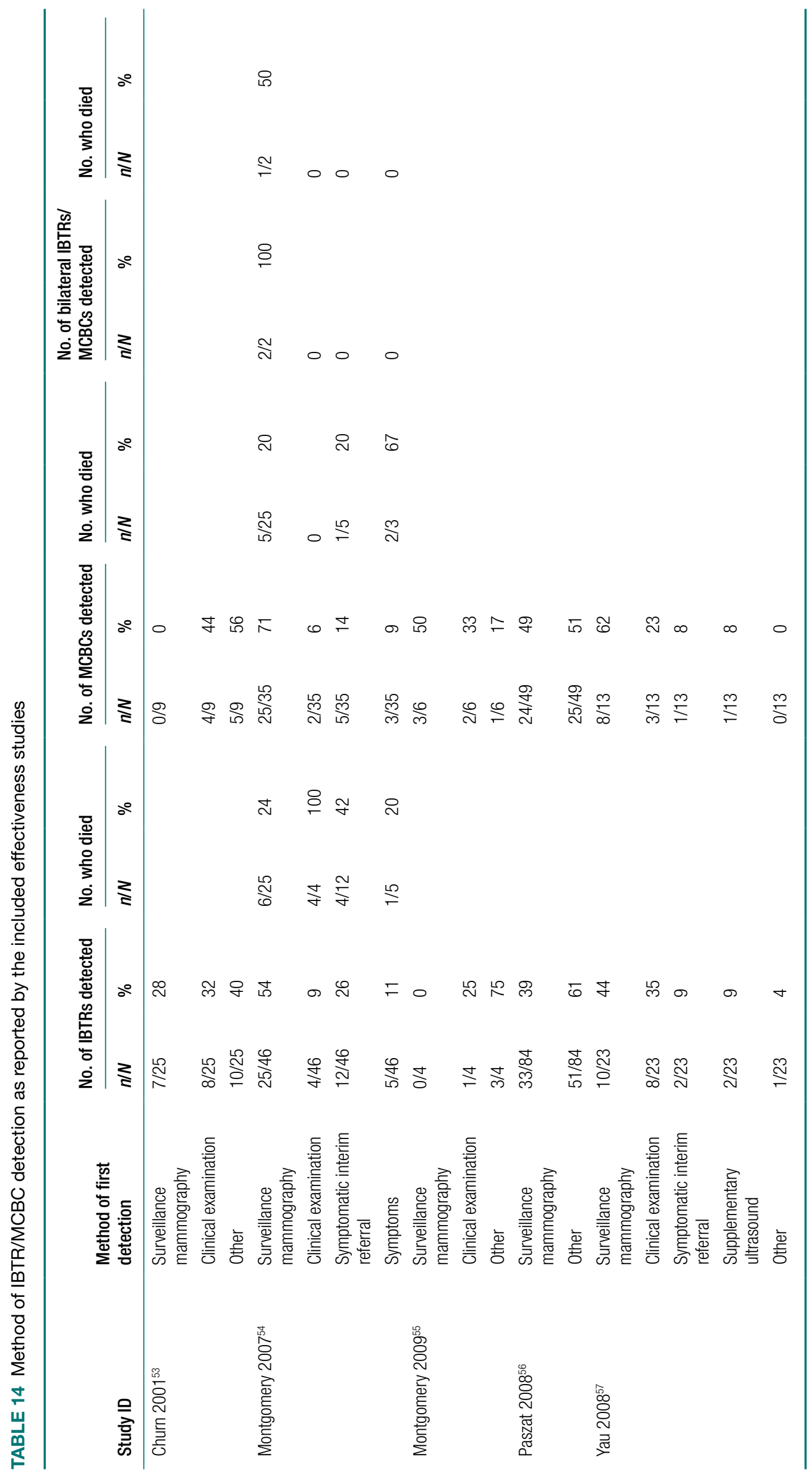




\section{Summary}

We conducted a systematic review to determine the clinical effectiveness of differing surveillance mammography regimens in detecting IBTR and MCBC in women who were previously diagnosed and treated for primary breast cancer without metastatic disease from 1990 onwards. We aimed to include RCT and non-randomised comparative studies of differing surveillance mammography and alternative breast cancer follow-up regimens. We also included prospective and retrospective cohort studies of differing surveillance mammography regimens or alternative breast cancer follow-up regimens containing 100 or more participants, where participants were followed up for at least a median time of 5 years. The methods used for conducting this review are systematic, explicit and comprehensive, and our inclusion criteria are both clinically relevant and scientifically rigorous.

Despite extensive searching, we identified only eight studies that met our inclusion criteria, all of which were cohort studies. Furthermore, none of the regimens described in these studies was identical. For this review, we sought data from the included studies on eight outcomes but for six of these outcomes no data were available. Only two studies ${ }^{54,55}$ provided time-to-event data for overall and disease-free survival in graph form. In terms of numbers of patients alive and dead, only five studies contributed data towards this outcome. ${ }^{53,54,56,59,60}$ Only six studies reported data on numbers of patients experiencing IBTR and/or MCBC events. ${ }^{53-58}$ The paucity of the underlying evidence base mirrors the findings of previous reviews ${ }^{4,29,43-47}$ described earlier. Nevertheless, a number of conclusions may be drawn.

Firstly, there is a suggestion from two studies that surveillance mammography offers a protective effect against death from all causes. ${ }^{59,60}$ The data reported by Paszat and colleagues ${ }^{56}$ also suggest a protective effect of surveillance mammography for breast cancer-specific death. Furthermore, the data reported by Montgomery and colleagues ${ }^{54}$ suggest a protective effect against death for surveillance mammography-detected IBTR when compared with clinical examination, although numbers of patients and events are small. In addition, the Montgomery data suggest that a high proportion of patients $(20 \%)$ were recalled in between routine appointments, in most cases to report a recurrence detected by the patients themselves. As the remaining studies did not include a comparator for surveillance mammography, neither in frequency, nor with an alternative regimen, it is not possible to make recommendations for the effectiveness of any surveillance regimen beyond these three studies.

Few studies met our eligibility criteria and those included in this review are prone to lead and length time bias. The limited and variable nature of the data provided by the included studies precluded formal quantitative synthesis, thus only a narrative synthesis of evidence was possible. The predefined inclusion criteria for this review did not include measures of psychological effects, which are known to be important to the patient experience of surveillance. We further discuss key findings and limitations of this review in Chapter 8. 


\section{Chapter 5}

\section{Systematic review of test performance of surveillance mammography and comparator tests in detecting IBTR/MCBC}

\section{Overview}

\section{Introduction}

The aim of this review was to determine the diagnostic accuracy of surveillance mammography for detecting IBTR and MCBC in women who were previously treated for primary breast cancer.

- Primary objective To determine the performance of surveillance mammography, alone or in combination with other tests, in detecting IBTR and/or MCBC in women undergoing routine surveillance.

- Secondary objective To determine the performance of surveillance mammography, alone or in combination with other tests, compared with alternative tests, alone or in combination, in detecting IBTR and/or MCBC in women with a prior diagnostic test result indicating suspicion of IBTR and/or MCBC (referred to subsequently as non-routine surveillance).

\section{Methods}

\section{Inclusion and exclusion criteria}

Types of study

The following types of study were considered for inclusion:

- RCTs in which women are randomised to receive either the index or comparator test(s) and all receive the reference standard test.

- Diagnostic consecutive cohort studies in which both index and comparator tests are evaluated against the reference standard test in the same women within the study (headto-head design).

- Indirect (between-study) comparisons by comparing cohort studies where women received either the index test, or the comparator test, or a combination of tests with the reference standard test, and where at least 100 participants were included in the analysis of test performance. This type of study design is less reliable than direct studies, as differences in diagnostic accuracy are susceptible to confounding factors between studies. ${ }^{61}$

We did not consider case reports and studies investigating technical aspects of a test.

\section{Types of participants}

The types of participants considered were women previously treated for primary breast cancer without detectable metastatic disease at initial presentation. 


\section{Index test}

The index test was surveillance mammography.

\section{Comparator test(s)}

The following comparator tests were considered:

- ultrasound

- magnetic resonance imaging

- specialist-led clinical examination:

- hospital clinician led

- hospital nurse led

- unstructured primary care follow-up (here defined as absence of formal routine secondary care follow-up, which may, or may not, involve mammography)

- GP-led follow-up:

- self-presentation

- self-examination.

We planned to consider comparisons of both individual and combinations of tests. We considered test performance in all settings.

\section{Target condition}

The target condition was IBTR and MCBC following treatment for primary breast cancer. For the purposes of diagnostic test accuracy, we made no distinction between detection of ipsilateral recurrent breast tumours and ipsilateral second primary tumours.

\section{Reference standard}

Histopathological examination is the commonly agreed reference standard for diagnosing recurrent breast cancer based on tissue obtained by biopsy, usually as a needle biopsy under local anaesthesia or in some patients following a surgical biopsy. In addition, in some breast clinics, fine-needle aspiration cytology (with or without immediate reporting) is available to allow a diagnosis of recurrence. This is usually confirmed by biopsy. Cytological and/or histopathological examination is undertaken if suspicion of malignancy is raised on a surveillance test. In contrast, there is no reference standard for ascertaining the true-negative and false-negative measures of a surveillance test for recurrent breast cancer, although this is usually ascertained by a negative result or a positive test result at subsequent testing after a period of follow-up has elapsed (e.g. 1-year mammography interval, 2- to 3-year MRI interval, etc.). A pragmatic reference standard is therefore diagnosis of IBTR or MCBC that occurs within a 3-year period of conducting a surveillance test.

The reference standard for this review was histopathological assessment for test positives, along with a follow-up period of up to 3 years for test negatives (in order to differentiate between truenegatives and false-negatives).

\section{Outcomes}

The following types of outcome were considered.

Test performance in diagnosing:

- IBTR in women undergoing routine surveillance

- IBTR in women undergoing non-routine surveillance

- MCBC in women undergoing routine surveillance

- MCBC in women undergoing non-routine surveillance. 
To be considered for inclusion, the studies had to report the absolute numbers of true-positives, false-positives, false-negatives and true-negatives, or provide information allowing their calculation, and report a per-patient analysis.

In studies reporting the above outcomes, we planned to record the following additional outcomes, if reported:

- adverse effects (defined as physical harms) of mammography and other tests

- acceptability of the tests

- reliability of the tests

- radiological/operator expertise (who conducts the test and previous experience)

- interpretability/readability of the tests.

\section{Search strategy}

Electronic searches were conducted to identify reports of published, unpublished and ongoing studies. The search strategies were designed to be highly sensitive, including both appropriate subject heading and text word terms to identify diagnostic accuracy studies of mammography and comparative tests when used in surveillance. To ensure data consistency with other areas of this project, searches were restricted to English-language reports published from 1990 onwards. Conference abstracts were not included. The following databases were searched for primary studies: MEDLINE, MEDLINE In-Process, EMBASE, BIOSIS, SCI and CANCERLIT, whereas Medion, the CDSR, DARE and the HTA database were searched for reports of evidence syntheses. Reports of ongoing and recently completed trials were sought from the CCT, Clinical Trials, WHO ICTRP, NCI Clinical Trials Database, NRR Archive and NIHR Portfolio Database. Appendix 17 gives full details of the search strategies used.

In addition, relevant websites were searched and included the National Cancer Institute, National Comprehensive Cancer Network, CancerWEB, Breast Cancer Surveillance Consortium, and National Library for Health, as well as relevant professional organisations including the RCR, ABS at the BASO, American Society of Clinical Oncology, American Society of Breast Disease, American College of Radiology, and European Society for Clinical Oncology. The reference lists of all included studies were also scanned for additional reports.

\section{Data extraction strategy}

One reviewer (from FG, GM, CR, SKA, RT or SZ) screened the titles and abstracts (if available) of all reports identified by the search strategy. One reviewer (from FG, JH, GM, CR, SKA, RT or SZ) assessed potentially relevant full text studies for inclusion. One reviewer (RT) conducted a $10 \%$ check of the inclusion assessment for all potentially relevant studies. Any disagreements were resolved by consensus or arbitration by a third party.

One reviewer (from JH, CR, SKA or SZ) independently extracted details of study design, participants, index, comparator and reference standard tests, and participant flow and outcome data (see Appendix 18 for details of the data extraction form). A second reviewer independently validated the data extraction. In the event of any uncertainty regarding the data extraction, a third reviewer gave advice and validated the data extraction (CB).

\section{Quality assessment strategy}

Two reviewers (from JH, CR, SKA or SZ) independently assessed the methodological quality of all included studies, using a modified version of the Quality Assessment of Diagnostic Accuracy Studies (QUADAS) tool (Appendix 19). ${ }^{62}$ QUADAS is a quality assessment tool for assessing the methodological quality of individual studies in systematic reviews of diagnostic accuracy. It is designed to be adapted to make it applicable to a specific review topic. QUADAS was developed 
through a formal consensus method and was based on empirical evidence. The original QUADAS checklist contained 14 questions. We adapted the QUADAS tool to make it more applicable for assessing reports of tests for IBTR/MCBC. We omitted three questions in the original QUADAS tool that related to the quality of reporting rather than methodological quality (questions 2, 8 and 9). These questions related to the description of (1) the selection criteria; (2) the execution of the index test; and (3) the execution of the reference standard test.

In question 1 we defined the spectrum of patients representative of those receiving the test in practice as women of all ages previously treated for primary breast cancer, without detectable metastatic disease. We defined the reference standard (question 2) of the modified tool as histopathological assessment for correctly identifying the target condition. We did not include follow-up time in this question, as follow-up alone will only identify a possible false-negative result but will not identify the target condition as a stand-alone test. We split question 3 (disease progression bias) into two parts for (1) test positives and (2) test negatives to assess whether the target condition was likely to have changed between the test and the two types of reference standard test. We split question 5 (differential verification bias) to assess whether (1) all test positives were verified by histopathological assessment and (2) whether all test negatives received appropriate follow-up. We added an additional item (question 9) to assess whether the index and comparator tests were independently interpreted.

We did not change the remaining questions. These concerned partial verification bias (question 4), incorporation bias (question 6), test review bias and diagnostic review bias (questions 7 and 8), clinical review bias (question 10), reporting of uninterpretable/intermediate results (question 11) and attrition bias (question 12).

Any disagreements were resolved by consensus or arbitration by a third party. Each of the items was checked as 'yes', 'no' or 'unclear'. Each item was worded so that a rating of 'yes' was the optimal rating of methodological quality. A study was considered to be of higher quality if it was checked as 'yes' to items assessing patient spectrum, partial verification and differential verification bias and test review bias. We planned to use a separate quality assessment tool using a checklist adapted from Verhagen and colleagues ${ }^{63}$ for any RCTs.

\section{Data analysis}

The results of the individual studies were tabulated into a $2 \times 2$ table, and sensitivity, specificity, positive and negative likelihood ratios and diagnostic odds ratio (DOR) were calculated along with $95 \%$ CIs. The intention was to combine the results of the included studies in a meta-analysis using the hierarchical summary receiver operating characteristic (HSROC) framework, where three or more studies reported sufficient data. However, due to limited data and variability across studies, we felt it unwise to perform a meta-analysis and therefore a narrative synthesis of the results is presented (see Table 16 and Appendix 22 for summary characteristics of the included studies).

\section{Results}

\section{Number and type of studies included and excluded}

Nine studies met our inclusion criteria. Figure 8 shows the number of potentially relevant reports of the studies identified, the number included and excluded and a summary of the exclusion criteria. Appendix 20 lists the bibliographic details of the nine studies that were included in the review. 
The bibliographic details of the potentially relevant studies identified by the search strategy, for which full text papers were obtained, but which subsequently failed to meet the inclusion criteria, are given in Appendix 21. These studies were excluded because they failed to meet one or more of the inclusion criteria in terms of types of studies, participants, test, reference standard or outcomes (see also Figure 8).

\section{Characteristics of the included studies}

Appendix 22 provides details of the characteristics of the included studies. Table 15 provides a summary of the overall characteristics of the included studies. Table 16 provides further details of the characteristics of study design, patient type, considered index/comparator test and follow-up periods for verifying test-negative results for the individual studies arranged alphabetically by author.

Overall, the nine studies enrolled 4002 participants. After exclusions, due to eligibility or participant dropout, the studies included 3724 participants in their analyses. The earliest study took place in $1995^{64}$ and the latest in $2009 .{ }^{65}$ The earliest participant enrolment date given was $1992^{64}$ and the latest was $2003 .{ }^{65}$ Four studies did not give any indication of the enrolment time period. ${ }^{66-69}$ One study took place in Sweden, ${ }^{64}$ two in the UK, ${ }^{67,68}$ two in Germany, ${ }^{69,70}$ two in South Korea ${ }^{65,71}$ one in Italy ${ }^{66}$ and one in France. ${ }^{72}$ The ages of the participants ranged from 22 to 82 years. ${ }^{65}$ Most participants were aged in their fifties. Details of mean, with standard deviations (SDs), and median ages for individual studies are shown, where these were reported, in Appendix 22. Reported follow-up of test negatives ranged from 5 to 32 months.

The studies by Rieber and colleagues ${ }^{69}$ and Shin and colleagues ${ }^{71}$ were cohort studies, in which participants received a comparator test (MRI in the study by Rieber and colleagues ${ }^{69}$ and ultrasound in the study by Shin and colleagues ${ }^{71}$ ) and the reference standard. The seven remaining studies ${ }^{64-68,70,72}$ were direct head-to-head studies, in which participants all received the index test, comparator test and reference standard.

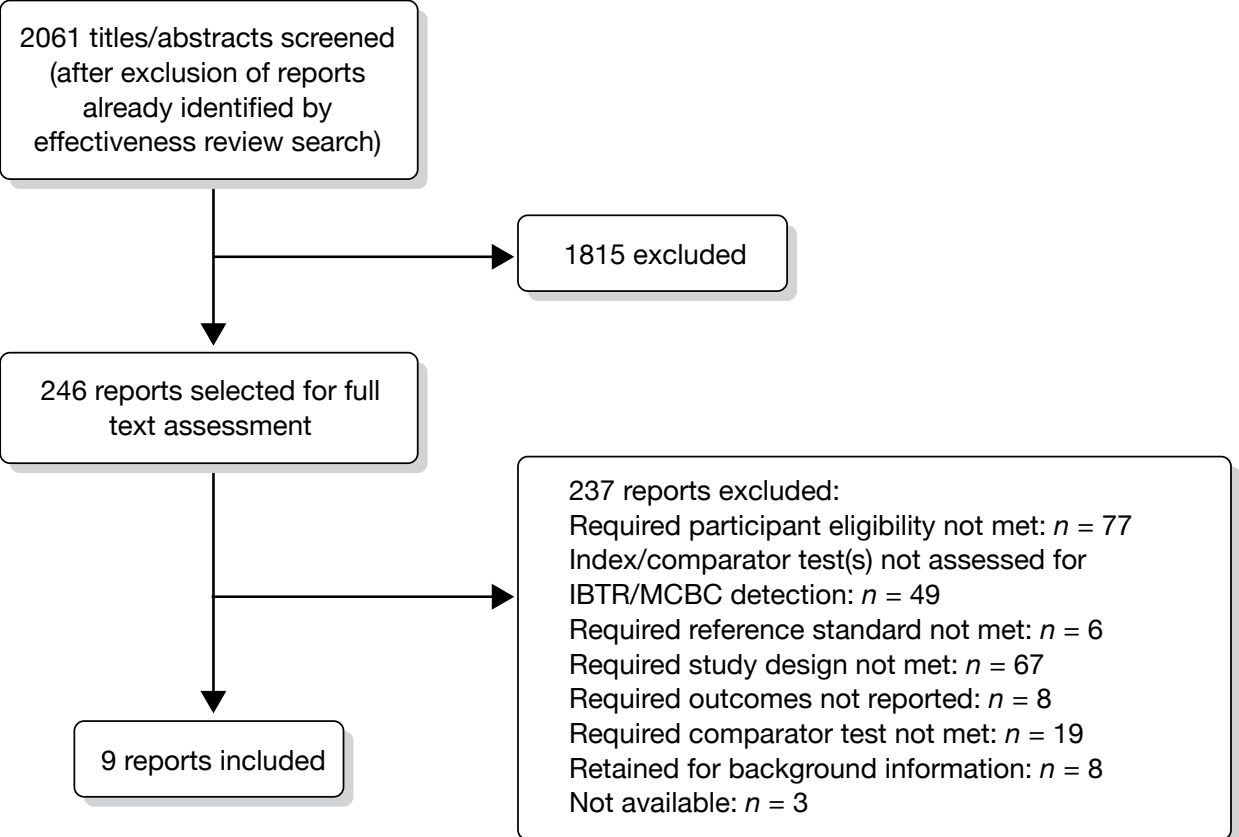

FIGURE 8 Flow chart of the number of potentially relevant reports of identified studies and the number subsequently included and excluded from the diagnostic accuracy review. 
TABLE 15 Summary of the total number of patients and number of studies by type of study, numbers analysed, type of surveillance and focus of surveillance for the included diagnostic accuracy studies

\begin{tabular}{|c|c|c|}
\hline Characteristic & No. of patients (\%) & No. of studies (\%) \\
\hline \multicolumn{3}{|l|}{ Type of study } \\
\hline Cohort & 2108 & 2 (22) \\
\hline Head to head & 1616 & $7(78)$ \\
\hline \multicolumn{3}{|l|}{ Patients } \\
\hline Enrolled & 4002 & 9 \\
\hline Analysed & 3724 & \\
\hline \multicolumn{3}{|l|}{ Type of surveillance } \\
\hline Routine surveillance & 3451 (93) & $6(77)$ \\
\hline Non-routine surveillance & $273(7)$ & $3(33)$ \\
\hline \multicolumn{3}{|l|}{ Focus of surveillance } \\
\hline Local recurrence (IBTR) & $298(8)$ & $5(56)$ \\
\hline Contralateral recurrence (MCBC) & $1375(37)$ & 2 (22) \\
\hline Both & 2051 (55) & $2(22)$ \\
\hline \multicolumn{3}{|l|}{ Type of test } \\
\hline XRM & 344 & 5 \\
\hline US & 2094 & 3 \\
\hline MRI & 500 & 6 \\
\hline CE & 314 & 4 \\
\hline Combined XRM, CE & 105 & 1 \\
\hline Combined XRM, US & 1256 & 1 \\
\hline Combined XRM, CE, US & 119 & 1 \\
\hline Combined XRM, CE, US, MRI & 119 & 1 \\
\hline
\end{tabular}

$\mathrm{CE}$, clinical examination; US, ultrasound; XRM, X-ray mammography.

a Multiple tests used within studies.

In breast cancer surveillance, the considered diagnostic tests can be used at different stages in the assessment pathway prior to a positive test result receiving verification via the reference standard test. As described earlier, test administration may be as a first routine surveillance test in a patient with no prior suspicion of IBTR/MCBC, or it may be used to evaluate a suspicious test finding on a prior diagnostic test (non-routine surveillance patients). Six studies assessed performance of the diagnostic test used as a routine first surveillance test. ${ }^{64-67,70,71}$ The three remaining studies, by Mumtaz and colleagues ${ }^{68}$ Rieber and colleagues ${ }^{69}$ and Ternier and colleagues ${ }^{72}$ assessed the performance of the diagnostic test as part of non-routine surveillance to evaluate a suspicious result from a prior diagnostic test.

Three studies reported data on ultrasound. ${ }^{66,71,72}$ Six studies reported data on MRI. ${ }^{64,66-70}$ Four reported data on specialist-led clinical examination. ${ }^{64,66,67,72}$ The studies did not explicitly state whether a hospital-based consultant or an alternative health-care professional conducted the clinical examination. We assumed in all cases that the examination was conducted at a consultant-supervised clinic. Drew and colleagues ${ }^{67}$ reported test performance for surveillance mammography, combined with clinical examination. Viehweg and colleagues ${ }^{70}$ reported test performance for combined surveillance mammography, clinical examination and ultrasound (known as conventional methods). This study also reported performance for combined MRI and 
TABLE 16 Summary of characteristics for individual diagnostic accuracy studies

\begin{tabular}{|c|c|c|c|c|c|}
\hline Study ID & Study design & $\begin{array}{l}\text { Type of primary } \\
\text { surgery }\end{array}$ & Index tests & Comparator test & $\begin{array}{l}\text { Follow-up time for verifying test- } \\
\text { negative results }\end{array}$ \\
\hline \multirow[t]{2}{*}{$\begin{array}{l}\text { Belli } \\
2002^{66}\end{array}$} & \multirow[t]{6}{*}{$\begin{array}{l}\text { Direct head-to- } \\
\text { head cohort }\end{array}$} & \multirow{2}{*}{$\begin{array}{l}\text { Non-routine } \\
\text { surveillance BCS } \\
\text { patients }\end{array}$} & \multirow[t]{2}{*}{$\begin{array}{l}\text { MRl for local } \\
\text { recurrence }\end{array}$} & \multirow[t]{2}{*}{$\begin{array}{l}\text { XRM, CE, US for } \\
\text { local recurrence }\end{array}$} & $\begin{array}{l}\text { MRI and CE follow-up performed at } \\
3 \text { months }\end{array}$ \\
\hline & & & & & $\begin{array}{l}\text { All MRI test negatives underwent cytological } \\
\text { examination }\end{array}$ \\
\hline $\begin{array}{l}\text { Boné } \\
1995^{64}\end{array}$ & & $\begin{array}{l}\text { Routine surveillance } \\
\text { mastectomy } \\
\text { patients, all with } \\
\text { breast reconstruction } \\
\text { and implants }\end{array}$ & $\begin{array}{l}\text { XRM for local } \\
\text { and contralateral } \\
\text { recurrence }\end{array}$ & $\begin{array}{l}\text { MRI, CE for local } \\
\text { and contralateral } \\
\text { recurrence }\end{array}$ & Median 10 months (range 5-18 months) \\
\hline $\begin{array}{l}\text { Drew } \\
1998^{67}\end{array}$ & & $\begin{array}{l}\text { Routine surveillance } \\
\text { BCS patients }\end{array}$ & $\begin{array}{l}\text { MRI for local } \\
\text { recurrence }\end{array}$ & $\begin{array}{l}\mathrm{XRM}, \mathrm{CE}, \mathrm{XRM}+\mathrm{CE} \\
\text { for local recurrence }\end{array}$ & Median 341 days (range 168-451 days) \\
\hline $\begin{array}{l}\text { Kim } \\
2009^{65}\end{array}$ & & $\begin{array}{l}\text { Routine surveillance } \\
\text { breast-conserving } \\
\text { and mastectomy } \\
\text { patients }\end{array}$ & $\begin{array}{l}\text { Adjunct US } \\
\text { (XRM + US) for } \\
\text { contralateral } \\
\text { recurrence }\end{array}$ & None & $1-2$ years \\
\hline $\begin{array}{l}\text { Mumtaz } \\
1997^{68}\end{array}$ & & \multirow{2}{*}{$\begin{array}{l}\text { Non-routine } \\
\text { surveillance BCS } \\
\text { patients }\end{array}$} & $\begin{array}{l}\text { XRM for local } \\
\text { recurrence }\end{array}$ & $\begin{array}{l}\text { MRI for local } \\
\text { recurrence }\end{array}$ & Median 12 months (range 6-15 months) \\
\hline $\begin{array}{l}\text { Rieber } \\
1997^{69}\end{array}$ & \multirow[t]{2}{*}{ Cohort } & & $\begin{array}{l}\text { MRI for local } \\
\text { recurrence }\end{array}$ & None & $\begin{array}{l}\text { CE, US performed at } 6 \text {-month follow-up. } \\
\text { XRM performed at 12-month follow-up. In } \\
22 \text { patients a control MRI was performed } \\
\text { at intervals of } 2-16 \text { months (mean } \\
7.2 \text { months) }\end{array}$ \\
\hline $\begin{array}{l}\text { Shin } \\
2005^{71}\end{array}$ & & $\begin{array}{l}\text { Routine surveillance } \\
\text { patients (primary } \\
\text { surgery type not } \\
\text { reported) }\end{array}$ & $\begin{array}{l}\text { US for local and } \\
\text { contralateral } \\
\text { recurrence }\end{array}$ & None & 6 months \\
\hline $\begin{array}{l}\text { Ternier } \\
2006^{72}\end{array}$ & \multirow[t]{2}{*}{$\begin{array}{l}\text { Direct head-to- } \\
\text { head cohort }\end{array}$} & $\begin{array}{l}\text { Non-routine } \\
\text { surveillance BCS } \\
\text { patients }\end{array}$ & $\begin{array}{l}\text { XRM for local } \\
\text { recurrence }\end{array}$ & $\begin{array}{l}\text { CE, US for local } \\
\text { recurrence }\end{array}$ & 6 months \\
\hline $\begin{array}{l}\text { Viehweg } \\
2004^{70}\end{array}$ & & $\begin{array}{l}\text { Routine surveillance } \\
\text { BCS patients }\end{array}$ & $\begin{array}{l}\text { MRI for } \\
\text { contralateral } \\
\text { recurrence }\end{array}$ & $\begin{array}{l}\mathrm{CM} \\
(\mathrm{XRM}+\mathrm{CE}+\mathrm{US}) \\
\mathrm{MRI}+\mathrm{CM} \text { for } \\
\text { contralateral } \\
\text { recurrence }\end{array}$ & 12 months \\
\hline
\end{tabular}

CE, clinical examination; CM, conventional methods; NS, not stated; US, ultrasound; XRM, X-ray mammography.

a Study authors considered computerised tomography as the index test in this study, but this test was not considered as an included comparator in this review.

conventional methods. Kim and colleagues ${ }^{65}$ reported performance for combined surveillance mammography and ultrasound. None of the studies meeting our inclusion criteria reported data on unstructured primary care follow-up.

The study by Boné and colleagues ${ }^{64}$ involved mastectomy patients who had all received breast reconstruction with implants. Currently, these patients are offered clinical follow-up for the ipsilateral breast and surveillance mammography for the contralateral breast annually or once every 2 years. Surveillance mammography of the ipsilateral reconstructed breast is performed if there is a clinical concern. A previous systematic review of surveillance mammography following breast reconstruction was published in 2007 by Barnsley and colleagues. ${ }^{43}$ This review considered case reports and case series literature published between January 1980 and August 2004. The included studies involved implants and immediate or delayed transverse rectus abdominis muscle (TRAM) flap reconstructions. The authors did not conduct a meta-analysis due to heterogeneity in study design, follow-up and surveillance mammography regimen. Review findings suggested 
that surveillance mammography is able to detect certain local recurrences, although the authors concluded that, due to the paucity of evidence, further research in this area is required. We therefore believe that this study merits inclusion in our review, as, although these women represent a subset of our considered population, they are an increasingly relevant subgroup who might receive surveillance mammography in the future.

\section{Quality of the included diagnostic accuracy studies}

The results of the quality assessment for the individual studies are shown in Appendix 23.

Figures 9 and 10 summarise the quality assessment of the included studies.

None of the studies met all of our quality criteria specified for higher-quality studies, although in five studies ${ }^{65,66,68,69,72}$ this was due to lack of clarity as to whether reference standard results were interpreted without knowledge of index test results only.

The study conducted by Boné and colleagues ${ }^{64}$ was considered to be unrepresentative of our considered patient population as a whole; as previously discussed, the participants had all received mastectomy for primary breast cancer with breast reconstruction and implants. As discussed earlier, it is not standard practice to offer routine surveillance of the treated breast to patients receiving either mastectomy alone, or mastectomy with breast reconstruction and implants. We therefore felt that, although this patient group represents a subset of our considered population, they differ from the wider spectrum of women who would receive surveillance in practice.

Only the Shin and colleagues ${ }^{71}$ study was judged as free of disease progression bias for positive index test results. Disease progression bias occurs when the time delay between the index and reference standard test is such that improvement or progression of the condition may occur in the intervening period. It was unclear whether the remaining eight (89\%) studies ${ }^{64-70,72}$ had

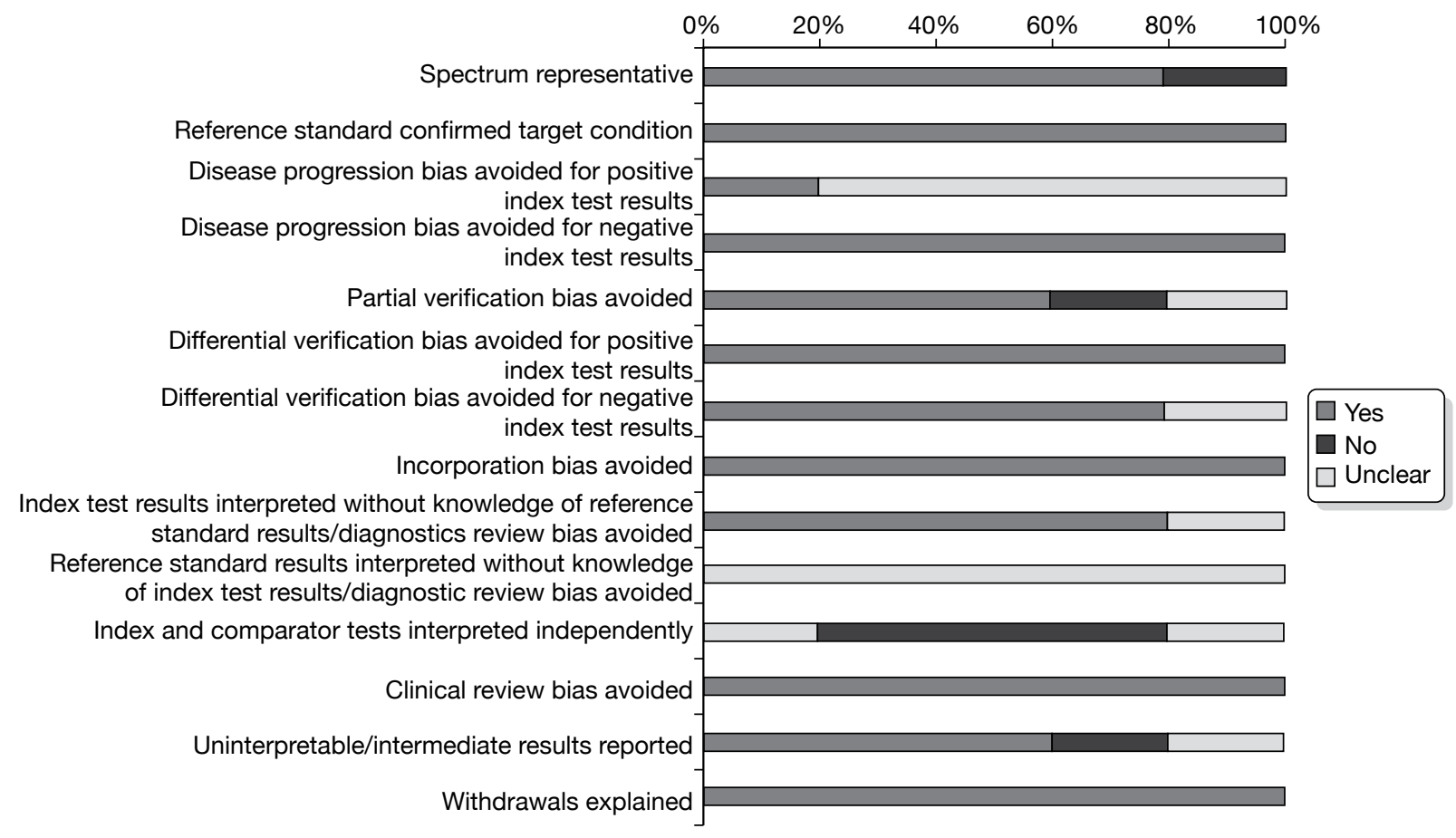

FIGURE 9 Summary of quality assessment of included diagnostic accuracy studies for routine surveillance. 


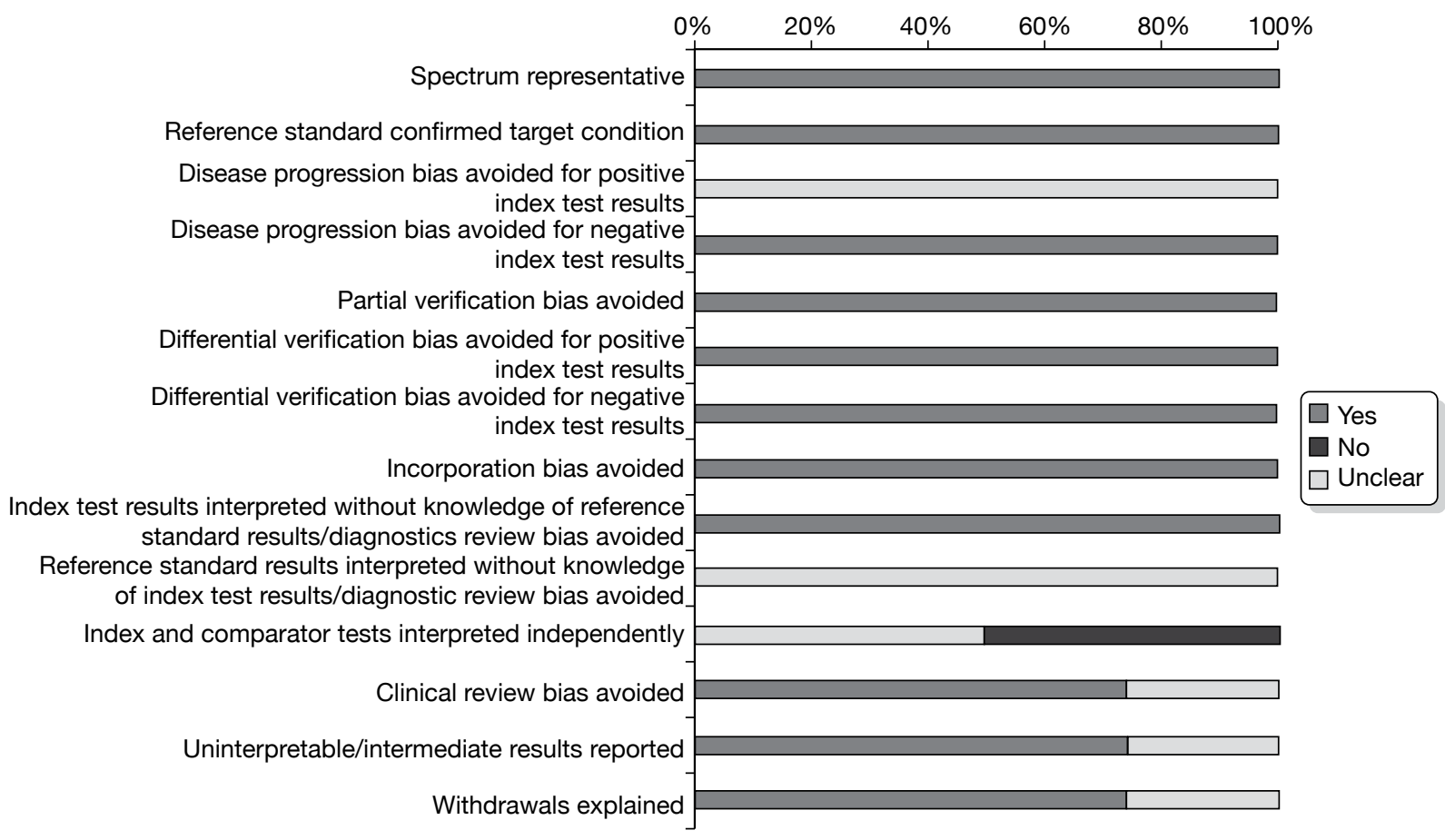

FIGURE 10 Summary of quality assessment of included diagnostic accuracy studies for non-routine surveillance.

avoided disease progression bias for positive index test results, whereas all studies successfully avoided disease progression bias for negative index test results.

Seven (78\%) studies ${ }^{64-66,68-70,72}$ (three routine surveillance and four non-routine surveillance) were free from partial verification bias. It was unclear in the study conducted by Shin and colleagues, ${ }^{71}$ however, whether test negatives received follow-up. The study by Drew and colleagues ${ }^{67}$ was considered to be vulnerable to partial verification bias, as only those participants testing positively on MRI received reference standard verification.

In all studies, positive index/comparator test results were verified by the same reference standard (histopathological assessment). In eight (89\%) studies (four routine surveillance and four non-routine surveillance), participants with negative index/comparator test results all received follow-up. ${ }^{64-70,72}$ In the study conducted by Shin and colleagues ${ }^{71}$ it was unclear whether all patients with negative test results received follow-up.

While it was unclear for all studies whether reference standard results had been interpreted without knowledge of the index/comparator test result, it was unclear in the study by Viehweg and colleagues ${ }^{70}$ whether index test results had been interpreted without knowledge of the reference standard. Five (56\%) studies (three routine surveillance and two non-routine surveillance) interpreted index and comparator test results independently. It was unclear in the study by Boné and colleagues ${ }^{64}$ whether index and comparator tests were interpreted independently. In the remaining studies, ${ }^{65,69-72}$ index and comparator test results were not analysed separately. Clinical examination and mammography were usually performed before MRI or ultrasound. Knowledge of a prior test result could influence the subjective assessment, and hence the diagnostic accuracy performance, of the subsequent test(s).

In the study conducted by Rieber and colleagues ${ }^{69}$ it was unclear whether the same clinical data were available as would be the case when the test is used in practice. It was also unclear 
in the studies by Belli and colleagues ${ }^{66}$ and Kim and colleagues ${ }^{65}$ whether uninterpretable or intermediate results had been reported, and in the study conducted by Belli and colleagues ${ }^{66}$ whether the number of or reason for withdrawals had been explained.

\section{Assessment of test performance}

\section{Test performance in diagnosing IBTR}

Table 17 shows test performance in detecting IBTR in routine surveillance patients. The studies by Boné and colleagues ${ }^{64}$ and Drew and colleagues ${ }^{67}$ involving a total of 188 patients reported the performance of surveillance mammography, MRI and clinical examination in routine surveillance patients. These studies reported sensitivities of $64 \%$ and $67 \%$, and specificities of $97 \%$ and $85 \%$, for surveillance mammography. For MRI the studies reported sensitivities of $86 \%$ and $100 \%$, and $50 \%$ and $89 \%$ for clinical examination. Boné and colleagues ${ }^{64}$ did not report specificity for MRI or clinical examination. The highest reported sensitivity was for MRI, and surveillance mammography combined with clinical examination (both 100\%), whereas the highest specificity was for surveillance mammography (97\%). Similarly, a high specificity of $93 \%$ was reported for MRI. The lowest reported sensitivity was for clinical examination (50\%) and the lowest specificity was for surveillance mammography combined with clinical examination (67\%).

Table 18 shows test performance in detecting IBTR in non-routine surveillance patients, as reported by Belli and colleagues, ${ }^{66}$ Mumtaz and colleagues, ${ }^{68}$ Rieber and colleagues ${ }^{69}$ and Ternier and colleagues. ${ }^{72}$ The studies by Boné and colleagues ${ }^{64}$ and Drew and colleagues ${ }^{67}$ involved a total of 156 patients and reported median (and range) sensitivity of $71 \%$ (50-83\%) and specificity of $63 \%$ (57-75\%) for surveillance mammography. For MRI, the studies by Belli and colleagues, ${ }^{66}$ Mumtaz and colleagues ${ }^{68}$ and Rieber and colleagues, ${ }^{69}$ involving a total of 193 patients, reported sensitivity of $93 \%$ and $100 \%$ and a median (and range) specificity of $94 \%$ (88-96\%). Belli and colleagues $^{66}$ and Ternier and colleagues ${ }^{72}$ reported the test performance of ultrasound sensitivities of $43 \%$ and $87 \%$, and specificities of $31 \%$ and $73 \%$, and clinical examination, sensitivities of $43 \%$ and $62 \%$, and specificities of $56 \%$ and $49 \%$. The highest reported sensitivity (100\%) and specificity (96\%) were for MRI. The lowest reported sensitivities were for both ultrasound and clinical examination (43\%). The lowest specificity was for ultrasound (31\%).

TABLE 17 Test performance as measured by sensitivity, specificity, likelihood ratio and DOR for detecting IBTR in routine surveillance patients

\begin{tabular}{|c|c|c|c|c|c|c|c|}
\hline Test & Study ID & $\begin{array}{l}\text { Primary } \\
\text { surgical } \\
\text { treatment }\end{array}$ & $\begin{array}{l}\text { Reported } \\
\text { sensitivity } \\
(\%)\end{array}$ & $\begin{array}{l}\text { Reported } \\
\text { specificity } \\
(\%)\end{array}$ & LR+ & LR- & DOR $(95 \% \mathrm{Cl})$ \\
\hline \multirow[t]{3}{*}{ XRM } & Boné $1995^{64}$ & Mastectomy & 64 & 97 & 22.2 & 0.4 & 60.3 (10.2 to 358.1) \\
\hline & Drew $1998^{67}$ & Breast & 67 & 85 & 4.6 & 0.4 & 11.7 (2.6 to 52.4 ) \\
\hline & & conserving & & & 1.9 & 0.5 & 4.2 (0.6 to 28.6$)$ \\
\hline \multirow[t]{2}{*}{ MRI } & Boné $1995^{64}$ & Mastectomy & 86 & NR & & & \\
\hline & Drew $1998^{67}$ & $\begin{array}{l}\text { Breast } \\
\text { conserving }\end{array}$ & 100 & 93 & 14.3 & IC & IC \\
\hline \multirow[t]{2}{*}{ CE } & Boné $1995^{64}$ & Mastectomy & 50 & NR & & & \\
\hline & Drew $1998^{67}$ & $\begin{array}{l}\text { Breast } \\
\text { conserving }\end{array}$ & 89 & 76 & 3.7 & 0.2 & 25.4 (3.0 to 213.9$)$ \\
\hline $\begin{array}{l}\text { Combined } \\
\text { XRM and CE }\end{array}$ & Drew $1998^{67}$ & $\begin{array}{l}\text { Breast } \\
\text { conserving }\end{array}$ & 100 & 67 & 3.0 & IC & IC \\
\hline
\end{tabular}

$\mathrm{CE}$, clinical examination; IC, incalculable; LR+, likelihood ratio of a positive test result; LR-, likelihood ratio of a negative test result; XRM, X-ray mammography. 
TABLE 18 Test performance as measured by sensitivity, specificity, likelihood ratio and DOR for detecting IBTR in nonroutine surveillance patients

\begin{tabular}{|c|c|c|c|c|c|c|c|}
\hline Test & Study ID & $\begin{array}{l}\text { Primary } \\
\text { surgical } \\
\text { treatment }\end{array}$ & $\begin{array}{l}\text { Reported } \\
\text { sensitivity } \\
(\%)\end{array}$ & $\begin{array}{l}\text { Reported } \\
\text { specificity } \\
(\%)\end{array}$ & $\mathrm{LR}+$ & LR- & DOR $(95 \% \mathrm{Cl})$ \\
\hline \multirow[t]{3}{*}{ XRM } & Belli $2002^{66}$ & \multirow{3}{*}{$\begin{array}{l}\text { Breast } \\
\text { conserving }\end{array}$} & 71 & 63 & 1.9 & 0.5 & 4.2 (2.6 to 52.4) \\
\hline & Mumtaz 199768 & & 50 & 75 & 2.0 & 0.7 & $3(0.6$ to 14.0$)$ \\
\hline & Ternier $2006^{72}$ & & 83 & 57 & 1.9 & 0.3 & 6.3 (2.5 to 15.6$)$ \\
\hline \multirow[t]{2}{*}{ US } & Belli $2002^{66}$ & & 43 & 31 & 0.6 & 1.8 & 0.3 (0.1 to 2.1$)$ \\
\hline & Ternier 2006 $6^{72}$ & & 87 & 73 & 3.2 & 0.2 & 17 (6.2 to 46.5$)$ \\
\hline \multirow[t]{3}{*}{ MRI } & Belli $2002^{66}$ & & 100 & 94 & 16.0 & IC & IC \\
\hline & Mumtaz $1997^{68}$ & & 93 & 88 & 7.4 & 0.1 & 91 (7.4 to 1126. 9) \\
\hline & Rieber $1997^{69}$ & & 100 & 96 & 24.2 & IC & IC \\
\hline \multirow[t]{2}{*}{ CE } & Belli $2002^{66}$ & & 43 & 56 & 1.0 & 1.0 & $1.0(0.2$ to 5.8$)$ \\
\hline & Ternier $2006^{72}$ & & 62 & 49 & 1.2 & 0.8 & 1.5 (0.7 to 3.4$)$ \\
\hline
\end{tabular}

CE, clinical examination; IC, incalculable; US, ultrasound; XRM, X-ray mammography.

\section{Test performance in diagnosing MCBC}

Table 19 shows test performance in detecting MCBC in routine surveillance patients. The studies by Boné and colleagues ${ }^{64}$ and Viehweg and colleagues ${ }^{70}$ involving a total of 202 patients, reported $67 \%$ and $91 \%$ sensitivity and $50 \%$ and $90 \%$ specificity, respectively, for MRI. Only individual studies reported the test performance of surveillance mammography, clinical examination, and combinations of tests involving surveillance mammography. The highest reported sensitivity (100\%) was for combined surveillance mammography, clinical examination, ultrasound and MRI,${ }^{70}$ whereas the highest reported specificity $(99 \%)$ was for combined surveillance mammography and ultrasound. ${ }^{65}$ The lowest reported sensitivity (0\%) was for clinical examination and the lowest specificity was for surveillance mammography, MRI and clinical examination (all 50\%). ${ }^{64}$

None of the studies reported diagnostic accuracy of the included tests for diagnosing $M C B C$ in non-routine surveillance patients with a prior suspicious test result.

\section{Test performance in diagnosing IBTR and MCBC}

The study conducted by Shin and colleagues ${ }^{71}$ was the sole study reporting overall test performance for diagnosing IBTR and MCBC. Shin and colleagues ${ }^{71}$ evaluated ultrasound in routine surveillance patients, reporting a sensitivity of $71 \%$ and a specificity of $98 \%$ [LR+ 41.4, LR- 0.3, OR 138.25 (95\% CI 61.26 to 312.04)].

\section{Adverse effects, acceptability of the tests, reliability, radiological/operator expertise and interpretability/readability of the tests}

None of the included studies reported data concerning these outcomes.

\section{Histology of cancers detected and not detected (true-positives and false-negatives)}

The histology of cancers detected and those that were not detected (true-positives and false-negatives), by each diagnostic test, where reported, are detailed in Appendix 24 (see Tables 60-63). We found no discernible pattern for cancers detected and not detected both within and between diagnostic tests. 
TABLE 19 Test performance as measured by sensitivity, specificity, likelihood ratio and DOR for detecting MCBC in routine surveillance patients

\begin{tabular}{|c|c|c|c|c|c|c|c|}
\hline Test & Study ID & $\begin{array}{l}\text { Primary surgical } \\
\text { treatment }\end{array}$ & $\begin{array}{l}\text { Reported } \\
\text { sensitivity (\%) }\end{array}$ & $\begin{array}{l}\text { Reported } \\
\text { specificity (\%) }\end{array}$ & $\mathrm{LR}+$ & LR- & DOR $(95 \%$ Cl) \\
\hline XRM & Boné $1995^{64}$ & Mastectomy & 67 & 50 & 1.3 & 0.7 & 2.0 (0.1 to 78.2 ) \\
\hline \multirow[t]{2}{*}{ MRI } & Boné $1995^{64}$ & Mastectomy & 67 & 50 & 1.3 & 0.7 & 2.0 (0.1 to 78.2 ) \\
\hline & Viehweg $2004^{70}$ & Breast conserving & 91 & 90 & 9.4 & 0.1 & 93.1 (11.0 to 786.2$)$ \\
\hline CE & Boné $1995^{64}$ & Mastectomy & 0 & 50 & & & \\
\hline $\begin{array}{l}\text { Combined XRM } \\
\text { and US }\end{array}$ & Kim $2009^{65}$ & Mastectomy & 95 & 99 & 61.5 & 0.05 & $\begin{array}{l}1149.2 \text { (148.0 to } \\
8937.8)\end{array}$ \\
\hline $\begin{array}{l}\text { Combined XRM, CE } \\
\text { and US }\end{array}$ & Viehweg $2004^{70}$ & Breast conserving & 64 & 84 & 3.9 & 0.4 & 8.9 (2.4 to 33.0$)$ \\
\hline $\begin{array}{l}\text { Combined XRM, CE, } \\
\text { US and MRI }\end{array}$ & Viehweg $2004^{70}$ & Breast conserving & 100 & 89 & 8.9 & IC & IC \\
\hline
\end{tabular}

CE, clinical examination; IC, incalculable; US, ultrasound; XRM, X-ray mammography.

\section{Summary}

Systematic reviews of diagnostic accuracy are highly complex and methodology in this area continues to evolve. We undertook a systematic review to determine the diagnostic accuracy of surveillance mammography for detecting IBTR and MCBC in women previously treated for primary breast cancer. We aimed to include RCT, head-to-head cohort and single cohort studies of women treated for primary breast cancer without detectable metastatic disease. The index test for our review was surveillance mammography and our comparator tests were ultrasound, MRI, specialist-led clinical examination and unstructured primary care follow-up. Our primary outcome was test performance in diagnosing IBTR and/or MCBC in routine and non-routine surveillance patients. The methods we adopted for this review are scientifically rigorous and compatible with current guidance in this area. Only nine studies, involving 3724 patients, evaluating the performance of tests for detecting IBTR and/or MCBC in women previously treated for primary breast cancer met our inclusion criteria. None of our considered tests was used for the same purpose (i.e. routine or non-routine surveillance) in all nine studies. Two studies evaluated the performance of surveillance mammography, and MRI, for detecting IBTR in routine surveillance patients. Three studies evaluated the performance of surveillance mammography, and MRI, for detecting IBTR in non-routine surveillance patients. None of the studies evaluated test performance for detecting $\mathrm{MCBC}$ in non-routine surveillance patients. Table 20 summarises the sensitivities and specificities of the included studies. Results for the Boné and colleagues ${ }^{64}$ study are excluded from this table due to the highly selected patient population of this study (mastectomy patients with breast reconstruction and implants).

For the detection of IBTR in routine surveillance patients with no prior suspicion of recurrence, the highest reported sensitivity was for MRI and surveillance mammography combined with clinical examination (both 100\%), whereas the highest reported specificity was for surveillance mammography (97\%) although it should be noted that this was reported for a highly select patient group (mastectomy patients with breast reconstruction and implants). The lowest reported sensitivity was for clinical examination (50\%), whereas the lowest specificity (67\%) was also for surveillance mammography combined with clinical examination. For the detection of IBTR in non-routine patients, with a suspicious result on a prior test, the highest reported sensitivity (100\%) and specificity (96\%) were for MRI. The lowest reported sensitivities were (43\%) for both ultrasound and clinical examination each, whereas the lowest specificity was for ultrasound (31\%). 
TABLE 20 Summary of test performance in detecting IBTR and MCBC in routine surveillance and non-routine surveillance patients

\begin{tabular}{|c|c|c|c|c|c|c|c|c|c|}
\hline \multirow[b]{2}{*}{ Test } & \multicolumn{3}{|c|}{ IBTR (routine surveillance) } & \multicolumn{3}{|c|}{ IBTR (non-routine surveillance) } & \multicolumn{3}{|c|}{ MCBC (routine surveillance) } \\
\hline & $\begin{array}{l}\text { No. of } \\
\text { studies }\end{array}$ & $\begin{array}{l}\text { Sensitivity } \\
(\%)\end{array}$ & $\begin{array}{l}\text { Specificity } \\
(\%)\end{array}$ & $\begin{array}{l}\text { No. of } \\
\text { studies }\end{array}$ & $\begin{array}{l}\text { Sensitivity } \\
(\%)\end{array}$ & $\begin{array}{l}\text { Specificity } \\
(\%)\end{array}$ & $\begin{array}{l}\text { No. of } \\
\text { studies }\end{array}$ & $\begin{array}{l}\text { Sensitivity } \\
(\%)\end{array}$ & $\begin{array}{l}\text { Specificity } \\
(\%)\end{array}$ \\
\hline XRM & 2 & $64-67$ & $85-97$ & 3 & (50-83) & $(57-75)$ & 1 & 67 & 50 \\
\hline US & & & & 2 & $(43-87)$ & $(31-73)$ & & & \\
\hline MRI & 2 & $86-100$ & 93 & 3 & $(93-100)$ & $(88-96)$ & 2 & $67-91$ & $50-90$ \\
\hline $\mathrm{CE}$ & 2 & $50-89$ & 76 & 2 & $(43-62)$ & $(49-56)$ & 1 & 0 & 50 \\
\hline $\begin{array}{l}\text { Combined } \\
\text { XRM, CE }\end{array}$ & 1 & 100 & 67 & & & & & & \\
\hline $\begin{array}{l}\text { Combined } \\
\text { XRM, US }\end{array}$ & & & & & & & 1 & 95 & 99 \\
\hline $\begin{array}{l}\text { Combined } \\
\text { XRM, CE, } \\
\text { US }\end{array}$ & & & & & & & 1 & 64 & 84 \\
\hline $\begin{array}{l}\text { Combined } \\
\text { XRM, CE, } \\
\text { US, MRI }\end{array}$ & & & & & & & 1 & 100 & 89 \\
\hline
\end{tabular}

CE, clinical examination; US, ultrasound; XRM, X-ray mammography.

For the detection of MCBC in routine surveillance patients, with no prior suspicion of contralateral cancer, the highest reported sensitivity (100\%) was for a combination of surveillance mammography, clinical examination, ultrasound and MRI, whereas the highest reported specificity (99\%) was for a combination of surveillance mammography and ultrasound. The lowest reported sensitivity ( $0 \%)$ was for clinical examination, with the lowest specificity for surveillance mammography, MRI and clinical examination (all 50\%), although these results were reported for the highly selected patient group mentioned above. No study reported test performance for detecting MCBC in suspicious patients.

From the available data, MRI can be considered as a highly sensitive test for diagnosing IBTR in both routine surveillance and non-routine surveillance patients. In both routine and nonroutine surveillance aimed at detecting IBTR, MRI ${ }^{67-69}$ achieved higher reported specificities than surveillance mammography. ${ }^{67,68,72}$ For the purposes of detecting routine surveillance MCBC, $\mathrm{MRI}^{70}$ had the highest reported sensitivity and specificity of any other individual test. From the data presented, combining tests increases both sensitivity and specificity for detecting MCBC. Combining surveillance mammography, clinical examination, ultrasound and $\mathrm{MRI}^{70}$ produced the highest reported sensitivity of $100 \%$. This test combination produced a lower reported specificity of $89 \%$ compared with combining surveillance mammography and ultrasound, ${ }^{65}$ which produced the highest reported specificity of $99 \%$ and the second highest reported sensitivity of $95 \%$. Of those test combinations reported here, surveillance mammography combined with ultrasound could be considered as the most accurate test combination for detecting $\mathrm{MCBC}$ via routine surveillance. No data were available to assess test performance for the detection of nonroutine surveillance MCBC.

These results should be interpreted with caution as they are based on a small evidence base of nine studies in total, with no one test evaluated by more than three studies, and most reported on by single studies only. Of the included studies, few have evaluated the performance of the considered tests for similar purposes. Due to differences between a 'treated' and 'untreated' breast (i.e. a breast that has undergone surgery and/or received radiotherapy) it is not appropriate to 
compare test accuracy in detecting IBTR and MCBC. Similarly, it is inappropriate to compare test accuracy between routine and non-routine surveillance patients, as the test operator is primed to evaluate a suspicious finding in the non-routine surveillance patient. It is unclear what effect this has on test accuracy but it is likely to focus attention on a particular area of the breast and may arguably increase sensitivity. Given differences in breast density following treatment for primary breast cancer, we felt it would also be inappropriate to include studies of test performance in the screening population to make inferences for surveillance of the contralateral breast, although parameter estimates for MRI in the screening population have been used in the economic evaluation in Chapter 7 of this report as it was felt that these would provide an indication of the relative value of a more costly but more effective test. Furthermore, no data were reported by the included studies on other test performance factors such as adverse effects or acceptability of the tests. We further discuss key findings and limitations of this review in Chapter 8. 


\section{Chapter 6}

\section{Analysis of Breast Cancer Registry database}

\section{Statement of overall aim and methods}

The purpose of surveillance mammography is to try to allow the earliest possible diagnosis of breast cancer and hence assume the tumour size at diagnosis is reduced. In order to ascertain the effectiveness of surveillance mammography we tried to identify a database with information on mammographic detection of IBTR and MCBC. Despite contacting a number of clinical and research groups and searching several databases, we were unable to locate a suitable data set with the required information. Furthermore, there was no information on how the IBTR or MCBC had been detected or on the follow-up mammography regimen of women diagnosed with breast cancer in any of the databases to which we had access. We required a representative UK cohort on which to base assumptions for our economic model (reported in Chapter 7). We also required a data set with a large number of IBTR and MCBC in a contemporary cohort with detailed information about the primary tumour to determine whether IBTR or MCBC constituted an independent risk of death. In the absence of mammography information, we wanted to determine whether size of the IBTR or MCBC was an independent risk factor for death, because if this is not the case then surveillance mammography is irrelevant.

We undertook an analysis of the West Midlands Cancer Intelligence Unit (WMCIU) breast cancer database to assess the generalisability of this database to UK breast cancer patients, to confirm risk factors for IBTR and MCBC, to determine if IBTR and MCBC were risk factors for all-cause death and breast cancer death, and to determine whether the size of the subsequent breast cancer tumour was a risk factor for death, given the risk factors of the primary tumour. If it was demonstrated that women who had an IBTR or MCBC were at an increased risk of death and that the size of the second tumour was associated with an elevated risk of death then it could be inferred that surveillance mammography may be an effective way to detect these second tumours and improve prognosis in breast cancer survivors.

\section{The WMCIU breast cancer primary tumour cohorts}

The WMCIU database contained information on 68,677 tumours in women diagnosed with breast cancer from 1 January 1990 to 31 December 2007. Follow-up data were available up until 31 January 2009. Tumours were excluded from analysis if the primary cancer was noninvasive or the treatment did not include surgical management in order to be able to distinguish future occurrences of ipsilateral local recurrence from progressive disease (Figure 11). Further exclusions were made if there was no information on risk factors recorded or if date of death was the same as the date of diagnosis. In the event of multiple primary tumours in the same breast or bilateral tumours, the worst tumour, as defined by the Nottingham Prognostic Index, was used. ${ }^{73}$ Prior to 1997 the WMCIU did not routinely collect information on all of the risk factors included in the analyses. For example, since 1997 the number of involved lymph nodes has been collected in addition to nodal status (positive or negative), which had not been collected prior to this date. Because of this, we took the pragmatic decision to include tumours diagnosed only after 1 January 1997 in the analyses. After exclusions, there were 32,877 women 


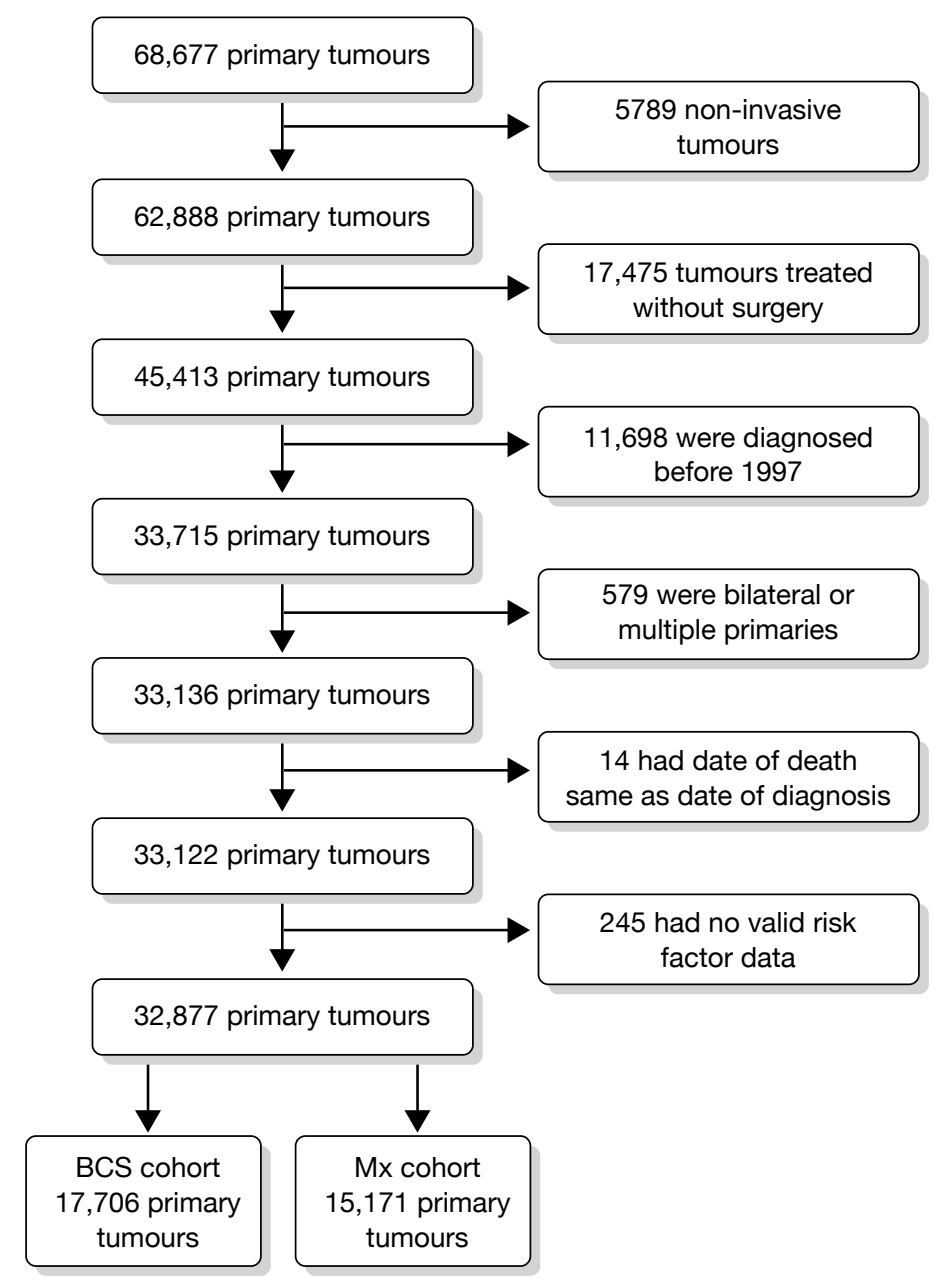

FIGURE 11 Flow chart of exclusion of primary tumours. Mx, mastectomy cohort.

with tumour data available for analysis. This total was split into two primary tumour cohorts to be analysed separately based on the type of surgery received: the BCS cohort of 17,706 women and the mastectomy cohort of 15,171 women. Descriptive information on both of the cohorts is contained in Table 21.

\section{Methods}

In both cohorts of primary tumours, Cox proportional hazards regression models ${ }^{74,75}$ were used to model four outcomes: time to IBTR, time to MCBC, time to death from all causes, and time to death from breast cancer. All risk factors were explored univariately in a simple Cox regression model and then simultaneously in a multiple Cox regression model. Risk factors modelled were age at diagnosis $(\leq 34,35-49,50-64,65-74,75-79, \geq 80$ years); grade of primary tumour (grade 1 , grade 2 , grade 3 , grade unknown); size of primary tumour $(\leq 10 \mathrm{~mm},>10 \mathrm{~mm}$ to $<20 \mathrm{~mm}$, $\geq 20 \mathrm{~mm}$, size unknown); nodal status (no nodes involved, one to three nodes involved, four or more nodes involved, nodal status unknown); and vascular invasion (no, yes, unknown). For all risk factors the level of the factor with the best prognosis was used as the reference category, with the exception of age at diagnosis where the screening age group (50-64 years of age) was used as the reference category. If a woman was indicated as having both an IBTR and MCBC then whichever event was detected earliest defined the event for that particular woman. Any IBTR or MCBC that occurred within 6 months of diagnosis was excluded, as this might be identified as 
TABLE 21 Description of the primary tumour cohorts

\begin{tabular}{|c|c|c|c|c|c|c|}
\hline Outcomes & \multicolumn{2}{|c|}{$\operatorname{BCS}(n=17,706)$} & \multicolumn{2}{|c|}{ Mastectomy $(n=15,171)$} & \multicolumn{2}{|c|}{ Total $(n=32,877)$} \\
\hline Death all cause & \multicolumn{2}{|l|}{2528} & \multicolumn{2}{|l|}{4345} & \multicolumn{2}{|l|}{6873} \\
\hline Death breast cancer & \multicolumn{2}{|l|}{1378} & \multicolumn{2}{|l|}{2713} & \multicolumn{2}{|l|}{4091} \\
\hline Ipsilateral event & \multicolumn{2}{|l|}{455} & \multicolumn{2}{|l|}{310} & \multicolumn{2}{|l|}{765} \\
\hline Contralateral event & \multicolumn{2}{|l|}{260} & \multicolumn{2}{|l|}{262} & \multicolumn{2}{|l|}{522} \\
\hline \multicolumn{7}{|l|}{ Age at diagnosis (years) } \\
\hline Mean (SD) & \multicolumn{2}{|l|}{$59(12)$} & \multicolumn{2}{|c|}{$61(14)$} & \multicolumn{2}{|l|}{$60(13)$} \\
\hline Median (25th, 75th centile) & \multicolumn{2}{|c|}{$58(50,67)$} & \multicolumn{2}{|c|}{$61(50,72)$} & \multicolumn{2}{|c|}{$59(50,69)$} \\
\hline \multirow{2}{*}{ Minimum, maximum } & 20,99 & & \multicolumn{2}{|c|}{20,97} & \multicolumn{2}{|c|}{20,99} \\
\hline & $n$ & $\%$ & $n$ & $\%$ & $n$ & $\%$ \\
\hline \multicolumn{7}{|l|}{ Age categories (years) } \\
\hline$\leq 34$ & 350 & 2.0 & 360 & 2.4 & 710 & 2.2 \\
\hline $35-49$ & 3513 & 19.8 & 3188 & 21.0 & 6701 & 20.4 \\
\hline 50-64 & 8511 & 48.1 & 5229 & 34.5 & 13,740 & 41.8 \\
\hline $65-74$ & 3491 & 19.7 & 3422 & 22.6 & 6913 & 21.0 \\
\hline $75-79$ & 1034 & 5.8 & 1547 & 10.2 & 2581 & 7.9 \\
\hline $80+$ & 807 & 4.6 & 1425 & 9.4 & 2232 & 6.8 \\
\hline Grade & & & & & & \\
\hline 1 & 4193 & 23.7 & 1827 & 12.0 & 6020 & 18.3 \\
\hline 2 & 7697 & 43.5 & 6918 & 45.6 & 14,615 & 44.5 \\
\hline 3 & 5388 & 30.4 & 5852 & 38.6 & 11,240 & 34.2 \\
\hline Unknown & 428 & 2.4 & 574 & 3.8 & 1002 & 3.0 \\
\hline Size $(m m)$ & & & & & & \\
\hline$\leq 10$ & 3473 & 19.6 & 1103 & 7.3 & 4576 & 13.9 \\
\hline$>10$ to $<20$ & 8663 & 48.9 & 4224 & 27.8 & 12,887 & 39.2 \\
\hline$\geq 20$ & 4885 & 27.6 & 8627 & 56.9 & 13,512 & 41.1 \\
\hline Size cat. unknown & 685 & 3.9 & 1217 & 8.0 & 1902 & 5.8 \\
\hline Nodes & & & & & & \\
\hline 0 & 11,630 & 65.7 & 6938 & 45.7 & 18,568 & 56.5 \\
\hline $1-3$ & 3367 & 19.0 & 4147 & 27.3 & 7514 & 22.9 \\
\hline $4+$ & 1232 & 7.0 & 3402 & 22.4 & 4634 & 14.1 \\
\hline Unknown & 1477 & 8.3 & 684 & 4.5 & 2161 & 6.6 \\
\hline Vascular invasion & & & & & & \\
\hline No & 12,887 & 72.8 & 8608 & 56.7 & 21,495 & 65.4 \\
\hline Yes & 3559 & 20.1 & 5341 & 35.2 & 8900 & 27.1 \\
\hline Unknown & 1260 & 7.1 & 1222 & 8.1 & 2482 & 7.5 \\
\hline
\end{tabular}

part of the management of the primary tumour and therefore would not be identifiable as part of a surveillance regimen.

Ipsilateral breast tumour recurrence (no, yes) and $\mathrm{MCBC}$ (no, yes) were entered into the multiple Cox regression for modelling death from all causes and death from breast cancer. HRs and 95\% CIs are presented. Complete tables of the univariate Cox regression models are reported in 
Appendix 25, summary tables of the multiple regression models are below. Kaplan-Meier ${ }^{76}$ failure curves are presented for selected risk factors and outcomes. Incidence rates were plotted against time for both IBTR and MCBC in both primary tumour cohorts.

\section{Results}

There were 32,877 women with primary breast cancer who met the inclusion criteria for analysis (see Table 21). These cohorts were younger on average than the total population of women diagnosed with breast cancer. This is because we excluded all women who were not treated with a surgical option and these tended to be older women. The BCS cohort consisted of 90,171 years of follow-up and the women had a median follow-up of 5 years. There were 73,500 years of follow-up in the mastectomy cohort and median follow-up was 4 years.

\section{Incidence of recurrences}

Incidence rates per 1000 persons are plotted against year of follow-up in Figures 12 and 13.

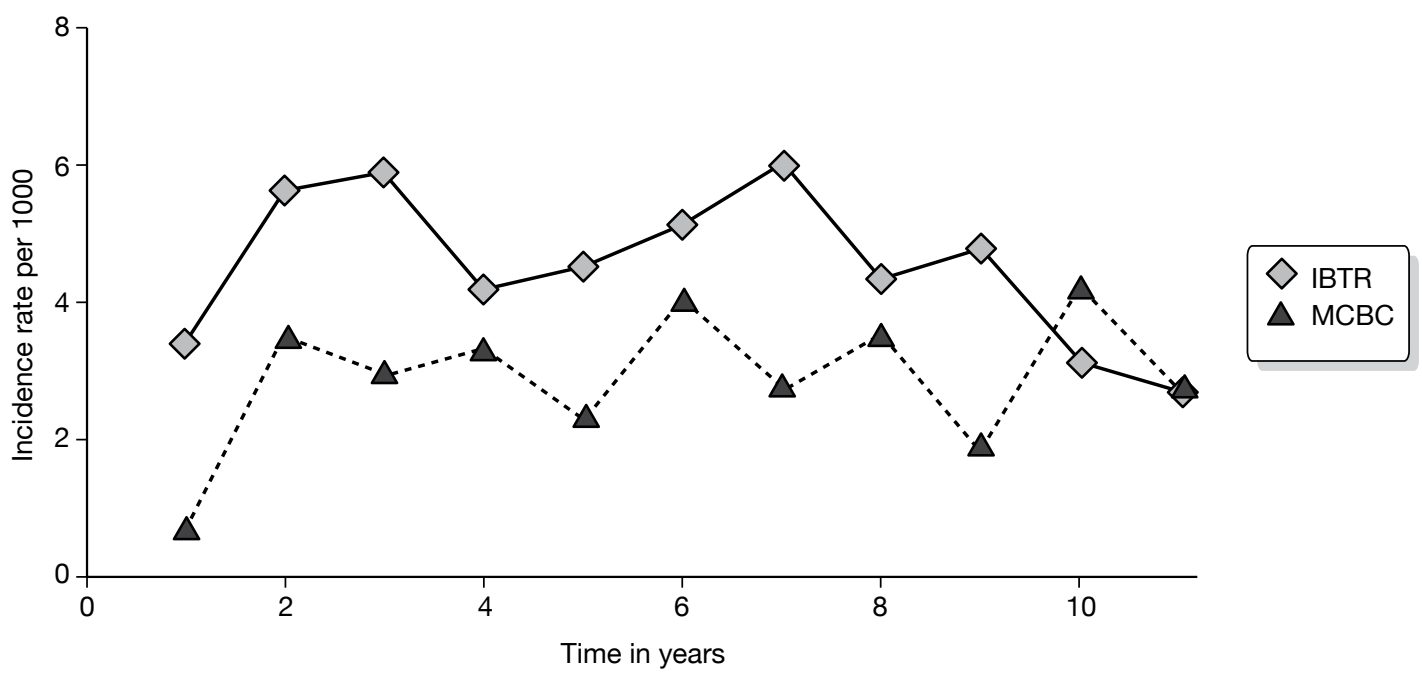

FIGURE 12 Incidence per 1000 per year of IBTR and MCBC occurrence for BCS cohort.

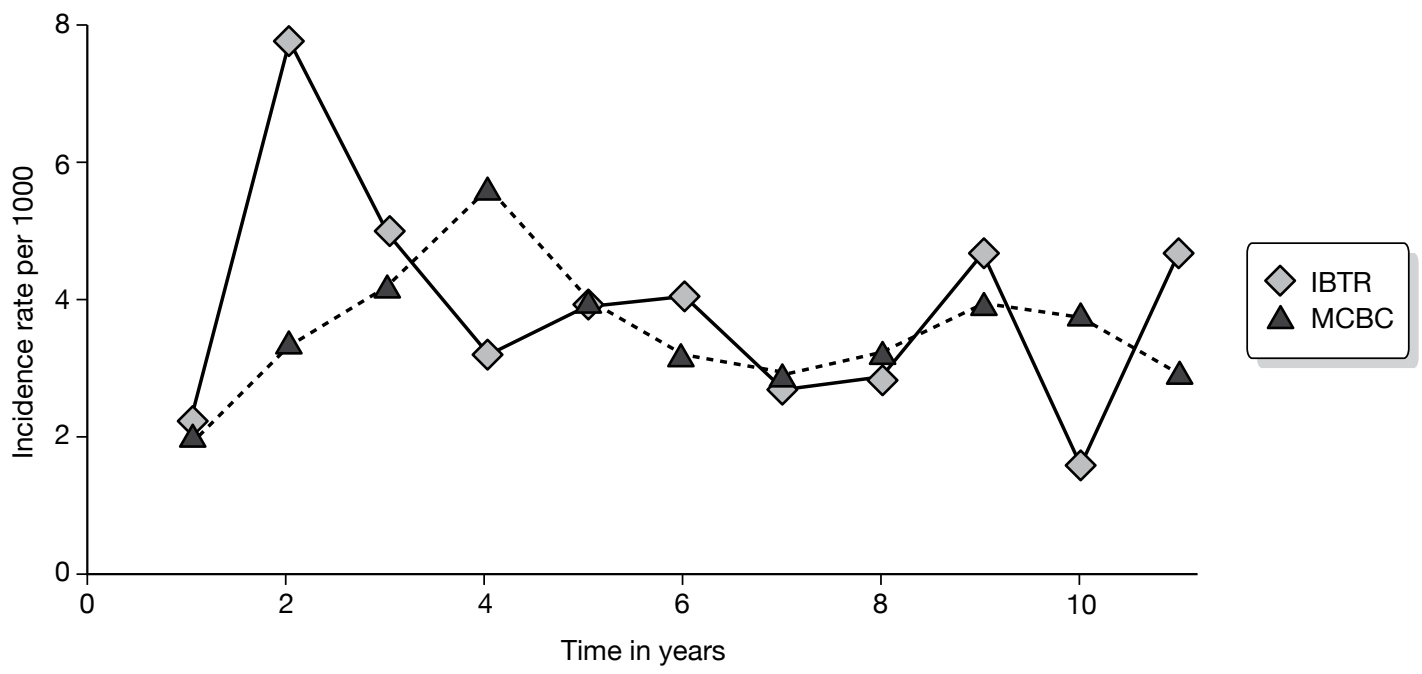

FIGURE 13 Incidence per 1000 per year of IBTR and MCBC occurrence for mastectomy cohort. 


\section{Breast-conserving surgery cohort}

Estimates from Cox proportional hazards regression models for time to outcome (IBTR, MCBC, death from all causes and death from breast cancer) in the BCS cohort are shown in Table 22. Tables with details of the univariate regression models are included in Appendix 25.

All risk factors were univariately associated with an increased hazard of IBTR. In particular, age $\leq 34$ years, grade 3 tumour, large tumour $(\geq 20 \mathrm{~mm}$ ), nodal involvement (four or more nodes) and vascular invasion (yes) all had HRs of approximately two or above (Appendix 25, Table 64). In the multiple Cox regression model (Table 22) the estimates were broadly consistent with the univariate models. Grade 3 tumour, age $\leq 34$ and nodal involvement (four or more nodes) in particular were associated with elevated risk of IBTR.

Univariately there was little that was associated with an increased risk of $\mathrm{MCBC}$ in the BCS group of women (Appendix 25, Table 65). Older women were at a reduced risk of MCBC. Women with a primary tumour $\geq 20 \mathrm{~mm}$ were at an increased risk of MCBC, HR 1.60 (95\% CI 1.08 to 2.38).

The Kaplan-Meier failure curves in Figure 14 show that women who experienced IBTR were at an increased risk of death from all causes. All risk factors were associated univariately with an increased risk of all-cause death although there was no evidence that the group aged 35-49 years was different in terms of all-cause mortality to the reference group (Appendix 25, Table 66). In the multiple Cox regression model (Table 22) all of the risk factors were associated with an increased risk of death, but the youngest age group (age $\leq 34$ years) was no longer different from the reference group. IBTR was a risk factor, which was associated with an increased risk of death univariately, HR 2.29 (95\% CI 1.97 to 2.67). In the multiple Cox regression model, adding IBTR was still associated with an increased risk of death, HR 1.76 (95\% CI 1.51 to 2.05). There was no evidence that MCBC was related to an elevated risk of death in the BCS cohort, HR 1.05 (95\% CI 0.80 to 1.39 ) (see Figure 14).

All risk factors were associated univariately with an increased risk of death from breast cancer (Appendix 25, Table 67). In particular, the worst prognostic categories of each risk factor were associated with an increased risk of death from breast cancer. IBTR was also associated with an increased risk of death from breast cancer (Figure 15). MCBC was excluded from the models as a risk factor as there were only three cases out of $260 \mathrm{MCBC}$ cases that were coded as having died from breast cancer. In the multiple Cox regression model (Table 22) there was no evidence that the age groups younger than the reference group were different in terms of risk of death from breast cancer. The remaining risk factors were associated with an increased risk of death from breast cancer and after adjusting for the other risk factors IBTR was an independent predictor, HR 2.13 (95\% CI 1.78 to 2.56 ).

\section{Mastectomy cohort}

Estimates from Cox proportional hazards regression models for time to outcome (IBTR, MCBC, death from all causes and death from breast cancer) in the mastectomy cohort are shown in Table 23. Tables with details of the univariate regression models are included in Appendix 25.

Univariately the worst prognosis levels of the risk factors were associated with an increased risk of IBTR in women treated with mastectomy (Appendix 25, Table 68). In the multiple Cox regression model the increased risk was associated with grade 3 primary tumour, nodal involvement (four or more nodes) and vascular invasion (yes).

Univariately the worst levels of risk factors were associated with an increased risk of MCBC in cases treated with mastectomy (Appendix 25, Table 69). In the multiple Cox regression model, older women were at reduced risk of contralateral tumour, adjusting for other risk factors 


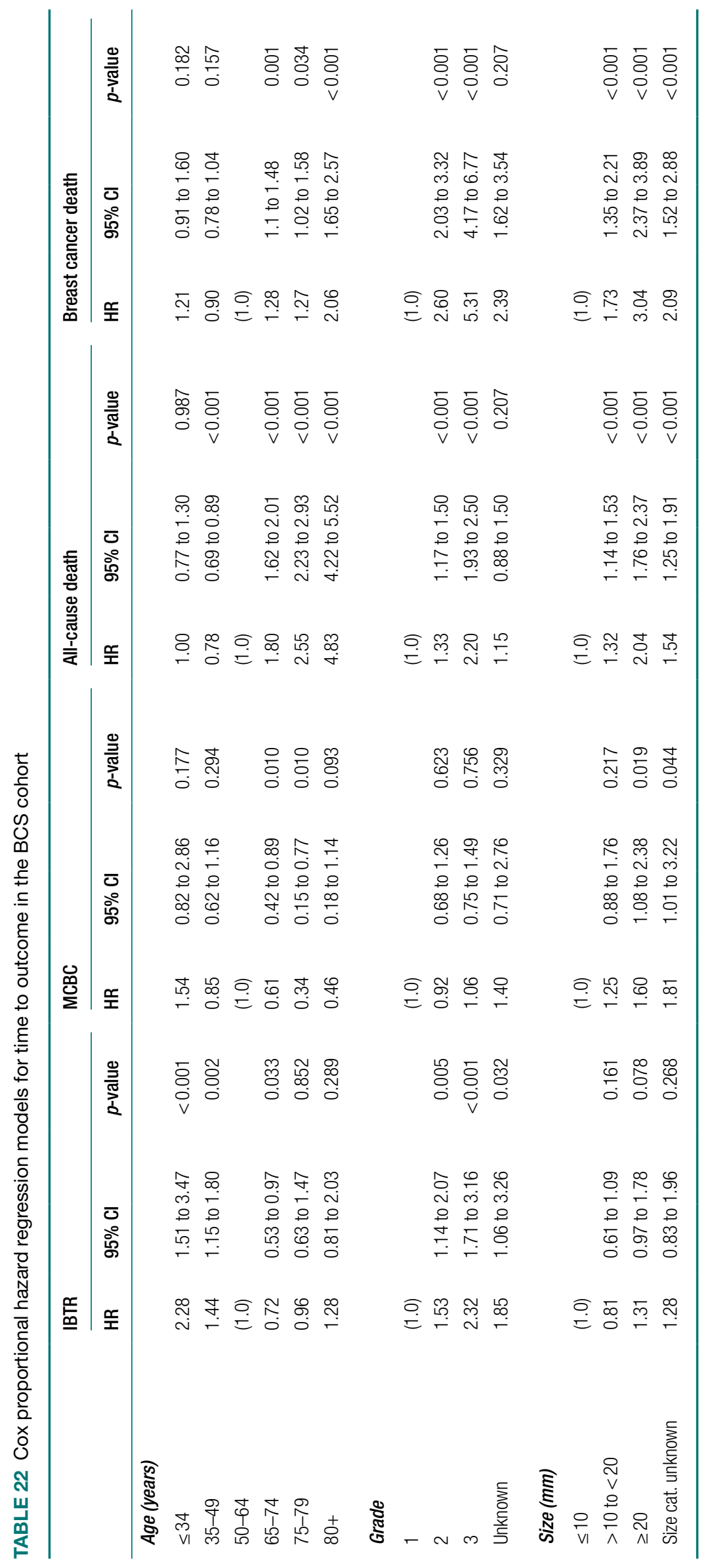




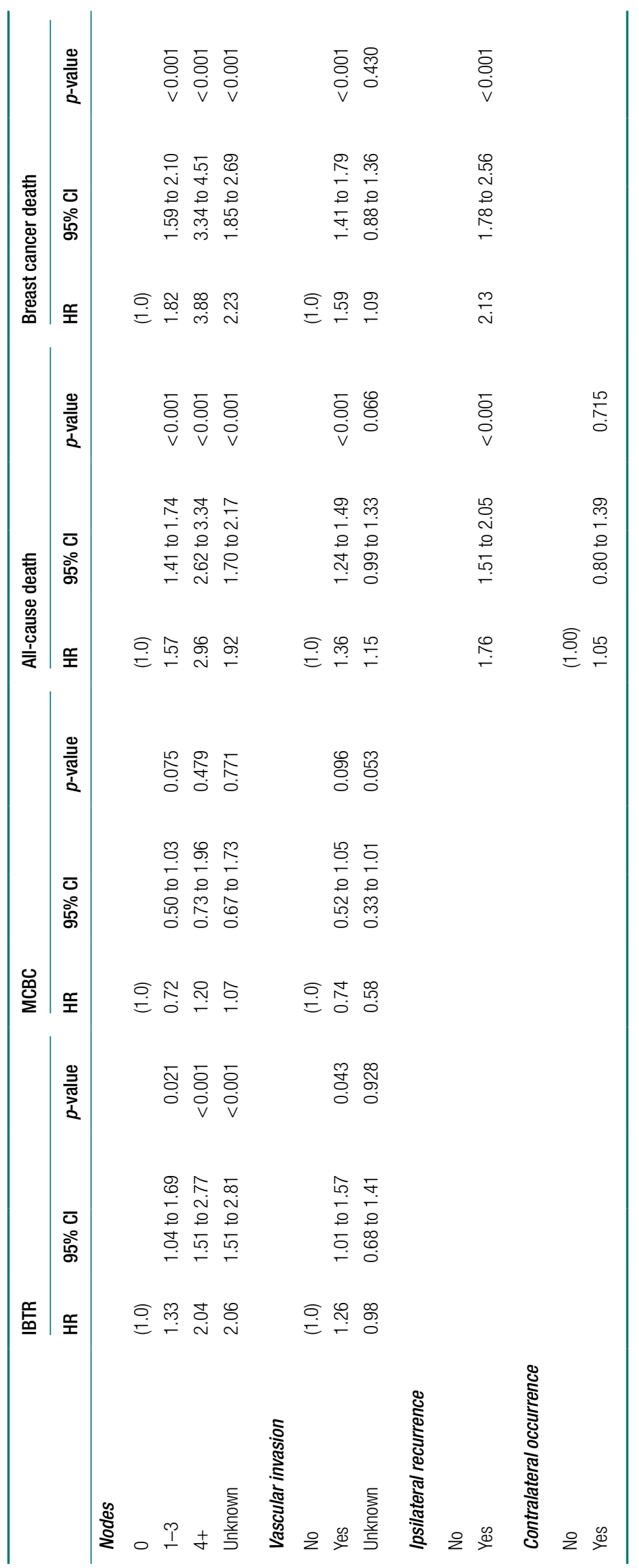




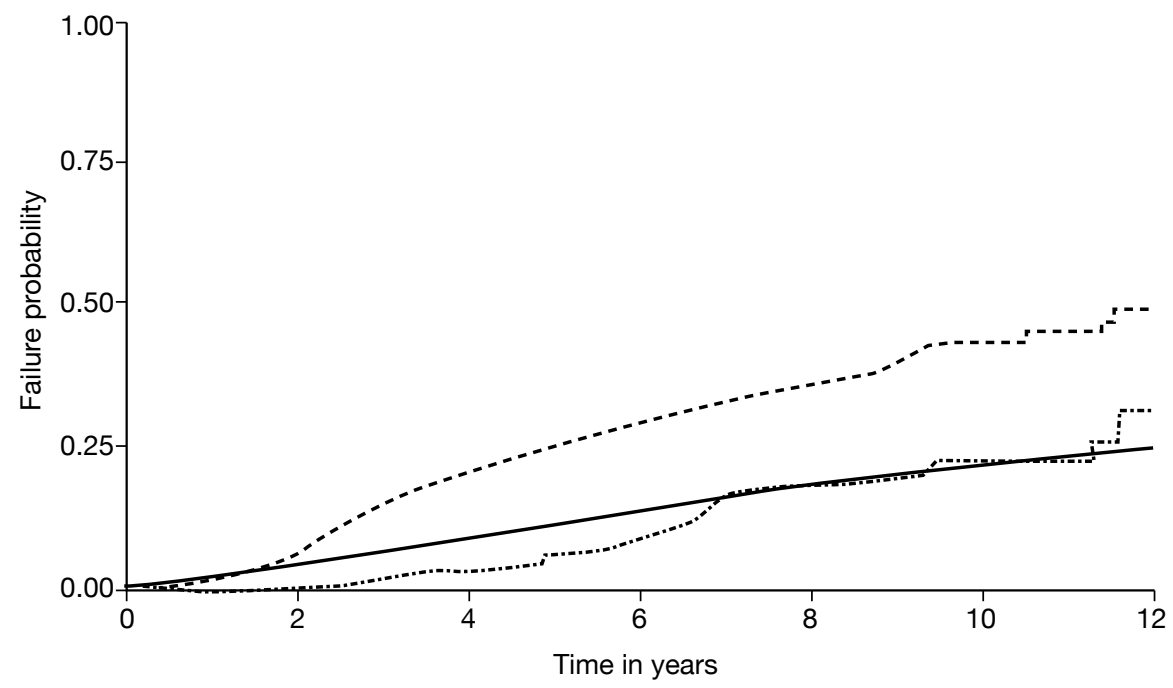

FIGURE 14 Kaplan-Meier failure curves for BCS cohort for time to death from all causes by disease status. BC, breast cancer.

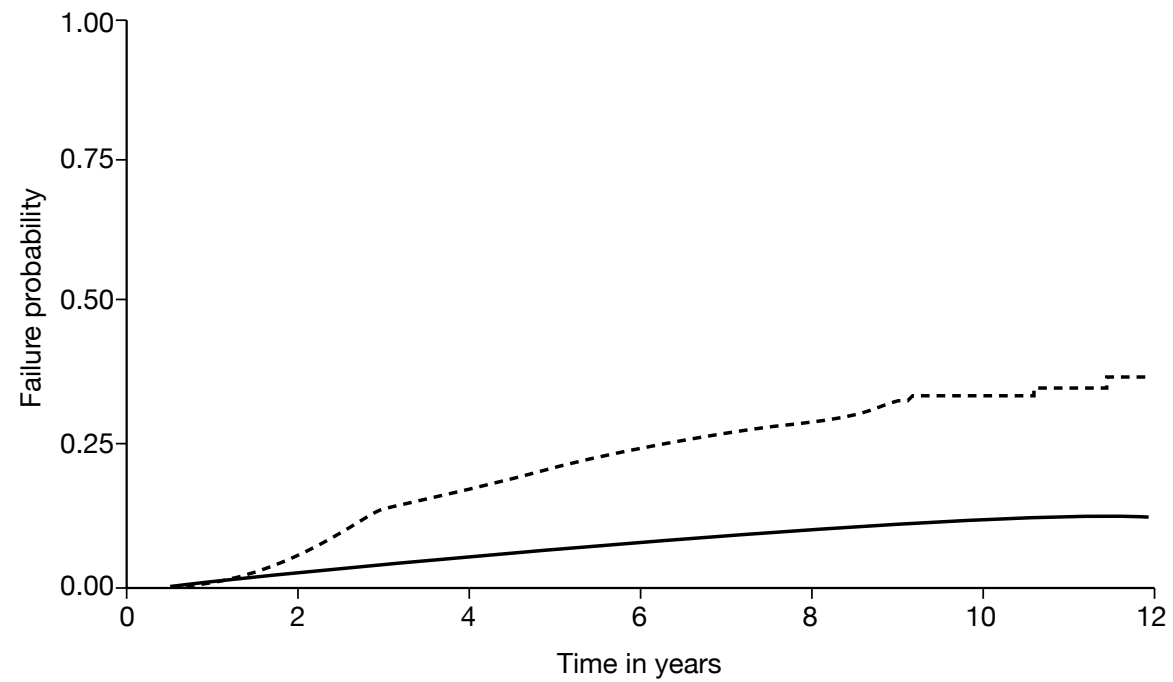

FIGURE 15 Kaplan-Meier failure curves for BCS cohort for time to death from breast cancer by disease status. BC, breast cancer.

(compared to the reference screening age group). Tumour size $(\geq 20 \mathrm{~mm})$ and nodal involvement (four or more nodes) were associated with an increased risk of MCBC.

All risk factors were associated univariately with increased risk of all-cause death, although there was evidence that risk of death from all causes in the 35- to 49-year age group of women was slightly reduced (Appendix 25, Table 70). In the multiple Cox regression model all the risk factors were associated with an increased risk of death, but the youngest age group ( $\leq 34$ years) had a similar risk of all-cause death as that of the reference age group. IBTR was a risk factor for death from all causes in a univariate analysis, HR 2.14 (95\% CI 1.86 to 2.47), but MCBC was not, HR 1.01 (95\% CI 0.83 to 1.24) (Figure 16). The estimates of the HRs for all risk factors from the univariate model were attenuated slightly in the multiple Cox model and IBTR was still associated with increased risk of death. However, a contralateral recurrence was not associated with an elevated risk of all-cause death in the mastectomy cohort. 


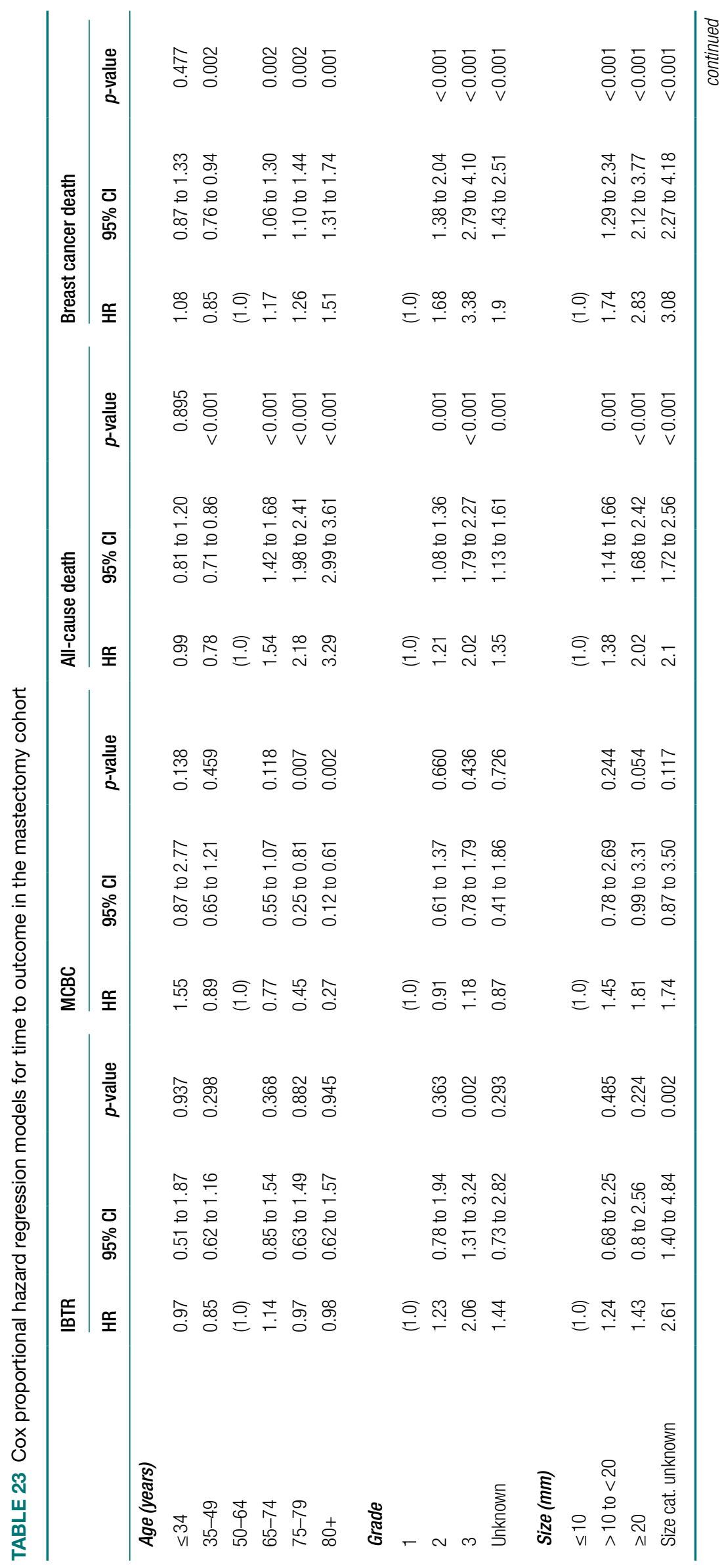




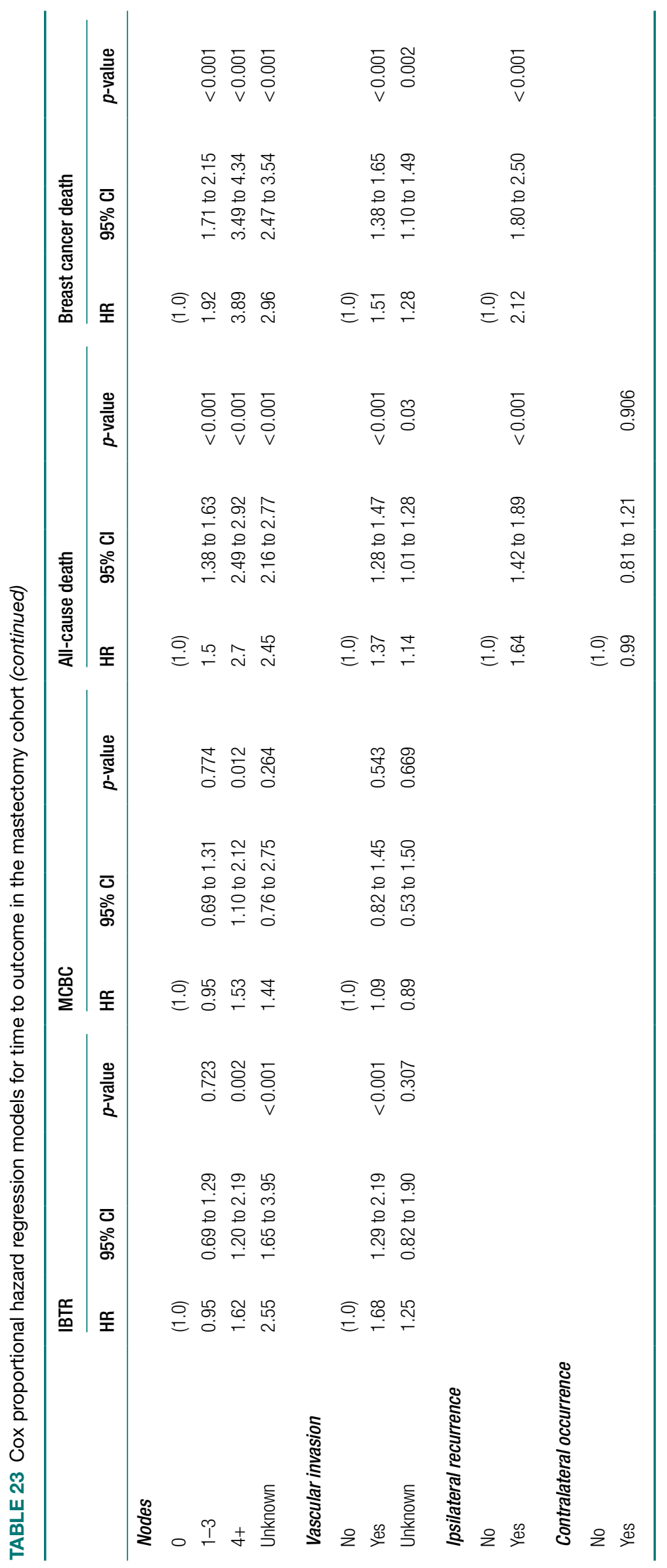


All risk factors were associated univariately with an increased risk of breast cancer death although there was no evidence that the group aged 35-49 years were different in terms of risk of death from breast cancer compared with the reference group (Appendix 25, Table 71). IBTR was a risk factor which was associated with an increased risk of death from breast cancer, HR 2.78 (95\% CI 2.37 to 3.27) (Figure 17). In the multiple Cox regression model (Table 23) all of the risk factors were associated with an increased risk of death, but in the youngest age group (age $\leq 34$ years) the risk of death from breast cancer was reduced. There was no evidence that, after correcting for other risk factors, this age group is at a different risk of death from breast cancer compared with the reference group. In the multiple Cox regression model IBTR was still associated with an increased risk of death from breast cancer, HR 2.12 (95\% CI 1.80 to 2.50). This indicates that an IBTR was related to an increased risk of death from breast cancer even after adjusting for all the other risk factors. MCBC was excluded from the models as a risk factor, as there were only six out of $262 \mathrm{MCBC}$ cases that were coded as having died from breast cancer.

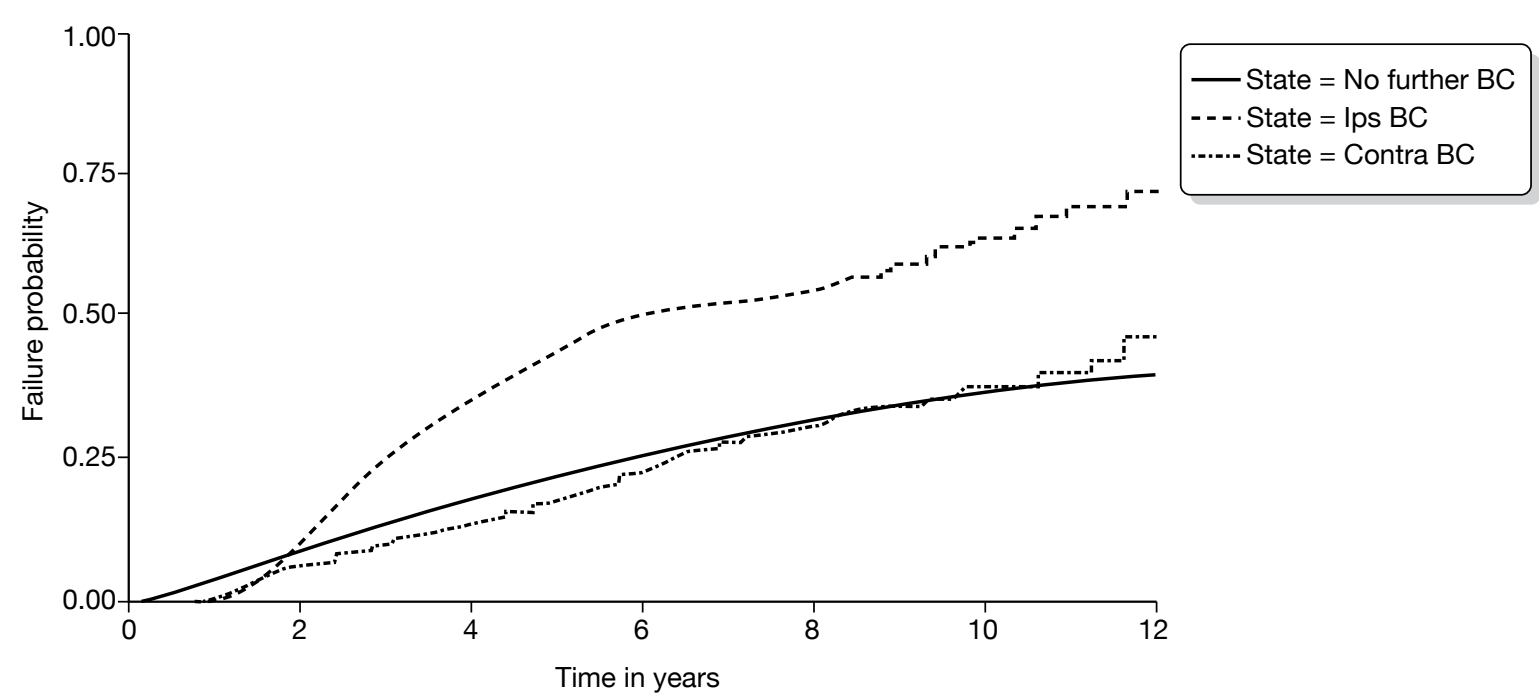

FIGURE 16 Kaplan-Meier failure curves for mastectomy cohort for time to death from all causes by disease status. BC, breast cancer.

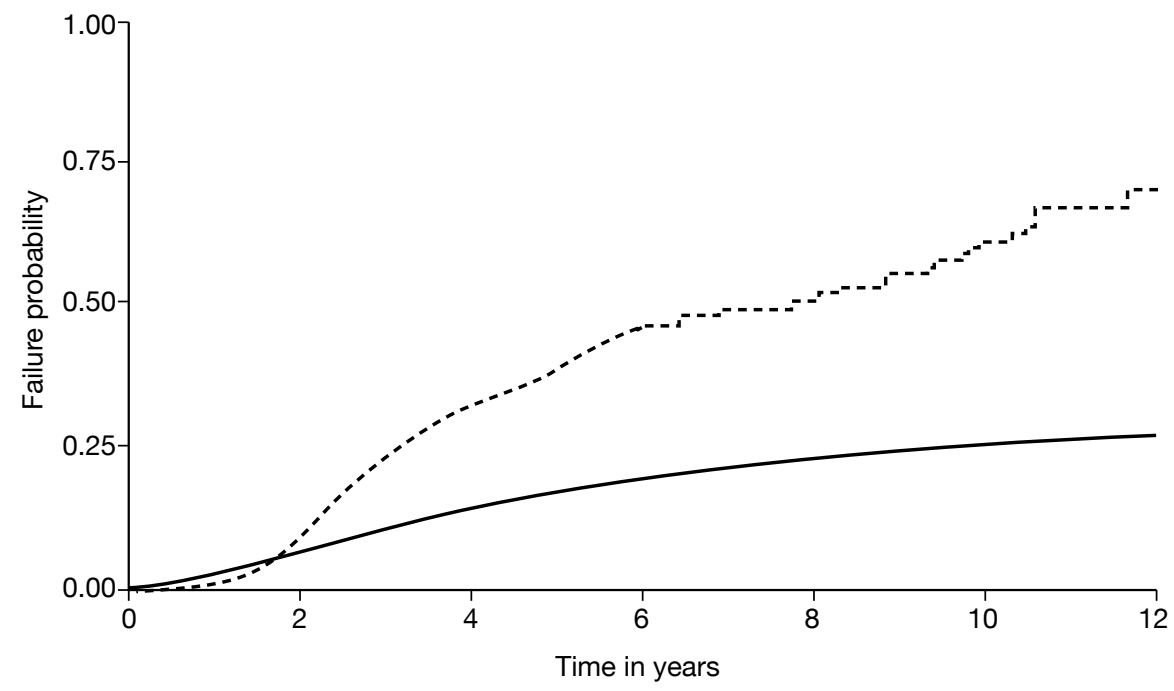

FIGURE 17 Kaplan-Meier failure curves for mastectomy cohort for time to death from breast cancer by disease status. $\mathrm{BC}$, breast cancer. 


\section{WMCIU second tumour cohorts}

The WMCIU database contained information on 2045 ipsilateral tumours and 1355 contralateral tumours, defined as 'second' tumours, in women diagnosed with breast cancer from 1 January 1990 to 31 January 2007. Second tumours were excluded from our analysis if the primary tumour could not be identified or management of the primary tumour did not include a surgical treatment (Figures 18 and 19). A further exclusion category was a non-invasive primary tumour. However, after exclusion on the previous criteria there were no women remaining who had experienced a non-invasive primary tumour. Further exclusions were made if there was no information on risk factors recorded or if date of death was the same as the date of diagnosis. In the event of multiple second tumours in the same breast, or in the contralateral breast, the tumour with the worst prognosis as defined by the Nottingham Prognostic Index was used. ${ }^{73}$ Descriptive information on both these cohorts of women is contained in Tables 24 and 25.

\section{Methods}

We modelled the following risk factors relating to characteristics of the primary tumour: age at diagnosis ( $\leq 34,35$ to $49,50-64,65-74,75$ to $79, \geq 80$ years), grade of primary tumour (grade 1 , grade 2 , grade 3 , grade unknown), size of primary tumour ( $\leq 10 \mathrm{~mm},>10 \mathrm{~mm}$ to $<20 \mathrm{~mm}$, $\geq 20 \mathrm{~mm}$, size unknown), nodal status (no nodes involved, one to three nodes involved, four or more nodes involved, nodal status unknown), vascular invasion (no, yes, unknown), and type of surgery (BCS or mastectomy). Modelled risk factors associated with the second tumour were time to second tumour ( $<60$ months or $\geq 60$ months) and size of second tumour $(\leq 10 \mathrm{~mm}$, $>10 \mathrm{~mm}$ to $<20 \mathrm{~mm}, \geq 20 \mathrm{~mm}$, size unknown). For all categorical risk factors the level of the factor with best prognosis was used as the reference category, with the exception of age at diagnosis where the screening age group (50-64 years of age) was used as the reference category.

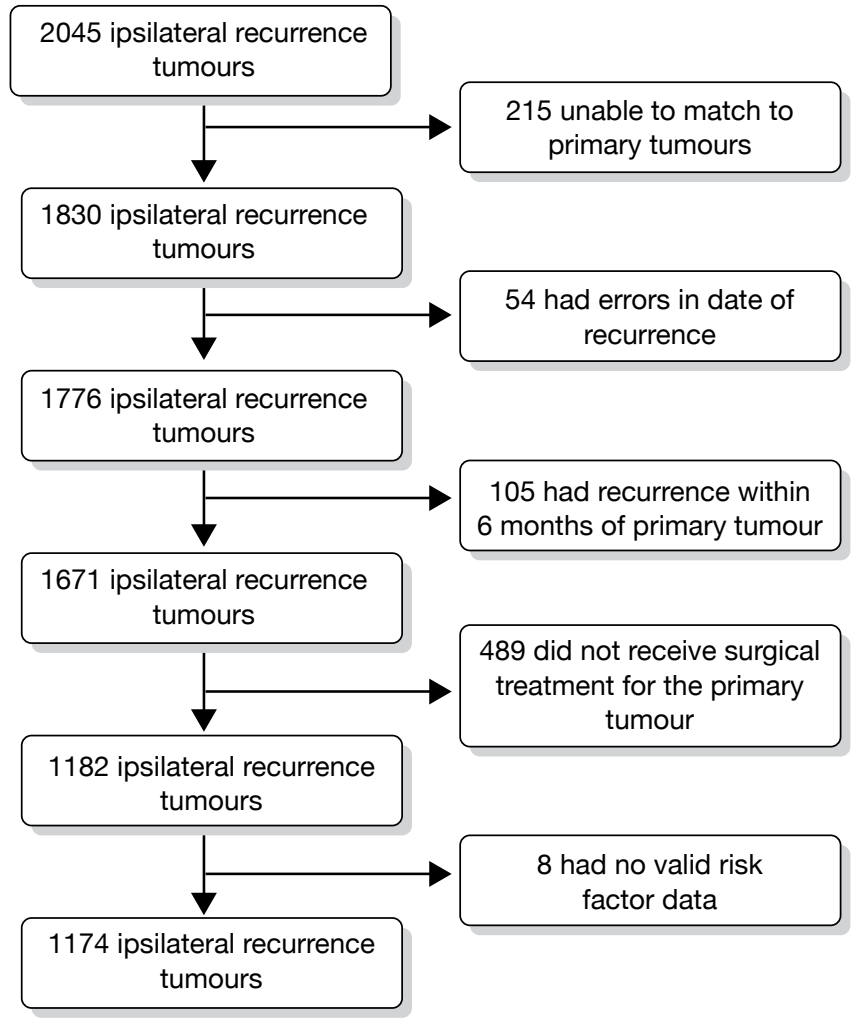

FIGURE 18 Flow chart of ipsilateral recurrence tumours (IBTR). 


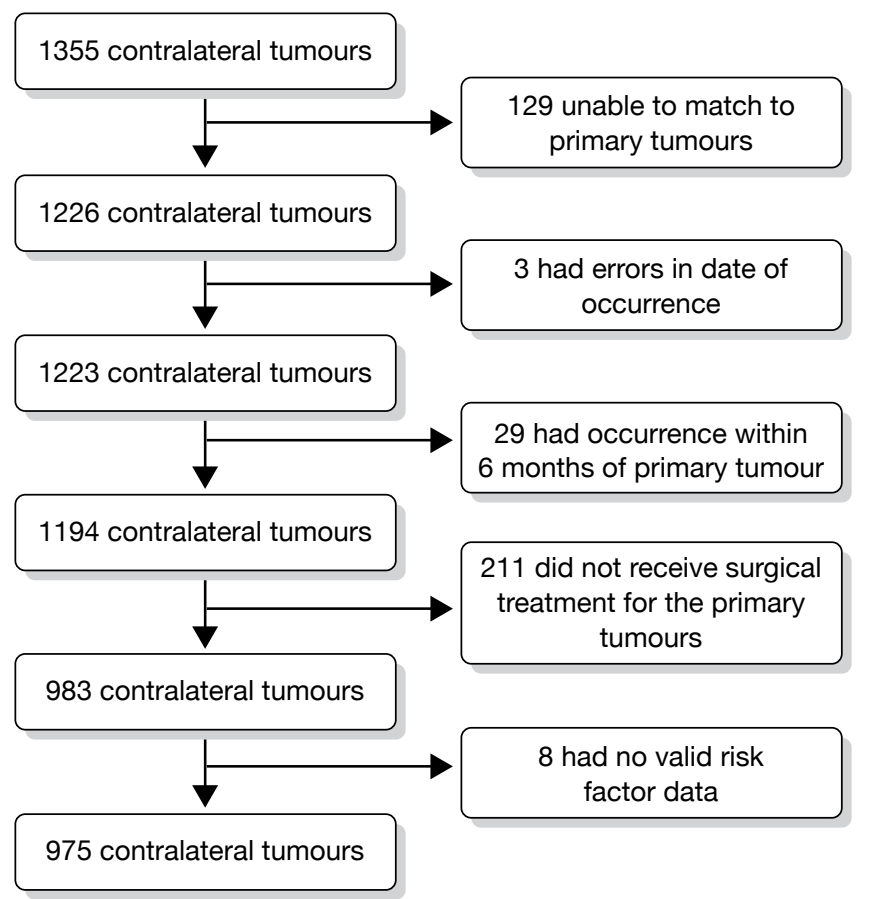

FIGURE 19 Flow chart of contralateral tumours.

TABLE 24 Description of the IBTR cohort

\begin{tabular}{|c|c|c|c|c|c|c|}
\hline & \multicolumn{2}{|c|}{$\begin{array}{l}\text { Received BCS: } 739 \\
(62.9 \%)\end{array}$} & \multicolumn{2}{|c|}{$\begin{array}{l}\text { Received mastectomy: } 435 \\
(37.1 \%)\end{array}$} & \multicolumn{2}{|c|}{ Total: 1174} \\
\hline Death all cause & \multicolumn{2}{|l|}{322} & \multicolumn{2}{|l|}{291} & \multicolumn{2}{|l|}{613} \\
\hline Death breast cancer & \multicolumn{2}{|l|}{219} & \multicolumn{2}{|l|}{223} & \multicolumn{2}{|l|}{442} \\
\hline \multicolumn{7}{|c|}{ Age at diagnosis of primary tumour (years) } \\
\hline Mean (SD) & \multicolumn{2}{|c|}{$55(13)$} & \multicolumn{2}{|c|}{$59(13)$} & \multicolumn{2}{|c|}{$57(14)$} \\
\hline Median (25th, 75th centile) & \multicolumn{2}{|c|}{$53(45,63)$} & \multicolumn{2}{|c|}{$58(49,70)$} & \multicolumn{2}{|c|}{$55(47,67)$} \\
\hline \multirow[t]{2}{*}{ Minimum, maximum } & 20,88 & & \multicolumn{2}{|c|}{24,93} & \multicolumn{2}{|c|}{20,93} \\
\hline & $n$ & $\%$ & $n$ & $\%$ & $n$ & $\%$ \\
\hline \multicolumn{7}{|l|}{ Age categories (years) } \\
\hline$\leq 34$ & 41 & 5.5 & 13 & 3.0 & 54 & 4.6 \\
\hline $35-49$ & 227 & 30.7 & 97 & 22.3 & 324 & 27.6 \\
\hline $50-64$ & 295 & 39.9 & 158 & 36.3 & 453 & 38.6 \\
\hline $65-74$ & 100 & 13.5 & 99 & 22.8 & 199 & 17.0 \\
\hline $75-79$ & 42 & 5.7 & 42 & 9.7 & 84 & 7.2 \\
\hline $80+$ & 34 & 4.6 & 26 & 6.0 & 60 & 5.1 \\
\hline \multicolumn{7}{|l|}{ Grade } \\
\hline 1 & 108 & 14.6 & 30 & 6.9 & 138 & 11.8 \\
\hline 2 & 270 & 36.5 & 178 & 40.9 & 448 & 38.2 \\
\hline 3 & 282 & 38.2 & 188 & 43.2 & 470 & 40.0 \\
\hline Unknown & 79 & 10.7 & 39 & 9.0 & 118 & 10.1 \\
\hline
\end{tabular}


TABLE 24 Description of the IBTR cohort (continued)

\begin{tabular}{|c|c|c|c|c|c|c|}
\hline Size $(m m)$ & \multicolumn{2}{|c|}{$\begin{array}{l}\text { Received BCS: } 739 \\
(62.9 \%)\end{array}$} & \multicolumn{2}{|c|}{$\begin{array}{l}\text { Received mastectomy: } 435 \\
(37.1 \%)\end{array}$} & \multicolumn{2}{|c|}{ Total: 1174} \\
\hline$\leq 10$ & 111 & 15.0 & 13 & 3.0 & 124 & 10.6 \\
\hline$>10$ to $<20$ & 287 & 38.8 & 91 & 20.9 & 378 & 32.2 \\
\hline$\geq 20$ & 247 & 33.4 & 244 & 56.1 & 491 & 41.8 \\
\hline Size unknown & 94 & 12.7 & 87 & 20.0 & 181 & 15.4 \\
\hline \multicolumn{7}{|l|}{ Nodes } \\
\hline 0 & 333 & 45.1 & 150 & 34.5 & 483 & 41.1 \\
\hline $1-3$ & 139 & 18.8 & 96 & 22.1 & 235 & 20.0 \\
\hline $4+$ & 72 & 9.7 & 126 & 29.0 & 198 & 16.9 \\
\hline Unknown & 195 & 26.4 & 63 & 14.5 & 258 & 22.0 \\
\hline \multicolumn{7}{|l|}{ Vascular invasion } \\
\hline No & 376 & 50.9 & 152 & 34.9 & 528 & 45.0 \\
\hline Yes & 194 & 26.3 & 193 & 44.4 & 387 & 33.0 \\
\hline Unknown & 169 & 22.9 & 90 & 20.7 & 259 & 22.1 \\
\hline \multicolumn{7}{|c|}{ Time to second tumour (months) } \\
\hline Median (25th, 75th centile) & 45 & 22,83 & 34 & 18,64 & 21 & 40.76 \\
\hline$<60$ & 447 & 60.5 & 309 & 71.0 & 756 & 64.4 \\
\hline \multicolumn{7}{|l|}{ Size of recurrence } \\
\hline$\leq 10$ & 140 & 18.9 & 51 & 11.7 & 191 & 16.3 \\
\hline$>10$ to $<20$ & 199 & 26.9 & 61 & 14.0 & 260 & 22.1 \\
\hline$\geq 20$ & 190 & 25.7 & 62 & 14.3 & 252 & 21.5 \\
\hline Size unknown & 210 & 28.4 & 261 & 60.0 & 471 & 40.1 \\
\hline
\end{tabular}

We undertook a multiple Cox regression model that included the risk factors associated with the primary tumour, time to the second tumour event and the size of second tumour. Outcomes for these models were time to death from all causes and time to death from breast cancer.

\section{Results}

There were 1174 women with 3870 years of follow-up in the IBTR cohort, of whom there were 613 deaths from all causes and 442 deaths from breast cancer. Median time from diagnosis of primary tumour to diagnosis of second tumour was 21 months.

Estimates from Cox regression models for time to death from all causes in the IBTR cohort are shown in Table 26. Women with a second tumour of $\geq 20 \mathrm{~mm}$ in maximum diameter were at an elevated risk of death compared with the reference group of $\leq 10 \mathrm{~mm}$, HR 1.75 (95\% CI 1.29 to 2.37). This was also evident from the Kaplan-Meier failure curves in Figure 20.

Estimates from Cox regression models for time to death from breast cancer in the IBTR cohort are shown in Table 26. Women with a second tumour of $\geq 20 \mathrm{~mm}$ were at an elevated risk of death compared with the reference group of $\leq 10 \mathrm{~mm}$, HR 1.99 (95\% CI 1.37 to 2.89). This was also evident from the Kaplan-Meier failure curves in Figure 21. 
TABLE 25 Description of the MCBC occurrence cohort

\begin{tabular}{|c|c|c|c|c|c|c|}
\hline & \multicolumn{2}{|c|}{ Received BCS: 534 (54.8\%) } & \multicolumn{2}{|c|}{$\begin{array}{l}\text { Received mastectomy: } 441 \\
(45.2 \%)\end{array}$} & \multicolumn{2}{|c|}{ Total: 975} \\
\hline Death all cause & 158 & & 200 & & 358 & \\
\hline Death breast cancer & 11 & & 12 & & 23 & \\
\hline \multicolumn{7}{|c|}{ Age at diagnosis of primary tumour (years) } \\
\hline Mean (SD) & $55(11)$ & & $56(12$ & & $55(1$ & \\
\hline Median (25th, 75th centile) & $55(47,62)$ & & $55(47$ & & $55(4$ & \\
\hline \multirow[t]{2}{*}{ Minimum, maximum } & 25,86 & & 26,86 & & 25,8 & \\
\hline & $n$ & $\%$ & $n$ & $\%$ & $n$ & $\%$ \\
\hline \multicolumn{7}{|l|}{ Age categories (years) } \\
\hline$\leq 34$ & 27 & 5.1 & 18 & 41 & 45 & 4.6 \\
\hline $35-49$ & 141 & 26.4 & 122 & 27.7 & 263 & 27.0 \\
\hline $50-64$ & 278 & 52.1 & 188 & 42.6 & 466 & 47.8 \\
\hline $65-74$ & 65 & 12.2 & 80 & 18.1 & 145 & 14.9 \\
\hline $75-79$ & 17 & 3.2 & 24 & 5.4 & 41 & 4.2 \\
\hline $80+$ & 6 & 1.1 & 9 & 2.0 & 15 & 1.5 \\
\hline \multicolumn{7}{|l|}{ Grade } \\
\hline 1 & 113 & 21.2 & 47 & 10.7 & 160 & 16.4 \\
\hline 2 & 199 & 37.3 & 165 & 37.4 & 364 & 37.3 \\
\hline 3 & 150 & 28.1 & 167 & 37.9 & 317 & 32.5 \\
\hline Unknown & 72 & 13.5 & 62 & 14.1 & 134 & 13.7 \\
\hline \multicolumn{7}{|l|}{ Size $(m m)$} \\
\hline$\leq 10$ & 84 & 15.7 & 22 & 5.0 & 106 & 10.9 \\
\hline$>10$ to $<20$ & 249 & 46.6 & 106 & 24.0 & 355 & 36.4 \\
\hline$\geq 20$ & 133 & 24.9 & 241 & 54.6 & 374 & 38.4 \\
\hline Size unknown & 68 & 12.7 & 72 & 16.3 & 140 & 14.4 \\
\hline \multicolumn{7}{|l|}{ Nodes } \\
\hline 0 & 298 & 55.8 & 187 & 42.4 & 485 & 49.7 \\
\hline $1-3$ & 74 & 13.9 & 103 & 23.4 & 177 & 18.2 \\
\hline $4+$ & 24 & 4.5 & 106 & 24.0 & 130 & 13.3 \\
\hline Unknown & 138 & 25.8 & 45 & 10.2 & 183 & 18.8 \\
\hline \multicolumn{7}{|l|}{ Vascular invasion } \\
\hline No & 288 & 53.9 & 187 & 42.4 & 475 & 48.7 \\
\hline Yes & 80 & 15.0 & 144 & 32.7 & 224 & 23.0 \\
\hline Unknown & 166 & 31.1 & 110 & 24.9 & 276 & 28.3 \\
\hline \multicolumn{7}{|c|}{ Time to second tumour (months) } \\
\hline Median (25th, 75th centile) & 60 & 33,95 & 44 & 26,79 & 52 & 29,88 \\
\hline$<60$ & 261 & 49.0 & 281 & 63.4 & 542 & 55.6 \\
\hline \multicolumn{7}{|l|}{ Size of recurrence $(\mathrm{mm})$} \\
\hline$\leq 10$ & 119 & 22.3 & 86 & 19.5 & 205 & 21.0 \\
\hline$>10$ to $<20$ & 185 & 36.6 & 124 & 28.1 & 309 & 31.7 \\
\hline$\geq 20$ & 135 & 25.3 & 119 & 27.0 & 254 & 26.1 \\
\hline Size unknown & 95 & 17.8 & 112 & 25.4 & 207 & 21.2 \\
\hline
\end{tabular}




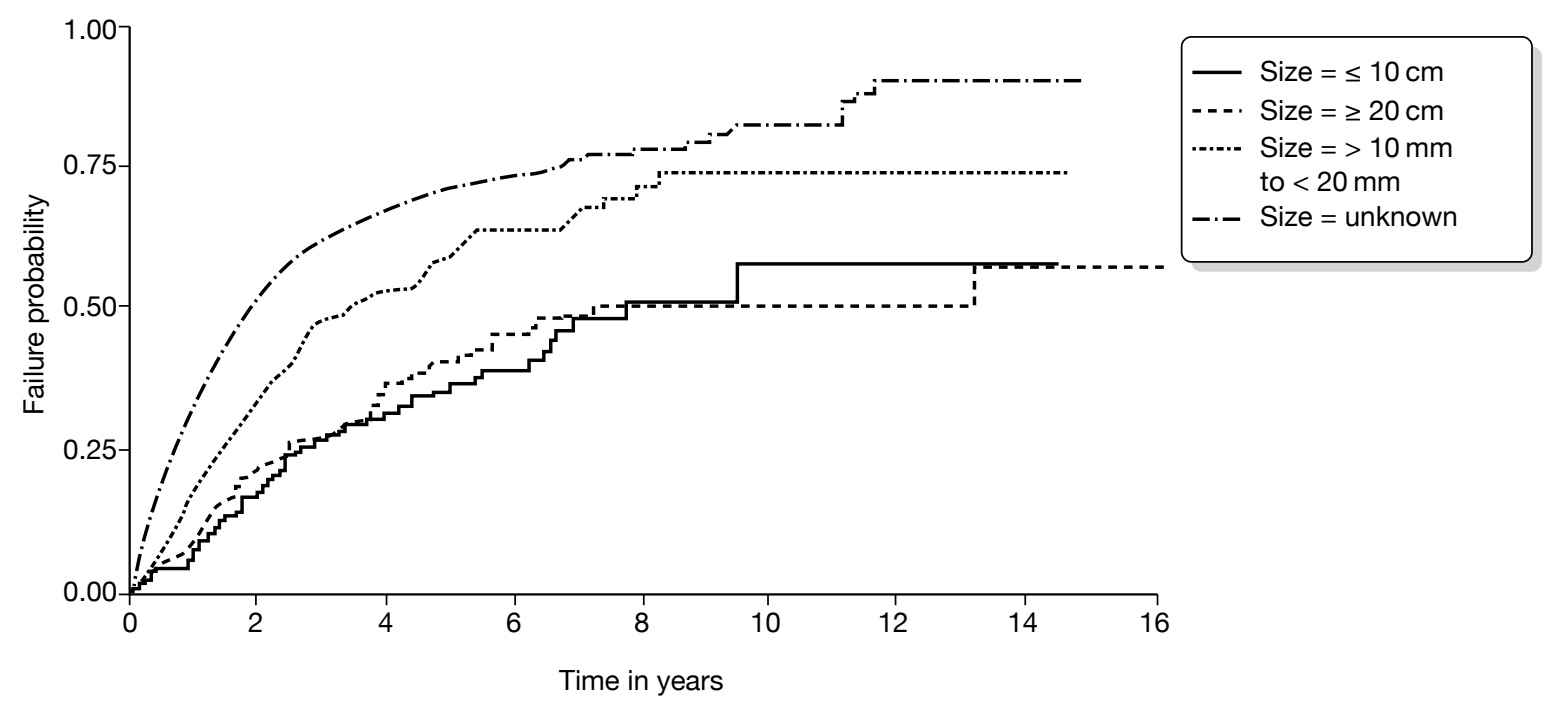

FIGURE 20 Kaplan-Meier failure curves for IBTR cohort for time to death from all causes by size of second tumour.

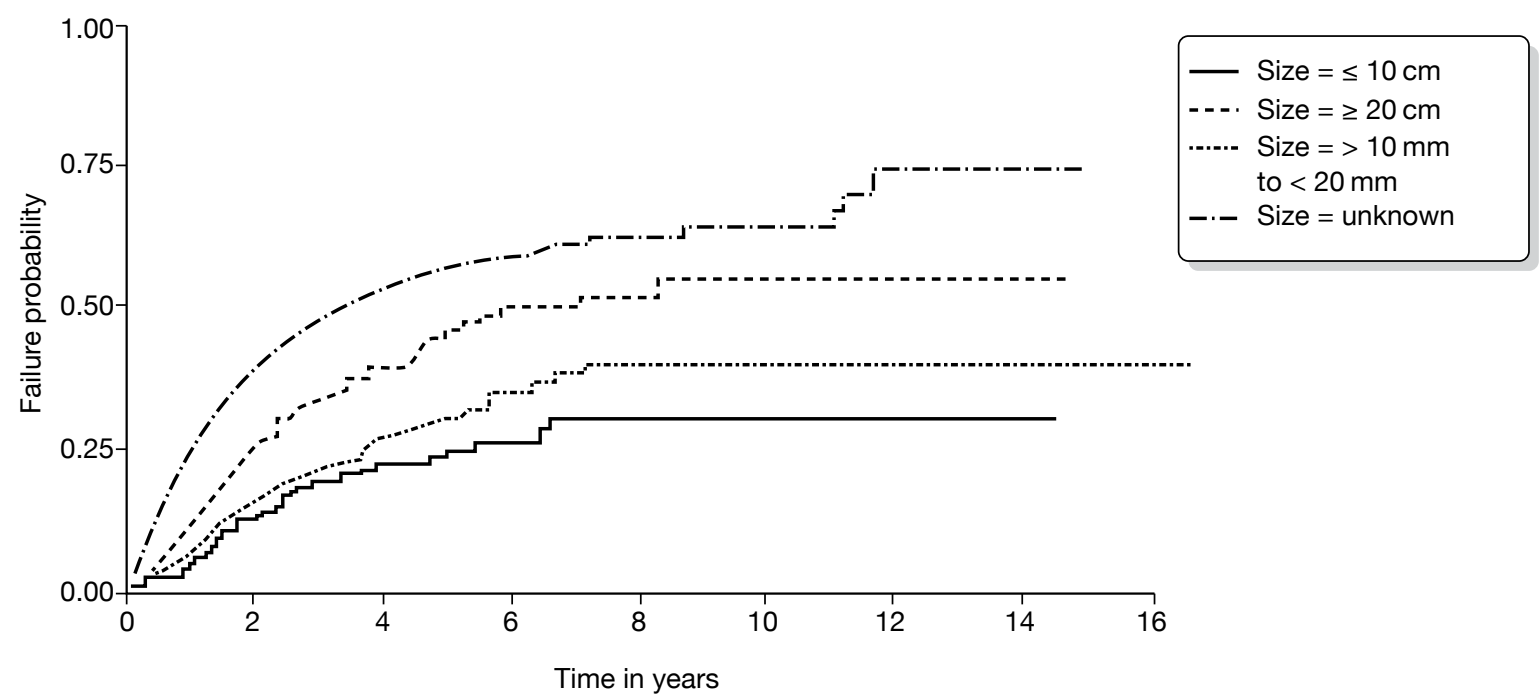

FIGURE 21 Kaplan-Meier failure curves for IBTR cohort for time to death from breast cancer by size of second tumour.

There were 975 women with 4268 years of follow-up in the MCBC tumour cohort, with 358 deaths from all causes and 23 deaths from breast cancer. Median time from diagnosis of primary tumour to diagnosis of second tumour was 52 months.

Estimates from Cox regression models for time to death from all causes in the MCBC cohort are shown in Table 26. Women with a second tumour of $\geq 20 \mathrm{~mm}$ in maximum diameter were at an elevated risk of death compared with the reference group of $\leq 10 \mathrm{~mm}$, HR 2.14 (95\% CI 1.49 to 3.06). This was also evident from the Kaplan-Meier failure curves shown in Figure 22.

Estimates from Cox proportional hazards regression models for time to death from breast cancer in the MCBC cohort are not included. This was because of the relatively few deaths recorded from breast cancer (see Table 25 and Figure 23). A univariate analysis of the size of second tumour showed that women with a second tumour of $\geq 20 \mathrm{~mm}$ in maximum diameter were at an 


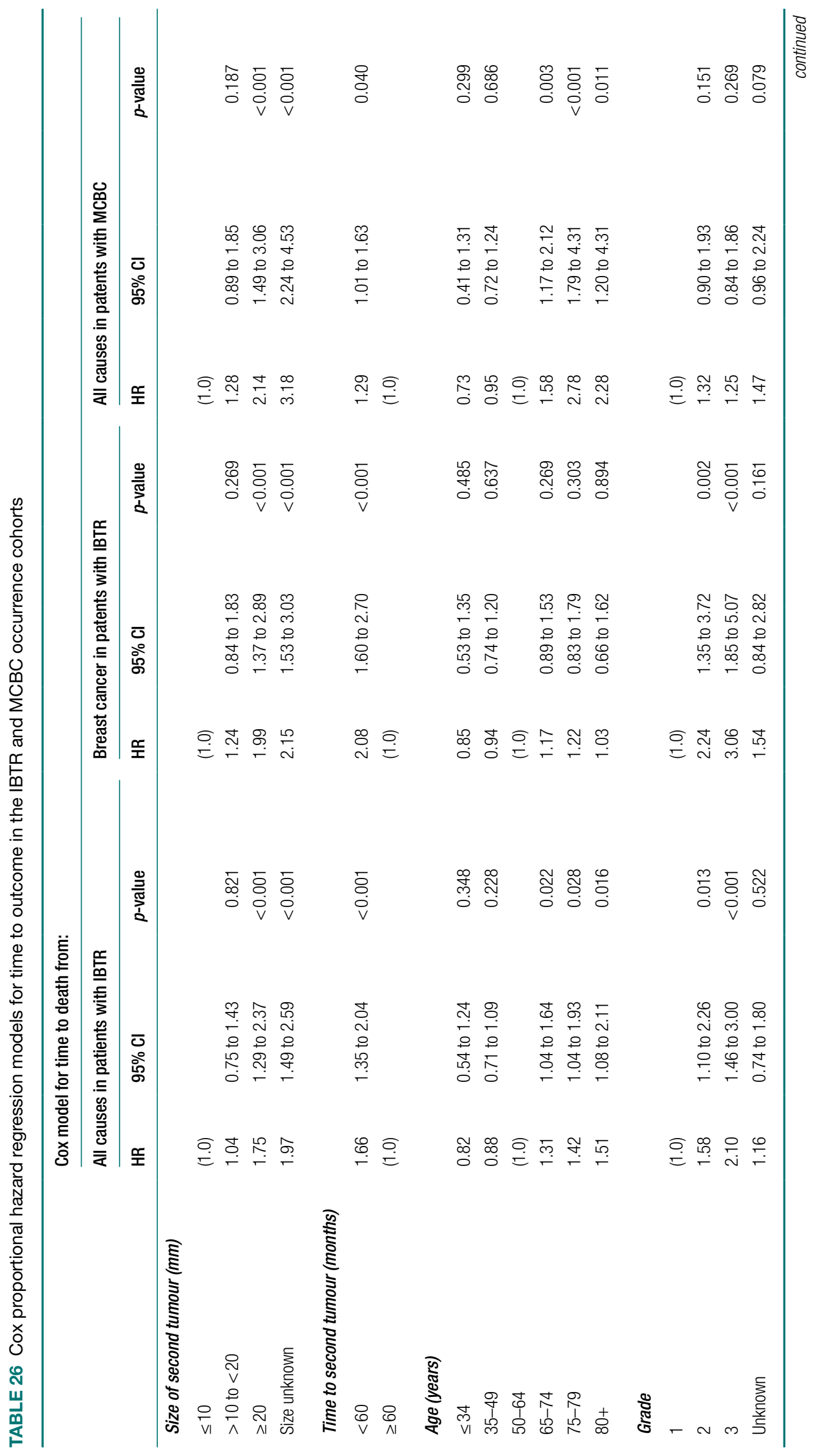




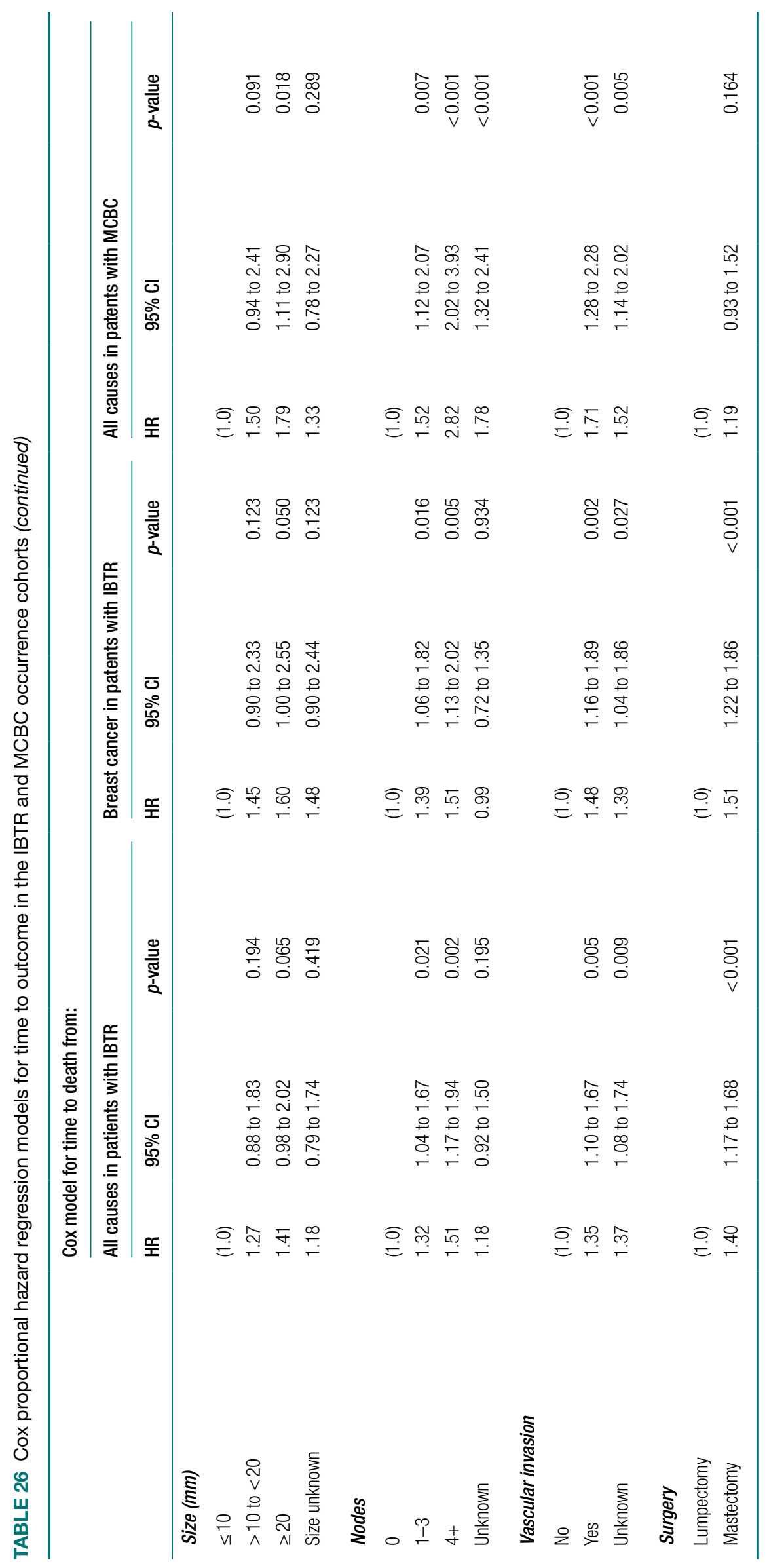




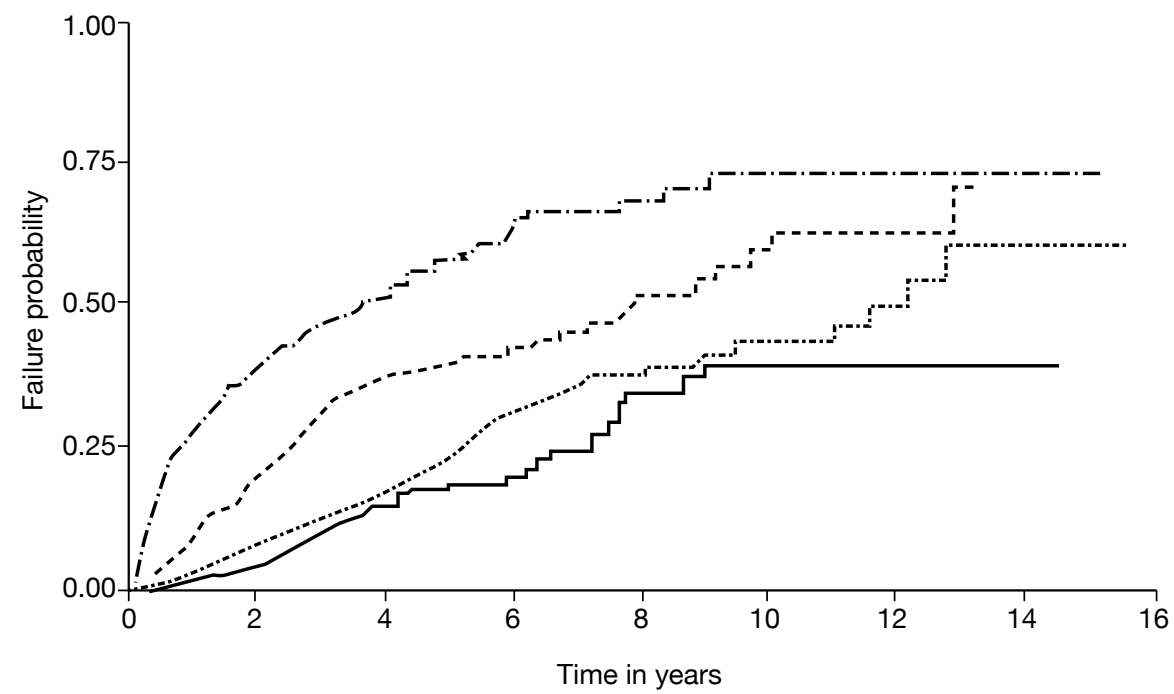

FIGURE 22 Kaplan-Meier failure curves for MCBC occurrence cohort for time to death from all causes by size of second tumour.

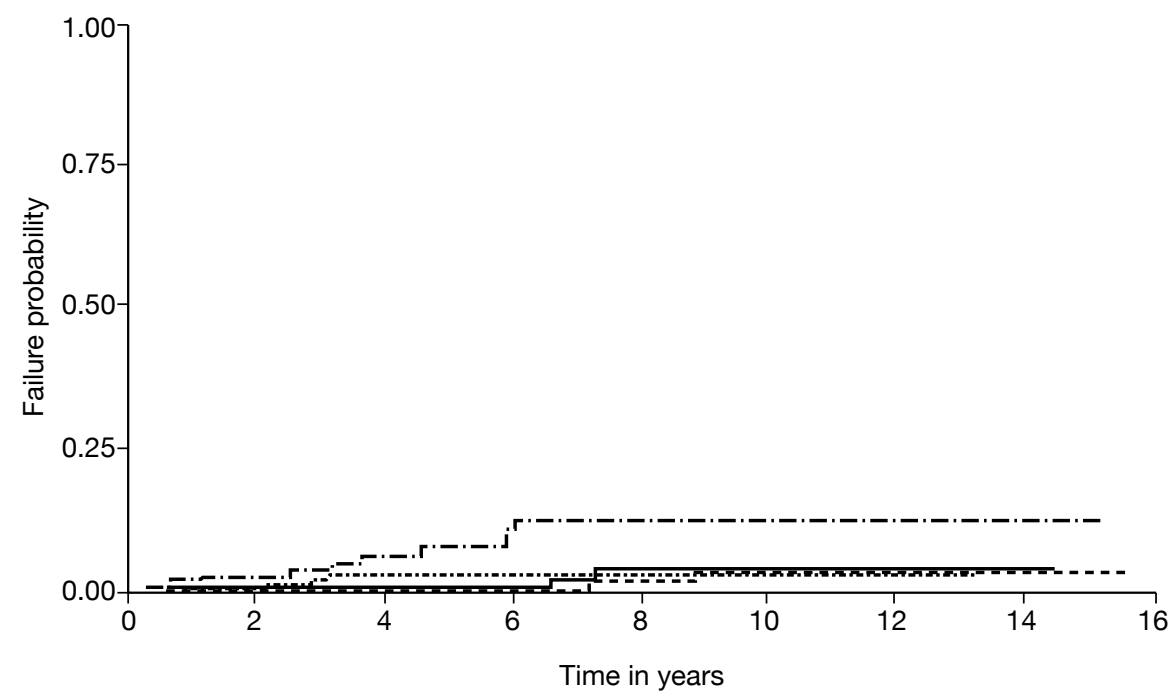

FIGURE 23 Kaplan-Meier failure curves for MCBC occurrence cohort for time to death from breast cancer by size of second tumour.

elevated risk of death compared with the reference group of $\leq 10 \mathrm{~mm}$, HR 1.99 (95\% CI 1.38 to 2.83) (see also Figure 23).

\section{Edinburgh breast cancer data set}

We also analysed a smaller but cleaner data set from Edinburgh to confirm risk factors for IBTR and MCBC, and also risk factors for all-cause death and breast cancer death. This analysis is included in Appendix 26. In summary, this analysis confirmed the findings from the WMCIU analysis. Women who experienced IBTR were at an increased risk of death from all causes (HR $1.87,95 \%$ CI 1.41 to 2.49 ) and of death from breast cancer (HR 2.69, 95\% CI 1.94 to 3.72). This was not true for women who experienced MCBC; there was no evidence that these women were at increased risk. 


\section{Summary}

The WMCIU data set confirms the known risk factors for death from breast cancer, which are tumour size, grade, lymph node involvement, lymphovascular invasion and age, and demonstrates that IBTR is an independent variable affecting survival. This was also confirmed in a second data set. However, the occurrence of MCBC did not adversely affect survival. In those women experiencing a second tumour, the size of the second tumour is important, with tumours of $>20 \mathrm{~mm}$ in maximum diameter being at a significantly greater risk of death than those with either no second tumour or those with a second tumour $<10 \mathrm{~mm}$ in maximum diameter.

We have shown that IBTR has an adverse effect on survival. This is independent of the primary tumour and in our analysis of BCS the HR was 2.13 (95\% CI 1.78 to 2.56). This has been found in several previously published series. ${ }^{20,77-80}$ Furthermore, the time to the event is important, with those events that happen later having a survival advantage compared with those that occur earlier. This was also found in a combined analysis of five National Surgical Adjuvant Breast and Bowel Project trials of women treated by BCS who had node-negative disease ${ }^{77}$ and the Tuscan second breast cancer case series. ${ }^{81}$ 


\section{Chapter 7}

\section{Economic evaluation}

\section{Introduction}

This chapter has four main sections: a brief outline of the principles of economic evaluation, followed by sections reporting the methods, results and summary of the findings of the economic evaluation.

The objectives of this chapter are to determine whether or not (1) any method of surveillance could potentially be cost-effective for the whole population of women eligible for the service and (2) the method of surveillance should be varied between subgroups of women who are eligible for surveillance following surgery for breast cancer.

\section{Principles of economic evaluation}

\section{A brief introduction to economic evaluation}

The decision to use resources to provide one method of breast cancer surveillance means that the opportunity to use these resources in other desirable ways (either to provide another method of surveillance or to meet an entirely different health need) is given up. The cost of this decision is the benefits (health gains, etc.) that could have been obtained had the resources been used in another desirable way. This is the economic notion of 'opportunity cost'. Strictly speaking, the opportunity cost of a decision to use resources in one way is equivalent to the benefits that could have been obtained had the resources been used to provide the next best alternative. Economic evaluation is a method of providing decision-makers with information about the opportunity cost of the decisions that could be made. It does this by comparing alternative courses of action in terms of both their costs and consequences. ${ }^{82}$

An economic evaluation in this context would involve assessing the relative costs and benefits associated with alternative surveillance regimens for breast cancer. The objective of such an economic evaluation would be to provide information to assist decision-makers in the allocation of available resources so that benefits could be maximised. A cost-effectiveness plane (Figure 24) illustrates how an economic evaluation brings together information on costs and benefits. The vertical axis represents the difference in costs between surveillance regimens (e.g. mammography vs MRI). The horizontal axis represents differences in effectiveness between the two regimens.

In the north-west and south-east quadrants of Figure 24 a clear decision about which surveillance regimen should be preferred is provided because one or other regimen is less costly but more effective (i.e. it dominates the other treatment). In the north-west quadrant the experimental regimen is more costly and provides less benefit, therefore the control regimen is more efficient (is dominant). In the south-east quadrant the opposite situation occurs and the experimental regimen is more efficient (is dominant), as it is less costly and provides more benefit. The circle in the centre of the figure represents the possibility that no meaningful differences in costs or benefits exist between the regimens and for practical purposes the two regimens are equally efficient. 


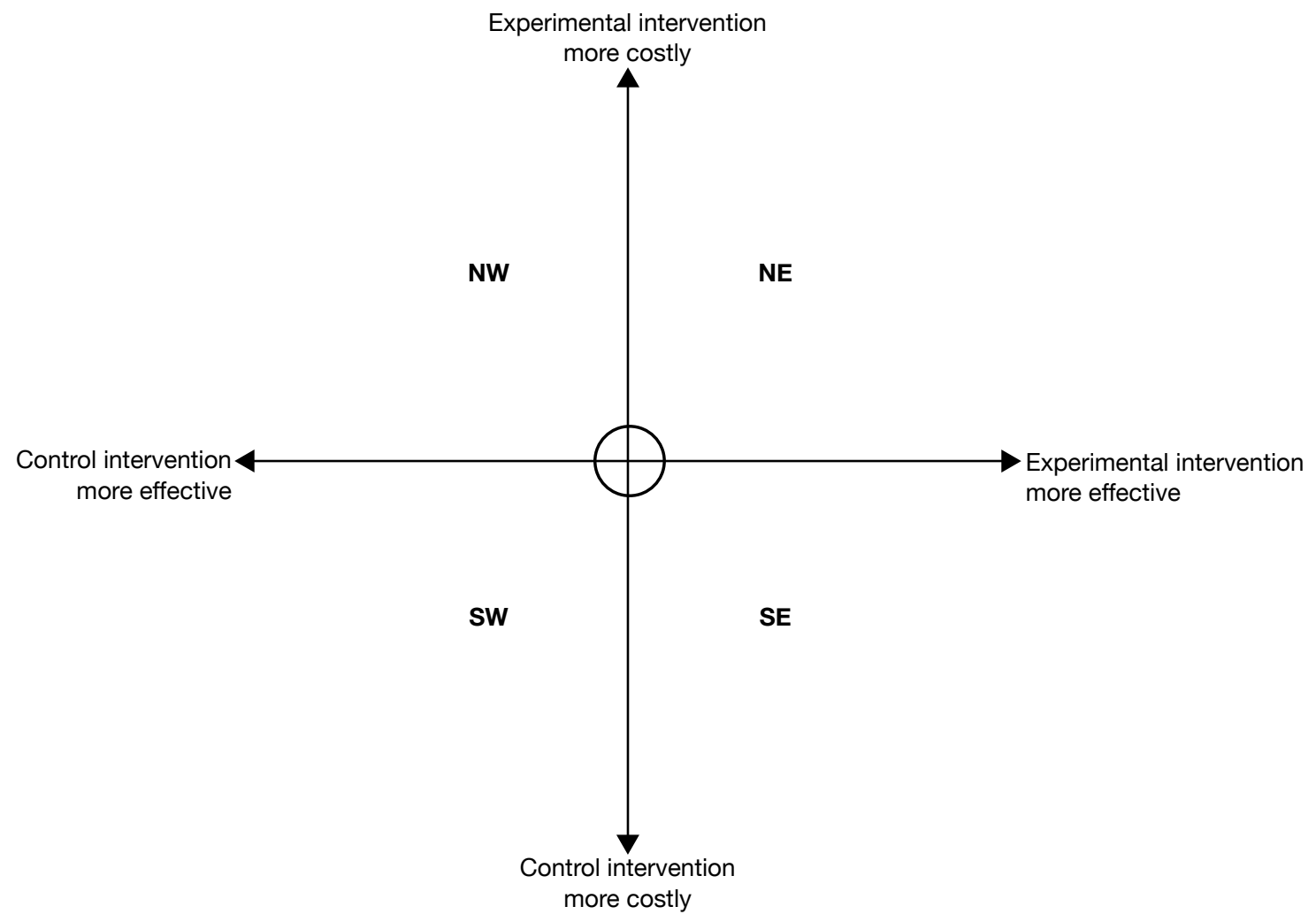

FIGURE 24 Relationship between the difference in costs and effects between a new (experimental) method of surveillance and an alternative (control) method. NE, north-east; NW, north-west; SE, south-east; SW, south-west.

In the two remaining areas of the figure, the north-east and south-west quadrants, a judgement is required as to whether the more effective regimen is worth the extra cost. To aid these judgements, information can be provided in terms of an incremental cost-effectiveness ratio (ICER). The higher the ICER of one intervention compared with another, the less likely it is that this intervention will be considered efficient.

\section{Methods}

\section{Economic modelling of alternative surveillance regimens}

A surveillance programme needs to be not only effective, but also cost-effective. Using Markov modelling methods, the cost-effectiveness of various surveillance programmes is compared. The economic model describes the pathway of care of individuals from the point where they received treatment for breast cancer and will receive some form of ongoing surveillance. This includes their longer-term (ideally their lifetime) costs and consequences, including those that might arise from any subsequent cancers. Surveillance can be considered as an event undertaken at discrete intervals and repeated over time and hence a Markov model was developed. This can be used to describe the logical and temporal sequence of events following the implementation of alternative surveillance regimens. We used the model to provide the estimated costs and outcomes for a selected period for a cohort of women for different surveillance regimens.

\section{The model}

Markov models comprise a set of states and at any point in time an individual will be in one of these states and will stay in that state for a defined period of time (the cycle length) before they 
are allowed to move to another state. The cycle length must be a period relevant to the condition considered (e.g. 6 months, 1 year, 18 months, etc.) At the end of each cycle, individuals can remain in the state in which they started the cycle or move to a different state. The probabilities of moving from one state to another are called transition probabilities. In each state, the model will assign costs and benefits for each individual according to different interventions and/or time spent in each state. In a Markov model, there must be at least one absorbing state, typically death, from which the person will not be able to leave.

Figure 25 shows a simplified version of the model presented for illustrative purposes (Appendix 27 contains a copy of a section of the full model structure). In this figure, the states are presented as ovals, whereas the arrows show the possible directions in which individuals could move at the end of each cycle. The rate at which an individual moves (makes a transition) between states is governed by the transition probabilities. The states considered in the model are thought to reflect possible paths of individuals. The top line in Figure 25 represents the possible path for individuals who start off after 'successful' (the belief being that the woman has been successfully treated for cancer but is at risk of developing subsequent disease) treatment free from cancer but who develop breast cancer over time but remain undiagnosed. The bottom section of Figure 25 represents those individuals who start in the model after 'successful' treatment free of cancer, but go on to develop IBTR or MCBC over time but are identified and treated for the disease.

If a woman initially has no evidence of IBTR or MCBC then over time she will have the chance of IBTR or MCBC occurring. The natural history of disease and the effectiveness of initial treatment determine the chance of this occurrence. Surveillance will not alter the chance of IBTR or MCBC occurring but may alter the chance of that cancer being detected, the stage at which it is found and hence the treatment and possible final outcome. Within the simplified version of the model shown in Figure 25 only three treatment states are depicted. These treatment states vary according to the risk profile of the breast cancer being treated. Once IBTR or MCBC is identified it is assumed that the cancer is treated and that subsequently individuals may have an altered life expectancy as a result of the recurrence. We also assumed that women who have had a further cancer will be judged as being at 'moderate' risk of developing further disease and so will have a more intensive follow-up. The absorbing state in the model is death. Any individual can move into this state from any other state within the model. The chance of moving into this state will be determined by the age of the woman through all-cause mortality and cancer-specific mortality. If a cancer is missed during surveillance then it is assumed that it will remain untreated until it is identified.

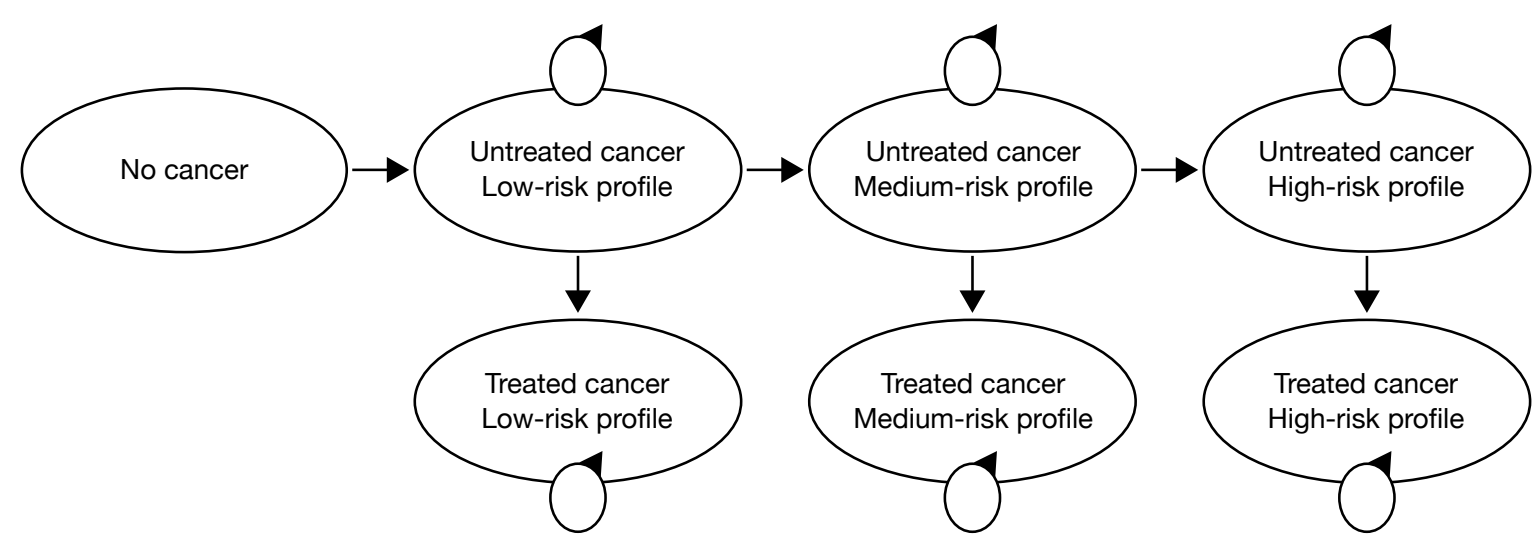

FIGURE 25 Depiction of a simplified version of the Markov model. Risk profile refers to the mortality risk for a given cancer. In this simplified figure, it is assumed that cancers differ in terms of the risk of death. 
The model will compare different regimens but, for each regimen, a cohort of women will pass through the different health states. The costs per woman and speed at which they progress through the states will vary between regimens. The intuitive idea behind the model is to identify the regimen that leads to the most effective and cost-effective surveillance regimen.

\section{Description of a woman's movement through a model regimen}

The model includes women who may develop IBTR and or MCBC. The model itself does not differentiate between these situations. The model starts with a surveillance year; based on information from the survey of current practice this frequency could be once every 6,12 , 18, 24 or 36 months. In regimen 1, individuals can either be followed up using surveillance mammography or present to their GP with a symptom, i.e. discover a lump. Women who have a mammogram can either be identified as free of disease or have a positive mammogram. The model structure allows this to be either a true- or false-positive or a true- or false-negative. If the mammogram is a true-negative, individuals will then go back to the surveillance programme. If the mammogram is a false-negative, individuals also return to the surveillance programme, but these individuals would not receive any treatment or care for that cancer although it remains undetected. Furthermore, it is possible that if a cancer is undiagnosed or untreated the severity of the cancer will increase and the prognosis worsen, for example over time the tumour may increase in size. We handle this within the model by increasing the severity of untreated disease over time. It is also possible for individuals who have had a true-negative, over the duration of the cycle, to go on to develop breast cancer in a subsequent cycle. The likelihood of this happening will be dictated by the expected incidence of IBTR and MCBC over the cycle length (for example, if the cycle length were 1 year then it would be based upon an estimate of the annual incidence of IBTR and MCBC).

If the mammogram is a true-positive, individuals will be diagnosed with invasive or non-invasive cancer and managed appropriately. If the mammogram is a false-positive it will be assumed that the individual will undergo further invasive tests and on a negative finding of these tests they will return to the surveillance programme at the end of the cycle.

\section{The regimens considered}

We outlined the alternative surveillance regimens in Chapter 3 (see Methods for the survey). The intention was to compare each of these within the economic model. We planned to combine surveillance regimens for hypothetical cohorts of the population defined in terms of the nature of primary disease, treatment and demographic characteristics, etc. These cohorts reflect the prior hypothesised risk of IBTR and MCBC in the population of women previously treated surgically for a primary breast cancer.

As described in Methods for the survey, we identified nine different surveillance regimens. We reduced these to three regimens, which we felt broadly represented the most relevant comparators. Furthermore, as reported in Chapter 5, few data on the diagnostic performance of the alternative methods of identifying a breast cancer were available. Consequently, it was not possible to model all of these options. However, some data were available to facilitate the modelling of mammographic surveillance with and without clinical follow-up organised either through secondary care or through the screening service. The presentation of the woman following referral from primary care following the identification of a suspicious lump on selfexamination was also modelled. We used this form of diagnosis in two specific ways within the model. First, we used it to define a situation where no formal surveillance is used. It is also used to model the possibility that a woman presents between surveillance points with symptoms suggestive to a GP of breast cancer, for example if surveillance is performed every 36 months then within this 36-month interval the model will allow a woman to present with clinical symptoms that are suggestive of breast cancer and for this cancer to be identified. 


\section{Populating the model with parameter estimates}

To provide estimates of relative cost-effectiveness, the model requires estimated values for a range of different types of parameters. Such parameter estimates should be derived in a systematic and reproducible manner to avoid bias caused by the distorted and selective use of data. ${ }^{50}$ The assembly of such data need not necessarily be comprehensive; rather, effort should focus on identifying the most relevant data to the decision problem, which in this case was the comparison of alternative surveillance regimens for women after treatment for primary breast cancer.

We assembled the different types of data required for the economic model from analyses of existing data sets, a series of systematic reviews, and focused searches for specific pieces of data. We report the methods and results of the reviews and analyses of existing data sets in detail in Chapters 4-6. In brief, the broad types of data required to populate the economic model relate to:

- the uptake of surveillance and follow-up

- the prevalence, incidence and risk of progression of the disease, i.e. its epidemiology and natural history

- the performance of different regimens (e.g. clinical examinations, mammograms, etc.) in terms of the accuracy of the diagnostic tests

- resource use and unit costs required to estimate the costs of alternative surveillance regimens; the specific parameters and methods used to provide estimates that are relevant to the UK context

- health-state utilities.

Within the model, we based estimates of uptake upon simplifying assumptions and advice from the members of the project Advisory Group. We derived the data on the natural history of women from the analysis of the large data set reported in Chapter 6. Further data relating to the management and outcomes came from the source data used to inform recent NICE guidelines. ${ }^{24}$

We derived information on the diagnostic performance of different types of clinical tests, for example the accuracy of mammography, from data reported in Chapter 5.

We derived data on the costs incurred for the different surveillance regimens and their consequences from structured reviews of the published literature, as well as routine data sources such as the NHS Reference Costs. ${ }^{40}$ The perspective for costs is the NHS.

Data on the utilities associated with differing severities of cancer and the possible differences in quality of life associated with various surveillance regimens were obtained from the published literature, including the review of economic evaluations, as described above, as well as a search of the Cost-Effectiveness Analysis Registry (CEA Registry: www.cearegistry.org/).

We report how we derived each of these sets of data and the values used in the model in more detail in the sections below.

\section{Uptake of surveillance and follow-up}

Within the model, we assumed that, if individuals are invited to attend surveillance, they do in fact attend. This may be too high, as approximately $75-80 \%$ of the normal population attend for breast screening. The other variable required for the model is the probability that a woman will present to the GP with symptoms that she thinks are suspicious. Based upon advice from the clinical members of the Advisory Group we assumed that $30 \%$ of women with prior treatment for breast cancer might present to the GP per annum. We then converted this percentage into a probability of presenting per 6-month cycle by fitting an exponential curve. The probability used within the model was 0.1393 , i.e. in the no surveillance arm of the model, and for during the 
surveillance interval in the surveillance arms of the model, just under $14 \%$ of surviving women who have not been diagnosed with a recurrent cancer will present to a GP every 6 months. The following formula assumes that events occur at a constant rate over time: $p=1-e^{-\mathrm{rt}}$, where $p=$ probability, $e=$ base of natural logarithm, $\mathrm{r}=$ rate and $\mathrm{t}=$ time period.

\section{Epidemiology and natural history of breast cancer}

Data relating to the natural history of breast cancer required for the model can be split into four components. These are:

1. recurrence/occurrence rates for women initially treated for breast cancer

2. estimated survival of women without and with IBTR or MCBC

3. estimated proportions of the different types of IBTR or MCBC occurring

4. estimated change in the severity of untreated cancer over time.

\section{IBTR and/or MCBC rates for women initially treated for breast cancer}

Part of the analysis reported in Chapter 6 related to the time to event for IBTR and MCBC cancers. Using the estimated 10-year survival and the same methods as described above, an exponential curve was fitted so that the probability of experiencing an event for IBTR or MCBC per cycle (a 6-month period) was estimated. We report the estimated 10-year incidence rate for women initially treated by BCS or mastectomy, as well as the incidence per cycle (per 6-month period) in Table 27. Within the model, we assumed IBTR and MCBC events were independent and that the rates summed within the model to provide a net rate of cancer incidence. As described below this assumption was relaxed in sensitivity analyses, where we modelled the impact on costs and benefits of the incidence of the more serious IBTR events separately.

The data reported in Table 27 are taken to be representative of the rates expected for the whole population of women who received either BCS or mastectomy as part of the treatment of their primary cancer. It should be noted that the rates of $\mathrm{MCBC}$ in women who were originally treated by mastectomy are higher than the rates in those who were originally treated using BCS. These estimates are based upon observed estimates and the difference may simply be a reflection of imprecision in estimates, i.e. in reality no difference exists. Whether this is true or not is a matter for debate. A finding from Chapter 6 was that the incidence of subsequent cancer events was predicted in part by the characteristics of the primary cancer. Using the same methods described we have estimated the incidence per cycle for the reference case used in Cox proportional hazard models reported in Chapter 6 (Table 28).

We calculated upper and lower estimates of incidence by combining estimates of the hazard rates obtained from the Cox proportional hazard models with the event rates and probabilities reported in Table 29. It was assumed that hazard rates were additive but upper and lower estimates were based only on proportional hazard rates for factors, for example tumour size, age, grade, etc., which were found to be statistically significant at the $5 \%$ level in the analyses reported in Chapter 6.

TABLE 27 Estimated incidence of IBTR and MCBC disease for women following surgery for primary disease

\begin{tabular}{llll}
\hline Initial form of surgery & Type of event & 10-year failure probability & $\begin{array}{l}\text { 6-month cycle failure } \\
\text { probability }\end{array}$ \\
\hline BCS & IBTR & 0.045 & 0.0023 \\
& MCBC & 0.030 & 0.0015 \\
\multirow{2}{*}{ Mastectomy } & IBTR & 0.035 & 0.0018 \\
& MCBC & 0.035 & 0.0018 \\
\hline
\end{tabular}


TABLE 28 Alternative values for the subgroup of women taken as the reference case in Cox proportional hazard model ${ }^{a}$

\begin{tabular}{llll}
\hline Initial form of surgery & Type of event & 10-year failure probability & $\begin{array}{l}\text { 6-month cycle failure } \\
\text { probability }\end{array}$ \\
\hline BCS & IBTR & 0.040 & 0.0020 \\
& MCBC & 0.020 & 0.0010 \\
\multirow{2}{*}{ Mastectomy } & IBTR & 0.038 & 0.0019 \\
& MCBC & 0.028 & 0.0014 \\
\hline
\end{tabular}

a The reference group in the Cox proportional hazard model was: age 50-64 years, grade 1 , size $<1 \mathrm{~cm}$, no lymph node involvement, no vascular invasion.

TABLE 29 Hazard rates for high and low estimates and the incidence per cycle estimated from these rates

\begin{tabular}{|c|c|c|c|c|c|}
\hline \multirow[b]{2}{*}{ Initial form of surgery } & \multirow[b]{2}{*}{ Type of event } & \multicolumn{2}{|c|}{ Cumulative hazard rate } & \multicolumn{2}{|c|}{$\begin{array}{l}\text { 6-month cycle failure } \\
\text { probability }\end{array}$} \\
\hline & & Low & High & Low & High \\
\hline \multirow[t]{2}{*}{ BCS } & IBTR & 0.72 & 6.64 & 0.0015 & 0.0135 \\
\hline & MCBC & 0.34 & 1.60 & 0.0003 & 0.0016 \\
\hline \multirow[t]{2}{*}{ Mastectomy } & IBTR & $0.85^{\mathrm{a}}$ & 5.36 & 0.0016 & 0.0104 \\
\hline & MCBC & 0.27 & 1.53 & $<0.0014$ & 0.0022 \\
\hline
\end{tabular}

a There was no evidence of a statistically significant difference. The smallest point estimate of a difference was for age 35-49 years.

\section{Estimated survival of women with and without IBTR and/or MCBC}

The economic model does not stop with the diagnosis of cancer. It seeks to model the impact on survival caused by delayed identification. To do this estimates of survival of women who develop a further case of cancer are required. Also required are estimates of survival for those women who do not develop further cancer. Estimates of the former depend upon whether or not the cancer was diagnosed and treated and the effectiveness of any treatment.

We conducted a structured review of relevant management guidelines to estimate data on the survival of women who go on to develop further cancer (reported in more detail in Appendix 28). The recent NICE guideline was identified as providing the best available evidence of treatments for early breast cancer relevant to the UK..$^{24}$ The data used to support the NICE guideline recommendations came from the EBCTCG ${ }^{83}$ and Adjuvant! Online computer program. ${ }^{84} \mathrm{We}$ prepared estimates of survival following various treatment options using the Adjuvant! Online computer program due to its flexibility. Adjuvant! Online draws on information from mortality statistics in the USA, the SEER database, and meta-analyses and individual clinical trials. Based on well-validated factors, such as age, menopausal status, oestrogen receptor (ER) status, tumour size and grade, nodes status, etc., predictions can be made about survival for alternative adjuvant treatment regimens, such as chemotherapy and hormone therapy. The programme derives survival estimates from the US population, however.

As survival estimates are linked to the effectiveness of treatments it was necessary to also define the therapy given for a cancer. The choice about what therapies would be adopted for which categories of cancer were based upon the recommendations in the NICE guideline ${ }^{24}$ and clinical advice from members of the study team about typical treatments within the UK. Based upon these data we derived specific therapies for cancers with specific characteristics. Using Adjuvant! Online, we estimated predictions of 10-year cancer-related mortality. Table 30 shows the simplified classification of prognostic factors used by Adjuvant! Online. We grouped these 
TABLE 30 The simplified classification of prognostic factors

\begin{tabular}{|c|c|c|c|c|}
\hline \multirow{2}{*}{$\begin{array}{l}\text { Comorbidity } \\
\text { ER status }\end{array}$} & \multicolumn{4}{|c|}{ Average for age } \\
\hline & Positive & & Negative & \\
\hline Tumour size (cm) & $0.1-2$ & & $2.1-5$ & $>5$ \\
\hline Positive nodes & 0 & $1-3$ & $4-9$ & $>9$ \\
\hline Tumour grade ${ }^{\mathrm{a}}$ & 1 & & 2 & 3 \\
\hline
\end{tabular}

a Excluded factors in current subgroups.

estimates, as described below, for cancers that had similar management costs and survival. This simplification was performed because Adjuvant! Online can provide more data than were readily manageable in the economic model, and the economic model itself was focused on surveillance regimens rather than treatments of women with breast cancer.

We grouped the different cancers into five different risk profiles, which had an increasingly worse prognosis. The cancers included in each risk profile had a predicted 10 -year mortality that fell into the range defined for the risk profile. We derived the range for each risk profile following consideration of the mortality data derived from Adjuvant! Online and discussions within the Advisory Group.

As each risk profile contained several different cancers (defined in terms of size, ER status, etc.) an average mortality had to be calculated. To calculate this average mortality we required data on the estimated proportion of each type of cancer in each risk profile. These data were derived from the further analysis of the WMCIU Breast Cancer Registry data set used in Chapter 6. Cases were included if they were invasive tumours and diagnosed from 1997 (due to the extent of missing data prior to that date). We considered only tumours that were surgically treated. For the selected cases, descriptive information about the proportions of women with cancers with the following combination of characteristics were derived: ER positive or negative, grade of cancer (grades 1, 2 or 3), tumour size $(0.1-2.0 \mathrm{~cm}, 2.1-5.0 \mathrm{~cm},>5.0 \mathrm{~cm}$ ) and number of positive lymph nodes (zero, one to three, four or more). Unfortunately, ER status was mostly missing within the data set so could not be provided. Therefore, using published information ${ }^{85}$ we considered that $70 \%$ of cancers would be ER positive with the remainder being ER negative. We report these data in detail in Appendix 28. We assumed that the proportions of the different types of IBTR and $\mathrm{MCBC}$ would be the same as those for primary cancer. We made this assumption because there were more cases of primary cancer and hence less likelihood of there being no data to provide estimates for the combination of tumour characteristics described below.

From the WMCIU Breast Cancer Registry data, the proportion of each type of cancer (in terms of the proportion with a particular tumour grade, size, nodal involvement, etc.) was defined in each risk profile. We then multiplied the proportions by the 10-year mortality estimates for the corresponding cancer. We then summed the product of these calculations to give an average 10 -year mortality rate for each risk profile.

Using the estimated 10-year mortality derived for each risk profile we fitted an exponential curve so that the probability of dying from cancer per cycle (a 6-month period) for each risk profile (Table 31) could be estimated using a formula similar to the one described above. For example, Table 31 shows that the average mortality rate from cancer at 10 years for risk profile state 1 was $4.86 \%$, and, using the formula reported above, the risk of dying from cancer in any 6-month cycle was estimated to be $0.002 \%$. Further detail of the data underpinning Table 31 is shown in Appendix 30. 
TABLE 31 Mortality rates (\%) from breast cancer at 10 years and per 6-month cycle for each risk profile (data used within the model)

\begin{tabular}{lcl}
\hline Risk profile & 10-year mortality rate & 6-month cycle mortality rate \\
\hline 1 & 4.86 & 0.002425 \\
2 & 12.47 & 0.006217 \\
3 & 21.19 & 0.010539 \\
4 & 36.81 & 0.018236 \\
5 & 57.20 & 0.028197 \\
\hline
\end{tabular}

This formula used to estimate the probability of dying from cancer per cycle (a 6-month period) assumes that deaths occur at a constant rate over time. If mortality is positively skewed then for a shorter time horizon of the model this may represent an underestimate of mortality, and it may overestimate mortality for longer time horizons. The cancer mortality data also assume that risk of death from cancer is independent of the women's age; this may underestimate the risks from cancer in younger women (i.e. those under 50 years of age).

These mortality rates are based on data for women who have received treatment for breast cancer. Therefore, they may not be applicable to women whose cancer is untreated because it is undetected. We hypothesised that at each time point a woman with an untreated cancer would face a higher risk of death in the next cycle (6-month period) than an identical woman whose cancer had been treated. This increased risk of death was proxied by comparing the estimated risk of dying at 5 years following a diagnosis of cancer for a woman diagnosed with cancer in the period 1980-4 with the risk for an identical woman from 2000 to 2004. The data used to derive the parameter value used in the model came from information produced by the Information and Statistics Division of NHS Scotland. ${ }^{86}$ The base-case value was based upon all women aged 15-74 years. In this group of women, expected 5-year mortality for women diagnosed between 1980 and 1984 was $34.9 \%$. In the period $2000-4$ the expected 5-year mortality was $14.9 \%$. The ratio of these two numbers gives a value of 2.34. This value was used to inflate the 6-month breast cancer mortality rates reported in Table 31 . Table 32 summarises the base-case and high and low values used within the model. Low and high values are based upon the lowest and highest values obtained for any age grouping reported by the Information and Statistics Division of NHS Scotland.

Within the base-case analysis we assumed that an IBTR that fits within a given risk profile will have the same probability of death per 6-month cycle period as an otherwise identical MCBC. The data reported in Chapter 6 suggests that mortality following IBTR may be substantially higher than the mortality for an otherwise identical MCBC, however. As noted above, we conducted sensitivity analyses to explore the impact of the increased risk of death from IBTR. We derived the increased risk of death per 6-month period by multiplying the HR for mortality from IBTR reported in Chapter 6 with the mortality rates for treated and untreated cancers. The point estimate for the hazard rate for death for IBTR was 1.76 (with an upper value from the $95 \% \mathrm{CI}$ of 2.13). We used the extremes of the CIs for this hazard rate to define low and high rates within the model.

Data were also required on all-cause mortality. These data were required because women who do not develop cancer still have a chance of dying from other causes. In addition, women who do develop cancer also have the risk of dying from other causes. For both sets of women as they age within the model, mortality will increase. Estimates of all-cause mortality were obtained from the published UK life tables for the years. ${ }^{87}$ From these data a mortality rate for each 6-monthly cycle was calculated using the previously defined formula. This is reported in detail in Appendix 31. 
TABLE 32 Summary of breast cancer mortality inflators used within the model to derive breast cancer mortality for those with undiagnosed breast cancer

\begin{tabular}{|c|c|c|c|c|}
\hline & \multicolumn{2}{|l|}{ 5-year mortality } & \multirow[b]{2}{*}{ Inflator } & \multirow[b]{2}{*}{ Notes } \\
\hline & $\begin{array}{l}\text { Cancer detected } \\
1980-4(\%)\end{array}$ & $\begin{array}{l}\text { Cancer detected } \\
2000-4(\%)\end{array}$ & & \\
\hline Base case & 34.9 & 14.9 & 2.340 & $\begin{array}{l}\text { Based on the cohorts of women aged } 15-74 \\
\text { years at time of diagnosis }\end{array}$ \\
\hline Low estimate & 36.4 & 12.5 & 1.508 & $\begin{array}{l}\text { Based on the cohorts of women aged } 75-84 \\
\text { years at time of diagnosis }\end{array}$ \\
\hline High estimate & 39.1 & 25.9 & 2.916 & $\begin{array}{l}\text { Based on the cohorts of women aged 55-64 } \\
\text { years at time of diagnosis }\end{array}$ \\
\hline
\end{tabular}

\section{Estimated proportions of the different types of IBTR and/or MCBC cancers occurring}

For the model, information was needed not just on whether a cancer occurs or not, but also on the severity of that cancer. We assumed that at the point where a cancer technically becomes detectable the size of the cancer is below $1 \mathrm{~cm}$ in diameter. In terms of the risk profile classification defined above we further assumed that all these people are initially in risk profile category 1 at the point when the cancer becomes detectable. Over time, an undetected cancer will increase in severity and the estimates used to model this are described below.

\section{Estimated change in the risk profile of untreated cancer over time}

One variable required for the economic evaluation is the rate at which an undiagnosed cancer may move to a worse risk profile (with a consequent reduction in life expectancy and quality of life and an increase in treatment costs). We sought data on which to base estimates for this variable from a structured review of the literature relating to doubling time of a breast cancer and the factors, for example grade of cancer, which might affect the doubling time of tumours. We sought these data as the individual patient analysis reported in Chapter 6 found that a significant predictor of mortality was tumour size, with larger-sized tumours having a shorter life expectancy than smaller tumours.

It is recognised that there is a considerable degree of uncertainty about the rates of growth of breast cancers. ${ }^{88}$ However, data were sought about plausible rates of growth and about potential range in the rates of growth that can be explored in a subsequent sensitivity analysis. A summary of the findings of this structured review is reported in Appendix 32. Given the information found in this review of the literature, it is clear that there is little consensus on the doubling times of breast cancer tumours. The data we have used in the economic model are based on the information provided by Peer and colleagues, ${ }^{89}$ taking the mean doubling time in tumour volume to be 157 days. We tested the consequences of this in a high/low sensitivity analysis where we will vary this rate between the plausible extremes of the data presented in Table 33.

To use information on the mean doubling time within the model we had to estimate how long it would take a tumour of the minimum technically identifiable size to increase in size, where an untreated tumour would move from one risk profile to a risk profile with a worse prognosis. We took the minimum diameter of a detectable cancer to be $0.75 \mathrm{~cm}$. This value was taken because the volume of a cancer with this diameter is close to the minimum volume size considered by Adjuvant! Online. The data on time to reach the threshold tumour size were converted into risks of increasing the risk profile by one level for each cycle that a cancer remains untreated. We performed this using the same methods described above to estimate incidence and mortality rates. 


\section{Diagnostic performance of tests}

As reported in Chapter 5, relatively few data were available on the diagnostic performance of any of the tests. Within the model, we assumed that at the time a woman receives a diagnostic test as part of surveillance she is asymptomatic.

For IBTR, we based data on data reported in Chapter 5, and summarised in Table 20 (Chapter 5), and on discussions with the clinical experts involved in the study. Where relevant published data were available in the absence of pooled data the study judged to be closest to the median of reported results was used to inform the values chosen for the base-case analysis. We used data from other studies to define plausible extremes. Where it was feasible for these tests to be used then they were also used for MCBC, as few additional data were available (Table 34).

For surveillance mammography the values used in the base-case analysis were based upon those derived from Drew and colleagues ${ }^{67}$ We based low and high estimates of sensitivity upon the ranges for these parameters reported in Table 20 (Chapter 5). These data represent extreme values that will be used in the sensitivity analysis.

As reported in Chapter 5, only one study provided data on the sensitivity and specificity of mammography and clinical follow-up. These data did not seem plausible (e.g. the reported sensitivity was $100 \%$ ). The values reported in Table 34 are assumptions derived following discussions with clinical experts. The consensus of opinion was that the combination of follow-up and mammography would slightly improve the sensitivity and specificity. In a sensitivity analysis we will explore the impact of changing these values between high and low estimates. We will also seek to identify whether there is a threshold in terms of diagnostic performance, which would make the additional cost of clinical follow-up worthwhile.

TABLE 33 Mean breast cancer doubling times by age

\begin{tabular}{llll}
\hline Age at diagnosis (years) & Mean doubling time (days) & $\begin{array}{l}\text { Time for a 0.75-cm diameter } \\
\text { tumour to move up one risk } \\
\text { profile (days) }\end{array}$ & $\begin{array}{l}\text { 6-month rate for increase in } \\
\text { risk profile }\end{array}$ \\
\hline$<50$ & 80 & 300 & 0.2623 \\
$50-70$ & 157 & 540 & $0.1555^{\mathrm{a}}$ \\
\hline
\end{tabular}

a Value taken in the base-case analysis.

Source: Peer and colleagues. ${ }^{89}$

TABLE 34 Diagnostic performance of the different tests

\begin{tabular}{lllll}
\hline Test & Parameter & $\begin{array}{l}\text { Base-case } \\
\text { value (\%) }\end{array}$ & Low (\%) & High (\%) \\
\hline Surveillance mammography & Sensitivity & 67 & 65 & 70 \\
\multirow{2}{*}{ Surveillance mammography and clinical follow-up } & Specificity & 85 & 65 & 90 \\
& Sensitivity & 75 & 70 & 80 \\
Clinical examination & Specificity & 90 & 70 & 50 \\
& Sensitivity & 35 & 25 & 50 \\
Rates available for other tests explored in a sensitivity analysis & Specificity & 35 & 25 & 70 \\
MRI & Sensitivity & & & 90 \\
\end{tabular}


Within the model, data are also required for the diagnostic performance of a clinical examination when performed by a GP. Again, few data were available and following discussions we assumed that the rates used within the model would be slightly lower than those reported in the systematic review of diagnostic performance (Chapter 5).

We considered the impact of using a higher cost but more effective diagnostic test. As a proxy for such a test data were based upon the performance of MRI. It should be noted that the values identified, especially at the upper level, are where MRI has been used in a higher risk group of women. Hence, the values are not necessarily illustrative of MRI itself but rather of a hypothetical test. The values for the base-case analysis were based upon those reported by Drew and colleagues ${ }^{67}$ but it was assumed that the sensitivity was slightly less than perfect (i.e. $95 \%$ vs the $100 \%$ reported by Drew and colleagues). ${ }^{65}$ Low values of sensitivity were based upon data from Warner and colleagues ${ }^{90}$ who conducted a systematic review of prospective studies in which women at very high risk for breast cancer were screened with both MRI and mammography. ${ }^{90}$ Hence even these data may not be fully representative of women eligible for surveillance mammography. The specificity values were informed by the estimates of one study ${ }^{91}$ included in the Warner and colleagues review. ${ }^{90}$ This study had the lowest specificity of any of the studies included in Warner and colleagues' 2008 study. ${ }^{90}$

\section{Costing data}

The costs of surveillance were broken down into the following cost categories:

- Cost of:

- inviting women for screening

- the surveillance test (e.g. mammogram, MRI, clinical examination)

- health-care professional time (e.g. GP consultation, clinical examination)

- further invasive tests (e.g. core biopsy)

- treatment (e.g. mastectomy, radiotherapy, drug treatment).

Tables 35-37 show the cost estimates used in the economic model. All costs are reported in 2008 pounds sterling. Table 35 shows the current cost of the alternative screening strategies. The cost of inviting women to attend screening was obtained from a recent HTA report. ${ }^{92}$ The cost of the alternative surveillance tests were all derived from routine sources. The cost of a mammogram was based on information from the NHSBSP $2009 .{ }^{93}$ The NHSBSP estimates the cost of a mammogram in England to be $£ 37.50$ per woman invited and $£ 45.50$ per woman screened. An alternative costing source was obtained from the Scottish Breast Screening Programme, which estimates the cost of a mammogram to be $£ 77.80 .{ }^{94}$ The implications of the variation in costs between Scotland and England were explored in a sensitivity analysis. The cost of an MRI was estimated as being twice the cost of that reported in the NHS Reference Costs ${ }^{40}$ for an outpatient MRI. This is because an MRI on a breast takes twice as long as a normal MRI and involves the use of a contrast. The lower quartile and upper quartile of the NHS Reference Costs ${ }^{40}$ for this category are used to inform sensitivity analysis. We derived the costs of a clinical examination from routine data sources. The cost of a GP clinical examination was obtained from the Personal Social Services Research Unit (PSSRU) ${ }^{95}$ and was based on the average cost of a GP consultation. In addition, we also included the cost of a clinical examination conducted in a secondary care setting by either a consultant or non-consultant. These costs were obtained from NHS Reference Costs. ${ }^{40}$ Information on the range of costs (lower and upper quartile) was also available and these were used as upper and lower estimates in sensitivity analysis.

The costs of further invasive tests were obtained from a NICE evidence review group (ERG) report $^{96}$ and inflated to current prices using the PSSRU inflation index. The cost of a mastectomy 
TABLE 35 Cost of screening regimens

\begin{tabular}{|c|c|c|}
\hline Type of test & $\begin{array}{l}\text { Cost (lower quartile/upper quartile), } \\
\text { [alternative] (£) }\end{array}$ & Source \\
\hline Clinical examination - GP & 36 & PSSRU ${ }^{95}$ \\
\hline Clinical examination - consultant & 86 (63 to 100) & NHS Reference Costs ${ }^{40}$ (ref. 103) \\
\hline Clinical examination - non-consultant & 65 (55 to 75) & NHS Reference Costs ${ }^{40}$ (ref. 103) \\
\hline Mammogram & $45.50[77.8]$ & $\begin{array}{l}\text { NHSBS93 (Scottish Breast Screening } \\
\text { Programme) })^{94}\end{array}$ \\
\hline MRI - outpatient & 232 (165 to 269) & $\begin{array}{l}\text { NHS Reference Costs }{ }^{40} \text { (ref. TDIAGIM_APC; } \\
\text { RA03Z) }\end{array}$ \\
\hline
\end{tabular}

TABLE 36 Cost of invasive tests and treatments ${ }^{98}$

\begin{tabular}{|c|c|c|}
\hline Type of treatment & Cost (low/high) (£) & Source \\
\hline Core biopsy & 126.6 & ERG report ${ }^{96}$ \\
\hline Mastectomy & 3429 & ERG report ${ }^{96}$ \\
\hline Radiotherapy & 2395.58 (1649.20 to 2784.80$)$ & NHS Reference Costs ${ }^{40}$ (based on 20 sessions) \\
\hline Hormone therapy (per year): tamoxifen & $29.92^{\mathrm{a}}$ & TA112 \\
\hline $\begin{array}{l}\text { Hormone therapy (per year): aromatase } \\
\text { inhibitor }\end{array}$ & 919.6 to 1115.4 & TA112 ${ }^{98}$ \\
\hline $\begin{array}{l}\text { Chemotherapy (per six treatment) } \\
\text { administrations }\end{array}$ & $4137^{a}$ to 9850 & TA10999 \\
\hline
\end{tabular}

a Value taken in breast cancer analysis.

TABLE 37 Surveillance regimen: clinical examination plus mammography for women receiving hormone therapy

\begin{tabular}{ll}
\hline Surveillance regimen & Cost (£) \\
\hline Screening invitation & 10.45 \\
Mammography + clinical examination (consultant) & $45.50+86$ \\
On positive finding & \\
Core biopsy & 126.6 \\
Mastectomy & 3429 \\
Radiotherapy & $2395.58\left(20\right.$ sessions $\left.^{\mathrm{a}}\right)$ \\
Drug treatment (tamoxifen) & 29.92 (per year) \\
\hline
\end{tabular}

a Based on information from the PRIME trial. ${ }^{97}$

was based on the same source, and inflated to 2008 prices. The cost of radiotherapy was based on the cost of complex treatment on a mega-voltage machine, ${ }^{40}$ assuming that women get on average 20 sessions of radiotherapy. This assumption was based on information from the PRIME trial, which reported that, on average, women receive 20 sessions of radiotherapy. ${ }^{97}$ Again, lower quartile and upper quartile estimates of the cost of a single session of radiotherapy will be used in a sensitivity analysis.

The costs of drug treatment, for example the cost of hormone treatment, chemotherapy and combined treatment, were obtained from recent NICE guidance. The cost of hormone treatment was based on information reported in the costing template for technology appraisal 
guidance 112. ${ }^{98}$ This included the costs of tamoxifen for 5 years and the cost of aromatase inhibitors (anastrozole or letrozole) for 5 years. The cost of chemotherapy was based on the costs reported in NICE technology appraisal guidance $109 .{ }^{99}$ The cost of chemotherapy is based on the cost of two different regimens (TAC - taxotere, adriamycin and cyclophosphamide; FEC fluorouracil, epirubicin and cyclophosphamide). This is based on six cycles of treatment.

Each risk profile consists of a series of different types of cancers (defined in terms of ER status, grade, size and number of lymph nodes involved). As described above, an average mortality for each risk profile was estimated by combining information on the expected mortality for each specific cancer within a risk profile with information on the proportion of women in that risk profile that had that specific type of cancer. Adjuvant! Online reports mortality by the type of adjuvant therapy used. The clinical members of the research team determined, based on UK practice, which specific cancer in a profile would receive hormone therapy and/or chemotherapy. Using information on the proportion of cancers in a given risk profile that would be treated with a given adjuvant therapy a proportion of a cost of a course of hormone treatment or radiotherapy was incorporated into the cost assigned to each risk profile.

Table 37 shows the costs of one surveillance regimen for a woman invited to screening and who received a clinical examination and a mammogram. The costs include the costs of screening, the mammogram and clinical examination, conducted by a consultant. On a positive mammogram, the woman would then go on to have further invasive tests to confirm the result (core biopsy). On a true-positive finding, the woman would have a mastectomy followed by radiotherapy, followed by drug treatment (depending on the severity of the IBTR or MCBC). We based the costs of treatment on a number of assumptions:

- It is assumed that all ER+ women will receive hormone treatment. It is assumed that those women who have an excellent prognosis (survival rate at 10 years of $96 \%$ or greater) and are postmenopausal will receive tamoxifen for 5 years. Women who are postmenopausal, with a poorer prognosis, will receive an aromatase inhibitor for 5 years.

- All women who are premenopausal and are ER+ will receive tamoxifen.

- All women who have grade 3 tumours will receive chemotherapy.

- Women who are ER+ and have positive lymph nodes will receive combined treatment (hormone + chemotherapy).

- Women who are ER- and have 0 nodes will receive no treatment (exception to this is that $15 \%$ might get hormone therapy).

- Women who are ER- and have positive lymph nodes will receive chemotherapy (exception to this is that $15 \%$ might receive combined therapy).

\section{Health-state utility values}

The primary purpose of the economic model was to inform decision-making in a UK setting, given that treatment for breast cancer affects not only survival, but also quality of life, for example different types and stages of cancer are likely to be associated with differences in quality of life, as would different treatment options. Therefore, we have also sought to assess the impact on quality of life, through the incorporation of health-state utility weights, which have been combined with estimates of survival to estimate QALYs.

Recent guidance suggests that estimates of QALYs should ideally be based on generic health-state valuation methods using UK population tariffs. ${ }^{100}$ Therefore, we conducted a focused search of the literature and other relevant sources such as the Harvard cost-utility database. We identified a number of studies reporting health-state utilities. In particular, we found a recent systematic review of breast cancer utility weights. ${ }^{101}$ In their systematic review, 59 studies were identified 
for review and nine studies included. Of the nine studies included, three were based on UK data. ${ }^{102-104}$ In addition, the utility values used in the paper by Sorensen and colleagues ${ }^{105}$ were based on a combination of UK and US data. ${ }^{105}$

It is difficult to determine how comprehensive this review is as, being available as a conference poster, the details provided on the literature searching are brief. The authors searched an appropriate selection of databases but the sensitivity of the search strategies used is unclear due to a lack of information. Missing information included whether MeSH terms were 'exploded' to include more specific terms, which Emtree terms were used in EMBASE, and how the terms were combined in the final search. From the information reported, one error was noted: 'breast neoplasms' was incorrectly described as a non-MeSH term.

Overall, the authors of the systematic review found considerable variability and inconsistency in the reported utility values. A selection of other studies eliciting health-state utilities was further identified. Overall, there was considerable variation in values and in definitions of health states; however, there is a general trend in the values reported in the literature. As would be expected, utilities decrease with increasing breast cancer severity and utilities are also found to be sensitive to treatment. For example, there is a general trend for those receiving chemotherapy to have lower utility values than those receiving hormone therapy, most likely due to the severity of the side effects of the respective treatments.

For the economic model, we have used the results reported in the systematic review of breast cancer utility weights. ${ }^{101}$ Using this information, we defined utilities for each of the five risk profiles in the model. For example, risk profile state 1 assumes a utility state with a low value of 0.75 and a high value of 0.85 (based on the distribution of values from the systematic review). We adjusted these utility states to include a decrement for those women who will receive chemotherapy. This decrement is based on the percentage of women in each of the five severity states who would receive chemotherapy. For example, 24\% of women in risk profile state 1 would receive chemotherapy. The chemotherapy decrement is based on information on patients' utilities for cancer treatments. ${ }^{106}$ In their study, using the time trade-off method utilities for chemotherapy were estimated to be 0.74 from an actual health state estimated to be 0.94 . All health-state utilities after treatment are assumed to be the same as the utilities defined before treatment without the chemotherapy decrement.

Utility values for risk profile states 3 and 4 are based on the health-state values in Tosteson and colleagues. ${ }^{107}$ This is based on the value for regional cancer in the age group 50-59 years. The utility value for risk profile state 5 is based on the value provided for distant rather than regional cancer in the age group 50-59 years. Each of these values has also been reduced by the decrement factor for chemotherapy. To achieve the high values reported in Table 38 for risk profile states 4 and 5 an additional 0.05 was added to the low value.

The values used in the base-case analysis are the low values reported in Table 38. Individuals in a 'no-cancer' state are assumed to have a health-state utility value of 0.80 in the base-case analysis.

\section{Key assumptions of the economic model}

This section provides a brief summary of the key assumptions made when developing the economic model.

\section{Structural assumptions}

The cycle length is assumed to be 6 months. 
TABLE 38 Health-state utilities

\begin{tabular}{|c|c|c|c|c|c|c|c|}
\hline \multirow[b]{3}{*}{ Severity } & \multirow{3}{*}{$\begin{array}{l}\text { Percentage on } \\
\text { chemotherapy }\end{array}$} & \multicolumn{4}{|l|}{ Managed } & & \\
\hline & & \multicolumn{2}{|c|}{ On treatment } & \multicolumn{2}{|c|}{ After treatment } & \multicolumn{2}{|c|}{ Unmanaged } \\
\hline & & Low & High & Low & High & Low & High \\
\hline 1 & 0.240 & 0.71165 & 0.80654 & 0.8 & 0.95 & 0.8 & 0.95 \\
\hline 2 & 0.759 & 0.59673 & 0.67629 & 0.8 & 0.95 & 0.8 & 0.95 \\
\hline 3 & 0.769 & 0.52693 & 0.60376 & 0.63 & 0.68 & 0.8 & 0.95 \\
\hline 4 & 0.991 & 0.49716 & 0.60807 & 0.63 & 0.68 & 0.8 & 0.95 \\
\hline 5 & 1 & 0.39362 & 0.50394 & 0.5 & 0.55 & 0.8 & 0.95 \\
\hline
\end{tabular}

It is assumed that, if individuals are invited to attend mammographic screening, they do in fact attend. This assumption may be too high, as approximately $75-80 \%$ of the normal population attend for breast screening.

Strategies compared are assumed to be homogeneous in that they do not change over time. More sophisticated strategies where the surveillance intervals and method of follow-up change over time have not been modelled.

Estimates of survival were based upon predictions derived from Adjuvant! Online and were grouped into five broad groupings based upon survival. This is a simplification of the different types of tumour that might occur, as well as how prognosis of untreated disease might change over time.

\section{Parameter value assumptions - natural history assumptions}

The incidence of IBTR and MCBC are assumed to be independent.

Incidence is assumed to have occurred at the point when a cancer could technically be identified.

The grade of IBTR does not have to be the same as that of the primary tumour but the grade of IBTR or MCBC does not have to change over time. There is some evidence to suggest that grade does not change. Should it occur as other evidence suggests, then, given the model structure, this would reduce the life expectancy of a woman. However, the impact on costeffectiveness is unclear as it depends upon the likelihood of a tumour progressing to a higher grade, the speed of progression, the diagnostic performance of the surveillance regimen and the surveillance interval.

Estimates of survival are assumed to be independent of age (age-adjusted all-cause mortality is included as a separate model parameter). This may underestimate risks to younger women whose cancers might be more aggressive.

Treatments for IBTR and MCBC were based upon NICE guidelines and expert opinion.

If a cancer is not detected in a given cycle (6 months) then it is assumed that it can only advance one risk profile level. The likelihood of this occurring was estimated from the literature on doubling times.

Many of the estimates used to model natural history of disease are assumed to be constant over time. Some of these assumptions are not consistent with the observed data. However, the consensus of opinion for other parameters, for example probability of progressing to a risk profile 
with a worse prognosis, is that they may decline over time. Allowing such probabilities to change over time would not greatly change costs but might be expected to increase QALYs over time for those strategies which have a better diagnostic performance.

\section{Parameter value assumptions - diagnostic performance}

Sensitivity and specificity for surveillance mammography and clinical follow-up were based upon expert opinion and assumed that it performed slightly better than surveillance mammography alone.

Sensitivity and specificity for clinical examination was based on clinical opinion. It was assumed that clinical examination has a lower sensitivity and specificity than reported in Chapter 5.

\section{Parameter value assumptions - cost assumptions}

Management packages previously prepared for UK guidance and HTAs have been used to estimate care, and hence costs. If treatment patterns have greatly altered in the last few years these data may not be applicable.

It is assumed that all women who have a breast cancer will incur the cost of a mastectomy. Obviously a woman who has had a previous mastectomy cannot have a further mastectomy on the same breast but the cost of a mastectomy has been used as a proxy for the cost of care such a woman would receive.

\section{Parameter value assumptions - utilities assumptions}

Data from different populations and elicited using different methods have been assumed to be sufficiently similar to the relevant UK population of women to be useful.

Utilities are assumed to reduce with increasing severity of disease and also on the use of chemotherapy. Other decrements to utility, for example complications of disease or treatment, are not modelled.

\section{Presentation of results}

The base-case analysis was run for a cohort of women (starting age in the model 57 years) with surveillance occurring once yearly. The starting age was chosen as this was the mean age of the women contributing to the analysis of data from the WMCIU Breast Cancer Registry, which was reported in Chapter 6. The model was run for different starting ages in further sensitivity analysis. The cycle length of the model is 6 months and cumulative costs and benefits are estimated over a maximum of 100 cycles, which is equivalent to a time horizon of 50 years. This time horizon was taken as a proxy for life expectancy of women treated for primary breast cancer. All costs are reported in 2008 pounds sterling and effectiveness in QALYS. A discount rate of 3.5\% for costs and benefits was used following guidelines for NICE. ${ }^{100}$ Results are presented as incremental cost per QALY gained. The modelling exercise will use a net benefit framework to combine cost and benefit estimates. The results of the analyses will be presented as point estimates of mean incremental costs, effects, incremental cost per QALY. This measure is a ratio of the difference in costs divided by the difference in effectiveness between two alternative strategies. These data can be interpreted as how much society would have to pay for an extra unit of effectiveness. Whether or not a more costly but more effective regimen is considered worthwhile depends upon society's willingness to pay for a QALY and, within England, the threshold adopted by NICE lies somewhere between $£ 20,000$ and $£ 30,000$.

Incremental cost per QALYs is a common way for presenting the results of an economic evaluation. They are, however, difficult to interpret when the choice is between several mutually exclusive options. In this circumstance the judgement can be informed by considering the net 
benefit statistic. The regimen with the greatest net benefit at a given value for society's willingness to pay for a QALY is considered to be most cost-effective. The net benefit statistic itself is defined as:

$$
\mathrm{NB}_{\mathrm{j}}=(\mathrm{QALY}, \mathrm{j})-\mathrm{cost}_{\mathrm{j}}
$$

where $\mathrm{NB}=$ net benefit, $\mathrm{QALY}_{\mathrm{i}}=\mathrm{QALY}$ for intervention $i, \cos _{\mathrm{i}}=\operatorname{cost}$ for intervention $i$, and $\lambda=$ society's willingness to pay per QALY.

Intervention $i$ would be chosen over intervention $j$ when $\mathrm{NB}_{\mathrm{i}}>\mathrm{NB}_{j}$.

\section{Sensitivity analysis}

We did not conduct probabilistic sensitivity analysis. The reason for this is that parameter values used are statistically imprecise and, as data are so limited, the model estimates may be unreliable. Therefore, the results of the economic evaluation should be interpreted cautiously and, at most, indicate situations where a particular method(s) of surveillance may be worthy of further consideration. Nevertheless, we conducted both one-way and multiway sensitivity analysis to assess how results may change as a consequence of plausible changes in parameter values. We also used deterministic sensitivity analysis to identify threshold values for key parameters. The methods used in the sensitivity analysis are described below.

\section{Probability of developing IBTR or MCBC}

We varied the probability of developing IBTR or $\mathrm{MCBC}$ in the sensitivity analysis from a low of 0.0030 [the lowest estimated 6-month cycle failure probability was for women who had a mastectomy and cumulatively had a risk of IBTR or MCBC of 0.0036 (Table 27)] to 0.0125 [the highest 6-month probability recurrence rate for IBTR and $\mathrm{MCBC}$ combined from the predicted HRs was 0.0125 (Table 29)].

\section{Inflating the risk of death from cancer for people who are unmanaged}

We also explored the effect of an increase in the risk of death for unmanaged individuals in sensitivity analyses. This was varied in the sensitivity analysis from the base-case assumption of 2.34 to a high of 2.916 (Table 32 in the base-case model).

We repeated the same analysis in the IBTR model, varying the increase in the risk of death in unmanaged states from 2.33 to 2.916 .

\section{Changes to the risk of progressing to a higher risk profile}

The consequences of changing the risk of unmanaged women progressing to higher-risk profiles was explored in both the base-case model and the IBTR model. The risk of progressing was altered from the base-case estimate of 0.1555 to 0.2623 in sensitivity analysis; 0.26 relates to a mean doubling time of 80 days and an estimated time of 300 days for a tumour to reach $2 \mathrm{~cm}^{3}$ (Table 33). In addition, in a further sensitivity analysis the risk of progressing was further lowered form the base-case assumption, to a mean doubling time of 942 days. This equates to a risk of 0.0923 per 6-month cycle.

\section{Sensitivity and specificity of the surveillance tests}

The diagnostic performance of the surveillance tests (sensitivity and specificity) was varied in the base-case model for both high and low sensitivity and specificity values (reported in Table 34). This was undertaken in multiway sensitivity analysis, varying all the tests simultaneously. 


\section{Sensitivity analysis on costs}

A range of sensitivity analyses on costs were performed. This included high treatment costs and surveillance costs. For example, the base-case model assumes that all women who receive hormone therapy receive tamoxifen. The consequences of this assumption were tested in sensitivity analyses. This involved re-estimating the model when all treatment costs were set to their highest estimates (highest cost for hormone and chemotherapy). In addition to treatment costs, higher surveillance costs were also incorporated into this sensitivity analysis. This included using the higher mammography cost ( $£ 77.80$ as opposed to base-case assumption of $£ 45.50)$ and also doubling the cost of an MRI. These cost estimates can be seen in Tables 35 and 36 . The same analyses were conducted for the IBTR model.

The cost of all clinical examinations conducted either at the point of surveillance or in follow-up visits were varied from the base-case assumption that these clinical examinations would be carried out by a consultant grade, to the alternative assumption that these examinations were carried out by a non-consultant (Table 35). This analysis was conducted for both the base-case model and the IBTR model.

\section{Sensitivity analysis on health-state utilities}

Health-state utility values were also tested in a range of sensitivity analyses. This included replacing the base-case values for quality of life (assumed to be the low values reported in Table 38) with high estimates of quality of life (Table 38).

In addition, quality of life was further tested in both the base-case model and the IBTR model by varying the quality of life in unmanaged states. The base-case model assumes that women in unmanaged states have the same quality of life as women free of disease. This assumption is tested in sensitivity analysis by giving women in unmanaged states the same quality of life as women after treatment.

\section{Age}

The effect of age was tested in the sensitivity analysis with the base-case model and IBTR model, which we ran for a starting age of 40 years and a starting age of 70 years. This analysis was conducted as multiway sensitivity analysis with a range of values varied in the analysis. For the younger age group (starting age 40 years) this included high chemotherapy and high hormone therapy costs (Table 36), high cancer incidence (0.0152, Table 29) and a short doubling time (0.2623, Table 33). In addition, for this age group, a further multiway analysis was conducted, repeating the analysis above, with the addition of the high inflation factor for unmanaged states (2.916, Table 32).

For a starting age of 70 years, sensitivity analysis was conducted for the base-case model and IBTR model using the new starting age of 70 years and the low hazard rate (0.0018, Table 29). All of the other variables were assumed to be as the base-case assumptions.

\section{Results}

\section{Base-case results}

Results for women who received BCS or mastectomy for their primary cancer

Tables 39 and 40 report the results of the base-case analyses for the average women treated for their primary breast cancer with BCS or with a mastectomy. These data can be used to inform 
TABLE 39 Results of the base-case analysis for women treated for their primary cancer with BCS

\begin{tabular}{|c|c|c|c|c|c|c|c|}
\hline \multirow[b]{2}{*}{ Regimen } & \multirow[b]{2}{*}{ Cost (£) } & \multirow[b]{2}{*}{ QALY } & \multirow[b]{2}{*}{ ICER (£) } & \multicolumn{4}{|c|}{ Net benefits (£) } \\
\hline & & & & $\lambda=£ 20,000$ & Rank & $\lambda=£ 30,000$ & Rank \\
\hline \multicolumn{8}{|l|}{ 12-month interval } \\
\hline No surveillance & 1033 & 12.925 & & 257,459 & 12 & 386,705 & 13 \\
\hline Mammography alone & 1970 & 13.123 & 4727 & 260,484 & 1 & 391,711 & 1 \\
\hline Mammography + clinical & 3396 & 13.129 & 236,826 & 259,178 & 8 & 390,465 & 7 \\
\hline MRI + clinical & 6499 & 13.139 & 297,848 & 256,283 & 13 & 387,675 & 12 \\
\hline \multicolumn{8}{|l|}{ 18-month interval } \\
\hline No surveillance & 1033 & 12.925 & & 257,459 & 12 & 386,705 & 13 \\
\hline Mammography alone & 1722 & 13.105 & 3811 & 260,385 & 2 & 391,438 & 2 \\
\hline Mammography + clinical & 2743 & 13.114 & 118,455 & 259,536 & 6 & 390,675 & 5 \\
\hline MRI + clinical & 4969 & 13.130 & 142,331 & 257,623 & 11 & 388,919 & 11 \\
\hline \multicolumn{8}{|l|}{ 24-month interval } \\
\hline No surveillance & 1033 & 12.925 & & 257,459 & 12 & 386,705 & 13 \\
\hline Mammography alone & 1587 & 13.089 & 3366 & 260,197 & 3 & 391,089 & 3 \\
\hline Mammography + clinical & 2408 & 13.100 & 78,167 & 259,586 & 5 & 390,583 & 6 \\
\hline MRI + clinical & 4200 & 13.120 & 90,382 & 258,191 & 10 & 389,387 & 10 \\
\hline \multicolumn{8}{|l|}{ 36-month interval } \\
\hline No surveillance & 1033 & 12.925 & & 257,459 & 12 & 386,705 & 14 \\
\hline Mammography alone & 1498 & 13.075 & 3099 & 259,994 & 4 & 390,740 & 4 \\
\hline Mammography + clinical & 2201 & 13.086 & 59,607 & 259,528 & 7 & 390,392 & 8 \\
\hline MRI + clinical & 3734 & 13.109 & 66,789 & 258,453 & 9 & 389,547 & 9 \\
\hline
\end{tabular}

judgements about what would be the single best regimen for the NHS to adopt for all women who had previously been treated with BCS or all women who had previously been treated with mastectomy. For both populations the results are shown for a range of surveillance intervals ranging from 12 months to 36 months. The costs and outcomes for the 'no surveillance' option are the same regardless of the surveillance interval. For the other surveillance regimens, both costs and QALYs fall as the surveillance interval increases. However, for each surveillance regimen the reduction in QALYs is more than compensated for by a reduction in cost. This is illustrated by the reduction in the incremental cost per QALY reported for each regimen as the surveillance interval increases. For example, for women who had received BCS the incremental cost per QALY for mammography alone compared with 'no surveillance' is $£ 4727$ for a 12 -month surveillance regimen and $£ 3811$ for an 18 -month regimen. Similarly, for a 12 -month surveillance regimen the incremental cost per QALY for mammography and clinical follow-up

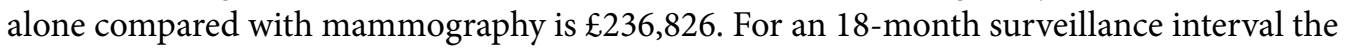
incremental cost per QALY falls to $£ 118,455$.

The results of the two sets of analyses reported in Tables 39 and 40 are very similar. As would be expected, the no surveillance regimen is least costly but also least effective. Whether or not a more costly but more effective regimen is considered worthwhile depends upon society's willingness to pay for a QALY. Within England, the threshold adopted by NICE lies somewhere between $£ 20,000$ and $£ 30,000$, and, as shown in Tables 39 and 40, only one regimen, mammography alone, is associated with an incremental cost per QALY below $£ 20,000$. 
TABLE 40 Results of the base-case analysis for women treated for their primary cancer with mastectomy

\begin{tabular}{|c|c|c|c|c|c|c|c|}
\hline \multirow[b]{2}{*}{ Regimen } & \multirow[b]{2}{*}{ Cost (£) } & \multirow[b]{2}{*}{ QALY } & \multirow[b]{2}{*}{ ICER (£) } & \multicolumn{4}{|c|}{ Net benefits $(£)$} \\
\hline & & & & $\lambda=20,000$ & Rank & $\lambda=30,000$ & Rank \\
\hline \multicolumn{8}{|l|}{ 12-month interval } \\
\hline No surveillance & 1012 & 12.938 & & 257,753 & 8 & 387,136 & 10 \\
\hline Mammography alone & 1927 & 13.127 & 4861 & 260,603 & 1 & 391,868 & 1 \\
\hline Mammography + clinical & 3360 & 13.132 & 250,230 & 259,285 & 8 & 390,608 & 7 \\
\hline \multicolumn{8}{|l|}{ 18-month interval } \\
\hline No surveillance & 1012 & 12.938 & & 257,753 & 8 & 387,136 & 9 \\
\hline Mammography alone & 1680 & 13.110 & 3885 & 260,521 & 2 & 391,621 & 2 \\
\hline Mammography + clinical & 2705 & 13.118 & 125,115 & 259,660 & 7 & 390,842 & 5 \\
\hline \multicolumn{8}{|l|}{ 24-month interval } \\
\hline No surveillance & 1012 & 12.938 & & 257,753 & 8 & 387,136 & 10 \\
\hline Mammography alone & 1545 & 13.095 & 3407 & 260,349 & 3 & 391,296 & 3 \\
\hline Mammography + clinical & 2369 & 13.105 & 82,525 & 259,725 & 5 & 390,772 & 6 \\
\hline \multicolumn{8}{|l|}{ 36-month interval } \\
\hline No surveillance & 1012 & 12.938 & & 257,753 & 8 & 387,136 & 10 \\
\hline Mammography alone & 1457 & 13.081 & 3117 & 260,160 & 4 & 390,969 & 4 \\
\hline Mammography + clinical & 2161 & 13.092 & 62,899 & 259,680 & 6 & 390,600 & 8 \\
\hline
\end{tabular}

Incremental cost per QALYs, as reported in Tables 39 and 40, can be difficult to interpret when the choice is between several different options (e.g. in Table 39 there are 13 different regimens and it is not immediately obvious which option might be considered most cost-effective). Therefore, we used the net benefit statistic to compare regimens. When society's willingness to pay for a QALY is $£ 20,000$, the regimen that is associated with the highest net benefit is mammography alone every year (Tables 39 and 40 ). When the threshold was increased to $£ 30,000$ mammography only had the greatest net benefit. Regardless of the surveillance interval, mammography alone had the highest net benefits and the regimen with the lowest net benefit was always no surveillance.

In Table 39 the impact of substituting a more effective but more costly surveillance technology (MRI) for mammography was considered. Regardless of the surveillance interval, this regimen was associated with a net benefit greater than that of the no surveillance regimen but less than that of all of the other regimens.

\section{Modelling IBTR alone}

The analyses reported in Tables 39 and 40 made the assumption that the consequences of an IBTR are the same as those of an otherwise identical contralateral recurrence. However, the analyses reported in Chapter 6 suggest that the mortality associated with IBTR is substantially higher than that associated with an otherwise identical recurrence in the contralateral breast. In this analysis this increased risk of death from IBTR is modelled (Table 41).

In these analyses the regimen mammography alone has an incremental cost per QALY compared with 'no surveillance' of $<£ 4000$ and the highest net benefit regardless of the surveillance interval. Mammography alone at 12 months has a marginally higher net benefit than mammography alone at 24 months for both a $£ 20,000$ and a $£ 30,000$ threshold for society’s willingness to pay for a QALY. 
TABLE 41 Ipsilateral breast tumour recurrence has a substantial higher risk of death than MCBC

\begin{tabular}{|c|c|c|c|c|c|c|c|}
\hline \multirow[b]{2}{*}{ Regimen } & \multirow[b]{2}{*}{ Cost $(£)$} & \multirow[b]{2}{*}{ QALY } & \multirow[b]{2}{*}{ ICER (£) } & \multicolumn{4}{|c|}{ Net benefits (£) } \\
\hline & & & & $\lambda=£ 20,000$ & Rank & $\lambda=£ 30,000$ & Rank \\
\hline \multicolumn{8}{|l|}{ 12-month interval } \\
\hline No surveillance & 978 & 12.830 & & 255,620 & 12 & 383,920 & 13 \\
\hline Mammography alone & 1956 & 13.076 & 3973 & 259,565 & 1 & 390,326 & 1 \\
\hline Mammography + clinical & 3384 & 13.084 & 177,150 & 258,298 & 8 & 389,139 & 5 \\
\hline MRI + clinical & 6491 & 13.098 & 216,537 & 255,478 & 13 & 386,463 & 12 \\
\hline \multicolumn{8}{|l|}{ 18-month interval } \\
\hline GP only & 978 & 12.830 & & 255,620 & 12 & 383,920 & 13 \\
\hline Mammography alone & 1703 & 13.053 & 3247 & 259,359 & 2 & 389,890 & 2 \\
\hline Mammography + clinical & 2727 & 13.064 & 92,265 & 258,557 & 5 & 389,198 & 4 \\
\hline MRI + clinical & 4958 & 13.085 & 108,007 & 256,739 & 11 & 387,587 & 11 \\
\hline \multicolumn{8}{|l|}{ 24-month interval } \\
\hline GP only & 978 & 12.830 & & 255,620 & 12 & 383,920 & 13 \\
\hline Mammography alone & 1563 & 13.032 & 2887 & 259,084 & 3 & 389,407 & 3 \\
\hline Mammography + clinical & 2387 & 13.046 & 62,482 & 258,523 & 6 & 388,978 & 6 \\
\hline MRI + clinical & 4185 & 13.071 & 70,315 & 257,237 & 10 & 387,947 & 10 \\
\hline \multicolumn{8}{|l|}{ 36-month interval } \\
\hline GP only & 978 & 12.830 & & 255,620 & 12 & 383,920 & 13 \\
\hline Mammography alone & 1468 & 13.014 & 2664 & 258,808 & 4 & 388,946 & 7 \\
\hline Mammography + clinical & 2175 & 13.028 & 48,551 & 258,393 & 7 & 388,676 & 8 \\
\hline MRI + clinical & 3716 & 13.058 & 52,811 & 257,435 & 9 & 388,010 & 9 \\
\hline
\end{tabular}

\section{Sensitivity analyses}

We conducted a range of different sensitivity analyses, as described above in Presentation of results. As the results for the analyses for women who received BCS for their primary cancer are similar to those obtained when we consider women who received a mastectomy for their primary cancer we present sensitivity analyses solely for the scenario where women received BCS for their primary cancer. However, we also report selected analyses for a model that considers IBTR alone.

\section{Sensitivity analysis around the breast-conserving model Probability of developing cancer}

Figures 26-29 illustrate the impact on incremental cost per QALYs as the incidence of cancer increases. In each figure, three lines are shown:

1. The incremental cost per QALY of mammography alone compared with no surveillance. This line can be used to inform the question: is it worth adopting the more effective but more costly mammography alone follow-up in place of the less costly and less effective no surveillance regimen?

2. The incremental cost per QALY of mammography plus clinical follow-up compared with mammography alone. This line can be used to inform the question: is it worth adopting the more effective but more costly mammography plus clinical follow-up in place of the less costly and less effective mammography alone regimen?

3. The incremental cost per QALY of MRI plus clinical follow-up compared with mammography plus clinical follow-up. This line can be used to inform the question: is it 


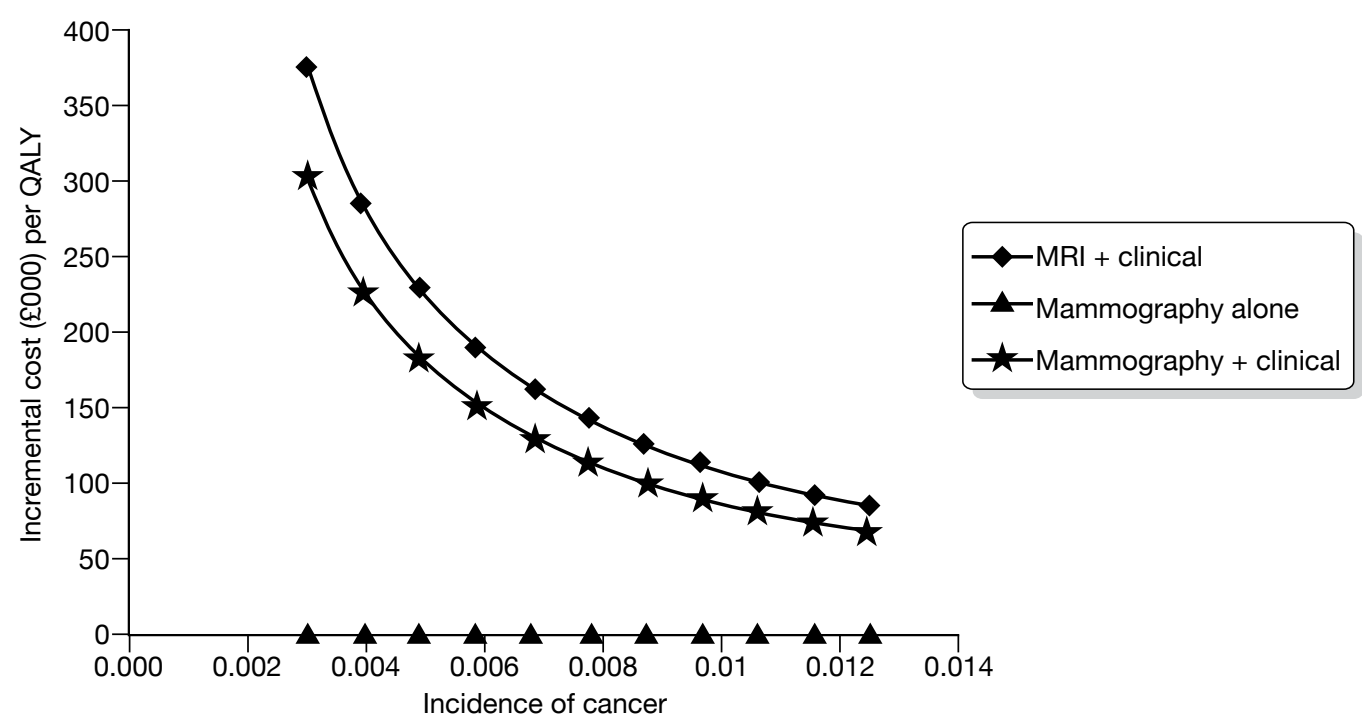

FIGURE 26 Incremental cost per QALYs for the different surveillance regimens at a 12-month surveillance interval.

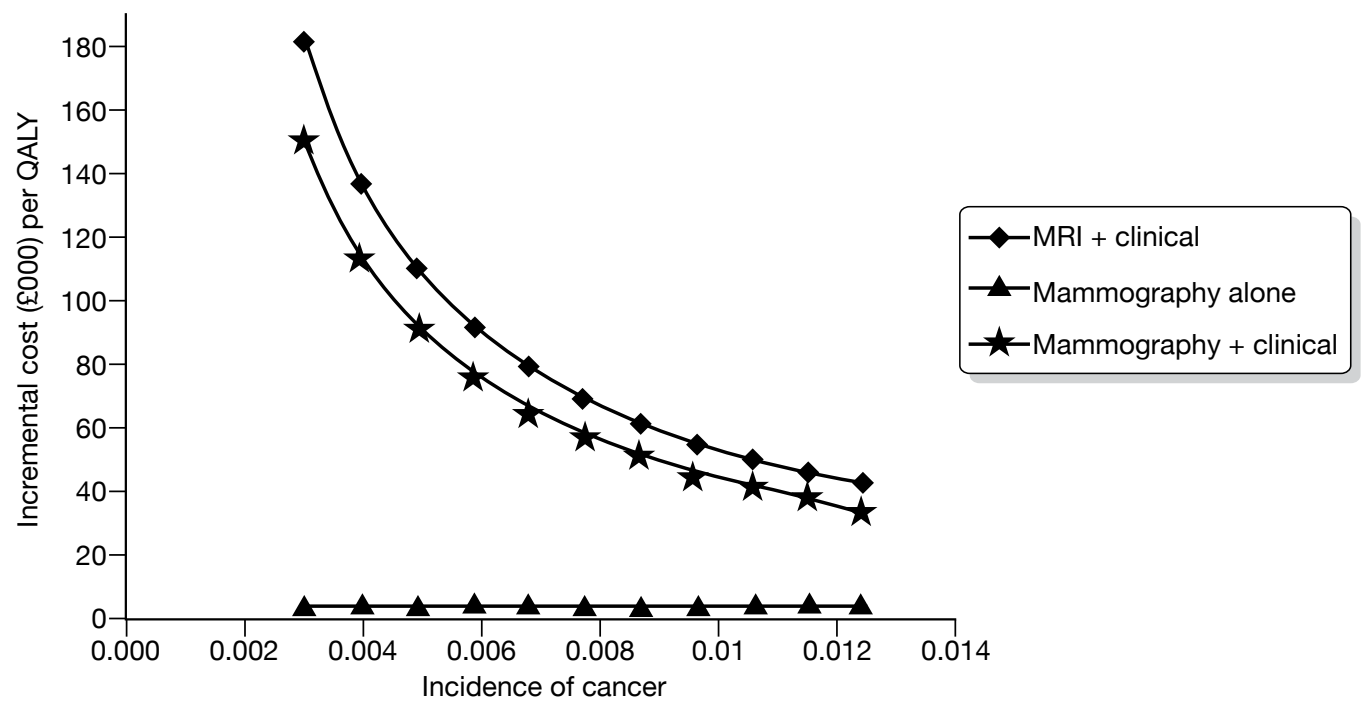

FIGURE 27 Incremental cost per QALYs for the different surveillance regimens at an 18-month surveillance interval.

worth adopting the more effective but more costly MRI plus clinical follow-up in place of the less costly and less effective mammography plus clinical follow-up regimen?

The results of the analysis shown in these figures suggest that:

- At all screening intervals considered some form of active surveillance might be considered cost-effective.

- Should the incidence of IBTR and MCBC increase towards the upper values of incidence considered, which are typical of those we might expect for higher risk women (e.g. those whose primary cancers were of higher grade, who were younger than 50 years and who had lymph node involvement), a regimen of clinical follow-up and mammography is more likely to be worthwhile. Furthermore, when the surveillance interval is 24 months the incremental 


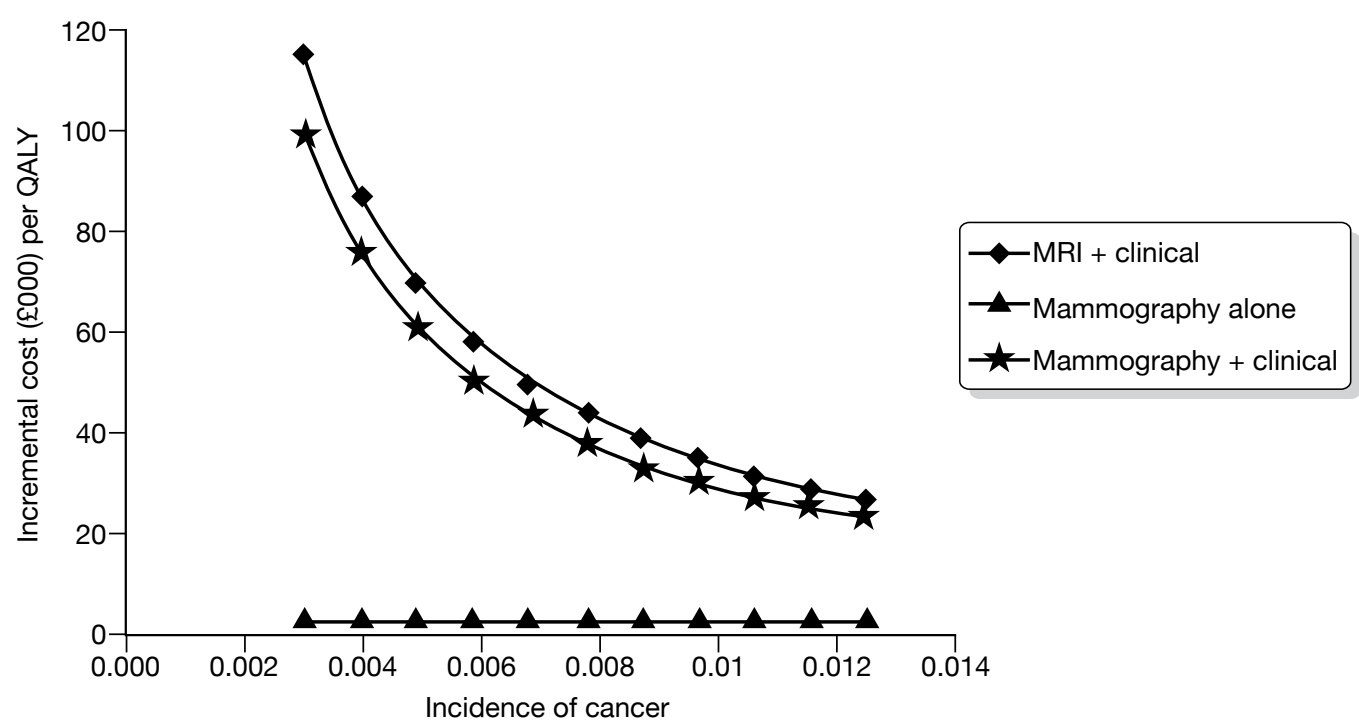

FIGURE 28 Incremental cost per QALYs for the different surveillance regimens at a 24-month surveillance interval.

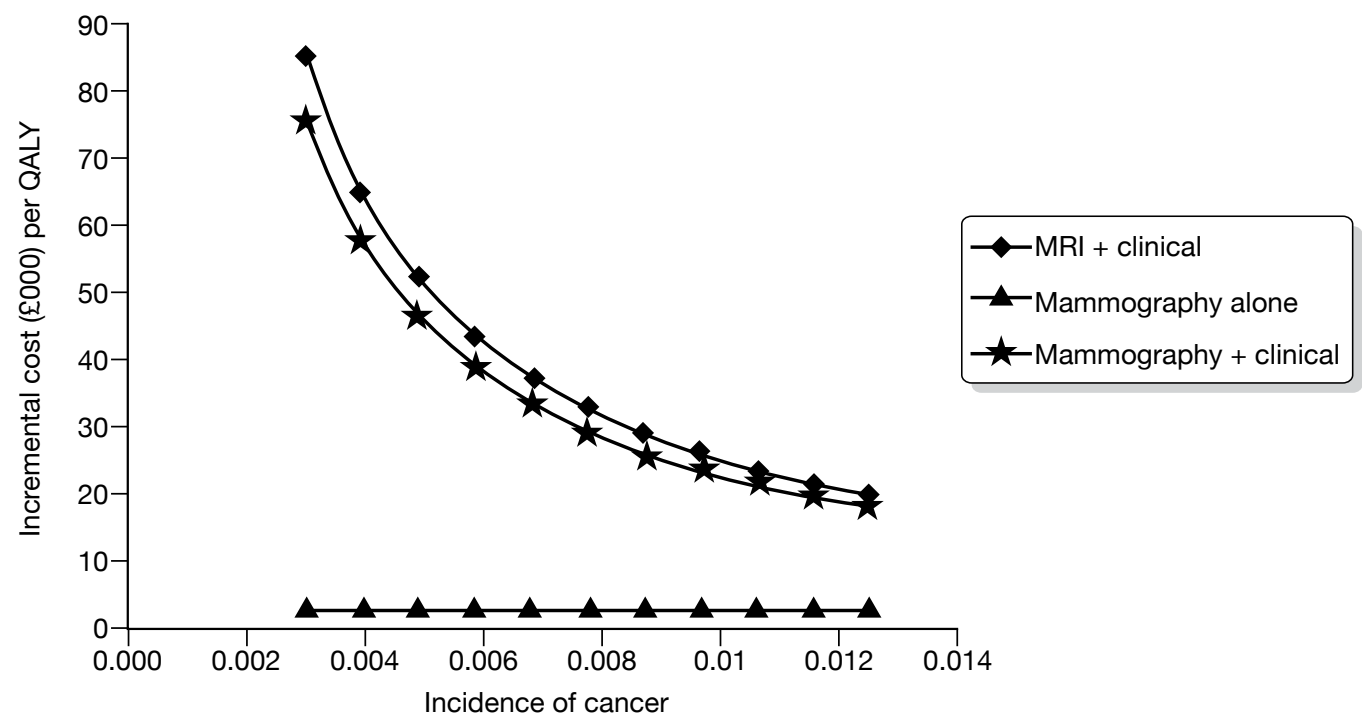

FIGURE 29 Incremental cost per QALYs for the different surveillance regimens at a 36-month surveillance interval.

cost per QALY compared with mammography alone approaches $£ 30,000$. At a surveillance interval of 36 months, it is approximately $£ 25,000$.

- As the screening interval and risk of IBTR and MCBC increases towards 36 months, it becomes more likely that a more costly but more effective surveillance intervention (in this analysis typified by MRI plus clinical follow-up) might be worthwhile.

Inflating the risk of death from untreated cancer

Sensitivity analysis was conducted to test the effect of inflating the risk of death for women who are unmanaged for cancer. In the sensitivity analysis the increased risk of death from cancer in unmanaged states was inflated from the base-case risk of 2.34 to a high of 2.196 (Table 42). The results of this analysis were broadly similar to the base-case analysis presented in Table 39 . We conducted this sensitivity analysis for both the base-case model and the IBTR model. 
TABLE 42 Increasing the risk of death for unmanaged disease

\begin{tabular}{|c|c|c|c|c|c|c|c|}
\hline \multirow[b]{2}{*}{ Regimen } & \multirow[b]{2}{*}{ Cost (£) } & \multirow[b]{2}{*}{ QALY } & \multirow[b]{2}{*}{ ICER (£) } & \multicolumn{4}{|c|}{ Net benefits (£) } \\
\hline & & & & $\lambda=£ 20,000$ & Rank & $\lambda=£ 30,000$ & Rank \\
\hline \multicolumn{8}{|l|}{ 12-month interval } \\
\hline GP only & 1014 & 12.898 & & 256,939 & 13 & 385,915 & 13 \\
\hline Mammography alone & 1967 & 13.120 & 4278 & 260,440 & 1 & 391,644 & 1 \\
\hline Mammography + clinical & 3394 & 13.127 & 217,826 & 259,145 & 8 & 390,414 & 7 \\
\hline MRI + clinical & 6498 & 13.138 & 274,030 & 256,267 & 14 & 387,649 & 12 \\
\hline \multicolumn{8}{|l|}{ 18-month interval } \\
\hline GP only & 1014 & 12.898 & & 256,939 & 12 & 385,915 & 13 \\
\hline Mammography alone & 1718 & 13.101 & 3451 & 260,312 & 2 & 391,326 & 2 \\
\hline Mammography + clinical & 2740 & 13.111 & 108,767 & 259,477 & 6 & 390,586 & 4 \\
\hline MRI + clinical & 4967 & 13.128 & 130,986 & 257,590 & 11 & 388,869 & 11 \\
\hline \multicolumn{8}{|l|}{ 24-month interval } \\
\hline GP only & 1014 & 12.898 & & 256,939 & 13 & 385,915 & 13 \\
\hline Mammography alone & 1581 & 13.084 & 3045 & 260,096 & 3 & 390,935 & 3 \\
\hline Mammography + clinical & 2403 & 13.095 & 71,562 & 259,504 & 5 & 390,457 & 6 \\
\hline MRI + clinical & 4197 & 13.117 & 83,055 & 258,142 & 10 & 389,312 & 10 \\
\hline \multicolumn{8}{|l|}{ 36-month interval } \\
\hline GP only & 1014 & 12.898 & & 256,939 & 13 & 385,915 & 13 \\
\hline Mammography alone & 1490 & 13.068 & 2796 & 259,867 & 4 & 390,546 & 5 \\
\hline Mammography + clinical & 2194 & 13.081 & 54,384 & 259,422 & 7 & 390,231 & 8 \\
\hline MRI + clinical & 3730 & 13.106 & 61,238 & 258,388 & 9 & 389,447 & 9 \\
\hline
\end{tabular}

\section{Changes to the probability of progressing to a more serious risk profile}

Table 43 reports the impact of increasing the speed that an untreated cancer progresses to a risk profile with a worse prognosis. As would be expected the higher the probability of progression (which would be analogous to a shorter doubling time of a tumour) the more likely earlier and more intensive follow-up becomes. Nevertheless, in this one-way sensitivity analysis none of the options, other than mammography alone, is associated with incremental costs per QALY approaching a value that society typically might be willing to pay.

\section{Changes to the sensitivity and specificity of the tests}

Tables 44 and 45 show multiway sensitivity analysis on the diagnostic performance of the surveillance tests. Again, changes in the sensitivities and specificities alone do not greatly alter the estimated cost-effectiveness of the different regimens.

\section{Changes to costs of tests and treatments}

Sensitivity analysis was also performed on costs. This included a high treatment cost and high surveillance cost sensitivity analysis. This involved re-estimating the model when all treatment costs were set to their highest estimates (highest cost for hormone and chemotherapy). In addition to treatment costs, higher surveillance costs were also incorporated into this sensitivity analysis. This included using the higher mammography cost ( $£ 78$ as opposed to base-case assumption of $£ 45.50$ ) and also doubling the cost of an MRI. These cost estimates can be seen in Table 46. The same analyses were conducted for the IBTR model (and are reported in the next subsection). 
TABLE 43 The impact of changing the probability of progressing to a higher risk profile

\begin{tabular}{|c|c|c|c|c|}
\hline \multirow[b]{2}{*}{ Surveillance interval } & \multirow[b]{2}{*}{ Regimen } & \multicolumn{3}{|c|}{$\begin{array}{l}\text { ICER at low and high probability of increasing to next risk } \\
\text { profile per cycle: }\end{array}$} \\
\hline & & 0.0923 & 0.1555 & 0.2623 \\
\hline \multirow[t]{4}{*}{12 months } & No surveillance & & & \\
\hline & Mammography alone & 6580 & 4727 & 3679 \\
\hline & Mammography + clinical & 389,207 & 236,826 & 137,745 \\
\hline & MRI + clinical & 487,420 & 297,848 & 170,226 \\
\hline \multirow[t]{4}{*}{18 months } & No surveillance & & & \\
\hline & Mammography alone & 5168 & 3811 & 3093 \\
\hline & Mammography + clinical & 193,537 & 118,455 & 71,767 \\
\hline & MRI + clinical & 235,502 & 142,331 & 83,040 \\
\hline \multirow[t]{4}{*}{24 months } & No surveillance & & & \\
\hline & Mammography alone & 4456 & 3366 & 2833 \\
\hline & Mammography + clinical & 125,343 & 78,167 & 49,644 \\
\hline & MRI + clinical & 148,299 & 90,382 & 54,714 \\
\hline \multirow[t]{4}{*}{36 months } & No surveillance & & & \\
\hline & Mammography alone & 4014 & 3099 & 2690 \\
\hline & Mammography + clinical & 93,489 & 59,607 & 39,604 \\
\hline & MRI + clinical & 107,873 & 66,789 & 42,079 \\
\hline
\end{tabular}

TABLE 44 High sensitivity and specificity values

\begin{tabular}{|c|c|c|c|c|c|c|c|}
\hline \multirow[b]{2}{*}{ Regimen } & \multirow[b]{2}{*}{ Cost (£) } & \multirow[b]{2}{*}{ QALY } & \multirow[b]{2}{*}{ ICER (£) } & \multicolumn{4}{|c|}{ Net benefits $(£)$} \\
\hline & & & & $\lambda=£ 20,000$ & Rank & $\lambda=£ 30,000$ & Rank \\
\hline \multicolumn{8}{|l|}{ 12-month interval } \\
\hline No surveillance & 1058 & 12.958 & & 258,097 & 11 & 387,674 & 13 \\
\hline Mammography alone & 1944 & 13.126 & 5280 & 260,568 & 1 & 391,823 & 1 \\
\hline Mammography + clinical & 3343 & 13.132 & 211,875 & 259,300 & 8 & 390,622 & 8 \\
\hline MRI + clinical & 6390 & 13.141 & 338,553 & 256,433 & 13 & 387,845 & 12 \\
\hline \multicolumn{8}{|l|}{ 18-month interval } \\
\hline No surveillance & 1058 & 12.958 & & 258,097 & 11 & 387,674 & 13 \\
\hline Mammography alone & 1690 & 13.110 & 4150 & 260,511 & 2 & 391,612 & 2 \\
\hline Mammography + clinical & 2676 & 13.120 & 104,218 & 259,715 & 7 & 390,910 & 5 \\
\hline MRI + clinical & 4826 & 13.133 & 159,306 & 257,835 & 12 & 389,165 & 11 \\
\hline \multicolumn{8}{|l|}{ 24-month interval } \\
\hline No surveillance & 1058 & 12.958 & & 258,097 & 11 & 387,674 & 13 \\
\hline Mammography alone & 1554 & 13.096 & 3597 & 260,360 & 3 & 391,317 & 3 \\
\hline Mammography + clinical & 2335 & 13.107 & 67,680 & 259,810 & 5 & 390,882 & 6 \\
\hline MRI + clinical & 4040 & 13.124 & 99,707 & 258,447 & 10 & 389,691 & 10 \\
\hline \multicolumn{8}{|l|}{ 36-month interval } \\
\hline No surveillance & 1058 & 12.958 & & 258,097 & 11 & 387,674 & 13 \\
\hline Mammography alone & 1466 & 13.083 & 3263 & 260,189 & 4 & 391,017 & 4 \\
\hline Mammography + clinical & 2126 & 13.096 & 50,836 & 259,789 & 6 & 390,746 & 7 \\
\hline MRI + clinical & 3566 & 13.116 & 72,694 & 258,745 & 9 & 389,900 & 9 \\
\hline
\end{tabular}


TABLE 45 Low sensitivity and specificity values

\begin{tabular}{|c|c|c|c|c|c|c|c|}
\hline \multirow[b]{2}{*}{ Regimen } & \multirow[b]{2}{*}{ Cost (£) } & \multirow[b]{2}{*}{ QALY } & \multirow[b]{2}{*}{ ICER (£) } & \multicolumn{4}{|c|}{ Net benefits $(£)$} \\
\hline & & & & $\lambda=£ 20,000$ & Rank & $\lambda=£ 30,000$ & Rank \\
\hline \multicolumn{8}{|l|}{ 12-month interval } \\
\hline No surveillance & 1061 & 12.900 & & 256,942 & 12 & 385,944 & 13 \\
\hline Mammography alone & 2023 & 13.121 & 4365 & 260,388 & 1 & 391,594 & 1 \\
\hline Mammography + clinical & 3467 & 13.125 & 342,589 & 259,029 & 8 & 390,277 & 6 \\
\hline MRI + clinical & 6600 & 13.128 & 859,244 & 255,969 & 13 & 387,253 & 12 \\
\hline \multicolumn{8}{|l|}{ 18-month interval } \\
\hline No surveillance & 1061 & 12.900 & & 256,942 & 12 & 385,944 & 13 \\
\hline Mammography alone & 1792 & 13.102 & 3622 & 260,246 & 2 & 391,264 & 2 \\
\hline Mammography + clinical & 2835 & 13.108 & 173,779 & 259,323 & 6 & 390,401 & 5 \\
\hline MRI + clinical & 5094 & 13.113 & 425,796 & 257,170 & 11 & 388,301 & 11 \\
\hline \multicolumn{8}{|l|}{ 24-month interval } \\
\hline No surveillance & 1061 & 12.900 & & 256,942 & 12 & 385,944 & 13 \\
\hline Mammography alone & 1664 & 13.084 & 3270 & 260,026 & 3 & 390,870 & 3 \\
\hline Mammography + clinical & 2508 & 13.092 & 116,098 & 259,327 & 5 & 390,244 & 7 \\
\hline MRI + clinical & 4331 & 13.098 & 278,691 & 257,634 & 10 & 388,617 & 10 \\
\hline \multicolumn{8}{|l|}{ 36-month interval } \\
\hline No surveillance & 1061 & 12.900 & & 256,942 & 12 & 385,944 & 13 \\
\hline Mammography alone & 1577 & 13.069 & 3063 & 259,796 & 4 & 390,483 & 4 \\
\hline Mammography + clinical & 2303 & 13.077 & 89,497 & 259,232 & 7 & 390,000 & 8 \\
\hline MRI + clinical & 3866 & 13.084 & 211,068 & 257,818 & 9 & 388,660 & 9 \\
\hline
\end{tabular}

In addition, Table 47 shows sensitivity analysis in which the cost of a clinical examination is priced at the consultant and non-consultant rate and the effect on the incremental cost per QALYs. Although the changes are minor, adopting a lower cost for a clinical examination makes the mammography alone regimen marginally less cost-effective compared with 'no surveillance'. This is because the lower cost is also incurred for all clinical examinations, including those during follow-up for those with IBTR or MCBC.

\section{Changes to utility estimates used}

The analysis presented in Table 48 is based on the high estimates of quality of life reported in Table 38. The results suggest that at a willingness-to-pay threshold of $£ 20,000$ the regimen with the highest net benefit is likely to be mammography alone at 12 -month surveillance intervals, followed by mammography alone at 18 -, then 24 - and then 36 -month intervals. At a willingnessto-pay threshold of $£ 30,000$ the ordering is very similar with the exception that mammography plus clinical examination has the fourth highest net benefit at a willingness-to-pay threshold of $£ 30,000$.

In addition to sensitivity analysis on higher quality-of-life values, we conducted further sensitivity analyses to test the base-case assumption that women in unmanaged states have the same quality of life as women who are disease free. We tested this assumption by giving all women in unmanaged states the same utility as women who had been treated for IBTR or MCBC. These results are presented in Table 49. Decreasing the quality of life of women in unmanaged states has no appreciable effect on the analysis presented above for a threshold value of $£ 20,000$ or $£ 30,000$. 
TABLE 46 High-cost sensitivity analysis

\begin{tabular}{|c|c|c|c|c|c|c|c|}
\hline \multirow[b]{2}{*}{ Regimen } & \multirow[b]{2}{*}{ Cost (£) } & \multirow[b]{2}{*}{ QALY } & \multirow[b]{2}{*}{$\operatorname{ICER}(£)$} & \multicolumn{4}{|c|}{ Net benefits $(£)$} \\
\hline & & & & $\lambda=£ 20,000$ & Rank & $\lambda=£ 30,000$ & Rank \\
\hline \multicolumn{8}{|l|}{ 12-month interval } \\
\hline No surveillance & 1704 & 12.925 & & 256,787 & 9 & 386,033 & 10 \\
\hline Mammography alone & 3940 & 13.123 & 11,284 & 258,514 & 3 & 389,741 & 1 \\
\hline Mammography + clinical & 5384 & 13.129 & 239,765 & 257,191 & 8 & 388,478 & 8 \\
\hline MRI + clinical & 11826 & 13.139 & 618,286 & 250,957 & 13 & 382,348 & 13 \\
\hline \multicolumn{8}{|l|}{ 18-month interval } \\
\hline No surveillance & 1704 & 12.925 & & 256,787 & 9 & 386,033 & 10 \\
\hline Mammography alone & 3483 & 13.105 & 9842 & 258,623 & 1 & 389,676 & 2 \\
\hline Mammography + clinical & 4532 & 13.114 & 121,600 & 257,747 & 7 & 388,887 & 6 \\
\hline MRI + clinical & 9173 & 13.130 & 296,783 & 253,419 & 12 & 384,715 & 12 \\
\hline \multicolumn{8}{|l|}{ 24-month interval } \\
\hline No surveillance & 1704 & 12.925 & & 256,787 & 9 & 386,033 & 10 \\
\hline Mammography alone & 3219 & 13.089 & 9199 & 258,565 & 2 & 389,458 & 3 \\
\hline Mammography + clinical & 4075 & 13.100 & 81,481 & 257,920 & 6 & 388,917 & 5 \\
\hline MRI + clinical & 7826 & 13.120 & 189,286 & 254,565 & 11 & 385,760 & 11 \\
\hline \multicolumn{8}{|l|}{ 36-month interval } \\
\hline No surveillance & 1704 & 12.925 & & 256,787 & 9 & 386,033 & 10 \\
\hline Mammography alone & 3034 & 13.075 & 8861 & 258,459 & 4 & 389,205 & 4 \\
\hline Mammography + clinical & 3777 & 13.086 & 63,077 & 257,951 & 5 & 388,815 & 7 \\
\hline MRI + clinical & 7,001 & 13.109 & 140,417 & 255,186 & 10 & 386,280 & 9 \\
\hline
\end{tabular}

TABLE 47 Consultant and non-consultant cost of clinical examinations

\begin{tabular}{|c|c|c|c|}
\hline \multirow[b]{2}{*}{ Surveillance interval } & \multirow[b]{2}{*}{ Regimen } & \multicolumn{2}{|c|}{ ICER at low and high clinical examination cost (£): } \\
\hline & & 65 & 86 \\
\hline \multirow[t]{4}{*}{12 months } & No surveillance & & \\
\hline & Mammography alone & 4945 & 4727 \\
\hline & Mammography + clinical & 178,716 & 236,826 \\
\hline & MRI + clinical & 297,829 & 297,848 \\
\hline \multirow[t]{4}{*}{18 months } & No surveillance & & \\
\hline & Mammography alone & 4057 & 3811 \\
\hline & Mammography + clinical & 89,362 & 118,455 \\
\hline & MRI + clinical & 142,296 & 142,331 \\
\hline \multirow[t]{4}{*}{24 months } & No surveillance & & \\
\hline & Mammography alone & 3644 & 3366 \\
\hline & Mammography + clinical & 59,002 & 78,167 \\
\hline & MRI + clinical & 90,339 & 90,382 \\
\hline \multirow[t]{4}{*}{36 months } & No surveillance & & \\
\hline & Mammography alone & 3410 & 3099 \\
\hline & Mammography + clinical & 45,043 & 59,607 \\
\hline & MRI + clinical & 66,741 & 66,789 \\
\hline
\end{tabular}


TABLE 48 Sensitivity analysis using high estimates of quality of life

\begin{tabular}{|c|c|c|c|c|c|c|c|}
\hline \multirow[b]{2}{*}{ Regimen } & \multirow[b]{2}{*}{ Cost (£) } & \multirow[b]{2}{*}{ QALY } & \multirow[b]{2}{*}{ ICER (£) } & \multicolumn{4}{|c|}{ Net benefits $(£)$} \\
\hline & & & & $\lambda=£ 20,000$ & Rank & $\lambda=£ 30,000$ & Rank \\
\hline \multicolumn{8}{|l|}{ 12-month interval } \\
\hline No surveillance & 1033 & 13.080 & & 260,573 & 12 & 391,376 & 13 \\
\hline Mammography alone & 1970 & 13.320 & 3902 & 264,436 & 1 & 397,638 & 1 \\
\hline Mammography + clinical & 3396 & 13.328 & 183,571 & 263,165 & 8 & 396,445 & 5 \\
\hline MRI + clinical & 6499 & 13.341 & 231,932 & 260,329 & 9 & 393,743 & 12 \\
\hline \multicolumn{8}{|l|}{ 18-month interval } \\
\hline No surveillance & 1033 & 13.080 & & 260,573 & & 391,376 & 13 \\
\hline Mammography alone & 1722 & 13.298 & 3164 & 264,237 & 2 & 397,216 & 2 \\
\hline Mammography + clinical & 2743 & 13.309 & 91,801 & 263,438 & 5 & 396,529 & 4 \\
\hline MRI + clinical & 4969 & 13.329 & 109,808 & 261,618 & 11 & 394,911 & 11 \\
\hline \multicolumn{8}{|l|}{ 24-month interval } \\
\hline No surveillance & 1033 & 13.080 & & 260,573 & 12 & 391,376 & 13 \\
\hline Mammography alone & 1587 & 13.277 & 2813 & 263,958 & 3 & 396,731 & 3 \\
\hline Mammography + clinical & 2408 & 13.291 & 61,015 & 263,407 & 6 & 396,314 & 6 \\
\hline MRI + clinical & 4200 & 13.316 & 69,854 & 262,128 & 10 & 395,292 & 10 \\
\hline \multicolumn{8}{|l|}{ 36-month interval } \\
\hline No surveillance & 1033 & 13.080 & & 260,573 & 12 & 391,376 & 13 \\
\hline Mammography alone & 1498 & 13.259 & 2606 & 263,676 & 4 & 396,263 & 7 \\
\hline Mammography + clinical & 2201 & 13.274 & 46,960 & 263,272 & 7 & 396,009 & 8 \\
\hline MRI + clinical & 3734 & 13.303 & 51,938 & 262,329 & 13 & 395,361 & 9 \\
\hline
\end{tabular}

\section{Exploration of the impact of age at the time surveillance starts}

Table 50 reports the results of sensitivity analysis based on a starting age in the model of 40 years old. This table not only reports multiway sensitivity analysis for a starting age of 40 years, but also includes high chemotherapy costs and high hormone therapy costs (Table 36). In addition, a higher incidence rate for cancer is used in this model (0.0152, Table 29) and high probability of moving to the next risk profile. This analysis was conducted for surveillance intervals ranging from 12 to 36 months. In this analysis, the option with the highest net benefit is mammography alone at a surveillance interval of 12 months for a willingness-to-pay threshold of $£ 20,000$ per QALY. At a higher willingness-to-pay threshold of $£ 30,000$ per QALY, MRI plus clinical examination at a surveillance interval of 12 months has the highest net benefit. The second highest net benefit at a threshold of $£ 20,000$ and $£ 30,000$ is mammography and clinical examination at 12 -month surveillance intervals.

In addition to a starting age of 40 years old, sensitivity analysis was also conducted on a higher starting age. In this model all parameters are assumed to be as the base-case assumptions with two differences: (1) starting age is 70 years old and (2) the incidence of cancer is based on the lowest hazard estimates (Table 29). The results of this analysis are presented in Table 51. At a willingness-to-pay threshold of $£ 20,000$ per QALY, the test with the highest net benefit is mammography alone at a 36-month interval. At a willingness-to-pay threshold of $£ 30,000$, the regimen with the highest net benefit is mammography alone at intervals of 24 months. 
TABLE 49 Sensitivity analysis on quality of life in unmanaged states: breast-conserving model

\begin{tabular}{|c|c|c|c|c|c|c|c|}
\hline \multirow[b]{2}{*}{ Regimen } & \multirow[b]{2}{*}{ Cost (£) } & \multirow[b]{2}{*}{ QALY } & \multirow[b]{2}{*}{ ICER (£) } & \multicolumn{4}{|c|}{ Net benefits $(£)$} \\
\hline & & & & $\lambda=£ 20,000$ & Rank & $\lambda=£ 30,000$ & Rank \\
\hline \multicolumn{8}{|l|}{ 12-month interval } \\
\hline No surveillance & 1033 & 12.880 & & 256,573 & 12 & 385,377 & 13 \\
\hline Mammography alone & 1970 & 13.122 & 3868 & 260,478 & 1 & 391,702 & 1 \\
\hline Mammography + clinical & 3396 & 13.129 & 230,558 & 259,175 & 8 & 390,461 & 6 \\
\hline MRI + clinical & 6499 & 13.139 & 293,795 & 256,283 & 13 & 387,674 & 12 \\
\hline \multicolumn{8}{|l|}{ 18-month interval } \\
\hline No surveillance & 1033 & 12.880 & & 256,573 & 12 & 385,377 & 13 \\
\hline Mammography alone & 1722 & 13.087 & 3075 & 260,021 & 3 & 390,892 & 3 \\
\hline Mammography + clinical & 2743 & 13.098 & 112,416 & 259,227 & 7 & 390,211 & 8 \\
\hline MRI + clinical & 4969 & 13.119 & 137,827 & 257,418 & 11 & 388,612 & 11 \\
\hline \multicolumn{8}{|l|}{ 24-month interval } \\
\hline No surveillance & 1033 & 12.880 & & 256,573 & 12 & 385,377 & 13 \\
\hline Mammography alone & 1587 & 13.087 & 2679 & 260,155 & 2 & 391,027 & 2 \\
\hline Mammography + clinical & 2408 & 13.098 & 72,359 & 259,561 & 5 & 390,546 & 5 \\
\hline MRI + clinical & 4200 & 13.119 & 85,807 & 258,188 & 10 & 389,381 & 10 \\
\hline \multicolumn{8}{|l|}{ 36-month interval } \\
\hline No surveillance & 1033 & 12.880 & 0 & 256,573 & 12 & 385,377 & 13 \\
\hline Mammography alone & 1498 & 13.071 & 2435 & 259,927 & 4 & 390,640 & 4 \\
\hline Mammography + clinical & 2201 & 13.084 & 53,905 & 259,485 & 6 & 390,328 & 7 \\
\hline MRI + clinical & 3734 & 13.109 & 62,168 & 258,445 & 9 & 389,535 & 9 \\
\hline
\end{tabular}

TABLE 50 Sensitivity analysis for a starting age of 40 years: breast-conserving model

\begin{tabular}{|c|c|c|c|c|c|c|c|}
\hline \multirow[b]{2}{*}{ Regimen } & \multirow[b]{2}{*}{ Cost (£) } & \multirow[b]{2}{*}{ QALY } & \multirow[b]{2}{*}{ ICER (£) } & \multicolumn{4}{|c|}{ Net benefits $(£)$} \\
\hline & & & & $\lambda=£ 20,000$ & Rank & $\lambda=£ 30,000$ & Rank \\
\hline \multicolumn{8}{|l|}{ 12-month interval } \\
\hline No surveillance & 5097 & 14.592 & & 286,742 & 13 & 432,661 & 13 \\
\hline Mammography alone & 11,885 & 16.364 & 3831 & 315,392 & 1 & 479,031 & 3 \\
\hline Mammography + clinical & 13,332 & 16.428 & 22,596 & 315,226 & 2 & 479,505 & 2 \\
\hline MRI + clinical & 16,389 & 16.540 & 27,173 & 314,419 & 3 & 479,823 & 1 \\
\hline \multicolumn{8}{|l|}{ 18-month interval } \\
\hline No surveillance & 5097 & 14.592 & & 286,742 & 13 & 432,661 & 13 \\
\hline Mammography alone & 11,308 & 16.183 & 3904 & 312,348 & 7 & 474,175 & 7 \\
\hline Mammography + clinical & 12,457 & 16.272 & 12,929 & 312,976 & 5 & 475,692 & 6 \\
\hline MRI + clinical & 14,850 & 16.437 & 14,459 & 313,893 & 4 & 478,265 & 4 \\
\hline \multicolumn{8}{|l|}{ 24-month interval } \\
\hline No surveillance & 5097 & 14.592 & & 286,742 & 13 & 432,661 & 13 \\
\hline Mammography alone & 10,829 & 16.019 & 4016 & 309,558 & 10 & 469,751 & 10 \\
\hline Mammography + clinical & 11,853 & 16.124 & 9769 & 310,630 & 9 & 471,871 & 9 \\
\hline MRI + clinical & 13,966 & 16.328 & 10,350 & 312,600 & 6 & 475,883 & 5 \\
\hline
\end{tabular}


TABLE 50 Sensitivity analysis for a starting age of 40 years: breast-conserving model (continued)

\begin{tabular}{|c|c|c|c|c|c|c|c|}
\hline \multirow[b]{2}{*}{ Regimen } & \multirow[b]{2}{*}{ Cost (£) } & \multirow[b]{2}{*}{ QALY } & \multirow[b]{2}{*}{ ICER (£) } & \multicolumn{4}{|c|}{ Net benefits $(£)$} \\
\hline & & & & $\lambda=£ 20,000$ & Rank & $\lambda=£ 30,000$ & Rank \\
\hline \multicolumn{8}{|l|}{ 36-month interval } \\
\hline No surveillance & 5097 & 14.592 & & 286,742 & 13 & 432,661 & 13 \\
\hline Mammography alone & 10,404 & 15.875 & 4135 & 307,100 & 12 & 465,852 & 12 \\
\hline Mammography + clinical & 11,363 & 15.989 & 8414 & 308,422 & 11 & 468,314 & 11 \\
\hline MRI + clinical & 13,337 & 16.219 & 8573 & 311,053 & 8 & 473,248 & 8 \\
\hline
\end{tabular}

TABLE 51 Sensitivity analysis for a starting age of 70 years: breast-conserving model

\begin{tabular}{|c|c|c|c|c|c|c|c|}
\hline \multirow[b]{2}{*}{ Regimen } & \multirow[b]{2}{*}{ Cost (£) } & \multirow[b]{2}{*}{ QALY } & \multirow[b]{2}{*}{ ICER (£) } & \multicolumn{4}{|c|}{ Net benefits (£) } \\
\hline & & & & $\lambda=£ 20,000$ & Rank & $\lambda=£ 30,000$ & Rank \\
\hline \multicolumn{8}{|l|}{ 12-month interval } \\
\hline No surveillance & 541 & 9.068 & & 180,828 & & 271,512 & 6 \\
\hline Mammography alone & 1047 & 9.100 & 16,182 & 180,947 & 4 & 271,944 & 4 \\
\hline Mammography + clinical & 2082 & 9.101 & 880,839 & 179,935 & 9 & 270,944 & 9 \\
\hline MRI + clinical & 4340 & 9.103 & $1,093,357$ & 177,719 & 12 & 268,749 & 13 \\
\hline \multicolumn{8}{|l|}{ 18-month interval } \\
\hline No surveillance & 541 & 9.068 & & 180,828 & 5 & 271,512 & 6 \\
\hline Mammography alone & 867 & 9.096 & 11,701 & 181,060 & 3 & 272,023 & 2 \\
\hline Mammography + clinical & 1603 & 9.098 & 451,156 & 180,357 & 8 & 271,337 & 8 \\
\hline MRI + clinical & 3211 & 9.101 & 529,198 & 178,810 & 11 & 269,820 & 12 \\
\hline \multicolumn{8}{|l|}{ 24-month interval } \\
\hline No surveillance & 541 & 9.068 & & 180,828 & 5 & 271,512 & 6 \\
\hline Mammography alone & 773 & 9.093 & 9331 & 181,093 & 2 & 272,026 & 1 \\
\hline Mammography + clinical & 1359 & 9.095 & 305,494 & 180,546 & 7 & 271,498 & 7 \\
\hline MRI + clinical & 2644 & 9.099 & 342,629 & 179,336 & 10 & 270,326 & 11 \\
\hline \multicolumn{8}{|l|}{ 36-month interval } \\
\hline No surveillance & 541 & 9.068 & & 180,828 & 5 & 271,512 & 6 \\
\hline Mammography alone & 713 & 9.091 & 7751 & 181,100 & 1 & 272,006 & 3 \\
\hline Mammography + clinical & 1210 & 9.093 & 238,858 & 180,644 & 6 & 271,571 & 5 \\
\hline MRI + clinical & 2302 & 9.097 & 258,696 & 179,636 & 10 & 270,606 & 10 \\
\hline
\end{tabular}

\section{Sensitivity analysis around model considering IBTR only Probability of developing cancer}

Figures 30-33 report the impact of increasing the incidence of IBTR only. In these analyses, the impact on costs and QALYs or MCBC is not considered. As described earlier, IBTR has a much worse prognosis than MCBC. As would be expected, as incidence increases the incremental cost per QALYs fall when we compare a more effective but more costly surveillance regimen with the next most costly and effective. For mammographic and clinical follow-up the incremental cost per QALY compared with mammography alone begins to fall below $£ 30,000$ once the incidence 


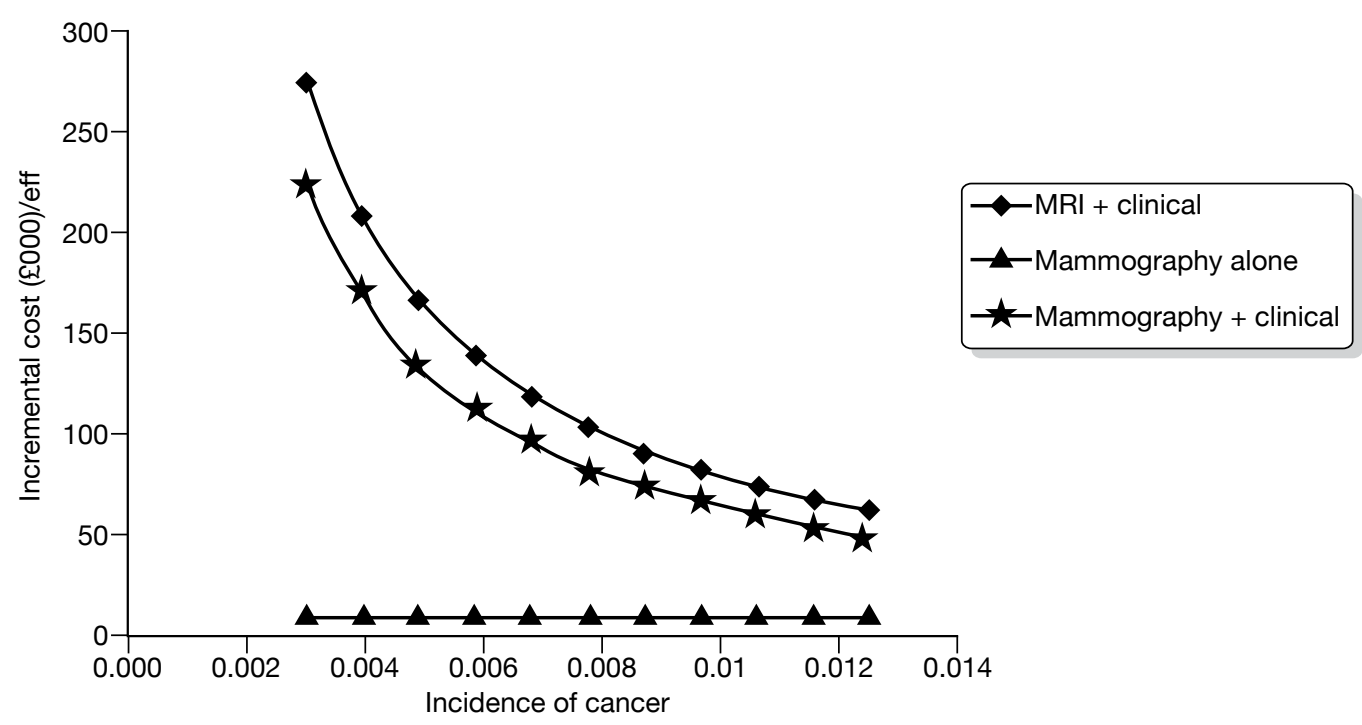

FIGURE 30 Incremental cost per QALYs for the different surveillance regimens at a 12-month surveillance interval.

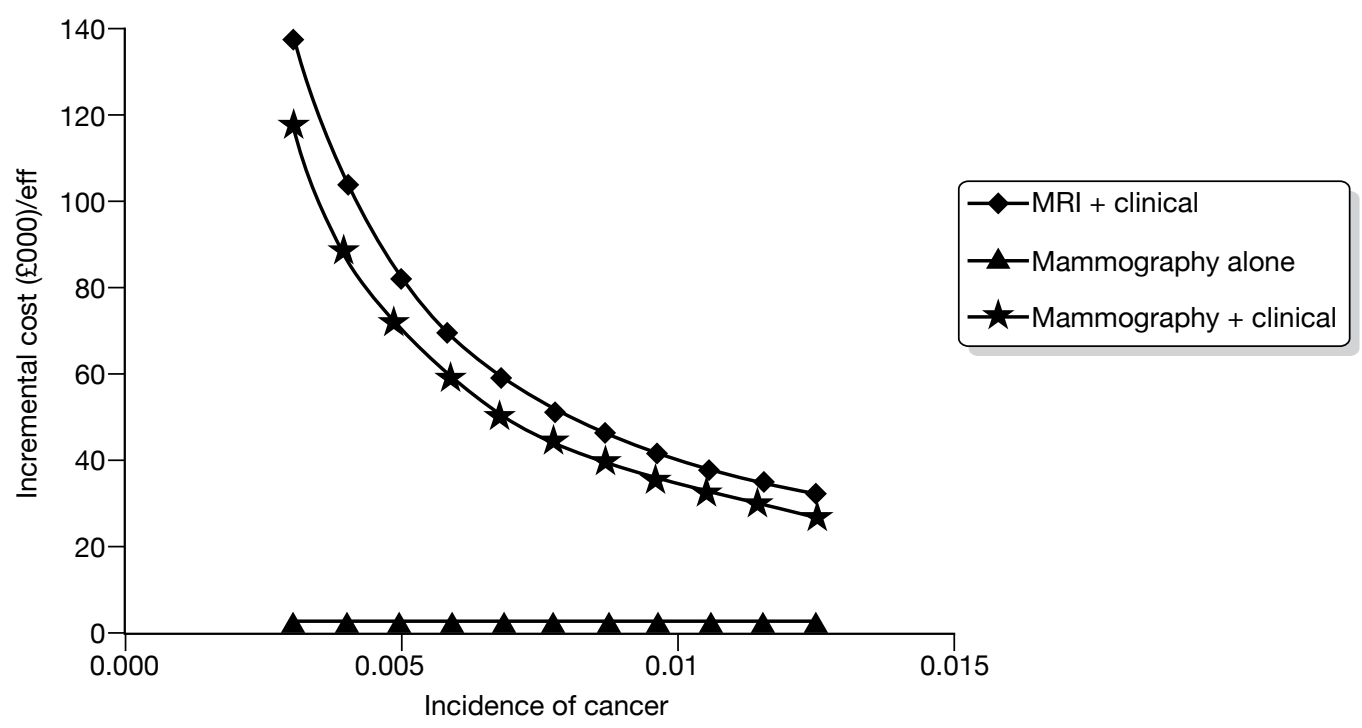

FIGURE 31 Incremental cost per QALYs for the different surveillance regimens at an 18-month surveillance interval.

of disease exceeds 0.00775 every 6 months and the surveillance interval is 24 months or longer. When the surveillance interval reaches 36 months, the adoption of a more effective but more costly regimen (again typified by MRI plus clinical follow-up) may be cost-effective once the incidence per cycle exceeds 0.0068 .

Inflating the risk of death from untreated cancer

In this sensitivity analysis, the increased risk of death from cancer in unmanaged states was inflated from the base-case risk of 2.34 to a high of 2.196 (Table 52). The results of this analysis were broadly similar to the base-case analysis presented in Table 41 . 


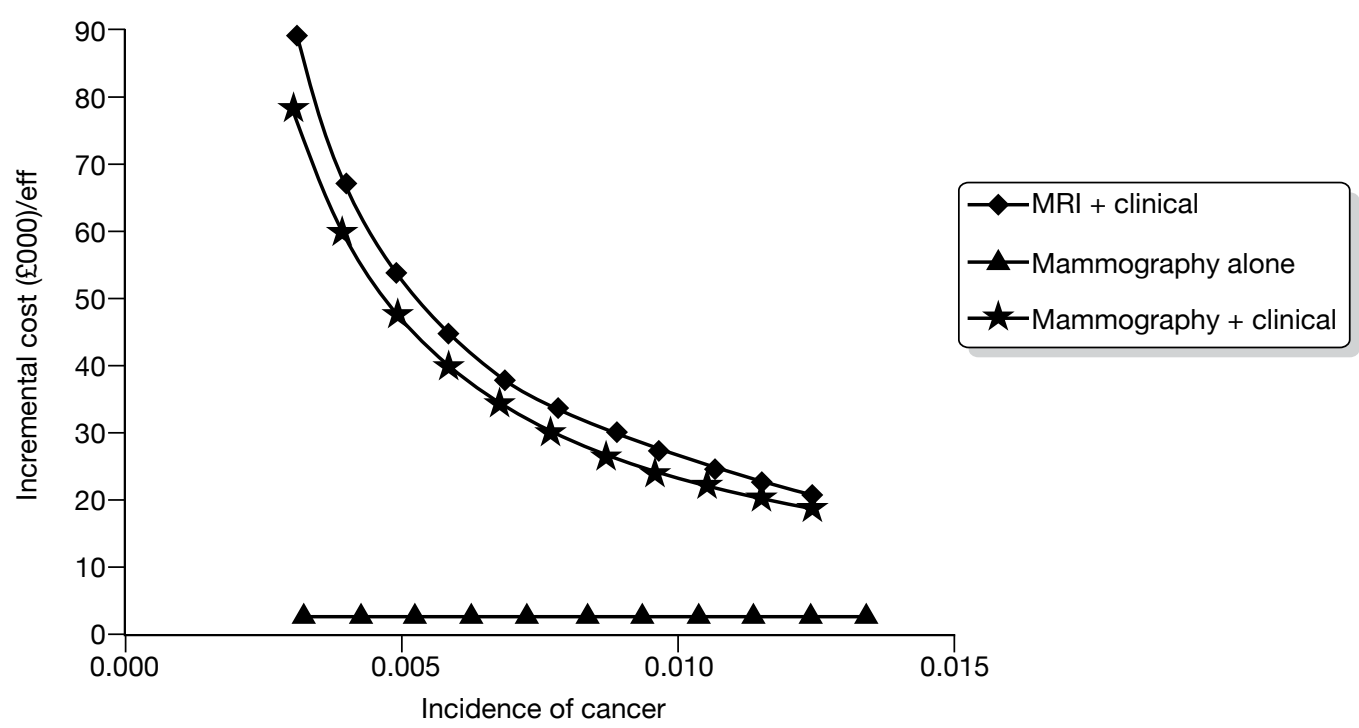

FIGURE 32 Incremental cost per QALYs for the different surveillance regimens at a 24-month surveillance interval.

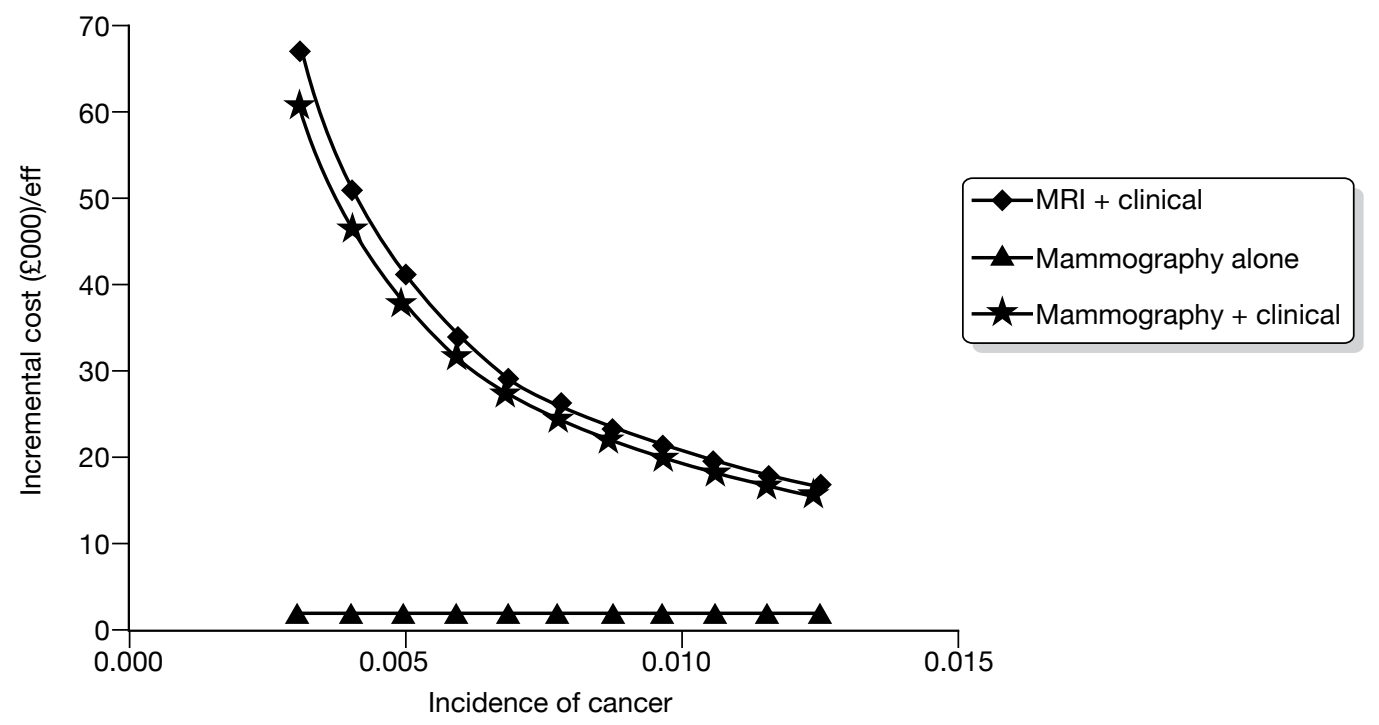

FIGURE 33 Incremental cost per QALYs for the different surveillance regimens at a 36-month surveillance interval.

\section{Changes to the probability of progressing to a more serious risk profile}

Table 53 reports the impact of increasing the speed that an untreated cancer progresses to a risk profile with a worse prognosis. The results of this analysis are similar to those reported above and it is unlikely that changes in this variable alone will result in any regimen other than mammography alone having an incremental cost per QALY that society might be willing to pay.

\section{Changes to costs of tests and treatments}

Table 54 shows a high treatment cost and high surveillance cost sensitivity analysis for the IBTR model. Again, the results of this sensitivity analysis are broadly similar to those reported in Table 41. 
TABLE 52 Increasing the risk of death for unmanaged disease

\begin{tabular}{|c|c|c|c|c|c|c|c|}
\hline \multirow[b]{2}{*}{ Regimen } & \multirow[b]{2}{*}{ Cost (£) } & \multirow[b]{2}{*}{ QALY } & \multirow[b]{2}{*}{ ICER (£) } & \multicolumn{4}{|c|}{ Net benefits (£) } \\
\hline & & & & $\lambda=£ 20,000$ & Rank & $\lambda=£ 30,000$ & Rank \\
\hline \multicolumn{8}{|l|}{ 12-month interval } \\
\hline GP only & 956 & 12.798 & & 255,010 & 13 & 382,994 & 12 \\
\hline Mammography alone & 1952 & 13.072 & 3634 & 259,494 & 1 & 390,217 & 1 \\
\hline Mammography + clinical & 3381 & 13.081 & 160,846 & 258,243 & 7 & 389,054 & 4 \\
\hline MRI + clinical & 6490 & 13.097 & 196,526 & 255,450 & 12 & 386,420 & 11 \\
\hline \multicolumn{8}{|l|}{ 18-month interval } \\
\hline GP only & 956 & 12.798 & & 255,010 & 13 & 382,994 & 12 \\
\hline Mammography alone & 1696 & 13.047 & 2973 & 259,243 & 2 & 389,713 & 2 \\
\hline Mammography + clinical & 2721 & 13.059 & 83,817 & 258,462 & 5 & 389,054 & 4 \\
\hline MRI + clinical & 4955 & 13.082 & 97,976 & 256,685 & 11 & 387,504 & 10 \\
\hline \multicolumn{8}{|l|}{ 24-month interval } \\
\hline GP only & 956 & 12.798 & & 255,010 & 13 & 382,994 & 12 \\
\hline Mammography alone & 1553 & 13.024 & 2642 & 258,928 & 3 & 389,168 & 3 \\
\hline Mammography + clinical & 2379 & 13.039 & 56,792 & 258,392 & 6 & 388,778 & 5 \\
\hline MRI + clinical & 4180 & 13.067 & 63,721 & 257,156 & 10 & 387,825 & 9 \\
\hline \multicolumn{8}{|l|}{ 36-month interval } \\
\hline GP only & 956 & 12.798 & & 255,010 & 13 & 382,994 & 12 \\
\hline Mammography alone & 1456 & 13.004 & 2433 & 258,615 & 4 & 388,651 & 6 \\
\hline Mammography + clinical & 2164 & 13.020 & 44,151 & 258,228 & 8 & 388,424 & 7 \\
\hline MRI + clinical & 3710 & 13.052 & 47,805 & 257,329 & 9 & 387,848 & 8 \\
\hline
\end{tabular}

TABLE 53 The impact of changing the probability of progressing to a higher risk profile

\begin{tabular}{|c|c|c|c|}
\hline \multirow[b]{2}{*}{ Surveillance interval } & \multirow[b]{2}{*}{ Regimen } & \multicolumn{2}{|c|}{$\begin{array}{l}\text { ICER at low and high probability of increasing to next risk profile } \\
\text { per cycle: }\end{array}$} \\
\hline & & 0.1555 & 0.2623 \\
\hline \multirow[t]{4}{*}{12 months } & No surveillance & & \\
\hline & Mammography alone & 3973 & 3231 \\
\hline & Mammography + clinical & 177,150 & 110,112 \\
\hline & MRI + clinical & 216,537 & 131,811 \\
\hline \multirow[t]{4}{*}{18 months } & No surveillance & & \\
\hline & Mammography alone & 3247 & 2523 \\
\hline & Mammography + clinical & 92,265 & 42,743 \\
\hline & MRI + clinical & 108,007 & 44973.0307 \\
\hline \multirow[t]{4}{*}{24 months } & No surveillance & & \\
\hline & Mammography alone & 2887 & 2523 \\
\hline & Mammography + clinical & 62,482 & 42,743 \\
\hline & MRI + clinical & 70,315 & 44,973 \\
\hline \multirow[t]{4}{*}{36 months } & No surveillance & & \\
\hline & Mammography alone & 2664 & 2391 \\
\hline & Mammography + clinical & 48,551 & 34,901 \\
\hline & MRI + clinical & 52,811 & 34,980 \\
\hline
\end{tabular}


TABLE 54 Ipsilateral breast tumour recurrence model: high costs

\begin{tabular}{|c|c|c|c|c|c|c|c|}
\hline \multirow[b]{2}{*}{ Regimen } & \multirow[b]{2}{*}{$\operatorname{Cost}(£)$} & \multirow[b]{2}{*}{ QALY } & \multirow[b]{2}{*}{ ICER (£) } & \multicolumn{4}{|c|}{ Net benefits $(£)$} \\
\hline & & & & $\lambda=£ 20,000$ & Rank & $\lambda=£ 30,000$ & Rank \\
\hline \multicolumn{8}{|l|}{ 12-month interval } \\
\hline No surveillance & 1578 & 12.830 & & 255,021 & 9 & 383,320 & 11 \\
\hline Mammography alone & 3869 & 13.076 & 9309 & 257,652 & 2 & 388,413 & 1 \\
\hline Mammography + clinical & 5317 & 13.084 & 179,674 & 256,365 & 8 & 387,206 & 6 \\
\hline MRI + clinical & 11,768 & 13.098 & 449,596 & 250,202 & 13 & 381,186 & 12 \\
\hline \multicolumn{8}{|l|}{ 18-month interval } \\
\hline No surveillance & 1578 & 12.830 & & 255,021 & 9 & 383,320 & 11 \\
\hline Mammography alone & 3400 & 13.053 & 8164 & 257,662 & 1 & 388,193 & 2 \\
\hline Mammography + clinical & 4454 & 13.064 & 95,002 & 256,830 & 7 & 387,471 & 4 \\
\hline MRI + clinical & 9107 & 13.085 & 225,253 & 252,590 & 12 & 383,438 & 10 \\
\hline \multicolumn{8}{|l|}{ 24-month interval } \\
\hline No surveillance & 1578 & 12.830 & & 255,021 & 9 & 383,320 & 11 \\
\hline Mammography alone & 3124 & 13.032 & 7638 & 257,523 & 3 & 387,846 & 3 \\
\hline Mammography + clinical & 3987 & 13.046 & 65,386 & 256,924 & 5 & 387,379 & 5 \\
\hline MRI + clinical & 7752 & 13.071 & 147,255 & 253,670 & 11 & 384,381 & 9 \\
\hline \multicolumn{8}{|l|}{ 36-month interval } \\
\hline No surveillance & 1578 & 12.830 & & 255,021 & 9 & 383,320 & 11 \\
\hline Mammography alone & 2929 & 13.014 & 7345 & 257,348 & 4 & 387,486 & 3 \\
\hline Mammography + clinical & 3679 & 13.028 & 51,600 & 256,888 & 6 & 387,172 & 7 \\
\hline MRI + clinical & 6919 & 13.058 & 110,989 & 254,232 & 10 & 384,808 & 8 \\
\hline
\end{tabular}

In addition, Table 55 shows sensitivity analysis in which the cost of a clinical examination is priced at the consultant and non-consultant rate. Although the incremental cost per QALYs changes, none is of sufficient magnitude to change conclusions.

\section{Changes to utility estimates used}

We tested the base-case assumption that women in unmanaged states have the same quality of life as women who are disease free (Table 56). In this analysis, all women in unmanaged states had the same utility as women who had been treated for IBTR or MCBC. A similar pattern of results is observed in the IBTR model sensitivity analysis as was observed in the breast-conserving model sensitivity analysis.

\section{Exploration of the impact of age at the time surveillance starts}

Table 57 reports the results of sensitivity analysis based on a starting age of 40 years old in the IBTR model. This table reports multiway sensitivity analysis for starting age 40 years, and includes the high chemotherapy costs and high hormone therapy costs (Table 36). In addition, the higher incidence rate for cancer is used in this model (0.0152, Table 29) and a short doubling time. This analysis was conducted for surveillance intervals ranging from 12 to 36 months. 
TABLE 55 Consultant and non-consultant cost of clinical examinations

\begin{tabular}{|c|c|c|c|}
\hline \multirow[b]{2}{*}{ Surveillance interval } & \multirow[b]{2}{*}{ Regimen } & \multicolumn{2}{|c|}{ ICER at low and high clinical examination cost (£): } \\
\hline & & 65 & 86 \\
\hline \multirow[t]{4}{*}{12 months } & No surveillance & & \\
\hline & Mammography alone & 4148 & 3973 \\
\hline & Mammography + clinical & 133,736 & 177,150 \\
\hline & MRI + clinical & 216,515 & 216,537 \\
\hline \multirow[t]{4}{*}{18 months } & No surveillance & & \\
\hline & Mammography alone & 3446 & 3247 \\
\hline & Mammography + clinical & 69,664 & 92,265 \\
\hline & MRI + clinical & 107,973 & 108,007 \\
\hline \multirow[t]{4}{*}{24 months } & No surveillance & & \\
\hline & Mammography alone & 3113 & 2887 \\
\hline & Mammography + clinical & 47,228 & 62,482 \\
\hline & MRI + clinical & 70,274 & 70,315 \\
\hline \multirow[t]{4}{*}{36 months } & No surveillance & & \\
\hline & Mammography alone & 2919 & 2664 \\
\hline & Mammography + clinical & 36,756 & 48,551 \\
\hline & MRI + clinical & 52,766 & 52,811 \\
\hline
\end{tabular}

TABLE 56 Sensitivity analysis on quality of life in unmanaged states: IBTR model

\begin{tabular}{|c|c|c|c|c|c|c|c|}
\hline \multirow[b]{2}{*}{ Regimen } & \multirow[b]{2}{*}{ Cost } & \multirow[b]{2}{*}{ QALY } & \multirow[b]{2}{*}{ ICER } & \multicolumn{4}{|l|}{ Net benefits } \\
\hline & & & & $\lambda=£ 20,000$ & Rank & $\lambda=£ 30,000$ & Rank \\
\hline \multicolumn{8}{|l|}{ 12-month interval } \\
\hline No surveillance & 978 & 12.797 & & 254,969 & 13 & 382,943 & 13 \\
\hline Mammography alone & 1956 & 13.076 & 3513 & 259,559 & 1 & 390,317 & 1 \\
\hline Mammography + clinical & 3384 & 13.084 & 173,840 & 258,295 & 7 & 389,135 & 5 \\
\hline MRI + clinical & 6491 & 13.098 & 214,485 & 255,478 & 12 & 386,463 & 12 \\
\hline \multicolumn{8}{|l|}{ 18-month interval } \\
\hline No surveillance & 978 & 12.797 & & 254,969 & 13 & 382,943 & 13 \\
\hline Mammography alone & 1703 & 13.052 & 2844 & 259,340 & 2 & 389,861 & 2 \\
\hline Mammography + clinical & 2727 & 13.064 & 88,891 & 258,546 & 5 & 389,183 & 4 \\
\hline MRI + clinical & 4958 & 13.085 & 105,565 & 256,738 & 10 & 387,586 & 6 \\
\hline \multicolumn{8}{|l|}{ 24-month interval } \\
\hline No surveillance & 978 & 12.797 & & 254,969 & 13 & 382,943 & 13 \\
\hline Mammography alone & 1703 & 13.030 & 2295 & 258,906 & 3 & 389,210 & 3 \\
\hline Mammography + clinical & 2727 & 13.044 & 45,211 & 258,161 & 8 & 388,604 & 8 \\
\hline MRI + clinical & 4958 & 13.071 & 50,179 & 256,460 & 11 & 387,170 & 11 \\
\hline \multicolumn{8}{|l|}{ 36-month interval } \\
\hline No surveillance & 978 & 12.797 & & 254,969 & 13 & 382,943 & 13 \\
\hline Mammography alone & 1468 & 13.011 & 2295 & 258,748 & 4 & 388,856 & 10 \\
\hline Mammography + clinical & 2175 & 13.026 & 45,211 & 258,354 & 6 & 388,619 & 7 \\
\hline MRI + clinical & 3716 & 13.057 & 50,179 & 257,427 & 9 & 387,999 & 9 \\
\hline
\end{tabular}


TABLE 57 Sensitivity analysis for starting age of 40 years: IBTR

\begin{tabular}{|c|c|c|c|c|c|c|c|}
\hline \multirow[b]{2}{*}{ Regimen } & \multirow[b]{2}{*}{ Cost (£) } & \multirow[b]{2}{*}{ QALY } & \multirow[b]{2}{*}{ ICER (£) } & \multicolumn{4}{|c|}{ Net benefits (£) } \\
\hline & & & & $\lambda=£ 20,000$ & Rank & $\lambda=£ 30,000$ & Rank \\
\hline \multicolumn{8}{|l|}{ 12-month interval } \\
\hline No surveillance & 4332 & 14.023 & & 276,121 & 13 & 416,347 & 13 \\
\hline Mammography alone & 11,383 & 16.013 & 3542 & 308,885 & 2 & 469,019 & 3 \\
\hline Mammography + clinical & 12,859 & 16.093 & 18,630 & 308,994 & 1 & 469,920 & 2 \\
\hline MRI + clinical & 15,970 & 16.237 & 21,569 & 308,767 & 3 & 471,136 & 1 \\
\hline \multicolumn{8}{|l|}{ 18-month interval } \\
\hline No surveillance & 4332 & 14.023 & & 276,121 & 13 & 416,347 & 13 \\
\hline Mammography alone & 10,724 & 15.792 & 3613 & 305,109 & 7 & 463,026 & 7 \\
\hline Mammography + clinical & 11,909 & 15.896 & 11,347 & 306,013 & 5 & 464,974 & 6 \\
\hline MRI + clinical & 14,376 & 16.099 & 12,171 & 307,600 & 4 & 468,588 & 4 \\
\hline \multicolumn{8}{|l|}{ 24-month interval } \\
\hline No surveillance & 4332 & 14.023 & & 276,121 & 13 & 416,347 & 13 \\
\hline Mammography alone & 10,176 & 15.598 & 3709 & 301,784 & 10 & 457,765 & 10 \\
\hline Mammography + clinical & 11,239 & 15.717 & 8945 & 303,098 & 9 & 460,266 & 9 \\
\hline MRI + clinical & 13,441 & 15.961 & 9033 & 305,772 & 6 & 465,378 & 5 \\
\hline \multicolumn{8}{|l|}{ 36-month interval } \\
\hline No surveillance & 4332 & 14.023 & & 276,121 & 13 & 416,347 & 13 \\
\hline Mammography alone & 9693 & 15.431 & 3807 & 298,921 & 12 & 453,228 & 12 \\
\hline Mammography + clinical & 10,692 & 15.556 & 7943 & 300,438 & 11 & 456,002 & 11 \\
\hline MRI + clinical & 12,764 & 15.827 & 7663 & 303,775 & 8 & 462,045 & 8 \\
\hline
\end{tabular}

\section{Summary}

In the base-case analysis the regimen with the highest net benefit and, therefore, most likely to be considered cost-effective was mammographic surveillance alone provided yearly. This result holds for women who had previously been treated for their primary cancer with either BCS or mastectomy or women who suffer IBTR.

As might be expected in a comparison of surveillance regimens, the results of the model are very sensitive to changes in the incidence of recurrent cancer. When the expected incidence is increased toward the maximum that could possibly be expected for any group of women mammography and clinical surveillance potentially becomes cost-effective when the surveillance interval is 24 months or longer. As the surveillance interval and incidence increase regimens that are more costly but more effective may also have incremental costs per QALY below typical threshold values. This suggests that there may be some scope for research into alternative technologies that could be used for surveillance.

The results of the analysis did not substantially alter for any of the other sensitivity analyses reported. The exception to this is when we changed several parameter values simultaneously. This was undertaken in an attempt to compare surveillance regimens for a hypothetical 40-year-old woman (who can be thought of as having a greater likelihood of developing 
IBTR or MCBC) and a hypothetical 70-year-old woman (representing a lower likelihood of developing IBTR or MCBC). In the sensitivity analysis conducted for a 40-year-old woman, the following changes were made: the incidence of recurrent cancer was increased and the time it took for an undetected cancer to progress to risk profiles with a worse prognosis was reduced. Furthermore, it was assumed that should IBTR or MCBC be detected then it would be treated more aggressively (and at higher cost). For 40-year-old women facing these risks and costs, mammographic surveillance every 12 months had the highest net benefit, although it was only slightly greater than mammography and clinical follow-up every 12 months. These results suggest that a more intensive follow-up of women judged to be at high risk may be costeffective. Conversely, for women at lower risk it may be more cost-effective for surveillance to be performed less often (every 2 or 3 years) with mammography alone or another similarly less intensive and costly test or tests. 


\section{Chapter 8}

\section{Discussion}

\section{Statement of overall aim and methods}

Our aim was to examine the clinical effectiveness and cost-effectiveness of different surveillance mammography regimens after the treatment for primary breast cancer in the UK in both primary and secondary care settings.

We addressed this by undertaking a survey of UK breast surgeons and radiologists to identify current practice and estimate resource consequences of the varying regimens. In addition, we undertook two discrete systematic reviews to determine the clinical effectiveness of differing surveillance mammography regimens carried out after treatment for primary breast cancer on patient health outcomes and the diagnostic accuracy of surveillance mammography in the detection of IBTR and MCBC. We undertook statistical analysis of individual patient data and economic modelling using the results of the systematic reviews and existing data sets, and focused searches for specific data analysis to determine the effectiveness and cost-utility of differing surveillance regimens.

We identified feasible management strategies for surveillance of women after treatment for breast cancer in a UK context, and have modelled the effectiveness and costs of these strategies. As is described later, we used these data to assess implications for clinical practice and to inform needs for future research.

\section{Summary of main findings}

The first two elements of research reported focused on describing and summarising current practice and current evidence with respect to mammographic surveillance after the treatment for primary breast cancer. Our survey of UK breast surgeons and radiologists described in Chapter 3 suggests that although common patterns in surveillance mammography practice exist there is considerable variation in the combinations of start, frequency, duration and discharge from surveillance mammography.

The most common approach was to start surveillance mammography 12 months after initial surgery for the treatment of the primary breast cancer $(87 \%)$, offer mammography annually (72\%), organised through a symptomatic breast service (96\%), discharging women (74\%) after either 5 years (35\%) or 10 years (55\%). Over 55\% stated that they discharged women to the NHSBSP for surveillance mammography to be carried out. Overall, $18 \%$ stated that they did not discharge patients from clinical follow-up but, of those who did, 65\% discharged after 5 years with $22 \%$ discharging at 10 years after initial treatment.

Although our response rate was low (17\%), which we discuss further below (see Strengths and limitations), we received responses from surgeons or radiologists working at 105 trusts across the UK. Our findings were concordant with previous surveys on this topic. ${ }^{26,27}$ Therefore, we feel that our results are valid and informative, as they represent the views of the surgeons and radiologists involved in delivering breast cancer care and treatment from throughout the UK. 
The plethora of different ways of conducting surveillance mammography in clinical practice means that there are potentially a large number of care pathways that are worthy of further consideration. It would not be possible to consider the merits of each of these options, especially when there are additional methods of follow-up that do not include mammography. Therefore, using the results of the survey and expert opinion from the project group a relatively small number of follow-up regimens was selected and defined. These differed in terms of the diagnostic technology used and the surveillance interval considered. It is possible, however, that potentially important pathways have been excluded from this list.

At the outset of this project, we felt that it would be important to evaluate the existing evidence regarding the effectiveness and cost-effectiveness of surveillance mammography, although we were unsure if the evidence base would be sufficient to guide any policy recommendations.

The systematic review we conducted to determine the effectiveness of differing surveillance mammography regimens after treatment for primary breast cancer in detecting IBTR and MCBC is described in Chapter 4. Our systematic review suggests that receipt of surveillance mammography offers a survival benefit compared with a surveillance regimen that does not include surveillance mammography. The surveillance regimens were complex and varied between studies (and only in broad terms could they be considered to fit within the different regimens of surveillance developed from the results of the survey). Furthermore, the influence of combinations of alternative surveillance regimens (e.g. clinical examination, ad hoc referral to the symptomatic service, etc.) remains unclear.

We adopted considerable and rigorous methods in an attempt to identify relevant studies. Despite this, only eight studies met our inclusion criteria, none of which was an RCT. We sought data from the included studies on eight outcomes, but few data on effectiveness, and none on costeffectiveness, were identified. The limited and variable data that were available precluded any quantitative analysis, and the paucity of the underlying evidence base mirrors the findings of previous reviews. ${ }^{4,29,43-47}$

As we anticipated that the existing evidence base would not be sufficiently informative, we planned a formal systematic review of diagnostic performance and analyses of existing individual patient data held in potentially appropriate data sets and registries. We conducted this work not only because we felt the data sets to be important in their own right, but also to inform an economic evaluation.

We conducted a systematic review to determine the performance (diagnostic accuracy) of surveillance mammography, alone or in combination with other tests, in detecting IBTR and/or $\mathrm{MCBC}$. The primary purpose of this review was to evaluate the test performance of surveillance mammography. Two studies reported sensitivity and specificity for surveillance mammography in detecting IBTR in patients undergoing routine surveillance; although we chose to describe results from the study by Boné and colleagues, ${ }^{64}$ they should be treated individually owing to their highly selected patient population. The remaining study $y^{66,67}$ reported a sensitivity of $67 \%$ and a specificity of $85 \%$ for surveillance mammography. The study authors included MRI as a comparator and reported $100 \%$ sensitivity and $93 \%$ specificity for MRI in this group of patients. Three studies ${ }^{66,68,72}$ reported the median (and range) sensitivity and specificity of surveillance mammography for detecting IBTR in non-routine surveillance patients as $71 \%(50-83 \%)$ and $63 \%(57-75 \%)$, respectively. Two of these studies ${ }^{68,72}$ included MRI and reported sensitivities of $93 \%$ and $100 \%$ and specificities of $88 \%$ and $96 \%$, respectively. In detecting MCBC in patients undergoing routine surveillance, only one study ${ }^{70}$ reported data for a single diagnostic test, MRI, with $91 \%$ sensitivity and $90 \%$ specificity. 
Again, few data were available regarding MRI. Nevertheless, our findings suggest that MRI is the most accurate test for detecting IBTR and MCBC. Of the test combinations reported, surveillance mammography combined with breast ultrasound could be considered as the most accurate combination of tests for detecting MCBC.

We decided that it was inappropriate to use studies of population-screening mammography to calculate diagnostic test performance, as patients with breast cancer will have had treatment that may affect their breast density and the operation site can show marked changes due to scar tissue, with the adjacent parenchymal tissue changed as a result of postoperative radiotherapy. The sensitivity of mammography is reduced when a more sensitive test (MRI) is also used and this accounts for the difference in the literature with respect to screening mammography. Parameter estimates for MRI in the screening population were used in the economic evaluation in Chapter 7 of this report, however, as it was felt that these would provide an indication of the relative value of a more costly but more effective test. Similarly, it was not possible to explore the effects of adjuvant treatments or any other subgroup effects that may have reduced breast density, and which theoretically would increase the sensitivity of surveillance mammography.

We have shown that IBTR has an adverse effect on survival. This is independent of the primary tumour and its characteristics, and in our analysis of BCS the HR was 2.13 (95\% CI 1.78 to 2.56). This has been found in several previously published series. ${ }^{20,77-80}$ Furthermore, the time from the treatment of the primary cancer to the event occurring is important, with those events that happen later having a survival advantage compared with those that occur earlier. This was also found in a combined analysis of five trials of women treated by BCS who had node-negative disease. ${ }^{77}$

We did not find that MCBC has an independent adverse effect on survival in either the BCS or mastectomy group. This is contrary to a large series of women with bilateral disease, who developed MCBC within 5 years of the primary tumour occurring and were under the age of 50 years. They were 3.9 times more likely to die than those who did not develop MCBC. ${ }^{108}$ Our result may be due to there being too few deaths in our cohort with $\mathrm{MCBC}$. We did find that older women are at reduced risk of $\mathrm{MCBC}$ and that women with larger tumours are at an increased risk of MCBC. It is known that women who have more advanced disease are at increased risk of developing contralateral disease. This needs to occur relatively early, as women with more advanced disease have poor survival. Therefore, it is likely that those women who develop MCBC later actually have a survival advantage from their primary disease. With respect to survival there was no evidence that women who develop $\mathrm{MCBC}$ have a different risk to those women who do not develop either IBTR or MCBC.

We have shown that there is a survival advantage if the IBTR or MCBC is detected at a smaller size compared with a larger size. The reference category was taken as $<10 \mathrm{~mm}$ in maximum diameter. Women with tumours of $>20 \mathrm{~mm}$ had an independent relative risk of death of 2.26 ( $95 \%$ CI 1.58 to 3.24). In those women for whom the data about size were missing (i.e. those women who did not have surgery) the risk of death is even greater at 3.19 (95\% CI 2.24 to 4.53 ). While we have no information as to how these events were detected in clinical practice, the implication is that surveillance mammography may be of value. We know from the NHSBSP that $80 \%$ of cancers detected by 3-yearly mammography are $<20 \mathrm{~mm}$ in maximum diameter. This suggests that surveillance mammography could be used to reduce the size at which IBTR or MCBC cancers are detected. In our primary tumour cohorts the incidences of IBTR and MCBC are fairly constant over the first 10 years, although we did not follow this for a longer time period. This supports the fact that surveillance mammography should be continued for at least 10 years. 
We used information derived from the prior analyses to inform the economic evaluation. The methods and results of this are reported in Chapter 7 . The structure for the economic evaluation was informed by our survey, together with detailed discussions with clinical members of the research team. Rigorous and systematic attempts were then made to identify data relevant to the UK. These included the reviews and individual patient data analyses from existing data sets, and also included a series of focused reviews to identify other necessary data (e.g. on aspects of epidemiology/natural history of disease, health-state utilities, etc.). Despite these efforts, few data were available to inform many of the estimates used by the model and consequently the economic analysis should be considered as exploratory, and hence interpreted cautiously. Despite this, the results of the economic analysis suggest that the regimen with the highest net benefit, and therefore most likely to be considered cost-effective, was mammographic surveillance alone provided yearly. This result holds for women who were previously treated for their primary cancer with either BCS or mastectomy or for women who suffer an IBTR.

The results of the model were very sensitive to changes in the incidence of IBTR and MCBC. The maximum expected incidence modelled was $1.25 \%$ every 6 months. This exceeds the maximum that might be expected based on an interpretation of the Kaplan-Meier survival curves and the HRs reported in Chapter 6 where there was moderate evidence of a statistically significant difference (i.e. the reported $p$-value was $\leq 5 \%$ ). As the incidence of recurrent cancer increased to $0.9 \%$ every 6 months, a surveillance regimen of mammography and clinical surveillance potentially becomes cost-effective when the surveillance interval is 24 months or longer. This is because as incidence increases there is more scope for more costly regimens that have a better diagnostic performance to prove their worth. When the surveillance interval was increased to 36 months and the modelled incidence of IBTR and MCBC every 6 months was approaching 1\%, a regimen of MRI and clinical follow-up also had an incremental cost per QALY below $£ 30,000$ (a typical threshold adopted by, for example, NICE). This suggests that there may be some scope for research into alternative technologies that could be used for surveillance for women at the highest risk of recurrence.

To test how robust the results were we explored the impact of changes in many of the other model parameters in a number of sensitivity analyses. The results of these analyses were not substantially different to the base-case results.

The exception to this was when we changed several parameter values simultaneously. This was carried out in an attempt to compare surveillance regimens for a hypothetical 40-year-old woman (where the model was changed so that a woman has a greater likelihood of developing IBTR and MCBC and has a worse prognosis) and a hypothetical 70-year-old woman (potentially representing a patient with less likelihood of developing IBTR and $M C B C$ ). In the sensitivity analysis conducted for a 40-year-old woman the following changes were made: the incidence of IBTR and MCBC cancer was increased and the time it took for an undetected cancer to progress to risk profiles with a worse prognosis was reduced. Furthermore, we assumed that should IBTR or MCBC be detected then it would be treated more aggressively (and at a higher cost).

For the scenario considering the cost-effectiveness of surveillance for a 40-year-old woman (a high-risk scenario) mammographic surveillance every 12 months had the highest net benefit, although it was only slightly greater than mammography combined with clinical follow-up every 12 months. These results suggest that a more intensive follow-up of women judged to be at high risk may be cost-effective. Conversely, for women at lower risk of IBTR or MCBC (the scenario modelled was for a 70-year-old woman) it may be more cost-effective for surveillance to be performed less often (every 2 or 3 years) with mammography alone or another similarly less intensive and less costly test or combination of tests. 
Taken together, the results of the economic analysis would suggest that were the NHS to adopt a single policy covering all women after treatment for a primary breast cancer then a policy of mammographic surveillance alone at 12,18 or 24 months might be the best policy. If the base-case analysis underestimated the risk of IBTR or MCBC then a more intensive regimen, for example mammography and clinical follow-up, might be more efficient, although because of the trade-off between cost and diagnostic performance the surveillance interval might increase to 24-36 months. Were the NHS to consider targeting intensive surveillance for those deemed at higher risk of recurrence then it is possible that more intensive methods of surveillance would be considered worthwhile given the conventional threshold for society's willingness to pay for a QALY. For women deemed at lower risk of recurrence a less intensive regimen may be the most cost-effective. This raises questions about the feasibility of providing different patterns of surveillance according to perceived risk of recurrence. It also raises questions about the ethics of treating women who have been treated for a primary breast cancer differently, based not just upon characteristics of their primary cancer, but also other demographic characteristics such as age.

\section{Strengths and limitations}

Our work focused on the question posed by the HTA programme: what is the clinical effectiveness and cost-effectiveness of different surveillance mammography regimens after the treatment for primary breast cancer? Mammography is the primary follow-up examination conducted in the UK at the present time in clinical practice. For this reason, our survey and systematic reviews concentrated on surveillance mammography, although other tests were considered when reported with mammography results in this particular patient population. Overall, we believe the main strengths of this project are the rigorous, systematic, explicit and comprehensive methods used. We have identified that there is limited evidence available to answer our research question and, as such, this is the main limitation of our findings.

\section{Identifying feasible management strategies for surveillance and follow-up of women after treatment for breast cancer (Chapter 3)}

Our survey findings reflect the different guidance given by the various professional organisations with an interest and expertise in surveillance following treatment for breast cancer in combination with 'local circumstances' and protocols of the respondents (see Table 10). Our findings are generally consistent with those of recent surveys addressing similar questions. Donnelly and colleagues' survey ${ }^{26}$ of 256 specialists registered to Cancer Trials Units suggested that the majority $(84 \%)$ adhered to locally developed protocols and that mammography at annual intervals was the most common surveillance interval for both invasive and non-invasive primary breast cancer. Respondents favoured a risk-adjusted protocol and their preferred median clinical follow-up time overall was 5 years. Maxwell and colleagues' survey ${ }^{27}$ of breast-screening units taking part in the Sloane project concluded that there was wide variation in follow-up practice after surgery (breast conservation or mastectomy) for non-invasive breast cancer, the most common frequency and duration of clinical follow-up being annually for the first 5 years after treatment, and surveillance mammography being annually for the first 10 years in patients undergoing breast conservation, and annually or 2-yearly in patients who had undergone a mastectomy, also for 10 years.

The final response rate to our survey was low compared with other recent similar surveys. ${ }^{26,27}$ Cook and colleagues ${ }^{109}$ recently reported in a review of response rates of surveys of health-care professionals that response rates are low and probably declining [average response rate in doctors was $57.5 \%$ (95\% CI $55.2 \%$ to $59.8 \%$ ), but this is significantly lower than the estimate for the 
prior 10-year period]. Rates are lower without a reminder and with larger surveys. Our survey was a 'cold-calling' mass e-mailing with no personal reminders to complete the survey, whereas the sample taking part in these previous surveys was a selected population with an interest in clinical trials or taking part in national audit. Our response rate also highlights the disadvantages of undertaking an anonymous survey, as it is probable that our low response rate would have been improved if we could have used personal reminders to non-responders. Our low response rate may mean our results are not representative of the population of health-care professionals organising, providing and overseeing the follow-up care of women after surgery for primary breast cancer. However, taken together with data from Donnelly and colleagues ${ }^{26}$ and Maxwell and colleagues, ${ }^{27}$ it has provided an insight into the more common patterns of surveillance mammography practice and the feasible strategies for follow-up.

\section{Systematic review of effectiveness of surveillance mammography after treatment for primary cancer (Chapter 4)}

The methods used for conducting the review of the clinical effectiveness and cost-effectiveness of surveillance mammography were systematic, explicit and comprehensive. We believe that our inclusion criteria were both clinically relevant and scientifically rigorous. The main limitation of the review relates to the limitations of the underlying evidence base. Few published studies met the inclusion criteria, none of which was an RCT. Few data were available and what data were available were potentially biased. Owing to limited data and variability across studies we felt it unwise to perform a meta-analysis, thus a narrative synthesis of evidence was presented. The included studies are likely to be prone to both lead and length time bias. There was no comparison of varying surveillance mammography regimens. An RCT study design with survival as the end point would take these biases into account as the random allocation of participants to intervention groups minimises the impact of bias due to confounding variables. Such a study would be possible, as equipoise exists as to the ideal interval between surveillance mammograms.

It is possible that there are unpublished reports that may have otherwise met our inclusion criteria. The clinical members of the research team did not suggest any potentially relevant work that we could have included and, at the time of writing this report, we are unaware of any unpublished studies meeting our inclusion criteria.

Health-related quality of life was included as a review outcome and we were not restrictive in the measures that we considered. Some of the potential methods could have captured the effects on the psychological health of the woman. Nevertheless, we did not explicitly consider psychological factors as outcomes. This may be of greater concern in the NHSBSP, in which women who are 'well' are invited for mammography, because psychological harm or anxiety due to the examination or a suspicious test result or a false reassurance could have greater adverse impact. It is, however, worth noting that none of the included studies reported quality-of-life data and it is unclear if any would have provided data regarding psychological outcomes.

\section{Systematic review of the test performance of mammography and comparator tests in detecting IBTR/MCBC (Chapter 5)}

We believe that the methods adopted for this review are scientifically rigorous and compatible with current guidance in this area. Very few studies met our review inclusion criteria. The included studies were restricted to those published in the English language. This potentially limits the evidence base available, although non-English-language studies were less likely to be relevant to UK practice. As with the review of clinical effectiveness and cost-effectiveness we deliberately chose broad participant inclusion criteria. We based our rationale for this decision on our preliminary knowledge of the limited evidence base available. Narrowing our inclusion criteria would further limit the amount of available data. Although broadening our inclusion criteria carried the risk of finding variation between studies we believe that the limited data presented 
have greater generalisability to the whole spectrum of women who would be offered surveillance in practice. Of the studies included here, few evaluated the performance of the considered tests for similar purposes. Furthermore, even where data were available it was not clinically appropriate to combine them. For example, because of anatomical differences between a 'treated' and an 'untreated' breast (due to the effects of treatments) it was not appropriate to combine data on test performance for the detection of IBTR and MCBC. Similarly, it was inappropriate to combine data from routine and non-routine surveillance patients, as the test operator is primed to evaluate a suspicious finding in the non-routine surveillance patient. It is unclear what effect this has on test accuracy but it is likely to focus their attention on a particular area of the breast and may conceivably increase the sensitivity of the diagnostic test. Furthermore, no data were reported by the studies included on other test performance factors, such as adverse effects or acceptability of the tests.

Systematic reviews of diagnostic accuracy are highly complex and methodology in this area continues to evolve. One of the problems with a diagnostic review in this area is that there is not an established reference standard for ascertaining the true- and false-negative results of a surveillance test for IBTR or MCBC breast cancer. One potential solution is that true- and false-negatives are ascertained by a negative result or a positive test result at subsequent testing after a period of follow-up has elapsed. While this represents a pragmatic reference standard for verifying negative test results it introduces the possibility of an interval cancer occurring during the time period between tests.

Disease progression bias refers to instances where there is a time delay between an index test result and administration of the reference standard test, thus allowing the possibility of a misclassified reference standard test result. In breast cancer, uncertainty might exist as to whether a false-negative index test truly represents a cancer missed at the time of administering the index test, or whether a positive test result on subsequent testing represents a cancer that has occurred in the time interval after administration of the index test and before verification via the subsequent surveillance test. The possibility of this bias can be minimised through short time intervals between test administrations, although it cannot be completely eliminated. All studies in this review were considered to have adequate time intervals for verifying negative results for all tests. Similarly, we defined a time interval of no longer than 3 months between a positive test result and confirmation by reference standard assessment by fine-needle aspiration cytology or tissue biopsy, and no longer than 6 months for histopathological assessment by biopsy. The time period between a positive test result and reference standard confirmation was unclear in all but one study. ${ }^{71}$

Results for the index and comparator tests evaluated in this review were ascertained by subjective operator interpretation, either by visual inspection of an image of the breast (surveillance mammography, ultrasound and MRI) or by clinical examination of the breast. We do not have available data on the level of operator expertise or intra/inter-rater reliability for the included studies. It is therefore unclear whether these factors had any influence on reported test accuracy within, and between, studies and therefore whether any potential test operator bias exists.

\section{Analysis of breast cancer data set (Chapter 6)}

The strength of this analysis is that it is based on a large consecutive cohort of women diagnosed with breast cancer in the UK and treated within the NHS. The data have been collected prospectively and without selection bias. There were a large number of IBTR and MCBC events. The results of this analysis are generalisable to the UK as the WMCIU collects information from a large geographical area with a population of over 5.3 million. We used the time period from 1997 onwards to ensure consistency in data coding. As discussed earlier, this time period reflects the current management and follow-up of breast cancer patients and was after the introduction 
of the screening programme. Some previous analyses, although having the advantage of longer follow-up, cover time periods where cancer diagnosis and treatment were different. ${ }^{108}$ Our large data set allowed us to model the effects of IBTR and MCBC, and the time when they occurred, on survival and to examine whether these were independent risk factors.

The limitations of this analysis are that the data set did not contain information on surveillance mammography required to assess the effectiveness of such a regimen on survival after breast cancer treatment (i.e. frequency and duration of surveillance regimen). We used an NHS cancer data set, which was reflective of routine data collection within the NHS, although not as intensively populated or meticulously recorded as a trial data set. It was only possible to have a maximum of 10 years of follow-up because we wanted to ensure that our results were relevant to current practice. The shorter median follow-up time will possibly have the effect of underestimating the long-term adverse effect of second cancers. The data set did not contain information on whether the IBTR/MCBC were detected by mammography or clinical examination or from the patient's symptoms but we could not expect this as this is not routinely recorded by cancer registries. Women who did not have surgery for their primary breast tumour were not included in our analysis. This was because curative intent was unlikely when they were treated. Logically, it would be inappropriate to offer them surveillance mammography in the same way as women following curative treatments. A small number of older women are treated with endocrine therapy and possible radiotherapy if the tumour progresses while on hormone therapy. In addition, those who are ER negative and unfit for surgery may have radiotherapy and are monitored by mammography but this was not felt to equate with surveillance mammography.

There were perhaps fewer IBTR and MCBC events than expected compared with many published studies. For example, the recent Guy's data set reported a cumulative 10-year combined IBTR/ MCBC risk of 0.11 (95\% CI 0.09 to 0.14 ) in patients undergoing BCS, whereas this was 0.071 (95\% CI 0.065 to 0.077 ) in the WMCIU data set. ${ }^{110}$ This is most probably due to the relatively short follow-up time compared with the Guy's data set (median 9 years compared with median 5 years). The WMCIU data set also only includes those patients who have had pathological confirmation of IBTR or MCBC, and this partly accounts for the apparent underascertainment of events in the WMCIU data set. The Guy's data set was meticulously collected and the IBTR or MCBC events continued to be collected even after distant metastases were found. In some clinical practices, once distant metastases are found, breast cancer follow-up, with respect to detection of IBTR and MCBC, is not consistently undertaken, thus such events may not be recorded. We did not incorporate information about distant metastases and so could not censor at this time point. It is possible that this accounts for the apparent lack of worse prognosis for those women who develop an MCBC compared with women who do not develop further breast cancer.

It is important to recognise that the second tumour cohort data set that we used to inform the risk of death from IBTR or MCBC was created by taking all events from 1990 onwards from women who had been treated surgically for primary breast cancer. This was performed in order to have a sufficiently long follow-up period to calculate long-term survival in a large sample. Although they contain related information, the data sets should be treated independently and therefore information not extrapolated from one to another.

\section{Economic evaluation (Chapter 7)}

The main strength of the economic evaluation is that it has attempted to use rigorous and systematic methods to obtain parameter inputs into the economic evaluation. These were then assembled in the economic model whose structure was informed by both detailed discussions with the clinical members of our research team and the results of the survey of current and desired practice, commissioned as part of this study. One of the most important challenges faced when conducting the economic evaluation was the limited evidence base available. This 
constrained the model in a number of key ways. First, there were insufficient data available to model all the potential strategies that we considered relevant, and, second, few data were available on many model parameters. Furthermore, what data were available were not ideally suited to the question being addressed. For example, for health-state utilities few data relevant to a UK decision-making context were identified. In response to this, we adapted our plans to focus on three basic patterns of surveillance, the cost-effectiveness of which was explored in extensive sensitivity analyses. The object of these sensitivity analyses was not necessarily to provide definitive conclusions about the best regimen for the UK to adopt but rather to identify 'best bets' that would be worthy of further consideration. Our economic evaluation did not model all the commonly used surveillance regimens in the UK. This, in part, was due to limitations in existing evidence, but also because there were so many variants.

A further limitation relates to the way the available data were structured in the economic analysis. The model structure contains a number of simplifying assumptions. First, IBTR and MCBC are treated identically and it is assumed that they are of the lowest level of severity at the point where it is technically possible to detect them. Severity of disease has also been defined in an arbitrary manner (by grouping cancers according to the expected 10-year survival). This was driven, in part, by a desire to keep this element of the model manageable. However, it may mean that relatively subtle differences between strategies might be missed.

The economic evaluation took the UK NHS as its perspective. This meant that only costs incurred by the NHS were included, and benefits were measured in terms of the effects on health (measured in QALYs) that were consequent on increased survival caused by earlier detection. This perspective is the one recommended by NICE, ${ }^{100}$ for instance, but it means that other potentially important costs and benefits are excluded. With respect to costs it might be expected that the women's costs of accessing surveillance services would increase as the frequency of surveillance increases. However, earlier detection and successful treatment would reduce time away from usual activities following treatment and those caused by ill health attributed to more advanced disease. The net effect of these aspects is uncertain.

Similarly, this project did not consider psychological implications for women and their families. Quality-of-life measures used as the basis of QALYs can capture some aspects of the effects on anxiety and mental health. However, the model only considered those effects relating to health effects from treatment/non-treatment of the cancer. It did not consider the whole patient experience of undergoing surveillance. We sought opinion from the patient representative member of our Advisory Group regarding our results. Variation in the manner in which surveillance is organised can be a source of anxiety to women due to concerns that differences in frequency and duration of follow-up have implications for risk of recurrence and survival. A woman could, for example, assume that 10 compared with 5 years of follow-up implies that she is at a heightened personal risk of developing IBTR or MCBC, rather than being an association with eligible screening age or local practice. Similarly, a patient undergoing mammography every 3 years compared with one having annual mammography may either feel reassured that she has less risk of developing further cancer or feel anxiety that any further cancer will go undetected for a greater length of time. With respect to the model, these aspects could be further explored using preference elicitation techniques that go beyond the QALY framework. In more practical terms, this concern highlights the potential need to ensure that patients with breast cancer have a greater understanding of how and why their follow-up regimen has been 'personalised' to match their personal breast cancer type and its risk factors. Greater standardisation of practice in timing of implementing and discharging surveillance regimens would also provide reassurance that patients throughout the UK are all receiving the same standard of care. 



\section{Chapter 9}

\section{Conclusions}

\section{Summary of findings}

The most common mammographic surveillance regimen identified by our survey was annual mammography commencing 12 months after surgical treatment of the primary breast cancer. There was considerable variation in the combinations of start, frequency, duration and discharge from different surveillance regimens, however. Prior to our work, there was little existing evidence on the effectiveness of surveillance mammography and follow-up after treatment for primary breast cancer. Our systematic review of clinical effectiveness suggests that surveillance mammography offers a survival benefit compared with a surveillance regimen that does not include surveillance mammography. However, the effects of combinations of alternative surveillance regimens (e.g. clinical examination, referral to secondary care between planned surveillance if the patient has symptoms, etc.) remain unclear. Few data were identified regarding the diagnostic performance. The few data we found suggest that mammography is associated with a high sensitivity and specificity but MRI is the most accurate test for detecting IBTR and MCBC. The results from the systematic reviews should be interpreted with caution due to the limited evidence base from which they are derived (effectiveness review, eight studies; test performance review, nine studies).

No existing data set was identified that was ideally suited to answering our research questions. Our analysis of the WMCIU data set confirmed the previously known risk factors for mortality from breast cancer. It also demonstrated that IBTR is an independent predictor of survival. For women experiencing IBTR size is important, with those women with tumours $>20 \mathrm{~mm}$ in size being at a significantly greater risk of death than those with IBTR of $<10 \mathrm{~mm}$. There was no evidence that $\mathrm{MCBC}$ is an independent predictor of survival; however, in women who do experience $\mathrm{MCBC}$ the size of the tumour is important. Women with tumours $>20 \mathrm{~mm}$ in size are at a significantly greater risk of death than those with $\mathrm{MCBC}$ tumours of $<10 \mathrm{~mm}$. Given the limited evidence base the results of the economic evaluation should be considered as exploratory. If it were decided that the NHS should adopt a single surveillance regimen for all women then the economic evaluation suggests that the regimen most likely to be considered cost-effective when the cost per QALY threshold was either $£ 20,000$ or $£ 30,000$ would be mammographic surveillance alone, provided every 12-24 months. The results of the economic model were sensitive to incidence and other factors, for example age, tumour characteristics, etc., that might define groups of women with greater or lesser likelihoods of developing a IBTR or MCBC. These results suggest that a more intensive follow-up of women judged to be at greater likelihood might be worthwhile. Conversely, for women with less likelihood it may be more cost-effective for surveillance to be performed less often (every 2 or 3 years) with mammography alone.

\section{Implications for the NHS and patients}

- Results suggest that surveillance, when combined with effective treatment of the cancers detected, is likely to improve survival.

- The evidence base on which to recommend any change in current practice is relatively weak. 
- The current evidence base suggests that should the NHS choose to standardise surveillance for all women then a regimen of mammography alone every 12-24 months appears to have the highest net benefits when society’s willingness to pay for a QALY is $£ 20,000$ or $£ 30,000$.

- Rather than offering the same regimen to all patients, careful consideration should be given to stratification of patients to ensure maximum benefit to ensure optimal use of resources. How best to deliver a varying surveillance regimen would be challenging, and consideration is also needed about how such a service could be best organised.

- The current evidence base suggests that should the NHS choose to tailor surveillance for those women with a greater likelihood of developing IBTR or MCBC then more comprehensive (e.g. mammography and clinical follow-up) and more frequent surveillance (every 12 months) would be associated with the greatest net benefit.

- The current evidence base suggests that should the NHS choose to tailor surveillance for those women least likely to develop IBTR or MCBC then only less frequent mammographic surveillance (e.g. every 36 months) would be associated with the greatest net benefit.

- Variation in surveillance practice (e.g. frequency and duration of follow-up regimen) can be a source of anxiety to women. This implies that efforts to improve the patient's understanding of how they will be followed up are important. Should the NHS choose to tailor surveillance then there would need to be the provision of information and reassurance about how the follow-up regimen has been tailored to match the type of breast cancer they have had and their risk factors for developing IBTR or MCBC.

- Although evidence is limited about how best to standardise practice greater standardisation of an agreed surveillance regimen would provide reassurance that patients throughout the UK are receiving the same standard of care.

\section{Suggested research priorities}

Further evidence is required to make a robust and informed judgement on the effectiveness of mammographic surveillance and follow-up. From a scoping search conducted on 25 August 2010, 14 months after conducting the search for the main effectiveness systematic review, we identified 192 potential titles and abstracts. Of these, we found no RCT studies directly addressing our research question. We found one RCT for which primary outcomes considered psychological morbidity for point of need compared with 6-monthly clinic surveillance. Only the secondary outcomes of this RCT, recurrences and method of detection, are relevant for this report. We found six non-RCT studies, two of which are referenced in the introductory section of this report, ${ }^{5,48}$ providing indirectly relevant evidence. We found no new HTA report or ongoing RCTs. The evidence trajectory for the clinical and cost-effectiveness of surveillance mammography therefore appears to be slow moving. Research is suggested in the following areas:

- The data sets that exist at the moment are an excellent resource for researchers and considerable further analysis, addressing both clinically and economically important questions, could be undertaken using the data they contain. For example, further work could focus on extending our analyses by incorporating the influence of distant metastases on survival. Where appropriate such analyses should be incorporated into an economic evaluation model.

- With respect to the research question posed within this report the utility of the data sets could be improved. The optimal data set would record details of mode of detection for IBTR or MCBC; the frequency of the clinical and mammographic surveillance regimen, and how this varies over time; and whether a woman's IBTR or MCBC was detected during routine surveillance or as a result of it causing symptoms for the patient. Some of this information is well collected currently and additional information might reasonably be captured in a thorough audit. It is important to record accurate and timely information on 
grade, size, tumour type, ER status and lymph node status of both the primary tumour and subsequent IBTR or MCBC tumour; details of the primary treatment given (i.e. type and extent of surgery, excision margins, radiotherapy, hormone therapy, chemotherapy and other treatments); details of IBTR or MCBC treatment; when and where distant metastases occurred; and cause of death. Once available such data should be incorporated into refined statistical and economic analyses addressing the research question posed within this report.

- The paucity of data on the diagnostic performance of tests in the group of women of interest needs to be addressed. There is a need for high-quality, direct head-to-head studies comparing the diagnostic accuracy of tests used in the surveillance population. Such studies are likely to be lower cost than a definitive RCT and provide data sooner. Such data should then be incorporated into an economic evaluation model.

- The economic evaluation suggested that there were circumstances where the use of tests with a better diagnostic performance but higher cost might be potentially cost-effective. Further primary work should also consider whether the use of existing technologies, such as MRI, which may have better performance, could be worthwhile for patients at high risk of IBTR or MCBC. An economic analysis should form part of such work.

- It would be useful to compare within an economic evaluation mixed clinical follow-up in lower cost settings combined with surveillance mammography in the long term. It would also be important to consider psychological factors associated with differing types, settings and frequency of surveillance regimens using a major health psychology theory so that the economic modelling could focus down on regimens that, based on the psychological analyses, look most promising.

- A definitive RCT would be ideal and, although costly, could focus on those women at higher risk of IBTR or MCBC. The interventions considered might include mammography and MRI, for those at the highest risk, or surveillance mammography of 1 year versus a longer time interval, for example 3 years. Such a trial might also compare more sophisticated surveillance regimens that vary not only in terms of the frequency of mammography, but also in terms of the frequency and setting of clinical follow-up. 



\title{
Acknowledgements
}

\author{
$\mathrm{W}_{\text {ethank: }}$
}

- Val Bain, breast cancer nurse specialist, Peter Donnelly, consultant breast surgeon, and Peter Murchie, general practitioner, for participating as members of the project Advisory Group.

- Jen Burr, senior clinical research fellow, for advice at the start of the study.

- Members of ABS at BASO and the RCR Breast Group for participating in the survey of current surveillance mammography practice.

- Daniel Barnett for programming, web and database set-up for the survey of current surveillance mammography practice; Lucy Davies, administrator, ABS at BASO, for administering the survey; Stewart Nicholson, honorary secretary, ABS at BASO, for giving permission to distribute the survey to ABS members; and Anthony Maxwell, RCR Breast Group, for giving permission and administering the survey.

- Jennifer Hislop, research fellow, for extracting data in the systematic review of test performance.

We also thank Lara Kemp, for secretarial support.

The HSRU and the Health Economics Research Unit are both core funded by the Chief Scientist Office of the Scottish Government Health Directorates. The views expressed are those of the authors.

\section{Contributions of authors}

Fiona Gilbert led the drafting of the Background chapter. Clare Robertson wrote the initial draft with advice from Steve Heys.

Luke Vale and Laura Ternent led the drafting of Chapter 2.

Ruth Thomas led the drafting of Chapter 3, identifying feasible regimens, with Luke Vale.

Clare Robertson developed the protocol and undertook the survey described in Chapter 3, working closely with Ruth Thomas.

Clare Robertson led the systematic reviews with advice from Graham Mowatt. She developed the protocols for the systematic reviews working closely with Ruth Thomas and led the drafting of the systematic review chapters (Chapters 4 and 5).

The systematic review team of Charles Boachie, Graeme MacLennan, Clare Robertson, Ruth Thomas and Shihua Zhu undertook the effectiveness review (Chapter 4).

The systematic review team for Chapter 5 was Charles Boachie, Fiona Gilbert, Graeme MacLennan, Jennifer Hislop, Clare Robertson, Senthil Kumar Arcot Ragupathy, Ruth Thomas and Shihua Zhu.

Graeme MacLennan led the drafting of Chapter 6, the analysis of the existing data sets, with advice from Steve Heys and Fiona Gilbert. Graeme MacLennan developed the protocol and led 
the analysis of the data set, drawing upon the advice of Steve Heys, Gill Lawrence, Wilma Jack and Gill Kerr.

Laura Ternent led the economic evaluation including the drafting of the economic evaluation, Chapter 7, in which she worked closely with Luke Vale, with assistance from Rodolfo Hernandez and Shihua Zhu.

Cynthia Fraser conducted the literature searches, drafted sections of the report related to this work and provided reference management for the whole project.

Mike Dixon, Steve Heys, Wilma Jack, Gill Kerr, Gill Lawrence, Anthony Maxwell, Jean McGregor, Sarah Pinder and Robin Wilson all provided advice in their own areas of expertise and provided critical comments throughout the project.

Fiona Gilbert and Luke Vale were involved in all elements of the project and provided project management, assisted by Ruth Thomas.

All authors assisted in preparing the manuscript, reading and commenting on drafts, and reading relevant sections of the final draft. 


\section{References}

1. Cancer Research UK. Prostate cancer: survival statistics. London: Cancer Research UK; 2009. URL: http://info.cancerresearchuk.org/cancerstats/types/prostate/survival/index.htm (accessed April 2010).

2. Clarke M, Collins R, Darby S, Davies C, Elphinstone P, Evans E, et al. Effects of radiotherapy and of differences in the extent of surgery for early breast cancer on local recurrence and 15-year survival: an overview of the randomised trials. Lancet 2005;366:2087-106.

3. Early Breast Cancer Trialists' Collaborative Group (EBCTCG). Effects of chemotherapy and hormonal therapy for early breast cancer on recurrence and 15-year survival: an overview of the randomised trials. Lancet 2005;365:1687-717.

4. Montgomery DA, Krupa K, Cooke TG. Follow-up in breast cancer: does routine clinical examination improve outcome? A systematic review of the literature. Br J Cancer 2007;97:1631-41.

5. Lu WL. Impact on survival of early detection of isolated breast recurrences after the primary treatment for breast cancer: a meta-analysis. Breast Cancer Res Treat 2009;114:403-12.

6. Schootman M, Fuortes L, Aft R. Prognosis of metachronous contralateral breast cancer according to stage at diagnosis: the importance of early detection. Breast Cancer Res Treat 2006;99:91-5.

7. della Rovere GQ, Benson JR. Ipsilateral local recurrence of breast cancer: determinant or indicator of poor prognosis? Lancet Oncol 2002;3:183-7.

8. Voogd AC, Nielsen M, Peterse JL, Blichert-Toft M, Bartelink H, Overgaard M, et al. Differences in risk factors for local and distant recurrence after breast-conserving therapy or mastectomy for stage I and II breast cancer: pooled results of two large European randomized trials. J Clin Oncol 2001;19:1688-97.

9. Houghton J, ATAC Trialists' Group. A detailed analysis of the risk factors influencing local and distant breast cancer recurrence during adjuvant endocrine therapy. J Clin Oncol 2007;25:S562.

10. Benson JR, della Rovere GQ. Ipsilateral breast cancer recurrence. Breast 2008;17:12-18.

11. Rossouw JE, Anderson GL, Prentice RL, LaCroix AZ, Kooperberg C, Stefanick ML, et al. Risks and benefits of estrogen plus progestin in healthy postmenopausal women: principal results from the Women's Health Initiative randomized controlled trial. JAMA 2002;288:321-33.

12. Ravdin PM, Cronin KA, Howlader N, Berg CD, Chlebowski RT, Feuer EJ, et al. The decrease in breast-cancer incidence in 2003 in the United States. N Engl J Med 2007;356:1670-4.

13. Parkin DM. Is the recent fall in incidence of post-menopausal breast cancer in UK related to changes in use of hormone replacement therapy? Eur J Cancer 2009;45:1649-53.

14. Cancer Research UK. Breast cancer: UK incidence statistics. London: Cancer Research UK; 2009. URL: http://info.cancerresearchuk.org/cancerstats/types/breast/incidence/index.htm (accessed January 2010).

15. Cajucom CC, Tsangaris TN, Nemoto T, Driscoll D, Penetrante RB, Holyoke ED. Results of salvage mastectomy for local recurrence after breast-conserving surgery without radiation therapy. Cancer 1993;71:1774-9. 
16. Chaudary MA, Millis RR, Hoskins EO, Halder M, Bulbrook RD, Cuzick J, et al. Bilateral primary breast cancer: a prospective study of disease incidence. Br J Surg 1984;71:711-14.

17. Chen Y, Thompson W, Semenciw R, Mao Y. Epidemiology of contralateral breast cancer. Cancer Epidemiol Biomarkers Prev 1999;8:855-61.

18. Maddams J, Brewster D, Gavin A, Steward J, Elliott J, Utley M, et al. Cancer prevalence in the United Kingdom: estimates for 2008. Br J Cancer 2009;101:541-7.

19. Abner AL, Recht A, Eberlein T, Come S, Shulman L, Hayes D, et al. Prognosis following salvage mastectomy for recurrence in the breast after conservative surgery and radiation therapy for early-stage breast cancer. J Clin Oncol 1993;11:44-8.

20. Haffty BG, Fischer D, Beinfield M, McKhann C. Prognosis following local recurrence in the conservatively treated breast cancer patient. Int J Radiat Oncol Biol Phys 1991;21:293-8.

21. Association of Breast Surgeons at the British Association of Surgical Oncology (ABS at BASO). Surgical guidelines for the management of breast cancer. Eur J Surg Oncol 2009;35(Suppl. 1):1-22.

22. Scottish Intercollegiate Guidelines Network (SIGN). Management of breast cancer in women. SIGN Clinical Guideline no. 84. Edinburgh: SIGN; 2005. URL: www.sign.ac.uk/pdf/sign84. pdf (accessed January 2010).

23. Board of the Faculty of Clinical Radiology. Guidance on screening and symptomatic breast imaging. London: The Royal College of Radiologists; 2003. URL: www.rcr.ac.ukindex.asp?Pag eID=310\&PublicationID=184 (accessed January 2010).

24. National Institute for Health and Clinical Excellence (NICE). Early and locally advanced breast cancer: full guideline. NICE CG80. London: NICE; 2009. URL: http://guidance.nice. org.uk/CG80/Guidance/pdf/English (accessed January 2010).

25. National Institute for Health and Clinical Excellence (NICE). Guidance on cancer services. Improving outcomes in breast cancer. London: NICE; 2002. URL: www.nice.org.uk/pdf/ Improving_outcomes_breastcancer_manual.pdf (accessed January 2010).

26. Donnelly P, Hiller L, Bathers S, Bowden S, Coleman R. Questioning specialists' attitudes to breast cancer follow-up in primary care. Ann Oncol 2007;18:1467-76.

27. Maxwell AJ, Evans AJ, Carpenter R, Dobson HM, Kearins O, Clements K, et al. Follow-up for screen-detected ductal carcinoma in situ: results of a survey of UK centres participating in the Sloane project. Eur J Surg Oncol 2009;35:1055-9.

28. Khatcheressian JL, Wolff AC, Smith TJ, Grunfeld E, Muss HB, Vogel VG, et al. American Society of Clinical Oncology 2006 update of the breast cancer follow-up and management guidelines in the adjuvant setting. J Clin Oncol 2006;24:5091-7.

29. Rojas MP, Telaro E, Russo A, Moschetti I, Coe L, Fossati R, et al. Follow-up strategies for women treated for early breast cancer. Cochrane Database Syst Rev 2000;4:CD001768. DOI: 10.1002/14651858.CD001768.pub2.

30. Flobbe K, van der Linden ES, Kessels AG, van Engelshoven JM. Diagnostic value of radiological breast imaging in a non-screening population. Int J Cancer 2001;92:616-18.

31. Orel SG, Troupin RH, Patterson EA, Fowble BL. Breast cancer recurrence after lumpectomy and irradiation: role of mammography in detection. Radiology 1992;183:201-6.

32. Fowble B, Solin LJ, Schultz DJ, Rubenstein J, Goodman RL. Breast recurrence following conservative surgery and radiation: patterns of failure, prognosis, and pathologic findings from mastectomy specimens with implications for treatment. Int J Radiat Oncol Biol Phys 1990;19:833-42. 
33. Joseph E, Hyacinthe M, Lyman GH, Busch C, Demps L, Reintgen DS, et al. Evaluation of an intensive strategy for follow-up and surveillance of primary breast cancer. Ann Surg Oncol 1998;5:522-8.

34. Bartella L, Smith CS, Dershaw DD, Liberman L. Imaging breast cancer. Radiol Clin North Am 2007;45:45-67.

35. Pisano ED, Hendrick RE, Yaffe MJ, Baum JK, Acharyya S, Cormack JB, et al. Diagnostic accuracy of digital versus film mammography: exploratory analysis of selected population subgroups in DMIST. Radiology 2008;246:376-83.

36. Berg WA, Blume JD, Cormack JB, Mendelson EB, Lehrer D, Bohm-Velez M, et al. Combined screening with ultrasound and mammography vs mammography alone in women at elevated risk of breast cancer. JAMA 2008;299:2151-63.

37. Crystal P, Strano SD, Shcharynski S, Koretz MJ. Using sonography to screen women with mammographically dense breasts. AJR Am J Roentgenol 2003;181:177-82.

38. Kaplan SS. Clinical utility of bilateral whole-breast US in the evaluation of women with dense breast tissue. Radiology 2001;221:641-9.

39. Buchberger W, DeKoekkoek-Doll P, Springer P, Obrist P, Dunser M. Incidental findings on sonography of the breast: clinical significance and diagnostic workup. AJR Am J Roentgenol 1999;173:921-7.

40. Department of Health. NHS reference costs 2008-09. London: Department of Health; 2010. URL: www.dh.gov.uk/en/Publicationsandstatistics/Publications/ PublicationsPolicyAndGuidance/DH_111591 (accessed January 2010).

41. Grunfeld E, Mant D, Yudkin P, Adewuyi-Dalton R, Cole D, Stewart J, et al. Routine follow up of breast cancer in primary care: randomised trial. BMJ 1996;313:665-9.

42. Breast Cancer.org. Breast self-examination. Ardmore, PA: Breastcancer.org; 2008. URL: www. breastcancer.org/symptoms/testing/types/self_exam/ (accessed January 2010).

43. Barnsley GP, Grunfeld E, Coyle D, Paszat L. Surveillance mammography following the treatment of primary breast cancer with breast reconstruction: a systematic review. Plast Reconstr Surg 2007;120:1125-32.

44. Collins RF, Bekker HL, Dodwell DJ. Follow-up care of patients treated for breast cancer: a structural review. Cancer Treat Rev 2004;30:19-35.

45. de Bock GH, Bonnema J, van der Hage J, Kievit J, van de Velde CJ. Effectiveness of routine visits and routine tests in detecting isolated locoregional recurrences after treatment for early-stage invasive breast cancer: a meta-analysis and systematic review. J Clin Oncol 2004;22:4010-18.

46. Montgomery DA, Krupa K, Cooke TG. Alternative methods of follow up in breast cancer: a systematic review of the literature. Br J Cancer 2007;96:1632-41.

47. Grunfeld E, Noorani H, McGahan L, Paszat L, Coyle D, Van Walraven C, et al. Surveillance mammography after treatment of primary breast cancer: a systematic review. Breast 2002;1 1:228-35.

48. Houssami N, Ciatto S, Martinelli F, Bonardi R, Duffy SW. Early detection of second breast cancers improves prognosis in breast cancer survivors. Ann Oncol 2009;20:1505-10.

49. Voogd AC. Local recurrence after breast conservation therapy for early stage breast carcinoma detection, treatment, and outcome in 266 patients. Cancer 1999;85:437-46. 
50. Philips Z, Ginnelly L, Sculpher M, Claxton K, Golder S, Riemsma R, et al. Review of guidelines for good practice in decision-analytic modelling in health technology assessment. Health Technol Assess 2004;8(36).

51. Higgins JP, Green S, editors. Cochrane handbook for systematic reviews of interventions. Version 5.0.2. The Cochrane Collaboration, 2009. URL: www.cochrane-handbook.org (accessed January 2010).

52. Centre for Reviews and Dissemination. NHS Economic Evaluation Database handbook. University of York: CRD; 2007. URL: www.york.ac.uk/inst/crd/pdf/nhseed-handb07.pdf (accessed January 2010).

53. Churn M, Kelly V. Outpatient follow-up after treatment for early breast cancer: updated results after 5 years. Clin Oncol 2001;13:187-94.

54. Montgomery DA, Krupa K, Jack WJ, Kerr GR, Kunkler IH, Thomas J, et al. Changing pattern of the detection of locoregional relapse in breast cancer: the Edinburgh experience. $\mathrm{Br} J$ Cancer 2007;96:1802-7.

55. Montgomery DA, Krupa K, Cooke TG. Locoregional relapse after breast cancer: most relapses occur late and are not clinically detected. Breast J 2009;15:163-7.

56. Paszat L, Sutradhar R, Grunfeld E, Gainford C, Benk V, Bondy S, et al. Outcomes of surveillance mammography after treatment of primary breast cancer: a population-based case series. Breast Cancer Res Treat 2008;114:169-78.

57. Yau TK, Sze H, Soong IS, Wong W, Chan K, Chang A, et al. Surveillance mammography after breast conservation therapy in Hong Kong: effectiveness and feasibility of risk-adapted approach. Breast 2008;17:132-7.

58. Lash TL, Fox MP, Buist DS, Wei F, Field TS, Frost FJ, et al. Mammography surveillance and mortality in older breast cancer survivors. J Clin Oncol 2007;25:3001-6.

59. Lash TL, Clough-Gorr K, Silliman RA. Reduced rates of cancer-related worries and mortality associated with guideline surveillance after breast cancer therapy. Breast Cancer Res Treat 2005;89:61-7.

60. Lash TL, Fox MP, Silliman RA. Reduced mortality rate associated with annual mammograms after breast cancer therapy. Breast J 2006;12:2-6.

61. Bossuyt PM, Leeflang MM. Developing criteria for included studies. Cochrane Handbook for Systematic Reviews of Diagnostic Test Accuracy. Version 0.4 (updated September 2008). The Cochrane Collaboration, 2008. URL: http://srdta.cochrane.org/en/authors.html (accessed January 2010).

62. Whiting P, Rutjes AW, Reitsma JB, Bossuyt PM, Kleijnen J. The development of QUADAS: a tool for the quality assessment of studies of diagnostic accuracy included in systematic reviews. BMC Med Res Methodol 2003;3:25.

63. Verhagen AP, de Vet HC, de Bie RA, Kessels AG, Boers M, Bouter LM, et al. The Delphi list: a criteria list for quality assessment of randomized clinical trials for conducting systematic reviews developed by Delphi consensus. J Clin Epidemiol 1998;51:1235-41.

64. Boné B, Aspelin P, Isberg B, Perbeck L, Veress B. Contrast-enhanced MR imaging of the breast in patients with breast implants after cancer surgery. Acta Radiol 1995;36:111-16.

65. Kim MJ, Kim EK, Kwak JY, Park BW, Kim SI, Sohn J, et al. Sonographic surveillance for the detection of contralateral metachronous breast cancer in an Asian population. AJR Am J Roentgenol 2009;192:221-8. 
66. Belli P, Pastore G, Romani M, Terribile D, Canade A, Costantini M. Role of magnetic resonance imaging in the diagnosis of recurrence after breast conserving therapy. Rays 2002;27:241-57.

67. Drew PJ, Kerin MJ, Turnbull LW, Imrie M, Carleton PJ, Fox JN, et al. Routine screening for local recurrence following breast-conserving therapy for cancer with dynamic contrastenhanced magnetic resonance imaging of the breast. Ann Surg Oncol 1998;5:265-70.

68. Mumtaz H, Davidson T, Hall-Craggs MA, Payley M, Walmsley K, Cowley G, et al. Comparison of magnetic resonance imaging and conventional triple assessment in locally recurrent breast cancer. Br J Surg 1997;84:1147-51.

69. Rieber A, Merkle E, Zeitler H, Gorich J, Kreienberg R, Brambs HJ, et al. Value of MR mammography in the detection and exclusion of recurrent breast carcinoma. J Comput Assist Tomogr 1997;21:780-4.

70. Viehweg P, Rotter K, Laniado M, Lampe D, Buchmann J, Kolbl H, et al. MR imaging of the contralateral breast in patients after breast-conserving therapy. Eur Radiol 2004;14:402-8.

71. Shin JH, Han BK, Choe YH, Nam SJ, Park W, Im YH. Ultrasonographic detection of occult cancer in patients after surgical therapy for breast cancer. J Ultrasound Med 2005;24:643-9.

72. Ternier F, Houvenaeghel G, Lecrivain F, Brigand BL, Margain D, Brunelle S, et al. Computed tomography in suspected local breast cancer recurrence. Breast Cancer Res Treat 2006;100:247-54.

73. Haybittle JL, Blamey RW, Elston CW, Johnson J, Doyle PJ, Campbell FC, et al. A prognostic index in primary breast cancer. Br J Cancer 1982;45:361-6.

74. Collett D. Modelling survival data in medical research. London: Chapman \& Hall/CRC; 2003.

75. Cox DR. Regression models and life tables. J Roy Stat Soc B 1972;34:187-220.

76. Kaplan EL, Meier P. Nonparametric estimation from incomplete observations. J Am Stat Assoc 1958;53:457-81.

77. Anderson SJ, Wapnir I, Dignam JJ, Fisher B, Mamounas EP, Jeong JH, et al. Prognosis after ipsilateral breast tumor recurrence and locoregional recurrences in patients treated by breastconserving therapy in five National Surgical Adjuvant Breast and Bowel Project protocols of node-negative breast cancer. J Clin Oncol 2009;27:2466-73.

78. Fisher B, Anderson S, Fisher ER, Redmond C, Wickerham DL, Wolmark N, et al. Significance of ipsilateral breast tumour recurrence after lumpectomy. Lancet 1991; 338:327-31.

79. Fortin A, Larochelle M, Laverdiere J, Lavertu S, Tremblay D. Local failure is responsible for the decrease in survival for patients with breast cancer treated with conservative surgery and postoperative radiotherapy. J Clin Oncol 1999;17:101-9.

80. Veronesi U, Marubini E, Del Vecchio M, Manzari A, Andreola S, Greco M, et al. Local recurrences and distant metastases after conservative breast cancer treatments: partly independent events. J Natl Cancer Inst 1995;87:19-27.

81. Ciatto S, Houssami N, Martinelli F, Bonardi R, Cafferty FH, Duffy SW. Second breast cancers in a Tuscan case series: characteristics, prognosis, and predictors of survival. Br J Cancer 2008;99:539-44.

82. Drummond MF, Sculpher MJ, Torrance GW, O'Brien BJ, Stoddart GL. Methods for the economic evaluation of health care programmes. 3rd edn. Oxford: Oxford University Press; 2005. 
83. Early Breast Cancer Trialists' Collaborative Group (EBCTCG) website. University of Oxford: Clinical Trial Service Unit and Epidemiological Studies Unit; 2008. URL: www.ctsu.ox.ac.uk/ projects/ebctcg (accessed January 2010).

84. Adjuvant! Online website. Adjuvant! Inc., 2008. URL: www.adjuvantonline.com/index.jsp (accessed January 2010).

85. Rhodes A, Jasani B, Balaton AJ, Barnes DM, Miller KD. Frequency of oestrogen and progesterone receptor positivity by immunohistochemical analysis in 7016 breast carcinomas: correlation with patient age, assay sensitivity, threshold value, and mammographic screening. J Clin Pathol 2000;53:688-96.

86. NHS Scotland Information and Statistics Division. Breast cancer: data analyses and reports. Edinburgh: NHS Scotland; 2010. URL: www.isdscotland.org/isd/1420.html (accessed January 2010).

87. Government Actuary's Department (GAD). Interim life tables 2006-08. London: GAD; 2008. URL: www.gad/gov.uk/Demography\%20Data/Life\%20Tables/index.html (accessed January 2010).

88. Spratt JA, von Fournier D, Spratt JS, Weber EE. Mammographic assessment of human breast cancer growth and duration. Cancer 1993;71:2020-6.

89. Peer PG, van Dijck JA, Hendriks JH, Holland R, Verbeek AL. Age-dependent growth rate of primary breast cancer. Cancer 1993;71:3547-51.

90. Warner E, Messersmith H, Causer P, Eisen A, Shumak R, Plewes D. Systematic review: using magnetic resonance imaging to screen women at high risk for breast cancer. Ann Intern Med 2008;148:671-9.

91. Leach MO, Boggis CR, Dixon AK, Easton DF, Eeles RA, Evans DG, et al. Screening with magnetic resonance imaging and mammography of a UK population at high familial risk of breast cancer: a prospective multicentre cohort study (MARIBS). Lancet 2005;365:1769-78.

92. Burr J, Mowatt G, Siddiqui MAR, Herandez R, Cook JA, Lourenco T, et al. The clinical and cost effectiveness of screening for open angle glaucoma: a systematic review and economic evaluation. Health Technol Assess 2007;11(41).

93. NHS Breast Screening Programme (NHSBSP) website. NHSBSP, 2009. URL: www. cancerscreening.nhs.uk/breastscreen/index.html\#cost (accessed January 2010).

94. Scottish National Breast Screening Programme spreadsheet. Edinburgh: Information Services Division Scotland; 2009. URL: www.isdscotland.org/isd/servlet/

FileBuffer?namedFile=Costs_R921_2009.xls\&pContentDispositionType=inline (accessed January 2010).

95. Personal Social Services Research Unit (PSSRU). Curtis L. Unit costs of health and social care 2008. Canterbury: PSSRU; 2008. URL: www.pssru.ac.uk.uc/uc/uc2008contents.htm\#contents (accessed January 2010).

96. Griffin S, Dunn GPS, Macfarlane K, Brent S, Dyker A, Erhorn S. The use of paclitaxel in the management of early stage breast cancer. The Evidence Review Group's report. London: NICE; 2006. URL: www.nice.org.uk/nicemedia/pdf/STA_report.pdf (accessed January 2010).

97. Prescott RJ, Kunkler IH, Williams LJ, King CC, Jack W, van der Pol M, et al. A randomised controlled trial of postoperative radiotherapy following breast-conserving surgery in a minimum-risk older population. The PRIME trial. Health Technol Assess 2007;11(31). 
98. National Institute for Health and Clinical Excellence (NICE). Breast cancer (early): hormonal treatments: costing template and report. London: NICE; 2006. URL: http://guidance.nice.org. uk/TA112/CostReport/xls/English (accessed January 2010).

99. National Institute for Health and Clinical Excellence (NICE). Docetaxel for the adjuvant treatment of early node-positive breast cancer. TA109. London: NICE; 2006. URL: http:// guidance.nice.org.uk/TA109/Guidance/pdf/English (accessed January 2010).

100. National Institute for Health and Clinical Excellence (NICE). Guide to the methods of technology appraisal. London: NICE; 2008. URL: www.nice.org.uk/media/B52/A7/ TAMethodsGuideUpdatedJune2008.pdf (accessed January 2010).

101. Brennan V, Wolowacz S. A systematic review of breast cancer utility weights. ISPOR 13th Annual International Meeting, Toronto, 3-7 May 2008.

102. Lloyd A, Nafees B, Narewska J, Dewilde S, Watkins J. Health state utilities for metastatic breast cancer. Br J Cancer 2006;95:683-90.

103. Mansel R, Locker G, Fallowfield L, Benedict A, Jones D. Cost-effectiveness analysis of anastrozole vs tamoxifen in adjuvant therapy for early stage breast cancer in the United Kingdom: the 5-year completed treatment analysis of the ATAC ('Arimidex', Tamoxifen alone or in combination) trial. Br J Cancer 2007;97:152-61.

104. Schleinitz MD, DePalo D, Blume J, Stein M. Can differences in breast cancer utilities explain disparities in breast cancer care? J Gen Intern Med 2006;21:1253-60.

105. Sorensen S, Brown R, Benedict A, Flood E, Revicki D. Patient-rated utilities in postmenopausal early breast cancer (EBC): a cross-country comparison. Value Health 2004;7:641-2.

106. Jansen SJ, Stiggelbout AM, Wakker PP, Vliet Vlieland TP, Leer JW, Nooy MA, et al. Patients' utilities for cancer treatments: a study of the chained procedure for the standard gamble and time trade-off. Med Decis Making 1998;18:391-9.

107. Tosteson AN, Stout NK, Fryback DG, Acharyya S, Herman BA, Hannah LG, et al. Cost-effectiveness of digital mammography breast cancer screening. Ann Intern Med 2008;148:1-10.

108. Hartman M, Czene K, Reilly M, Adolfsson J, Bergh J, Adami HO, et al. Incidence and prognosis of synchronous and metachronous bilateral breast cancer. J Clin Oncol 2007;25:4210-16.

109. Cook JV, Dickinson HO, Eccles MP. Response rates in postal surveys of healthcare professionals between 1996 and 2005: an observational study. BMC Health Serv Res 2009;9:160.

110. Kontos M, Allen D, Trafalis DT, Jones G, Garmo H, Holmberg L, et al. Follow-up may not be beneficial after treatment of grade 1 breast cancer. Br J Surg 2009;96:999-1004.

111. National Breast and Ovarian Cancer Centre (NBOCC). Clinical practice guideline for the management of early breast cancer. Canberra, Australia: National Breast and Ovarian Cancer Centre; 2001. URL: http://nbocc.org.au/view-document-details/cpg-clinical-practiceguidelines-for-the-management-of-early-breast-cancer-2nd-ed (accessed January 2010).

112. National Comprehensive Cancer Network (NCCN). Clinical practice guidelines in oncology, breast cancer. v.1.2009. Fort Washington, PA: NCCN; 2009. URL: www.nccn.org/ professionals/physician_gls/f_guidelines.asp (accessed January 2010). 
113. Grunfeld E, Dhesy-Thind S, Levine M, Steering Committee on Clinical Practice Guidelines for the Care and Treatment of Breast Cancer. Clinical practice guidelines for the care and treatment of breast cancer: follow-up after treatment for breast cancer (summary of the 2005 update). CMAJ 2005;172:1319-20.

114. Pestalozzi B, Castiglione M, ESMO Guidelines Working Group. Primary breast cancer: ESMO clinical recommendations for diagnosis, treatment and follow-up. Ann Oncol 2008;19(Suppl. 2):7-10.

115. Temple LK, Wang EE, McLeod RS. Preventive health care, 1999 update: 3. Follow-up after breast cancer. Canadian Task Force on Preventive Health Care. CMAJ 1999;161:1001-8.

116. National Institute of Health and Clinical Excellence (NICE). Breast cancer (early): paclitaxel: guidance. TA108. London: NICE; 2006. URL: http://guidance.nice.org.uk/TA108/Guidance/ pdf/English (accessed January 2010).

117. National Institute of Health and Clinical Excellence (NICE). Breast cancer (early): trastuzumab: guidance. TA107. London: NICE; 2006. URL: http://guidance.nice.org.uk/ TA108/Guidance/pdf/English (accessed January 2010).

118. Early Breast Cancer Trialists' Collaborative Group (EBCTCG), Clarke M, Coates AS, Darby SC, Davies C, Gelber RD, et al. Adjuvant chemotherapy in oestrogen-receptor-poor breast cancer: patient-level meta-analysis of randomised trials. Lancet 2008;371:29-40.

119. Galante E, Guzzon A, Gallus G, Mauri M, Bono A, De Carli A, et al. Prognostic significance of the growth rate of breast cancer: preliminary evaluation on the follow-up of 196 breast cancers. Tumori 1981;67:333-40.

120. Lundgren B. Observations on growth rate of breast carcinomas and its possible implications for lead time. Cancer 1977;40:1722-5.

121. Spratt JS. The relationship between the rates of growth of cancers and the intervals between screening examinations necessary for effective discovery. Cancer Prev Detect 1981;4:301-5.

122. Arnerlöv C, Emdin SO, Lundgren B, Roos G, Söderström J, Bjersing L, et al. Breast carcinoma growth rate described by mammographic doubling time and S-phase fraction. Correlations to clinical and histopathologic factors in a screened population. Cancer 1992;70:1928-34.

123. Weedon-Fekjaer H, Lindqvist BH, Vatten LJ, Aalen OO, Tretli S. Breast cancer tumor growth estimated through mammography screening data. Breast Cancer Res 2008;10:R41.

124. Brekelmans CT, van Gorp JM, Peeters PH, Collette HJ. Histopathology and growth rate of interval breast carcinoma. Characterization of different subgroups. Cancer 1996;78:1220-8.

125. Tilanus-Linthorst MM, Obdeijn IM, Hop WC, Causer PA, Leach MO, Warner E, et al. BRCA1 mutation and young age predict fast breast cancer growth in the Dutch, United Kingdom, and Canadian magnetic resonance imaging screening trials. Clin Cancer Res 2007;13:7357-62. 


\title{
Appendix 1
}

\section{Membership and individual area of expertise of the project Advisory Group}

\author{
Aberdeen working group \\ Charles Boachie (Statistician) \\ Cynthia Fraser (Information Specialist) \\ Fiona Gilbert (Roland Sutton Chair of Radiology/Chief Investigator) \\ Steve Heys (Professor of Surgical Oncology, Chair of Applied Medicine) \\ Graeme MacLennan (Senior Statistician) \\ Graham Mowatt (Senior Research Fellow - Lead Systematic Review Team) \\ Clare Robertson (Research Fellow - Systematic Reviewer) \\ Laura Ternent (Research Fellow - Health Economics) \\ Ruth Thomas (Senior Research Fellow - Project Manager) \\ Luke Vale (Senior Research Fellow - Health Economics)
}

\section{HTA grant co-applicants}

Mike Dixon (Consultant Breast Surgeon)

Sarah Pinder (Professor of Breast Pathology)

Robin Wilson (Consultant Radiologist)

\section{Expert panel}

\author{
Val Bain (Breast Care Nurse) \\ Peter Donnelly (Consultant Breast Surgeon) \\ Wilma Jack (Senior Clinical Research Fellow, Edinburgh data set) \\ Gill Kerr (Statistician, Edinburgh data set)
}


Gill Lawrence (Director, WCMIU)

Anthony Maxwell (Consultant Radiologist)

Jean McGregor (Patient Representative, Breast Cancer Care Support Volunteer)

Peter Murchie (GP, Clinical Senior Lecturer) 


\section{Appendix 2}

Questionnaire content: a survey of UK surveillance mammography practice after treatment for primary breast cancer 

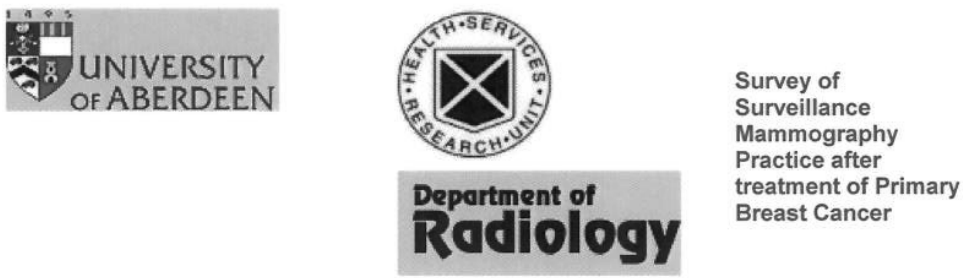

Clare Robertson

Research Fellow

Health Services Research Unit

University of Aberdeen 3rd Floor

Health Sciences Building

Aberdeen AB25 2ZD

Tel: 01224551100

Fax: 01224554580

Breast Cancer

c.robertson@abdn.ac.uk

This questionnaire is about your current and ideal surveillance mammography practice for women who have had breast conserving surgery or mastectomy for primary breast cancer. This survey should take no longer than 5-10 minutes to complete.

\section{Page 1}

Page 2

Page 3

Page 4

Section A : Background

1. What is your speciality

2. Please name your NHS Trust (please write in full instead of abbreviating)

3. How many women with newly diagnosed breast cancer does your unit manage annually (include both symptomatically and screen-detected)

4. How soon after completing surgery for primary breast cancer do you initiate surveillance mammography? (answer in months)

\section{Section B : Your Standard Surveillance Mammography Practice}

\section{For women who have had breast conserving surgery}

5. Please describe your standard surveillance mammography for women who have had breast conserving surgery

I arrange surveillance mammography

Annually
Next $\gg$ If other, please
describe

This project is funded by the NHS R\&D Programme Health Technology Assessment Programme (HTA Project 07/47/01). The views and opinions expressed therein are those of the authors and do not necessarily reflect those of the Department of Health. HSRU is funded by the Chief Scientist Office of the Scottish Government Health Directorates. The authors accept full responsibility for this survey.

NIHR Heatth Technology Assessment
programme funded project
$\begin{aligned} & \text { This questionnaire is about your current and ideal surveillance mammography practice for women who } \\ & \text { have had breast conserving surgery or mastectomy for primary breast cancer. This survey should take } \\ & \text { no longer than 5-10 minutes to complete. }\end{aligned}$




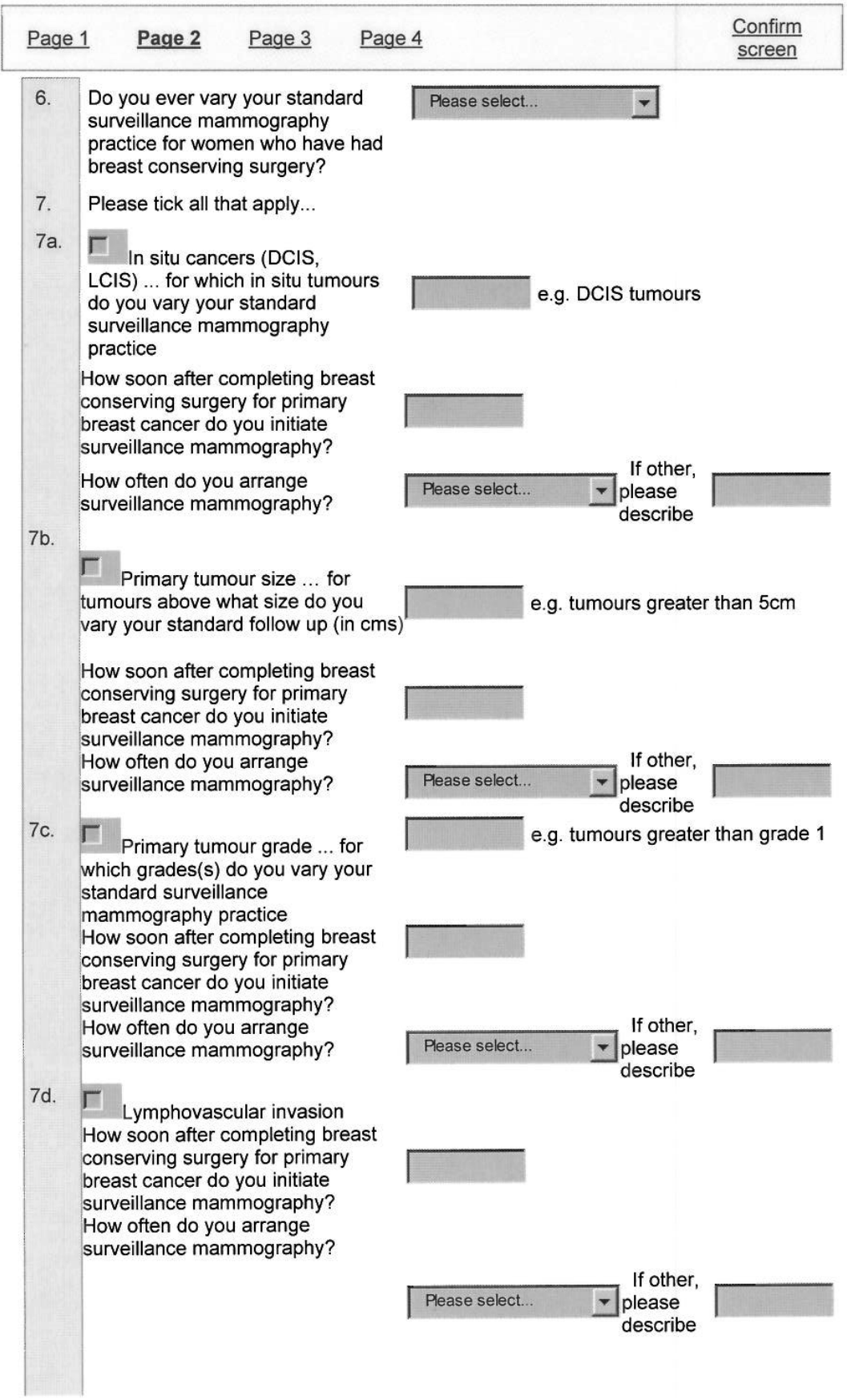


$7 e$

Age ... for which ages do you vary your standard surveillance mammography practice

How soon after completing breas

conserving surgery for primary

breast cancer do you initiate surveillance mammography?

How often do you arrange surveillance mammography?

$7 f$.

$\Gamma$ Absence of radiotherapy

How soon after completing breas

conserving surgery for primary

breast cancer do you initiate

surveillance mammography?

How often do you arrange

surveillance mammography?

$7 g$

I Combination of criteria above Please describe which criteria you combine

How soon after completing breast conserving surgery for primary breast cancer do you initiate surveillance mammography?

How often do you arrange surveillance mammography?

7h. $\Gamma$ Other ... Other criteria not listed above

How soon after completing breast conserving surgery for primary breast cancer do you initiate surveillance mammography?

How often do you arrange

surveillance mammography? e.g. under 30 years

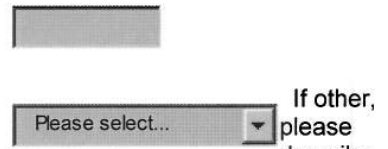
describe

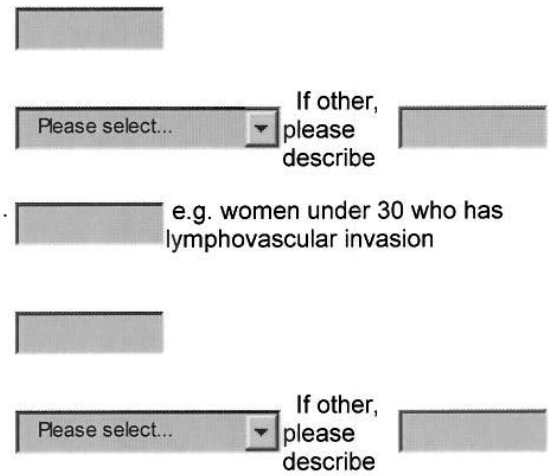




\begin{tabular}{|c|c|c|c|c|}
\hline Page 1 & Page 2 & Page 3 & Page 4 & $\frac{\text { Confirm }}{\text { screen }}$ \\
\hline
\end{tabular}

\section{For women who have had mastectomy}

8. Please describe your surveillance mammography practice for the contralateral breast for women who have had mastectomy

8a. I initiate surveillance mammography

$8 b$. I arrange surveillance mammography

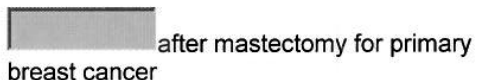
breast cancer

\begin{tabular}{|l|l} 
If other, \\
please \\
describe
\end{tabular}

9. Do you ever vary your standard surveillance mammography practice for the contralateral breast for women who have had mastectomy?

10. Please describe the critieria you use for varying your standard

surveillance mammography for the contralateral breast for women who have had mastectomy How soon after completing breast conserving surgery for primary breast cancer do you initiate surveillance mammography? surveillance mammography?

Please select...

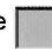

Pease select...

$\longrightarrow$

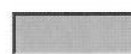

$$
\text { How often do you arrange }
$$

If othe
Please select.. $\quad$ please




Page 1 Page 2 Page 3 Page 4

Section C: Surveillance Mammography Scheduling and Discharge

11. Through which service is your surveillance mammography scheduled?

12. If eligible do you discharge women into the screening programme for surveillance mammography?

13. Do you discharge women from clinical follow up?

If yes, please indicate when

13b. Do you discharge women from surveillance mammography follow up?

If yes, please indicate when
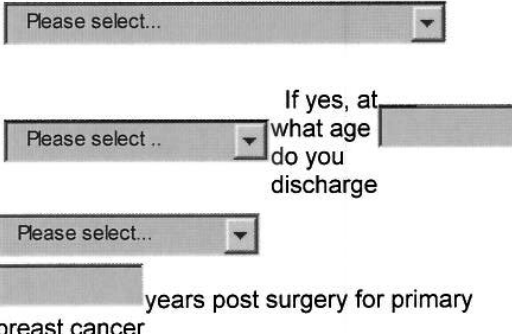

breast cancer

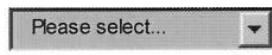

years post surgery for primary

breast cancer

14. Are there any exceptions to your normal surveillance mammography practice (e.g. patient participating in a clinical trial)?

If yes, can you specify what this would involve?

screen

Durveillance

mammography practice differ from

how you would ideally like to arrange

surveillance mammography?

16. Can you please indicate how often you would ideally like to arrange surveillance mammography?

16a. How often would you ideally arrange surveillance mammography for women who have had breast

.

conserving surgery?

16b. How often would you ideally arrange surveillance mammography for women who have had mastectomy?

Please select... $\begin{gathered}\text { If other, } \\ \text { please } \\ \text { describe }\end{gathered}$

If your current surveillance

mammography practice differs from your ideal surveillance

mammography practice what factor

18. influence this difference?

If other,
please
describe

Please use this space to provide any comments

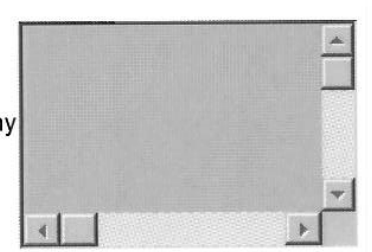


This project is funded by the NHS R\&D Programme Health Technology Assessment Programme (HTA Project 07/47/01).

The views and opinions expressed therein are those of the authors and do not necessarily reflect those of the Department of

Health.

HSRU is funded by the Chief Scientist Office of the Scottish Government Health Directorates. The authors accept full responsibility for this survey.

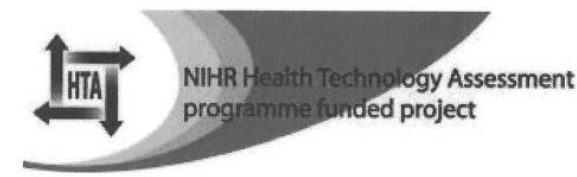





\title{
Appendix 3
}

\section{E-mail invitation to participate in a survey of UK surveillance mammography practice after treatment for primary breast cancer}

From: hsru.notification.service@abdn.ac.uk

Sent:

To:

Subject: Survey of UK Surveillance Mammography practice after treatment for primary breast cancer

\begin{abstract}
Survey of UK Surveillance Mammography practice after treatment for primary breast cancer
We are inviting you to complete an online questionnaire about your current surveillance mammography practice (please see web link below). We are surveying members of the Association of Breast Surgery at BASO and the Royal College of Radiologists Breast Group. The survey is part of a National Institute for Health Research, Health Technology Assessment programme commissioned study (HTA Project 07/47/01 - to determine the clinical effectiveness and cost-effectiveness of different surveillance mammography regimens after the treatment of primary breast cancer in the UK). The study is being conducted by a multidisciplinary research team led by Professor Fiona Gilbert (Roland Sutton Chair of Radiology) at the University of Aberdeen.
\end{abstract}

The questionnaire should take no longer than 5-10 mins to complete. Your valuable participation will contribute towards identifying the clinical and cost-effectiveness of differing surveillance mammography regimens, and help to identify future research priorities in this area. We would be very grateful if you would consider helping us with our important research.

The questionnaire is strictly confidential and anonymous. In order to ensure anonymity, please note that you will not be able to save your responses and return to the survey at a later stage. Please review your responses before clicking 'submit' to send your completed survey. You will not be able to return to your responses after submitting the survey.

Please click on the web link below to begin the questionnaire. Thank you very much for giving your time to help us with our research.

https://viis.abdn.ac.uk/HSRU/htamammography/default.aspx?uid=52b54a34-12d1-41f8-86d8b60786762426 
If you have any queries or comments about the questionnaire or the research study, please contact:

Clare Robertson

Research Fellow

Health Services Research Unit

University of Aberdeen

3rd Floor, Health Sciences Building

Aberdeen AB25 2ZD 


\section{Appendix 4}

\section{Reminder e-mail invitation to participate in a survey of UK surveillance mammography practice after treatment for primary breast cancer}

From: hsru.notification.service@abdn.ac.uk

Sent:

To:

Subject: REMINDER: Survey of UK Surveillance Mammography practice after treatment for primary breast cancer

REMINDER: Survey of UK Surveillance Mammography practice after treatment for primary breast cancer

You may have already received an e-mail inviting you to participate in this survey. If you have already completed and returned the questionnaire, please accept our thanks and delete this e-mail as no further involvement is required. If you have not completed the questionnaire please take the time to consider helping us with this important research.

We are inviting you to complete an online questionnaire about your current surveillance mammography practice (please see web link below). We are surveying members of the Association of Breast Surgery at BASO and the Royal College of Radiologists Breast Group. The survey is part of a National Institute for Health Research, Health Technology Assessment programme commissioned study (HTA Project 07/47/01 - to determine the clinical effectiveness and cost-effectiveness of different surveillance mammography regimens after the treatment of primary breast cancer in the UK). The study is being conducted by a multidisciplinary research team led by Professor Fiona Gilbert (Roland Sutton Chair of Radiology) at the University of Aberdeen.

The questionnaire should take no longer than 5-10 mins to complete. Your valuable participation will contribute towards identifying the clinical and cost-effectiveness of differing surveillance mammography regimens, and help to identify future research priorities in this area. We would be very grateful if you would consider helping us with our important research.

The questionnaire is strictly confidential and anonymous. In order to ensure anonymity, please note that you will not be able to save your responses and return to the survey at a later stage. Please review your responses before clicking 'submit' to send your completed survey. You will not be able to return to your responses after submitting the survey. 
Please click on the web link below to begin the questionnaire. Thank you very much for giving your time to help us with our research.

https://viis.abdn.ac.uk/HSRU/htamammography/default.aspx?uid=52b54a34-12d1-41f8-86d8b60786762426\&Reminder=true

If you have any queries or comments about the questionnaire or the research study, please contact:

Clare Robertson

Research Fellow

Health Services Research Unit

University of Aberdeen

3rd Floor, Health Sciences Building

Aberdeen AB25 2ZD 


\section{Appendix 5}

\section{Number and percentage of respondents completing questions in the survey of UK surveillance mammography practice after treatment for primary breast cancer}

\begin{tabular}{|c|c|c|c|}
\hline & Question & $n=183$ & $\begin{array}{l}\text { Percentage } \\
\text { of } n\end{array}$ \\
\hline 1 & What is your specialty? & 183 & 100 \\
\hline 2 & Please name your NHS trust & 182 & 99 \\
\hline 3 & $\begin{array}{l}\text { How many women with newly diagnosed breast cancer does your unit manage annually (include both } \\
\text { symptomatically and screen detected)? }\end{array}$ & 183 & 100 \\
\hline 4 & $\begin{array}{l}\text { How soon after completing surgery for primary breast cancer do you initiate surveillance mammography? } \\
\text { (answer in months) }\end{array}$ & 181 & 99 \\
\hline \multirow[t]{2}{*}{5} & Please describe your standard surveillance mammography for women who have had BCS: & & \\
\hline & I arrange surveillance mammography (give frequency) & 182 & 99 \\
\hline 6 & $\begin{array}{l}\text { Do you ever vary your standard surveillance mammography practice for women who have had BCS? } \\
\text { (filter question) }\end{array}$ & 180 & 98 \\
\hline 7 & If yes please tick all below that apply: & & \\
\hline \multirow[t]{4}{*}{$7 a$} & In situ cancers (DCIS, LCIS): & 14 & 8 \\
\hline & For which in situ tumours do you vary your standard surveillance mammography practice? & 11 & 6 \\
\hline & How soon after completing BCS for primary breast cancer do you initiate surveillance mammography? & 14 & 8 \\
\hline & How often do you arrange surveillance mammography? & 13 & 7 \\
\hline \multirow[t]{4}{*}{$7 \mathrm{~b}$} & Primary tumour size: & 5 & 3 \\
\hline & For tumours above what size do you vary your standard follow up (in cm) & 4 & 2 \\
\hline & How soon after completing BCS for primary breast cancer do you initiate surveillance mammography? & 4 & 2 \\
\hline & How often do you arrange surveillance mammography? & 4 & 2 \\
\hline \multirow[t]{4}{*}{ 7c } & Primary tumour grade: & 4 & 2 \\
\hline & For which grades(s) do you vary your standard surveillance mammography practice? & 4 & 2 \\
\hline & How soon after completing BCS for primary breast cancer do you initiate surveillance mammography? & 4 & 2 \\
\hline & How often do you arrange surveillance mammography? & 4 & 2 \\
\hline \multirow[t]{3}{*}{$7 \mathrm{~d}$} & Lymphovascular invasion: & 4 & 2 \\
\hline & How soon after completing BCS for primary breast cancer do you initiate surveillance mammography? & 4 & 2 \\
\hline & How often do you arrange surveillance mammography? & 3 & 2 \\
\hline \multirow[t]{4}{*}{$7 e$} & Age: & 9 & 5 \\
\hline & For which ages do you vary your standard surveillance mammography practice? & 9 & 5 \\
\hline & How soon after completing BCS for primary breast cancer do you initiate surveillance mammography? & 9 & 5 \\
\hline & How often do you arrange surveillance mammography? & 9 & 5 \\
\hline \multirow[t]{3}{*}{$7 f$} & Absence of radiotherapy: & 3 & 2 \\
\hline & How soon after completing BCS for primary breast cancer do you initiate surveillance mammography? & 3 & 2 \\
\hline & How often do you arrange surveillance mammography? & 2 & 1 \\
\hline
\end{tabular}




\begin{tabular}{|c|c|c|c|}
\hline & Question & $n=183$ & $\begin{array}{l}\text { Percentage } \\
\text { of } n\end{array}$ \\
\hline \multirow[t]{4}{*}{$7 \mathrm{~g}$} & Combination of criteria above: & 2 & 1 \\
\hline & Please describe which criteria you combine & 1 & 1 \\
\hline & How soon after completing BCS for primary breast cancer do you initiate surveillance mammography? & 1 & 1 \\
\hline & How often do you arrange surveillance mammography? & 1 & 1 \\
\hline \multirow[t]{4}{*}{$7 \mathrm{~h}$} & Other: & 10 & 5 \\
\hline & Other criteria not listed above & 8 & 4 \\
\hline & How soon after completing BCS for primary breast cancer do you initiate surveillance mammography? & 6 & 3 \\
\hline & How often do you arrange surveillance mammography? & 7 & 4 \\
\hline 8 & $\begin{array}{l}\text { Please describe your surveillance mammography practice for the contralateral breast for women who } \\
\text { have had mastectomy }\end{array}$ & & \\
\hline $8 a$ & I initiate surveillance mammography $x$ months after mastectomy for primary breast cancer & 159 & 87 \\
\hline $8 b$ & I arrange surveillance mammography (give frequency) & 181 & 99 \\
\hline 9 & $\begin{array}{l}\text { Do you ever vary your standard surveillance mammography practice for the contralateral breast for } \\
\text { women who have had mastectomy? (filter question) }\end{array}$ & 180 & 98 \\
\hline \multirow[t]{3}{*}{10} & $\begin{array}{l}\text { Please describe the criteria you use for varying your standard surveillance mammography for the } \\
\text { contralateral breast for women who have had mastectomy }\end{array}$ & 20 & 11 \\
\hline & $\begin{array}{l}\text { How soon after completing mastectomy surgery for primary breast cancer do you initiate surveillance } \\
\text { mammography? }\end{array}$ & 23 & 13 \\
\hline & How often do you arrange surveillance mammography? & 24 & 13 \\
\hline 11 & Through which service is your surveillance mammography scheduled? & 182 & 99 \\
\hline \multirow[t]{2}{*}{12} & If eligible do you discharge women into the screening programme for surveillance mammography? & 179 & 98 \\
\hline & If yes, at what age? & 39 & 21 \\
\hline \multirow[t]{2}{*}{13} & Do you discharge women from clinical follow up? & 180 & 98 \\
\hline & If yes, please indicate when & 130 & 88 \\
\hline \multirow[t]{2}{*}{$13 b$} & Do you discharge women from surveillance mammography follow-up? & 182 & 99 \\
\hline & If yes, please indicate when & 121 & 89 \\
\hline \multirow[t]{2}{*}{14} & $\begin{array}{l}\text { Are there any exceptions to your normal surveillance mammography practice (e.g. patient participating in } \\
\text { a clinical trial)? }\end{array}$ & 178 & 97 \\
\hline & If yes, can you specify what this would involve? & 38 & 21 \\
\hline 15 & $\begin{array}{l}\text { Does your current surveillance mammography practice differ from how you would ideally like to arrange } \\
\text { surveillance mammography? }\end{array}$ & 180 & 98 \\
\hline 16 & Can you please indicate how often you would ideally like to arrange surveillance mammography? & & \\
\hline $16 \mathrm{a}$ & How often would you ideally arrange surveillance mammography for women who have had BCS? & 106 & 58 \\
\hline $16 \mathrm{~b}$ & How often would you ideally arrange surveillance mammography for women who have had mastectomy? & 106 & 58 \\
\hline 17 & $\begin{array}{l}\text { If your current surveillance mammography practice differs from your ideal surveillance mammography } \\
\text { practice what factors influence this difference? }\end{array}$ & 53 & 29 \\
\hline 18 & Please use this space to provide any comments & 63 & 34 \\
\hline
\end{tabular}

DCIS, ductal carcinoma in situ; LCIS, lobular carcinoma in situ. 


\title{
Appendix 6
}

\section{List of NHS trusts of respondents to the survey of UK surveillance mammography practice after the treatment for primary breast cancer}

\author{
Abertawe Bro Morgannwg University NHS Trust \\ Barking, Havering \& Redbridge NHS Trust \\ Barts and the London NHS Trust \\ Basingstoke and North Hampshire NHS Foundation Trust \\ Royal United Hospital Bath NHS Trust \\ Bedford Hospital NHS Trust \\ Belfast Health \& Social Care Trust \\ Blackpool Fylde and Wyre NHS Foundation Trust \\ Bradford Teaching Hospitals NHS Foundation Trust \\ Brighton and Sussex University Hospitals NHS Trust \\ Buckinghamshire Hospitals NHS Trust \\ Cambridge University Hospitals NHS Foundation Trust \\ Cardiff and Vale NHS Trust \\ City Hospitals Sunderland NHS Foundation Trust \\ Co. Durham and Darlington NHS Foundation Trust \\ Countess of Chester NHS Foundation Trust \\ Derby Hospitals NHS Foundation Trust \\ Doncaster and Bassetlaw Hospitals NHS Foundation Trust \\ East and North Hertfordshire NHS Trust \\ East Kent Hospitals University NHS Foundation Trust
}


Frimley Park Hospital NHS Foundation Trust

George Eliot Hospital NHS Trust

Gloucestershire Hospitals NHS Foundation Trust

Great Western Hospitals NHS Foundation Trust

Gwent Healthcare NHS Trust

Harrogate and District NHS Foundation Trust

Heart of England NHS Foundation Trust

Heatherwood and Wexham Park Hospitals NHS Foundation Trust

Hereford Hospitals NHS Trust

Hinchingbrooke Health Care NHS Trust

Homerton University Hospital NHS Foundation Trust

Hull and East Yorkshire Hospitals NHS Trust

Ipswich Hospital NHS Trust

James Paget University Hospitals NHS Foundation Trust

Kingston Hospital NHS Trust

Leeds Teaching Hospitals NHS Trust

Maidstone and Tunbridge Wells NHS Trust

Mayday Healthcare NHS Trust

Medway NHS Foundation Trust

Mid Cheshire Hospitals NHS Foundation Trust

Mid Yorkshire Hospitals NHS Trust

NHS Ayrshire and Arran

NHS Dumfries and Galloway

NHS Grampian

NHS Greater Glasgow and Clyde

NHS Highland 
NHS Lanarkshire

NHS Lothian

NHS Tayside

Noble's Hospital, Isle of Man

Norfolk and Norwich University Hospitals NHS Foundation Trust

North Bristol NHS Trust

North Cumbria University Hospitals NHS Trust

North Wales NHS Trust

North West London Hospitals NHS Trust

North West Wales NHS Trust

Northampton General Hospitals NHS Trust

Northumbria Healthcare NHS Foundation Trust

Nottingham University Hospitals NHS Trust

Oxford Radcliffe Hospitals NHS Trust

Pennine Acute Hospital NHS Trust

Peterborough and Stamford Hospitals Foundation Trust

Plymouth Hospitals NHS Trust

Poole Hospital NHS Trust

Portsmouth Hospitals NHS Trust

Queen Elizabeth Healthcare Trust

Royal Bolton Hospitals NHS Foundation Trust

Royal Cornwall Hospitals NHS Trust

Royal Liverpool and Broadgreen University Hospitals NHS Trust

Royal Surrey County Hospital NHS Trust

Salford Royal NHS Foundation Trust

Sandwell and West Birmingham Hospitals NHS Trust 
Sheffield Teaching Hospitals NHS Foundation Trust

Shrewsbury and Telford Hospital NHS Trust

South Devon and Exeter NHS Foundation Trust

South Devon Healthcare NHS Foundation Trust

South London Healthcare NHS Trust, London

South Warwickshire NHS Trust

Southampton University Hospitals NHS Trust

Southern Health and Social Care Trust (Northern Ireland)

Southport and Ormskirk NHS Hospital Trust

St Georges Healthcare NHS Trust

St Helens \& Knowsley Hospitals NHS Foundation Trust

Taunton and Somerset NHS Foundation Trust

The Dudley Group of Hospitals NHS Foundation Trust

The Hillingdon Hospital NHS Trust

The Newcastle upon Tyne Hospitals NHS Foundation Trust

The Princess Alexandra Hospital NHS Trust

The Royal Marsden NHS Foundation Trust

The Royal Wolverhampton Hospitals NHS Trust

United Lincolnshire Hospitals NHS Trust

University College London Hospitals NHS Foundation Trust

University Hospital Birmingham NHS Foundation Trust

University Hospital of South Manchester NHS Foundation Trust

University Hospitals Coventry and Warwickshire NHS Trust

University Hospitals of Leicester NHS Trust

University Hospitals of Morecambe Bay NHS Foundation Trust

Walsall Hospitals NHS Trust 
Warrington and Halton Hospitals NHS Foundation Trust

West Hertfordshire Hospitals NHS Trust

West Middlesex University Hospital NHS Trust

Winchester and Eastleigh Healthcare NHS Trust

Worcestershire Acute Hospitals NHS Trust

Wrightington, Wigan and Leigh NHS Foundation Trust

York Hospitals NHS Foundation Trust 



\section{Appendix 7}

\section{Description of mammography surveillance regimens for women following breast- conserving surgery}

\begin{tabular}{|c|c|c|c|c|c|c|}
\hline \multirow[b]{4}{*}{ Regimen no. } & \multicolumn{6}{|c|}{ Surveillance regimens for women following BCS } \\
\hline & \multirow[b]{3}{*}{$\begin{array}{l}\text { Initiation: } \\
\text { post surgery } \\
\text { (months) }\end{array}$} & \multirow{3}{*}{$\begin{array}{l}\text { Frequency } \\
\text { (annual, } \\
\text { 18-monthly, } \\
\text { 2-/3- yearly, } \\
\text { annual for } \\
5 \text { years then } \\
\text { biennial, other) }\end{array}$} & \multicolumn{4}{|l|}{ Duration } \\
\hline & & & \multicolumn{2}{|c|}{$\begin{array}{l}\text { Discharge from surveillance } \\
\text { mammography follow-up }\end{array}$} & \multicolumn{2}{|c|}{ Discharge from clinical follow-up } \\
\hline & & & $\begin{array}{l}\text { Years post } \\
\text { surgery }\end{array}$ & $\begin{array}{l}\text { No. of } \\
\text { respondents: } \\
n=165\end{array}$ & $\begin{array}{l}\text { Years post } \\
\text { surgery }\end{array}$ & $\begin{array}{l}\text { No. of } \\
\text { respondents: } \\
n=154\end{array}$ \\
\hline 1 & 6 & Annual & 10 & 3 & 3 & 1 \\
\hline 2 & 6 & Annual & 10 & & 5 & 1 \\
\hline 3 & 6 & Annual & 10 & & 10 & 1 \\
\hline 4 & 6 & Other & 5 & 1 & 2 & 1 \\
\hline 5 & 6 & Other & 10 & 2 & No & 1 \\
\hline 6 & 6 & Other & 10 & & 10 & 1 \\
\hline 7 & 8 & Annual & 10 & 1 & 5 & 1 \\
\hline 8 & 9 & Annual & 5 & 1 & 5 & 1 \\
\hline 9 & 11 & Annual & No & 1 & 5 & 1 \\
\hline 10 & 11 & Annual & 10 & 2 & 10 & 2 \\
\hline 11 & 12 & Annual & No & 32 & No & 19 \\
\hline 12 & 12 & Annual & No & & 3 & 1 \\
\hline 13 & 12 & Annual & No & & 5 & 7 \\
\hline 14 & 12 & Annual & No & & 10 & 4 \\
\hline 15 & 12 & Annual & 3 & 1 & 3 & 1 \\
\hline 16 & 12 & Annual & 5 & 31 & No & 4 \\
\hline 17 & 12 & Annual & 5 & & 2 & 1 \\
\hline 18 & 12 & Annual & 5 & & 5 & 22 \\
\hline 19 & 12 & Annual & 9 & 3 & 5 & 1 \\
\hline 20 & 12 & Annual & 10 & 44 & No & 2 \\
\hline 21 & 12 & Annual & 10 & & 3 & 2 \\
\hline 22 & 12 & Annual & 10 & & 5 & 20 \\
\hline 23 & 12 & Annual & 10 & & 10 & 18 \\
\hline 24 & 12 & Every 18 months & 10 & 6 & No & 1 \\
\hline 25 & 12 & Every 18 months & 10 & & 5 & 4 \\
\hline 26 & 12 & Every 18 months & 10 & & 10 & 1 \\
\hline 27 & 12 & Every 2 years & No & 3 & 5 & 2 \\
\hline 28 & 12 & Every 2 years & 5 & 3 & 5 & 3 \\
\hline 29 & 12 & Every 2 years & 6 & 1 & No & 1 \\
\hline 30 & 12 & Every 2 years & 9 & 2 & 1 & 2 \\
\hline 31 & 12 & Every 2 years & 10 & 4 & No & 1 \\
\hline
\end{tabular}




\begin{tabular}{|c|c|c|c|c|c|c|}
\hline \multirow[b]{4}{*}{ Regimen no. } & \multicolumn{6}{|c|}{ Surveillance regimens for women following BCS } \\
\hline & \multirow[b]{3}{*}{$\begin{array}{l}\text { Initiation: } \\
\text { post surgery } \\
\text { (months) }\end{array}$} & \multirow{3}{*}{$\begin{array}{l}\text { Frequency } \\
\text { (annual, } \\
\text { 18-monthly, } \\
2-/ 3-\text { yearly, } \\
\text { annual for } \\
5 \text { years then } \\
\text { biennial, other) }\end{array}$} & \multicolumn{4}{|l|}{ Duration } \\
\hline & & & \multicolumn{2}{|c|}{$\begin{array}{l}\text { Discharge from surveillance } \\
\text { mammography follow-up }\end{array}$} & \multicolumn{2}{|c|}{ Discharge from clinical follow-up } \\
\hline & & & $\begin{array}{l}\text { Years post } \\
\text { surgery }\end{array}$ & $\begin{array}{l}\text { No. of } \\
\text { respondents: } \\
n=165\end{array}$ & $\begin{array}{l}\text { Years post } \\
\text { surgery }\end{array}$ & $\begin{array}{l}\text { No. of } \\
\text { respondents: } \\
n=154\end{array}$ \\
\hline 32 & 12 & Every 2 years & 10 & & 1 & 1 \\
\hline 33 & 12 & Every 2 years & 10 & & 5 & 2 \\
\hline 34 & 12 & $\begin{array}{l}\text { Annual to } 5 \text { years } \\
\text { then biennial }\end{array}$ & No & 2 & 5 & 2 \\
\hline 35 & 12 & $\begin{array}{l}\text { Annual to } 5 \text { years } \\
\text { then biennial }\end{array}$ & 5 & 4 & No & 1 \\
\hline 36 & 12 & $\begin{array}{l}\text { Annual to } 5 \text { years } \\
\text { then biennial }\end{array}$ & 5 & & 3 & 1 \\
\hline 37 & 12 & $\begin{array}{l}\text { Annual to } 5 \text { years } \\
\text { then biennial }\end{array}$ & 5 & & 5 & 2 \\
\hline 38 & 12 & $\begin{array}{l}\text { Annual to } 5 \text { years } \\
\text { then biennial }\end{array}$ & 10 & 1 & 5 & 1 \\
\hline 39 & 12 & $\begin{array}{l}\text { Annual to } 5 \text { years } \\
\text { then biennial }\end{array}$ & 11 & 1 & 5 & 1 \\
\hline 40 & 12 & Other & No & 2 & 3 & 1 \\
\hline 41 & & Other & No & & 5 & 1 \\
\hline 42 & 12 & Other & 5 & 1 & 5 & 1 \\
\hline 43 & 12 & Other & 10 & 2 & 3 & 1 \\
\hline 44 & 12 & Other & 10 & & 5 & 1 \\
\hline 45 & 18 & Annual & 9 & 1 & 5 & 1 \\
\hline 46 & 18 & Every 18 months & No & 2 & No & 1 \\
\hline 47 & 18 & Every 18 months & & & 5 & 1 \\
\hline 48 & 18 & Every 18 months & 5 & 1 & 5 & 1 \\
\hline 49 & 18 & Every 18 months & 6 & 2 & No & 1 \\
\hline 50 & 18 & Other & No & 1 & 5 & 1 \\
\hline 51 & 24 & Every 2 years & No & 1 & 6 & 1 \\
\hline 52 & 24 & Every 2 years & 5 & 1 & 5 & 1 \\
\hline 53 & 24 & Every 2 years & 8 & 1 & 8 & 1 \\
\hline 54 & 24 & Every 2 years & 10 & 1 & 10 & 1 \\
\hline
\end{tabular}




\section{Appendix 8}

\section{Description of mammography surveillance regimens for women following mastectomy}

\begin{tabular}{|c|c|c|c|c|c|c|}
\hline \multirow[b]{4}{*}{ Regimen no. } & \multicolumn{6}{|c|}{ Surveillance regimens for women following mastectomy } \\
\hline & \multirow[b]{3}{*}{$\begin{array}{l}\text { Initiation: post } \\
\text { surgery (months) }\end{array}$} & \multirow{3}{*}{$\begin{array}{l}\text { Frequency (annual, } \\
\text { 18-monthly, 2-/ } \\
\text { 3-yearly, annual } \\
\text { for } 5 \text { years then } \\
\text { biennial, other) }\end{array}$} & \multicolumn{4}{|l|}{ Duration } \\
\hline & & & \multicolumn{2}{|c|}{$\begin{array}{l}\text { Discharge from surveillance } \\
\text { mammography follow-up }\end{array}$} & \multicolumn{2}{|c|}{ Discharge from clinical follow-up } \\
\hline & & & $\begin{array}{l}\text { Years post } \\
\text { surgery }\end{array}$ & $\begin{array}{l}\text { No. of } \\
\text { respondents: } \\
n=146\end{array}$ & $\begin{array}{l}\text { Years post } \\
\text { surgery }\end{array}$ & $\begin{array}{l}\text { No. of } \\
\text { respondents: } \\
n=136\end{array}$ \\
\hline 1 & 6 & Annual & 10 & 2 & 3 & 1 \\
\hline 2 & 6 & Annual & 10 & & 5 & 1 \\
\hline 3 & 11 & Annual & 10 & 1 & 10 & 1 \\
\hline 4 & 12 & Annual & 10 & & 3 & 1 \\
\hline 5 & 12 & Annual & No & 19 & No & 10 \\
\hline 6 & 12 & Annual & No & & 5 & 5 \\
\hline 7 & 12 & Annual & No & & 10 & 4 \\
\hline 8 & 12 & Annual & 3 & 1 & 3 & 1 \\
\hline 9 & 12 & Annual & 5 & 20 & No & 4 \\
\hline 10 & 12 & Annual & 5 & & 2 & 1 \\
\hline 11 & 12 & Annual & 5 & & 5 & 13 \\
\hline 12 & 12 & Annual & 9 & 2 & & \\
\hline 13 & 12 & Annual & 10 & 30 & No & 2 \\
\hline 14 & 12 & Annual & 10 & & 3 & 1 \\
\hline 15 & 12 & Annual & 10 & & 5 & 11 \\
\hline 16 & 12 & Annual & 10 & & 10 & 15 \\
\hline 17 & 12 & Every 18 months & No & 1 & No & 1 \\
\hline 18 & 12 & Every 18 months & 5 & 1 & 5 & 1 \\
\hline 19 & 12 & Every 18 months & 10 & 8 & No & 1 \\
\hline 20 & 12 & Every 18 months & 10 & & 3 & 1 \\
\hline 21 & 12 & Every 18 months & 10 & & 5 & 5 \\
\hline 22 & 12 & Every 18 months & 10 & & 10 & 1 \\
\hline 23 & 12 & Every 2 years & No & 4 & 3 & 1 \\
\hline 24 & 12 & Every 2 years & No & & 5 & 3 \\
\hline 25 & 12 & Every 2 years & 5 & 8 & No & 1 \\
\hline 26 & 12 & Every 2 years & 5 & & 5 & 7 \\
\hline 27 & 12 & Every 2 years & 9 & 2 & 1 & 2 \\
\hline 28 & 12 & Every 2 years & 10 & 9 & No & 2 \\
\hline 29 & 12 & Every 2 years & 10 & & 1 & 1 \\
\hline 30 & 12 & Every 2 years & 10 & & 5 & 4 \\
\hline 31 & 12 & Every 2 years & 10 & & 10 & 2 \\
\hline
\end{tabular}


Surveillance regimens for women following mastectomy

\begin{tabular}{|c|c|c|c|c|c|c|}
\hline \multirow[b]{3}{*}{ Regimen no. } & \multirow[b]{3}{*}{$\begin{array}{l}\text { Initiation: post } \\
\text { surgery (months) }\end{array}$} & \multirow{3}{*}{$\begin{array}{l}\text { Frequency (annual, } \\
\text { 18-monthly, 2-/ } \\
\text { 3-yearly, annual } \\
\text { for } 5 \text { years then } \\
\text { biennial, other) }\end{array}$} & \multicolumn{4}{|l|}{ Duration } \\
\hline & & & \multicolumn{2}{|c|}{$\begin{array}{l}\text { Discharge from surveillance } \\
\text { mammography follow-up }\end{array}$} & \multicolumn{2}{|c|}{ Discharge from clinical follow-up } \\
\hline & & & $\begin{array}{l}\text { Years post } \\
\text { surgery }\end{array}$ & $\begin{array}{l}\text { No. of } \\
\text { respondents: } \\
n=146\end{array}$ & $\begin{array}{l}\text { Years post } \\
\text { surgery }\end{array}$ & $\begin{array}{l}\text { No. of } \\
\text { respondents: } \\
n=136\end{array}$ \\
\hline 32 & 12 & Every 3 years & 5 & 1 & 5 & 1 \\
\hline 33 & 12 & Every 3 years & 6 & 1 & No & 1 \\
\hline 34 & 12 & $\begin{array}{l}\text { Annual to } 5 \text { years } \\
\text { then biennial }\end{array}$ & 5 & 2 & 3 & 1 \\
\hline 35 & 12 & $\begin{array}{l}\text { Annual to } 5 \text { years } \\
\text { then biennial }\end{array}$ & 5 & & 5 & 1 \\
\hline 36 & 12 & $\begin{array}{l}\text { Annual to } 5 \text { years } \\
\text { then biennial }\end{array}$ & 10 & 1 & 5 & 1 \\
\hline 37 & 12 & $\begin{array}{l}\text { Annual to } 5 \text { years } \\
\text { then biennial }\end{array}$ & 11 & 1 & 5 & 1 \\
\hline 38 & 12 & Other & No & 2 & 3 & 1 \\
\hline 39 & 12 & Other & No & & 5 & 1 \\
\hline 40 & 12 & Other & 5 & 1 & 5 & 1 \\
\hline 41 & 18 & Annual & 9 & 1 & 5 & 1 \\
\hline 42 & 18 & Every 18 months & No & 1 & No & 1 \\
\hline 43 & 18 & Every 18 months & 6 & 2 & No & 1 \\
\hline 44 & 18 & Every 18 months & 10 & 2 & 5 & 2 \\
\hline 45 & 18 & Every 2 years & No & 1 & 5 & 1 \\
\hline 46 & 18 & Other & No & 1 & 5 & 1 \\
\hline 47 & 24 & Every 2 years & No & 6 & No & 4 \\
\hline 48 & 24 & Every 2 years & No & & 6 & 1 \\
\hline 49 & 24 & Every 2 years & 5 & 5 & 2 & 1 \\
\hline 50 & 24 & Every 2 years & 5 & & 5 & 3 \\
\hline 51 & 24 & Every 2 years & 8 & 1 & 8 & 1 \\
\hline 52 & 24 & Every 2 years & 10 & 7 & 3 & 1 \\
\hline 53 & 24 & Every 2 years & 10 & & 5 & 3 \\
\hline 54 & 24 & Every 2 years & 10 & & 10 & 2 \\
\hline 55 & 24 & Every 3 years & No & 1 & & \\
\hline 56 & 24 & Every 3 years & 10 & 1 & 10 & 1 \\
\hline
\end{tabular}




\section{Appendix 9}

\section{List of questions used to help define alternative care pathways}

$\mathrm{F}$

or mammographic surveillance:

- For which women is the issue of mammographic surveillance relevant?

- What mammography surveillance should be used?

- Does it vary between women and if so why would it vary?

- How often is it performed?

- Where does it take place?

For clinical follow-up:

- What clinical follow-up is used?

- Does it vary between women and if so why?

- Where does it take place?

- How often?

For unstructured primary care follow-up:

- How might a diagnosis be made?

- At what point would these women enter the care pathway described in Figures 2-4 (see Chapter 2)?

More specifically, what factors might influence the choices made about mammographic surveillance and clinical follow-up?

Other factors to consider:
- age
- risk factors
- type of primary disease
- type of treatment. 



\section{Appendix 10}

\section{Search strategies: systematic review of clinical effectiveness of surveillance mammography}

MEDLINE (1990 - May, week 4, 2009), EMBASE (1990-2009, week 22), MEDLINE In-Process (1 June 2009)

Ovid multifile search: http://shibboleth.ovid.com/

1. exp ${ }^{\star}$ breast neoplasms/

2. breast.ti.

3. or $/ 1-2$

4. neoplasm recurrence, local/

5. neoplasms, second primary/

6. (recur\$ or second or secondary or contralateral or ipsilateral or ibtr or mcbc).tw.

7. or $/ 4-6$

8. exp mammography/

9. (mammograph\$ or mammogram\$).tw.

10. physical examination/

11. breast self-examination/

12. ((physical or clinical or self) adj1 (exam? or examin\$)).tw.

13. surveillance.hw,tw.

14. follow up.ti.

15. (routine adj3 (visit or follow up)).tw.

16. or $/ 8-15$

17. exp clinical trial/

18. randomized controlled trial.pt.

19. controlled clinical trial.pt.

20. randomization/use emez

21. randomi?ed.ab.

22. randomly.ab.

23. trial.ab.

24. groups.ab.

25. comparative study/use mesz

26. follow-up studies/use mesz

27. time factors/use mesz

28. Treatment outcome/use emez

29. major clinical study/use emez

30. controlled study/use emez

31. clinical trial/use emez

32. (chang $\$$ or evaluat $\$$ or reviewed or baseline).tw.

33. (prospective\$ or retrospective\$).tw.

34. (cohort\$ or case series).tw.

35. (compare $\$$ or compara $\$$ ).tw.

36. or $/ 17-35$

37. human/

38. 36 and 37 

39. 3 and 7 and 16 and 38
40. limit 39 to $\mathrm{yr}=“ 1990-2009$ "
41. remove duplicates from 40

\section{Science Citation Index (1990 - 3 June 2009), BIOSIS (1990 - 4 June 2009)}

ISI Web of Knowledge: http://wok.mimas.ac.uk/

\# 1 TS=(breast neoplasm ${ }^{\star}$ AND (recur ${ }^{\star}$ or secondary or contralateral or ipsilateral or ibtr or mcbc)) AND Document Type $=($ Article $)$

\# 2 TS=(breast cancer ${ }^{\star}$ AND (recur ${ }^{\star}$ or secondary or contralateral or ipsilateral or ibtr or mcbc))

AND Document Type $=($ Article $)$

\# 3 TS=(breast carcin* AND (recur* or secondary or contralateral or ipsilateral or ibtr or mcbc))

AND Document Type $=($ Article $)$

\# 4 TS=(breast tumour ${ }^{\star}$ AND (recur ${ }^{\star}$ or secondary or contralateral or ipsilateral or ibtr or mcbc) $)$ AND Document Type $=($ Article $)$

\# 5 TS=(breast tumor ${ }^{\star}$ AND (recur ${ }^{\star}$ or secondary or contralateral or ipsilateral or ibtr or mcbc)

AND Document Type $=($ Article $)$

\# 6 \#1 or \#2 or \#3 or \#4 or \#5 AND Document Type $=($ Article $)$

$\# 7$ \#6 and TS=mammogram ${ }^{*}$ AND Document Type $=($ Article $)$

\# 8 \#6 and TS=mammograph ${ }^{*}$ AND Document Type $=($ Article $)$

\# 9 \#6 and TS=surveillance AND Document Type $=($ Article $)$

\# 10 \#6 and TS=routine AND Document Type $=($ Article $)$

\# $11 \mathrm{TS}=$ "physical exam*" AND Document Type $=($ Article $)$

\# 12 \#6 AND \#11 AND Document Type=(Article)

\# 13 TS="self exam"” AND Document Type $=($ Article $)$

\# 14 \#6 and \#13 AND Document Type $=($ Article $)$

\# $15 \mathrm{TS}=$ "clinical exam ${ }^{\star}$ AND Document Type $=($ Article $)$

\# 16 \#6 and \#15 AND Document Type $=($ Article $)$

\# 17 TI="follow up" AND Document Type $=($ Article $)$

\# 18 \# 6 and \#17 AND Document Type $=($ Article $)$

\# 19 \#18 OR \#16 OR \#14 OR \#12 OR \#10 OR \#9 OR \#8 OR \#7

\# $20 \mathrm{TI}=$ breast AND Document Type $=($ Article $)$

\# 21 \#19 and \#20 AND Document Type=(Article)

\section{Cochrane Library Issue 2, 2009 (Cochrane Central Registry of Controlled}

Trials and Cochrane Database of Systematic Reviews)

www3.interscience.wiley.com/

\#1 MeSH descriptor Breast Neoplasms explode all trees

$\# 2$ (breast):ti

\#3 (\#1 OR \#2)

\#4 MeSH descriptor Neoplasm Recurrence, Local explode all trees

\#5 MeSH descriptor Neoplasms, Second Primary explode all trees

\#6 (recur* or second or secondary):ti,ab,kw or (contralateral or ipsilateral):ti,ab,kw or (ibtr or $\mathrm{mcbc}): \mathrm{ti}, \mathrm{ab}, \mathrm{kw}$

\#7 (\#4 OR \#5 OR \#6)

\#8 MeSH descriptor Mammography explode all trees

\#9 (mammograph ${ }^{\star}$ or mammogram $\left.{ }^{\star}\right): \mathrm{ti}, \mathrm{ab}, \mathrm{kw}$

\#10 MeSH descriptor Physical Examination, this term only

\#11 MeSH descriptor Breast Self-Examination, this term only

\#12 (follow up):ti

\#13 (routine near/3 visit*):ti,ab,kw or (routine near/3 follow up):ti,ab,kw 
\#14 (physical near/1 exam):ti,ab,kw or (clinical near/1 exam):ti,ab,kw or (self near/1 exam $\left.{ }^{\star}\right): \mathrm{ti}, \mathrm{ab}, \mathrm{kw}$ \#15 (\#8 OR \#9 OR \#10 OR \#11 OR \#12 OR \#13 OR \#14)

\#16 (\#3 AND \#7 AND \#15)

\section{CANCERLIT (3 June 2009)}

The US National Library of Medicine: www.ncbi.nlm.nih.gov

\#1 Search (breast neoplasms[majr] AND human[mh] AND english[la]) OR dcis[ti] OR lcis[ti] OR ((breast[ti] OR breasts[ti] OR mammary[ti] OR nipple[ti] OR nipples[ti]) AND ((cancer ${ }^{\star}[\mathrm{ti}]$ OR carcinoma ${ }^{\star}[\mathrm{ti}]$ OR adenocarcinoma ${ }^{*}[\mathrm{ti}]$ OR malignan ${ }^{*}[\mathrm{ti}]$ OR tumor ${ }^{*}[\mathrm{ti}]$ OR tumour ${ }^{*}[\mathrm{ti}]$ OR neoplasm*[ti]) OR in situ[ti]))

\#2 Search recur ${ }^{\star}$ or secondary or contralateral or ipsilateral or ibtr or mcbc Limits: Cancer \#3 Search \#1 and \#2 Limits: Cancer \#4 Search (("mammogram* “[Title]) OR ("examin*”[Title])) \#5 Search ((“surveillance"[Title]) OR ("follow up"[Title]) OR ("routine visit*” [Title])) \#6 \#4 OR \#5 \#7 \#3 AND \#6

\section{The Health Technology Assessment/Database of Abstracts of Reviews and} Effects/NHS Economic Evaluation databases, June 2009

Centre for Reviews and Dissemination: http://nhscrd.york.ac.uk/ welcome.htm

\# 1 MeSH Breast Neoplasms EXPLODE 12

\# 2 MeSH Mammography EXPLODE 1

\# 3 mammography OR mammogram

\# 4 MeSH Population Surveillance EXPLODE 1234

\# 5 surveillance OR monitor*

\# 6 \#2 or \#3 or \#4 or \#5

$\# 7 \# 1$ and \#6

\# 8 MeSH Postoperative Care EXPLODE 123

\# 9 MeSH Follow-Up Studies EXPLODE 123

\# $10 \mathrm{MeSH}$ Neoplasm Recurrence, Local EXPLODE 12

\# $11 \mathrm{MeSH}$ Recurrence EXPLODE 1

\# 12 recurrence OR secondary OR ipsilateral OR contralateral

\# 13 \#8 OR \#9 OR \#10 OR \#11 OR \#12

\# 14 \#7 AND \#13

\# 15 "follow-up strategies"

\# 16 follow AND up

\# 17 "follow up"

\#18 \#15 OR \#16 OR \#17

\# $19 \# 13$ or \#18

\# 20 \#7 AND \#19

\section{Clinical Trials, June 2009}

http://clinicaltrials.gov/ct/gui/c/r

"breast diseases" AND (mammography OR surveillance)

\section{Current Controlled Trials, June 2009}

www.controlled-trials.com/

breast AND (mammography OR surveillance) 
The World Health Organization International Clinical Trials Registry

Platform, June 2009

World Health Organization: www.who.int/ictrp/en/

breast AND mammography OR breast AND surveillance

National Research Register Archive

National Institute for Health Research portal: https://portal.nihr. ac.uk/Pages/NRRArchiveSearch.aspx

NIHR portfolio, June 2009

National Institute for Health Research portal: https://portal.nihr. ac.uk/Search/Advanced.aspx

breast AND mammography OR breast AND surveillance

\section{National Cancer Institute Clinical Trials, June 2009}

The US National Institutes of Health: www.cancer.gov

breast cancer (female) AND mammography OR surveillance

\section{Websites consulted}

American College of Radiology

www.acr.org/

American Society of Breast Disease

www.asbd.org/

American Society of Clinical Oncology

www.asco.org/

Breast Cancer Surveillance Consortium

http://breastscreening.cancer.gov/

CancerWEB

www.infoventures.com/cancer/

Canadian Task Force on Preventive Health Care www.ctfphc.org/

Cancer Research UK www.cancerresearchuk.org/

European Society for Medical Oncology www.esmo.org/

National Breast and Ovarian Cancer Centre www.nbocc.org.au

National Cancer Institute www.cancer.gov/cancertopics/types/breast

National Comprehensive Cancer Network www.nccn.org/index.asp 
National Library for Health

www.library.nhs.uk/default.aspx

Royal College of Radiologists

www.rcr.ac.uk/

Surveillance, Epidemiology, and End Results Program

http://seer.cancer.gov/ 



\section{Appendix 11}

\section{Reports of included studies: systematic review of clinical effectiveness of surveillance mammography}

\section{Churn 2001}

Churn M, Kelly V. Outpatient follow-up after treatment for early breast cancer: updated results after 5 years. Clin Oncol 2001;13:187-94.

\section{Lash 2005}

Lash TL, Clough-Gorr K, Silliman RA. Reduced rates of cancer-related worries and mortality associated with guideline surveillance after breast cancer therapy. Breast Cancer Res Treat 2005;89:61-7.

Lash 2006

Lash TL, Fox MP, Silliman RA. Reduced mortality rate associated with annual mammograms after breast cancer therapy. Breast J 2006;12:2-6.

\section{Lash 2007}

Lash TL, Fox MP, Buist DS, Wei F, Field TS, Frost FJ, et al. Mammography surveillance and mortality in older breast cancer survivors. J Clin Oncol 2007;25:3001-6.

\section{Montgomery 2007}

Montgomery DA, Krupa K, Jack WJ, Kerr GR, Kunkler IH, Thomas J, et al. Changing pattern of the detection of locoregional relapse in breast cancer: the Edinburgh experience. Br J Cancer 2007;96:1802-7.

\section{Montgomery 2009}

Montgomery DA, Krupa K, Cooke TG. Locoregional relapse after breast cancer: most relapses occur late and are not clinically detected. Breast J 2009;15:163-7.

\section{Paszat 2008}

Paszat L, Sutradhar R, Grunfeld E, Gainford C, Benk V, Bondy S, et al. Outcomes of surveillance mammography after treatment of primary breast cancer: a population-based case series. Breast Cancer Res Treat 2008;114:169-78.

\section{Yau 2008}

Yau TK, Sze H, Soong IS, Wong W, Chan K, Chang A, et al. Surveillance mammography after breast conservation therapy in Hong Kong: effectiveness and feasibility of risk-adapted approach. Breast 2008;17:132-7. 



\section{Appendix 12}

\section{Excluded studies: systematic review of clinical effectiveness of surveillance mammography}

Surveillance regimens not included $(\mathrm{n}=76)$

Adedapo KS, Choudhury PS. Scintimammography screening for recurrent breast cancer in women. Afr J Med Sci 2007;36:279-82.

Altomaa S, Lipponen P, Eskelinen M, Kosma VM, Marin S, Alhava E, et al. Prognostic factors after 5 years follow-up in female breast-cancer. Oncology 1992;49:93-8.

Aniorte FM. Breast scintigraphy with Tc-MIBI in the diagnosis of breast cancer recurrence after conservative treatment. Rev Esp Med Nucl 1997;16:250-4.

Ash DV, Benson EA, Sainsbury JR, Round C, Head C. Seven-year follow-up on 334 patients treated by breast conserving surgery and short course radical postoperative radiotherapy: a report of the Yorkshire Breast Cancer Group. Clin Oncol 1995;7:93-6.

Bernstein JL, Thompson WD, Risch N, Holford TR. Risk factors predicting the incidence of second primary breast cancer among women diagnosed with a first primary breast cancer. Am J Epidemiol 1992;136:925-36.

Bernstein JL, Thompson WD, Risch N, Holford TR. The genetic epidemiology of second primary breast cancer. Am J Epidemiol 1992;136:937-48.

Bobo JK, Lawson HW, Lee NC. Risk factors for failure to detect a cancer during clinical breast examinations (United States). Cancer Causes Control 2003;14:461-8.

Bohmer C, Jager W, Lang N. CA 125 measurement in the follow-up of breast cancer patients. Anticancer Res 1997;17:3099-100.

Bokobsa J. Breast pathology: personal experience in color Doppler sonography. Le Sein 1994;4:182-7.

Bucchi L, Piatesi A, Falcini F, Nanni O, Amadori D. Effect of routine mammography practice on tumour size of a registry-based series of breast cancer cases compared with those observed in a screening cohort. Br J Radiol 1997;70:1174-7.

Buiatti E, Crocetti E, Acciai S, Gafà L, Falcini F, Milandri C, et al. Incidence of second primary cancers in three Italian population-based cancer registries. Eur J Cancer 1997;33:1829-34.

Cabioglu N, Hunt KK, Buchholz TA, Mirza N, Singletary SE, Kuerer HM, et al. Improving local control with breast-conserving therapy: a 27-year single-institution experience. Cancer 2005;104:20-9.

Campi R, Vanoli C, Belli L, Carlotto M, Bernasconi P, Tufarulo L. [Imaging of the breast treated with quadrantectomy and radiotherapy. Interpretative difficulties in diagnosing asymptomatic recurrences.] Radiol Med 1991;82:427-9.

Chuba PJ, Hamre MR, Yap J, Severson RK, Lucas D, Shamsa F, et al. Bilateral risk for subsequent breast cancer after lobular carcinoma-in-situ: analysis of surveillance, epidemiology, and end results data. J Clin Oncol 2005;23:5534-41. 
Ciatto S. Detection of breast cancer local recurrences. Ann Oncol 1995;6(Suppl. 2):23-6.

Cook LS, White E, Schwartz SM, McKnight B, Daling JR, Weiss NS. A population-based study of contralateral breast cancer following a first primary breast cancer (Washington, United States). Cancer Causes Control 1996;7:382-90.

Coppola V, Brunese L, Gatta G, Coppola M, Alfano L, Cariello S et al. [Follow-up chest radiography in surgical breast cancer patients.] Radiol Med 1999;98:264-7.

de la Rochfordière A, Mouret-Fourme E, Asselain B, Scholl SM, Campana F, Broet P, et al. Metachronous contralateral breast cancer as first event of relapse. Int J Radiat Oncol Biol Phys 1996;36:615-21.

Dershaw DD. Evaluation of the breast undergoing lumpectomy and radiation therapy. Radiol Clin North Am 1995;33:1147-60.

Dershaw DD. Mammography in patients with breast cancer treated by breast conservation (lumpectomy with or without radiation). AJR Am J Roentgenol 1995;164:309-16.

Deshpande AH, Munshi M, Gaikwad S, Deshpande KM. Triple approach method for screening of contralateral breast in treated cases of breast cancer. Ind J Pathol Microbiol 2003;46:385-9.

Duffy CM, Clark MA, Allsworth JE. Health maintenance and screening in breast cancer survivors in the United States. Cancer Detect Prevent 2006;30:52-7.

Earle CC, Burstein HJ, Winer EP, Weeks JC. Quality of non-breast cancer health maintenance among elderly breast cancer survivors. J Clin Oncol 2003;21:1447-51.

Elmore JG, Wells CK, Howard DH, Feinstein AR. The impact of clinical history on mammographic interpretations. JAMA 1997;277:49-52.

Fliquete Peris MV, Gimenez CJ, Vazquez FC, Mas AC, Vazquez AC. [Role of 99mTC-Sestamibi in the diagnosis of breast cancer. Report on 100 cases.] Rev Esp Med Nucl 1999;18:436-41.

Freedman GM, Anderson PR, Li T, Nicolaou N. Locoregional recurrence of triple-negative breast cancer after breast-conserving surgery and radiation. Cancer 2009;115:946-51.

Gage I, Recht A, Gelman R, Nixon AJ, Silver B, Bornstein BA, et al. Long-term outcome following breast-conserving surgery and radiation therapy. Int J Radiat Oncol Biol Phys 1995;33:245-51.

Gerlach B. Breast ultrasound in pre- and postoperative patients. Ultrasound Q 1995;13:27-40.

Gimotty PA, Burack RC, George J. A randomized design for repeated binary outcomes used to evaluate continued effectiveness of a breast cancer control intervention strategy. Control Clin Trials 1999;20:280-93.

Goldstein NS, Kestin L, Vicini F. Intraductal carcinoma of the breast: pathologic features associated with local recurrence in patients treated with breast-conserving therapy. Am J Surg Pathol 2000;24:1058-67.

Grahek D. Role and impact of [18F]-fluorodeoxyglucose positron emission tomography in recurrent breast cancer. Med Nucl 2004;28:291-6.

Gross CP, Filardo G, Singh HS, Freedman AN, Farrell MH. The relation between projected breast cancer risk, perceived cancer risk, and mammography use. Results from the National Health Interview Survey. J Gen Intern Med 2006;21:158-64.

Hall SA. Routine follow up of breast cancer in primary care. Open door and listening ear are best support for patients. BMJ 1996;313:1548.

Helvie MA, Bailey JE, Roubidoux MA, Pass HA, Chang AE, Pierce LJ, et al. Mammographic screening of TRAM flap breast reconstructions for detection of nonpalpable recurrent cancer. Radiology 2002;224:211-16. 
Hurley SF, Jolley DJ, Livingston PM, Reading D, Cockburn J, Flint-Richter D. Effectiveness, costs, and cost-effectiveness of recruitment strategies for a mammographic screening program to detect breast cancer. J Natl Cancer Inst 1992;84:855-63.

Hurria A, Leung D, Trainor K, Norton L, Hudis C. Screening chest imaging studies are not effective in the follow-up of breast cancer patients. J Oncol Manag 2003;12:13-15.

Huston TL. Locally recurrent breast cancer after conservation therapy. Am J Surg 2005; 189:229-35.

Kauczor HU, Voges EM, Wieland-Schneider C, Mitze M, Thelen M. Value of routine abdominal and lymph node sonography in the follow-up of breast cancer patients. Eur J Radiol 1994;18:104-8.

Klimberg VS. The path to recurrence is paved with good intentions. Ann Surg Oncol 2000;7:402-3.

Kopans DB, Moore RH, McCarthy KA, Hall DA, Hulka CA, Whitman GJ, et al. Should women with implants or a history of treatment for breast cancer be excluded from mammography screening programs? AJR Am J Roentgenol 1997;168:29-31.

Kreager JA, Kornguth PJ. Mammography for screening high-risk patients for cancer: value of including a lateral projection. AJR Am J Roentgenol 1994;162:295-7.

Kwon S, Kang SH, Ro J, Jeon CH, Park JW, Lee ES. The melanoma antigen gene as a surveillance marker for the detection of circulating tumor cells in patients with breast carcinoma. Cancer 2005;104:251-6.

Lauro S, Trasatti L, Bordin F, Lanzetta G, Bria E, Gelibter A, et al. Comparison of CEA, MCA, CA 15-3 and CA 27-29 in follow-up and monitoring therapeutic response in breast cancer patients. Anticancer Res 1999;19:3511-15.

Leong C. Effect of margins on ipsilateral breast tumor recurrence after breast conservation therapy for lymph node-negative breast carcinoma. Women Oncol Rev 2004;4:229-30.

Luck AA. Special investigations of the breast. Surgery 2008;26:231-4.

Lumachi F, Brandes AA, Boccagni P, Polistina F, Favia G, D'Amico DF. Long-term follow-up study in breast cancer patients using serum tumor markers CEA and CA 15-3. Anticancer Res 1999;19:4485-9.

Mansour OA, Zekri AR, Harvey J, Teramoto Y, el Ahmady O. Tissue and serum c-erbB-2 and tissue EGFR in breast carcinoma: three years follow-up. Anticancer Res 1997;17:3101-16.

Mollick JA, Carlson RW. Rational surveillance programs for early stage breast cancer patients after primary treatment. Breast Dis 2004;21:47-54.

Montravers F, Younsi N, Rousseau C, Philippe C, Seddiki M, Uzan S, et al. Comparison of bone scanning and CA 15-3 serum concentration in the follow-up of breast cancer. Anticancer Res 1997;17:1683-6.

Mould RF, Asselain B, De Rycke Y. Methodology to predict a maximum follow-up period for breast cancer patients without significantly reducing the chance of detecting a local recurrence. Physics Med Biol 2004;49:1079-83.

Munhoz AM. Breast cancer local recurrence after mastectomy and TRAM flap reconstruction: incidence and treatment options. Plast Reconstr Surg 2006;118:1664-5.

Navarro MA, Mesia R, Diez-Gibert O, Rueda A, Ojeda B, Alonso MC. Epidermal growth factor in plasma and saliva of patients with active breast cancer and breast cancer patients in follow-up compared with healthy women. Breast Cancer Res Treat 1997;42:83-6. 
Nicolini A, Carpi A, Ferrari P, Anselmi L. Clinical use of tumor markers in the postoperative management of breast cancer patients: new concepts. Int J Biol Markers 1997;12:181-6.

Oh S, Heflin L, Meyerowitz BE, Desmond KA, Rowland JH, Ganz PA. Quality of life of breast cancer survivors after a recurrence: a follow-up study. Breast Cancer Res Treat 2004;87:45-57.

Olivotto IA, Mates D, Kan L, Fung J, Samant R, Burhenne LJ. Prognosis, treatment, and recurrence of breast cancer for women attending or not attending the Screening Mammography Program of British Columbia. Breast Cancer Res Treat 1999;54:73-81.

Pace BW, Tinker MA. Follow-up of patients with breast cancer. Clin Obstet Gynecol 1994;37:998-1002.

Quan G, Pommier SJ, Pommier RF. Incidence and outcomes of contralateral breast cancers. Am J Surg 2008;195:645-50.

Rial M, Garbovesky C, Wons B, Tena M, Abella O. Usefulness of scintimammography to investigate local recurrences of breast cancer. Prensa Med Argent 1998;85:849-51.

Roussel-Andry EG. Ultrasonographic aspects of breast cancer recurrence. Le Sein 1995;5:287-90.

Schneider G, Steindorfer P, Fotter R. [The place of mammography following breast-conserving therapy of breast cancer.] ROFO-Fortschr Rontg 1992;156:582-6.

Sellers TA, Anderson VE, Potter JD, Bartow SA, Chen PL, Everson L, et al. Epidemiologic and genetic follow-up study of 544 Minnesota breast cancer families: design and methods. Genet Epidemiol 1995;12:417-29.

Stokes ME, Thompson D, Montoya EL, Weinstein MC, Winer EP, Earle CC. Ten-year survival and cost following breast cancer recurrence: estimates from SEER-medicare data. Value Health 2008;11:213-20.

Tempfer C, Hanzal E, Zeillinger R, Koelbl H, Dadak C, Kainz C. The serum tumor marker M3/ M21 in the follow-up of breast cancer patients. Anticancer Res 1996;16:3049-52.

Thurfjell E. Mammography screening methods and diagnostic results. Acta Radiol 1995;395(Suppl.):1-22.

Tofighi M. F-FDG coincidence imaging in patients with increased CA 15-3 levels during follow-up for breast cancer. Med Nucl 2002;26:87-94.

Tomiak EM, Piccart MJ. Routine follow-up of patients following primary therapy for early breast cancer: what is useful? Acta Clinica Belgica 1993;15(Suppl.):38-42.

Tranquart F, Samardzija V, Body G, Gallay X, Lansac J, Rouleau P, et al. Color-coded Doppler assessment of recurrent breast cancer. J Radiol 1996;77:171-5.

Trask PC, Pahl L, Begeman M. Breast self-examination in long-term breast cancer survivors. J Cancer Surv 2008;2:243-52.

Vennin P, Belkacemi Y, Chauvet MP. [Follow-up of patients treated for localized invasive breast carcinoma.] Gynecol Obstet Fertil 2008;36:183-9.

Voogd AC, van der Horst F, Crommelin MA, Peterse JL, van Beek MW, Repelaer van Driel OJ, et al. The relationship between findings on pre-treatment mammograms and local recurrence after breast-conserving therapy for invasive breast cancer. Eur J Surg Oncol 1999;25:273-9.

Wagman LD, Sanders RD, Terz JJ, Beatty JD, Kokal WA, Riihimaki DU. The value of symptom directed evaluation in the surveillance for recurrence of carcinoma of the breast. Surg Gynecol Obstet 1991;172:191-6. 
Weidenmaier W, Schnarkowski P, Heuchemer T, Schumacher KA. [Visible mammographic changes following breast-conserving therapy: radiation effects or recurrence?] Rontgenpraxis 1992;45:253-6.

Wertheimer MD. Against minimalism in breast cancer follow-up. JAMA 1991;265:396-7.

Wood WC. Nonimaging aspects of follow-up in breast cancer reconstruction. Cancer 1991;68:1164-6.

Wright T. Breast cancer: new technologies for risk assessment and diagnosis. Mol Diagn 2002;7:49-55.

Younsi N, Montravers F, Philippe C, Seddiki M, Uzan S, Izrael V, et al. CA 15-3 and bone scintigraphy in the follow-up of breast cancer. Int J Biol Markers 1997;12:154-7.

\section{Study design not met $(\mathrm{n}=156)$}

Albert U-S. From symptom- to problem-oriented follow-up care. Improvement in clinical practice management of breast cancer. Gynäkologe 2002;35:1105-13.

Andersen MR. The use of mammography by survivors of breast cancer. Am J Public Health 1998;88:1713-15.

Andersen MR, Urban N. Involvement in decision-making and breast cancer survivor quality of life. Ann Behav Med 1999;21:201-9.

Ashkanani F, Sarkar T, Needham G, Coldwells A, Ah-See AK, Gilbert FJ, et al. What is achieved by mammographic surveillance after breast conservation treatment for breast cancer? Am J Surg 2001;182:207-10.

Barkhausen J. Magnetic-resonance-mammography in the assessment of early and late recurrence after breast conserving therapy. Tumor Diagnostik Therapie 1997;18:65-70.

Beaver K. Comparing hospital and telephone follow-up after treatment for breast cancer: randomised equivalence trial. BMJ 2009;338:337-40.

Beaver K, Luker KA. Follow-up in breast cancer clinics: reassuring for patients rather than detecting recurrence. Psychooncology 2005;14:94-101.

Beinart G, Gonzalez-Angulo AM, Broglio K, Mejia J, Ruggeri A, Mininberg E, et al. Clinical course of 771 patients with bilateral breast cancer: characteristics associated with overall and recurrence-free survival. Clin Breast Cancer 2007;7:867-74.

Birrenbach S, Miller S, Stern W, Xydeas T, Pietsch-Breitfeld B, Belka C, et al. [Clinical value of mammography, ultrasound and $\mathrm{MR}$ imaging during the first year after breast conserving therapy of breast cancer.] ROFO-Fortschr Rontg 2004;176:1423-30.

Bock E, Bock C, Belli P, Campioni P, Manfredi R, Pastore G. [Role of diagnostic imaging of the breast in patients treated with postsurgical radiotherapy or presurgical radiotherapy or chemotherapy.] Radiol Med 1998;95:38-43.

Bonifacino A. Ultrasonography of local recurrent breast cancer. JEMU 1998;19:81-5.

Braw M, Erlandsson I, Ewers SB, Samuelsson L. Mammographic follow-up after breast conserving surgery and postoperative radiotherapy without boost irradiation for mammary carcinoma. Acta Radiol 1991;32:398-402.

Briggs J. Mammographic evaluation of the postsurgical and irradiated breast. Radiol Technol 1997;68:287-304. 
Brown L, Payne S, Royle G. Patient initiated follow up of breast cancer. Psychooncology 2002;11:346-55.

Brown M. Imaging in the follow-up of patients with breast cancer. Seminar Radiol Technol 2002;10:146-54.

Buchberger W, Hamberger L, Schon G, Steixner G, Fritsch E. [Mammography and sonography in the diagnosis of recurrence after breast-preserving therapy of breast carcinoma.] ROFO-Fortschr Rontg 1991;154:650-6.

Butsch WS, Munoz AJ, Heimburger DC. Follow-up of patients with early breast cancer. N Engl J Med 2007;357:1053.

Cappellini M, Fallai C, Pacini P, Mungai R. [Follow-up of breast carcinoma.] Radiol Med 1991;81:718-24.

Carlotti GA, Siragusa A, Grillo RF, Vitali ML, Grimaldi A, Barone D. [The mammographic images of the irradiated breast after conservative therapy for carcinoma.] Radiol Med 1993;86:101-5.

Cawson J, Billson V, Russell I. Mammographic follow up: a vital component of breast cancer management. Aust N Z J Surg 1993;63:551-3.

Chen C, Orel SG, Harris EE, Hwang WT, Solin LJ. Relation between the method of detection of initial breast carcinoma and the method of detection of subsequent ipsilateral local recurrence and contralateral breast carcinoma. Cancer 2003;98:1596-602.

Cochet S, Favet L, Sappino AP. [Surveillance following curative therapy for breast cancer.] Rev Med Suisse 2008;4:1258, 1260-2.

Costa SD, Souchon R, Scharl A. [Ipsilateral breast tumor recurrence after conservative breast cancer surgery: diagnosis and therapy.][Erratum appears in Zentralbl Gynakol 2004;126:336.] Zentralbl Gynakol 2004;126:244-51.

Dalberg K, Azavedo E, Svane G, Sandelin K. Mammographic features, predictors of early ipsilateral breast tumour recurrences? Eur J Surg Oncol 1996;22:483-90.

Decrombrugghe G, Tristant H. Imaging of the treated breast: the locoregional recurrence. Int Congr Senol 1992;1005:59-60.

Dershaw DD, McCormick B, Osborne MP. Detection of local recurrence after conservative therapy for breast carcinoma. Cancer 1992;70:493-6.

Dewar J. Follow up in breast cancer. BMJ 1995;310:685-6.

Di Cosimo S, Ferretti G, Alimonti A, Gravante G, Silvestris N, D’Aprile M. Long-term follow-up in breast cancer survivors: a single institution survey. J Womens Health 2003;12:599-600.

Dixon JM, Montgomery D. Follow-up after breast cancer. BMJ 2008;336:107-8.

Dixon JM, Montgomery DA. Extended follow-up of breast cancer patients in clinic wastes time for both patients and doctors: the case for. Breast Cancer Res 2008;10(Suppl. 4):S7.

Donegan WL. Follow-up after treatment for breast cancer: how much is too much? J Surg Oncol 1995;59:211-14.

Donnelly J, Mack P, Donaldson LA. Follow-up of breast cancer: time for a new approach? Int $J$ Clin Pract 2001;55:431-3.

Doyle T, Schultz DJ, Peters C, Harris E, Solin LJ. Long-term results of local recurrence after breast conservation treatment for invasive breast cancer. Int J Radiat Oncol Biol Phys 2001;51:74-80. 
Duran MAC, Gomez AG, Palmer MAS, Guerrero RB, Izquierdo JAD, Dominguez AR, et al. Clinical and mammography follow-up of breast-cancer after conservative treatment. Rev Clin Esp 1993;192:309-14.

Earnshaw JJ, Stephenson Y. First two years of a follow-up breast clinic led by a nurse practitioner. J R Soc Med 1997;90:258-9.

Ebell MH. Follow-up after surgically treated breast cancer. Am Fam Physician 2005;72:77.

Elder EE, Kennedy CW, Gluch L, Carmalt HL, Janu NC, Joseph MG, et al. Patterns of breast cancer relapse. Eur J Surg Oncol 2006;32:922-7.

Elsamaloty H, Elzawawi MS, Mohammad S, Herial N. Increasing accuracy of detection of breast cancer with 3-T MRI. AJR Am J Roentgenol 2009;192:1142-8.

Emens LA. The follow-up of breast cancer. Semin Oncol 2003;30:338-48.

Eusebi V, Feudale E, Foschini MP, Micheli A, Conti A, Riva C, et al. Long-term follow-up of in situ carcinoma of the breast. Semin Diagn Pathol 1994;11:223-35.

Fallowfield L, George D. Introduction to the sessions on 'Debate: extended follow-up of breast cancer patients in clinic wastes time for both patients and doctors'. Breast Cancer Res 2008;10(Suppl. 4):S6.

Field TS, Doubeni C, Fox MP, Buist DS, Wei F, Geiger AM, et al. Under utilization of surveillance mammography among older breast cancer survivors. J Gen Intern Med 2008;23:158-63.

Fisher B. From Halsted to prevention and beyond: advances in the management of breast cancer during the twentieth century. Eur J Cancer 1999;35:1963-73.

Friedrich M. MRI of the breast: state of the art. Eur Radiol 1998;8:707-25.

Fries MH, Hailey BJ, Flanagan J, Licklider D. Outcome of five years of accelerated surveillance in patients at high risk for inherited breast/ovarian cancer: report of a phase II trial. Mil Med 2004;169:411-16.

Gao X. Risk of second primary cancer in the contralateral breast in women treated for early-stage breast cancer: a population-based study. Int J Radiat Oncol Biol Phys 2003;56:1038-45.

Geller BM. Mammography surveillance following breast cancer. Breast Cancer Res Treat 2003;81:107-15.

Giess CS, Keating DM, Osborne MP, Rosenblatt R. Local tumor recurrence following breastconservation therapy: correlation of histopathologic findings with detection method and mammographic findings. Radiology 1999;212:829-35.

Gilles R. Local recurrence of breast cancer: clinical and mammographic features. Rev Imager Med 1993;5:761-5.

Grogan M. The value of follow-up of patients with early breast cancer treated with conservative surgery and radiation therapy. Breast 2002;11:163-9.

Grosse A, Schreer I, Frischbier HJ, Maass H, Loening T, Bahnsen J. Results of breast conserving therapy for early breast cancer and the role of mammographic follow-up. Int J Radiat Oncol Biol Phys 1997;38:761-7.

Grunfeld E, Gray A, Mant D, Yudkin P, Adewuyi-Dalton R, Coyle D, et al. Follow-up of breast cancer in primary care vs specialist care: results of an economic evaluation. $\mathrm{Br} \mathrm{J}$ Cancer 1999;79:1227-33.

Guastalla JP, Kerbrat P. [Primary malignant tumor of the breast. Monitoring after treatment.] Presse Med 1995;24:1415-17. 
Gundersen S, Kvaloy S, Hannisdal E. [Check up of breast cancer stages 1 and 2.] Tidsskr Nor Laegeforen 1997;117:1079-81.

Haid A. Follow-up for cancer patients. Eur Surg 2006;38:15-20.

Hannisdal E, Gundersen S, Kvaloy S, Lindegaard MW, Aas M, Finnanger AM, et al. Follow-up of breast cancer patients stage I-II: a baseline strategy. Eur J Cancer 1993;29A:992-7.

Hassell PR, Olivotto IA, Mueller HA, Kingston GW, Basco VE. Early breast cancer: detection of recurrence after conservative surgery and radiation therapy. Radiology 1990;176:731-5.

Hill-Kayser CE, Harris EE, Hwang WT, Solin LJ. Twenty-year incidence and patterns of contralateral breast cancer after breast conservation treatment with radiation. Int J Radiat Oncol Biol Phys 2006;66:1313-19.

Hiramanek N. Breast cancer recurrence: follow up after treatment for primary breast cancer. Postgrad Med J 2004;80:172-6.

Hurley SF, Huggins RM, Snyder RD, Bishop JF. The cost of breast cancer recurrences. Br J Cancer 1992;65:449-55.

Hussian ST, Gui GP, Lee KS, Plowman PN, Gilmore OJ, Allum WH. Detection of loco-regional recurrence after breast-conserving surgery and radiotherapy. J R Coll Surg Edinb 1995;40:163-6.

Imoto $S$, Jitsuiki Y. Detection of the first recurrence during intensive follow-up of breast cancer patients. Jpn J Clin Oncol 1998;28:597-600.

Jack WJL. Long-term follow-up after breast conservation: the Edinburgh experience. Breast 1998;7:80-4.

Jacobs HJM, van Dijck JAAM, de Kleijn EMHA, Kiemeney LALM, Verbeek ALM. Routine follow-up examinations in breast cancer patients have minimal impact on life expectancy: a simulation study. Ann Oncol 2001;12:1107-13.

Jiwa M, Thompson J, Coleman R, Reed M. Breast cancer follow-up: could primary care be the right venue? Curr Med Res Opin 2006;22:625-30.

Johnson RC. Mastectomy follow-up by biennial mammograms: is it worthwhile? Breast 2000;9:93-5.

Joseph E, Hyacinthe M, Lyman GH, Busch C, Demps L, Reintgen DS, et al. Evaluation of an intensive strategy for follow-up and surveillance of primary breast cancer. Ann Surg Oncol 1998;5:522-8.

Jubelirer SJ. Surveillance testing in patients with early stage breast cancer: a review. $W V$ Med $J$ 1998;94:14-17.

Kaas R, Hart AA, Besnard AP, Peterse JL, Rutgers EJ. Impact of mammographic interval on stage and survival after the diagnosis of contralateral breast cancer. Br J Surg 2001;88:123-7.

Kaas R, Muller SH, Hart AA, Rutgers EJ. Stage of breast cancers found during the surveillance of women with a familial or hereditary risk. Eur J Surg Oncol 2008;34:501-7.

Kagan AR, Steckel RJ. Routine imaging studies for the post-treatment surveillance of breast and colorectal carcinoma. J Clin Oncol 1991;9:837-42.

Kamby C, Sengelov L. Pattern of dissemination and survival following isolated locoregional recurrence of breast cancer. A prospective study with more than 10 years of follow up. Breast Cancer Res Treat 1997;45:181-92.

Karadayi B. Surveillance of breast cancer after primary treatment. THOD - Turk HematolojiOnkoloji Dergisi 2004;14:224-31. 
Kauhava L, Immonen-Raiha P, Parvinen I, Holli K, Pylkkanen L, Kaljonen A, et al. Lower recurrence risk through mammographic screening reduces breast cancer treatment costs. Breast 2008; 17:550-4.

Khandekar JD. Recommendations on follow-up of breast cancer patients following primary therapy. Semin Surg Oncol 1996;12:346-51.

Kini VR, Vicini FA, Frazier R, Victor SJ, Wimbish K, Martinez AA. Mammographic, pathologic, and treatment-related factors associated with local recurrence in patients with early-stage breast cancer treated with breast conserving therapy. Int J Radiat Oncol Biol Phys 1999;43:341-6.

Kini VR, Vicini FA, Victor SJ, Dmuchowski CF, Rebner M, Martinez AA. Impact of the mode of detection on outcome in breast cancer patients treated with breast-conserving therapy. Am J Clin Oncol 1999;22:429-35.

Kirkbride P, Vallis K. Routine follow up of breast cancer in primary care. Study of open access centre for patients with breast cancer is planned. BMJ 1996;313:1547.

Kollias J, Evans A, Wilson AR, Ellis IO, Elston CW, Blamey RW. Value of contralateral surveillance mammography for primary breast cancer follow-up. World J Surg 2000;24:983-7.

Kontos M, Hamed H. Why individualize breast cancer survivors' follow-up? J BUON 2007;12:553-4.

Kopans DB. Mammographic follow-up to detect breast cancer recurrence after conservative treatment. Radiology 1994;190:286.

Krengli M, Pastore G, Maffei S. [The importance of the follow-up in patients operated on for breast cancer. A retrospective analysis of 2482 cases.] Minerva Med 1993;84:409-15.

Krishnaraj A, Yankaskas BC, Stearns SC. Screening mammography after breast cancer treatment: patterns in community practice. Breast Cancer Res Treat 2006;97:73-80.

Kroll SS, Khoo A, Singletary SE, Ames FC, Wang BG, Reece GP, et al. Local recurrence risk after skin-sparing and conventional mastectomy: a 6-year follow-up. Plastic Recon Surg 1999;104:421-5.

Kunkler I, Kerr G, Jack W. Intensity of surveillance after breast conservation. J Clin Oncol 1997;15:3420.

Kurtz JM, Spitalier J-M. Local recurrence after breast-conserving surgery and radiotherapy: what have we learned. Int J Radiat Oncol Biol Phys 1990;19:1087-90.

Lansac J, Diouf A. [Follow-up of women treated for breast cancer. State of the art.] J Gynecol Obstet Biol Reprod (Paris) 1998;27:21-33.

Lash TL, Silliman RA. Medical surveillance after breast cancer diagnosis. Med Care 2001;39:945-55.

Latteri M, Cipolla C, Pantuso G, Amato C, Cassano T, Salanitro L, et al. [The value and limits of the follow-up in patients operated on for breast carcinoma. Our experience.] Minerva Chir 1990;45:1379-84.

Lee JM, Georgian-Smith D, Gazelle GS, Halpern EF, Rafferty EA, Moore RH, et al. Detecting nonpalpable recurrent breast cancer: the role of routine mammographic screening of transverse rectus abdominis myocutaneous flap reconstructions. Radiology 2008;248:398-405.

Lees A, Gabos A, Jenkins H. Investigations for staging and follow-up of breast cancer patients. Cancer Prevent Control 1997;1:157-60.

Lelli G, Indelli M, Modonesi C, Gulmini L, Durante E. [Clinical postoperative surveillance of breast carcinoma.] Recenti Prog Med 2002;93:637-41. 
Li S, Li L. A retrospective study of first indicators of breast cancer recurrence. Zhongguo Zhongliu Linchuang 2003;30:791-2.

Loomer L, Brockschmidt JK, Muss HB, Saylor G. Postoperative follow-up of patients with early breast cancer. Patterns of care among clinical oncologists and a review of the literature. Cancer 1991;67:55-60.

Loong S, Wilkins M, Bliss JM, Davidson J, Ebbs SR, Regan J, et al. The effectiveness of the routine clinic visit in the follow-up of breast cancer patients: analysis of a defined patient cohort. Clin Oncol (R Coll Radiol) 1998;10:103-6.

$\mathrm{Lu}$ WL. Impact on survival of early detection of isolated breast recurrences after the primary treatment for breast cancer: a meta-analysis. Breast Cancer Res Treat 2009;114:403-12.

Mallol N, Desandes E, Lesur-Schwander A, Guillemin F. Disease-specific and event-free survival in breast cancer patients: a hospital-based study between 1990 and 2001. Rev Epidemiol Santé Publique 2006;54:313-25.

Mann GB, Port ER, Rizza C, Tan LK, Borgen PI, Van Zee KJ. Six-year follow-up of patients with microinvasive, T1a, and T1b breast carcinoma. Ann Surg Oncol 1999;6:591-8.

Marret H, Bougnoux P, Fignon A, Hubert B, Fetissof F, Le Floch O, et al. Prognosis of locoregional recurrence following conservative treatment of breast cancer. J Gynecol Obstet Biol Reprod 1997;26:144-7.

Martinez PM. Monitoring to patients with breast cancer after multimodal treatment. Ginecol Obstet Mex 2008;76:107-12.

Mason BH, Holdaway IM, Benton NM, Benson-Cooper DM, Hadden WE, Kay RG. Detection of contralateral breast cancer by mammography in women with previous breast cancer and the impact of endocrine therapy. NZ Med J 1993;106:23-5.

McKee MD. Breast cancer follow-up. Prob Gen Surg 2000;17:87-100.

Mellink WA, Holland R, Hendriks JH, Peeters PH, Rutgers EJ, van Daal WA. The contribution of routine follow-up mammography to an early detection of asynchronous contralateral breast cancer. Cancer 1991;67:1844-8.

Menke H, Schreckenberger C, Restel J, Olbrisch RR. [Manifestation, therapy and follow-up of locoregional breast carcinoma recurrence.] Langenbecks Arch Chir Suppl Kon 1996;113:108-10.

Mennel C, Tulusan AH, Reitzenstein M, Ronay G, Willgeroth F, Lang N. [Diagnostic problems in local recurrence after breast saving therapy of breast cancer.] Geburtsh Frauenheilk 1991;51:262-6.

Molino A. What is the best follow-up methodology in early breast cancer? Breast 2008;17:1-2.

Morgan RJ, Bristol JB. Detection of metachronous breast carcinoma: the role of follow-up? Ann $R$ Coll Surg Engl 1999;81:97-9.

Morris J, Wheatley D, Ingram C. An audit of follow-up clinics for breast cancer patients in Cornwall: is the time and effort worth it? Eur J Cancer Care 2008;17:578-84.

Morris S, Corder AP, Taylor I. What are the benefits of routine breast cancer follow-up? Postgrad Med J 1992;68:904-7.

Moskovic E, Parsons C, Baum M. Chest radiography in the management of breast cancer. $\mathrm{Br} \mathrm{J}$ Radiol 1992;65:30-2.

Mundinger A, Martini C, Madjar H, Laubenberger J, Gufler H, Langer M. [Ultrasound and mammography follow-up of findings after breast saving operation and adjuvant irradiation.] Ultraschall Med 1996;17:7-13. 
Muss HB, Tell GS, Case LD, Robertson P, Atwell BM. Perceptions of follow-up care in women with breast cancer. Am J Clin Oncol 1991;14:55-9.

Muttarak M, Pojchamarnwiputh S, Padungchaichote W, Chaiwun B. Evaluation of the contralateral breast in patients with ipsilateral breast carcinoma: the role of mammography. Singapore Med J 2002;43:229-33.

Neises M. Results of the follow-up of outpatients with breast cancer: a program of the Kassenarztliche Vereinigung Nordbaden and the Oncologic Center at Mannheim University Hospital. Tumor Diagnostik und Therapie 1995;16:7-12.

Nelson NJ. Do follow-up tests actually help detect recurrent disease? J Natl Cancer Inst 2000;92:1798-800.

Palli D, Russo A, Saieva C, Ciatto S, Rosselli DT, Distante V, et al. Intensive vs clinical follow-up after treatment of primary breast cancer: 10-year update of a randomized trial. National Research Council Project on Breast Cancer Follow-up. JAMA 1999;281:1586.

Park-Simon TW, Hillemanns P. Breast cancer follow-up. Onkologie 2008;14:520-5.

Peppercorn J. Standards for follow-up care of patients with breast cancer. Breast 2005;14:500-8.

Perrone MA, Musolino A, Michiara M, Di Blasio B, Bella M, Franciosi V, et al. Early detection of recurrences in the follow-up of primary breast cancer in an asymptomatic or symptomatic phase. Tumori 2004;90:276-9.

Poplack SP, Maurer LH. Reduction in size of a breast mass due to concurrent chemotherapy: pitfalls in mammographic follow-up. AJR Am J Roentgenol 1996;167:392-3.

Potemski P. Follow-up of breast cancer patients after curative treatment. A review of literature. Onkologia Polska 2002;5:87-91.

Raspall MJF, Serra EP, Verdum JI, Munoz CA, Gonzalez BO, Gomez AG, et al. Locoregional recurrences after conservative treatment of breast cancer stage I-II. Med Clin (Barc) 2002;118:161-5.

Rebner M, Grills I, Vicini F. Should screening MRI be included in surveillance for patients treated with breast-conserving therapy? Nature Clin Pract Oncol 2009;6:8-9.

Rieber A, Schramm K, Helms G, von Puckler S, Nuessle K, Kreienberg R, et al. Breast-conserving surgery and autogenous tissue reconstruction in patients with breast cancer: efficacy of MRI of the breast in the detection of recurrent disease. Eur Radiol 2003;13:780-7.

Ritter N, Love N, Osman D. After breast cancer: implications for long-term primary care. Postgrad Med 1999;105:103.

Robinson E, Rennert G, Rennert HS, Neugut AI. Survival of first and second primary breast cancer. Cancer 1993;71:172-6.

Rosselli DT, Palli D, Cariddi A, Ciatto S, Pacini P, Distante V. Intensive diagnostic follow-up after treatment of primary breast cancer. A randomized trial. National Research Council Project on Breast Cancer Follow-up. JAMA 1994;271:1593-7.

Rosselli DT, Palli D, Cariddi A, Ciatto S, Pacini P, Distante V. The efficacy of intensive follow-up testing in breast cancer cases. Ann Oncol 1995;6(Suppl. 2):37-9.

Rutgers EJ, van Rossum AB, Peterse JL, Cohen P, Borger JH, Kemperman HW. Breast-conserving therapy for invasive carcinoma: diagnosis of local recurrence. Neth J Surg 1991;43:110-3.

Salas AP, Helvie MA, Wilkins EG, Oberman HA, Possert PW, Yahanda AM, et al. Is mammography useful in screening for local recurrences in patients with TRAM flap breast reconstruction after mastectomy for multifocal DCIS? Ann Surg Oncol 1998;5:456-63. 
Samant RS, Olivotto IA, Jackson JS, Mates D. Diagnosis of metachronous contralateral breast cancer. Breast J 2001;7:405-10.

Sardi A, Eckholdt G, McKinnon WM, Bolton JS. The significance of mammographic findings after breast-conserving therapy for carcinoma of the breast. Surg Gynecol Obstet 1991;173:309-12.

Sardi A, Facundus EC, Eckholdt GJ, McKinnon WM, Skenderis BS, Bolton JS. Management of cancer of the opposite breast following breast preservation. Int Surg 1992;77:289-92.

Sauer H, Holzel D. [Breast carcinoma - revision of after-care recommendations. 1: Results of many large studies from the literature are in agreement.] Fortschr Med 1995;113:183-7.

Sauer H, Holzel D. [Breast carcinoma - revision of follow-up recommendations. 2: Special diagnostic value of bone scintigraphy, thoracic radiography, liver ultrasonic, mammography and laboratory tests.] Fortschr Med 1995;113:210-14.

Schapira DV. Breast cancer surveillance: a cost-effective strategy. Breast Cancer Res Treat 1993;25:107-11.

Schapira MM, McAuliffe TL, Nattinger AB. Underutilization of mammography in older breast cancer survivors. Med Care 2000;38:281-9.

Schmoor C, Olschewski M, Sauerbrei W, Schumacher M. Long-term follow-up of patients in four prospective studies of the German Breast Cancer Study Group (GBSG): a summary of key results. Onkologie 2002;25:143-50.

Schwartz GF. The role of excision and surveillance alone in subclinical DCIS of the breast. Oncology 1994;8:21-6.

Shaikh N, LaTrenta G, Swistel A, Osborne FM. Detection of recurrent breast cancer after TRAM flap reconstruction. Ann Plast Surg 2001;47:602-7.

Snee M. Routine follow-up of breast cancer patients. Clin Oncol 1994;6:154-6.

Son HJ, Oh KK. Significance of follow-up mammography in estimating the effect of tamoxifen in breast cancer patients who have undergone surgery. AJR Am J Roentgenol 1999;173:905-9.

Soot ML, Nielsen DL, Kamby C. [Follow-up after surgery for breast cancer.] Ugeskr Laeger 2002;164:2918-22.

Spaulding CA. Detection of recurrence after breast conservation treatment with radiotherapy. Breast Dis 1992;5:75-90.

Stutte H. [Soft-tissue sonography in the follow-up care of breast cancer: indications of liver metastases caused by lymphatic spread.] Ultraschall Med 1999;20:150-7.

Tangkaratt S. Mammographic findings in breast cancer patients, who were treated with breast conserving therapy. J Med Assoc Thai 2004;87:1439-43.

Tella S, Occhiato R, Speziale AM, Poggi R, Grasso F, Frasca F, et al. [Metachronous tumors of the breast. Mammographic findings.] Radiol Med 1994;87:245-9.

Tomiak E, Piccart M. Routine follow-up of patients after primary therapy for early breast cancer: changing concepts and challenges for the future. Ann Oncol 1993;4:199-204.

Tsodikov AD, Asselain B, Fourque A, Hoang T, Yakovlev AY. Discrete strategies of cancer posttreatment surveillance. Estimation and optimization problems. Biometrics 1995;51:437-47.

Vannetzel JM. [Against an intensive strategy for surveillance after treatment of breast cancer.] Gynecol Obstet Fertil 2006;34:271-3. 
Villella JA. Long-term surveillance of previously treated breast cancer survivors by primary care physicians. Primary Care Update Ob/Gyns 2002;9:110-15.

Voogd AC. Local recurrence after breast conservation therapy for early stage breast carcinoma detection, treatment, and outcome in 266 patients. Cancer 1999;85:437-46.

Weight SC, Windle R, Stotter AT. Optimizing surveillance mammography following breast conservation surgery. Eur J Surg Oncol 2002;28:11-13.

Wheeler T, Stenning S, Negus S, Picken S, Metcalfe S. Evidence to support a change in follow-up policy for patients with breast cancer: time to first relapse and hazard rate analysis. Clin Oncol ( $R$ Coll Radiol) 1999;11:169-73.

Wieland-Schneider C. Analysis of a radiologic follow-up program for breast cancer patients. Tumor Diagnostik und Therapie 1995;16:13-15.

Zuber M, Viehl CT, Guller U, Langer I. [Follow-up recommendations for patients after curative treatment of invasive breast cancer.] Ther Umsch 2008;65:319-21.

\section{Outcomes not reported $(\mathrm{n}=12)$}

Bornhak S, Heidemann E, Herschlein HJ, Simon W, Merkle E, Widmaier G, et al. Symptomoriented follow-up of early breast cancer is not inferior to conventional control. Results of a prospective multicentre study. Onkologie 2007;30:443-9.

Gardner B. Surveillance in the management of the cancer patient with special reference to breast and colon cancer. Am J Surg 1997;173:141-4.

Grunfeld E, Julian J, Levine M, Pritchard K. A randomized controlled trial (RCT) of long-term follow-up for early stage breast cancer comparing family physician to specialist care: a report of secondary outcomes. J Clin Oncol 2006;24:301s.

Gulliford T, Opomu M, Wilson E, Hanham I, Epstein R. Popularity of less frequent follow up for breast cancer in randomised study: initial findings from the hotline study. $B M J$ 1997;314:174-7.

Khatcheressian JL, Smith TJ. Randomized trial of long-term follow-up for early-stage breast cancer: a comparison of family physician versus specialist care. J Clin Oncol 2006;24:835-7.

Koinberg IL, Fridlund B, Engholm GB, Holmberg L. Nurse-led follow-up on demand or by a physician after breast cancer surgery: a randomised study. Eur J Oncol Nurs 2004;8:109-17.

Kokko R, Hakama M, Holli K. Follow-up cost of breast cancer patients with localized disease after primary treatment: a randomized trial. Breast Cancer Res Treat 2005;93:255-60.

Orel SG, Troupin RH, Patterson EA, Fowble BL. Breast cancer recurrence after lumpectomy and irradiation: role of mammography in detection. Radiology 1992;183:201-6.

Pinsky RW, Rebner M, Pierce LJ, Ben David MA, Vicini F, Hunt KA, et al. Recurrent cancer after breast-conserving surgery with radiation therapy for ductal carcinoma in situ: mammographic features, method of detection, and stage of recurrence. AJR Am J Roentgenol 2007;189:140-4.

Robinson A, Speers C, Olivotto I, Chia S. Method of detection of new contralateral primary breast cancer in younger versus older women. Clin Breast Cancer 2007;7:705-9.

Robinson E, Adler Z, Nasrallah S, Rennert G, Neugut AI. Clinical characteristics of second primary tumors following breast cancer. Isr J Med Sci 1995;31:169-71.

Simon MS, Stano M, Severson RK, Hoff MS, Smith DW. Clinical surveillance for early stage breast cancer: an analysis of claims data. Breast Cancer Res Treat 1996;40:119-28. 


\section{Not available $(\mathrm{n}=7)$}

Ben-Haim S, Radan L, Bar-Shalom R, Guralnik L, Israel O. FDG-PET/CT in suspected recurrence of breast cancer. Am J Hematol Oncol 2007;6:279-83.

Climent Duran MA, Gomez GA, Segui Palmer MA, Blanco GR, Marco Izquierdo JA, Rueda DA, et al. [The clinico-mammographic follow-up of breast cancer after conservative treatment.] Rev Clin Esp 1993;192:309-14.

Edwards AS. After breast cancer. Patient follow-up in the primary care setting. Adv Nurse Pract 2004;12:37-42.

Martinez-Regueira F, Diez-Caballero A, Sierra A, Espi A, Baixauli J, Zornoza A. [Follow-up of breast cancer: review of 750 cases after 5 years.] Rev Med Univ Navarra 1998;42:7-13.

McLaren B, Wynne C. Breast cancer follow up; how much is enough? NZ Med J 1996;109:125-6.

Menendez C. Management and follow-up of the breast. Rev Iberoamericana 2002;4:15-21.

Reitzig P. [Tumor follow-up care in breast carcinoma - what is sensible?.] Z Arztl Fortbild (Jena) 1991;85:1050-3.

\section{Retained for background information $(\mathrm{n}=49)$}

Abel U, Wollermann C. Methodological aspects of the evaluation of postoperative cancer surveillance. Part II: Efficacy. Clin Lab 2003;49:379-98.

American Society of Clinical Oncology. Recommended breast cancer surveillance guidelines. J Clin Oncol 1997;15:2149-56.

Arriagada R, Le MG, Dunant A, Tubiana M, Contesso G. Twenty-five years of follow-up in patients with operable breast carcinoma - correlation between clinicopathologic factors and the risk of death in each 5-year period. Cancer 2006;106:743-50.

Barnsley GP, Grunfeld E, Coyle D, Paszat L. Surveillance mammography following the treatment of primary breast cancer with breast reconstruction: a systematic review. Plastic Reconst Surg 2007;120:1125-32.

Bartella L. Advances in breast imaging: magnetic resonance imaging. Curr Oncol Rep 2006;8:7-13.

Cameron DA. Extended follow-up of breast cancer patients in clinic wastes time for both patients and doctors: the case against. Breast Cancer Res 2008;10(Suppl. 4):8.

Collins RF, Bekker HL, Dodwell DJ. Follow-up care of patients treated for breast cancer: a structural review. Cancer Treat Rev 2004;30:19-35.

Dawson LA, Chow E, Goss PE. Evolving perspectives in contralateral breast cancer. Eur J Cancer 1998;34:2000-9.

de Bock GH, Bonnema J, van der Hage J, Kievit J, van de Velde CJ. Effectiveness of routine visits and routine tests in detecting isolated locoregional recurrences after treatment for early-stage invasive breast cancer: a meta-analysis and systematic review. J Clin Oncol 2004;22:4010-18.

Dixon JM, Norman B. Routine follow up of breast cancer in primary care. Most recurrences after breast conservation are detected by regular hospital visits. BMJ 1996;313:1548.

Donnelly P, Hiller L, Bathers S, Bowden S, Coleman R. Questioning specialists' attitudes to breast cancer follow-up in primary care. Ann Oncol 2007;18:1467-76.

Easson AM. Management of local recurrence of breast cancer. Expert Rev Anticancer Ther 2004;4:219-26. 
GIVIO Investigators. Impact of follow-up testing on survival and health-related quality of life in breast cancer patients. A multicenter randomized controlled trial. JAMA 1994;271:1587-92.

Grunfeld E, Mant D, Vessey MP, Fitzpatrick R. Specialist and general practice views on routine follow-up of breast cancer patients in general practice. Fam Pract 1995;12:60-5.

Grunfeld E, Mant D, Vessey MP, Yudkin P. Evaluating primary care follow-up of breast cancer: methods and preliminary results of three studies. Ann Oncol 1995;6(Suppl. 2):47-52.

Grunfeld E, Mant D, Yudkin P, Adewuyi-Dalton R, Cole D, Stewart J, et al. Routine follow up of breast cancer in primary care: randomised trial. BMJ 1996;313:665-9.

Grunfeld E, Fitzpatrick R, Mant D, Yudkin P, Adewuyi-Dalton R, Stewart J, et al. Comparison of breast cancer patient satisfaction with follow-up in primary care versus specialist care: results from a randomized controlled trial. Br J Gen Pract 1999;49:705-10.

Grunfeld E, Noorani H, McGahan L, Paszat L, Coyle D, van Walraven C, et al. Surveillance mammography after treatment of primary breast cancer: a systematic review. Breast 2002;11:228-35.

Harries SA, Lawrence RN, Scrivener R, Fieldman NR, Kissin MW. A survey of the management of breast cancer in England and Wales. Ann R Coll Surg Engl 1996;78:197-202.

Hayes DF. Clinical practice. Follow-up of patients with early breast cancer. N Engl J Med 2007;356:2505-13.

Hurria A. Follow-up care of breast cancer survivors. Crit Rev Oncol Hematol 2003;48:89-99.

Kataja VV. ESMO minimum clinical recommendations for diagnosis, treatment and follow-up of locally recurrent or metastatic breast cancer (MBC). Ann Oncol 2005;16:i10-12.

Kataja VV. Recurrent or metastatic breast cancer: ESMO clinical recommendations for diagnosis, treatment and follow-up. Ann Oncol 2007;18:9-11.

Kataja V, Castiglione M, ESMO Guidelines Working Group. Locally recurrent or metastatic breast cancer: ESMO clinical recommendations for diagnosis, treatment and follow-up. Ann Oncol 2008;19(Suppl. 2):ii11-13.

Kelemen LE, Pankratz VS, Sellers TA, Brandt KR, Wang A, Janney C, et al. Age-specific trends in mammographic density: the Minnesota Breast Cancer Family Study. Am J Epidemiol 2008;167:1027-36.

Khatcheressian JL, Smith TJ. Randomized trial of long-term follow-up for early-stage breast cancer: a comparison of family physician versus specialist care. J Clin Oncol 2006;24:835-7.

Khatcheressian J, Swainey C. Breast cancer follow-up in the adjuvant setting. Curr Oncol Rep 2008;10:38-46.

Khatcheressian JL, Wolff AC, Smith TJ, Grunfeld E, Muss HB, Vogel VG, et al. American Society of Clinical Oncology 2006 update of the breast cancer follow-up and management guidelines in the adjuvant setting. J Clin Oncol 2006;24:5091-7.

Kimman ML, Voogd AC, Dirksen CD, Falger P, Hupperets P, Keymeulen K, et al. Follow-up after curative treatment for breast cancer: why do we still adhere to frequent outpatient clinic visits? Eur J Cancer 2007;43:647-53.

Liberati A. The GIVIO trial on the impact of follow-up care on survival and quality of life in breast cancer patients. Interdisciplinary Group for Cancer Care Evaluation. Ann Oncol 1995;6(Suppl. 2):41-6. 
Maher EJ. Non-surgical management of early breast cancer in the United Kingdom: follow-up. Clinical Audit Sub-committee of the Faculty of Clinical Oncology, Royal College of Radiologists, and the Joint Council for Clinical Oncology. Clin Oncol (R Coll Radiol) 1995;7:227-31.

Mertens WC. Contralateral breast cancer: factors associated with stage and size at presentation. Breast J 2004;10:304-12.

Montgomery DA, Krupa K, Cooke TG. Alternative methods of follow up in breast cancer: a systematic review of the literature. Br J Cancer 2007;96:1625-32.

Montgomery DA, Krupa K, Cooke TG. Follow-up in breast cancer: does routine clinical examination improve outcome? A systematic review of the literature. Br J Cancer 2007;97:1632-41.

Palmer A. Disease overview: breast cancer. Drugs in Context 2006;2:285-303.

Riebe E, Gunther K, Schulz K, Kohler G, Schimming A, Schwesinger G, et al. Recurrent disease after breast preserving therapy (BPT) and radiation therapy for breast cancer: diagnostic yield of palpation, mammography and ultrasonography. Ultraschall Med 2007;28:394-400.

Roche N. Follow-up after treatment for breast cancer in young women. Breast 2006;15:S71-5.

Rojas MP, Telaro E, Russo A, Moschetti I, Coe L, Fossati R, et al. Follow-up strategies for women treated for early breast cancer. Cochrane Database Syst Rev 2005;1:CD001768.

Rutgers EJ. Follow-up care in breast cancer. Expert Rev Anticancer Ther 2004;4:212-18.

Sakorafas GH, Tsiotou AG, Pavlakis G. Follow-up after primary treatment for breast cancer. Acta Oncol 2000;39:935-40.

Schootman M, Fuortes L, Aft R. Prognosis of metachronous contralateral breast cancer according to stage at diagnosis: the importance of early detection. Breast Cancer Res Treat 2006;99:91-5.

Sebban E. [For an intensive strategy for surveillance after treatment of breast cancer.] Gynecol Obstet Fertil 2006;34:268-70.

Simon MS, Stano M, Hussein M, Hoff M, Smith D. An analysis of the cost of clinical surveillance after primary therapy for women with early stage invasive breast cancer. Breast Cancer Res Treat 1996;37:39-48.

Smith TE, Lee D, Turner BC, Carter D, Haffty BG. True recurrence vs. new primary ipsilateral breast tumor relapse: an analysis of clinical and pathologic differences and their implications in natural history, prognoses, and therapeutic management. Int J Radiat Oncol Biol Phys 2000;48:1281-9.

Smith TJ, Davidson NE, Schapira DV, Grunfeld E, Muss HB, Vogel VG, III, et al. American Society of Clinical Oncology 1998 update of recommended breast cancer surveillance guidelines. J Clin Oncol 1999;17:1080-2.

Snee M. Follow-up of women treated for breast cancer. Clin Oncol 1996;8:85-9.

Steering Committee on Clinical Practice Guidelines for the Care and Treatment of Breast Cancer. Follow-up after treatment for breast cancer. CMAJ 1998;158(Suppl. 3):65-70.

Temple LK, Wang EE, McLeod RS. Preventive health care, 1999 update: 3. Follow-up after breast cancer. Canadian Task Force on Preventive Health Care. CMAJ 1999;161:1001-8.

Tolaney SM, Winer EP. Follow-up care of patients with breast cancer. Breast 2007; 16(Suppl. 2):S45-50. 


\section{Retained for screening for diagnostic accuracy review $(\mathrm{n}=114)$}

Adler LP, Bakale G, Schnur KC, Shenk RR. Innovations in breast cancer imaging: PET for diagnosis and follow-up. Medscape Womens Health 1998;3:5.

Auguste LJ, Gerold T, Rothenberg H, Litvak S, Gentin S. Detection of recurrent carcinoma in the irradiated breast. Complication Surg 1994;13:556-61.

Bahri S, Chen J-H, Yu HJ, Kuzucan A, Nalcioglu O, Su M-Y. Can dynamic contrast-enhanced MRI (DCE-MRI) predict tumor recurrence and lymph node status in patients with breast cancer? Ann Oncol 2008;19:822-4.

Balu-Maestro C. [Magnetic resonance imaging of the breast.] J Radiol 2001;82:17-26.

Balu-Maestro C, Bruneton JN, Geoffray A, Chauvel C, Rogopoulos A, Bittman O.

Ultrasonographic posttreatment follow-up of breast cancer patients. J Ultrasound Med 1991;10:1-7.

Balu-Maestro C, Bruneton JN, Geoffray A, Chauvel C, Rogopoulos A, Hery MD. [Ultrasonographic surveillance of treated breast cancer.] J Radiol 1991;72:655-61.

Bartella L, Morris EA. Advances in breast imaging: magnetic resonance imaging. Curr Oncol Rep 2006;8:7-13.

Bartella L, Smith CS, Dershaw DD, Liberman L. Imaging breast cancer. Radiol Clin North Am 2007;45:45-67.

Baz E, Madjar H, Reuss C, Vetter M, Hackeloer B, Holz K. The role of enhanced Doppler ultrasound in differentiation of benign vs. malignant scar lesion after breast surgery for malignancy. Ultrasound Obstet Gynecol 2000;15:377-82.

Belli P, Costantini M, Romani M, Marano P, Pastore G. Magnetic resonance imaging in breast cancer recurrence. Breast Cancer Res Treat 2002;73:223-35.

Belli P, Pastore G, Romani M, Terribile D, Canade A, Costantini M. Role of magnetic resonance imaging in the diagnosis of recurrence after breast conserving therapy. Rays 2002;27:241-57.

Boné B, Aspelin P, Isberg B, Perbeck L, Veress B. Contrast-enhanced MR imaging of the breast in patients with breast implants after cancer surgery. Acta Radiol 1995;36:111-16.

Bongers V, Perre C, de Hooge P. The use of scintimammography for detecting the recurrence of loco-regional breast cancer: histopathologically proven results. Nucl Med Commun 2004;25:145-9.

Buckley DL, Mussurakis S, Horsman A. Effect of temporal resolution on the diagnostic efficacy of contrast-enhanced MRI in the conservatively treated breast. J Comput Assist Tomogr 1998;22:47-51.

Buscombe JR, Cwikla JB, Thakrar DS, Parbhoo SP, Hilson AJ. Prone SPET scintimammography. Nucl Med Commun 1999;20:237-45.

Buscombe JR, Holloway B, Roche N, Bombardieri E. Position of nuclear medicine modalities in the diagnostic work-up of breast cancer. Quart J Nucl Med Mol Imaging 2004;48:109-18.

Buthiau D, Rixe O, Nizri D, Piette JC, Khayat D. [Breast cancer: role of MRI in follow-up of cases treated.] Bull Acad Natl Med 1995;179:693-705.

Chen SL. The survival impact of the choice of surgical procedure after ipsilateral breast cancer recurrence. Am J Surg 2008;196:495-9. 
Cheng JC, Cheng SH, Lin KJ, Jian JJ, Chan KY, Huang AT. Diagnostic thoracic-computed tomography in radiotherapy for loco-regional recurrent breast carcinoma. Int J Radiation Oncol Biol Phys 1998;41:607-13.

Ciatto S, Ambrogetti D, Muraca MG. [Combined diagnosis of breast cancer recurrences after conservative treatment. Critical review of 143 consecutive cases.] Radiol Med 1995;90:383-5.

Ciatto S, Catallotti L, Distante V, Rontini M, Muraca MG. Diagnostic features of 225 consecutive cases of cancer recurrence in the conserved breast. Breast 1997;6:367-70.

Cid JA, Rampaul RS, Ellis IO, Wilson AR, Burrell HC, Evans AJ, et al. Woman feels breast lump: surgeon cannot: the role of ultrasound in arbitration. Eur J Cancer 2004;40:2053-5.

Claus EB, Stowe M, Carter D, Holford T. The risk of a contralateral breast cancer among women diagnosed with ductal and lobular breast carcinoma in situ: data from the Connecticut Tumor Registry. Breast 2003;12:451-6.

Cohen EK, Leonhardt CM, Shumak RS, Souter IC, Bukhanov K, Fishell EK, et al. Magnetic resonance imaging in potential postsurgical recurrence of breast cancer: pitfalls and limitations. Can Assoc Radiol J 1996;47:171-6.

Coulthard A, Beveridge CJ, Potterton AJ. MRI in routine breast cancer follow-up: correlation with clinical outcome. Clin Radiol 1999;54:459-61.

Crystal P, Strano SD, Shcharynski S, Koretz MJ. Using sonography to screen women with mammographically dense breasts. AJR Am J Roentgenol 2003;181:177-82.

Cwikla JB, Buscombe JR, Parbhoo SP, Kelleher SM, Thakrar DS, Hinton J, et al. Use of 99TcmMIBI in the assessment of patients with suspected recurrent breast cancer. Nucl Med Commun 1998;19:649-55.

Cwikla JB, Kolasinska A, Buscombe JR, Hilson AJ. Tc-99m MIBI in suspected recurrent breast cancer. Cancer Biotherap Radiopharmaceut 2000;15:367-72.

Dao TH, Rahmouni A, Servois V, Nguyen-Tan T. MR imaging of the breast in the follow-up evaluation of conservative nonoperatively treated breast cancer. Magn Reson Imaging Clin N Am 1994;2:605-22.

Datoc PD, Hayes CW, Conway WF, Bosch HA, Neal MP. Mammographic follow-up of nonpalpable low-suspicion breast abnormalities: one versus two views. Radiology 1991; 180:387-91.

Davis PL, McCarty KS, Jr. Sensitivity of enhanced MRI for the detection of breast cancer: new, multicentric, residual, and recurrent. Eur Radiol 1997;7(Suppl. 5):289-98.

Delorme S. [Breast cancer. Sonography and magnetic resonance mammography.] Radiologie 2004;44:621-37.

Dinh P, Cardoso F, Sotiriou C, Piccart-Gebhart MJ. New tools for assessing breast cancer recurrence. Cancer Treat Res 2008;141:99-118.

Emens LA, Davidson NE. The follow-up of breast cancer. Semin Oncol 2003;30:338-48.

Eubank WB, Mankoff DA, Vesselle HJ, Eary JF, Schubert EK, Dunnwald LK, et al. Detection of locoregional and distant recurrences in breast cancer patients by using FDG PET. Radiographics 2002;22:5-17.

Fajardo LL, Roberts CC, Hunt KR. Mammographic surveillance of breast cancer patients: should the mastectomy site be imaged? AJR Am J Roentgenol 1993;161:953-5.

Flanagan FL, Dehdashti F, Siegel BA. PET in breast cancer. Semin Nucl Med 1998;28:290-302. 
Flobbe K, Kessels AGH, Severens JL, Beets GL, de Koning HJ, von Meyenfeldt MF, et al. Costs and effects of ultrasonography in the evaluation of palpable breast masses. Int $J$ Technol Assess Health Care 2004;20:440-8.

Fowble B, Solin LJ, Schultz DJ, Rubenstein J, Goodman RL. Breast recurrence following conservative surgery and radiation: patterns of failure, prognosis, and pathologic findings from mastectomy specimens with implications for treatment. Int J Radiat Oncol Biol Phys 1990;19:833-42.

Gallowitsch HJ, Kresnik E, Gasser J, Kumnig G, Igerc I, Mikosch P, et al. F-18 fluorodeoxyglucose positron-emission tomography in the diagnosis of tumor recurrence and metastases in the follow-up of patients with breast carcinoma: a comparison to conventional imaging. Invest Radiol 2003;38:250-6.

Gilles R, Guinebretiere JM, Shapeero LG, Lesnik A, Contesso G, Sarrazin D, et al. Assessment of breast-cancer recurrence with contrast-enhanced subtraction MR-imaging - preliminary-results in 26 patients. Radiology 1993;188:473-8.

Giuseppetti GM, Baldassarre S, Manna P. [Echography of the operated breast.] Radiol Med 1992;83:402-6.

Goerres GW, Michel SC, Fehr MK, Kaim AH, Steinert HC, Seifert B, et al. Follow-up of women with breast cancer: comparison between MRI and FDG PET. Eur Radiol 2003;13:1635-44.

Gojkovic Z. Comparison of ultrasound controlled aspiration puncture to clinical examination in detection of breast cancer recurrence. Arch Oncol 2006;14:118-21.

Gorechlad JW, McCabe EB, Higgins JH, Likosky DS, Lewis PJ, Rosenkranz KM, et al. Screening for recurrences in patients treated with breast-conserving surgery: is there a role for MRI? Ann Surg Oncol 2008;15:1703-9.

Gunhan-Bilgen I, Oktay A. Mammographic features of local recurrence after conservative surgery and radiation therapy: comparison with that of the primary tumor. Acta Radiol 2007;48:390-7.

Hagay C, Cherel PJ, de Maulmont CE, Plantet MM, Gilles R, Floiras JL, et al. Contrast-enhanced $\mathrm{CT}$ : value for diagnosing local breast cancer recurrence after conservative treatment. Radiology 1996;200:631-8.

Hall NJ, Evans AJ, Kollias J, Denley H, Pinder SE, Ellis IO, et al. Bilateral breast carcinomas: do they have similar mammographic features? Clin Radiol 1999;54:434-7.

Hermans J. Scintimammography: a new imaging technique for diagnosis and follow-up of breast cancer. Bull Cancer 2000;87:334-40.

Heywang-Kobrunner SH, Schlegel A, Beck R, Wendt T, Kellner W, Lommatzsch B, et al. Contrast-enhanced MRI of the breast after limited surgery and radiation therapy. J Comput Assist Tomogr 1993;17:891-900.

Jager JJ, Langendijk JA, Dohmen JP, Schreutelkamp IL, Volovics L, Vanengelshoven JM, et al. Mammography in the follow-up after breast-conserving treatment in cancer of the breast: suitability for mammographic interpretation, validity and interobserver variation. Br J Radiol 1995;68:754-60.

Kato K. Diagnostic accuracy and limitations of breast sonography. Geburtsh Frauenheilk 1998;58:1-5.

Kim MJ, Kim EK, Kwak JY, Park BW, Kim SI, Sohn J, et al. Role of sonography in the detection of contralateral metachronous breast cancer in an Asian population. AJR Am J Roentgenol 2008;190:476-80. 
Kleit AN, Ruiz JF. False positive mammograms and detection controlled estimation. Health Serv Res 2003;38:1207-28.

Kramer S, Schulz-Wendtland R, Hagedorn K, Bautz W, Lang N. Magnetic resonance imaging in the diagnosis of local recurrences in breast cancer. Anticancer Res 1998;18:2159-61.

Lafata JE, Simpkins J, Schultz L, Chase GA, Johnson CC, Yood MU, et al. Routine surveillance care after cancer treatment with curative intent. Med Care 2005;43:592-9.

Lee VW, Sax EJ, McAneny DB, Pollack S, Blanchard RA, Beazley RM, et al. A complementary role for thallium-201 scintigraphy with mammography in the diagnosis of breast cancer. $J \mathrm{Nucl}$ Med 1993;34:2095-100.

Leikola J, Saarto T, Joensuu H, Sarvas K, Vironen J, Von Smitten K, et al. Ultrasonography of the axilla in the follow-up of breast cancer patients who have a negative sentinel node biopsy and who avoid axillary clearance. Acta Oncol 2006;45:571-5.

Leivo T, Salminen T, Sintonen H, Tuominen R, Auerma K, Partanen K, et al. Incremental costeffectiveness of double-reading mammograms. Breast Cancer Res Treat 1999;54:261-7.

Lewis-Jones HG, Whitehouse GH, Leinster SJ. The role of magnetic resonance imaging in the assessment of local recurrent breast carcinoma. Clin Radiol 1991;43:197-204.

Liberman L. Breast MR imaging in assessing extent of disease. Magn Reson Imaging Clin N Am 2006;14:339-49.

Lind P, Igerc I, Beyer T, Reinprecht P, Hausegger K. Advantages and limitations of FDG PET in the follow-up of breast cancer. Eur J Nucl Med Mol Imaging 2004;31(Suppl. 1):125-34.

Mangkharak J. Scintimammography (SMM) in breast cancer patients. J Med Assoc Thai 1999;82:242-9.

Margolese RG. Recent trends in the management of breast cancer. 4. Diagnosis and management of local recurrence after breast-conservation surgery. Can J Surg 1992;35:378-81.

Mathieu I, Mazy S, Willemart B, Destine M, Mazy G, Lonneux M. Inconclusive triple diagnosis in breast cancer imaging: is there a place for scintimammography? J Nucl Med 2005;46:1574-81.

Melani E, Sardanelli F, Ottonello C, Parodi RC, Castaldi A, Succi S. [Magnetic resonance mammography in suspected tumor recurrences.] Radiol Med 1995;89:219-24.

Molino A. What is the best follow-up methodology in early breast cancer? Breast 2008;29:1-2.

Moskovic E, Curtis S, A'Hern RP, Harmer CL, Parsons C. The role of diagnostic CT scanning of the brachial plexus and axilla in the follow-up of patients with breast cancer. Clin Oncol 1992;4:74-7.

Moy L, Murphy-Walcott AD. Improving specificity of breast MRI using prone PET and fused MRI and PET 3D volume datasets. J Nucl Med 2007;48:528-37.

Mumtaz H, Davidson T, Hall-Craggs MA, Payley M, Walmsley K, Cowley G, et al. Comparison of magnetic resonance imaging and conventional triple assessment in locally recurrent breast cancer. Br J Surg 1997;84:1147-51.

Musumeci R. Radiologic procedures for the diagnosis of distant recurrences of breast cancer. Ann Oncol 1995;6(Suppl. 2):27-9.

Muuller RD, Barkhausen J, Sauerwein W, Langer R. Assessment of local recurrence after breastconserving therapy with MRI. J Comput Assist Tomogr 1998;22:408-12.

Newstead GM. MR imaging in the management of patients with breast cancer. Semin Ultrasound CT MRI 2006;27:320-32. 
Noh DY, Yun IJ, Kim JS, Kang HS, Lee DS, Chung JK, et al. Diagnostic value of positron emission tomography for detecting breast cancer. World J Surg 1998;22:223-7.

Nowicki A, Olszewska A, Humanska M. [Self-examination influence on breast cancer detection. Research conducted in women after breast amputation.] Ginekol Pol 2007;78:293-8.

Orel SG, Fowble BL, Solin LJ, Schultz DJ, Conant EF, Troupin RH. Breast cancer recurrence after lumpectomy and radiation therapy for early-stage disease: prognostic significance of detection method. Radiology 1993; 188:189-94.

Patel MR, Whitman GJ. Negative mammograms in symptomatic patients with breast cancer. Acad Radiol 1998;5:26-33.

Pediconi F, Catalano C, Roselli A, Padula S, Altomari F, Moriconi E, et al. Contrast-enhanced MR mammography for evaluation of the contralateral breast in patients with diagnosed unilateral breast cancer or high-risk lesions. Radiology 2007;243:670-80.

Pepe MS, Urban N, Rutter C, Longton G. Design of a study to improve accuracy in reading mammograms. J Clin Epidemiol 1997;50:1327-38.

Potterton AJ, Yuille F, Tinkler S, Chippindale AJ, Wilsdon JB, Lucraft HH, et al. MRI appearances of the axilla in treated breast cancer. Br J Radiol 1999;72:250-7.

Prasad A. A radiologist's perspective in breast cancer. J Int Med Sci Acad 2006;19:15-19.

Preda L, Villa G, Rizzo S, Bazzi L, Origgi D, Cassano E, et al. Magnetic resonance mammography in the evaluation of recurrence at the prior lumpectomy site after conservative surgery and radiotherapy. Breast Cancer Res 2006;8:R53.

Propeck PA, Scanlan KA. Utility of axillary views in postmastectomy patients. Radiology 1993;187:769-71.

Rajkovaca Z, Vuleta G, Matavulj A, Kovacevic P, Ponorac N. 99m Tc-sestamibi scintimammography in detection of recurrent breast cancer. Bosnian J Basic Med Sci 2007;7:256-60.

Rieber A, Merkle E, Zeitler H, Gorich J, Kreienberg R, Brambs HJ, et al. Value of MR mammography in the detection and exclusion of recurrent breast carcinoma. J Comput Assist Tomogr 1997;21:780-4.

Rissanen TJ, Makarainen HP, Mattila SI, Lindholm EL, Heikkinen MI, Kiviniemi HO. Breast cancer recurrence after mastectomy: diagnosis with mammography and US. Radiology 1993;188:463-7.

Rissanen TJ, Makarainen HP, Apaja-Sarkkinen MA, Lindholm EL. Mammography and ultrasound in the diagnosis of contralateral breast cancer. Acta Radiol 1995;36:358-66.

Rissanen TJ, Apaja-Sarkkinen MA, Makarainen HP, Heikkinen MI. Ultrasound-guided fine needle aspiration biopsy in the diagnosis of breast cancer recurrence after mastectomy. Acta Radiol 1997;38:232-9.

Rostom AY, Powe J, Kandil A, Ezzat A, Bakheet S, el Khwsky F, et al. Positron emission tomography in breast cancer: a clinicopathological correlation of results. Br J Radiol 1999;72:1064-8.

Roubidoux MA, Helvie MA, Lai NE, Paramagul C. Bilateral breast cancer: early detection with mammography. Radiology 1995;196:427-31.

Safir J, Zito JL, Gershwind ME, Faegenburg D, Tobin CE, Cayea PD, et al. Contrast-enhanced breast MRI for cancer detection using a commercially available system: a perspective. Clin Imaging 1998;22:162-79. 
Schulz-Wendtland R. Follow-up after breast-conserving therapy: comparison of conventional imaging methods with MRI. Geburtsh Frauenheilk 2001;61:396-9.

Seely JM, Nguyen ET, Jaffey J. Breast MRI in the evaluation of locally recurrent or new breast cancer in the postoperative patient: correlation of morphology and enhancement features with the BI-RADS category. Acta Radiol 2007;48:838-45.

Shin JH, Han BK, Choe YH, Nam SJ, Park W, Im YH. Ultrasonographic detection of occult cancer in patients after surgical therapy for breast cancer. J Ultrasound Med 2005;24:643-9.

Sickles EA. Periodic mammographic follow-up of probably benign lesions: results in 3,184 consecutive cases. Radiology 1991;179:463-8.

Siggelkow W, Zimny M, Faridi A, Petzold K, Buell U, Rath W. The value of positron emission tomography in the follow-up for breast cancer. Anticancer Res 2003;23:1859-67.

Simonetti G, Cossu E, Montanaro M, Caschili C, Giuliani V. What's new in mammography. Eur J Radiol 1998;27(Suppl. 2):234-41.

Snider HC, Rubin E, Henson R. Axillary ultrasonography to detect recurrence after sentinel node biopsy in breast cancer. Ann Surg Oncol 2006;13:501-7.

Snow RD, Dyess DL, Harpen MD, Kreisberg CN, Tucker JA. Dynamic magnetic resonance imaging in evaluating suspicious breast lesions: correlation with pathologic findings. South Med J 1998;91:527-32.

Spanu A, Farris A, Schillaci O, Chessa F, Solinas ME, Falchi A, et al. The usefulness of 99mTc tetrofosmin scintigraphy in patients with breast cancer recurrences. Nucl Med Commun 2003;24:145-54.

Stickeler E. Diagnosis, staging and follow-up in breast cancer patients. Breast Care 2007;2:158-62.

Strax P. Imaging. Follow-up of breast cancer reconstruction cases. Cancer 1991;68:1157-8.

Suissa M. Imaging the breast after surgery, radiation therapy or medical treatment. Imagerie de la Femme 2006;16:237-49.

Susini T, Nori J, Vanzi E, Livi L, Pecchioni S, Bazzocchi M, et al. Axillary ultrasound scanning in the follow-up of breast cancer patients undergoing sentinel node biopsy. Breast 2007;16:190-6.

Taourel P, Hoquet-Devaux M, Curros-Doyon F, Merigeaud S, Prat X. Does breast density have an impact on the indications for MRI? J Radiol 2008;89:1187-95.

Turnbull LW. Magnetic resonance imaging of the breast: potential for gain in the detection, staging and diagnosis of primary and recurrent tumour? Breast 1998;7:1-4.

Usmani S, Niaz K, Maseeh UZ, Kamal S, Niyaz K, Mehboob J, et al. Role of 99mTc-MIBI scintimammography and X-ray mammography in the diagnosis of locoregional recurrence of breast cancer. JPMA 2007;57:172-5.

Valenzuela P, Mateos S, Tello E, Lopez-Bueno MJ, Garrido N, Gaspar MJ. The contribution of the CEA marker to CA 15.3 in the follow-up of breast cancer. Eur J Gynaecol Oncol 2003;24:60-2.

Viehweg P, Heinig A, Lampe D, Buchmann J, Heywang-Kobrunner SH. Retrospective analysis for evaluation of the value of contrast-enhanced MRI in patients treated with breast conservative therapy. MAGMA 1998;7:141-52.

Viehweg P, Rotter K, Laniado M, Lampe D, Buchmann J, Kolbl H, et al. MR imaging of the contralateral breast in patients after breast-conserving therapy. Eur Radiol 2004;14:402-8.

Warren RM, Duffy SW. Comparison of single reading with double reading of mammograms, and change in effectiveness with experience. Br J Radiol 1995;68:958-62. 
Wieland-Schneider C, Voges E, Kauczor H, Thelen M. [The aftercare of breast carcinoma. The value of plain thoracic radiography, abdominal sonography and mammography.] ROFO-Fortschr Rontg 1994;160:513-17.

Williams LJ, Hartswood M, Prescott RJ. Methodological issues in mammography double reading studies. J Med Screen 1998;5:202-6.

Winehouse J, Douek H, Holz K, Madjar H, Gillams A, Lees W, et al. Contrast-enhanced colour Doppler ultrasonography in suspected breast cancer recurrence. Br J Surg 1999;86:1198-201. 



\section{Appendix 13}

\section{Data extraction form for the systematic review of clinical effectiveness of surveillance mammography}


HTA Mammography Surveillance - Clinical Effectiveness Review 1 data extraction form Version 3

Study id:

Extractor initials:

Date:

Study ids of linked reports:

Aim of study:

Study design:

$\square \quad R C T$

$\square \quad$ Non-randomised comparison

$\square \quad$ Prospective/Retrospective cohort (please underline)

Multicentre study? $\square \quad$ Yes If Yes number of centres:

$$
\square \quad \text { No }
$$

Length of follow-up:

Study start/end dates:

Duration of study:

Country:

Source of funding:

Additional information on study design:

Types of participants:

$\square \quad$ Women without detectable metastatic disease who have received breast conserving surgery for primary breast cancer

$\square \quad$ Women without detectable metastatic disease who have received mastectomy for primary breast cancer 


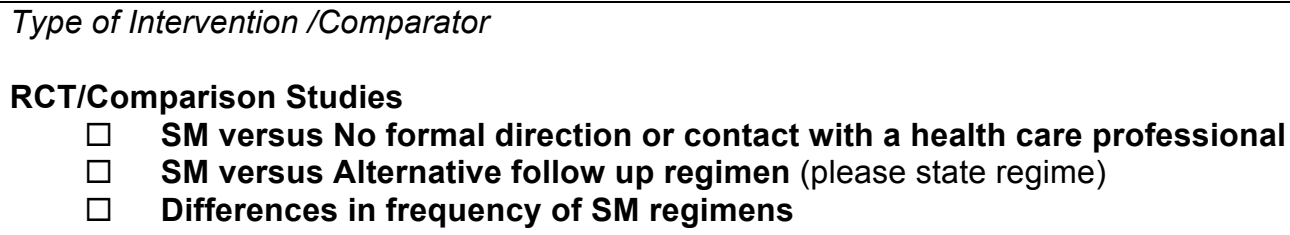

Cohorts:
$\square \quad$ Surveillance Mammography
$\square \quad$ Alternative follow up regimen (please state regimen)

\section{Outcomes reported:}

\section{$\square \quad$ IBTR}

$\square \quad$ Overall survival

$\square \quad$ Disease free survival

$\square \quad$ QOL

$\square \quad$ Harms of mammography

$\square \quad$ Uptake of mammography $\square \quad$ МсвС
$\square \quad$ Overall survival
$\square \quad$ Disease free survival
$\square \quad$ QOL
$\square \quad$ Harms of mammography

\section{Participant Characteristics}

Study inclusion criteria:

\section{Study exclusion criteria:}

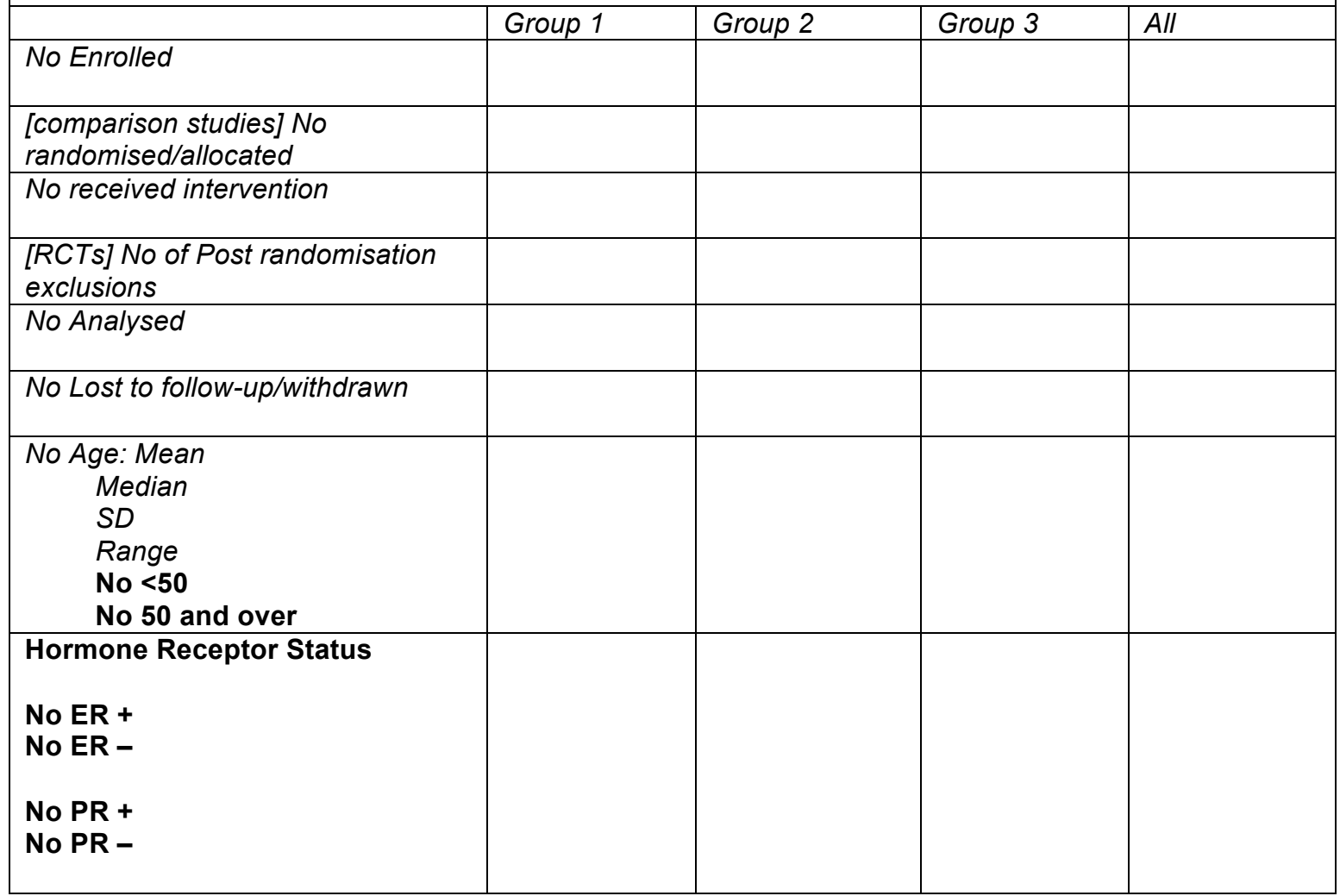




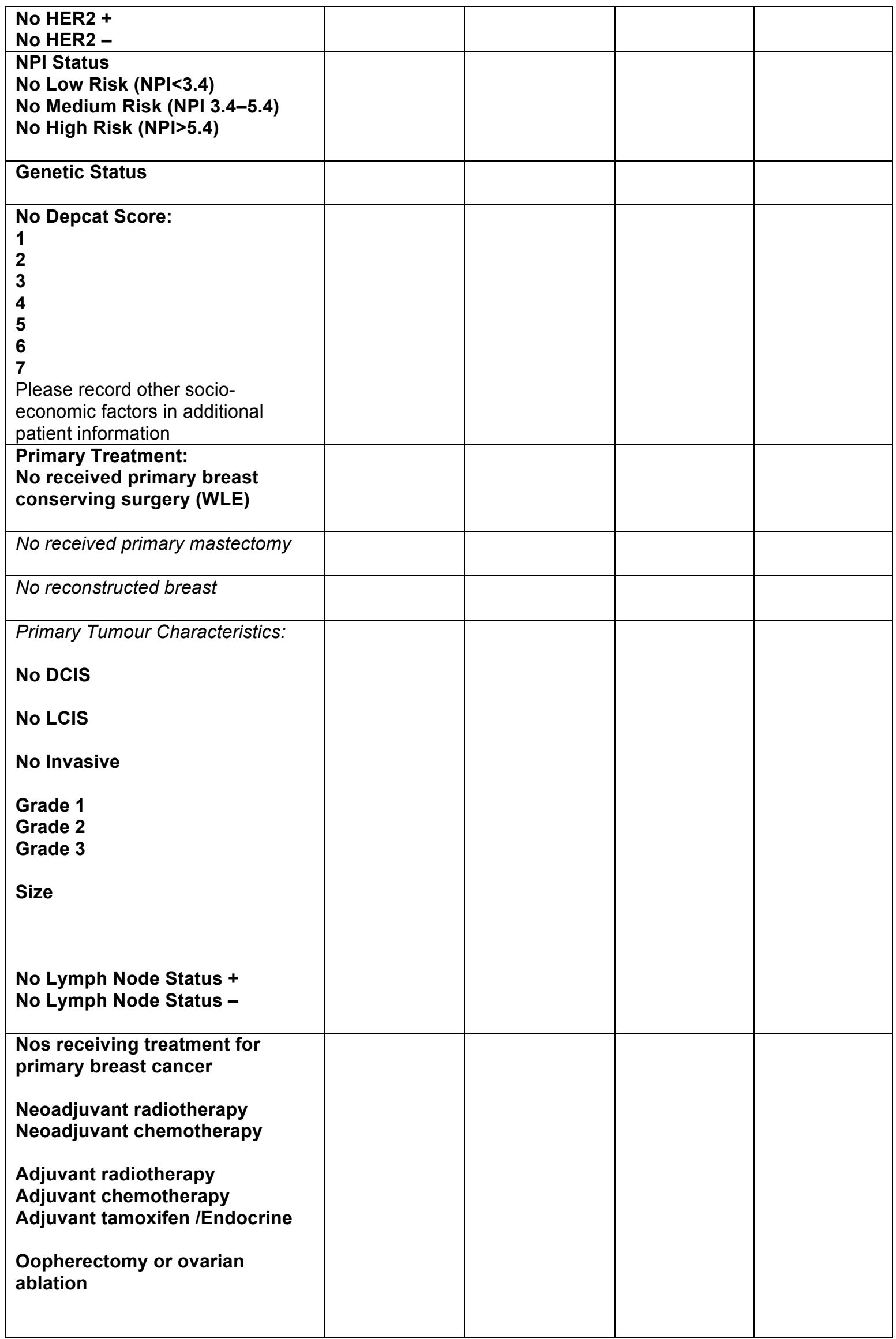




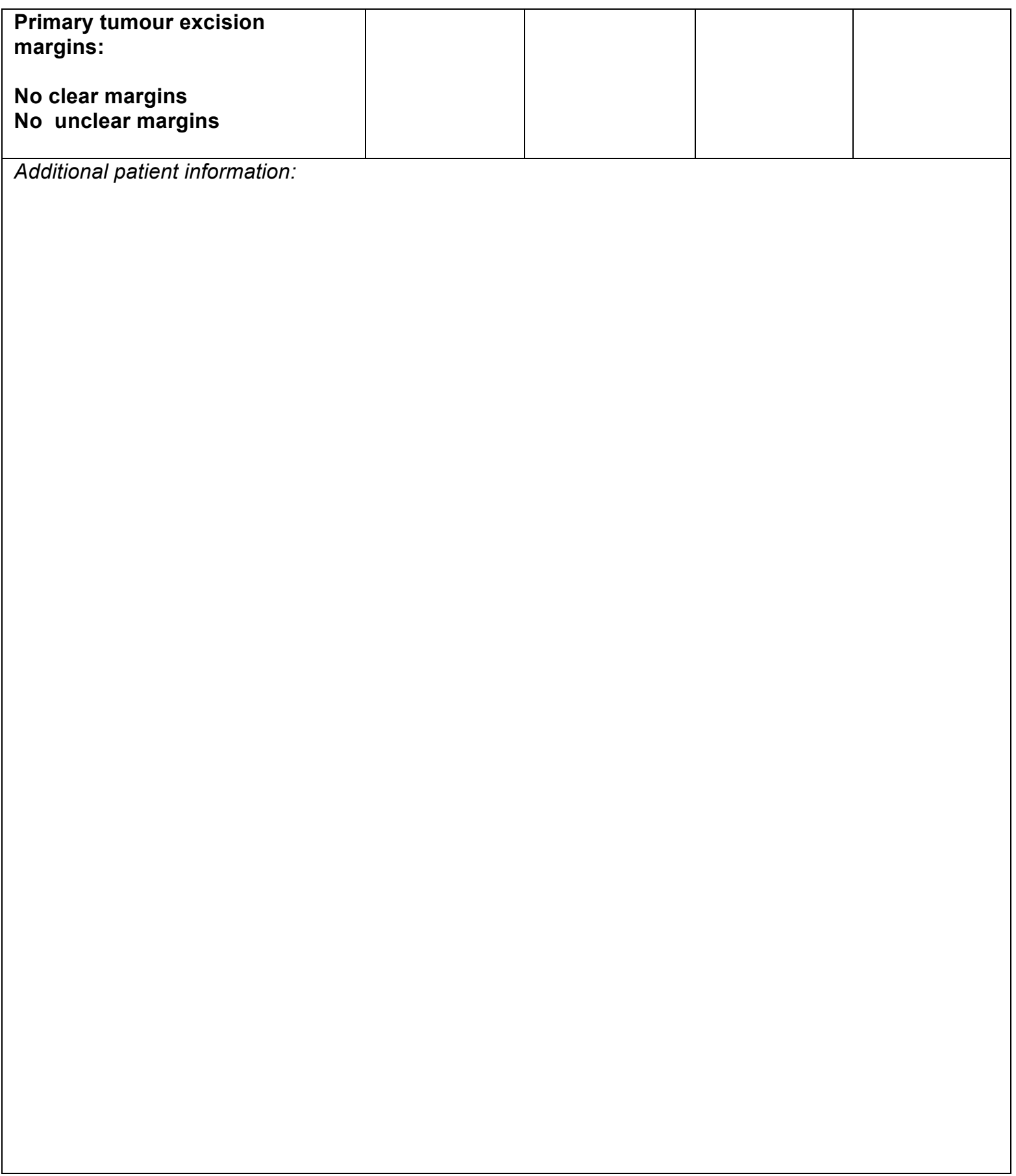


Intervention Group - Group 1

Components of the Intervention

\section{Component 1}

$\square \quad$ Surveillance Mammography

Unstructured Primary Care Follow Up

Structured Primary Care Follow Up

$\square \quad$ Specialist led Clinical Exam

$\square \quad$ Healthcare Professional directed self-exam

$\square \quad$ Alternative surveillance regimen (please state)

Intervention Setting

$\square \quad$ Secondary Care

$\square \quad$ Primary Care

$\square \quad$ Other (please state)

Who administered the intervention? (please give experience level if recorded)
$\square \quad$ Radiologist
$\square \quad$ Hospital Clinician
$\square \quad$ Breast Care Nurse
$\square \quad$ Patient
$\square \quad$ Other (please state)

How long after primary treatment/at what time point was the intervention (or component 1 of the intervention) initiated?

Frequency of the intervention:

Duration of the intervention: 
Components of the Intervention

\section{Component 2}

$\square \quad$ Surveillance Mammography

Unstructured Primary Care Follow Up

$\square \quad$ Structured Primary Care Follow Up

$\square \quad$ Specialist led Clinical Exam

$\square \quad$ Healthcare Professional directed self-exam

$\square \quad$ Alternative surveillance regimen (please state)

Intervention Setting
$\square \quad$ Secondary Care
$\square \quad$ Primary Care
$\square \quad$ Other (please state)

Who administered the intervention? (please give experience level if recorded)
$\square \quad$ Radiologist
$\square \quad$ Hospital Clinician
$\square \quad$ Breast Care Nurse
$\square \quad$ Patient
$\square \quad$ Other (please state)

How long after primary treatment/at what time point was the intervention (or component 2 of the intervention) initiated?

Frequency of the intervention:

Duration of the intervention: 
Components of the Intervention

\section{Component 3}

$\square \quad$ Surveillance Mammography

Unstructured Primary Care Follow Up

$\square \quad$ Structured Primary Care Follow Up

$\square \quad$ Specialist led Clinical Exam

$\square \quad$ Healthcare Professional directed self-exam

$\square \quad$ Alternative surveillance regimen (please state)

Intervention Setting
$\square \quad$ Secondary Care
$\square \quad$ Primary Care
$\square \quad$ Other (please state)

Who administered the intervention? (please give experience level if recorded)
$\square \quad$ Radiologist
$\square \quad$ Hospital Clinician
$\square \quad$ Breast Care Nurse
$\square \quad$ Patient
$\square \quad$ Other (please state)

How long after primary treatment/at what time point was the intervention (or component 3 of the intervention) initiated?

Frequency of the intervention:

Duration of the intervention:

Please use a separate sheet for any additional Group 1 intervention components. 
Comparison Group - Group 2

Components of the Comparator

\section{Component 1}

$\square \quad$ Surveillance Mammography

Unstructured Primary Care Follow Up

$\square \quad$ Structured Primary Care Follow Up

$\square \quad$ Specialist led Clinical Exam

$\square \quad$ Healthcare Professional directed self-exam

$\square \quad$ Alternative surveillance regimen (please state)

Comparator Setting

$\square \quad$ Secondary Care

$\square \quad$ Primary Care

$\square \quad$ Other (please state)

Who administered the comparator? (please give experience level if recorded)
$\square \quad$ Radiologist
$\square \quad$ Hospital Clinician
$\square \quad$ Breast Care Nurse
$\square \quad$ Patient
$\square \quad$ Other (please state)

How long after primary treatment/at what time point was the comparator (or component 1 of the comparator) initiated?

Frequency of the comparator:

Duration of the comparator: 
Components of the Comparator

\section{Component 2}

$\square \quad$ Surveillance Mammography

Unstructured Primary Care Follow Up

$\square \quad$ Structured Primary Care Follow Up

$\square \quad$ Specialist led Clinical Exam

$\square \quad$ Healthcare Professional directed self-exam

$\square \quad$ Alternative surveillance regimen (please state)

Intervention Setting
$\square$ Secondary Care
$\square \quad$ Primary Care
$\square \quad$ Other (please state)

Who administered the intervention? (please give experience level if recorded)
$\square \quad$ Radiologist
$\square \quad$ Hospital Clinician
$\square \quad$ Breast Care Nurse
$\square \quad$ Patient
$\square \quad$ Other (please state)

How long after primary treatment/at what time point was the comparator (or component 2 of the comparator) initiated?

Frequency of the comparator:

Duration of the comparator: 
Components of the Comparator

\section{Component 3}

$\square \quad$ Surveillance Mammography

Unstructured Primary Care Follow Up

$\square \quad$ Structured Primary Care Follow Up

$\square \quad$ Specialist led Clinical Exam

$\square \quad$ Healthcare Professional directed self-exam

$\square \quad$ Alternative surveillance regimen (please state)

Intervention Setting
$\square \quad$ Secondary Care
$\square \quad$ Primary Care
$\square \quad$ Other (please state)

Who administered the comparator? (please give experience level if recorded)
$\square \quad$ Radiologist
Hospital Clinician
Breast Care Nurse
Patient
$\square \quad$ Other (please state)

How long after primary treatment/at what time point was the comparator (or component 3 of the comparator) initiated?

Frequency of the comparator:

Duration of the comparator:

Please use a separate sheet for any additional Group2 comparator components. 


\section{Comparison Group - Group 3}

Components of the Comparator

\section{Component 1}

$\square \quad$ Surveillance Mammography

$\square \quad$ Unstructured Primary Care Follow Up

$\square \quad$ Structured Primary Care Follow Up

$\square \quad$ Specialist led Clinical Exam

$\square \quad$ Healthcare Professional directed self-exam

$\square \quad$ Alternative surveillance regimen (please state)

\section{Comparator Setting}
$\square \quad$ Secondary Care
$\square \quad$ Primary Care
$\square \quad$ Other (please state)

Who administered the comparator? (please give experience level if recorded)
$\square \quad$ Radiologist
$\square \quad$ Hospital Clinician
$\square \quad$ Breast Care Nurse
$\square \quad$ Patient
$\square \quad$ Other (please state)

How long after primary treatment/at what time point was the comparator (or component 1 of the comparator) initiated?

Frequency of the comparator:

Duration of the comparator: 
Components of the Comparator

\section{Component 2}

$\square \quad$ Surveillance Mammography

Unstructured Primary Care Follow Up

Structured Primary Care Follow Up

Specialist led Clinical Exam

Healthcare Professional directed self-exam

Alternative surveillance regimen (please state)

Intervention Setting

$\square \quad$ Secondary Care

Primary Care

$\square \quad$ Other (please state)

Who administered the intervention? (please give experience level if recorded)
$\square \quad$ Radiologist
Hospital Clinician
$\square \quad$ Breast Care Nurse
$\square \quad$ Patient
$\square \quad$ Other (please state)

How long after primary treatment/at what time point was the comparator (or component 2 of the comparator) initiated?

Frequency of the comparator:

Duration of the comparator: 
Components of the Comparator

\section{Component 3}

$\square \quad$ Surveillance Mammography

Unstructured Primary Care Follow Up

$\square \quad$ Structured Primary Care Follow Up

$\square \quad$ Specialist led Clinical Exam

$\square \quad$ Healthcare Professional directed self-exam

$\square \quad$ Alternative surveillance regimen (please state)

Intervention Setting
$\square \quad$ Secondary Care
$\square \quad$ Primary Care
$\square \quad$ Other (please state)

Who administered the comparator? (please give experience level if recorded)
$\square \quad$ Radiologist
$\square \quad$ Hospital Clinician
$\square \quad$ Breast Care Nurse
$\square \quad$ Patient
$\square \quad$ Other (please state)

How long after primary treatment/at what time point was the comparator (or component 3 of the comparator) initiated?

Frequency of the comparator:

Duration of the comparator:

Please use a separate sheet for any additional intervention components.

Outcome(s) reported and time point(s)

How were outcome data collected/measured?

Did the analysis adjust for any confounding factors (if yes please state the confounding factor(s)?

How was the confounding factor categorised? (e.g. Age, $<50$ or $50>$ ) 


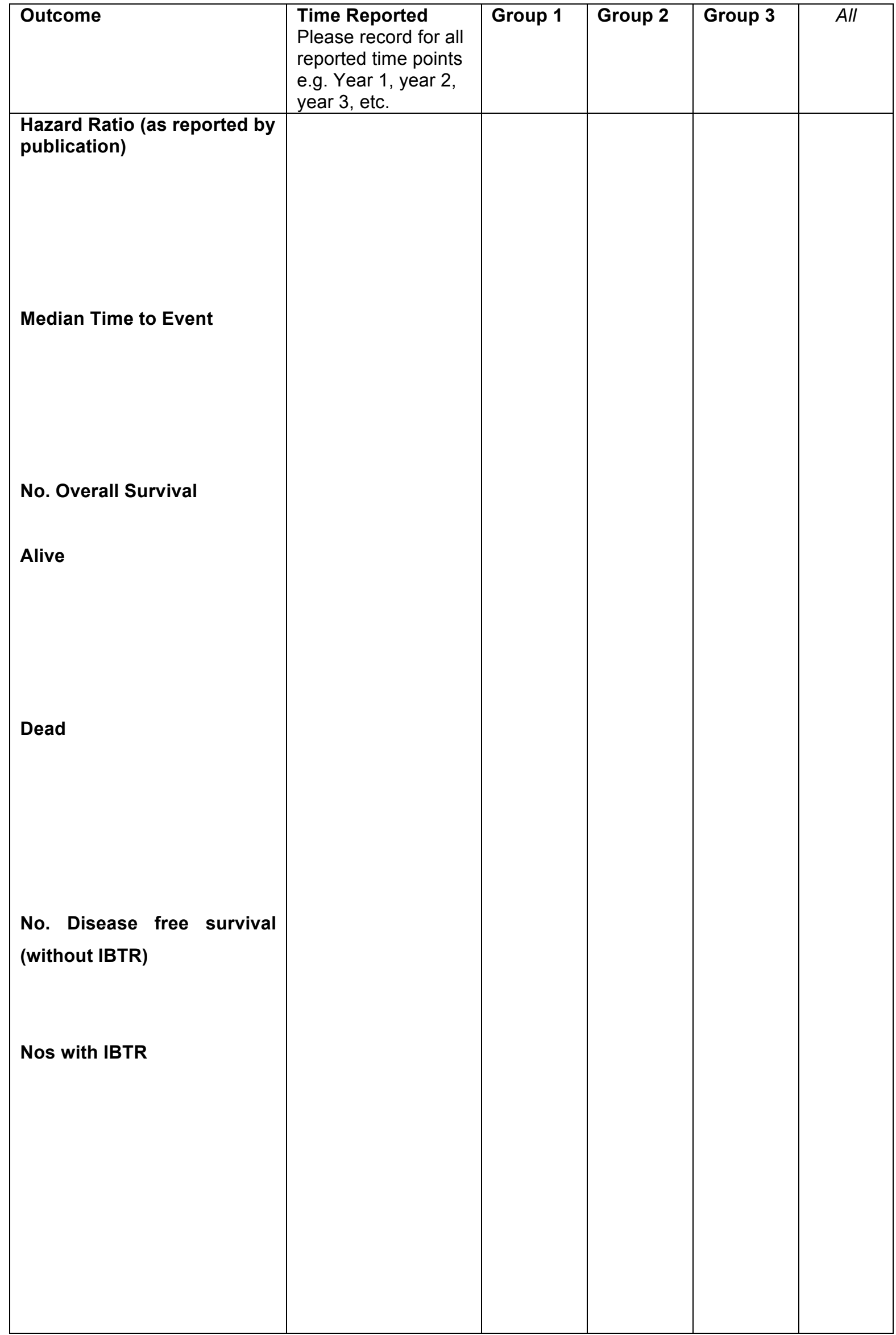




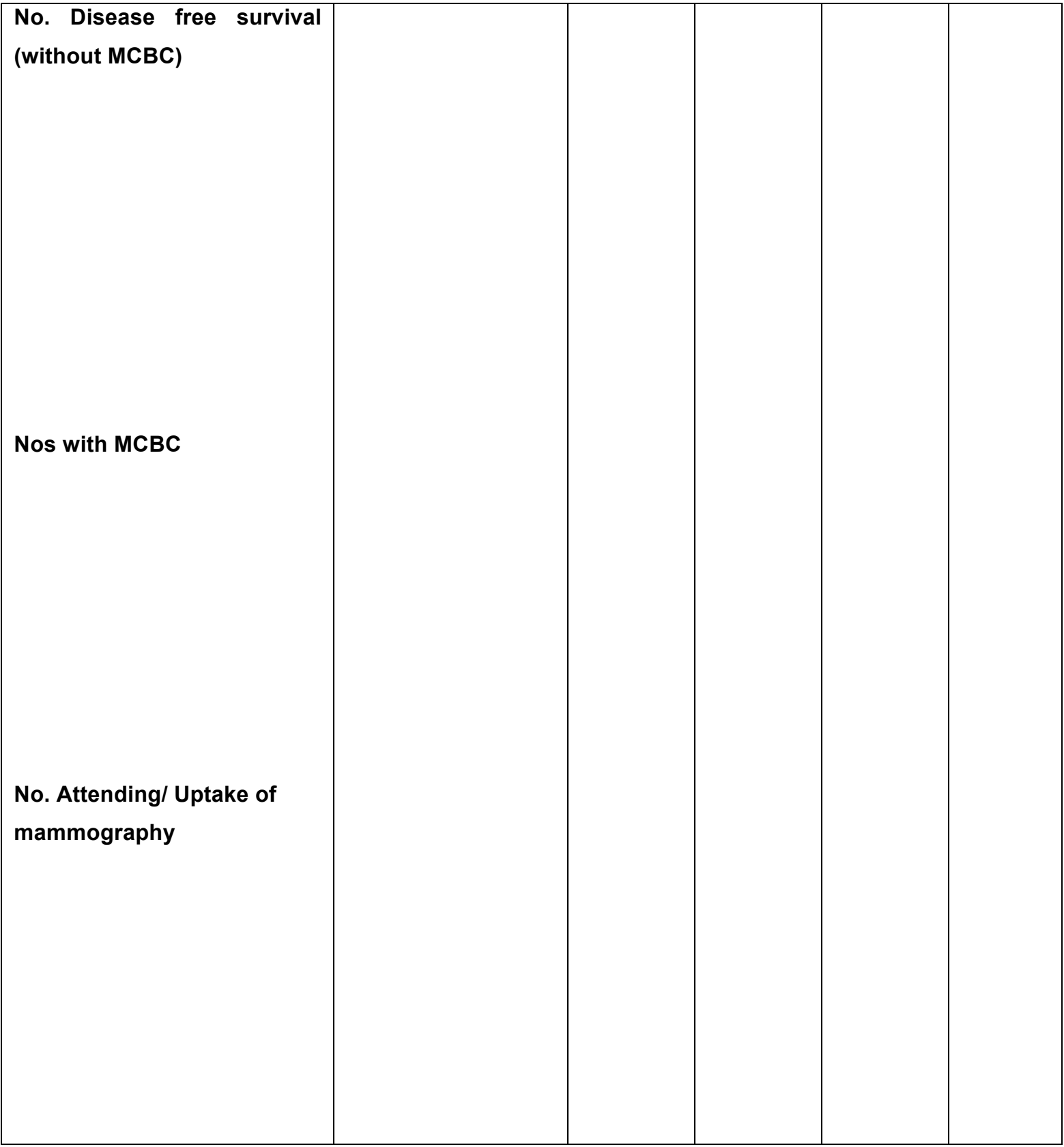




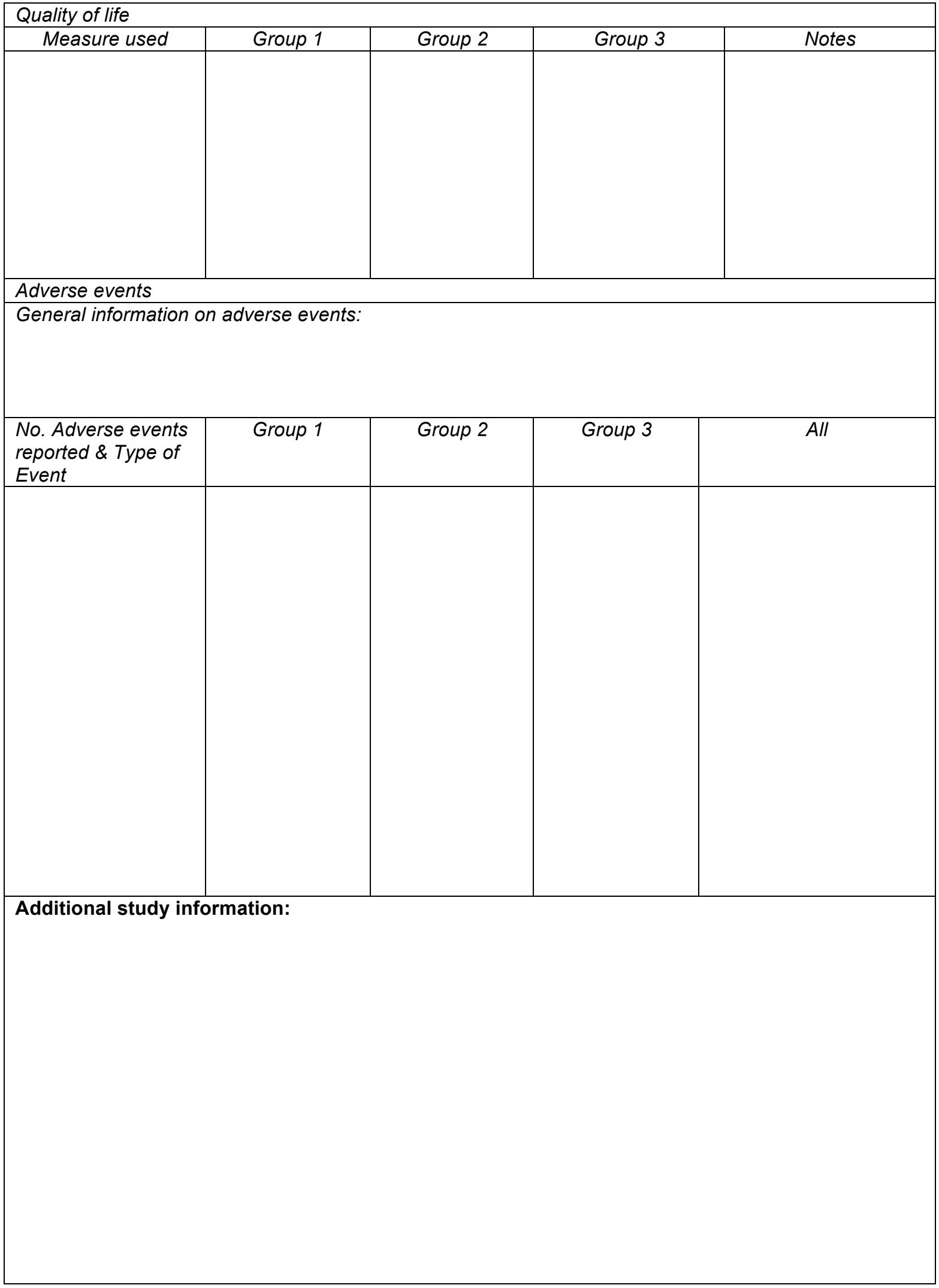


HTA Mammography Surveillance - Clinical Effectiveness Review 1 Data Extraction Form for Outcomes by Subgroup

Version 2

Study ID

List of possible subgroups:

Grade of primary breast cancer tumour, Size of primary breast cancer tumour, Lymphovascular Invasion, NPI status, ER/PR/HER2 status, Genetic status (BRCA genes), Age (Under 50 years, 50 years and over), Type and extent of surgery for primary breast cancer tumour (Breast conserving, Mastectomy, Excision margins), Primary neoadjuvant / adjuvant treatment (Radiotherapy, Chemotherapy, Tamoxifen/Endocrine treatment, Oopherectomy or ovarian ablation) Depcat status

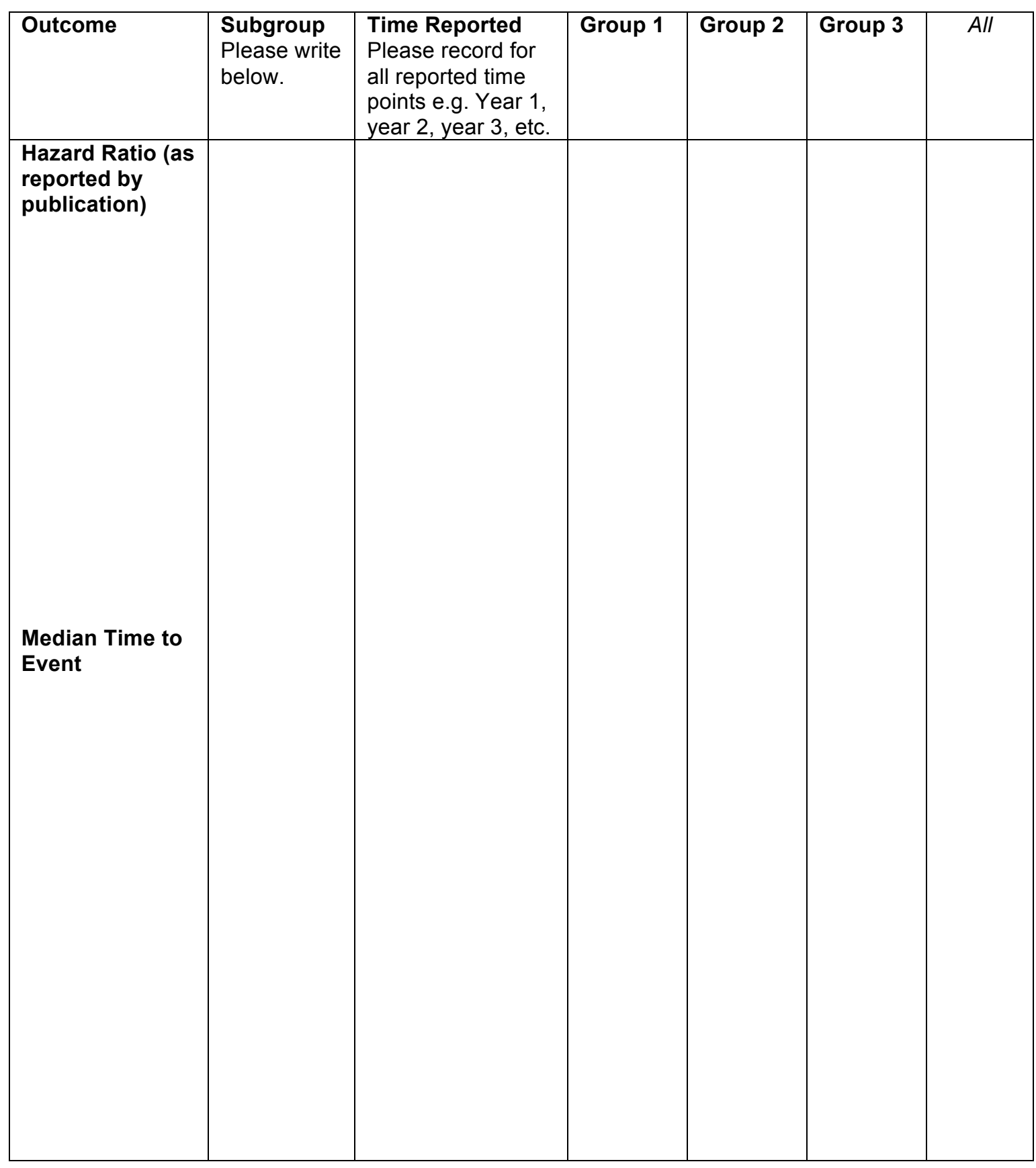




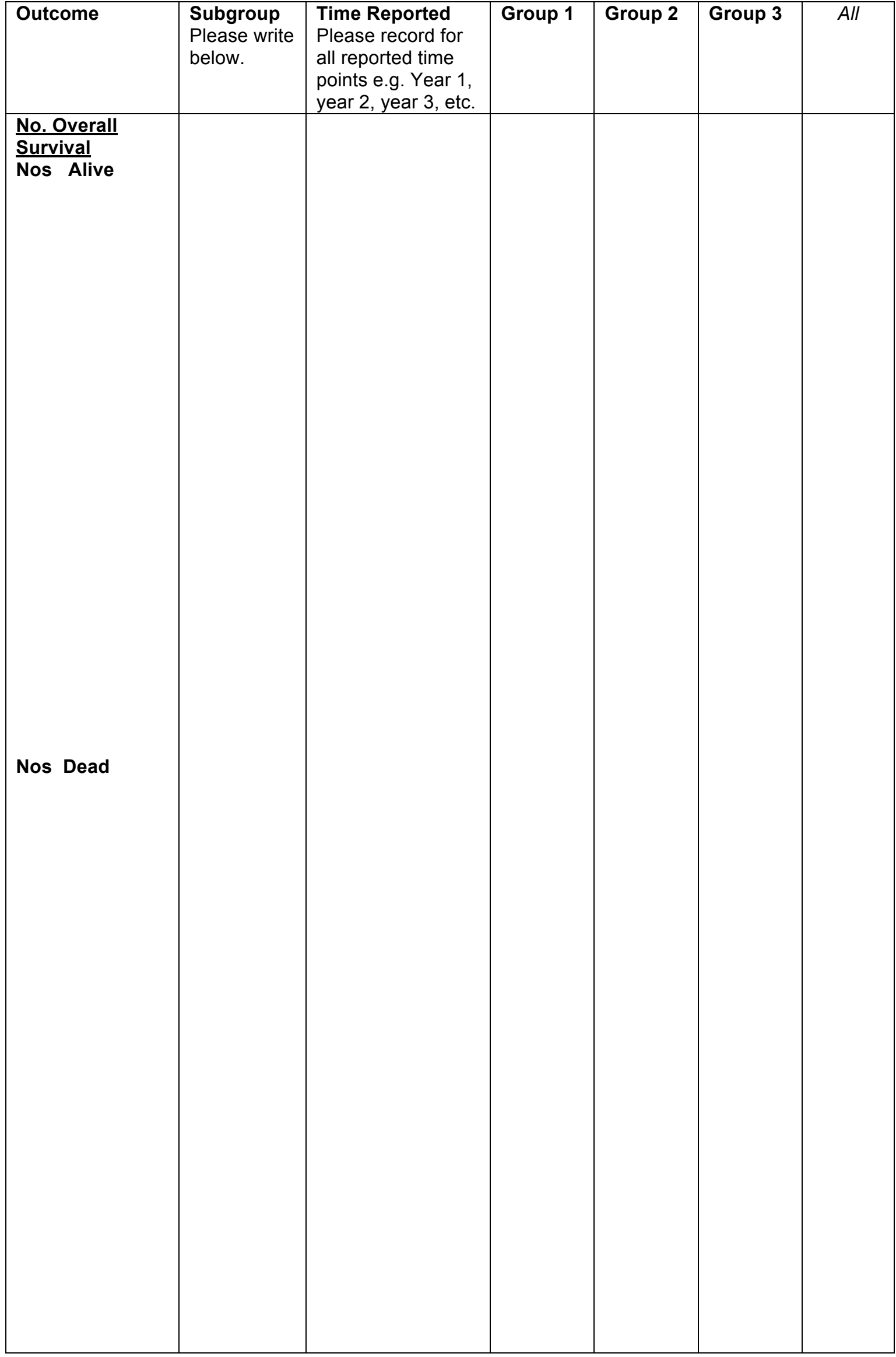




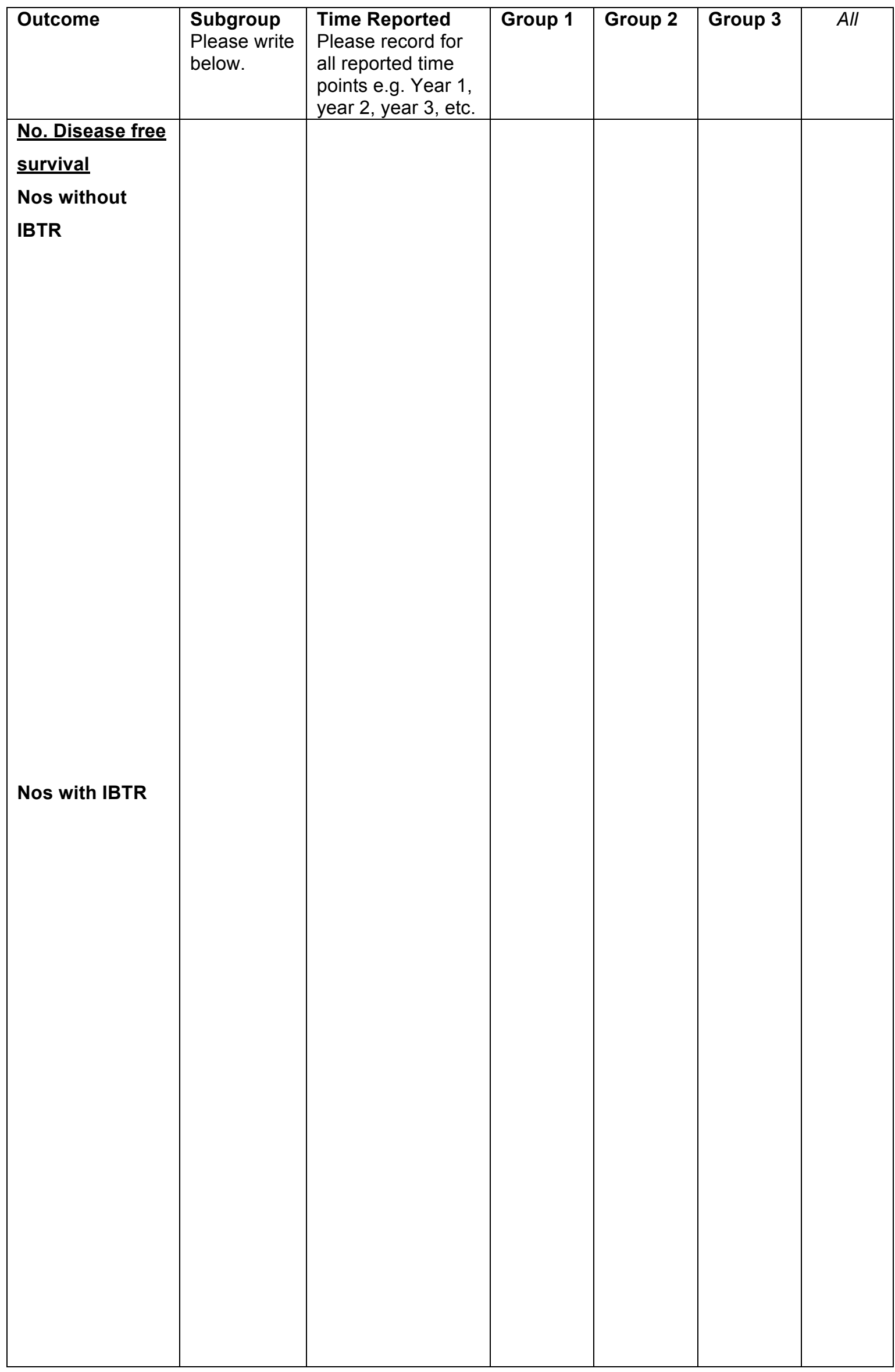




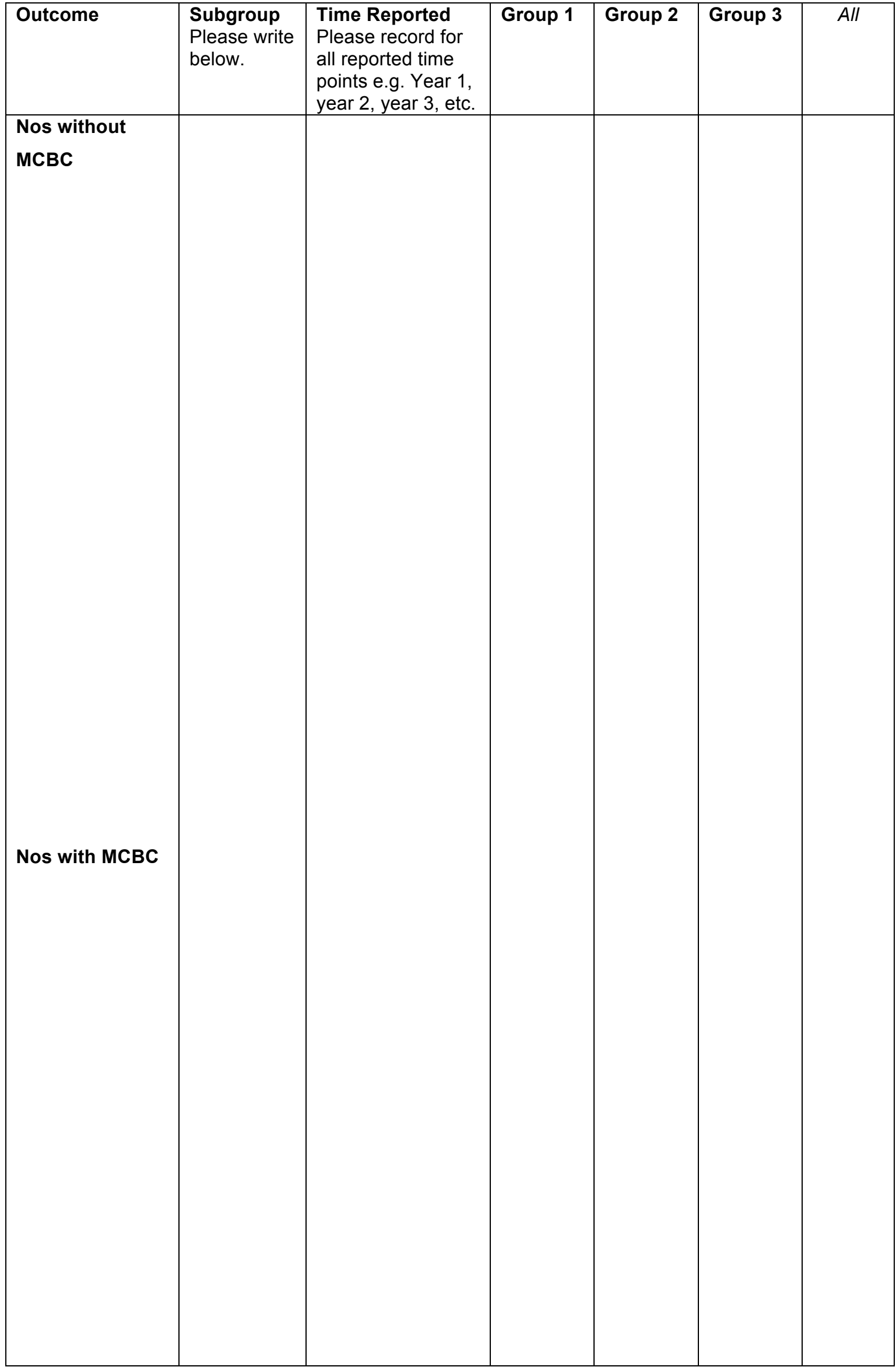




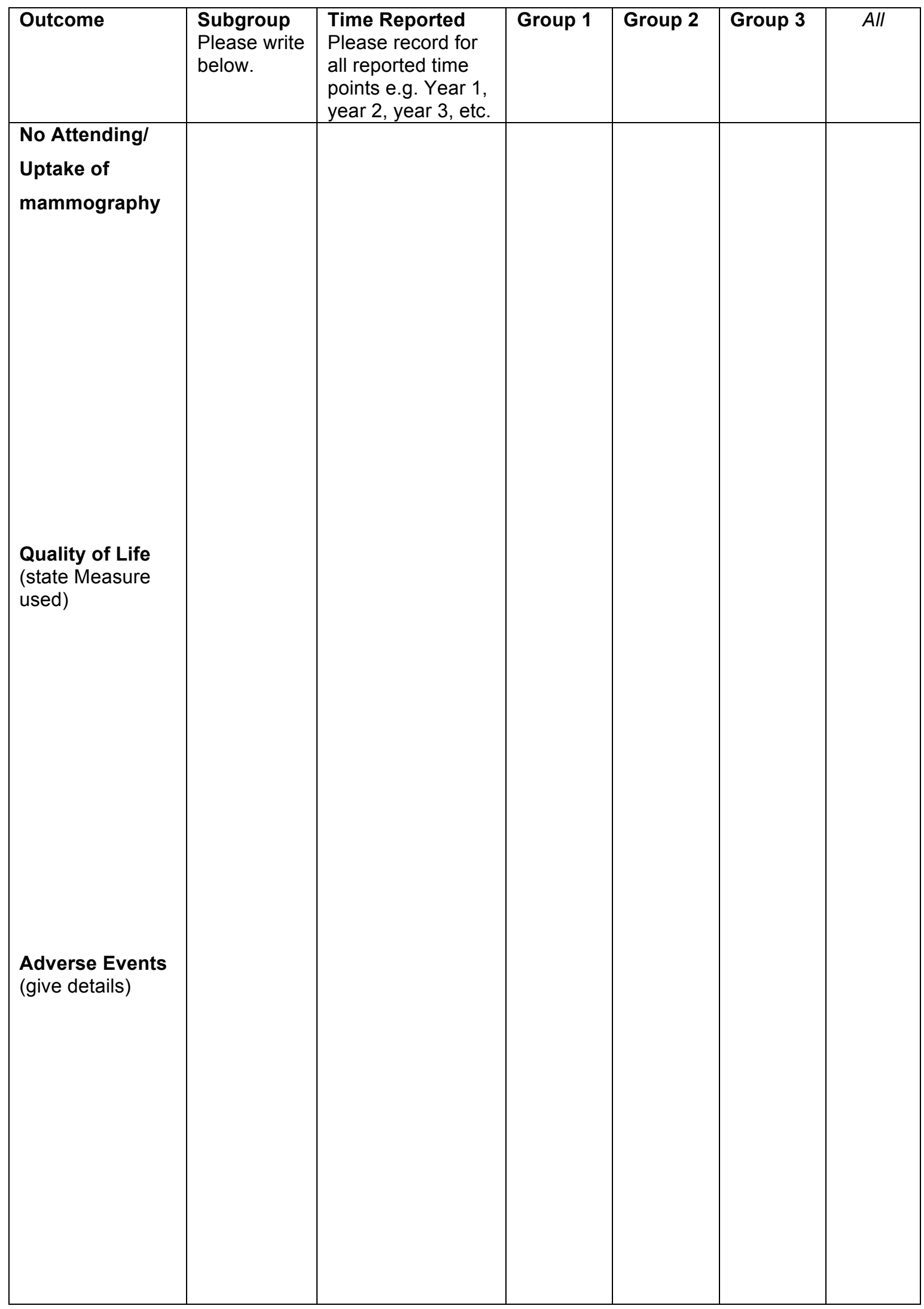




\section{Appendix 14}

\section{Quality assessment tool used in the systematic review of clinical effectiveness of surveillance mammography}

\section{HTA mammography surveillance - clinical effectiveness and cost-effectiveness review 1}

Checklist for quality assessment of non-randomised studies (comparative and cohort studies). Items specific to comparative studies are in italic text.

Version 3

Study ID:
Reviewer initials:
May 2010

Date:

\begin{tabular}{lllll}
\hline Criteria & Yes & No & Unclear Comments \\
\hline
\end{tabular}

\section{Participants: sample definition and selection}

1. Were participants a representative sample selected from a relevant patient population?

2. Were the inclusion/exclusion criteria of participants clearly described?

3. Were participants entering the study at a similar point in their disease progression?

4. Was selection of patients consecutive?

5. Was data collection undertaken prospectively?

6. Were the groups comparable on demographic characteristics and clinical features?

\section{Intervention}

7. Was the intervention clearly defined?

8. Was the comparison clearly defined?

\section{Outcome measures}

9. Were overall survival, disease-free survival and relative survival outcomes considered?

10. Were objective (valid and reliable) outcome measure/s used for ascertaining disease-free survival?

11. Was the assessment of disease-free outcomes blind? 


\begin{tabular}{llll}
\hline Criteria & Yes & No & Unclear Comments \\
\hline Follow-up & Comm
\end{tabular}

Follow-up

12. Was follow-up long enough to detect important effects on outcomes of interest? Follow-up must be a minimum of 5 years to account for changes in surveillance policy after this time due to most recurrences occurring within first 5 years following initial breast cancer

13. Was information provided on non-respondents, dropouts?

14. Were participants lost to follow-up likely to introduce bias? (e.g. high dropout rate, differential dropout, no description of those lost)

15. Was length of follow-up similar between comparison groups?

\section{Analysis}

16. Were important prognostic factors identified?

17. Were the analyses adjusted for confounding factors? Importance of confounding factor as judged by study authors?

18. Did authors attempt to correct for lead time bias?

19. Did authors attempt to correct for length time bias? 


\section{Appendix 15}

\section{Characteristics of the studies included in the systematic review of clinical effectiveness of surveillance mammography}

\begin{tabular}{|c|c|c|c|c|}
\hline Study ID & Participants & $\begin{array}{l}\text { Description of surveillance } \\
\text { regimen }\end{array}$ & Study comparator & Outcomes summary ${ }^{a}$ \\
\hline $\begin{array}{l}{\text { Churn } 2001^{53}} \\
\text { Study design: } \\
\text { retrospective } \\
\text { cohort } \\
\text { Follow-up: } 5 \text { years } \\
\text { Primary treatment. } \\
\text { all } 1993 \\
\text { Country. UK }\end{array}$ & $\begin{array}{l}\text { Enrolled: } n=612 \\
\text { Excluded: } n=0 \\
\text { Analysed: } n=612 \\
\text { Mastectomy: } n=104 \\
\text { analysed } \\
\text { BCS: } n=505 \text { analysed } \\
\text { No primary surgery. } n=3 \\
\text { analysed } \\
\text { Age (years): } \\
\text { Mean NR, SD NR, range } \\
\text { NR } \\
<50 \text { years } n=189 \\
50 \text { years and over } \\
n=423\end{array}$ & $\begin{array}{l}\text { Clinical examination 3- to } \\
\text { 4-monthly for first 2-3 } \\
\text { years, } 6 \text {-monthly for up to } \\
5 \text { years, annually thereafter. } \\
\text { System involved alternate } \\
\text { appointments with the } \\
\text { surgical and oncological } \\
\text { teams for } 3-5 \text { years, } \\
\text { followed by discharge to } \\
\text { continue follow-up in one } \\
\text { or other of these clinics. } \\
\text { Surveillance mammography } \\
\text { according to individual } \\
\text { clinician preference (usually } \\
\text { less frequently than annually) }\end{array}$ & None & $\begin{array}{l}\text { Type of recurrence: local recurrence } \\
\text { (IBTR and MCBC?) } \\
\text { Local recurrence at } 5 \text { years post } \\
\text { primary treatment. } \\
\text { BCS (IBTR) } n=25 \text { (four concurrent with } \\
\text { metastases, two after presentation with } \\
\text { systemic disease) } \\
\text { Mastectomy (MCBC): } n=9 \text { (three } \\
\text { concurrent metastases) } \\
\text { Overall survival at } 5 \text { years: } \\
\text { Alive all } n=500 \\
\text { Alive breast cancer recurrence NR } \\
\text { Dead all cause } n=112 \\
\text { Dead breast cancer NR } \\
\text { Dead breast cancer recurrence NR } \\
\text { Mode of detection: } \\
\text { IBTR: } \\
\text { SM } n=7 \\
\text { CE } n=8 \\
\text { Symptomatic interim referral } n=9 \\
\text { MCBC: } \\
\text { CE } n=6 \\
\text { Symptomatic interim referral } n=1 \\
\text { Uncertain } n=2\end{array}$ \\
\hline $\begin{array}{l}\text { Lash } 2005^{59} \\
\text { Study design: } \\
\text { multicentre } \\
\text { prospective cohort } \\
\text { Follow-up: median } \\
7.4 \text { years, range } \\
10 \text { months to } 9.5 \\
\text { years } \\
\text { Primary treatment: } \\
\text { October } 1992 \text { to } \\
\text { March } 1994 \\
\text { Country: USA }\end{array}$ & $\begin{array}{l}\text { Enrolled: } n=388 \\
\text { Excluded: } n=85 \\
\text { Analysed: } n=303 \\
\text { Mastectomy: } n=71 \\
\text { analysed } \\
\text { BCS: } n=228 \text { analysed } \\
\text { Primary surgery details } \\
\text { missing: } n=4 \text { analysed } \\
\text { Age (years): } \\
\text { Mean NR, SD NR, range } \\
\text { NR } \\
<50 \text { years } n=0 \\
50 \text { years and over } \\
n=388\end{array}$ & $\begin{array}{l}\text { Guideline surveillance } \\
\text { - defined as annual CE } \\
\text { and XRM for patients with } \\
\text { asymptomatic history }\end{array}$ & $\begin{array}{l}\text { Number of } \\
\text { consecutive } \\
\text { years of receipt } \\
\text { of guideline } \\
\text { surveillance } \\
\text { compared with not } \\
\text { receiving guideline } \\
\text { surveillance }\end{array}$ & $\begin{array}{l}\text { Recurrence: NR } \\
\text { Overall survival: } \\
\text { Alive all } n=240 \\
\text { Alive breast cancer recurrence NR } \\
\text { Dead (all cause) } n=63 \\
\text { Dead breast cancer } n=27 \\
\text { Dead other } n=36 \\
\text { Dead breast cancer recurrence NR }\end{array}$ \\
\hline
\end{tabular}




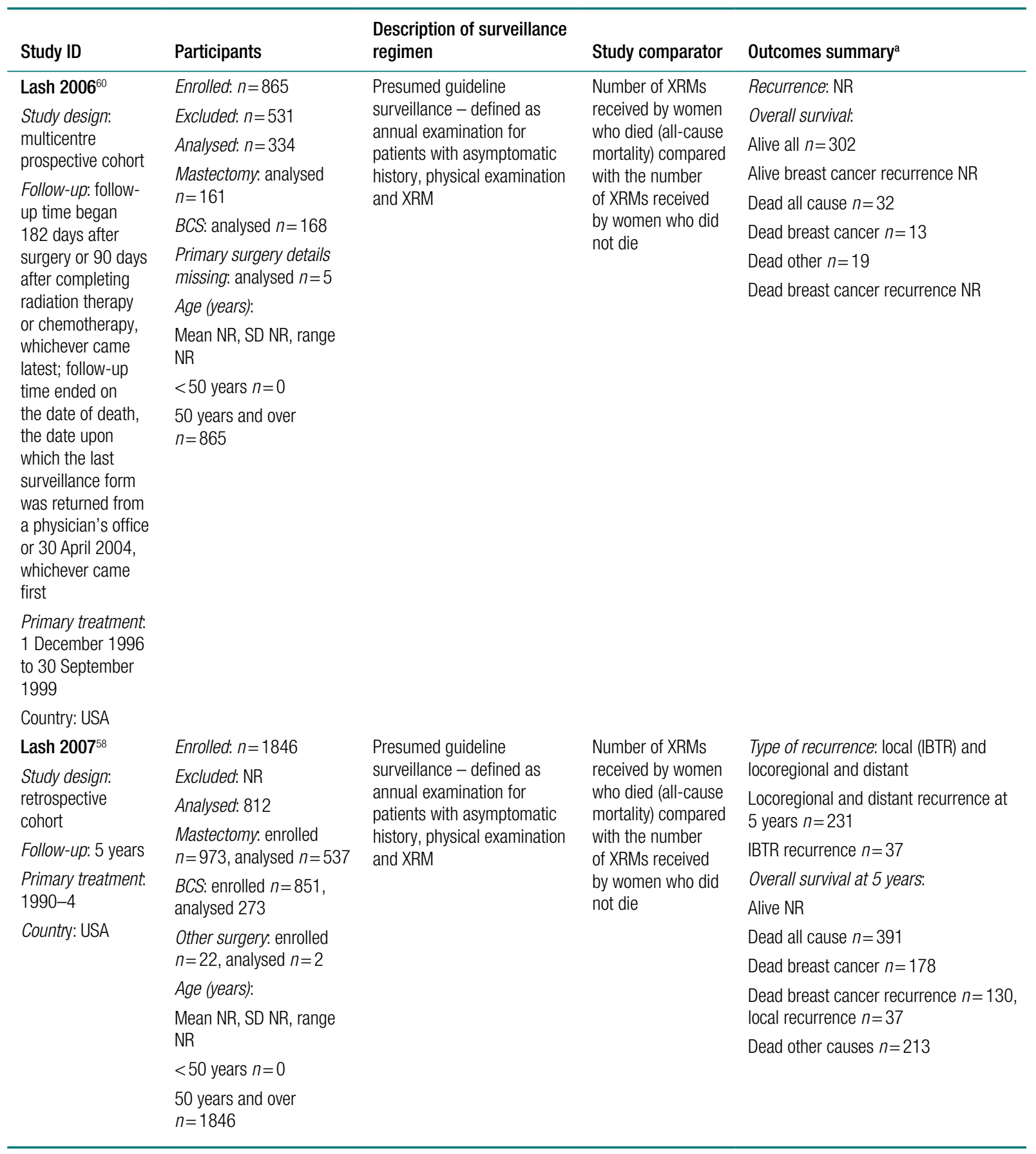




\begin{tabular}{|c|c|c|c|c|}
\hline Study ID & Participants & $\begin{array}{l}\text { Description of surveillance } \\
\text { regimen }\end{array}$ & Study comparator & Outcomes summary ${ }^{\mathrm{a}}$ \\
\hline \multirow{2}{*}{$\begin{array}{l}\text { Montgomery } \\
2007^{54}\end{array}$} & Enrolled: $n=1312$ & \multirow{28}{*}{$\begin{array}{l}\text { Outpatient clinical } \\
\text { examination performed } \\
\text { 3- to 4-monthly for first } 2 \\
\text { years, } 6 \text {-monthly for } 3 \text { years } \\
\text { then annually until } 10 \text { years } \\
\text { post primary treatment } \\
\text { from } 1991 \text { to } 1998 \text {. From } \\
2000 \text {, all patients received } \\
\text { annual clinic visits only. } \\
\text { Annual bilateral surveillance } \\
\text { mammography performed. } \\
\text { All patients received } \\
\text { instruction in breast self- } \\
\text { examination. Additional } \\
\text { interval visits arranged as } \\
\text { required }\end{array}$} & \multirow[t]{28}{*}{ None } & Type of recurrence: local recurrence \\
\hline & Excluded: $n=42$ & & & (IBTR) and contralateral second primary \\
\hline Study design: & Analysed: $n=110$ & & & cancer (MCBC) \\
\hline retrospective & Mastectomy: $n=0$ & & & Recurrence at 5 years: \\
\hline & BCS: enrolled $n=1312$ & & & IBTR $n=48$ \\
\hline Follow-up: median & analysed $n=110$ & & & $\operatorname{MCBC} n=35$ \\
\hline $1.5-15$ years & Age (years): & & & Bilateral IBTR + MCBC $n=2$ \\
\hline Primary treatment. & Mean 56, SD NR, range & & & Ipsilateral axilla $n=25$ \\
\hline $1991-8$ & $24-91$ & & & Overall survival at 5 years: \\
\hline \multirow[t]{19}{*}{ Country: UK } & $<50 \mathrm{NR}$ & & & Alive all NR \\
\hline & 50 and over NR & & & Dead breast cancer recurrence $n=24$ \\
\hline & & & & Dead IBTR recurrence $n=15$ \\
\hline & & & & Dead MCBC $n=8$ \\
\hline & & & & Dead bilateral $n=1$ \\
\hline & & & & Dead other NR \\
\hline & & & & ${ }^{b}$ Mode of detection: ${ }^{6}$ \\
\hline & & & & IBTR: \\
\hline & & & & SM $n=25$ (6 died) \\
\hline & & & & CE $n=4$ (4 died) \\
\hline & & & & $\begin{array}{l}\text { Symptomatic interim referral } n=12 \\
\text { ( } 4 \text { died) }\end{array}$ \\
\hline & & & & Symptoms $n=5$ (1 died) \\
\hline & & & & MCBC: \\
\hline & & & & SM $n=25$ (5 died $)$ \\
\hline & & & & $\mathrm{CE} n=2$ \\
\hline & & & & $\begin{array}{l}\text { Symptomatic interim referral } n=5 \\
\text { ( } 1 \text { died) }\end{array}$ \\
\hline & & & & Symptoms $n=3$ (2 died) \\
\hline & & & & Bilateral IBTR/MCBC: \\
\hline & & & & SM $n=2(1$ died $)$ \\
\hline
\end{tabular}




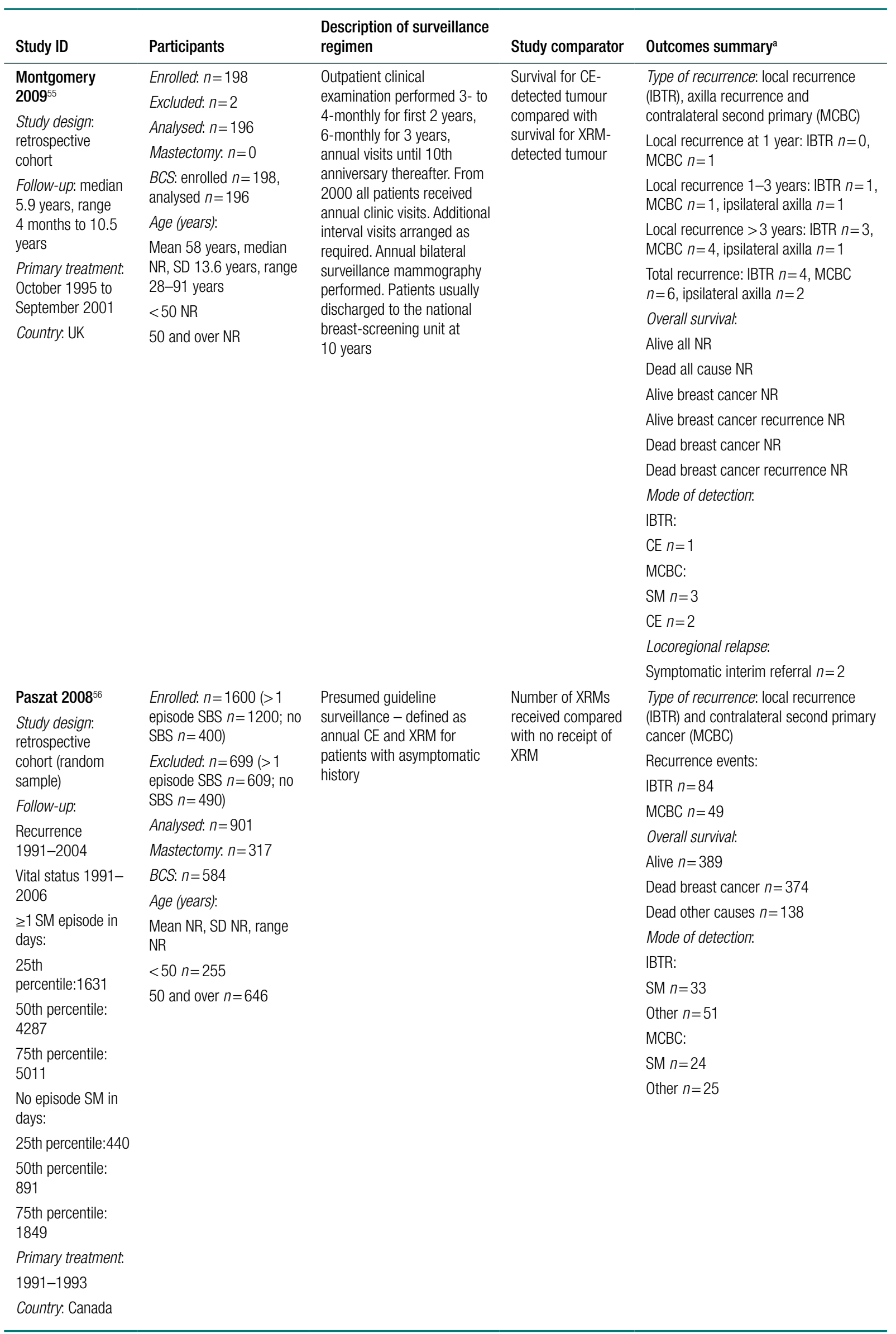




\begin{tabular}{|c|c|c|c|c|}
\hline Study ID & Participants & $\begin{array}{l}\text { Description of surveillance } \\
\text { regimen }\end{array}$ & Study comparator & Outcomes summary ${ }^{\mathrm{a}}$ \\
\hline $\begin{array}{l}\text { Yau 2008 } \\
\text { Study design: } \\
\text { retrospective } \\
\text { cohort } \\
\text { Follow-up: median } \\
\text { 5.9 years, range } \\
0.2-13 \text { years } \\
\text { Primary treatment: } \\
\text { 1994-2003 } \\
\text { Country: Hong } \\
\text { Kong }\end{array}$ & $\begin{array}{l}\text { Enrolled: } n=507 \\
\text { Excluded: } n=0 \\
\text { Analysed: } n=507 \\
\text { Mastectomy: } n=0 \\
\text { BCS: } n=507 \\
\text { Age (years): } \\
\text { Median 46, SD NR, range } \\
25-90 \\
<50 \text { NR } \\
50 \text { and over NR }\end{array}$ & $\begin{array}{l}\text { Clinical examination every } \\
2-3 \text { months in the first } 2 \\
\text { years, every } 4-6 \text { months } \\
\text { in the third to fifth years, } \\
\text { annually thereafter. Bilateral } \\
\text { surveillance mammography } \\
\text { performed annually first } \\
5 \text { years, once every } 1-2 \\
\text { years for next } 5 \text { years. } \\
\text { Supplementary ultrasound } \\
\text { performed at clinician's } \\
\text { discretion }\end{array}$ & None & $\begin{array}{l}\text { Type of recurrence: local recurrence } \\
\text { (IBTR) and contralateral second primary } \\
\text { cancer (MCBC) } \\
\text { Recurrence events: } \\
\text { IBTR } n=23 \\
\text { MCBC } n=13 \\
\text { Overall survival: } \\
\text { Alive all NR } \\
\text { Alive breast cancer recurrence NR } \\
\text { Dead all cause NR } \\
\text { Dead breast cancer NR } \\
\text { Dead breast cancer recurrence NR } \\
\text { Mode of detection: } \\
\text { IBTR: } \\
\text { SM } n=10 \\
\text { CE } n=8 \\
\text { Symptomatic interim referral } n=2 \\
\text { Supplementary ultrasound } n=2 \\
\text { MCBC: } \\
\text { SM } n=8 \\
\text { CE } n=3 \\
\text { Symptomatic interim referral } n=1 \\
\text { Supplementary ultrasound } n=1\end{array}$ \\
\hline
\end{tabular}

CE, clinical examination; SBS, subsequent breast surgery; SM, surveillance mammography; XRM, X-ray mammography.

a The included studies did not include data on the following outcomes considered in our review: quality of life, harms of mammography (adverse events), uptake of mammography, economic outcomes.

b Two patients with IBTR were excluded from mode of detection data by the study authors. Both were diagnosed incidentally during breast reshaping procedures. Both patients subsequently died. 



\section{Appendix 16}

Quality assessment of individual studies included in the systematic review of clinical effectiveness of surveillance mammography 


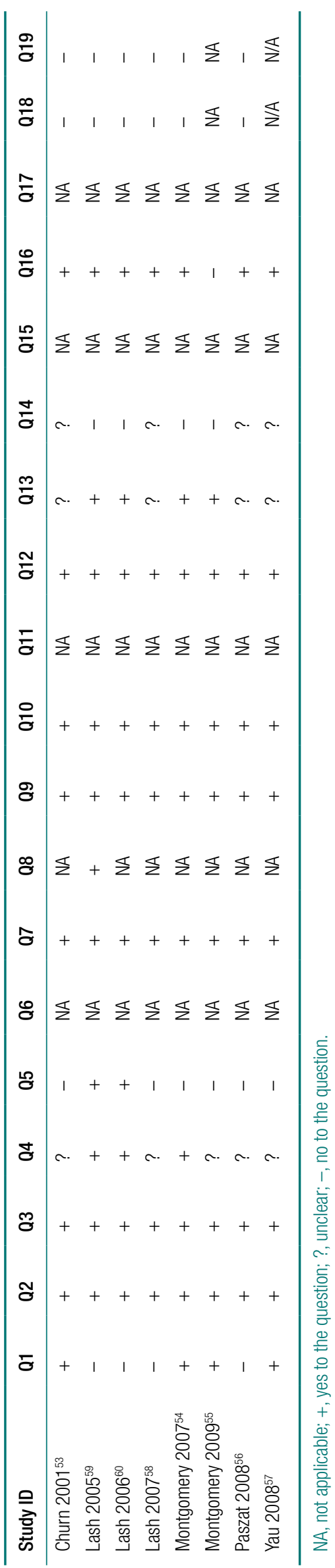




\section{Appendix 17}

\section{Search strategies: systematic review of test performance of surveillance mammography and comparator tests in detecting IBTR/MCBC}

MEDLINE (1990 - March, week 1, 2009), EMBASE (1990-2009, week 11) MEDLINE In-Process (13 March 2009)

Ovid multifile search: https://shibboleth.ovid.com/

1. exp ${ }^{\star}$ breast neoplasms/use mesz

2. exp ${ }^{\star}$ breast tumor/use emez

3. breast.ti.

4. or/1-3

5. neoplasm recurrence, local/use mesz

6. tumor recurrence/use emez

7. cancer recurrence/use emez

8. neoplasms, second primary/use mesz

9. second cancer/use emez

10. (recur $\$$ or second or secondary or contralateral or ipsilateral or ibtr or mcbc).tw.

11. or $/ 5-10$

12. exp mammography/

13. (mammograph\$ or mammogram\$).tw.

14. physical examination/

15. breast self-examination/

16. breast examination/use emez

17. ((physical or clinical or self) adj1 (exam? or examin\$)).tw.

18. surveillance.hw,tw.

19. follow up.ti.

20. (routine adj3 (visit or follow up)).tw.

21. Magnetic resonance imaging/use mesz

22. Nuclear Magnetic resonance imaging/use emez

23. (magnetic resonance imag $\$$ or $\mathrm{mri}$ ).tw.

24. ultrasonography, mammary/use mesz

25. echomammography/use emez

26. (ultrasound or ultrasonograph\$ or echo mammogra $\$$ or echomammogra $\$$ ).tw.

27. or $/ 12-26$

28. neoplasm recurrence, local/di use mesz

29. tumor recurrence/di use emez

30. cancer recurrence/di use emez

31. neoplasms, second primary/di use mesz

32. second cancer/di use emez

33. or $/ 28-32$

34. 4 and 33

35. "sensitivity and specificity"।

36. roc curve/ 
37. receiver operating characteristic/use emez

38. predictive value of tests/

39. diagnostic errors/use emez

40. false positive reactions/use mesz

41. false negative reactions/use mesz

42. diagnostic accuracy/use emez

43. diagnostic value/use emez

44. du.fs. use mesz

45. sensitivity.tw.

46. distinguish\$.tw.

47. differentiat\$.tw.

48. identif\$.tw.

49. detect\$.tw.

50. diagnos\$.tw.

51. (predictive adj4 value\$).tw.

52. accura\$.tw.

53. comparison.tw.

54. or/35-53

55. 27 and 11 and 4 and 54

56. 34 or 55

57. remove duplicates from 56

58. limit 57 to $\mathrm{yr}=" 1990-2009$ "

59. limit 58 to english language

\section{Science Citation Index (1990 -14 March 2009), BIOSIS}

(1990 - 12 March 2009)

ISI Web of Knowledge:http://wok.mimas.ac.uk/

\# 1 TS=(breast neoplasm ${ }^{\star}$ AND (recur* or secondary or contralateral or ipsilateral or ibtr or $\mathrm{mcbc})$ )

\# 2 TS=(breast cancer ${ }^{\star}$ AND (recur ${ }^{*}$ or secondary or contralateral or ipsilateral or ibtr or mcbc))

\# 3 TS=(breast carcin* AND (recur ${ }^{*}$ or secondary or contralateral or ipsilateral or ibtr or mcbc))

\# 4 TS=(breast tumour ${ }^{\star}$ AND (recur ${ }^{\star}$ or secondary or contralateral or ipsilateral or ibtr or $\mathrm{mcbc})$ )

\# $5 \mathrm{TS}=$ (breast tumor ${ }^{\star}$ AND (recur ${ }^{\star}$ or secondary or contralateral or ipsilateral or ibtr or mcbc))

$\# 6 \# 1$ or \#2 or \#3 or \#4 or \#5

\# 7 \#6 AND TS=mammogram ${ }^{\star}$

\# 8 \#6 AND TS=mammograph ${ }^{*}$

\# 9 \#6 and TS=surveillance

$\# 10 \# 6$ and TS=routine

\# $11 \# 6$ and TS=magnetic resonance imag*

\# 12 \#6 AND TS=mri

$\# 13 \# 6$ and TS=ultrasound

\# 14 \#6 and TS=ultrasonograph ${ }^{*}$

\# $15 \# 6$ and TS=echo mammogra*

\# 16 \#6 and TS=echomammogra ${ }^{*}$

\# 17 \#6 and TS $=\left(\right.$ cliniCAL EXAM ${ }^{\star}$ OR SELF EXAM ${ }^{\star}$ OR PHYSICAL EXAM $\left.{ }^{\star}\right)$

\# 18 \# OR \#8 OR \#9 OR \#10 OR \#11 OR \#12 OR \#13 OR \#14 OR \#15 OR \#16 OR \#17

\# 19 \#18 AND TS=(sensitivity or specificity)

\# 20 \#18 AND TS $=\left(\right.$ ROC or receiver operat ${ }^{*}$ characteristic)

\# 21 \#18 AND TS $=\left(\right.$ diagnos $^{*}$ or $\left.\operatorname{detect}^{\star}\right)$

\# 22 \#18 AND TS=(differentiat ${ }^{\star}$ or distinguish $\left.{ }^{\star}\right)$

\# 23 \#18 AND TS $=\left(\right.$ accura $^{\star}$ or identif $\left.{ }^{\star}\right)$ 
\# 24 \#23 OR \#22 OR \#21 OR \#20 OR \#19 AND Language=(English) AND Document

Type $=($ Article $)$

Databases=SCI-EXPANDED Timespan $=1990-2009$

\section{Cochrane Library Issue 2, 2009 (Cochrane Central Registry of Controlled \\ Trials and Cochrane Database of Systematic Reviews)}

www3.interscience.wiley.com/

\#1 MeSH descriptor Breast Neoplasms explode all trees

$\# 2$ (breast):ti

\#3 (\#1 OR \#2)

\#4 MeSH descriptor Neoplasm Recurrence, Local, this term only

\#5 MeSH descriptor Neoplasms, Second Primary, this term only

\#6 (recur* or second or secondary):ti,ab,kw or (contralateral or ipsilateral):ti,ab,kw or (ibtr or

$\mathrm{mcbc}): \mathrm{ti}, \mathrm{ab}, \mathrm{kw}$

\#7 (\#4 OR \#5 OR \#6)

\#8 MeSH descriptor Mammography explode all trees

\#9 $\left(\right.$ mammograph $^{\star}$ or mammogram $\left.{ }^{\star}\right)$ :ti,ab,kw

\#10 MeSH descriptor Physical Examination, this term only

\#11 MeSH descriptor Breast Self-Examination, this term only

\#12 (physical NEAR/1 exam):ti,ab,kw or (clinical NEAR/1 exam):ti,ab,kw or (self NEAR/1

exam $\left.{ }^{*}\right): \mathrm{ti}, \mathrm{ab}, \mathrm{kw}$

\#13 (surveillance):ti,ab,kw or (follow up):ti or (routine NEAR/3 visit*):ti,ab,kw or (routine

NEAR/3 follow up):ab

\#14 MeSH descriptor Magnetic Resonance Imaging, this term only

\#15 MeSH descriptor Ultrasonography, Mammary, this term only

\#16 (magnetic resonance imag ${ }^{\star}$ or mri):ti,ab,kw or (ultrasound or ultrasonograph ${ }^{\star}$ ):ti,ab,kw or

(echo mammogra ${ }^{\star}$ or echomammogra $\left.{ }^{\star}\right): \mathrm{ti}, \mathrm{ab}, \mathrm{kw}$

\#17 (\#8 OR \#9 OR \#10 OR \#11 OR \#12 OR \#13 OR \#14 OR \#15 OR \#16)

\#18 (\#3 AND \#7 AND \#17)

\#19 MeSH descriptor Neoplasm Recurrence, Local explode all trees with qualifier: DI

\#20 MeSH descriptor Neoplasms, Second Primary explode all trees with qualifier: DI

\#21 (\#3 AND (\#19 OR \#20))

\#22 \#18 OR \#21 (1990-2009)

\section{CANCERLIT (20 March 2009)}

The US National Library of Medicine: www.ncbi.nlm.nih.gov

\#1 Search (breast neoplasms[majr] AND human[mh] AND english[la]) OR dcis[ti] OR lcis[ti]

OR ((breast[ti] OR breasts[ti] OR mammary[ti] OR nipple[ti] OR nipples[ti]) AND ((cancer ${ }^{\star}[\mathrm{ti}]$

OR carcinoma ${ }^{\star}[\mathrm{ti}]$ OR adenocarcinoma ${ }^{\star}[\mathrm{ti}]$ OR malignan ${ }^{\star}[\mathrm{ti}]$ OR tumor ${ }^{\star}[\mathrm{ti}]$ OR tumour ${ }^{\star}[\mathrm{ti}]$

OR neoplasm $\left.{ }^{\star}[\mathrm{ti}]\right)$ OR in situ[ti])) Limits: Cancer

\#2 Search recur ${ }^{\star}$ or secondary or contralateral or ipsilateral or ibtr or mcbc Limits: Cancer

\#3 Search \#1 AND \#2 Limits: Cancer

\#4 Search ((((Magnetic resonance imaging[MeSH Terms]) OR (ultrasonography,

mammary[MeSH Terms])) OR (“magnetic resonance imag* or mri”[Text Word])) OR

("ultrasound or ultrasonograph* or echo mammogra* or echomammogra*"[Text Word])) AND

(\#1 AND \#2) Limits: English

\section{Health Technology Assessment/Database of Abstracts of Reviews and Effects/NHS Economic Evaluation databases, March 2009 Centre for Reviews \& Dissemination: http://nhscrd.york.ac.uk/ welcome.htm \\ \# 1 MeSH Breast Neoplasms EXPLODE 12}


\# 2 (recur* OR ipsilateral OR contralateral OR ibtr OR mcbc OR second OR secondary)

$\# 3 \# 1$ and $\# 2$

MEDION, March 2009

www.mediondatabase.nl/

ICPC CODE=Female genital

AND Signssymp=Medical Imaging

AND Textword=breast

\section{Clinical Trials, March 2009}

http://clinicaltrials.gov/ct/gui/c/r

"Breast neoplasms"

AND

Mammography OR ultrasonography OR magnetic resonance imaging

AND

Recurrence or ipsilateral or ccontralateral or ibtr or mcbc

Current Controlled Trials, March 2009

www.controlled-trials.com/

Breast AND (mammography OR magnetic resonance imaging OR ultrasonography)

\section{The World Health Organization International Clinical Trials Registry}

Platform, March 2009

World Health Organization: www.who.int/ictrp/en/

Breast AND (mammography OR magnetic resonance imaging OR ultrasonography)

\section{National Research Register Archive}

National Institute for Health Research portal: https://portal.nihr.

ac.uk/Pages/NRRArchiveSearch.aspx

NIHR portfolio, March 2009

National Institute for Health Research portal: https://portal.nihr. ac.uk/Search/Advanced.aspx

Breast AND (mammography OR magnetic resonance imaging OR ultrasonography)

\section{National Cancer Institute Clinical Trials, March 2009}

\section{The US National Institutes of Health: www.cancer.gov}

breast cancer (female) AND mammography OR magnetic resonance imaging

OR ultrasonography

Websites consulted

American College of Radiology

www.acr.org/

American Society of Breast Disease

www.asbd.org/

American Society of Clinical Oncology

www.asco.org/

Breast Cancer Surveillance Consortium http://breastscreening.cancer.gov/ 


\section{CancerWEB}

www.infoventures.com/cancer/

Canadian Task Force on Preventive Health Care www.ctfphc.org/

Cancer Research UK

www.cancerresearchuk.org/

European Society for Medical Oncology

www.esmo.org/

National Breast and Ovarian Cancer Centre www.nbocc.org.au/

National Cancer Institute www.cancer.gov/cancertopics/types/breast

National Comprehensive Cancer Network www.nccn.org/index.asp

National Library for Health www.library.nhs.uk/default.aspx

Royal College of Radiologists www.rcr.ac.uk/

Surveillance, Epidemiology, and End Results (SEER) Program http://seer.cancer.gov/ 



\section{Appendix 18}

\section{Reports of included studies: systematic review of test performance of surveillance mammography and comparator tests in detecting IBTR/MCBC}

\section{Belli 2003}

Belli P, Pastore G, Romani M, Terribile D, Canade A, Costantini M. Role of magnetic resonance imaging in the diagnosis of recurrence after breast conserving therapy. Rays 2002;27:241-57.

Boné 1995

Boné B, Aspelin P, Isberg B, Perbeck L, Veress B. Contrast-enhanced MR imaging of the breast in patients with breast implants after cancer surgery. Acta Radiol 1995;36:111-16.

Drew 1998

Drew PJ, Kerin MJ, Turnbull LW, Imrie M, Carleton PJ, Fox JN, et al. Routine screening for local recurrence following breast-conserving therapy for cancer with dynamic contrast-enhanced magnetic resonance imaging of the breast. Ann Surg Oncol 1998;5:265-70.

Kim 2009

Kim MJ, Kim EK, Kwak JY, Park BW, Kim SI, Sohn J, et al. Sonographic surveillance for the detection of contralateral metachronous breast cancer in an Asian population. AJR Am J Roentgenol 2009;92:221-8.

\section{Mumtaz 1997}

Mumtaz H, Davidson T, Hall-Craggs MA, Payley M, Walmsley K, Cowley G, et al. Comparison of magnetic resonance imaging and conventional triple assessment in locally recurrent breast cancer. Br J Surg 1997;84:1147-51.

Rieber 1997

Rieber A, Merkle E, Zeitler H, Gorich J, Kreienberg R, Brambs HJ, et al. Value of MR mammography in the detection and exclusion of recurrent breast carcinoma. J Comput Assist Tomogr 1997;21:780-4.

\section{Shin 2005}

Shin JH, Han BK, Choe YH, Nam SJ, Park W, Im YH. Ultrasonographic detection of occult cancer in patients after surgical therapy for breast cancer. J Ultrasound Med 2005;24:643-9.

\section{Ternier 2006}

Ternier F, Houvenaeghel G, Lecrivain F, Brigand BL, Margain D, Brunelle S, et al. Computed tomography in suspected local breast cancer recurrence. Breast Cancer Res Treat 2006;100:247-54. 


\section{Viehweg 2004}

Viehweg P, Rotter K, Laniado M, Lampe D, Buchmann J, Kolbl H, et al. MR imaging of the contralateral breast in patients after breast-conserving therapy. Eur Radiol 2004;14:402-8. 


\section{Appendix 19}

\section{Reports of excluded studies: systematic review of test performance of surveillance mammography and comparator tests in detecting IBTR/MCBC}

\section{Participants ineligible $(\mathrm{n}=77)$}

Bader W, Bohmer S, van Leeuwen P, Hackmann J, Westhof G, Hatzmann W. Does texture analysis improve breast ultrasound precision? Ultrasound Obstet Gynecol 2000;15:311-16.

Berg WA, Gutierrez L, NessAiver MS, Carter WB, Bhargavan M, Lewis RS, et al. Diagnostic accuracy of mammography, clinical examination, US, and MR imaging in preoperative assessment of breast cancer. Radiology 2004;233:830-49.

Blair S, McElroy M, Middleton MS, Comstock C, Wolfson T, Kamrava M, et al. The efficacy of breast MRI in predicting breast conservation therapy. J Surg Oncol 2006;94:220-5.

Boerner S, Fornage BD, Singletary E, Sneige N. Ultrasound-guided fine-needle aspiration (FNA) of nonpalpable breast lesions: a review of 1885 FNA cases using the National Cancer Institutesupported recommendations on the uniform approach to breast FNA. Cancer 1999;87:19-24.

Buxant F, Scuotto F, Hottat N, Noel JC, Simon P. Does preoperative magnetic resonance imaging modify breast cancer surgery? Acta Chir Belg 2007;107:288-91.

Chang RF, Kuo WJ, Chen DR, Huang YL, Lee JH, Chou YH. Computer-aided diagnosis for surgical office-based breast ultrasound. Arch Surg 2000;135:696-9.

Chen DR, Kuo WJ, Chang RF, Moon WK, Lee CC. Use of the bootstrap technique with small training sets for computer-aided diagnosis in breast ultrasound. Ultrasound Med Biol 2002;28:897-902.

Cooney CS, Khouri NF, Tsangaris TN. The role of breast MRI in the management of patients with breast disease. Adv Surg 2008;42:299-312.

Crowe JP. The importance of preoperative breast MRI for patients newly diagnosed with breast cancer. Breast J 2009;15:52-60.

Datoc PD, Hayes CW, Conway WF, Bosch HA, Neal MP. Mammographic follow-up of nonpalpable low-suspicion breast abnormalities: one versus two views. Radiology 1991;180:387-91.

Del Frate C, Borghese L, Cedolini C, Bestagno A, Puglisi F, Isola M, et al. Role of pre-surgical breast MRI in the management of invasive breast carcinoma. Breast 2007;16:469-81.

Delorme S, Zuna I, Huber S, Albert B, Bahner ML, Junkermann H, et al. Colour Doppler sonography in breast tumours: an update. Eur Radiol 1998;8:189-93.

Drew PJ. Magnetic-resonance imaging for breast cancer. Lancet 1998;351:1661-2.

Fischer U, Kopka L, Grabbe E. Breast carcinoma: effect of preoperative contrast-enhanced MR imaging on the therapeutic approach. Radiology 1999;213:881-8. 
Fischer U, Zachariae O, Baum F, von Heyden D, Funke M, Liersch T. The influence of preoperative MRI of the breasts on recurrence rate in patients with breast cancer. Eur Radiol 2004;14:1725-31.

Flobbe K, Kessels AGH, Severens JL, Beets GL, de Koning HJ, von Meyenfeldt MF, et al. Costs and effects of ultrasonography in the evaluation of palpable breast masses. Int $J$ Technol Assess Health Care 2004;20:440-8.

Galinsky D, Kisselgoff D, Sella T, Peretz T, Libson E, Sklair-Levy M. Effect of breast magnetic resonance imaging on the clinical management of breast cancer. Isr Med Assoc J 2005;7:700-3.

Golshan M, Fung BB, Wolfman J, Rademaker A, Morrow M. The effect of ipsilateral whole breast ultrasonography on the surgical management of breast carcinoma. Am J Surg 2003;186:391-6.

Hitchcock YJ. Contrast-enhanced MRI for the assessment of local recurrence after breast conservation. Am J Clin Oncol 2002;25:428-9.

Hollingsworth AB. Breast magnetic resonance imaging for preoperative locoregional staging. Am J Surg 2008;196:389-97.

Hrung JM. Cost-effectiveness of MR imaging and core-needle biopsy in the preoperative work-up of suspicious breast lesions. Radiology 1999;213:39-49.

Khatri VP, Stuppino JJ, Espinosa MH, Pollack MS. Improved accuracy in differentiating malignant from benign mammographic abnormalities: a simple, improved magnetic resonance imaging method. Cancer 2001;92:471-8.

Kinkel K, Hylton NM. Challenges to interpretation of breast MRI. J Magn Reson Imaging 2001;13:821-9.

Klein S. Evaluation of palpable breast masses. Am Fam Physician 2005;71:1731-8.

Kleit AN, Ruiz JF. False positive mammograms and detection controlled estimation. Health Serv Res 2003;38:1207-28.

Kuhl C, Kuhn W, Schild H. Management of women at high risk for breast cancer: new imaging beyond mammography. Breast 2005;14:480-6.

Kuhl C, Kuhn W, Braun M, Schild H. Pre-operative staging of breast cancer with breast MRI: one step forward, two steps back? Breast 2007;16(Suppl. 2):S34-44.

Kumar R. F-18 FDG positron emission tomography in non-Hodgkin lymphoma of the breast. Clin Nucl Med 2005;30:246-8.

Lee JM. MRI before re-excision surgery in patients with breast cancer. AJR Am J Roentgenol 2004; 182:473-80.

Lee SG, Orel SG, Woo IJ, Cruz-Jove E, Putt ME, Solin LJ, et al. MR imaging screening of the contralateral breast in patients with newly diagnosed breast cancer: preliminary results. Radiology 2003;226:773-8.

Lehman CD, Blume JD, Thickman D, Bluemke DA, Pisano E, Kuhl C, et al. Added cancer yield of MRI in screening the contralateral breast of women recently diagnosed with breast cancer: results from the International Breast Magnetic Resonance Consortium (IBMC) trial. J Surg Oncol 2005;92:9-15.

Lehman CD, Gatsonis C, Kuhl CK, Hendrick RE, Pisano ED, Hanna L, et al. MRI evaluation of the contralateral breast in women with recently diagnosed breast cancer. $N$ Engl J Med 2007;356:1295-303.

Leivo T, Salminen T, Sintonen H, Tuominen R, Auerma K, Partanen K, et al. Incremental costeffectiveness of double-reading mammograms. Breast Cancer Res Treat 1999;54:261-7. 
Liberman L, Morris EA, Dershaw DD, Abramson AF, Tan LK. MR imaging of the ipsilateral breast in women with percutaneously proven breast cancer. AJR Am J Roentgenol 2003;180:901-10.

Liberman L, Morris EA, Dershaw DD, Abramson AF, Tan LK. Ductal enhancement on MR imaging of the breast. AJR Am J Roentgenol 2003;181:519-25.

Luini A, Galimberti V, Gatti G, Arnone P, Vento AR, Trifiro G, et al. The sentinel node biopsy after previous breast surgery: preliminary results on 543 patients treated at the European Institute of Oncology. Breast Cancer Res Treat 2005;89:159-63.

Manton DJ. Neoadjuvant chemotherapy in breast cancer: early response prediction with quantitative MR imaging and spectroscopy. Br J Cancer 2006;94:427-35.

McMahon KE. Role of breast magnetic resonance imaging in difficult diagnostic situations. Med $J$ Aust 2001;175:494-7.

Meisamy S, Bolan PJ, Baker EH, Pollema MG, Le CT, Kelcz F, et al. Adding in vivo quantitative $1 \mathrm{H}$ MR spectroscopy to improve diagnostic accuracy of breast MR imaging: preliminary results of observer performance study at 4.0 T. Radiology 2005;236:465-75.

Mobbs LM. The accuracy of sonography in detecting abnormal axillary lymph nodes when breast cancer is present. J Diagn Med Sonograph 2005;21:297-303.

Moon WK, Noh DY, Im JG. Multifocal, multicentric, and contralateral breast cancers: bilateral whole-breast US in the preoperative evaluation of patients. Radiology 2002;224:569-76.

Morris EA. Breast MRI: ready for general use? Breast J 1999;5:219-20.

Morris EA. Diagnostic breast MR imaging: current status and future directions. Radiol Clin North Am 2007;45:863-80.

Murray N. Breast MRI in nonpalpable breast lesions: a randomized trial with diagnostic and therapeutic outcome: MONET-study. Adv Breast Cancer 2008;5:24.

Ozaki S, Tozaki M, Fukuma E, Kawano N, Suzuki T, Yamashiro N, et al. Bilateral breast MR imaging: is it superior to conventional methods for the detection of contralateral breast cancer? Breast Cancer 2008;15:169-74.

Park SY, Kim EK, Oh KK, Lee KS, Park BW. The role of sonography in patients with breast cancer presenting as an axillary mass. Korean J Radiol 2002;3:189-93.

Pediconi F, Venditti F, Padula S, Roselli A, Moriconi E, Giacomelli L, et al. CE-magnetic resonance mammography for the evaluation of the contralateral breast in patients with diagnosed breast cancer. Radiol Med 2005;110:61-8.

Pediconi F, Catalano C, Padula S, Roselli A, Moriconi E, Dominelli V, et al. Contrast-enhanced magnetic resonance mammography: does it affect surgical decision-making in patients with breast cancer? Breast Cancer Res Treat 2007;106:65-74.

Pediconi F, Catalano C, Roselli A, Padula S, Altomari F, Moriconi E, et al. Contrast-enhanced MR mammography for evaluation of the contralateral breast in patients with diagnosed unilateral breast cancer or high-risk lesions. Radiology 2007;243:670-80.

Pegg S, Furnival C, Fryar BG. Mammography after mastectomy. Australas Radiol 1990;34:280.

Peintinger F, Kuerer HM, Anderson K, Boughey JC, Meric-Bernstam F, Singletary SE, et al. Accuracy of the combination of mammography and sonography in predicting tumor response in breast cancer patients after neoadjuvant chemotherapy. Ann Surg Oncol 2006;13:1443-9.

Pepe MS, Urban N, Rutter C, Longton G. Design of a study to improve accuracy in reading mammograms. J Clin Epidemiol 1997;50:1327-38. 
Perlet C, Becker C, Sittek H, Pistitsch C, Jäger L, Kessler M, et al. A comparison of digital luminescence mammography and conventional film-screen system: preliminary results of clinical evaluation. Eur J Med Res 1998;3:165-71.

Peters NH, Borel Rinkes, I, Mali WP, van den Bosch MA, Storm RK, Plaisier PW, et al. Breast MRI in nonpalpable breast lesions: a randomized trial with diagnostic and therapeutic outcome MONET - study. Trials 2007;8:40.

Pisano ED, Fajardo LL, Caudry DJ, Sneige N, Frable WJ, Berg WA, et al. Fine-needle aspiration biopsy of nonpalpable breast lesions in a multicenter clinical trial: results from the radiologic diagnostic oncology group V. Radiology 2001;219:785-92.

Puggioni G. Joint modeling of sensitivity and specificity. Stat Med 2008;27:1745-61.

Quan ML, Sclafani L, Heerdt AS, Fey JV, Morris EA, Borgen PI. Magnetic resonance imaging detects unsuspected disease in patients with invasive lobular cancer. Ann Surg Oncol 2003;10:1048-53.

Ranieri E, D'Andrea MR, D'Alessio A, Bergomi S, Virno F. The integration of diagnostic tests and the role of outpatient surgery in the management of breast disease. Int Surg 1995;80:181-4.

Rhodes DJ, O'Connor MK, Phillips SW, Smith RL, Collins DA. Molecular breast imaging: a new technique using technetium Tc $99 \mathrm{~m}$ scintimammography to detect small tumors of the breast. Mayo Clin Proc 2005;80:24-30.

Rieber A, Merkle E, Bohm W, Brambs HJ, Tomczak R. MRI of histologically confirmed mammary carcinoma: clinical relevance of diagnostic procedures for detection of multifocal or contralateral secondary carcinoma. J Comput Assist Tomogr 1997;21:773-9.

Roubidoux MA, Helvie MA, Lai NE, Paramagul C. Bilateral breast cancer: early detection with mammography. Radiology 1995;196:427-31.

Sardanelli F. MR dynamic enhancement of breast lesions: High temporal resolution during the first-minute versus eight-minute study. J Comput Assist Tomogr 2000;24:724-31.

Shankar PM, Piccoli CW, Reid JM, Forsberg F, Goldberg BB. Application of the compound probability density function for characterization of breast masses in ultrasound B scans. Phys Med Biol 2005;50:2241-8.

Sickles EA. Periodic mammographic follow-up of probably benign lesions: results in 3,184 consecutive cases. Radiology 1991;179:463-8.

Slanetz PJ. Occult contralateral breast carcinoma incidentally detected by breast magnetic resonance imaging. Breast J 2002;8:145-8.

Soerjomataram I. Rising incidence of breast cancer among female cancer survivors: implications for surveillance. Br J Cancer 2009;100:77-81.

Stokes ME. Ten-year survival and cost following breast cancer recurrence: estimates from SEERMedicare data. Value Health 2008;11:213-20.

Sun X, Qian W, Song D. Ipsilateral-mammogram computer-aided detection of breast cancer. Comput Med Imaging Graph 2004;28:151-8.

Sundararajan S, Tohno E, Kamma H, Ueno E, Minami M. Detection of intraductal component around invasive breast cancer using ultrasound: correlation with MRI and histopathological findings. Radiat Med 2006;24:108-14.

The JS, Schilling KJ, Hoffmeister JW, Friedmann E, McGinnis R, Holcomb RG. Detection of breast cancer with full-field digital mammography and computer-aided detection. AJR Am J Roentgenol 2009;192:337-40. 
Thomas A. Real-time elastography - an advanced method of ultrasound: first results in 108 patients with breast lesions. Ultrasound Obstet Gynecol 2006;28:335-40.

Trecate G, Vergnaghi D, Manoukian S, Bergonzi S, Scaperrotta G, Marchesini M, et al. MRI in the early detection of breast cancer in women with high genetic risk. Tumori 2006;92:517-23.

Vahabi M. Breast cancer screening methods: a review of the evidence. Health Care Women Int 2003;24:773-93.

Van Goethem M, Schelfout K, Kersschot E, Colpaert C, Verslegers I, Biltjes I, et al. MR mammography is useful in the preoperative locoregional staging of breast carcinomas with extensive intraductal component. Eur J Radiol 2007;62:273-82.

Van Ongeval C, Bosmans H, Van Steen A. Current challenges of full field digital mammography. Rad Prot Dosim 2005;117:148-53.

Viehweg P, Bernerth T, Heinig A, Kiechle M, Buchmann J, Koelbl H, et al. MR-guided intervention in women at high hereditary risk of breast cancer due to both family and personal history of breast cancer. Breast J 2006;12:549-58.

Williams LJ, Hartswood M, Prescott RJ. Methodological issues in mammography double reading studies. J Med Screen 1998;5:202-6.

\section{Index/comparator test(s) not assessed for IBTR/MCBC detection ( $\mathrm{n}=49$ )}

Abel U. Methodological aspects of the evaluation of postoperative cancer surveillance. Part II: Efficacy. Clin Lab 2003;49:379-98.

Bongers V, Perre C, de Hooge P. The use of scintimammography for detecting the recurrence of loco-regional breast cancer: histopathologically proven results. Nucl Med Commun 2004;25:145-9.

Bradley AJ. Accuracy of axillary MR imaging in treated breast cancer for distinguishing between recurrent tumour and treatment effects: does intravenous Gd-DTPA enhancement help in cases of diagnostic dilemma? Clin Radiol 2000;55:921-8.

Buscombe JR, Holloway B, Roche N, Bombardieri E. Position of nuclear medicine modalities in the diagnostic work-up of breast cancer. Q J Nucl Med Mol Imaging 2004;48:109-18.

Casselman JW. Contrast-enhanced MRI of the breast: technique and indications. J Belge Radiol 1996;79:76-81.

Cazzaniga M. Patterns of relapse and modalities of treatment of breast cancer: the 'IRIS' project, a multicenter observational study. Oncology 2004;66:260-8.

Chen CH, Orel SG, Harris EER, Hwang WT, Solin LJ. Relation between the method of detection of initial breast carcinoma and the method of detection of subsequent ipsilateral local recurrence and contralateral breast carcinoma. Cancer 2003;98:1596-602.

Chen SL. The survival impact of the choice of surgical procedure after ipsilateral breast cancer recurrence. Am J Surg 2008;196:495-9.

Christian MC, Mccabe MS, Korn EL, Abrams JS, Kaplan RS, Friedman MA. The NationalCancer-Institute Audit of the National-Surgical-Adjuvant-Breast-And-Bowel-ProjectProtocol-B-06. N Engl J Med 1995;333:1469-74.

Ciatto S, Bravetti P, Cecchini S, Grazzini G, Iossa A, Cariaggi P, et al. The role of fine needle aspiration cytology in the differential diagnosis of suspected breast cancer local recurrences. Tumori 1990;76:225-6.

Ciatto S, Miccinesi G, Zappa M. Prognostic impact of the early detection of metachronous contralateral breast cancer. Eur J Cancer 2004;40:1496-501. 
Claus EB, Stowe M, Carter D, Holford T. The risk of a contralateral breast cancer among women diagnosed with ductal and lobular breast carcinoma in situ: data from the Connecticut Tumor Registry. Breast 2003;12:451-6.

Crowe J, Gordon NH, Brumberg DJ. Primary tumor size: relevance to breast cancer survival. Arch Surg 1992;127:910-16.

Dinh P, Cardoso F, Sotiriou C, Piccart-Gebhart MJ. New tools for assessing breast cancer recurrence. Cancer Treat Res 2008;141:99-118.

Eubank WB. Impact of FDG PET on defining the extent of disease and on the treatment of patients with recurrent or metastatic breast cancer. Am J Roentgenol 2004;183:479-86.

Gallowitsch HJ, Kresnik E, Gasser J, Kumnig G, Igerc I, Mikosch P, et al. F-18 fluorodeoxyglucose positron-emission tomography in the diagnosis of tumor recurrence and metastases in the follow-up of patients with breast carcinoma: a comparison to conventional imaging. Invest Radiol 2003;38:250-6.

Geiger AM, Thwin SS, Lash TL, Buist DSM, Prout MN, Wei FF, et al. Recurrences and second primary breast cancers in older women with initial early-stage disease. Cancer 2007;109:966-74.

Geller BM, Kerlikowske K, Carney PA, Abraham LA, Yankaskas BC, Taplin SH, et al. Mammography surveillance following breast cancer. Breast Cancer Res Treat 2003;81:107-15.

Grahek D, Montravers F, Kerrou K, Aide N, Lotz JP, Talbot JN. [18F]FDG in recurrent breast cancer: diagnostic performances, clinical impact and relevance of induced changes in management. Eur J Nucl Med Mol Imaging 2004;31:179-88.

Gunhan-Bilgen I, Oktay A. Mammographic features of local recurrence after conservative surgery and radiation therapy: comparison with that of the primary tumor. Acta Radiol 2007;48:390-7.

Hagay C, Cherel PJ, de Maulmont CE, Plantet MM, Gilles R, Floiras JL, et al. Contrast-enhanced CT: value for diagnosing local breast cancer recurrence after conservative treatment. Radiology 1996;200:631-8.

Jarvenpaa R, Holli K, Hakama M. Double-reading of plain radiographs - no benefit with regard to earliness of diagnosis of cancer recurrence: a randomised follow-up study. Eur J Cancer 2004;40:1668-73.

Jarvenpaa R, Holli K, Hakama M. Resource savings in the single reading of plain radiographs by oncologist only in cancer patient follow-up: a randomized study. Acta Oncol 2005;44:149-54.

Kuo WH, Yen AMF, Lee PH, Hou MF, Chen SC, Chen KM, et al. Incidence and risk factors associated with bilateral breast cancer in area with early age diagnosis but low incidence of primary breast cancer: analysis of 10-year longitudinal cohort in Taiwan. Breast Cancer Res Treat 2006;99:221-8.

Kuo WJ, Chang RF, Lee CC, Moon WK, Chen DR. Retrieval technique for the diagnosis of solid breast tumors on sonogram. Ultrasound Med Biol 2002;28:903-9.

Lafata JE, Simpkins J, Schultz L, Chase GA, Johnson CC, Yood MU, et al. Routine surveillance care after cancer treatment with curative intent. Med Care 2005;43:592-9.

Leibman AJ, Styblo TM, Bostwick J. Mammography of the postreconstruction breast. Plast Reconstr Surg 1997;99:698-704.

Marshall C, Eremin J, El Sheemy M, Eremin O, Griffiths PA. Monitoring the response of large $(>3 \mathrm{~cm})$ and locally advanced (T3-4, N0-2) breast cancer to neoadjuvant chemotherapy using (99m)Tc-Sestamibi uptake. Nucl Med Commun 2005;26:9-15. 
McDonough MD. The role of positron emission tomographic imaging in breast cancer. Curr Oncol Rep 2004;6:62-8.

Nielsen HM, Overgaard M, Grau C, Jensen AR, Overgaard J. Loco-regional recurrence after mastectomy in high-risk breast cancer - risk and prognosis. An analysis of patients from the DBCG 82 b\&c randomization trials. Radiother Oncol 2006;79:147-55.

Noh DY, Yun IJ, Kim JS, Kang HS, Lee DS, Chung JK, et al. Diagnostic value of positron emission tomography for detecting breast cancer. World J Surg 1998;22:223-7.

Oltra A, Santaballa A, Munarriz B, Pastor M, Montalar J. Cost-benefit analysis of a follow-up program in patients with breast cancer: a randomized prospective study. Breast J 2007;13:571-4.

Patel MR, Whitman GJ. Negative mammograms in symptomatic patients with breast cancer. Acad Radiol 1998;5:26-33.

Putter H, van der Hage J, De Bock GH, Elgalta R, van de Velde CJ. Estimation and prediction in a multi-state model for breast cancer. Biom J 2006;48:366-80.

Radan L. The role of FDG-PET/CT in suspected recurrence of breast cancer. Cancer 2006;107:2545-51.

Rajkovaca Z, Vuleta G, Matavulj A, Kovacevic P, Ponorac N. 99m Tc-sestamibi scintimammography in detection of recurrent breast cancer. Bosnian J Basic Med Sci 2007;7:256-60.

Santiago RJ, Wu L, Harris E, Fox K, Schultz D, Glick J, et al. Fifteen-year results of breastconserving surgery and definitive irradiation for Stage I and II breast carcinoma: the University of Pennsylvania experience. Int J Radiat Oncol Biol Phys 2004;58:233-40.

Schlechter BL. Quantitative DNA fingerprinting may distinguish new primary breast cancer from disease recurrence. J Clin Oncol 2004;22:1830-8.

Shen J, Hunt KK, Mirza NQ, Buchholz TA, Babiera GV, Kuerer HM, et al. Predictors of systemic recurrence and disease-specific survival after ipsilateral breast tumor recurrence. Cancer 2005; 104:479-90.

Snider HC, Rubin E, Henson R. Axillary ultrasonography to detect recurrence after sentinel node biopsy in breast cancer. Ann Surg Oncol 2006;13:501-7.

Soldin MG. Routine histological examination of the mastectomy scar at the time of breast reconstruction: important oncological surveillance? Br J Plast Surg 2004;57:143-5.

Spanu A, Farris A, Schillaci O, Chessa F, Solinas ME, Falchi A, et al. The usefulness of 99mTc tetrofosmin scintigraphy in patients with breast cancer recurrences. Nucl Med Commun 2003;24:145-54.

Takeuchi H, Baba H, Kano T, Maehara Y. The time-related changes of the importance of prognostic factors in breast cancer. A sequential multivariate analysis of 1423 Japanese patients. Breast Cancer Res Treat 2005;94:273-8.

Tamaki T, Naito A, Nishio M, Kawahara K. FDG-PET for evaluation of recurrent lymph node metastases in patients with surgically resected breast cancer: adding spot images to whole body images. Breast Cancer 2004;11:60-4.

Truong PT, Berthelet E, Lee J, Kader HA, Olivotto IA. The prognostic significance of the percentage of positive/dissected axillary lymph nodes in breast cancer recurrence and survival in patients with one to three positive axillary lymph nodes. Cancer 2005;103:2006-14.

Warren RM, Duffy SW. Comparison of single reading with double reading of mammograms, and change in effectiveness with experience. Br J Radiol 1995;68:958-62. 
Wu D. Positron emission tomography in diagnosis and management of invasive breast cancer: current status and future perspectives. Clin Breast Cancer 2003;4:S55-63.

Yilmaz MH, Esen G, Ayarcan Y, Aydogan F, Ozguroglu M, Demir G, et al. The role of US and MR imaging in detecting local chest wall tumor recurrence after mastectomy. Diagn Interv Radiol 2007;13:13-18.

Younis T, Rayson D, Dewar R, Skedgel C. Modeling for cost-effective-adjuvant aromatase inhibitor strategies for postmenopausal women with breast cancer. Ann Oncol 2007;18:293-8.

\section{Reference standard not met $(\mathrm{n}=6)$}

Baz EM. The role of enhanced Doppler ultrasound in differentiation of benign vs. malignant scar lesion after breast surgery for malignancy. Ultrasound Obstet Gynecol 2000;15:377-82.

Ciatto S, Cataliotti L, Pacini P, Distante V, Bianchi S, Simoncini R, et al. Cancer reoccurrence in the conserved breast: diagnostic features in a consecutive series of 102 cases. Int J Oncol 1993;3:337-9.

Cohen EK, Leonhardt CM, Shumak RS, Souter IC, Bukhanov K, Fishell EK, et al. Magnetic resonance imaging in potential postsurgical recurrence of breast cancer: pitfalls and limitations. Can Assoc Radiol J 1996;47:171-6.

Gilles R, Guinebretiere JM, Shapeero LG, Lesnik A, Contesso G, Sarrazin D, et al. Assessment of breast-cancer recurrence with contrast-enhanced subtraction MR-imaging: preliminary results in 26 patients. Radiology 1993;188:473-8.

Kramer S, Schulz-Wendtland R, Hagedorn K, Bautz W, Lang N. Magnetic resonance imaging in the diagnosis of local recurrences in breast cancer. Anticancer Res 1998;18:2159-61.

Lee JM. Detecting nonpalpable recurrent breast cancer: the role of routine mammographic screening of transverse rectus abdominis myocutaneous flap reconstructions. Radiology 2008;248:398-405.

\section{Not relevant comparator test $(\mathrm{n}=19)$}

Adler LP, Bakale G, Schnur KC, Shenk RR. Innovations in breast cancer imaging: PET for diagnosis and follow-up. Medscape Womens Health 1998;3:5.

Buscombe JR, Cwikla JB, Thakrar DS, Parbhoo SP, Hilson AJ. Prone SPET scintimammography. Nucl Med Commun 1999;20:237-45.

Cid JA, Rampaul RS, Ellis IO, Wilson AR, Burrell HC, Evans AJ, et al. Woman feels breast lump surgeon cannot: the role of ultrasound in arbitration. Eur J Cancer 2004;40:2053-5.

Cwikla JB, Buscombe JR, Parbhoo SP, Kelleher SM, Thakrar DS, Hinton J, et al. Use of 99TcmMIBI in the assessment of patients with suspected recurrent breast cancer. Nucl Med Commun 1998;19:649-55.

Cwikla JB, Kolasinska A, Buscombe JR, Hilson AJ. Tc-99m MIBI in suspected recurrent breast cancer. Cancer Biother Radiopharm 2000;15:367-72.

Eubank WB, Mankoff DA, Vesselle HJ, Eary JF, Schubert EK, Dunnwald LK, et al. Detection of locoregional and distant recurrences in breast cancer patients by using FDG PET. Radiographics 2002;22:5-17.

Flanagan FL, Dehdashti F, Siegel BA. PET in breast cancer. Semin Nucl Med 1998;28:290-302.

Hermans J. Scintimammography: a new imaging technique for diagnosis and follow-up of breast cancer. Bull Cancer 2000;87:334-40. 
Lee VW, Sax EJ, McAneny DB, Pollack S, Blanchard RA, Beazley RM, et al. A complementary role for thallium-201 scintigraphy with mammography in the diagnosis of breast cancer. J Nucl Med 1993;34:2095-100.

Lind P, Igerc I, Beyer T, Reinprecht P, Hausegger K. Advantages and limitations of FDG PET in the follow-up of breast cancer. Eur J Nucl Med Mol Imaging 2004;31 (Suppl. 1):S125-34.

Mangkharak J. Scintimammography (SMM) in breast cancer patients. J Med Assoc Thai 1999;82:242-9.

Mathieu I, Mazy S, Willemart B, Destine M, Mazy G, Lonneux M. Inconclusive triple diagnosis in breast cancer imaging: is there a place for scintimammography? J Nucl Med 2005;46:1574-81.

Moskovic E, Curtis S, A'Hern RP, Harmer CL, Parsons C. The role of diagnostic CT scanning of the brachial plexus and axilla in the follow-up of patients with breast cancer. Clin Oncol ( $R$ Coll Radiol) 1992;4:74-7.

Moy L, Murphy-Walcott AD. Improving specificity of breast MRI using prone PET and fused MRI and PET 3D volume datasets. J Nucl Med 2007;48:528-37.

Propeck PA, Scanlan KA. Utility of axillary views in postmastectomy patients. Radiology 1993;187:769-71.

Rostom AY, Powe J, Kandil A, Ezzat A, Bakheet S, el Khwsky F, et al. Positron emission tomography in breast cancer: a clinicopathological correlation of results. Br J Radiol 1999;72:1064-8.

Siggelkow W, Zimny M, Faridi A, Petzold K, Buell U, Rath W. The value of positron emission tomography in the follow-up for breast cancer. Anticancer Res 2003;23:1859-67.

Usmani S, Niaz K, Maseeh UZ, Kamal S, Niyaz K, Mehboob J, et al. Role of 99mTc-MIBI scintimammography and $\mathrm{X}$-ray mammography in the diagnosis of locoregional recurrence of breast cancer. JPMA 2007;57:172-5.

Viehweg P, Heinig A, Lampe D, Buchmann J, Heywang-Kobrunner SH. Retrospective analysis for evaluation of the value of contrast-enhanced MRI in patients treated with breast conservative therapy. MAGMA 1998;7:141-52.

\section{Study design not met $(\mathrm{n}=67)$}

Athanasiou A. How to optimize breast ultrasound. Eur J Radiol 2009;69:6-13.

Auguste LJ, Gerold T, Rothenberg H, Litvak S, Gentin S. Detection of recurrent carcinoma in the irradiated breast. Comp Surg 1994;13:556-561.

Balu-Maestro C, Bruneton JN, Geoffray A, Chauvel C, Rogopoulos A, Bittman O.

Ultrasonographic posttreatment follow-up of breast cancer patients. J Ultrasound Med 1991;10:1-7.

Bartella L, Smith CS, Dershaw DD, Liberman L. Imaging breast cancer. Radiol Clin North Am 2007;45:45-67.

Belli P, Costantini M, Romani M, Marano P, Pastore G. Magnetic resonance imaging in breast cancer recurrence. Breast Cancer Res Treat 2002;73:223-35.

Broët P, de la Rochefordière A, Scholl SM, Fourquet A, De Rycke Y, Pouillart P, et al. Analyzing prognostic factors in breast cancer using a multistate model. Breast Cancer Res Treat 1999;54:83-9. 
Buckley DL, Mussurakis S, Horsman A. Effect of temporal resolution on the diagnostic efficacy of contrast-enhanced MRI in the conservatively treated breast. J Comput Assist Tomogr 1998;22:47-51.

Ciatto S. Re: for debate: post-treatment mammography following breast-conserving treatment. Breast 1994;3:130.

Ciatto S. Detection of breast cancer local recurrences. Ann Oncol 1995;6:23-6.

Ciatto S, Bonardi R, Cataliotti L, Cardona G. Intraductal breast carcinoma. Review of a multicenter series of 350 cases. Coordinating Center and Writing Committee of FONCAM (National Task Force for Breast Cancer), Italy. Tumori 1990;76:552-4.

Ciatto S, Catallotti L, Distante V, Rontini M, Muraca MG. Diagnostic features of 225 consecutive cases of cancer recurrence in the conserved breast. Breast 1997;6:367-70.

Coulthard A, Beveridge CJ, Potterton AJ. MRI in routine breast cancer follow-up: correlation with clinical outcome. Clin Radiol 1999;54:459-61.

Davis PL, McCarty KS, Jr. Sensitivity of enhanced MRI for the detection of breast cancer: new, multicentric, residual, and recurrent. Eur Radiol 1997;7(Suppl. 5):289-98.

Davis PL, McCarty KS, Jr. Magnetic resonance imaging in breast cancer staging. Top Magn Reson Imaging 1998;9:60-75.

Dipaola RS. Ipsilateral breast tumor recurrence following conservative surgery and radiation therapy. Oncology 1994;8:59-68.

Dixon JM, Norman B. Routine follow up of breast cancer in primary care. Most recurrences after breast conservation are detected by regular hospital visits. BMJ 1996;313:1548.

Douek M, Davidson T, Taylor I. Breast cancer imaging: what are the optimal modalities? Eur J Surg Oncol 1998;24:573-82.

Duffy SW, Chen HH, Tabar L, Day NE. Estimation of mean sojourn time in breast cancer screening using a Markov chain model of both entry to and exit from the preclinical detectable phase. Stat Med 1995;14:1531-43.

Emens LA, Davidson NE. The follow-up of breast cancer. Semin Oncol 2003;30:338-48.

Fowble B, Solin LJ, Schultz DJ, Rubenstein J, Goodman RL. Breast recurrence following conservative surgery and radiation: patterns of failure, prognosis, and pathologic findings from mastectomy specimens with implications for treatment. Int J Radiat Oncol Biol Phys 1990; 19:833-42.

Giger ML. Computerized image analysis in breast cancer detection and diagnosis. Semin Breast Dis 2002;5:199-210.

Goerres GW, Michel SC, Fehr MK, Kaim AH, Steinert HC, Seifert B, et al. Follow-up of women with breast cancer: comparison between MRI and FDG PET. Eur Radiol 2003;13:1635-44.

Gojkovic Z. Comparison of ultrasound controlled aspiration puncture to clinical examination in detection of breast cancer recurrence. Arch Oncol 2006;14:118-21.

Hall NJ, Evans AJ, Kollias J, Denley H, Pinder SE, Ellis IO, et al. Bilateral breast carcinomas: do they have similar mammographic features? Clin Radiol 1999;54:434-7.

Heywang-Kobrunner SH, Schlegel A, Beck R, Wendt T, Kellner W, Lommatzsch B, et al. Contrast-enhanced MRI of the breast after limited surgery and radiation therapy. J Comput Assist Tomogr 1993;17:891-900. 
Jacobs BP, Burns B, Marya R, Chapman J, Stone B, Hwang S, et al. Integrative tumor board: recurrent breast cancer or new primary? UCSF Osher Center for Integrative Medicine and UCSF Carol Franc Buck Breast Care Center. Integr Cancer Ther 2003;2:289-300.

Jager JJ, Langendijk JA, Dohmen JP, Schreutelkamp IL, Volovics L, Vanengelshoven JM, et al. Mammography in the follow-up after breast-conserving treatment in cancer of the breast suitability for mammographic interpretation, validity and interobserver variation. $\mathrm{Br} J$ Radiol 1995;68:754-60.

Kumar NA. MR imaging: its current and potential utility in the diagnosis and management of breast cancer. Magn Reson Imaging Clin N Am 2000;8:715-28.

Lehman CD. Indications for breast MRI in the patient with newly diagnosed breast cancer. JNCCN 2009;7:193-200.

Lewis-Jones HG, Whitehouse GH, Leinster SJ. The role of magnetic resonance imaging in the assessment of local recurrent breast carcinoma. Clin Radiol 1991;43:197-204.

Liberman L. Breast MR imaging in assessing extent of disease. Magn Reson Imaging Clin N Am 2006;14:339-49.

Lu WL, Jansen L, Post WJ, Bonnema J, Van de Velde JC, De Bock GH. Impact on survival of early detection of isolated breast recurrences after the primary treatment for breast cancer: a metaanalysis. Breast Cancer Res Treat 2009;114:403-12.

Molino A. What is the best follow-up methodology in early breast cancer? Breast 2008;29:1-2.

Morakkabati N, Leutner CC, Schmiedel A, Schild HH, Kuhl CK. Breast MR imaging during or soon after radiation therapy. Radiology 2003;229:893-901.

Morgan RJ. Detection of metachronous breast carcinoma: the role of follow-up? Ann R Coll Surg Engl 1999;81:97-9.

Mould RF. Methodology to predict a maximum follow-up period for breast cancer patients without significantly reducing the chance of detecting a local recurrence. Phys Med Biol 2004;49:1079-83.

Murphy KC, Coppin CML, Kader HEHA, Norris BD, Weir LM, Hugi MR, et al. Follow-up after treatment for breast cancer. Can Med Assoc J 1998;158:S65-70.

Mussurakis S, Buckley DL, Bowsley SJ, Carleton PJ, Fox JN, Turnbull LW, et al. Dynamic contrast-enhanced magnetic resonance imaging of the breast combined with pharmacokinetic analysis of gadolinium-DTPA uptake in the diagnosis of local recurrence of early stage breast carcinoma. Invest Radiol 1995;30:650-62.

Musumeci R. Radiologic procedures for the diagnosis of distant recurrences of breast cancer. Ann Oncol 1995;6(Suppl. 2):27-9.

Muuller RD, Barkhausen J, Sauerwein W, Langer R. Assessment of local recurrence after breastconserving therapy with MRI. J Comput Assist Tomogr 1998;22:408-12.

Newstead GM. MR imaging in the management of patients with breast cancer. Semin Ultrasound CT MRI 2006;27:320-32.

Nguyen BD. Intraductal papilloma of the breast: F-18 FDG PET demonstration. Clin Nucl Med 2005;30:481-2.

Orel SG, Fowble BL, Solin LJ, Schultz DJ, Conant EF, Troupin RH. Breast cancer recurrence after lumpectomy and radiation therapy for early-stage disease: prognostic significance of detection method. Radiology 1993;188:189-94. 
Potterton AJ, Yuille F, Tinkler S, Chippindale AJ, Wilsdon JB, Lucraft HH, et al. MRI appearances of the axilla in treated breast cancer. Br J Radiol 1999;72:250-7.

Preda L, Villa G, Rizzo S, Bazzi L, Origgi D, Cassano E, et al. Magnetic resonance mammography in the evaluation of recurrence at the prior lumpectomy site after conservative surgery and radiotherapy. Breast Cancer Res 2006;8:R53.

Rankin SC. MRI of the breast. Br J Radiol 2000;73:806-18.

Rissanen TJ, Makarainen HP, Mattila SI, Lindholm EL, Heikkinen MI, Kiviniemi HO. Breast cancer recurrence after mastectomy: diagnosis with mammography and US. Radiology 1993; 188:463-7.

Rodger A. Routine follow up of breast cancer in primary care. Br Med J 1997;314:1129-30.

Safir J, Zito JL, Gershwind ME, Faegenburg D, Tobin CE, Cayea PD, et al. Contrast-enhanced breast MRI for cancer detection using a commercially available system: a perspective. Clin Imaging 1998;22:162-79.

Schmidt GP. Comprehensive imaging of tumor recurrence in breast cancer patients using wholebody MRI at 1.5 and 3 T compared to FDG-PET-CT. Eur J Radiol 2008;65:47-58.

Seely JM, Nguyen ET, Jaffey J. Breast MRI in the evaluation of locally recurrent or new breast cancer in the postoperative patient: correlation of morphology and enhancement features with the BI-RADS category. Acta Radiol 2007;48:838-45.

Singh V. New diagnostic techniques for breast cancer detection. Future Oncol 2008;4:501-13.

Siva N. Using MRI to detect contralateral breast cancer. Lancet Oncol 2007;8:377.

Snow RD, Dyess DL, Harpen MD, Kreisberg CN, Tucker JA. Dynamic magnetic resonance imaging in evaluating suspicious breast lesions: correlation with pathologic findings. South Med J 1998;91:527-32.

Soo MS, Rosen EL, Baker JA, Vo TT, Boyd BA. Negative predictive value of sonography with mammography in patients with palpable breast lesions. AJR Am J Roentgenol 2001;177:1167-70.

Stickeler E. Diagnosis, staging and follow-up in breast cancer patients. Breast Care 2007;2:158-62.

Strax P. Imaging. Follow-up of breast cancer reconstruction cases. Cancer 1991;68:1157-8.

Susini T, Nori J, Vanzi E, Livi L, Pecchioni S, Bazzocchi M, et al. Axillary ultrasound scanning in the follow-up of breast cancer patients undergoing sentinel node biopsy. Breast 2007;16:190-6.

Turnbull LW. Magnetic resonance imaging of the breast: potential for gain in the detection, staging and diagnosis of primary and recurrent tumour? Breast 1998;7:1-4.

Valdes EK. Diagnosis of recurrent breast cancer by ductoscopy. Breast J 2005;11:506.

Van Goethem M. Role of MRI of the breast in the evaluation of the symptomatic patient. Curr Opin Obstet Gynecol 2009;21:74-9.

Van Steen AM. The problems of imaging and interpretation of the treated breast. Acta Clinica Belgica 1993;15:S32-7.

Vogel VG. Identifying and screening patients at risk of second cancers. Cancer Epidemiol Biomarker Prevent 2006;15:2027-32.

Warner E, Messersmith H, Causer P, Elsen A, Shumack R, Plewes D. Systematic review: using magnetic resonance imaging to screen women at high risk for breast cancer. Ann Intern Med 2008; 148:671-9.

Winehouse J, Douek H, Holz K, Madjar H, Gillams A, Lees W, et al. Contrast-enhanced colour Doppler ultrasonography in suspected breast cancer recurrence. Br J Surg 1999;86:1198-201. 
Wright T, McGechan A. Breast cancer: new technologies for risk assessment and diagnosis. Mol Diagn 2003;7:49-55.

Zakhireh J, Gomez R, Esserman L. Converting evidence to practice: a guide for the clinical application of MRI for the screening and management of breast cancer. Eur J Cancer 2008;44:2742-52.

\section{Outcomes not reported $(\mathrm{n}=8)$}

Bahri S, Chen J-H, Yu HJ, Kuzucan A, Nalcioglu O, Su M-Y. Can dynamic contrast-enhanced MRI (DCE-MRI) predict tumor recurrence and lymph node status in patients with breast cancer? Ann Oncol 2008;19:822-4.

Crystal P, Strano SD, Shcharynski S, Koretz MJ. Using sonography to screen women with mammographically dense breasts. AJR Am J Roentgenol 2003;181:177-82.

Fajardo LL, Roberts CC, Hunt KR. Mammographic surveillance of breast cancer patients: should the mastectomy site be imaged? AJR Am J Roentgenol 1993;161:953-5.

Gorechlad JW, McCabe EB, Higgins JH, Likosky DS, Lewis PJ, Rosenkranz KM, et al. Screening for recurrences in patients treated with breast-conserving surgery: is there a role for MRI? Ann Surg Oncol 2008;15:1703-9.

Kim MJ, Kim EK, Kwak JY, Park BW, Kim SI, Sohn J, et al. Role of sonography in the detection of contralateral metachronous breast cancer in an Asian population. AJR Am J Roentgenol 2008;190:476-80.

Liberman L, Morris EA, Kim CM, Kaplan JB, Abramson AF, Menell JH, et al. MR imaging findings in the contralateral breast of women with recently diagnosed breast cancer. AJR Am J Roentgenol 2003;180:333-41.

Rissanen TJ, Makarainen HP, Apaja-Sarkkinen MA, Lindholm EL. Mammography and ultrasound in the diagnosis of contralateral breast cancer. Acta Radiol 1995;36:358-66.

Sardanelli F, Melani E, Ottonello C, Parodi RC, Imperiale A, Massa T, et al. Magnetic resonance imaging of the breast in characterizing positive or uncertain mammographic findings. Cancer Detect Prevent 1998;22:39-42.

\section{Retained for background information ( $\mathrm{n}=8$ )}

Bartella L. Advances in breast imaging: magnetic resonance imaging. Curr Oncol Rep 2006;8:7-13.

BlueCross BlueShield Association (BCBSA). Breast MRI for detection or diagnosis of primary or recurrent breast cancer. Technology Evaluation Center Assessment Program (executive summary). Chicago, IL: BCBSA, vol. 19, pp. 1-9; 2004.

Dershaw DD. Mammography in patients with breast cancer treated by breast conservation (lumpectomy with or without radiation). AJR Am J Roentgenol 1995;164:309-16.

Kataja VV, Colleoni M, Bergh J, ESMO Guidelines TF. ESMO Minimum Clinical Recommendations for diagnosis, treatment and follow-up of locally recurrent or metastatic breast cancer (MBC). Ann Oncol 2005;16(Suppl. 1):i10-12.

Kopans DB. Mammographic follow-up to detect breast cancer recurrence after conservative treatment. Radiology 1994;190:286.

Margolese RG. Recent trends in the management of breast cancer. 4. Diagnosis and management of local recurrence after breast-conservation surgery. Can J Surg 1992;35:378-81.

Rebner M. Should screening MRI be included in surveillance for patients treated with breastconserving therapy? Nature Clin Pract Oncol 2009;6:8-9. 
Rissanen TJ, Apaja-Sarkkinen MA, Makarainen HP, Heikkinen MI. Ultrasound-guided fine needle aspiration biopsy in the diagnosis of breast cancer recurrence after mastectomy. Acta Radiol 1997;38:232-9.

\section{Not available $(\mathrm{n}=3)$}

Ahmed I, Nazir R, Chaudhary MY, Kundi S. Triple assessment of breast lump. JCPSP 2007; 17:535-8.

Edwards AS. After breast cancer. Patient follow-up in the primary care setting. Adv Nurse Pract 2004;12:37-42.

Zwiggelaar R, Parr TC, Schumm JE, Hutt IW, Taylor CJ, Astley SM, et al. Model-based detection of spiculated lesions in mammograms. Med Image Anal 1999;3:39-62. 


\section{Appendix 20}

Data extraction form for the systematic review of test performance of surveillance mammography and comparator tests in detecting IBTR/MCBC 
HTA Mammography Surveillance

Diagnostic Accuracy (review 2) data extraction form

Version 4

May 2009

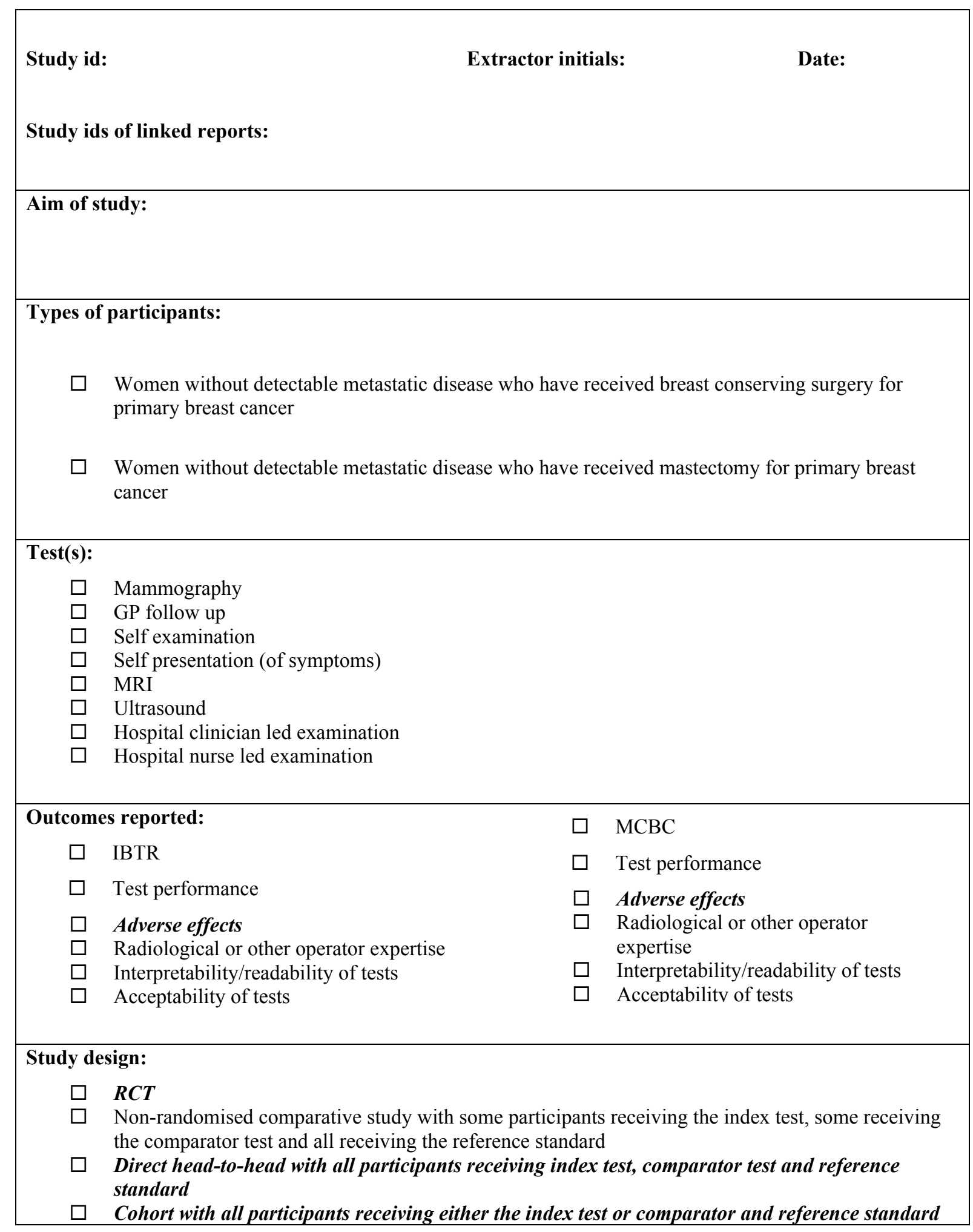




\section{Multicentre study? $\quad \square \quad$ No $\quad \square \quad$ Yes $\quad$ If yes, number of centres:}

Study start/end dates: Duration of study:

Country:

Source of funding:

Additional information on study design:

\section{Inclusion criteria:}

\section{Exclusion criteria:}

Characteristics of the participants

\begin{tabular}{|l|l|l|l|}
\hline & Group 1 & Group 2 & All \\
\hline Enrolled & & & \\
\hline $\begin{array}{l}\text { [For RCTs - number } \\
\text { randomised] }\end{array}$ & & & \\
\hline $\begin{array}{l}\text { Received tests } \\
\text { Received rence } \\
\text { standard }\end{array}$ & & & \\
\hline $\begin{array}{l}\text { [Post randomisation } \\
\text { exclusions] }\end{array}$ & & & \\
\end{tabular}




\begin{tabular}{|c|c|c|c|}
\hline Analysed & & & \\
\hline Lost to follow-up & & & \\
\hline $\begin{array}{c}\text { No Age: } \text { Mean } \\
\text { Median } \\
\text { SD } \\
\text { Range } \\
\text { No. }<50 \\
\text { No. } 50 \text { and over }\end{array}$ & & 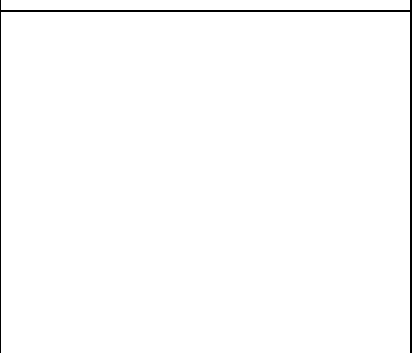 & 列 \\
\hline $\begin{array}{l}\text { Menopausal status: } \\
\text { No. premenopausal } \\
\text { No. postmenopausal }\end{array}$ & & 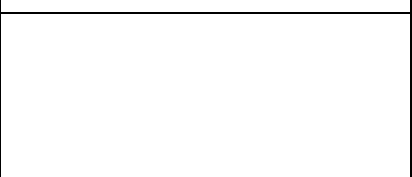 & \\
\hline $\begin{array}{l}\text { HRT Status: } \\
\text { No. currently receiving } \\
\text { HRT } \\
\text { No. previously received } \\
\text { HRT } \\
\text { No. never received } \\
\text { HRT }\end{array}$ & & & \\
\hline Primary Treatment: & & & \\
\hline $\begin{array}{l}\text { No. received primary } \\
\text { breast conserving } \\
\text { surgery (WLE) }\end{array}$ & & & \\
\hline $\begin{array}{l}\text { No. received primary } \\
\text { mastectomy }\end{array}$ & & & \\
\hline $\begin{array}{ll}\text { No. } & \text { reconstructed } \\
\text { breast } & \end{array}$ & & & \\
\hline $\begin{array}{l}\text { No. receiving treatment } \\
\text { for primary breast } \\
\text { cancer: }\end{array}$ & & & \\
\hline
\end{tabular}




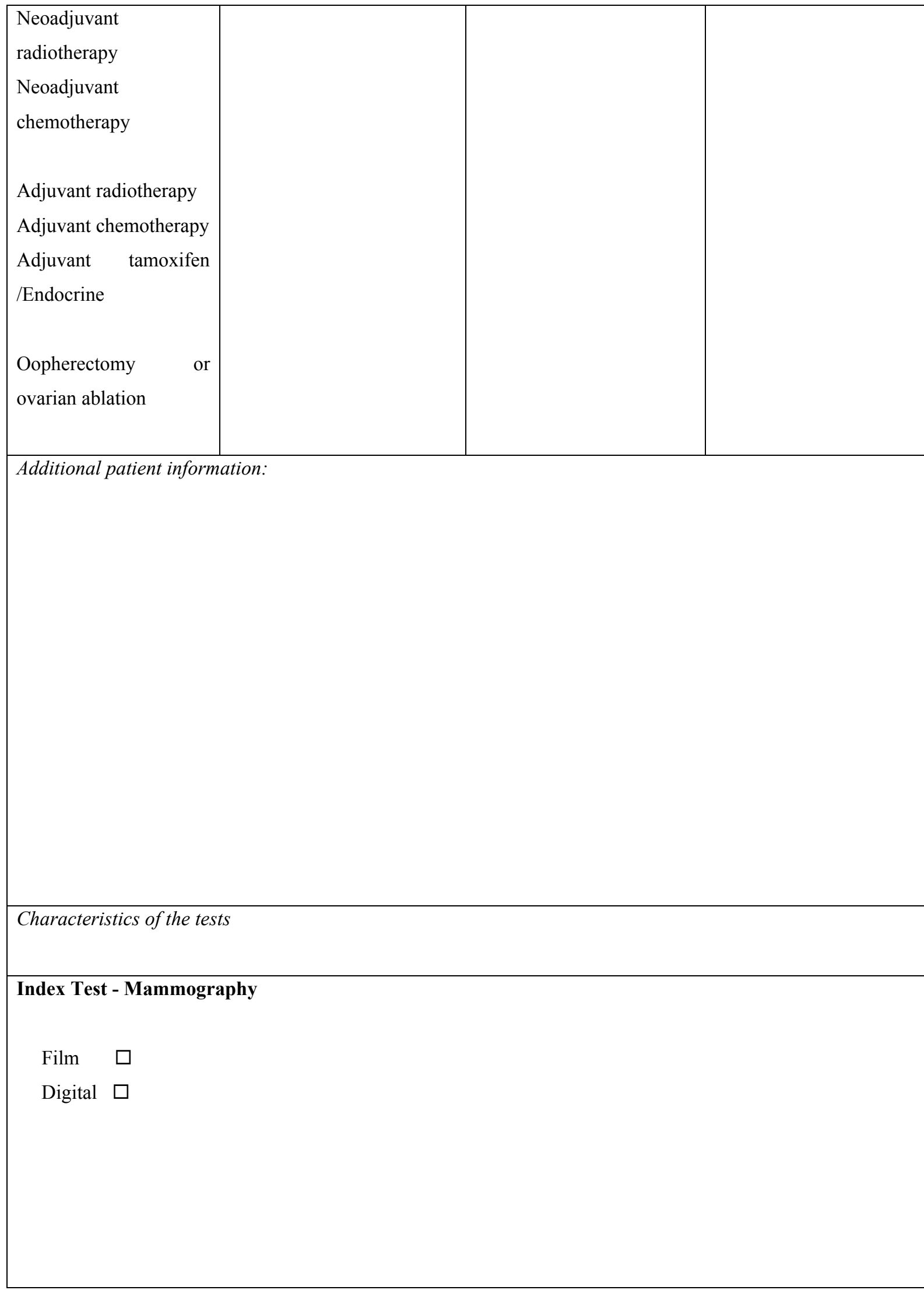


Scoring system and positive test result defined as:

Details of interpreter/reader experience if reported:

Additional information on test (e.g. radiation dose, time taken, etc): 
Comparator test:
MRI
Ultrasound

For the following comparators, a positive test result (e.g. lump identified by palpation) will initiate an imaging test prior to biopsy or Fine Needle Aspiration Cytology (FNAC). Please indicate whether a mammogram or other imaging test was conducted prior to biopsy/ FNAC for people with positive test results. Reported test performance (sensitivity/specificity) should reflect the comparator test and not the imaging test alone.

Mammo/Other prior to biopsy/FNAC
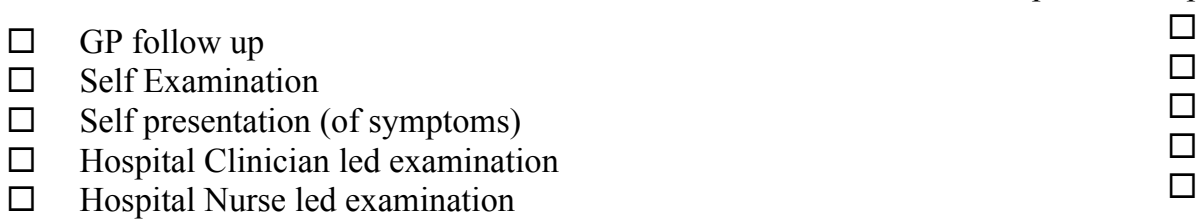

Positive test result defined as:

Details of operator experience if reported: 


\section{Additional information on comparator test:}

Reference standard:

Positive Index/Comparator test results verified by:

$\square \quad$ Histopathological assessment of biopsied tissue

$\square \quad$ Fine Needle Aspiration Cytology

Negative Index/Comparator test results verified by:

$\square \quad$ Subsequent testing within a 3 year follow up period

Length of follow-up time for verifying negative index/comparator test results:

How was tumour size determined?

How was tumour grade determined? 
Additional information on reference standard: 


\section{Results \\ IBTR/MCBC Tumour Type}

Please record the number of women with IBTR and/or MCBC

No of women with:

No of women with:

IBTR

$\mathrm{MCBC}$

Please record the associated the number of women with the following for IBTR and/or MCBC:

IBTR - No of women with:

DCIS

LCIS

Invasive

Grade 1

Grade 1
MCBC - No of women with:

DCIS

LCIS

Invasive 
Grade 2

Grade 2

Grade 3

Grade 3

If reported, please record the number of women with the following:

IBTR

$\mathrm{MCBC}$

$\underline{\text { Size }}$

$\underline{\text { Size }}$

Not measurable

Invasive tumor in $\mathrm{mm}$

(largest dimension of

dominant invasive

tumour focus)

Whole size of tumor

(invasive plus

surrounding DCIS if DCIS

extends $>1 \mathrm{~mm}$ beyond

invasive)

Morphologic type

a. Ductal/no specific (ductal NST) 
b. Lobular

c. Other 


\section{Test performance (true and false positives and negatives)}

Record data for each level of analysis e.g. patient, all biopsies, e.g. Size, grade, DCIS, Invasive, etc on separate sheet(s) containing $2 \times 2$ tables

\section{General information on IBTR/MCBC:}

\section{General information on adverse events:}

\begin{tabular}{|l|l|l|l|}
\hline $\begin{array}{c}\text { Adverse events } \\
\text { reported }\end{array}$ & $\begin{array}{l}\text { Group 1 } \\
\text { no. of women with event } \\
\text { and \% of total women in } \\
\text { group }\end{array}$ & $\begin{array}{l}\text { Goup } 2 \\
\text { and \% of total women in } \\
\text { group }\end{array}$ & $\begin{array}{l}\text { All } \\
\text { and \% of total women in } \\
\text { study }\end{array}$ \\
\hline & & & \\
& & & \\
\end{tabular}




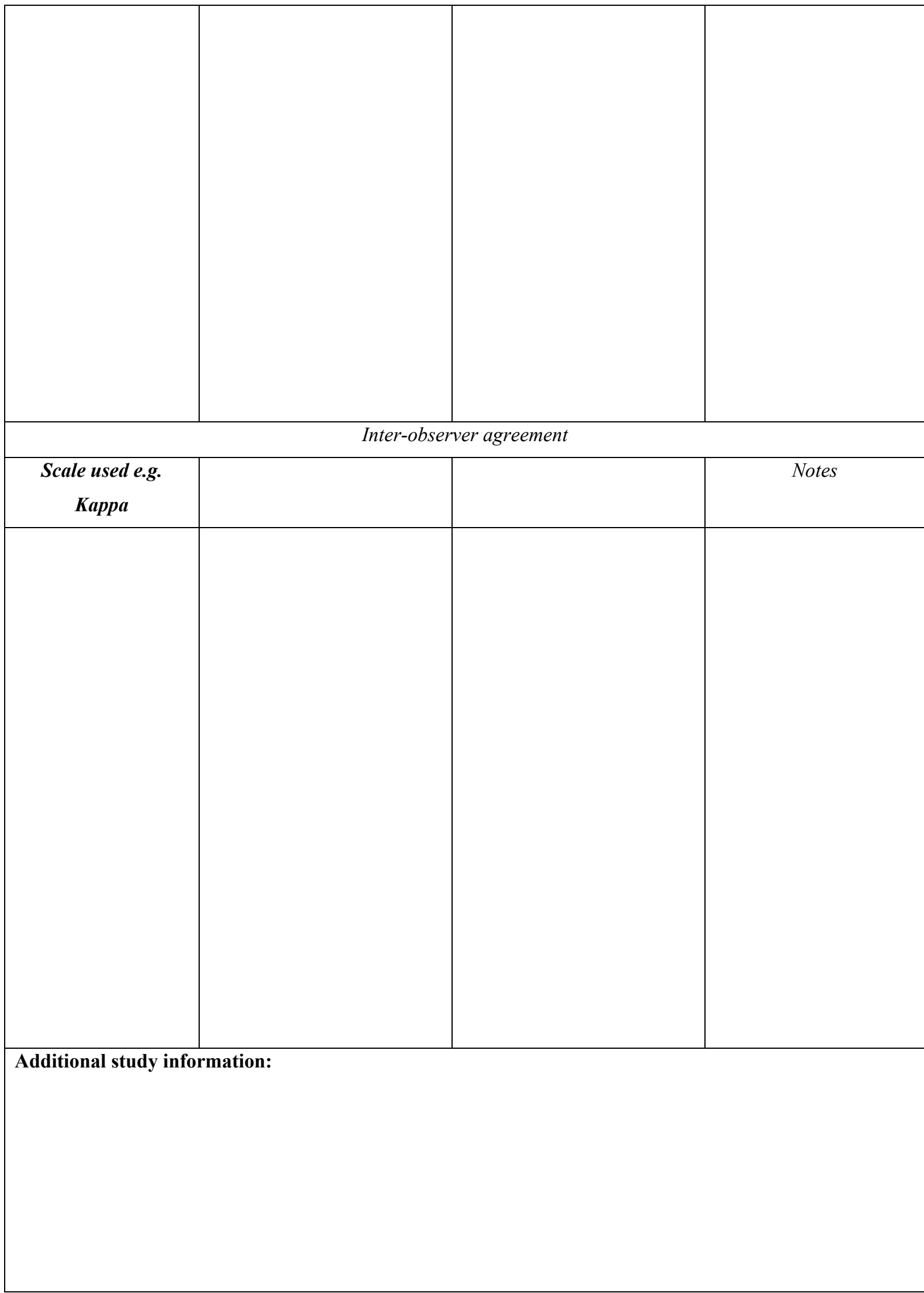


HTA Mammography Surveillance- Diagnostic Accuracy (review 2) 2x2 form Version 2, March 2009

Study id:

Extractor initials:

Date:

Study ids of linked reports:

Please record the unit of analysis as reported by the study authors - e.g. women level, biopsy level. If given, please record unit of analysis by our considered sub-groups: Age, menopausal status, HRT status, primary treatment, second tumour type.

Test:

IBTR/MCBC

\begin{tabular}{|l|l|l|l|}
\hline & \multicolumn{2}{|l|}{ Unit of analysis: } & \\
\hline & With disease & Without disease & \\
\hline Positive test & TP & FP & Total testing positive \\
\hline Negative test & FN & TN & Total testing negative \\
\hline & Total with & $\begin{array}{l}\text { Total } \\
\text { disease without }\end{array}$ & \\
\hline & & & \\
\hline
\end{tabular}

Sensitivity:

Specificity:

Test:

IBTR/MCBC 


\begin{tabular}{|l|l|l|l|}
\hline & \multicolumn{2}{|l|}{ Unit of analysis: } & \\
\hline & With disease & Without disease & \\
\hline Positive test & TP & FP & Total testing positive \\
\hline Negative test & FN & TN & Total testing negative \\
\hline & Total with & $\begin{array}{l}\text { Total without } \\
\text { disease }\end{array}$ & \\
& & & \\
\hline
\end{tabular}

\section{Sensitivity:}

Specificity: 



\section{Appendix 21}

Quality assessment tool used in the systematic review of test performance of surveillance mammography and comparator tests in detecting IBTR/MCBC 
HTA Mammography Surveillance - Diagnostic Accuracy review 2

Quality Assessment Form

Version 4

Study id:
May 2010

Extractor initials: Date:

\begin{tabular}{|c|c|c|c|c|}
\hline Item & & Yes & No & Unclear \\
\hline 1. & $\begin{array}{l}\text { Was the spectrum of patients representative of the } \\
\text { patients who will receive the test in practice? } \\
\text { (women previously treated for primary breast } \\
\text { cancer) }\end{array}$ & & & \\
\hline 2. & $\begin{array}{l}\text { Is the reference standard likely to correctly classify } \\
\text { the target condition? }\end{array}$ & & & \\
\hline $3 a$ & $\begin{array}{l}\text { For positive test results, is the time period } \\
\text { between reference standard and } \\
\text { index/comparator test short enough to be } \\
\text { reasonably sure that the target condition did not } \\
\text { change between the two tests? (biopsy or FNAC } \\
\text { within } 3 \text { months, histopathology within } 6 \text { months) }\end{array}$ & & & \\
\hline $3 b$ & $\begin{array}{l}\text { For negative test results, is the time period } \\
\text { between the index/comparator test and the } \\
\text { reference standard short enough to be reasonably } \\
\text { sure that the target condition did not change } \\
\text { between the two tests? (follow-up within } 3 \text { years) }\end{array}$ & & & \\
\hline 4. & $\begin{array}{l}\text { Did the whole sample or a random selection of the } \\
\text { sample receive verification using a reference } \\
\text { standard of diagnosis? }\end{array}$ & & & \\
\hline $5 a$ & $\begin{array}{l}\text { Did patients testing positively on the } \\
\text { index/comparator test receive the same reference } \\
\text { standard (i.e. FNAC or biopsy)? }\end{array}$ & & & \\
\hline $5 b$ & $\begin{array}{l}\text { Did patients testing negatively on the } \\
\text { index/comparator test receive the same reference } \\
\text { standard (i.e. follow up)? }\end{array}$ & & & \\
\hline 6. & $\begin{array}{l}\text { Was the reference standard independent of the } \\
\text { index test (i.e. the index test did not form part of } \\
\text { the reference standard)? }\end{array}$ & & & \\
\hline 7. & $\begin{array}{l}\text { Were the index test results interpreted without } \\
\text { knowledge of the results of the reference } \\
\text { standard? }\end{array}$ & & & \\
\hline 8. & $\begin{array}{l}\text { Were the reference standard results interpreted } \\
\text { without knowledge of the results of the index test? }\end{array}$ & & & \\
\hline 9. & $\begin{array}{l}\text { Were index and comparator tests interpreted } \\
\text { independently (if no record the sequence)? }\end{array}$ & & & \\
\hline 10. & $\begin{array}{l}\text { Were the same clinical data available when test } \\
\text { results were interpreted as would be available } \\
\text { when the test is used in practice? }\end{array}$ & & & \\
\hline 11. & $\begin{array}{l}\text { Were uninterpretable/intermediate test results } \\
\text { reported? }\end{array}$ & & & \\
\hline 12. & Were withdrawals from the study explained? & & & \\
\hline
\end{tabular}




\section{Appendix 22}

\section{Characteristics of the included studies for the systematic review of test performance of surveillance mammography and comparator tests in detecting IBTR/MCBC}

\begin{tabular}{|c|c|c|c|c|}
\hline Study ID & Study design & Index tests & Comparator test & Test purpose \\
\hline $\begin{array}{l}\text { Belli } \mathbf{2 0 0 2 ^ { 6 6 }} \\
\text { Time period: NR } \\
\text { Country: Italy } \\
\text { Study design: direct head-to- } \\
\text { head cohort }\end{array}$ & $\begin{array}{l}\text { Enrolled: } 23 \\
\text { Analysed: } 23 \\
\text { BCT: } 23 \\
\text { Mastectomy: } 0 \\
\text { Age (years): mean } 52.9 \\
\text { years; median NR; SD, } \\
\text { 10.8; range 35-75 years }\end{array}$ & $\begin{array}{l}\text { Index test. MRI for local } \\
\text { recurrence }\end{array}$ & $\begin{array}{l}\text { XRM, CE, US for local } \\
\text { recurrence }\end{array}$ & $\begin{array}{l}\text { Surveillance for detecting } \\
\text { IBTR }\end{array}$ \\
\hline $\begin{array}{l}\text { Boné } \text { 1995 }^{64} \\
\text { Time period: September } \\
1992 \text { to September } 1993 \\
\text { Country: Sweden } \\
\text { Study design: direct head-to- } \\
\text { head cohort }\end{array}$ & $\begin{array}{l}\text { Enrolled: } 83 \\
\text { Analysed: } 83 \\
\text { BCT: } 0 \\
\text { Mastectomy. 83, all with } \\
\text { breast reconstruction and } \\
\text { implants } \\
\text { Age (years): mean NR; } \\
\text { median } 50 \text { years; SD, NR; } \\
\text { range 33-75 years }\end{array}$ & $\begin{array}{l}\text { Index test. XRM for } \\
\text { local and contralateral } \\
\text { recurrence }\end{array}$ & $\begin{array}{l}\text { MRI, CE for local } \\
\text { and contralateral } \\
\text { recurrence }\end{array}$ & $\begin{array}{l}\text { Surveillance for detecting } \\
\text { IBTR and MCBC }\end{array}$ \\
\hline $\begin{array}{l}\text { Drew } 1998^{67} \\
\text { Time period: NR } \\
\text { Country: UK } \\
\text { Study design: direct head-to- } \\
\text { head cohort }\end{array}$ & $\begin{array}{l}\text { Enrolled: } 105 \\
\text { Analysed: } 105 \\
\text { BCT: } 105 \\
\text { Mastectomy. } 0 \\
\text { Age (years): mean NR; } \\
\text { median } 58 \text { years; SD, NR; } \\
\text { range 50-65 years }\end{array}$ & $\begin{array}{l}\text { Index test: MRI for local } \\
\text { recurrence }\end{array}$ & $\begin{array}{l}\text { XRM, CE, XRM +CE for } \\
\text { local recurrence }\end{array}$ & $\begin{array}{l}\text { Surveillance for detecting } \\
\text { IBTR }\end{array}$ \\
\hline $\begin{array}{l}\text { Kim } \mathbf{2 0 0 9}^{65} \\
\text { Time period: January } 2003 \\
\text { to December } 2003 \\
\text { Country: South Korea } \\
\text { Study design: direct head-to- } \\
\text { head cohort }\end{array}$ & $\begin{array}{l}\text { Enrolled: } 1431 \\
\text { Analysed: } 1256 \\
\text { BCT: } 292 \\
\text { Mastectomy: } 964 \\
\text { Age (years): mean } 50 \text { years; } \\
\text { median } 49 \text { years; SD, } 10 \\
\text { years; range 22-82 years }\end{array}$ & $\begin{array}{l}\text { Index test. adjunct } \\
\text { US (XRM + US) for } \\
\text { contralateral recurrence }\end{array}$ & None & $\begin{array}{l}\text { Surveillance for detecting } \\
\text { MCBC }\end{array}$ \\
\hline
\end{tabular}




\begin{tabular}{|c|c|c|c|c|}
\hline Study ID & Study design & Index tests & Comparator test & Test purpose \\
\hline $\begin{array}{l}\text { Mumtaz } 1997^{68} \\
\text { Time period: NR } \\
\text { Country. UK } \\
\text { Study design: direct head-to- } \\
\text { head cohort }\end{array}$ & $\begin{array}{l}\text { Enrolled: } 30 \\
\text { Analysed: } 30 \\
\text { BCT: } 30 \\
\text { Mastectomy: } 0 \\
\text { Age (years): recurrence } \\
\text { patients mean NR; median } \\
52 \text { years; SD, NR; range } \\
34-80 \text { years; recurrence- } \\
\text { free patients mean NR; } \\
\text { median } 62 \text { years; SD, NR; } \\
\text { range } 47-79 \text { years }\end{array}$ & $\begin{array}{l}\text { Index test: XRM for local } \\
\text { recurrence }\end{array}$ & $\begin{array}{l}\text { MRI for local } \\
\text { recurrence }\end{array}$ & $\begin{array}{l}\text { Confirmation of suspicious } \\
\text { result on prior diagnostic } \\
\text { test (previous clinical } \\
\text { suspicion) }\end{array}$ \\
\hline $\begin{array}{l}\text { Rieber } 1997^{69} \\
\text { Time period: NR } \\
\text { Country: Germany } \\
\text { Study design: cohort }\end{array}$ & $\begin{array}{l}\text { Enrolled: } 140 \\
\text { Analysed: } 140 \\
\text { BCT: } 140 \\
\text { Mastectomy: } 0 \\
\text { Age (years): mean } 52.8 \\
\text { years; median NR; SD, NR; } \\
\text { range 32-81 years }\end{array}$ & $\begin{array}{l}\text { Index test. MRI for local } \\
\text { recurrence }\end{array}$ & None & $\begin{array}{l}\text { Confirmation of suspicious } \\
\text { result on prior diagnostic } \\
\text { test (suspicious finding } \\
\text { on at least one of } \\
\text { the following: clinical } \\
\text { examination, ultrasound or } \\
\text { mammography) }\end{array}$ \\
\hline $\begin{array}{l}\text { Shin } 2005^{71} \\
\text { Time period: January } 2001 \\
\text { to February } 2004 \\
\text { Country. South Korea } \\
\text { Study design: cohort }\end{array}$ & $\begin{array}{l}\text { Enrolled: } 1968 \\
\text { Analysed: } 1968 \\
\text { BCT: NR } \\
\text { Mastectomy: NR } \\
\text { Age (years): mean } 49 \text { years; } \\
\text { median NR; SD, NR; range } \\
\text { 32-69 years }\end{array}$ & $\begin{array}{l}\text { Index test: US for } \\
\text { local and contralateral } \\
\text { recurrence }\end{array}$ & None & $\begin{array}{l}\text { Surveillance for detecting } \\
\text { IBTR and MCBC }\end{array}$ \\
\hline $\begin{array}{l}\text { Ternier 2006 } \\
\text { Time period: May } 1997 \text { to } \\
\text { May } 2002 \\
\text { Country. France } \\
\text { Study design: direct head-to- } \\
\text { head cohort }\end{array}$ & $\begin{array}{l}\text { Enrolled: } 103 \\
\text { Analysed: } 103 \\
\text { BCT: } 103 \\
\text { Mastectomy: } 0 \\
\text { Age (years): mean NR; } \\
\text { median } 60 \text { years; SD, NR; } \\
\text { range 32-82 years }\end{array}$ & $\begin{array}{l}\text { Index test. XRM for local } \\
\text { recurrence }\end{array}$ & $\begin{array}{l}\text { CE, US for local } \\
\text { recurrence }\end{array}$ & $\begin{array}{l}\text { Confirmation of suspicious } \\
\text { result on prior diagnostic } \\
\text { test (suspicious finding } \\
\text { on at least one of } \\
\text { the following: clinical } \\
\text { examination, ultrasound or } \\
\text { mammography) }\end{array}$ \\
\hline $\begin{array}{l}\text { Viehweg } 2004^{70} \\
\text { Time period: August } 1994 \text { to } \\
\text { April } 2001 \\
\text { Country. Germany } \\
\text { Study design: direct head-to- } \\
\text { head cohort }\end{array}$ & $\begin{array}{l}\text { Enrolled: } 119 \\
\text { Analysed: } 119 \\
\text { BCT: } 119 \\
\text { Mastectomy: } 0 \\
\text { Age (years): mean NR; } \\
\text { median } 55.7 \text { years; SD, 9.8; } \\
\text { range 25-78 years }\end{array}$ & $\begin{array}{l}\text { Index test. MRI for } \\
\text { contralateral recurrence }\end{array}$ & $\begin{array}{l}\mathrm{CM}(\mathrm{XRM}+\mathrm{CE}+\mathrm{US}) \text {; } \\
\mathrm{MRI}+\mathrm{CM} \text { for } \\
\text { contralateral } \\
\text { recurrence }\end{array}$ & $\begin{array}{l}\text { Surveillance for detecting } \\
\text { MCBC }\end{array}$ \\
\hline
\end{tabular}

$\mathrm{CE}$, clinical examination; CM, conventional methods; US, ultrasound; XRM, X-ray mammography.

a Study authors considered computerised tomography as the index test in this study but this test was not considered as an included comparator in this review. 


\section{Appendix 23}

\section{Quality assessment of individual studies included in the systematic review of test performance of surveillance mammography and comparator tests in detecting IBTR/MCBC}

TABLE 58 Quality assessment for included studies of routine surveillance tests

\begin{tabular}{lllllllllllllll}
\hline Study ID & Q1 & Q2 & Q3a & Q3b & Q4 & Q5a & Q5b & Q6 & Q7 & Q8 & Q9 & Q10 & Q11 & Q12 \\
\hline Boné 199564 & - & + & $?$ & + & + & + & + & + & + & $?$ & $?$ & + & + & + \\
Drew 199867 & + & + & $?$ & + & - & + & + & + & + & $?$ & + & + & + & + \\
Viehweg 200470 & + & + & $?$ & + & + & + & + & + & $?$ & $?$ & - & + & - & + \\
Shin 200571 & + & + & + & + & $?$ & + & $?$ & + & + & $?$ & - & + & + & + \\
Kim 200965 & + & + & $?$ & + & + & + & + & + & + & $?$ & - & + & $?$ & + \\
\hline
\end{tabular}

NA, not applicable; +, yes to the question; - , no to the question; ?, unclear.

TABLE 59 Quality assessment for included studies of non-routine surveillance tests

\begin{tabular}{lllllllllllllll}
\hline Study ID & Q1 & Q2 & Q3a & Q3b & Q4 & Q5a & Q5b & Q6 & Q7 & Q8 & Q9 & Q10 & Q11 & Q12 \\
\hline Mumtaz 199768 & + & + & $?$ & + & + & + & + & + & + & $?$ & + & + & + & + \\
Rieber 199769 & + & + & $?$ & + & + & + & + & + & + & $?$ & - & $?$ & + & + \\
Belli 200266 & + & + & $?$ & + & + & + & + & + & + & $?$ & + & + & $?$ & $?$ \\
Ternier 2006 & + & + & $?$ & + & + & + & + & + & + & $?$ & - & + & + & + \\
\hline
\end{tabular}

NA, not applicable; +, yes to the question; - , no to the question; ?, unclear. 



\section{Appendix 24}

Histology of cancers detected and not detected (true-positives and falsenegatives): systematic review of test performance of surveillance mammography and comparator tests in detecting IBTR/MCBC 
TABLE 60 Histology of true-positive and false-negative test results for surveillance mammography

\begin{tabular}{|c|c|c|c|}
\hline Test & Study ID & True-positives & False-negatives \\
\hline \multirow{13}{*}{$\begin{array}{l}\text { Surveillance } \\
\text { mammography }\end{array}$} & \multirow[t]{3}{*}{ Boné $1995^{64}$} & DCIS $n=7$ & IDC $n=4$ \\
\hline & & $\| \mathrm{LC} n=1$ & $\| \mathrm{LC} n=1$ \\
\hline & & IDC $n=2$ & \\
\hline & $\begin{array}{l}\text { Mumtaz } \\
1997^{68}\end{array}$ & & $\begin{array}{l}\text { Mammographic findings: deformed dense breast } n=4 \text {; } \\
\text { scarring and benign linear microcalcifications } n=3\end{array}$ \\
\hline & \multirow{6}{*}{$\begin{array}{l}\text { Viehweg } \\
2004^{70}\end{array}$} & Minimally invasive carcinoma plus: & \multirow{2}{*}{$\begin{array}{l}\text { Minimally invasive carcinoma plus DCIS grade } 3 \text { comedo } \\
n=1\end{array}$} \\
\hline & & DCIS grade 1 non-comedo $n=1$ & \\
\hline & & DCIS grade 1 comedo $n=1$ & DCIS grade 1 non-comedo $n=1$ \\
\hline & & IDC grade $2 n=2$ & DCIS grade 2 non-comedo $n=3$ \\
\hline & & DCIS grade 2 non-comedo $n=1$ & IDC grade $2 n=1$ \\
\hline & & $\begin{array}{l}\text { Ductolobular carcinoma plus DCIS grade } 2 \text { non- } \\
\text { comedo } n=1\end{array}$ & \\
\hline & \multirow[t]{3}{*}{ Kim 200965} & IDC $n=12$ & \multirow[t]{3}{*}{$\mathrm{DCIS} n=2$} \\
\hline & & $\| \mathrm{LC} n=1$ & \\
\hline & & DCIS $n=3$ & \\
\hline
\end{tabular}

DCIS, ductal carcinoma in situ; IDC, invasive ductal carcinoma; ILC, invasive lobular carcinoma.

TABLE 61 Histology of true-positive and false-negative test results for ultrasound

\begin{tabular}{|c|c|c|c|}
\hline Test & Study ID & True-positives & False-negatives \\
\hline \multirow[t]{10}{*}{ Ultrasound } & \multirow[t]{3}{*}{ Kim $2009^{65}$} & $\mathrm{IDC} n=12$ & IDC $n=1$ \\
\hline & & $\operatorname{ILC} n=1$ & \multirow[t]{3}{*}{$\mathrm{DCIS} n=2$} \\
\hline & & DCIS $n=2$ & \\
\hline & Shin $2005^{71}$ & $\begin{array}{l}\text { Most frequently lymphadenopathy (non- } \\
\text { palpable?) }\end{array}$ & \\
\hline & \multirow{6}{*}{$\begin{array}{l}\text { Viehweg } \\
2004^{70}\end{array}$} & DCIS grade 2 non-comedo $n=1$ & $\begin{array}{l}\text { Minimally invasive carcinoma plus DCIS grade } 1 \text { non- } \\
\text { comedo } n=1\end{array}$ \\
\hline & & \multirow[t]{5}{*}{$\begin{array}{l}\text { Ductolobular carcinoma plus DCIS grade } 2 \text { non- } \\
\text { comedo } n=1\end{array}$} & $\begin{array}{l}\text { Comedo } n=1 \\
\text { Minimally invasive carcinoma plus DCIS grade } 3 \text { comedo } \\
n=1\end{array}$ \\
\hline & & & DCIS grade 1 non-comedo $n=1$ \\
\hline & & & DCIS grade 2 non-comedo $n=2$ \\
\hline & & & DCIS grade 1 comedo $n=1$ \\
\hline & & & IDC grade $2 n=3$ \\
\hline
\end{tabular}

DCIS, ductal carcinoma in situ; IDC, invasive ductal carcinoma; ILC, invasive lobular carcinoma. 
TABLE 62 Histology of true-positive and false-negative test results for MRI

\begin{tabular}{|c|c|c|c|}
\hline Test & Study ID & True-positives & False-negatives \\
\hline \multirow[t]{10}{*}{ MRI } & Boné $1995^{64}$ & IDC $n=5$ & DCIS, $n=1$ \\
\hline & & DCIS $n=5$ & IDC, $n=1$ \\
\hline & & $\operatorname{ILC} n=2$ & \\
\hline & $\begin{array}{l}\text { Mumtaz } \\
1997^{68}\end{array}$ & & $\begin{array}{l}\text { Diffuse scattered cancer cells within a dense fibrotic } \\
\text { stroma with no discrete tumour focus } n=1\end{array}$ \\
\hline & $\begin{array}{l}\text { Viehweg } \\
2004^{70}\end{array}$ & $\begin{array}{l}\text { Minimally invasive carcinoma plus DCIS grade } 1 \\
\text { non-comedo } n=1\end{array}$ & DCIS grade 1 non-comedo $n=1$ \\
\hline & & $\begin{array}{l}\text { Minimally invasive carcinoma plus DCIS grade } 3 \\
\text { comedo } n=1\end{array}$ & \\
\hline & & DCIS grade 2 non-comedo $n=2$ & \\
\hline & & Minimally invasive carcinoma plus DCIS grade 1 : & \\
\hline & & comedo $n=1$ & \\
\hline & & IDC grade $2 n=3$ & \\
\hline
\end{tabular}

DCIS, ductal carcinoma in situ; IDC, invasive ductal carcinoma; ILC, invasive lobular carcinoma.

TABLE 63 Histology of true-positive and false-negative test results for clinical examination

\begin{tabular}{|c|c|c|c|}
\hline Test & Study ID & True-positives & False-negatives \\
\hline \multirow[t]{14}{*}{ CE } & \multirow[t]{3}{*}{ Boné $1995^{64}$} & $\mathrm{ILC} n=2$ & IDC $n=2$ \\
\hline & & $\operatorname{IDC} n=4$ & DCIS $n=5$ \\
\hline & & DCIS $n=1$ & \\
\hline & \multirow{8}{*}{$\begin{array}{l}\text { Viehweg } \\
2004^{70}\end{array}$} & \multirow{8}{*}{$\begin{array}{l}\text { IDC grade } 2 n=1 \\
\text { Ductolobular carcinoma plus DCIS grade } 2 \text { non- } \\
\text { comedo } n=1\end{array}$} & Minimally invasive carcinoma plus DCIS grade 1 non- \\
\hline & & & comedo $n=1$ \\
\hline & & & $\begin{array}{l}\text { Minimally invasive carcinoma plus DCIS grade } 3 \text { comedo } \\
n=1\end{array}$ \\
\hline & & & DCIS grade 1 non-comedo $n=1$ \\
\hline & & & DCIS grade 2 non-comedo $n=2$ \\
\hline & & & Minimally invasive carcinoma plus DCIS grade 1: \\
\hline & & & comedo $n=1$ \\
\hline & & & IDC grade $2 n=2$ \\
\hline & \multirow[t]{3}{*}{ Kim $2009^{65}$} & \multirow[t]{3}{*}{$\operatorname{IDC} n=5$} & IDC $n=8$ \\
\hline & & & $\operatorname{ILC} n=1$ \\
\hline & & & DCIS $n=4$ \\
\hline
\end{tabular}

CE, clinical examination; DCIS, ductal carcinoma in situ; IDC, invasive ductal carcinoma; ILC, invasive lobular carcinoma. 



\section{Appendix 25}

\section{Univariate Cox regression models for the WMCIU cohorts}

TABLE 64 Cox proportional hazards regression models for time to IBTR in BCS cohort

\begin{tabular}{|c|c|c|c|c|c|c|c|c|c|}
\hline & \multirow[b]{2}{*}{$n$} & \multirow[b]{2}{*}{$N$} & \multirow[b]{2}{*}{$\%$} & \multicolumn{3}{|c|}{ Univariate Cox regression } & \multicolumn{3}{|c|}{ Multiple Cox regression } \\
\hline & & & & HR & $95 \% \mathrm{Cl}$ & $p$-value & HR & $95 \% \mathrm{Cl}$ & $p$-value \\
\hline \multicolumn{10}{|c|}{ Age (years) } \\
\hline$\leq 34$ & 26 & 350 & 7.4 & 3.27 & 2.17 to 4.92 & $<0.001$ & 2.28 & 1.51 to 3.47 & $<0.001$ \\
\hline $35-49$ & 138 & 3513 & 3.9 & 1.80 & 1.45 to 2.24 & $<0.001$ & 1.44 & 1.15 to 1.80 & 0.002 \\
\hline $50-64$ & 189 & 8511 & 2.2 & $(1.00)$ & & & $(1.00)$ & & \\
\hline $65-74$ & 54 & 3491 & 1.5 & 0.80 & 0.59 to 1.09 & 0.154 & 0.72 & 0.53 to 0.97 & 0.033 \\
\hline $75-79$ & 25 & 1034 & 2.4 & 1.25 & 0.83 to 1.89 & 0.284 & 0.96 & 0.63 to 1.47 & 0.852 \\
\hline $80+$ & 23 & 807 & 2.9 & 1.93 & 1.27 to 2.93 & 0.002 & 1.28 & 0.81 to 2.03 & 0.289 \\
\hline \multicolumn{10}{|l|}{ Grade } \\
\hline 1 & 60 & 4193 & 1.4 & $(1.00)$ & & & $(1.00)$ & & \\
\hline 2 & 175 & 7697 & 2.3 & 1.75 & 1.31 to 2.35 & $<0.001$ & 1.53 & 1.14 to 2.07 & 0.005 \\
\hline 3 & 204 & 5388 & 3.8 & 3.15 & 2.36 to 4.21 & $<0.001$ & 2.32 & 1.71 to 3.16 & $<0.001$ \\
\hline Unknown & 16 & 428 & 3.7 & 2.75 & 1.69 to 4.49 & $<0.001$ & 1.85 & 1.06 to 3.26 & 0.032 \\
\hline \multicolumn{10}{|c|}{ Size $(\mathrm{mm})$} \\
\hline$\leq 10$ & 68 & 3473 & 2.0 & (1.00) & & & $(1.00)$ & & \\
\hline $\begin{array}{l}>10 \text { to } \\
<20\end{array}$ & 173 & 8663 & 2.0 & 1.02 & 0.77 to 1.35 & 0.892 & 0.81 & 0.61 to 1.09 & 0.161 \\
\hline$\geq 20$ & 179 & 4885 & 3.7 & 2.11 & 1.60 to 2.80 & $<0.001$ & 1.31 & 0.97 to 1.78 & 0.078 \\
\hline $\begin{array}{l}\text { Size cat. } \\
\text { unknown }\end{array}$ & 35 & 685 & 5.1 & 2.23 & 1.51 to 3.30 & $<0.001$ & 1.28 & 0.83 to 1.96 & 0.268 \\
\hline \multicolumn{10}{|l|}{ Nodes } \\
\hline 0 & 231 & 11,630 & 2.0 & (1.00) & & & $(1.00)$ & & \\
\hline $1-3$ & 103 & 3367 & 3.1 & 1.64 & 1.30 to 2.07 & $<0.001$ & 1.33 & 1.04 to 1.69 & 0.021 \\
\hline $4+$ & 59 & 1232 & 4.8 & 2.98 & 2.23 to 3.96 & $<0.001$ & 2.04 & 1.51 to 2.77 & $<0.001$ \\
\hline Unknown & 62 & 1477 & 4.2 & 2.14 & 1.63 to 2.80 & $<0.001$ & 2.06 & 1.51 to 2.81 & $<0.001$ \\
\hline \multicolumn{10}{|c|}{ Vascular invasion } \\
\hline No & 277 & 12,887 & 2.1 & (1.00) & & & $(1.00)$ & & \\
\hline Yes & 140 & 3559 & 3.9 & 1.96 & 1.60 to 2.40 & $<0.001$ & 1.26 & 1.01 to 1.57 & 0.043 \\
\hline Unknown & 38 & 1260 & 3.0 & 1.40 & 1.02 to 1.93 & 0.040 & 0.98 & 0.68 to 1.41 & 0.928 \\
\hline
\end{tabular}

HR, hazard ratio.

$N$ is the size of the subgroup, $n$ is the number of events in that subgroup. 
TABLE 65 Cox proportional hazards regression models for time to MCBC occurrence in BCS cohort

\begin{tabular}{|c|c|c|c|c|c|c|c|c|c|}
\hline & \multirow[b]{2}{*}{$n$} & \multirow[b]{2}{*}{$N$} & \multirow[b]{2}{*}{$\%$} & \multicolumn{3}{|c|}{ Univariate Cox regression } & \multicolumn{3}{|c|}{ Multiple Cox regression } \\
\hline & & & & $\mathrm{HR}$ & $95 \% \mathrm{Cl}$ & $p$-value & HR & $95 \% \mathrm{Cl}$ & $p$-value \\
\hline \multicolumn{10}{|c|}{ Age (years) } \\
\hline$\leq 34$ & 11 & 350 & 3.1 & 1.62 & 0.88 to 2.98 & 0.124 & 1.54 & 0.82 to 2.86 & 0.177 \\
\hline $35-49$ & 55 & 3513 & 1.6 & 0.87 & 0.64 to 1.19 & 0.390 & 0.85 & 0.62 to 1.16 & 0.294 \\
\hline 50-64 & 150 & 8511 & 1.8 & $(1.00)$ & & & & & \\
\hline $65-74$ & 33 & 3491 & 0.9 & 0.62 & 0.43 to 0.90 & 0.012 & 0.61 & 0.42 to 0.89 & 0.010 \\
\hline $75-79$ & 6 & 1034 & 0.6 & 0.36 & 0.16 to 0.81 & 0.014 & 0.34 & 0.15 to 0.77 & 0.010 \\
\hline $80+$ & 5 & 807 & 0.6 & 0.49 & 0.20 to 1.19 & 0.116 & 0.46 & 0.18 to 1.14 & 0.093 \\
\hline \multicolumn{10}{|l|}{ Grade } \\
\hline 1 & 69 & 4193 & 1.6 & $(1.00)$ & & & (1.00) & & \\
\hline 2 & 102 & 7697 & 1.3 & 0.91 & 0.67 to 1.24 & 0.558 & 0.92 & 0.68 to 1.26 & 0.623 \\
\hline 3 & 79 & 5388 & 1.5 & 1.11 & 0.81 to 1.53 & 0.524 & 1.06 & 0.75 to 1.49 & 0.756 \\
\hline Unknown & 10 & 428 & 2.3 & 1.27 & 0.69 to 2.35 & 0.439 & 1.40 & 0.71 to 2.76 & 0.329 \\
\hline \multicolumn{10}{|c|}{ Size (mm) } \\
\hline$\leq 10$ & 45 & 3473 & 1.3 & $(1.00)$ & & & (1.00) & & \\
\hline $\begin{array}{l}>10 \text { to } \\
<20\end{array}$ & 125 & 8663 & 1.4 & 1.13 & 0.80 to 1.59 & 0.485 & 1.25 & 0.88 to 1.76 & 0.217 \\
\hline$\geq 20$ & 74 & 4885 & 1.5 & 1.35 & 0.93 to 1.96 & 0.109 & 1.60 & 1.08 to 2.38 & 0.019 \\
\hline $\begin{array}{l}\text { Size cat. } \\
\text { unknown }\end{array}$ & 16 & 685 & 2.3 & 1.54 & 0.90 to 2.63 & 0.115 & 1.81 & 1.01 to 3.22 & 0.044 \\
\hline \multicolumn{10}{|l|}{ Nodes } \\
\hline 0 & 183 & 11,630 & 1.6 & $(1.00)$ & & & $(1.00)$ & & \\
\hline $1-3$ & 38 & 3367 & 1.1 & 0.75 & 0.53 to 1.06 & 0.100 & 0.72 & 0.50 to 1.03 & 0.075 \\
\hline $4+$ & 19 & 1232 & 1.5 & 1.19 & 0.74 to 1.91 & 0.473 & 1.20 & 0.73 to 1.96 & 0.479 \\
\hline Unknown & 20 & 1477 & 1.4 & 0.89 & 0.58 to 1.38 & 0.608 & 1.07 & 0.67 to 1.73 & 0.771 \\
\hline \multicolumn{10}{|c|}{ Vascular invasion } \\
\hline No & 204 & 12,887 & 1.6 & $(1.00)$ & & & $(1.00)$ & & \\
\hline Yes & 43 & 3559 & 1.2 & 0.80 & 0.57 to 1.11 & 0.175 & 0.74 & 0.52 to 1.05 & 0.096 \\
\hline Unknown & 13 & 1260 & 1.0 & 0.68 & 0.41 to 1.13 & 0.139 & 0.58 & 0.33 to 1.01 & 0.053 \\
\hline
\end{tabular}

HR, hazard ratio.

$N$ is the size of the subgroup, $n$ is the number of events in that subgroup. 
TABLE 66 Cox proportional hazards regression models for time to all-cause death in BCS cohort

\begin{tabular}{|c|c|c|c|c|c|c|c|c|c|}
\hline & \multirow[b]{2}{*}{$n$} & \multirow[b]{2}{*}{$N$} & \multirow[b]{2}{*}{$\%$} & \multicolumn{3}{|c|}{ Univariate Cox regression } & \multicolumn{3}{|c|}{ Multiple Cox regression } \\
\hline & & & & $\mathrm{HR}$ & $95 \% \mathrm{Cl}$ & $p$-value & HR & $95 \% \mathrm{Cl}$ & $p$-value \\
\hline \multicolumn{10}{|l|}{ Age (years) } \\
\hline$\leq 34$ & 60 & 350 & 17.1 & 1.57 & 1.21 to 2.04 & 0.001 & 1.00 & 0.77 to 1.30 & 0.987 \\
\hline $35-49$ & 371 & 3513 & 10.6 & 1.05 & 0.93 to 1.18 & 0.463 & 0.78 & 0.69 to 0.89 & $<0.001$ \\
\hline $50-64$ & 848 & 8511 & 10.0 & $(1.00)$ & & & $(1.00)$ & & \\
\hline $65-74$ & 594 & 3491 & 17.0 & 1.96 & 1.76 to 2.17 & $<0.001$ & 1.80 & 1.62 to 2.01 & $<0.001$ \\
\hline $75-79$ & 293 & 1034 & 28.3 & 3.16 & 2.77 to 3.61 & $<0.001$ & 2.55 & 2.23 to 2.93 & $<0.001$ \\
\hline $80+$ & 362 & 807 & 44.9 & 6.49 & 5.74 to 7.33 & $<0.001$ & 4.83 & 4.22 to 5.52 & $<0.001$ \\
\hline \multicolumn{10}{|l|}{ Grade } \\
\hline 1 & 344 & 4193 & 8.2 & $(1.00)$ & & & $(1.00)$ & & \\
\hline 2 & 1002 & 7697 & 13.0 & 1.77 & 1.57 to 2.00 & $<0.001$ & 1.33 & 1.17 to 1.50 & $<0.001$ \\
\hline 3 & 1114 & 5388 & 20.7 & 3.04 & 2.69 to 3.43 & $<0.001$ & 2.20 & 1.93 to 2.50 & $<0.001$ \\
\hline Unknown & 68 & 428 & 15.9 & 2.05 & 1.64 to 2.57 & $<0.001$ & 1.15 & 0.88 to 1.50 & 0.207 \\
\hline \multicolumn{10}{|l|}{ Size $(m m)$} \\
\hline$\leq 10$ & 230 & 3473 & 6.6 & $(1.00)$ & & & $(1.00)$ & & \\
\hline$>10$ to $<20$ & 1030 & 8663 & 11.9 & 1.81 & 1.57 to 2.09 & $<0.001$ & 1.32 & 1.14 to 1.53 & $<0.001$ \\
\hline$\geq 20$ & 1117 & 4885 & 22.9 & 3.92 & 3.40 to 4.52 & $<0.001$ & 2.04 & 1.76 to 2.37 & $<0.001$ \\
\hline $\begin{array}{l}\text { Size cat. } \\
\text { unknown }\end{array}$ & 151 & 685 & 22.0 & 2.83 & 2.33 to 3.45 & $<0.001$ & 1.54 & 1.25 to 1.91 & $<0.001$ \\
\hline \multicolumn{10}{|l|}{ Nodes } \\
\hline 0 & 1073 & 11,630 & 9.2 & $(1.00)$ & & & $(1.00)$ & & \\
\hline $1-3$ & 573 & 3367 & 17.0 & 1.94 & 1.75 to 2.15 & $<0.001$ & 1.57 & 1.41 to 1.74 & $<0.001$ \\
\hline $4+$ & 416 & 1232 & 33.8 & 4.44 & 3.96 to 4.97 & $<0.001$ & 2.96 & 2.62 to 3.34 & $<0.001$ \\
\hline Unknown & 466 & 1477 & 31.6 & 3.27 & 2.94 to 3.63 & $<0.001$ & 1.92 & 1.70 to 2.17 & $<0.001$ \\
\hline \multicolumn{10}{|c|}{ Vascular invasion } \\
\hline No & 1460 & 12,887 & 11.3 & $(1.00)$ & & & $(1.00)$ & & \\
\hline Yes & 847 & 3559 & 23.8 & 2.22 & 2.04 to 2.41 & $<0.001$ & 1.36 & 1.24 to 1.49 & $<0.001$ \\
\hline Unknown & 221 & 1260 & 17.5 & 1.50 & 1.31 to 1.71 & $<0.001$ & 1.15 & 0.99 to 1.33 & 0.066 \\
\hline \multicolumn{10}{|c|}{ Ipsilateral recurrence } \\
\hline No & 2350 & 17,251 & 13.6 & $(1.00)$ & & & & & \\
\hline Yes & 178 & 455 & 39.1 & 2.29 & 1.97 to 2.67 & $<0.001$ & 1.76 & 1.51 to 2.05 & $<0.001$ \\
\hline \multicolumn{10}{|c|}{ Contralateral occurrence } \\
\hline No & 2475 & 17,446 & 14.2 & $(1.00)$ & & & $(1.00)$ & & \\
\hline Yes & 53 & 260 & 20.4 & 0.99 & 0.76 to 1.30 & 0.97 & 1.05 & 0.80 to 1.39 & 0.715 \\
\hline
\end{tabular}

HR, hazard ratio.

$N$ is the size of the subgroup, $n$ is the number of events in that subgroup. 
TABLE 67 Cox proportional hazards regression models for time to death from breast cancer in BCS cohort

\begin{tabular}{|c|c|c|c|c|c|c|c|c|c|}
\hline & \multirow[b]{2}{*}{$n$} & \multirow[b]{2}{*}{$N$} & \multirow[b]{2}{*}{$\%$} & \multicolumn{3}{|c|}{ Univariate Cox regression } & \multicolumn{3}{|c|}{ Multiple Cox regression } \\
\hline & & & & HR & $95 \% \mathrm{Cl}$ & $p$-value & HR & $95 \% \mathrm{Cl}$ & $p$-value \\
\hline \multicolumn{10}{|c|}{ Age (years) } \\
\hline$\leq 34$ & 55 & 350 & 15.7 & 2.33 & 1.77 to 3.08 & $<0.001$ & 1.21 & 0.91 to 1.60 & 0.182 \\
\hline $35-49$ & 310 & 3513 & 8.8 & 1.39 & 1.21 to 1.60 & $<0.001$ & 0.90 & 0.78 to 1.04 & 0.157 \\
\hline $50-64$ & 535 & 8511 & 6.3 & $(1.00)$ & & & $(1.00)$ & & \\
\hline $65-74$ & 278 & 3491 & 8.0 & 1.43 & 1.24 to 1.65 & $<0.001$ & 1.28 & 1.10 to 1.48 & 0.001 \\
\hline $75-79$ & 96 & 1034 & 9.3 & 1.63 & 1.32 to 2.03 & $<0.001$ & 1.27 & 1.02 to 1.58 & 0.034 \\
\hline $80+$ & 104 & 807 & 12.9 & 2.85 & 2.32 to 3.51 & $<0.001$ & 2.06 & 1.65 to 2.57 & $<0.001$ \\
\hline \multicolumn{10}{|l|}{ Grade } \\
\hline 1 & 76 & 4193 & 1.8 & $(1.00)$ & & & $(1.00)$ & & \\
\hline 2 & 489 & 7697 & 6.4 & 3.87 & 3.04 to 4.92 & $<0.001$ & 2.60 & 2.03 to 3.32 & $<0.001$ \\
\hline 3 & 786 & 5388 & 14.6 & 9.56 & 7.55 to 12.10 & $<0.001$ & 5.31 & 4.17 to 6.77 & $<0.001$ \\
\hline Unknown & 27 & 428 & 6.3 & 4.21 & 2.90 to 6.12 & $<0.001$ & 2.39 & 1.62 to 3.54 & 0.207 \\
\hline \multicolumn{10}{|c|}{ Size $(\mathrm{mm})$} \\
\hline$\leq 10$ & 75 & 3473 & 2.2 & $(1.00)$ & & & $(1.00)$ & & \\
\hline $\begin{array}{l}>10 \text { to } \\
<20\end{array}$ & 510 & 8663 & 5.9 & 2.75 & 2.16 to 3.50 & $<0.001$ & 1.73 & 1.35 to 2.21 & $<0.001$ \\
\hline$\geq 20$ & 717 & 4885 & 14.7 & 7.66 & 6.04 to 9.71 & $<0.001$ & 3.04 & 2.37 to 3.89 & $<0.001$ \\
\hline $\begin{array}{l}\text { Size cat. } \\
\text { unknown }\end{array}$ & 76 & 685 & 11.1 & 4.61 & 3.39 to 6.25 & $<0.001$ & 2.09 & 1.52 to 2.88 & $<0.001$ \\
\hline \multicolumn{10}{|l|}{ Nodes } \\
\hline 0 & 505 & 11,630 & 4.3 & $(1.00)$ & & & $(1.00)$ & & \\
\hline $1-3$ & 366 & 3367 & 10.9 & 2.63 & 2.30 to 3.01 & $<0.001$ & 1.82 & 1.59 to 2.10 & $<0.001$ \\
\hline $4+$ & 332 & 1232 & 26.9 & 7.42 & 6.46 to 8.53 & $<0.001$ & 3.88 & 3.34 to 4.51 & $<0.001$ \\
\hline Unknown & 175 & 1477 & 11.8 & 2.72 & 2.30 to 3.22 & $<0.001$ & 2.23 & 1.85 to 2.69 & $<0.001$ \\
\hline \multicolumn{10}{|c|}{ Vascular invasion } \\
\hline No & 679 & 12,887 & 5.3 & $(1.00)$ & & & $(1.00)$ & & \\
\hline Yes & 606 & 3559 & 17.0 & 3.40 & 3.04 to 3.79 & $<0.001$ & 1.59 & 1.41 to 1.79 & $<0.001$ \\
\hline Unknown & 93 & 1260 & 7.4 & 1.44 & 1.17 to 1.76 & $<0.001$ & 1.09 & 0.88 to 1.36 & 0.430 \\
\hline \multicolumn{10}{|c|}{ Ipsilateral recurrence } \\
\hline No & 1247 & 17,251 & 7.2 & & & & & & \\
\hline Yes & 131 & 455 & 28.8 & 3.30 & 2.76 to 3.94 & $<0.001$ & 2.13 & 1.78 to 2.56 & $<0.001$ \\
\hline \multicolumn{10}{|c|}{ Contralateral occurrence } \\
\hline No & 1375 & 17,446 & 7.9 & $(1.00)$ & & & & & \\
\hline Yes & 3 & 260 & 1.2 & 1.13 & 0.86 to 1.49 & 0.378 & & & \\
\hline
\end{tabular}

HR, hazard ratio.

$N$ is the size of the subgroup, $n$ is the number of events in that subgroup. 
TABLE 68 Multiple Cox proportional hazards regression models for time to IBTR in mastectomy cohort

\begin{tabular}{|c|c|c|c|c|c|c|c|c|c|}
\hline & \multirow[b]{2}{*}{$n$} & \multirow[b]{2}{*}{$N$} & \multirow[b]{2}{*}{$\%$} & \multicolumn{3}{|c|}{ Univariate Cox regression } & \multicolumn{3}{|c|}{ Multiple Cox regression } \\
\hline & & & & HR & $95 \% \mathrm{Cl}$ & $p$-value & HR & $95 \% \mathrm{Cl}$ & $p$-value \\
\hline \multicolumn{10}{|c|}{ Age (years) } \\
\hline$\leq 34$ & 10 & 360 & 2.8 & 1.36 & 0.71 to 2.60 & 0.352 & 0.97 & 0.51 to 1.87 & 0.937 \\
\hline $35-49$ & 65 & 3188 & 2.0 & 0.97 & 0.72 to 1.32 & 0.854 & 0.85 & 0.62 to 1.16 & 0.298 \\
\hline $50-64$ & 110 & 5229 & 2.1 & $(1.00)$ & & & $(1.00)$ & & \\
\hline $65-74$ & 77 & 3422 & 2.3 & 1.11 & 0.83 to 1.48 & 0.498 & 1.14 & 0.85 to 1.54 & 0.368 \\
\hline $75-79$ & 26 & 1547 & 1.7 & 0.90 & 0.59 to 1.38 & 0.629 & 0.97 & 0.63 to 1.49 & 0.882 \\
\hline $80+$ & 22 & 1425 & 1.5 & 1.05 & 0.67 to 1.63 & 0.834 & 0.98 & 0.62 to 1.57 & 0.945 \\
\hline \multicolumn{10}{|l|}{ Grade } \\
\hline 1 & 23 & 1827 & 1.3 & $(1.00)$ & & & $(1.00)$ & & \\
\hline 2 & 116 & 6918 & 1.7 & 1.48 & 0.95 to 2.32 & 0.085 & 1.23 & 0.78 to 1.94 & 0.363 \\
\hline 3 & 157 & 5852 & 2.7 & 2.78 & 1.79 to 4.31 & $<0.001$ & 2.06 & 1.31 to 3.24 & 0.002 \\
\hline Unknown & 14 & 574 & 2.4 & 2.34 & 1.28 to 4.30 & 0.006 & 1.44 & 0.73 to 2.82 & 0.293 \\
\hline \multicolumn{10}{|l|}{ Size $(\mathrm{mm})$} \\
\hline$\leq 10$ & 13 & 1103 & 1.2 & $(1.00)$ & & & $(1.00)$ & & \\
\hline $\begin{array}{l}>10 \text { to } \\
<20\end{array}$ & 68 & 4224 & 1.6 & 1.38 & 0.76 to 2.51 & 0.283 & 1.24 & 0.68 to 2.25 & 0.485 \\
\hline$\geq 20$ & 175 & 8627 & 2.0 & 2.04 & 1.16 to 3.59 & 0.013 & 1.43 & 0.80 to 2.56 & 0.224 \\
\hline $\begin{array}{l}\text { Size cat. } \\
\text { unknown }\end{array}$ & 54 & 1217 & 4.4 & 3.82 & 2.09 to 6.97 & $<0.001$ & 2.61 & 1.40 to 4.84 & 0.002 \\
\hline \multicolumn{10}{|l|}{ Nodes } \\
\hline 0 & 114 & 6938 & 1.6 & $(1.00)$ & & & $(1.00)$ & & \\
\hline $1-3$ & 68 & 4147 & 1.6 & 1.10 & 0.82 to 1.49 & 0.518 & 0.95 & 0.69 to 1.29 & 0.723 \\
\hline $4+$ & 97 & 3402 & 2.9 & 2.33 & 1.78 to 3.06 & $<0.001$ & 1.62 & 1.20 to 2.19 & 0.002 \\
\hline Unknown & 31 & 684 & 4.5 & 3.42 & 2.35 to 4.98 & $<0.001$ & 2.55 & 1.65 to 3.95 & $<0.001$ \\
\hline \multicolumn{10}{|c|}{ Vascular invasion } \\
\hline No & 126 & 8608 & 1.5 & $(1.00)$ & & & $(1.00)$ & & \\
\hline Yes & 150 & 5341 & 2.8 & 2.24 & 1.76 to 2.84 & $<0.001$ & 1.68 & 1.29 to 2.19 & $<0.001$ \\
\hline Unknown & 34 & 1222 & 2.8 & 2.14 & 1.49 to 3.06 & $<0.001$ & 1.25 & 0.82 to 1.90 & 0.307 \\
\hline
\end{tabular}

HR, hazard ratio.

$N$ is the size of the subgroup, $n$ is the number of events in that subgroup. 
TABLE 69 Multiple Cox proportional hazards regression models for time to MCBC occurrence in mastectomy cohort

\begin{tabular}{|c|c|c|c|c|c|c|c|c|c|}
\hline & \multirow[b]{2}{*}{$n$} & \multirow[b]{2}{*}{$N$} & \multirow[b]{2}{*}{$\%$} & \multicolumn{3}{|c|}{ Univariate Cox regression } & \multicolumn{3}{|c|}{ Multiple Cox regression } \\
\hline & & & & HR & $95 \% \mathrm{Cl}$ & $p$-value & HR & $95 \% \mathrm{Cl}$ & $p$-value \\
\hline \multicolumn{10}{|c|}{ Age (years) } \\
\hline$\leq 34$ & 13 & 360 & 3.6 & 1.79 & 1.01 to 3.17 & 0.048 & 1.55 & 0.87 to 2.77 & 0.138 \\
\hline $35-49$ & 64 & 3188 & 2.0 & 0.96 & 0.71 to 1.31 & 0.817 & 0.89 & 0.65 to 1.21 & 0.459 \\
\hline 50-64 & 114 & 5229 & 2.2 & $(1.00)$ & & & (1.00) & & \\
\hline $65-74$ & 52 & 3422 & 1.5 & 0.79 & 0.57 to 1.09 & 0.156 & 0.77 & 0.55 to 1.07 & 0.118 \\
\hline $75-79$ & 13 & 1547 & 0.8 & 0.45 & 0.25 to 0.80 & 0.007 & 0.45 & 0.25 to 0.81 & 0.007 \\
\hline $80+$ & 6 & 1425 & 0.4 & 0.31 & 0.15 to 0.68 & 0.003 & 0.27 & 0.12 to 0.61 & 0.002 \\
\hline \multicolumn{10}{|l|}{ Grade } \\
\hline 1 & 31 & 1827 & 1.7 & $(1.00)$ & & & (1.00) & & \\
\hline 2 & 110 & 6918 & 1.6 & 1.03 & 0.69 to 1.54 & 0.870 & 0.91 & 0.61 to 1.37 & 0.660 \\
\hline 3 & 111 & 5852 & 1.9 & 1.52 & 1.02 to 2.26 & 0.040 & 1.18 & 0.78 to 1.79 & 0.436 \\
\hline Unknown & 10 & 574 & 1.7 & 1.12 & 0.57 to 2.18 & 0.742 & 0.87 & 0.41 to 1.86 & 0.726 \\
\hline \multicolumn{10}{|c|}{ Size (mm) } \\
\hline$\leq 10$ & 14 & 1103 & 1.3 & $(1.00)$ & & & (1.00) & & \\
\hline $\begin{array}{l}>10 \text { to } \\
<20\end{array}$ & 67 & 4224 & 1.6 & 1.42 & 0.77 to 2.63 & 0.263 & 1.45 & 0.78 to 2.69 & 0.244 \\
\hline$\geq 20$ & 156 & 8627 & 1.8 & 1.96 & 1.09 to 3.53 & 0.025 & 1.81 & 0.99 to 3.31 & 0.054 \\
\hline $\begin{array}{l}\text { Size cat. } \\
\text { unknown }\end{array}$ & 25 & 1217 & 2.1 & 2.01 & 1.03 to 3.94 & 0.042 & 1.74 & 0.87 to 3.50 & 0.117 \\
\hline \multicolumn{10}{|l|}{ Nodes } \\
\hline 0 & 110 & 6938 & 1.6 & $(1.00)$ & & & (1.00) & & \\
\hline $1-3$ & 64 & 4147 & 1.5 & 1.08 & 0.79 to 1.47 & 0.634 & 0.95 & 0.69 to 1.31 & 0.774 \\
\hline $4+$ & 75 & 3402 & 2.2 & 1.90 & 1.42 to 2.55 & $<0.001$ & 1.53 & 1.10 to 2.12 & 0.012 \\
\hline Unknown & 13 & 684 & 1.9 & 1.40 & 0.81 to 2.45 & 0.231 & 1.44 & 0.76 to 2.75 & 0.264 \\
\hline \multicolumn{10}{|c|}{ Vascular invasion } \\
\hline No & 137 & 8608 & 1.6 & $(1.00)$ & & & $(1.00)$ & & \\
\hline Yes & 105 & 5341 & 2.0 & 1.44 & 1.12 to 1.86 & 0.005 & 1.09 & 0.82 to 1.45 & 0.543 \\
\hline Unknown & 20 & 1222 & 1.6 & 1.07 & 0.67 to 1.69 & 0.784 & 0.89 & 0.53 to 1.50 & 0.669 \\
\hline
\end{tabular}

HR, hazard ratio.

$N$ is the size of the subgroup, $n$ is the number of events in that subgroup. 
TABLE 70 Multiple Cox proportional hazards regression models for time to death from all causes in mastectomy cohort

\begin{tabular}{|c|c|c|c|c|c|c|c|c|c|}
\hline & \multirow[b]{2}{*}{$n$} & \multirow[b]{2}{*}{$N$} & \multirow[b]{2}{*}{$\%$} & \multicolumn{3}{|c|}{ Univariate Cox regression } & \multicolumn{3}{|c|}{ Multiple Cox regression } \\
\hline & & & & $H R$ & $95 \% \mathrm{Cl}$ & $p$-value & HR & $95 \% \mathrm{Cl}$ & $p$-value \\
\hline \multicolumn{10}{|c|}{ Age (years) } \\
\hline$\leq 34$ & 108 & 360 & 30.0 & 1.37 & 1.13 to 1.67 & 0.002 & 0.99 & 0.81 to 1.20 & 0.895 \\
\hline $35-49$ & 647 & 3188 & 20.3 & 0.91 & 0.82 to 1.00 & 0.042 & 0.78 & 0.71 to 0.86 & $<0.001$ \\
\hline $50-64$ & 1179 & 5229 & 22.5 & $(1.00)$ & & & $(1.00)$ & & \\
\hline $65-74$ & 1088 & 3422 & 31.8 & 1.51 & 1.39 to 1.64 & $<0.001$ & 1.54 & 1.42 to 1.68 & $<0.001$ \\
\hline $75-79$ & 608 & 1547 & 39.3 & 2.06 & 1.86 to 2.27 & $<0.001$ & 2.18 & 1.98 to 2.41 & $<0.001$ \\
\hline $80+$ & 715 & 1425 & 50.2 & 3.14 & 2.87 to 3.45 & $<0.001$ & 3.29 & 2.99 to 3.61 & $<0.001$ \\
\hline \multicolumn{10}{|l|}{ Grade } \\
\hline 1 & 336 & 1827 & 18.4 & $(1.00)$ & & & $(1.00)$ & & \\
\hline 2 & 1697 & 6918 & 24.5 & 1.50 & 1.34 to 1.69 & $<0.001$ & 1.21 & 1.08 to 1.36 & 0.001 \\
\hline 3 & 2148 & 5852 & 36.7 & 2.68 & 2.39 to 3.01 & $<0.001$ & 2.02 & 1.79 to 2.27 & $<0.001$ \\
\hline Unknown & 164 & 574 & 28.6 & 1.85 & 1.56 to 2.19 & $<0.001$ & 1.35 & 1.13 to 1.61 & 0.001 \\
\hline \multicolumn{10}{|c|}{ Size $(m m)$} \\
\hline$\leq 10$ & 125 & 1103 & 11.3 & $(1.00)$ & & & $(1.00)$ & & \\
\hline $\begin{array}{l}>10 \text { to } \\
<20\end{array}$ & 846 & 4224 & 20.0 & 1.79 & 1.48 to 2.15 & $<0.001$ & 1.38 & 1.14 to 1.66 & 0.001 \\
\hline$\geq 20$ & 2926 & 8627 & 33.9 & 3.60 & 3.01 to 4.31 & $<0.001$ & 2.02 & 1.68 to 2.42 & $<0.001$ \\
\hline $\begin{array}{l}\text { Size cat. } \\
\text { unknown }\end{array}$ & 448 & 1217 & 36.8 & 3.39 & 2.78 to 4.12 & $<0.001$ & 2.10 & 1.72 to 2.56 & $<0.001$ \\
\hline \multicolumn{10}{|l|}{ Nodes } \\
\hline 0 & 1286 & 6938 & 18.5 & $(1.00)$ & & & $(1.00)$ & & \\
\hline $1-3$ & 1115 & 4147 & 26.9 & 1.61 & 1.49 to 1.75 & $<0.001$ & 1.50 & 1.38 to 1.63 & $<0.001$ \\
\hline $4+$ & 1589 & 3402 & 46.7 & 3.45 & 3.20 to 3.71 & $<0.001$ & 2.70 & 2.49 to 2.92 & $<0.001$ \\
\hline Unknown & 355 & 684 & 51.9 & 3.42 & 3.06 to 3.82 & $<0.001$ & 2.45 & 2.16 to 2.77 & $<0.001$ \\
\hline \multicolumn{10}{|c|}{ Vascular invasion } \\
\hline No & 1866 & 8608 & 21.7 & $(1.00)$ & & & $(1.00)$ & & \\
\hline Yes & 2079 & 5341 & 38.9 & 2.09 & 1.96 to 2.23 & $<0.001$ & 1.37 & 1.28 to 1.47 & $<0.001$ \\
\hline Unknown & 400 & 1222 & 32.7 & 1.67 & 1.51 to 1.85 & $<0.001$ & 1.14 & 1.01 to 1.28 & 0.030 \\
\hline \multicolumn{10}{|c|}{ Ipsilateral recurrence } \\
\hline No & 4150 & 14,861 & 27.9 & & & & & & \\
\hline Yes & 195 & 310 & 62.9 & 2.14 & 1.86 to 2.47 & $<0.001$ & 1.64 & 1.42 to 1.89 & $<0.001$ \\
\hline \multicolumn{10}{|c|}{ Contralateral occurrence } \\
\hline No & 4245 & 14,909 & 28.5 & & & & & & \\
\hline Yes & 100 & 262 & 38.2 & 1.01 & 0.83 to 1.24 & 0.901 & 0.99 & 0.81 to 1.21 & 0.906 \\
\hline
\end{tabular}

HR, hazard ratio.

$N$ is the size of the subgroup, $n$ is the number of events in that subgroup. 
TABLE 71 Multiple Cox proportional hazards regression models for time to death from breast cancer in mastectomy cohort

\begin{tabular}{|c|c|c|c|c|c|c|c|c|c|}
\hline & \multirow[b]{2}{*}{$n$} & \multirow[b]{2}{*}{$N$} & \multirow[b]{2}{*}{$\%$} & \multicolumn{3}{|c|}{ Univariate Cox regression } & \multicolumn{3}{|c|}{ Multiple Cox regression } \\
\hline & & & & HR & $95 \% \mathrm{Cl}$ & $p$-value & HR & $95 \% \mathrm{Cl}$ & $p$-value \\
\hline \multicolumn{10}{|c|}{ Age (years) } \\
\hline$\leq 34$ & 96 & 360 & 26.7 & 1.61 & 1.30 to 1.98 & $<0.001$ & 1.08 & 0.87 to 1.33 & 0.477 \\
\hline $35-49$ & 562 & 3188 & 17.6 & 1.03 & 0.93 to 1.15 & 0.561 & 0.85 & 0.76 to 0.94 & 0.002 \\
\hline $50-64$ & 901 & 5229 & 17.2 & $(1.00)$ & & & $(1.00)$ & & \\
\hline $65-74$ & 632 & 3422 & 18.5 & 1.14 & 1.03 to 1.27 & 0.009 & 1.17 & 1.06 to 1.30 & 0.002 \\
\hline $75-79$ & 269 & 1547 & 17.4 & 1.17 & 1.03 to 1.34 & 0.020 & 1.26 & 1.10 to 1.44 & 0.002 \\
\hline $80+$ & 253 & 1425 & 17.8 & 1.39 & 1.21 to 1.60 & $<0.001$ & 1.51 & 1.31 to 1.74 & 0.001 \\
\hline \multicolumn{10}{|l|}{ Grade } \\
\hline 1 & 117 & 1827 & 6.4 & $(1.00)$ & & & $(1.00)$ & & \\
\hline 2 & 924 & 6918 & 13.4 & 2.32 & 1.91 to 2.81 & $<0.001$ & 1.68 & 1.38 to 2.04 & $<0.001$ \\
\hline 3 & 1585 & 5852 & 27.1 & 5.55 & 4.60 to 6.70 & $<0.001$ & 3.38 & 2.79 to 4.10 & $<0.001$ \\
\hline Unknown & 87 & 574 & 15.2 & 2.98 & 2.32 to 3.84 & $<0.001$ & 1.90 & 1.43 to 2.51 & $<0.001$ \\
\hline \multicolumn{10}{|c|}{ Size $(\mathrm{mm})$} \\
\hline$\leq 10$ & 49 & 1103 & 4.4 & $(1.00)$ & & & $(1.00)$ & & \\
\hline $\begin{array}{l}>10 \text { to } \\
<20\end{array}$ & 438 & 4224 & 10.4 & 2.36 & 1.75 to 3.17 & $<0.001$ & 1.74 & 1.29 to 2.34 & $<0.001$ \\
\hline$\geq 20$ & 1916 & 8627 & 22.2 & 5.93 & 4.46 to 7.87 & $<0.001$ & 2.83 & 2.12 to 3.77 & $<0.001$ \\
\hline $\begin{array}{l}\text { Size cat. } \\
\text { unknown }\end{array}$ & 310 & 1217 & 25.5 & 6.00 & 4.45 to 8.09 & $<0.001$ & 3.08 & 2.27 to 4.18 & $<0.001$ \\
\hline \multicolumn{10}{|l|}{ Nodes } \\
\hline 0 & 568 & 6938 & 8.2 & $(1.00)$ & & & $(1.00)$ & & \\
\hline $1-3$ & 714 & 4147 & 17.2 & 2.32 & 2.07 to 2.59 & $<0.001$ & 1.92 & 1.71 to 2.15 & $<0.001$ \\
\hline $4+$ & 1250 & 3402 & 36.7 & 5.99 & 5.42 to 6.62 & $<0.001$ & 3.89 & 3.49 to 4.34 & $<0.001$ \\
\hline Unknown & 181 & 684 & 26.5 & 4.07 & 3.48 to 4.76 & $<0.001$ & 2.96 & 2.47 to 3.54 & $<0.001$ \\
\hline \multicolumn{10}{|c|}{ Vascular invasion } \\
\hline No & 942 & 8608 & 10.9 & $(1.00)$ & & & $(1.00)$ & & \\
\hline Yes & 1533 & 5341 & 28.7 & 3.02 & 2.79 to 3.28 & $<0.001$ & 1.51 & 1.38 to 1.65 & $<0.001$ \\
\hline Unknown & 238 & 1222 & 19.5 & 2.00 & 1.75 to 2.29 & $<0.001$ & 1.28 & 1.10 to 1.49 & 0.002 \\
\hline \multicolumn{10}{|c|}{ Ipsilateral recurrence } \\
\hline No & 2558 & 14,861 & 17.2 & & & & & & \\
\hline Yes & 155 & 310 & 50.0 & 2.78 & 2.37 to 3.27 & $<0.001$ & 2.12 & 1.80 to 2.50 & $<0.001$ \\
\hline \multicolumn{10}{|c|}{ Contralateral occurrence } \\
\hline No & 2707 & 14,909 & 18.2 & & & & & & \\
\hline Yes & 6 & 262 & 2.3 & 0.09 & 0.04 to 0.19 & $<0.001$ & & & \\
\hline
\end{tabular}

HR, hazard ratio.

$N$ is the size of the subgroup, $n$ is the number of events in that subgroup. 


\section{Appendix 26}

\section{Univariate Cox regression models for the Edinburgh cohort}

TABLE 72 Description of the Edinburgh breast cancer cohort

\begin{tabular}{|c|c|c|}
\hline & $n=1439$ & \\
\hline \multicolumn{3}{|l|}{ Age at diagnosis (years) } \\
\hline Mean (SD) & $56(11)$ & \\
\hline Median (25th, 75th centile) & $56(50,63)$ & \\
\hline \multirow[t]{2}{*}{ Minimum, maximum } & 24,91 & \\
\hline & $n$ & $\%$ \\
\hline \multicolumn{3}{|l|}{ Age categories (years) } \\
\hline$\leq 34$ & 40 & 2.8 \\
\hline $35-49$ & 308 & 21.4 \\
\hline $50-64$ & 826 & 57.4 \\
\hline $65-74$ & 201 & 14.0 \\
\hline $75+$ & 64 & 4.4 \\
\hline \multicolumn{3}{|l|}{ Grade } \\
\hline 1 & 363 & 25.2 \\
\hline 2 & 642 & 44.6 \\
\hline 3 & 402 & 27.9 \\
\hline Unknown & 32 & 2.2 \\
\hline \multicolumn{3}{|l|}{ Size $(\mathrm{mm})$} \\
\hline$\leq 10$ & 339 & 23.6 \\
\hline$>10$ to $<20$ & 632 & 43.9 \\
\hline$\geq 20$ & 414 & 28.8 \\
\hline Size cat. unknown & 54 & 3.8 \\
\hline \multicolumn{3}{|l|}{ Nodes } \\
\hline 0 & 1052 & 73.1 \\
\hline $1-3$ & 312 & 21.7 \\
\hline $4+$ & 75 & 5.2 \\
\hline \multicolumn{3}{|l|}{ Outcomes } \\
\hline Death all cause & 124 & 8.6 \\
\hline Death breast cancer & 67 & 4.7 \\
\hline Ipsilateral event & 434 & 30.2 \\
\hline Contralateral event & 234 & 16.3 \\
\hline
\end{tabular}

Note that all of the women in the Edinburgh breast cancer cohort were treated surgically with BCS. 


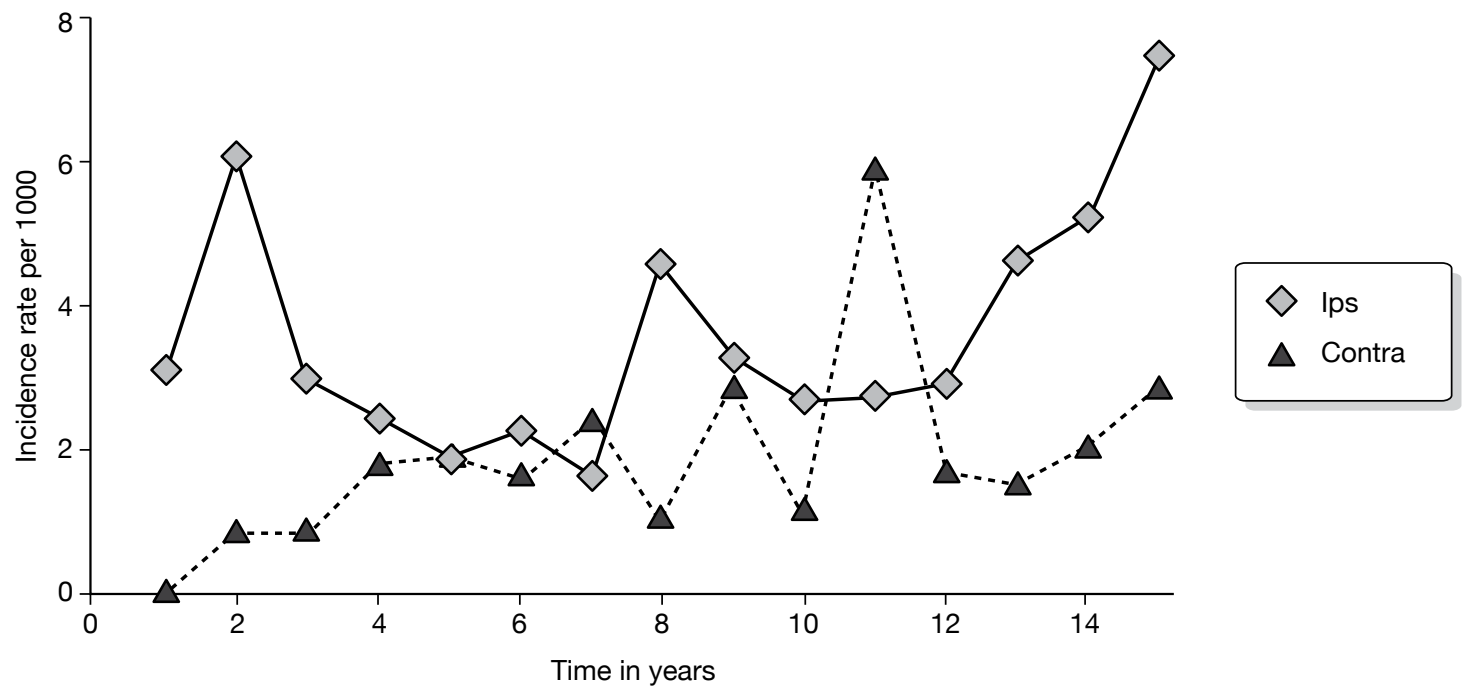

FIGURE 34 Incidence per 1000 per year of ipsilateral recurrence and contralateral occurrence for Edinburgh breast cancer cohort.

TABLE 73 Multiple Cox regression models for IBTR in Edinburgh breast cancer cohort

\begin{tabular}{|c|c|c|c|c|c|c|c|c|c|}
\hline & \multirow[b]{2}{*}{$n$} & \multirow[b]{2}{*}{$N$} & \multirow[b]{2}{*}{$\%$} & \multicolumn{3}{|c|}{ Univariate Cox regression } & \multicolumn{3}{|c|}{ Multiple Cox regression } \\
\hline & & & & HR & $95 \% \mathrm{Cl}$ & $p$-value & HR & $95 \% \mathrm{Cl}$ & $p$-value \\
\hline \multicolumn{10}{|c|}{ Age (years) } \\
\hline$\leq 34$ & 15 & 40 & 37.5 & 6.60 & 3.75 to 11.62 & $<0.001$ & 4.65 & 2.58 to 8.37 & $<0.001$ \\
\hline $35-49$ & 35 & 308 & 11.4 & 1.55 & 1.02 to 2.35 & 0.039 & 1.18 & 0.77 to 1.80 & 0.452 \\
\hline $50-64$ & 61 & 826 & 7.4 & $(1.00)$ & & & $(1.00)$ & & \\
\hline $65-74$ & 11 & 201 & 5.5 & 0.81 & 0.43 to 1.54 & 0.516 & 0.77 & 0.41 to 1.48 & 0.439 \\
\hline $75+$ & 2 & 64 & 3.1 & 0.60 & 0.15 to 2.47 & 0.482 & 0.51 & 0.12 to 2.09 & 0.349 \\
\hline \multicolumn{10}{|l|}{ Grade } \\
\hline 1 & 16 & 363 & 4.4 & $(1.00)$ & & & $(1.00)$ & & \\
\hline 2 & 55 & 642 & 8.6 & 2.19 & 1.25 to 3.82 & 0.006 & 1.86 & 1.06 to 3.27 & 0.030 \\
\hline 3 & 50 & 402 & 12.4 & 3.56 & 2.03 to 6.26 & $<0.001$ & 2.36 & 1.31 to 4.27 & 0.004 \\
\hline Unknown & 3 & 32 & 9.4 & 2.09 & 0.61 to 7.16 & 0.243 & 1.72 & 0.49 to 6.07 & 0.402 \\
\hline \multicolumn{10}{|c|}{ Size $(\mathrm{mm})$} \\
\hline$\leq 10$ & 20 & 339 & 5.9 & $(1.00)$ & & & (1.00) & & \\
\hline $\begin{array}{l}>10 \text { to } \\
<20\end{array}$ & 24 & 632 & 3.8 & 1.23 & 0.72 to 2.09 & 0.450 & 0.90 & 0.52 to 1.57 & 0.720 \\
\hline$\geq 20$ & 56 & 414 & 13.5 & 2.80 & 1.68 to 4.67 & $<0.001$ & 1.55 & 0.89 to 2.73 & 0.124 \\
\hline $\begin{array}{l}\text { Size cat. } \\
\text { unknown }\end{array}$ & 6 & 54 & 11.1 & 2.14 & 0.86 to 5.32 & 0.103 & 1.53 & 0.61 to 3.89 & 0.367 \\
\hline \multicolumn{10}{|l|}{ Nodes } \\
\hline 0 & 69 & 1052 & 6.6 & $(1.00)$ & & & (1.00) & & \\
\hline $1-3$ & 36 & 312 & 11.5 & 2.02 & 1.35 to 3.02 & 0.001 & 1.75 & 1.15 to 2.67 & 0.009 \\
\hline $4+$ & 19 & 75 & 25.3 & 5.68 & 3.41 to 9.45 & $<0.001$ & 4.18 & 2.45 to 7.12 & $<0.001$ \\
\hline
\end{tabular}

HR, hazard ratio.

$N$ is the size of the subgroup, $n$ is the number of events in that subgroup. 
TABLE 74 Multiple Cox regression models for MCBC occurrence in Edinburgh breast cancer cohort

\begin{tabular}{|c|c|c|c|c|c|c|c|c|c|}
\hline & \multirow[b]{2}{*}{$n$} & \multirow[b]{2}{*}{$N$} & \multirow[b]{2}{*}{$\%$} & \multicolumn{3}{|c|}{ Univariate Cox regression } & \multicolumn{3}{|c|}{ Multiple Cox regression } \\
\hline & & & & HR & $95 \% \mathrm{Cl}$ & $p$-value & HR & $95 \% \mathrm{Cl}$ & $p$-value \\
\hline \multicolumn{10}{|c|}{ Age (years) } \\
\hline$\leq 34$ & 6 & 40 & 6 & 4.79 & 2.01 to 11.43 & $<0.001$ & 3.94 & 1.60 to 9.74 & 0.003 \\
\hline $35-49$ & 19 & 308 & 19 & 1.55 & 0.88 to 2.73 & 0.128 & 1.51 & 0.84 to 2.71 & 0.165 \\
\hline $50-64$ & 33 & 826 & 33 & $(1.00)$ & & & & & \\
\hline $65-74$ & 7 & 201 & 7 & 0.99 & 0.44 to 2.25 & 0.990 & 1.03 & 0.45 to 2.36 & 0.936 \\
\hline $75-79$ & 2 & 64 & 2 & 1.28 & 0.30 to 5.34 & 0.738 & 1.29 & 0.31 to 5.49 & 0.726 \\
\hline \multicolumn{10}{|l|}{ Grade } \\
\hline 1 & 14 & 363 & 3.9 & (1.00) & & & $(1.00)$ & & \\
\hline 2 & 25 & 642 & 3.9 & 0.38 & 0.59 to 2.19 & 0.695 & 1.10 & 0.57 to 2.14 & 0.769 \\
\hline 3 & 24 & 402 & 6.0 & 0.67 & 1.03 to 3.86 & 0.041 & 1.81 & 0.90 to 3.64 & 0.098 \\
\hline Unknown & 4 & 32 & 12.5 & 1.95 & 1.13 to 10.47 & 0.030 & 2.61 & 0.82 to 8.30 & 0.104 \\
\hline \multicolumn{10}{|c|}{ Size $(\mathrm{mm})$} \\
\hline$\leq 10$ & 17 & 339 & 5.0 & $(1.00)$ & & & $(1.00)$ & & \\
\hline $\begin{array}{l}>10 \text { to } \\
<20\end{array}$ & 27 & 632 & 4.3 & 0.93 & 0.51 to 1.71 & 0.817 & 0.81 & 0.43 to 1.52 & 0.508 \\
\hline$\geq 20$ & 19 & 414 & 4.6 & 1.13 & 0.59 to 2.17 & 0.720 & 0.83 & 0.40 to 1.69 & 0.601 \\
\hline $\begin{array}{l}\text { Size cat. } \\
\text { unknown }\end{array}$ & 4 & 54 & 7.4 & 1.73 & 0.58 to 5.15 & 0.323 & 1.37 & 0.45 to 4.16 & 0.580 \\
\hline \multicolumn{10}{|l|}{ Nodes } \\
\hline 0 & 55 & 1052 & 5.2 & (1.00) & & & $(1.00)$ & & \\
\hline $1-3$ & 15 & 312 & 4.8 & 1.16 & 0.65 to 2.06 & 0.617 & 1.18 & 0.65 to 2.15 & 0.580 \\
\hline $4+$ & 1 & 75 & 1.3 & 0.40 & 0.05 to 2.88 & 0.362 & 0.39 & 0.05 to 2.88 & 0.357 \\
\hline
\end{tabular}

HR, hazard ratio.

$N$ is the size of the subgroup, $n$ is the number of events in that subgroup. 
TABLE 75 Multiple Cox regression models for all-cause death in Edinburgh breast cancer cohort

\begin{tabular}{|c|c|c|c|c|c|c|c|c|c|}
\hline & \multirow[b]{2}{*}{$n$} & \multirow[b]{2}{*}{$N$} & \multirow[b]{2}{*}{$\%$} & \multicolumn{3}{|c|}{ Univariate Cox regression } & \multicolumn{3}{|c|}{ Multiple Cox regression } \\
\hline & & & & HR & $95 \% \mathrm{Cl}$ & $p$-value & HR & $95 \% \mathrm{Cl}$ & $p$-value \\
\hline \multicolumn{10}{|c|}{ Age (years) } \\
\hline$\leq 34$ & 14 & 40 & 35.0 & 1.58 & 0.92 to 2.71 & 0.099 & 0.97 & 0.55 to 1.70 & 0.917 \\
\hline $35-49$ & 78 & 308 & 25.3 & 0.98 & 0.76 to 1.27 & 0.871 & 0.77 & 0.59 to 1.00 & 0.050 \\
\hline $50-64$ & 215 & 826 & 26.0 & $(1.00)$ & & & $(1.00)$ & & \\
\hline $65-74$ & 87 & 201 & 43.3 & 1.90 & 1.48 to 2.44 & $<0.001$ & 1.89 & 1.47 to 2.44 & $<0.001$ \\
\hline $75-79$ & 40 & 64 & 62.5 & 3.78 & 2.69 to 5.32 & $<0.001$ & 3.33 & 2.35 to 4.72 & $<0.001$ \\
\hline \multicolumn{10}{|l|}{ Grade } \\
\hline 1 & 80 & 363 & 22.0 & $(1.00)$ & & & $(1.00)$ & & \\
\hline 2 & 193 & 642 & 30.1 & 1.53 & 1.18 to 1.99 & 0.001 & 1.25 & 0.96 to 1.62 & 0.105 \\
\hline 3 & 150 & 402 & 37.3 & 2.11 & 1.61 to 2.77 & $<0.001$ & 1.72 & 1.29 to 2.28 & $<0.001$ \\
\hline Unknown & 11 & 32 & 34.4 & 1.50 & 0.80 to 2.81 & 0.210 & 1.86 & 0.97 to 3.56 & 0.061 \\
\hline \multicolumn{10}{|c|}{ Size $(m m)$} \\
\hline$\leq 10$ & 60 & 339 & 17.7 & $(1.00)$ & & & $(1.00)$ & & \\
\hline $\begin{array}{l}>10 \text { to } \\
<20\end{array}$ & 184 & 632 & 29.1 & 1.80 & 1.35 to 2.41 & $<0.001$ & 1.44 & 1.06 to 1.94 & 0.018 \\
\hline$\geq 20$ & 168 & 414 & 40.6 & 2.76 & 2.06 to 3.71 & $<0.001$ & 1.79 & 1.30 to 2.47 & $<0.001$ \\
\hline $\begin{array}{l}\text { Size cat. } \\
\text { unknown }\end{array}$ & 22 & 54 & 40.7 & 2.58 & 1.58 to 4.21 & $<0.001$ & 1.93 & 1.16 to 3.20 & 0.011 \\
\hline \multicolumn{10}{|l|}{ Nodes } \\
\hline 0 & 266 & 1052 & 25.3 & $(1.00)$ & & & $(1.00)$ & & \\
\hline $1-3$ & 124 & 312 & 39.7 & 1.79 & 1.44 to 2.21 & $<0.001$ & 1.59 & 1.28 to 1.98 & $<0.001$ \\
\hline $4+$ & 44 & 75 & 58.7 & 3.22 & 2.33 to 4.45 & $<0.001$ & 2.72 & 1.94 to 3.81 & $<0.001$ \\
\hline \multicolumn{10}{|c|}{ Ipsilateral recurrence } \\
\hline No & 372 & 1315 & 28.3 & $(1.00)$ & & & & & \\
\hline Yes & 62 & 124 & 50.0 & 1.94 & 1.48 to 2.54 & $<0.001$ & 1.87 & 1.41 to 2.49 & $<0.001$ \\
\hline \multicolumn{10}{|c|}{ Contralateral occurrence } \\
\hline No & 416 & 1372 & 30.3 & $(1.00)$ & & & (1.00) & & \\
\hline Yes & 18 & 67 & 26.9 & 0.77 & 0.48 to 1.23 & 0.277 & 0.70 & 0.43 to 1.13 & 0.140 \\
\hline
\end{tabular}

HR, hazard ratio.

$N$ is the size of the subgroup, $n$ is the number of events in that subgroup. 
TABLE 76 Multiple Cox regression models for death from breast cancer in Edinburgh breast cancer cohort

\begin{tabular}{|c|c|c|c|c|c|c|c|c|c|}
\hline & \multirow[b]{2}{*}{$n$} & \multirow[b]{2}{*}{$N$} & \multirow[b]{2}{*}{$\%$} & \multicolumn{3}{|c|}{ Univariate Cox regression } & \multicolumn{3}{|c|}{ Multiple Cox regression } \\
\hline & & & & HR & $95 \% \mathrm{Cl}$ & $p$-value & HR & $95 \% \mathrm{Cl}$ & $p$-value \\
\hline \multicolumn{10}{|c|}{ Age (years) } \\
\hline$\leq 34$ & 14 & 40 & 35.0 & 2.70 & 1.55 to 4.69 & $<0.001$ & 1.17 & 0.65 to 2.10 & 0.599 \\
\hline $35-49$ & 57 & 308 & 18.5 & 1.25 & 0.91 to 1.71 & 0.163 & 0.89 & 0.65 to 1.23 & 0.487 \\
\hline $50-64$ & 123 & 826 & 14.9 & $(1.00)$ & & & $(1.00)$ & & \\
\hline $65-74$ & 27 & 201 & 13.4 & 0.98 & 0.65 to 1.49 & 0.935 & 1.00 & 0.65 to 1.52 & 0.987 \\
\hline $75-79$ & 13 & 64 & 20.3 & 1.84 & 1.04 to 3.27 & 0.037 & 1.52 & 0.85 to 2.72 & 0.160 \\
\hline \multicolumn{10}{|l|}{ Grade } \\
\hline 1 & 26 & 363 & 7.2 & $(1.00)$ & & & $(1.00)$ & & \\
\hline 2 & 101 & 642 & 15.7 & 2.38 & 1.55 to 3.67 & $<0.001$ & 1.79 & 1.16 to 2.77 & 0.009 \\
\hline 3 & 101 & 402 & 25.1 & 4.18 & 2.71 to 6.43 & $<0.001$ & 2.86 & 1.83 to 4.47 & $<0.001$ \\
\hline Unknown & 6 & 32 & 18.8 & 2.64 & 1.08 to 6.41 & 0.032 & 3.30 & 1.32 to 8.21 & 0.010 \\
\hline \multicolumn{10}{|c|}{ Size $(m m)$} \\
\hline$\leq 10$ & 21 & 339 & 6.2 & $(1.00)$ & & & $(1.00)$ & & \\
\hline $\begin{array}{l}>10 \text { to } \\
<20\end{array}$ & 91 & 632 & 14.4 & 2.48 & 1.55 to 3.99 & $<0.001$ & 1.86 & 1.14 to 3.02 & 0.013 \\
\hline$\geq 20$ & 111 & 414 & 26.8 & 5.07 & 3.18 to 8.09 & $<0.001$ & 2.61 & 1.58 to 4.29 & $<0.001$ \\
\hline $\begin{array}{l}\text { Size cat. } \\
\text { unknown }\end{array}$ & 11 & 54 & 20.4 & 3.70 & 1.78 to 7.68 & $<0.001$ & 2.08 & 0.96 to 4.48 & 0.062 \\
\hline \multicolumn{10}{|l|}{ Nodes } \\
\hline 0 & 120 & 1052 & 11.4 & $(1.00)$ & & & $(1.00)$ & & \\
\hline $1-3$ & 78 & 312 & 25.0 & 2.45 & 1.84 to 3.26 & $<0.001$ & 2.05 & 1.53 to 2.76 & $<0.001$ \\
\hline $4+$ & 36 & 75 & 48.0 & 5.50 & 3.77 to 8.02 & $<0.001$ & 3.42 & 2.29 to 5.11 & $<0.001$ \\
\hline \multicolumn{10}{|c|}{ Ipsilateral recurrence } \\
\hline No & 179 & 1315 & 13.6 & & & & & & \\
\hline Yes & 55 & 124 & 44.4 & 3.59 & 2.65 to 4.86 & $<0.001$ & 2.69 & 1.94 to 3.72 & $<0.001$ \\
\hline \multicolumn{10}{|c|}{ Contralateral occurrence } \\
\hline No & 223 & 1372 & 16.3 & $(1.00)$ & & & & & \\
\hline Yes & 11 & 67 & 16.4 & 0.91 & 0.49 to 1.66 & 0.747 & 0.66 & 0.35 to 1.24 & 0.199 \\
\hline
\end{tabular}

HR, hazard ratio.

$N$ is the size of the subgroup, $n$ is the number of events in that subgroup. 



\section{Appendix 27}

\section{Example of detailed outline of economic model structure}




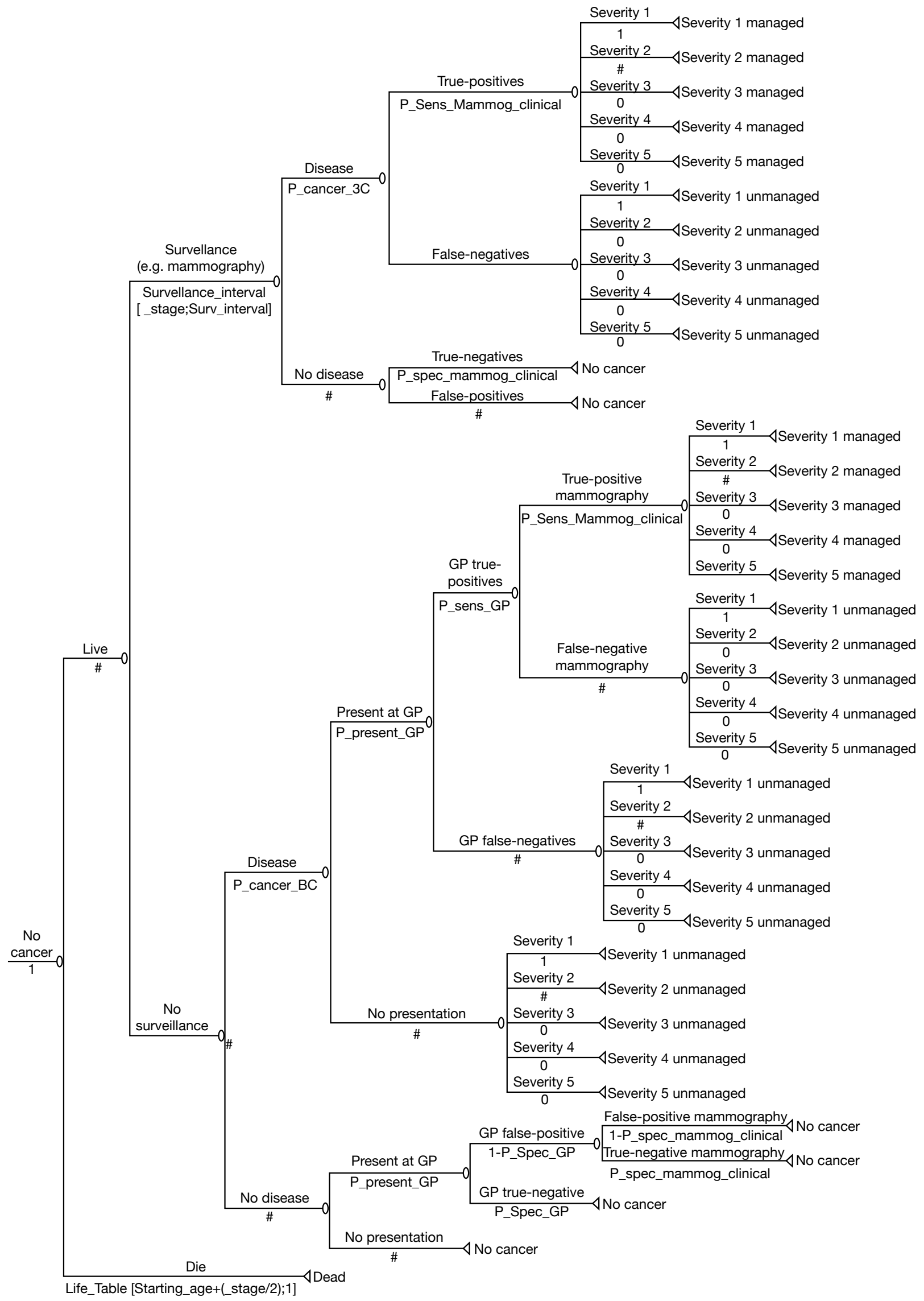




\section{Appendix 28}

\section{Review of management guidelines for breast cancer}

\section{Overview}

In order to assess the cost-effectiveness of different surveillance mammography regimens after the treatment for primary breast cancer, the clinical effectiveness of standard treatment options of any subsequent cancers were required for the economic model.

The objective of this review is to determine the effect of treatment options on survival in patients who are identified as having breast cancer during surveillance. After searching relevant guidelines, the recent updated NICE guideline Early and Locally Advanced Breast Cancer, published in February 2009, was identified as providing the best available evidence of treatments for early breast cancer relevant to the UK. ${ }^{24}$ This review, therefore, is mainly based on this guideline and the source data used to inform the guideline: the EBCTCG ${ }^{83}$ and Adjuvant! Online computer program. ${ }^{84}$ Using these sources, estimates of survival following various treatment options were prepared using the Adjuvant! Online computer program due to its flexibility.

\section{Methods}

At the beginning of this review, initial scoping searches were carried out into identify relevant local, national or international guidelines. Eleven guidelines were identified describing various treatments or managements of primary breast cancer. ${ }^{21-25,28,111-115}$ The most recent of these, and most applicable to the UK, were the NICE guidelines published in February 2009. ${ }^{24}$ Few data were available on the effectiveness of treatments for cancers identified following treatment for a primary cancer. As a consequence, it was judged that, in the absence of data in the literature directly relevant to our study question, the best source of information would relate to treatment of primary cancer. It was judged that, of the guidelines available, the best available summary of existing evidence on the clinical effectiveness of treatments of breast cancer, including early, locally advanced and advanced disease, was provided by the NICE guidelines. The literature searches used to inform these guidelines considered papers published up to July 2008.

\section{NICE guideline: Early and Locally Advanced Breast Cancer}

The NICE guideline Early and Locally Advanced Breast Cancer, ${ }^{24}$ published in February 2009, updated and developed guidance from three NICE technology appraisals: 109 (docetaxel), 108 (paclitaxel) and 107 (trastuzumab).99,116,117 The evidence on clinical effectiveness of diagnoses and treatments for early and locally advanced breast cancer is based on the systematic review of relevant clinical literatures and critical appraisal.

\section{Search strategy}

Papers that were published or accepted for publication in peer-reviewed journals were considered as relevant. Search filters, such as those to identify systematic reviews and RCTs, were applied to the search strategies when there was a wealth of these types of studies. No language restrictions were applied to the search; however, foreign language papers were not requested or reviewed 
(unless of particular importance to the question). Any evidence published before July 2008 was included. The following databases were included in the literature search:

- The Cochrane Library

- MEDLINE and PREMEDLINE 1950 onwards

- Excerpta Medica (EMBASE) 1980 onwards

- Cumulative Index to Nursing and Allied Health Literature (CINAHL) 1982 onwards

- Allied \& Complementary Medicine (AMED) 1985 onwards

- British Nursing Index (BNI) 1994 onwards

- PsycINFO 1806 onwards

- Web of Science 1970 onwards [specifically SCI Expanded and Social Sciences Citation Index (SSCI)]

- System for Information on Grey Literature In Europe (SIGLE) 1980-2005

- BioMed Central 1997 onwards

- NRR

- CCT.

\section{Types of studies included in the guideline}

Relevant guidelines, systematic reviews and RCTs of different treatments for early or locally advanced breast cancer. In the absence of RCT evidence, the observational studies were considered in the review such as cohort, case-controls, etc.

\section{Types of interventions considered in the guideline}

Table 77 summarises the treatment interventions included in the NICE guideline. Surgery is considered as the first line of treatment in primary breast cancer, such as mastectomy, BCT and surgery to the axilla. Adjuvant therapies were used in management of breast cancer after the surgery, including hormonal therapy, chemotherapy, biological therapy and radiotherapy.

\section{Critical appraisal of studies included in the NICE guideline}

One researcher independently scanned the titles and abstracts of every article. Full texts were obtained for any papers that were considered potentially relevant or where there was insufficient information. The researcher then applied the inclusion/exclusion criteria to determine which studies were relevant. Included papers were critically appraised and data extracted. Quality assessment was based on the SIGN criteria.

\section{Summary}

The NICE guideline overviewed the best clinical evidence of treatment options derived from the studies that it reviewed and appraised. ${ }^{24}$ In addition to BCS or mastectomy, adjuvant treatments are used and the selection of adjuvant therapies depends on tumour factors (ER and HER2 status) and patient characteristics (age and menopausal status). However, the NICE guideline did not report the detailed data but rather made a recommendation on the use of adjuvant treatments. The guideline suggested that two sources were more likely to provide reliable data on the effectiveness of adjuvant treatments: (1) a series of overviews and meta-analyses of different treatments in the EBCTCG that provides the effectiveness of adjuvant treatment after surgical therapy for early breast cancer, and (2) Adjuvant! Online, based on a US population, to estimate the efficacy of adjuvant therapies (hormonal or chemotherapy) after initial surgical treatment.

\section{Effect of adjuvant treatments on survival based on overview in EBCTCG}

The NICE guideline suggested that overviews of meta-analyses in EBCTCG appear to provide the best evidence for estimating risk for treating breast cancer in the UK. The EBCTCG was established in 1984. The most recent publications from the EBCTCG relate to: 
TABLE 77 Treatments considered in the NICE guideline

\begin{tabular}{|c|c|c|}
\hline Interventions & $\begin{array}{l}\text { Population setting: early and locally } \\
\text { advanced breast cancer }\end{array}$ & Studies \\
\hline Surgery to breast & DCIS & 33 observation studies \\
\hline \multicolumn{3}{|l|}{ Mastectomy } \\
\hline \multicolumn{3}{|l|}{ Breast-conserving therapy } \\
\hline Surgery to the axilla & & Invasive breast cancer SLNB vs clearance or \\
\hline \multirow[t]{2}{*}{ Sentinel lymph node biopsy (SNLB) } & & $\begin{array}{l}\text { axillary sampling: } 33 \text { RCTs and case series, a } \\
\text { systematic review (69 studies) }\end{array}$ \\
\hline & & $\begin{array}{l}\text { Axillary sampling as staging surgery: } 15 \text { studies } \\
\text { (two RCTs, } 13 \text { case series) }\end{array}$ \\
\hline Adjuvant endocrine therapy (hormonal) & $\begin{array}{l}\text { Invasive disease with hormone receptor } \\
\text { positive }\end{array}$ & \\
\hline Tamoxifen & ER alpha positive & \\
\hline \multicolumn{3}{|l|}{ Aromatase inhibitors: } \\
\hline Anastrozole & ER or PR positive & Nine RCTs \\
\hline Letrozole & ER or PR positive & Five RCTs \\
\hline Exemestane & ER or PR positive & Four RCTs \\
\hline Adjuvant chemotherapy & & A meta-analysis from EBCTCG \\
\hline Docetaxel & Early lymph node positive & $\begin{array}{l}\text { Four RCTs, one Cochrane, a HTA report, a meta- } \\
\text { analysis, a pooled analysis }\end{array}$ \\
\hline Paclitaxel & Early lymph node positive & $\begin{array}{l}\text { Four RCTs, one Cochrane, a HTA report, a meta- } \\
\text { analysis, a pooled analysis }\end{array}$ \\
\hline Taxanes & & $\begin{array}{l}\text { Two RCTs, a meta-analysis and a pooled analysis, } \\
\text { a TACT study }\end{array}$ \\
\hline Adjuvant biological therapy & HER2 receptor positive & \\
\hline Humanised monoclonal antibody (trastuzumab) & & $\begin{array}{l}\text { Four RCTs (HERA, NSABP B-31, NCCTG N9831 } \\
\text { and E2198) }\end{array}$ \\
\hline Adjuvant radiotherapy & Invasive breast, DCIS & \\
\hline BCS and radiotherapy & Invasive breast, DCIS & $\begin{array}{l}\text { One RCT, two non-RCTs, three reviews and four } \\
\text { guidelines for invasive, three studies (two RCTs, } \\
\text { one retrospective) for DCIS }\end{array}$ \\
\hline Postmastectomy radiotherapy & & EBCTCG and five additional RCTs \\
\hline Primary systematic therapy & & $\begin{array}{l}\text { Three systematic reviews of RCTs and a published } \\
\text { review }\end{array}$ \\
\hline Neoadjuvant endocrine therapy & & One systematic review of RCTs \\
\hline Neoadjuvant chemotherapy & & $\begin{array}{l}\text { One systematic review of RCTs and a published } \\
\text { review }\end{array}$ \\
\hline Neoadjuvant radiotherapy & & \\
\hline
\end{tabular}

PR, progesterone receptor; SNLB, sentinel lymph node biopsy.

- adjuvant polychemotherapy in ER-poor breast cancer: meta-analysis of individual patient data from the randomised trials ${ }^{118}$

- chemotherapy and hormonal therapy for early breast cancer: effects on recurrence and 15 -year survival in an overview of the randomised trials ${ }^{3}$

- effects of radiotherapy and of differences in the extent of surgery for early breast cancer on local recurrence and 15-year survival: an overview of the randomised trials. ${ }^{2}$

Some adjuvant treatments tested in the 1980s have clear evidence that they substantially reduce 5 -year recurrence rates and also substantially reduce 15 -year overall mortality rates (e.g. tamoxifen, polychemotherapy regimens and radiotherapy). Further improvements in long-term 
survival could be available from newer drugs, or better use of older drugs. The reviews, however, although excellent, do not provide data readily useable in an economic model, as it is not possible to estimate survivals for specific types of cancer. However, for completeness a summary of key findings is presented below.

\section{Adjuvant chemotherapy}

The number of randomised trials of chemotherapy increased substantially over the first two decades of the EBCTCG overview, with a shift from trials comparing chemotherapy with no chemotherapy, to trials of different types of chemotherapy. In the first cycle of the overview, 31 randomised trials of no chemotherapy versus chemotherapy using one or more drugs were included. This involved a total of 9000 women, of whom 2900 had died. Subsequent cycles refined this to focus on trials of prolonged multiagent chemotherapy. By the third cycle of the EBCTCG overview, this analysis was based on 18,000 women from 47 trials, and it had risen further to 60 trials (29,000 women and 10,000 deaths) by the fourth cycle. The recent metaanalysis from randomised trials is to assess the long-term effects of adjuvant polychemotherapy regimens in ER-poor breast cancer, and the extent to which these effects are modified by age or tamoxifen use. ${ }^{118}$ This study analysed 6000 patients with ER-poor breast cancer in 46 trials of polychemotherapy against not, and about 14,000 women with ER-poor breast cancer in 50 trials of tamoxifen versus not.

\section{Adjuvant hormonal therapy}

The first EBCTCG review of tamoxifen, in the mid-1980s, included data from a total of 16,500 women in 28 randomised trials, of whom nearly 3800 were known to have died. This grew to 40 trials (30,000 women and 8200 deaths) in the second cycle; and then to 55 trials, involving $>12,000$ deaths among 37,000 women. By the 2000-5 cycle of the EBCTCG overview, the growth in the number of trials included had levelled off at 56, but further accrual to some of these trials and five more years of follow-up for many of them meant that the number of women in the analysis had increased to 48,000 , with a total of 18,000 deaths. ${ }^{3}$ This represented $88 \%$ of the total number of women randomised into eligible trials of adjuvant tamoxifen versus no immediate tamoxifen worldwide.

\section{Adjuvant radiotherapy}

The most recent overview of radiotherapy and differences of surgery published in $2005^{2}$ was based on the analysis of 42,000 women in 78 randomised treatment comparisons (radiotherapy vs no radiotherapy, 23,500; more surgery vs less surgery, 9300; more surgery vs radiotherapy, 9300). A total of 24 types of local treatment comparison were identified to help relate the effect on local (i.e. locoregional) recurrence to that on breast cancer mortality.

\section{Effect of treatments on survival for breast cancer using the Adjuvant! Online computer program}

Adjuvant! Online is a tool of assessment of risk of an individual patient developing recurrent disease and/or dying within 10 years. Adjuvant! Online draws information from mortality statistics in the USA, the SEER database, and meta-analyses and individual clinical trials. Based on well-validated factors, such as age, menopausal status, ER status, tumour size and grade, nodes status, etc., predictions can be made about survival for alternative adjuvant treatment regimens, such as chemotherapy, endocrine, etc. However, survival estimates are derived from the US population. Version 8 of this tool may underestimate the risk of mortality and the benefit of trastuzumab in HER-2-positive patients. Table 78 describes the information used to predict recurrence and mortality. 
TABLE 78 Prognostic factors in Adjuvant! Online

\begin{tabular}{lllllll}
\hline Comorbidity & Perfect & $\begin{array}{l}\text { Minor } \\
\text { problems }\end{array}$ & Average for age & Major 10 & Major 20 & Major 30 \\
\hline ER status & Undefined & Positive & Negative & & \\
Tumour size (cm) & $0.1-1$ & $1.1-2$ & $2.1-3$ & $3.1-5$ & $>5$ \\
Positive nodes & 0 & $1-3$ & $4-9$ & $>9$ & \\
Tumour grade & Undefined & 1 & 2 & & \\
Age (years) & $1-99$ & & & & \\
\hline
\end{tabular}

The NICE guideline summarised the following issues in its critical appraisal of this tool:

- The predictions made by Adjuvant! Online are based on the published methodology, which has been updated periodically as evidence of treatment effectiveness and data on risk factors become available.

- Help files and published descriptions of the tool make clear some of the assumptions and limitations that underpin the methodology. The impact of these individual assumptions is difficult to assess. Adjuvant! Online deals with key uncertainties by alerting the user to them at relevant points.

- Survival estimates are derived from the US population. Quantifying the impact on survival of socioeconomic background and of ethnic differences between US and UK populations is difficult.

- Adjuvant! Online is already used in the UK and is designed to incorporate the Oxford overview meta-analyses.

- The strongest evidence of Adjuvant! Online validity for the UK is derived from comparisons between predictions and observed outcomes using a Canadian population. This study found its predications to be reliable for most groups. Further validation is under way using a European population.

\section{Survival estimates for treatment options using the Adjuvant! Online computer program}

Adjuvant! Online integrates patient-related information (age and comorbidity) and tumourrelated information (nodal status, tumour size, histological grade, ER status and histological subtype) to make estimates of mortality caused by cancer or from other causes. Table 79 reports an example of mortality estimates for a woman with breast cancer depending upon her characteristics and tumour factors when the woman is 40 years old, has perfect health, tumour grade is undefined and oestrogen status is positive.

\section{Summary}

Decisions on the treatment for every woman with breast cancer should be based on the best evidence. This requires a combination of information about the patient and tumour along with evidence on the effectiveness of the treatments being considered. This evidence needs to be as reliable as possible. It was judged by the research team that the NICE guideline Early and Locally Advanced Breast Cancer provided the best available summary of evidence of breast cancer treatments. However, the NICE guideline did not report estimates of clinical effectiveness of treatment options. Based on the NICE guideline, there are two reliable sources that produce the 


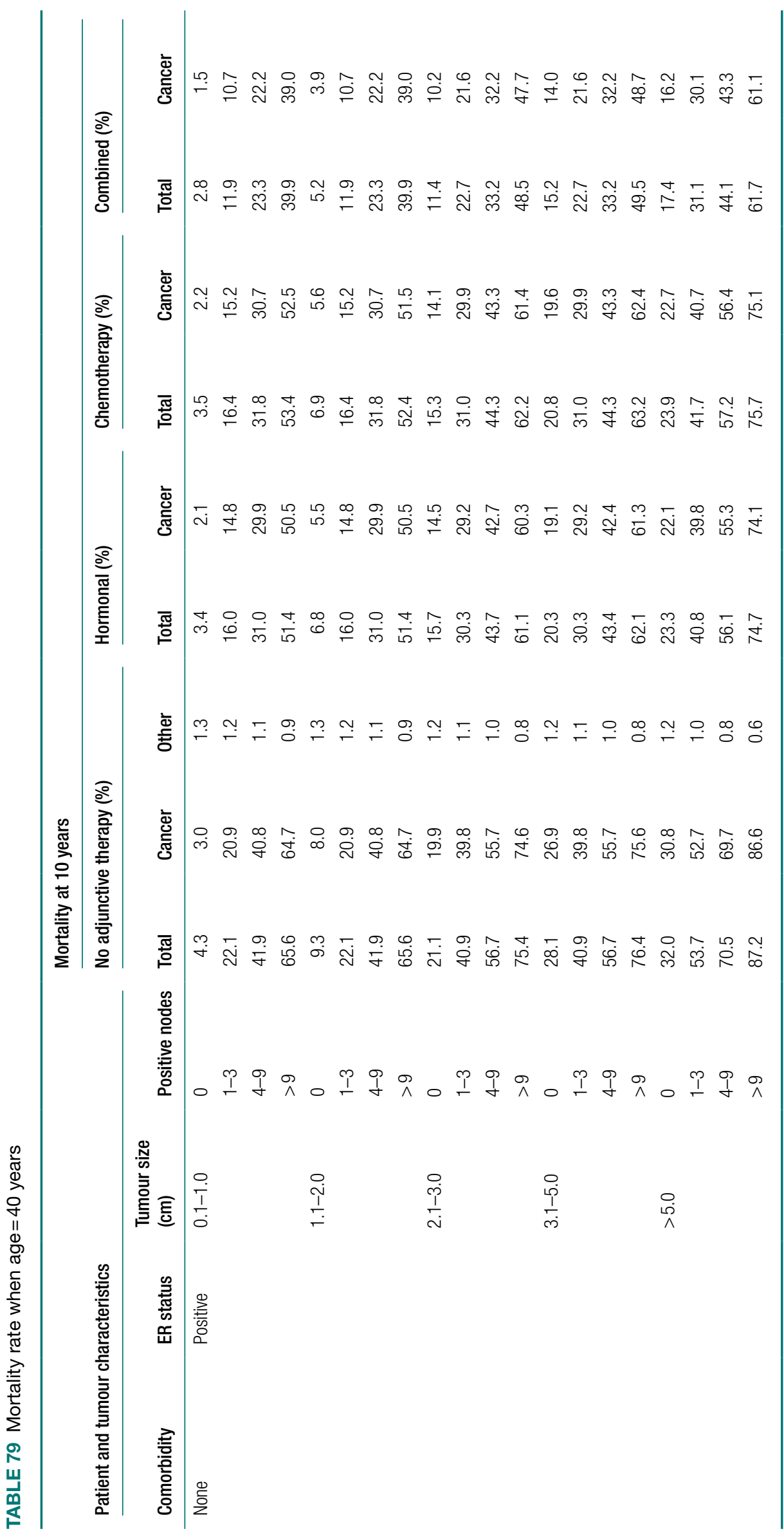


estimates of effectiveness of adjuvant treatments: EBCTCG overviews and Adjuvant! Online. Of these Adjuvant! Online was sufficiently flexible to allow relevant data to be estimated for the economic model. Estimates from Adjuvant! Online provide similar/dissimilar estimates to the EBCTCG overviews, which, arguably, are more applicable to a UK population. However, as EBCTCG overview subset analyses are carried out largely as a set of univariate subset analyses it is impossible to tell if two univariate effects are independent.

Adjuvant! Online integrates patient-related information (age and comorbidity) and tumourrelated information (nodal status, tumour size, histological grade, ER status and histological subtype) to make estimates of mortality and recurrence. However, survival estimates are derived from the US population. 



\section{Appendix 29}

\section{Estimates of the different types of cancer that exist}

\begin{tabular}{|c|c|c|c|c|c|c|}
\hline \multicolumn{4}{|l|}{ Categories } & \multirow[b]{2}{*}{ Count } & \multirow[b]{2}{*}{ Total } & \multirow[b]{2}{*}{ Percentage } \\
\hline Size (mm) & Grade & Nodes & $\begin{array}{l}\text { Vascular } \\
\text { invasion }\end{array}$ & & & \\
\hline \multirow[t]{18}{*}{$0-20$} & 1 & 0 & No & 3207 & 3326 & 12.28 \\
\hline & & & Yes & 119 & & \\
\hline & & $1-3$ & No & 503 & 589 & 2.18 \\
\hline & & & Yes & 86 & & \\
\hline & & $4+$ & No & 54 & 88 & 0.32 \\
\hline & & & Yes & 34 & & \\
\hline & 2 & 0 & No & 4522 & 5178 & 19.12 \\
\hline & & & Yes & 656 & & \\
\hline & & $1-3$ & No & 1031 & 1471 & 5.43 \\
\hline & & & Yes & 440 & & \\
\hline & & $4+$ & No & 253 & 450 & 1.66 \\
\hline & & & Yes & 197 & & \\
\hline & 3 & 0 & No & 2105 & 2695 & 9.95 \\
\hline & & & Yes & 590 & & \\
\hline & & $1-3$ & No & 491 & 916 & 3.38 \\
\hline & & & Yes & 425 & & \\
\hline & & $4+$ & No & 161 & 462 & 1.71 \\
\hline & & & Yes & 301 & & \\
\hline \multirow[t]{18}{*}{ 20.001-49.999 } & 1 & 0 & No & 477 & 528 & 1.95 \\
\hline & & & Yes & 51 & & \\
\hline & & $1-3$ & No & 209 & 283 & 1.05 \\
\hline & & & Yes & 74 & & \\
\hline & & $4+$ & No & 59 & 109 & 0.40 \\
\hline & & & Yes & 50 & & \\
\hline & 2 & 0 & No & 1781 & 2233 & 8.25 \\
\hline & & & Yes & 452 & & \\
\hline & & $1-3$ & No & 912 & 1541 & 5.69 \\
\hline & & & Yes & 629 & & \\
\hline & & $4+$ & No & 392 & 1018 & 3.76 \\
\hline & & & Yes & 626 & & \\
\hline & 3 & 0 & No & 1526 & 2209 & 8.16 \\
\hline & & & Yes & 683 & & \\
\hline & & $1-3$ & No & 628 & 1467 & 5.42 \\
\hline & & & Yes & 839 & & \\
\hline & & $4+$ & No & 292 & 1256 & 4.64 \\
\hline & & & Yes & 964 & & \\
\hline
\end{tabular}




\begin{tabular}{|c|c|c|c|c|c|c|}
\hline \multicolumn{4}{|l|}{ Categories } & \multirow[b]{2}{*}{ Count } & \multirow[b]{2}{*}{ Total } & \multirow[b]{2}{*}{ Percentage } \\
\hline Size $(\mathrm{mm})$ & Grade & Nodes & $\begin{array}{l}\text { Vascular } \\
\text { invasion }\end{array}$ & & & \\
\hline \multirow[t]{19}{*}{$50+$} & 1 & 0 & No & 22 & 24 & 0.09 \\
\hline & & & Yes & 2 & & \\
\hline & & $1-3$ & No & 11 & 15 & 0.06 \\
\hline & & & Yes & 4 & & \\
\hline & & $4+$ & No & 9 & 26 & 0.10 \\
\hline & & & Yes & 17 & & \\
\hline & 2 & 0 & No & 92 & 110 & 0.41 \\
\hline & & & Yes & 18 & & \\
\hline & & $1-3$ & No & 105 & 172 & 0.64 \\
\hline & & & Yes & 67 & & \\
\hline & & $4+$ & No & 95 & 280 & 1.03 \\
\hline & & & Yes & 185 & & \\
\hline & 3 & 0 & No & 76 & 118 & 0.44 \\
\hline & & & Yes & 42 & & \\
\hline & & $1-3$ & No & 44 & 161 & 0.59 \\
\hline & & & Yes & 117 & & \\
\hline & & $4+$ & No & 45 & 355 & 1.31 \\
\hline & & & Yes & 310 & & \\
\hline & & & Total & 27,080 & & 100 \\
\hline
\end{tabular}




\section{Appendix 30}

\section{Summary of data from Adjuvant! Online used - estimated 10-year survival}




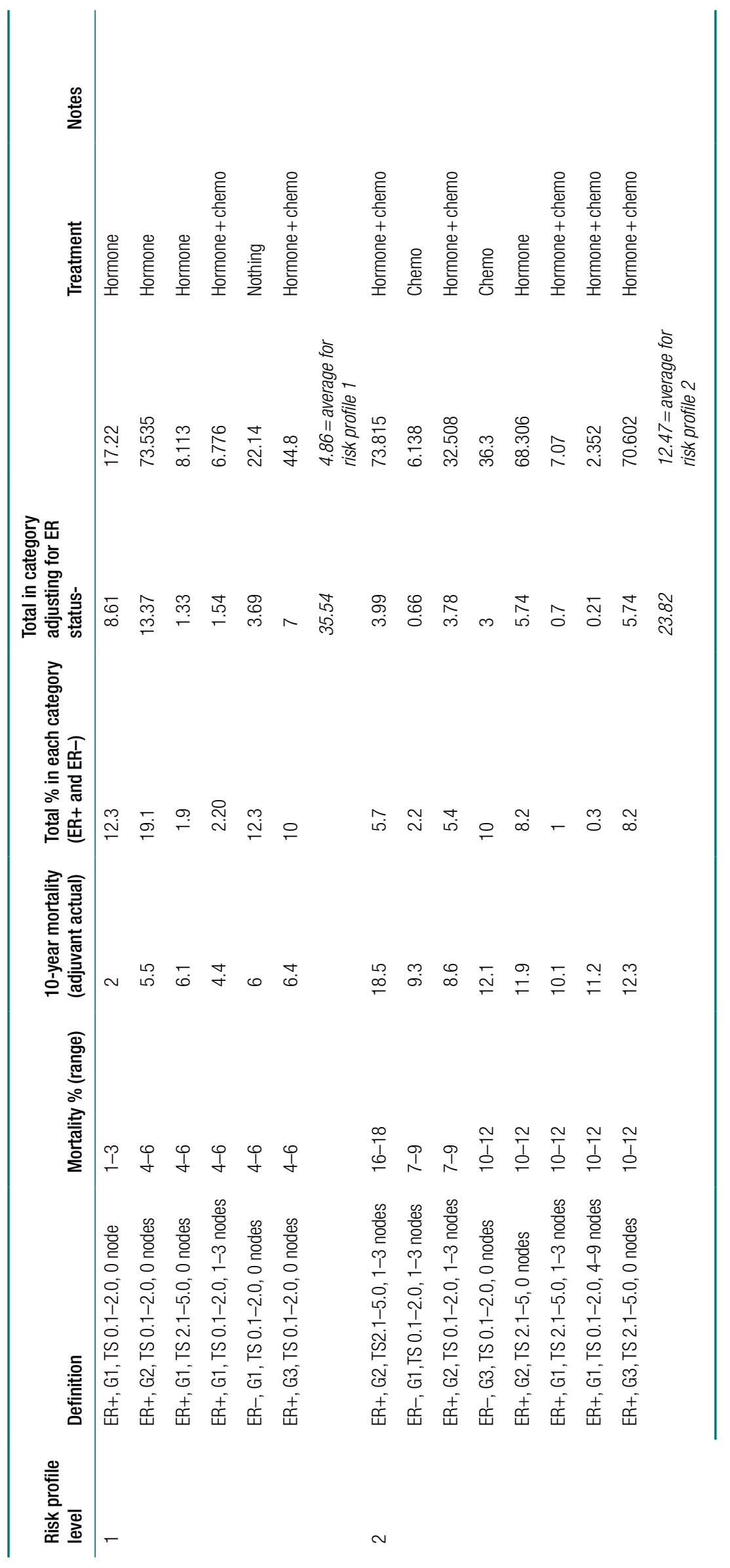




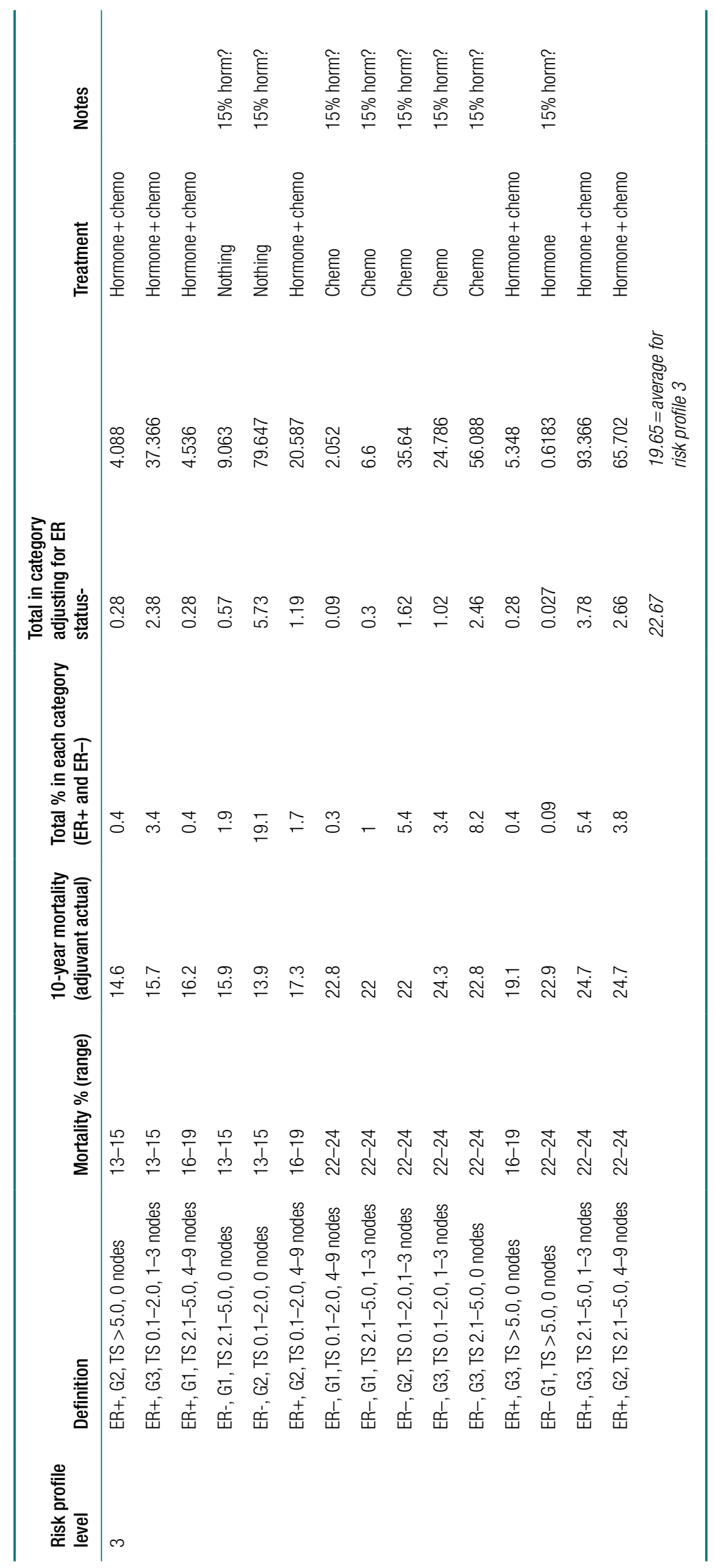




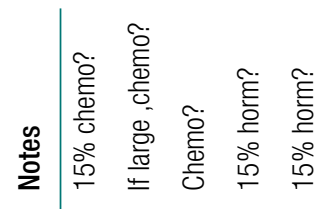

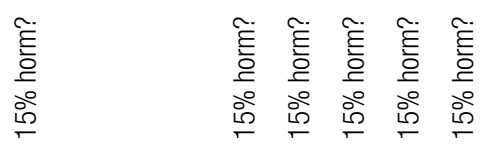

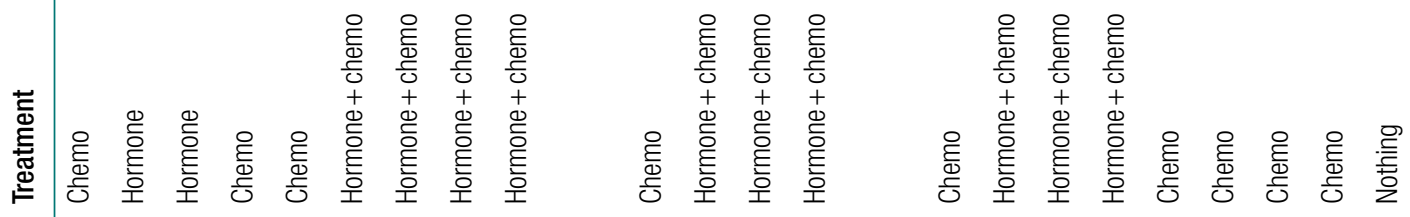

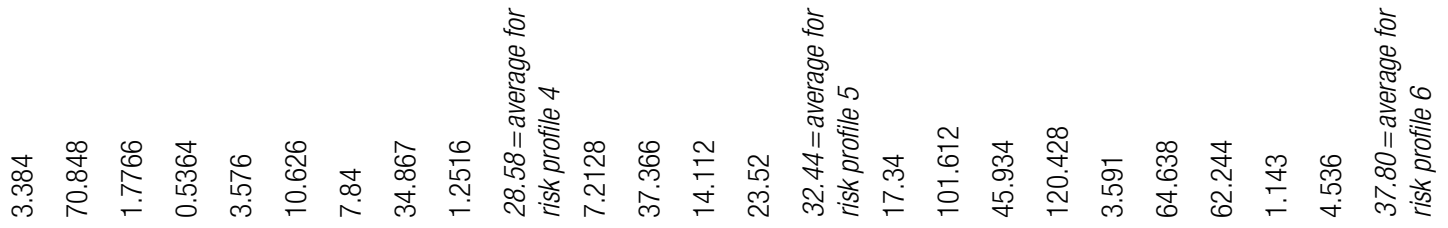

호ㅇㅝㅕㄴ

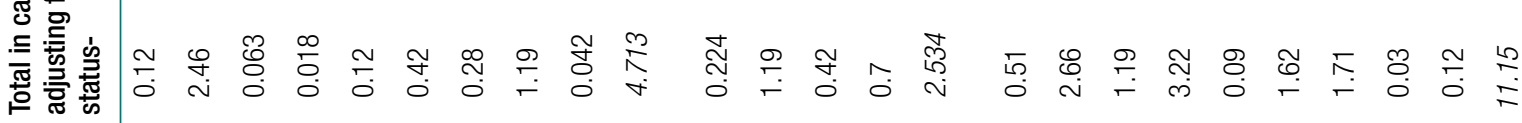

㐔

密 $\frac{T}{4}$

응 응

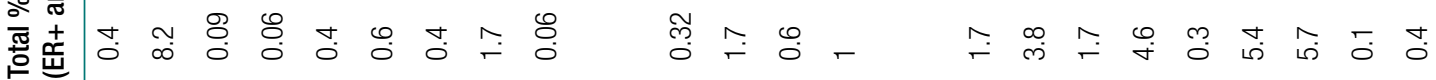

疍高

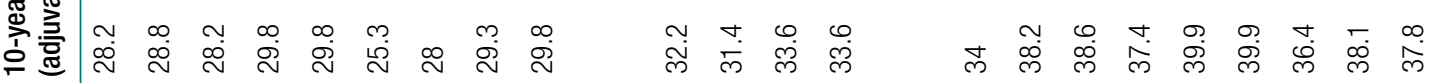

离

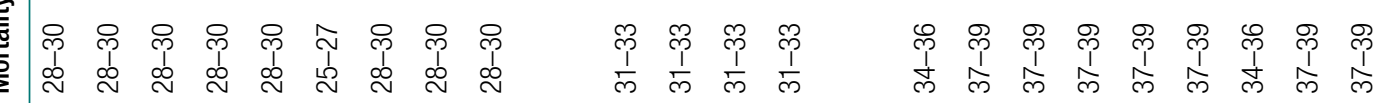

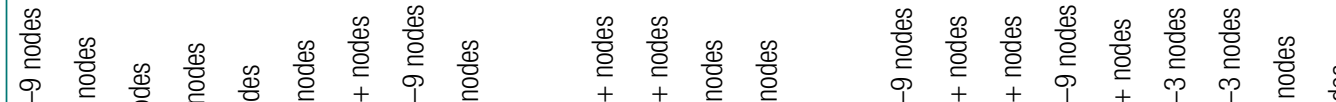

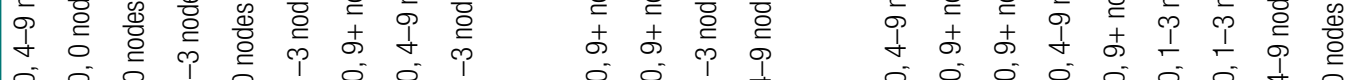

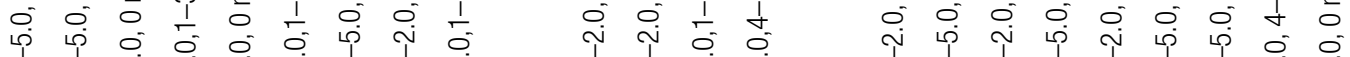

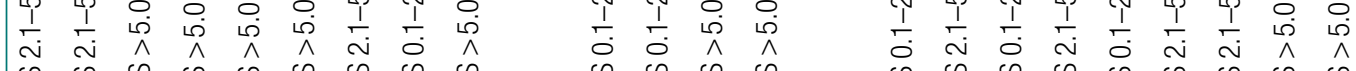

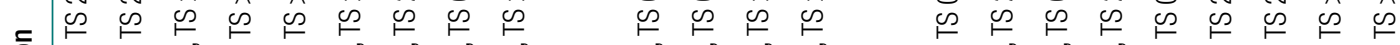

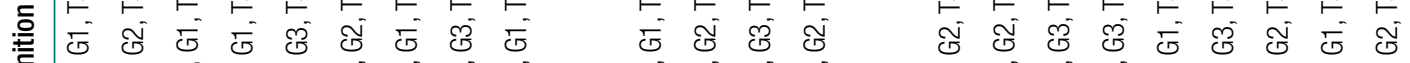

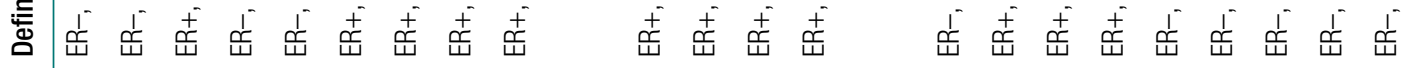

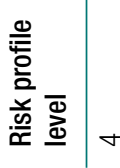




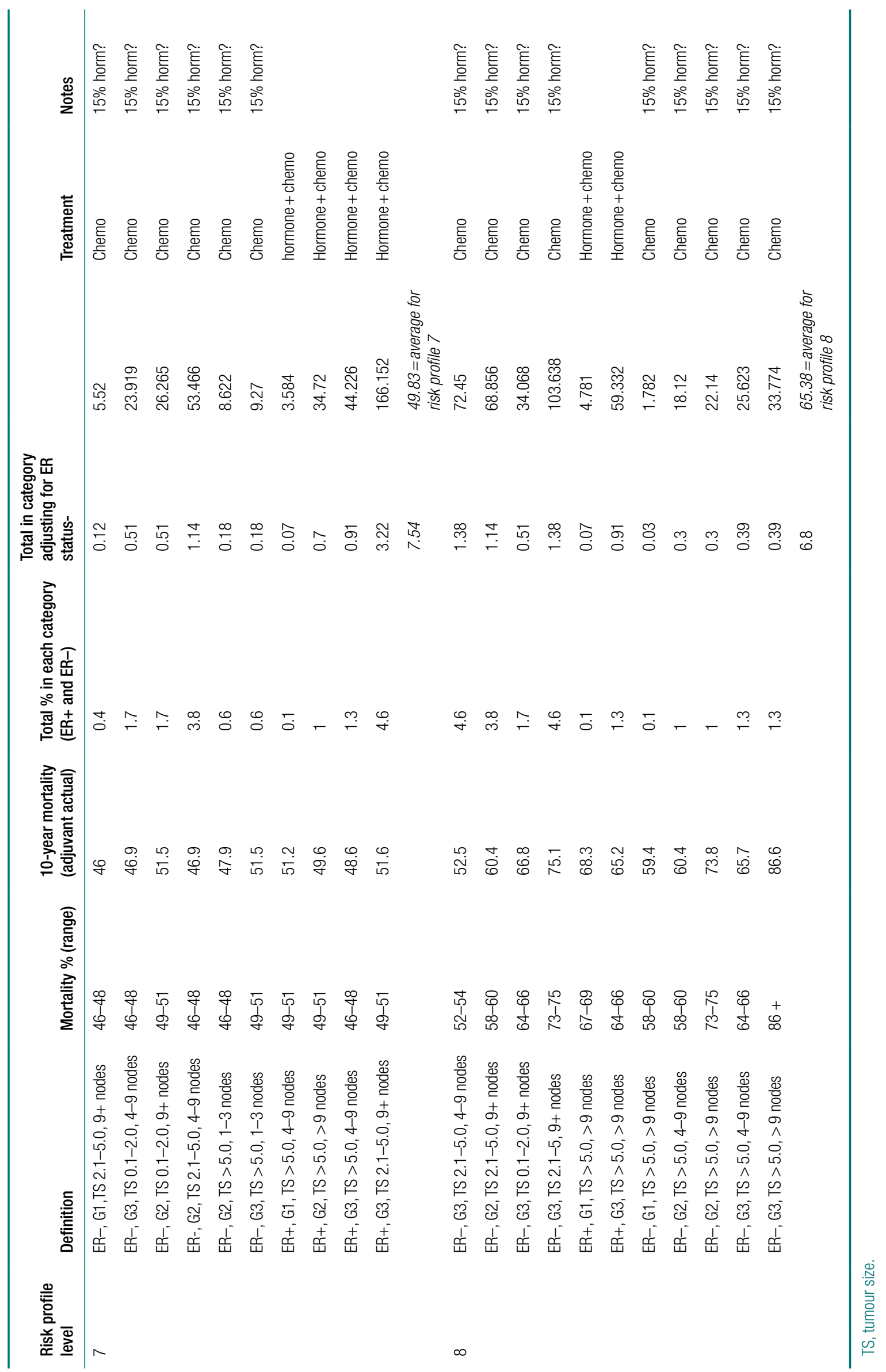




\section{Assumptions}

Decision rules for treatment

1. $E R+=$ hormone therapy

2. Grade $3=$ chemotherapy

3. ER+ and positive nodes $=$ hormone + chemotherapy

4. ER-, 0 nodes $=$ nothing

5. ER-= chemotherapy (15\% might get hormone therapy in addition) 


\section{Appendix 31}

\section{All-cause mortality rates}

\begin{tabular}{|c|c|c|c|}
\hline Index (cycle) & Value & Index (cycle) & Value \\
\hline 0 & 0.002185889 & 43 & 0.000633701 \\
\hline 1 & 0.000176516 & 44 & 0.000670725 \\
\hline 2 & $9.65047 \mathrm{E}-05$ & 45 & 0.000765293 \\
\hline 3 & $8.05032 \mathrm{E}-05$ & 46 & 0.00082434 \\
\hline 4 & 5.85017E-05 & 47 & 0.000914418 \\
\hline 5 & 4.80012E-05 & 48 & 0.001034035 \\
\hline 6 & 4.90012E-05 & 49 & 0.001076079 \\
\hline 7 & 4.10008E-05 & 50 & 0.00128032 \\
\hline 8 & $4.5001 \mathrm{E}-05$ & 51 & 0.001346907 \\
\hline 9 & $3.90008 \mathrm{E}-05$ & 52 & 0.001431525 \\
\hline 10 & 4.65011E-05 & 53 & 0.001580249 \\
\hline 11 & 4.85012E-05 & 54 & 0.001770067 \\
\hline 12 & $5.00013 \mathrm{E}-05$ & 55 & 0.001879266 \\
\hline 13 & $5.90017 \mathrm{E}-05$ & 56 & 0.002072648 \\
\hline 14 & $5.95018 \mathrm{E}-05$ & 57 & 0.002197414 \\
\hline 15 & $7.90031 \mathrm{E}-05$ & 58 & 0.002361288 \\
\hline 16 & $8.65037 \mathrm{E}-05$ & 59 & 0.002655025 \\
\hline 17 & 0.000122508 & 60 & 0.002852067 \\
\hline 18 & 0.000135509 & 61 & 0.00323122 \\
\hline 19 & 0.000128508 & 62 & 0.003459484 \\
\hline 20 & 0.000120507 & 63 & 0.003906631 \\
\hline 21 & 0.000132009 & 64 & 0.004267104 \\
\hline 22 & 0.000131509 & 65 & 0.004599578 \\
\hline 23 & 0.000123508 & 66 & 0.005054775 \\
\hline 24 & 0.000147011 & 67 & 0.005641413 \\
\hline 25 & 0.00014351 & 68 & 0.006184122 \\
\hline 26 & 0.000168514 & 69 & 0.006790556 \\
\hline 27 & 0.000155512 & 70 & 0.007564612 \\
\hline 28 & 0.000179016 & 71 & 0.00831507 \\
\hline 29 & 0.000190518 & 72 & 0.009229593 \\
\hline 30 & 0.000208022 & 73 & 0.010470819 \\
\hline 31 & 0.000203021 & 74 & 0.011811253 \\
\hline 32 & 0.00024353 & 75 & 0.013020264 \\
\hline 33 & 0.000269536 & 76 & 0.01471527 \\
\hline 34 & 0.000287541 & 77 & 0.016491993 \\
\hline 35 & 0.000299045 & 78 & 0.018562279 \\
\hline 36 & 0.000322052 & 79 & 0.021135862 \\
\hline 37 & 0.000363566 & 80 & 0.023809957 \\
\hline 38 & 0.000397579 & 81 & 0.026686587 \\
\hline 39 & 0.000448601 & 82 & 0.029678404 \\
\hline 40 & 0.00049012 & 83 & 0.033678625 \\
\hline 41 & 0.000528139 & 84 & 0.037974533 \\
\hline 42 & 0.000575666 & 85 & 0.04250483 \\
\hline
\end{tabular}




\begin{tabular}{ll}
\hline Index (cycle) & Value \\
\hline 86 & 0.047340565 \\
87 & 0.05188872 \\
88 & 0.058742862 \\
89 & 0.06434889 \\
90 & 0.072877031 \\
91 & 0.083887561 \\
92 & 0.093932122 \\
93 & 0.104376753 \\
94 & 0.114247213 \\
95 & 0.125022286 \\
96 & 0.13718484 \\
97 & 0.146604429 \\
98 & 0.163656171 \\
99 & 0.172317694 \\
100 & 0.188410202 \\
\hline
\end{tabular}




\title{
Appendix 32
}

\section{Summary of findings of the structured review of doubling times}

\begin{abstract}
The identified papers are summarised in Table 80 and described below. Peer and colleagues ${ }^{89}$ conducted a study in the Netherlands using data from the Breast Screening Programme from the period 1980-91 to determine the growth rate of primary breast cancer stratified by age. The authors report the mean breast cancer doubling times by age, based on information from 236 cancers with a measurable tumour nucleus (Table 81). The authors reported that tumour doubling time was age dependent, with those of a younger age having a shorter tumour doubling time. In addition to the data reported in Table 81 the authors also present doubling times for other studies. Doubling times from these studies range from a low of 60 days ${ }^{119}$ to 268 days. ${ }^{120}$
\end{abstract}

Spratt ${ }^{121}$ undertook a study to determine the association of doubling times of primary breast cancer and other histopathological and mammography characteristics. The study was undertaken at the University of Louisville Breast Cancer Detection and Demonstration Project (BCDDP). The data used consisted of 23 tumours with doubling times ranging from 109 days to 944 days (median 324 days). The authors found an association between faster growth rate and three factors, one of which was the nuclear grade of tumour; however, no information was presented on the actual increased doubling time by grade. A further study ${ }^{88}$ used data from both the University of Louisville and University of Heidelberg of women who had undergone routine mammography as part of a breast-screening programme. In total, 448 observations were obtained and used to measure the growth rate of tumours. The authors found that the median doubling time was 260 days (mean age 53 years). A large variation in doubling time was observed, however, ranging from 10 days to 7051 days.

Arnerlöv and colleagues ${ }^{122}$ analysed the doubling time of tumours based on information from 158 patients with breast cancer who were diagnosed between 1974 and 1987 in Sweden. The authors found large variations in tumour doubling rates. The median doubling time was 11 months. In contrast with Spratt's study, the authors found no association between doubling time and grade, although they did find that short doubling times were significantly correlated with tumour size.

In a recent study, Weedon-Fekjaer and colleagues ${ }^{123}$ used a likelihood-based estimating procedure to estimate tumour growth, using data from the Norwegian Breast Cancer Screening Programme. The data relate to 503 women aged between 50 and 69 years, over the period 1985-94. The authors found a large variation in tumour growth rates, with $5 \%$ of tumours taking $<1.2$ months to double in size from 10 to $20 \mathrm{~mm}$. At the other extreme, the authors found that $5 \%$ of tumours had a doubling time of 6.3 years. Mean tumour doubling time was found to be 1.7 years, with doubling time increasing with age. Overall, evidence from these studies shows a large variation in doubling times.

Brekelmans and colleagues ${ }^{124}$ conducted a study based on data from a breast-screening programme in the Netherlands. The results were based on 104 invasive tumours detected between 1975 and 1986. The mean doubling time was reported to be 99 days (range 6-309 days). 
TABLE 80 Summary of studies reporting data on doubling times of breast cancer

\begin{tabular}{|c|c|c|}
\hline $\begin{array}{l}\text { Author (date) } \\
\text { Location }\end{array}$ & Methods & Results \\
\hline \multirow{4}{*}{$\begin{array}{l}\text { Peer et al. (1993) } \\
\text { Nijmegen, Netherlands }\end{array}$} & \multirow{2}{*}{$\begin{array}{l}\text { Study based on data from the Breast Cancer Screening } \\
\text { Programme conducted in the Netherlands since } 1975 \text {. The } \\
\text { study data are from the period 1980-91 and are based on } \\
236 \text { cancers with measurable tumour nucleus }\end{array}$} & $\begin{array}{l}\text { The tumour doubling time was found to be age } \\
\text { dependent: }\end{array}$ \\
\hline & & $\begin{array}{l}<50 \text { years' doubling time was } 80 \text { days ( } 95 \% \mathrm{Cl} 44 \text { to } \\
147 \text { days) }\end{array}$ \\
\hline & \multirow{2}{*}{$\begin{array}{l}\text { The authors calculated the doubling time of tumours by age } \\
\text { group: }<50 \text { years ( } n=32 \text { cancers); } 50-70 \text { years ( } n=154 \\
\text { cancers); }>70 \text { years ( } n=50 \text { cancers) }\end{array}$} & $\begin{array}{l}50-70 \text { years' doubling time was } 157 \text { days }(95 \% \mathrm{Cl} \\
121 \text { to } 204 \text { days) }\end{array}$ \\
\hline & & $\begin{array}{l}>70 \text { years' doubling time } 188 \text { days }(95 \% \mathrm{Cl} 120 \text { to } \\
\text { 295) }\end{array}$ \\
\hline $\begin{array}{l}\text { Brekelmans et al. } \\
(1996)^{124}\end{array}$ & $\begin{array}{l}\text { Study based on data from a breast-screening programme } \\
\text { (DOM project) in the Netherlands }\end{array}$ & $\begin{array}{l}\text { The mean doubling time of the whole group was } 99 \\
\text { days (range 6-309 days) }\end{array}$ \\
\hline \multirow[t]{4}{*}{ Netherlands } & \multirow{3}{*}{$\begin{array}{l}\text { Based on } 139 \text { cancers detected between } 1975 \text { and } 1986 . \\
\text { After exclusions the analysis was based on } 104 \text { invasive } \\
\text { tumours }\end{array}$} & By subgroup analysis this was: \\
\hline & & 51 days in the masked group \\
\hline & & 128 days in the intermediate group \\
\hline & $\begin{array}{l}\text { The analysis was subdivided into four groups: missed } \\
\text { cancers; masked cancers; intermediate growth rate; and fast } \\
\text { growth rate }\end{array}$ & 79 days in the fast-growing group \\
\hline Arnerlöv et al. (1992) ${ }^{122}$ & \multirow{2}{*}{$\begin{array}{l}\text { Study analyses the doubling time of tumours based on } \\
\text { information form } 158 \text { patients with breast cancer who were } \\
\text { diagnosed between } 1974 \text { and } 1987\end{array}$} & \multirow{2}{*}{$\begin{array}{l}\text { The mean age of patients was } 65 \text { years. The authors } \\
\text { found variations in the tumour doubling rate ( } 0.6 \\
\text { months to indefinite time). Median doubling time } \\
\text { was } 11 \text { months (approximately } 330 \text { days) and mean } \\
\text { doubling time was } 10.9 \text { months }\end{array}$} \\
\hline Sweden & & \\
\hline Spratt et al. $(1993)^{88}$ & \multirow{2}{*}{$\begin{array}{l}\text { Used data (from both Heidelberg and Louisville) of women } \\
\text { who had undergone routine mammography as part of a } \\
\text { breast-screening programme. In total, } 448 \text { observations were } \\
\text { obtained and used to measure the growth rate of breast } \\
\text { cancers }\end{array}$} & \multirow{2}{*}{$\begin{array}{l}\text { The mean age of patients was } 53 \text { years. The authors } \\
\text { found the median doubling time to be } 260 \text { days, } \\
\text { ranging from the shortest doubling time of } 10 \text { days to } \\
7051 \text { days }\end{array}$} \\
\hline $\begin{array}{l}\text { Heidelberg and } \\
\text { Louisville }\end{array}$ & & \\
\hline Spratt $(1981)^{121}$ & \multirow{2}{*}{$\begin{array}{l}\text { Spratt undertook a study to determine the association } \\
\text { of doubling times of primary breast cancer and other } \\
\text { histopathologic and mammography characteristics. The study } \\
\text { was undertaken at the University of Louisville Breast Cancer } \\
\text { Detection and Demonstration Project (BCDDP) }\end{array}$} & \multirow[b]{2}{*}{$\begin{array}{l}\text { The data used consisted of } 23 \text { tumours with doubling } \\
\text { times ranging from } 109 \text { to } 944 \text { days (median } 324 \\
\text { days). The authors found an association between faster } \\
\text { growth rate and three factors, one of which was the } \\
\text { nuclear grade of tumour; however, no information was } \\
\text { presented on the actual increased doubling time by } \\
\text { grade }\end{array}$} \\
\hline University of Louisville & & \\
\hline $\begin{array}{l}\text { Tilanus-Linthorst et al. } \\
(2007)^{125}\end{array}$ & \multirow{3}{*}{$\begin{array}{l}\text { Analysed breast cancer growth rates found during screening } \\
\text { for patients in the UK, Holland and Canada. The authors were } \\
\text { able to assess doubling time of tumours in } 100 \text { cases. These } \\
\text { cases were subdivided into BRCA1 mutation ( } 43 \text { women); } \\
\text { BRCA2 mutation ( } 16 \text { women) and high risk ( } 41 \text { women) }\end{array}$} & \multirow{2}{*}{$\begin{array}{l}\text { The authors found an inverse relationship between } \\
\text { growth rate and age. The authors grouped their } \\
\text { analysis into three age groups: } \leq 40,41-50 \text { and } \\
>50 \text { years There were differences in doubling times } \\
\text { between the subgroups by severity and age: doubling } \\
\text { time in } B R C A 1 / 2 \text { mutations in the three age categories } \\
\text { was } 28,68 \text { and } 81 \text { days, respectively }\end{array}$} \\
\hline \multirow[t]{2}{*}{$\begin{array}{l}\text { UK, the Netherlands and } \\
\text { Canada }\end{array}$} & & \\
\hline & & $\begin{array}{l}\text { In the high-risk group, without mutations, the doubling } \\
\text { time in the three age groups was } 83,121 \text { and } 173 \\
\text { days, respectively }\end{array}$ \\
\hline $\begin{array}{l}\text { Weedon-Fekjaer et al. } \\
(2008)^{123}\end{array}$ & \multirow{3}{*}{$\begin{array}{l}\text { The authors used a likelihood-based modelling method to } \\
\text { estimate the growth rate of cancers using mammography } \\
\text { screening data. The data pertains to } 503 \text { women aged } \\
\text { between } 50 \text { and } 69 \text { years over the period 1985-94 }\end{array}$} & $\begin{array}{l}\text { The authors found large variation in the growth rates } \\
\text { of tumours }\end{array}$ \\
\hline \multirow[t]{2}{*}{ Norway } & & $\begin{array}{l}\text { In women aged } 50-59 \text { years the mean doubling time } \\
\text { (from } 10 \text { to } 20 \mathrm{~mm} \text { ) was } 1.4 \text { years. This increased to } \\
2.1 \text { years in women aged } 60-69 \text { years }\end{array}$ \\
\hline & & $\begin{array}{l}\text { Over both age groups this was estimated to be } 1.7 \\
\text { years (SD } 2.2 \text { years) }\end{array}$ \\
\hline
\end{tabular}


TABLE 81 Mean breast cancer doubling times by age

\begin{tabular}{ll}
\hline Age at diagnosis (years) & Mean $(\mathbf{9 5 \%} \mathrm{Cl})$ \\
\hline$<50$ & $80(44$ to 147$)$ days \\
$50-70$ & $157(121$ to 204$)$ days \\
$>70$ & $188(120$ to 295$)$ days \\
\hline
\end{tabular}

Source: Peer and colleagues..$^{89}$

Tilanus-Linthorst and colleagues ${ }^{125}$ analysed breast cancer growth rates found during screening patients in the UK, Holland and Canada. The authors assessed doubling time of tumours in 100 cases. These cases were subdivided into BRCA1 mutation (43 women), BRCA2 mutation (16 women) and high risk (41 women). The mean age of patients was 65 years. The authors found variations in the tumour doubling rate ( 0.6 months to indefinite time). Median doubling time was 11 months (approximately 330 days) and mean doubling time was 10.9 months. 



\section{Health Technology Assessment programme}

\section{Director,}

Professor Tom Walley, CBE

Director, NIHR HTA programme, Professor of Clinical Pharmacology,

University of Liverpool

\author{
Deputy Director, \\ Professor Hywel Williams, \\ Professor of Dermato-Epidemiology, \\ Centre of Evidence-Based Dermatology, \\ University of Nottingham
}

\section{Prioritisation Group}

\section{Members}

\section{Chair,}

Professor Tom Walley, CBE, Director, NIHR HTA programme, Professor of Clinical Pharmacology, University of Liverpool

Professor Imti Choonara, Professor in Child Health, Academic Division of Child Health, University of Nottingham Chair - Pharmaceuticals Panel

Dr Bob Coates,

Consultant Advisor - Disease

Prevention Panel

Dr Andrew Cook

Consultant Advisor - Intervention

Procedures Panel

Dr Peter Davidson,

Director of NETSCC, Health

Technology Assessment
Dr Nick Hicks,
Consultant Adviser - Diagnostic

Technologies and Screening Panel, Consultant Advisor-Psychological and Community Therapies Panel

Ms Susan Hird,

Consultant Advisor, External

Devices and Physical Therapies Pane

Professor Sallie Lamb,

Director, Warwick Clinical Trial Unit, Warwick Medical School, University of Warwick

Chair - HTA Clinical Evaluation and Trials Board

Professor Jonathan Michaels, Professor of Vascular Surgery, Sheffield Vascular Institute, University of Sheffield Chair - Interventional Procedures Panel
Professor Ruairidh Milne,

Director - External Relations

Dr John Pounsford,

Consultant Physician, Directorate of Medical Services, North Bristol NHS Trust

Chair - External Devices and

Physical Therapies Panel

Dr Vaughan Thomas

Consultant Advisor -

Pharmaceuticals Panel, Clinical

Lead - Clinical Evaluation Trials

Prioritisation Group

Professor Margaret Thorogood,

Professor of Epidemiology, Health

Sciences Research Institute,

University of Warwick

Chair - Disease Prevention Panel
Professor Lindsay Turnbull

Professor of Radiology, Centre for the MR Investigations, University of Hull

Chair - Diagnostic Technologies and Screening Panel

Professor Scott Weich,

Professor of Psychiatry, Health

Sciences Research Institute

University of Warwick

Chair - Psychological and

Community Therapies Panel

Professor Hywel Williams,

Director of Nottingham Clinical Trials Unit, Centre of Evidence-

Based Dermatology, University of

Nottingham

Chair - HTA Commissioning

Board

Deputy HTA Programme Director

\section{HTA Commissioning Board}

\section{Chair}

Professor Hywel Williams,

Professor of Dermato-Epidemiology, Centre of Evidence-Based Dermatology, University of Nottingham
Deputy Chair,

Professor Jon Deeks,

Department of Public Health and

Epidemiology, University of Birmingham
Professor Tom Walley, CBE,

Professor of Clinical Pharmacology, Director,

NIHR HTA programme, University of

Liverpool

\section{Members}

Professor Ann Ashburn,

Professor of Rehabilitation and

Head of Research, Southampton

General Hospital

Professor Peter Brocklehurst, Professor of Women's Health, Institute for Women's Health, University College London

Professor Jenny Donovan Professor of Social Medicine, University of Bristol

Professor Jonathan Green, Professor and Acting Head of Department, Child and Adolescent Psychiatry, University of Manchester Medical School
Professor John W Gregory,

Professor in Paediatric

Endocrinology, Department of Child Health, Wales School of Medicine, Cardiff University

Professor Steve Halligan, Professor of Gastrointestinal Radiology, University College Hospital, London

Professor Freddie Hamdy, Professor of Urology, Head of Nuffield Department of Surgery, University of Oxford

Professor Allan House, Professor of Liaison Psychiatry, University of Leeds
Dr Martin J Landray, Reader in Epidemiology, Honorary Consultant Physician, Clinical Trial Service Unit, University of Oxford

Professor Stephen Morris, Professor of Health Economics, University College London, Research Department of Epidemiology and Public Health, University College London

Professor Irwin Nazareth, Professor of Primary Care and Head of Department, Departmen of Primary Care and Population Sciences, University College London
Professor E Andrea Nelson, Professor of Wound Healing an Director of Research, School of Healthcare, University of Leeds

Professor John David Norrie, Chair in Clinical Trials and Biostatistics, Robertson Centre for Biostatistics, University of Glasgow

Dr Rafael Perera,

Lecturer in Medical Statisitics, Department of Primary Health Care, University of Oxford 


\section{HTA Commissioning Board (continued)}

Professor Barney Reeves,

Professorial Research Fellow

in Health Services Research,

Department of Clinical Science,

University of Bristo
Professor Martin Underwood, Professor of Primary Care

Research, Warwick Medical

School, University of Warwick
Professor Marion Walker, Professor in Stroke Rehabilitation, Associate Director UK Stroke Research Network, University of Nottingham
Dr Duncan Young,

Senior Clinical Lecturer and Consultant, Nuffield Department of Anaesthetics, University of Oxford

\section{Observers}

Dr Tom Foulks,

Medical Research Council
Dr Kay Pattison,

Senior NIHR Programme

Manager, Department of Health

\section{HTA Clinical Evaluation and Trials Board}

\section{Chair,}

Professor Sallie Lamb,

Director,

Warwick Clinical Trials Unit,

Warwick Medical School,

University of Warwick and Professor of

Rehabilitation,

Nuffield Department of Orthopaedic,

Rheumatology and Musculoskeletal Sciences,

University of Oxford

\section{Deputy Chair,}

Professor Jenny Hewison,

Professor of the Psychology of Health Care,

Leeds Institute of Health Sciences,

University of Leeds
Programme Director,

Professor Tom Walley, CBE,

Director, NIHR HTA programme, Professor of

Clinical Pharmacology, University of Liverpool

\section{Members}

Professor Keith Abrams, Professor of Medical Statistics, Department of Health Sciences, University of Leicester

Professor Martin Bland, Professor of Health Statistics, Department of Health Sciences, University of York

Professor Jane Blazeby, Professor of Surgery and Consultant Upper GI Surgeon, Department of Social Medicine, University of Bristol

Professor Julia M Brown, Director, Clinical Trials Research Unit, University of Leed

Professor Alistair Burns, Professor of Old Age Psychiatry, Psychiatry Research Group, Schoo of Community-Based Medicine, The University of Manchester \& National Clinical Director for Dementia, Department of Health

\section{Dr Jennifer Burr,} Director, Centre for Healthcare Randomised trials (CHART), University of Aberdeen

Professor Linda Davies, Professor of Health Economics, Health Sciences Research Group, University of Manchester

Professor Simon Gilbody, Prof of Psych Medicine and Health Services Research, Department of Health Sciences, University of York

Professor Steven Goodacre, Professor and Consultant in Emergency Medicine, School of Health and Related Research, University of Sheffield

Professor Dyfrig Hughes, Professor of Pharmacoeconomics, Centre for Economics and Policy in Health, Institute of Medical and Social Care Research, Bangor University
Professor Paul Jones, Professor of Respiratory Medicine, Department of Cardiac and Vascular Science, St George's Hospital Medical School, University of London

Professor Khalid Khan, Professor of Women's Health and Clinical Epidemiology, Barts and the London School of Medicine, Queen Mary, University of London

Professor Richard J McManus, Professor of Primary Care Cardiovascular Research, Primary Care Clinical Sciences Building,

University of Birmingham

Professor Helen Rodgers, Professor of Stroke Care, Institute for Ageing and Health, Newcastle University

Professor Ken Stein,

Professor of Public Health,

Peninsula Technology Assessment

Group, Peninsula College

of Medicine and Dentistry,

Universities of Exeter and

Plymouth
Professor Jonathan Sterne, Professor of Medical Statistics and Epidemiology, Department of Social Medicine, University of Bristol

Mr Andy Vail,

Senior Lecturer, Health Sciences Research Group, University of Manchester

Professor Clare Wilkinson, Professor of General Practice and Director of Research North Wales Clinical School, Department of Primary Care and Public Health Cardiff University

Dr Ian B Wilkinson,

Senior Lecturer and Honorary Consultant, Clinical Pharmacology Unit, Department of Medicine, University of Cambridge

\section{Observers}

Ms Kate Law, Director of Clinical Trials, Cancer Research UK
Dr Morven Roberts,

Clinical Trials Manager, Health

Services and Public Health

Services Board, Medical Research

Council 


\section{Diagnostic Technologies and Screening Panel}

Members

\section{Chair,}

Professor Lindsay Wilson

Turnbull,

Scientific Director of the

Centre for Magnetic Resonance

Investigations and YCR Professor

of Radiology, Hull Royal Infirmary

Professor Judith E Adams,

Consultant Radiologist,

Manchester Royal Infirmary,

Central Manchester \& Manchester

Children's University Hospitals

NHS Trust, and Professor of

Diagnostic Radiology, University

of Manchester

Mr Angus S Arunkalaivanan,

Honorary Senior Lecturer,

University of Birmingham and

Consultant Urogynaecologist

and Obstetrician, City Hospital,

Birmingham

Dr Diana Baralle,

Consultant and Senior Lecturer

in Clinical Genetics, University of

Southampton
Dr Stephanie Dancer,

Consultant Microbiologist,

Hairmyres Hospital, East Kilbride

Dr Diane Eccles,

Professor of Cancer Genetics,

Wessex Clinical Genetics Service,

Princess Anne Hospital

Dr Trevor Friedman,

Consultant Liason Psychiatrist,

Brandon Unit, Leicester General Hospital

Dr Ron Gray,

Consultant, National Perinatal Epidemiology Unit, Institute of Health Sciences, University of Oxford

Professor Paul D Griffiths, Professor of Radiology, Academic

Unit of Radiology, University of Sheffield

Mr Martin Hooper,

Public contributor
Professor Anthony Robert

Kendrick,

Associate Dean for Clinical

Research and Professor of Primary

Medical Care, University of

Southampton

Dr Nicola Lennard,

Senior Medical Officer, MHRA

Dr Anne Mackie,

Director of Programmes, UK

National Screening Committee,

London

Mr David Mathew,

Public contributor

Dr Michael Millar,

Consultant Senior Lecturer in

Microbiology, Department of

Pathology \& Microbiology, Barts

and The London NHS Trust, Royal

London Hospital

Mrs Una Rennard,

Public contributor
Dr Stuart Smellie,

Consultant in Clinical Pathology, Bishop Auckland General Hospital

Ms Jane Smith,

Consultant Ultrasound

Practitioner, Leeds Teaching

Hospital NHS Trust, Leeds

Dr Allison Streetly,

Programme Director, NHS Sickle

Cell and Thalassaemia Screening

Programme, King's College School of Medicine

Dr Matthew Thompson,

Senior Clinical Scientist and GP

Department of Primary Health

Care, University of Oxford

Dr Alan J Williams,

Consultant Physician, General and

Respiratory Medicine, The Royal

Bournemouth Hospital

\section{Observers}

\section{Dr Tim Elliott,}

Team Leader, Cancer Screening,

Department of Health

Dr Joanna Jenkinson,

Board Secretary, Neurosciences

and Mental Health Board

(NMHB), Medical Research

Council
Professor Julietta Patnick,

Director, NHS Cancer Screening

Programme, Sheffield

Dr Kay Pattison,

Senior NIHR Programme

Manager, Department of Health
Professor Tom Walley, CBE, Director, NIHR HTA

programme, Professor of Clinical

Pharmacology, University of

Liverpool
Dr Ursula Wells,

Principal Research Officer, Policy Research Programme, Department of Health

\section{Disease Prevention Panel}

Members

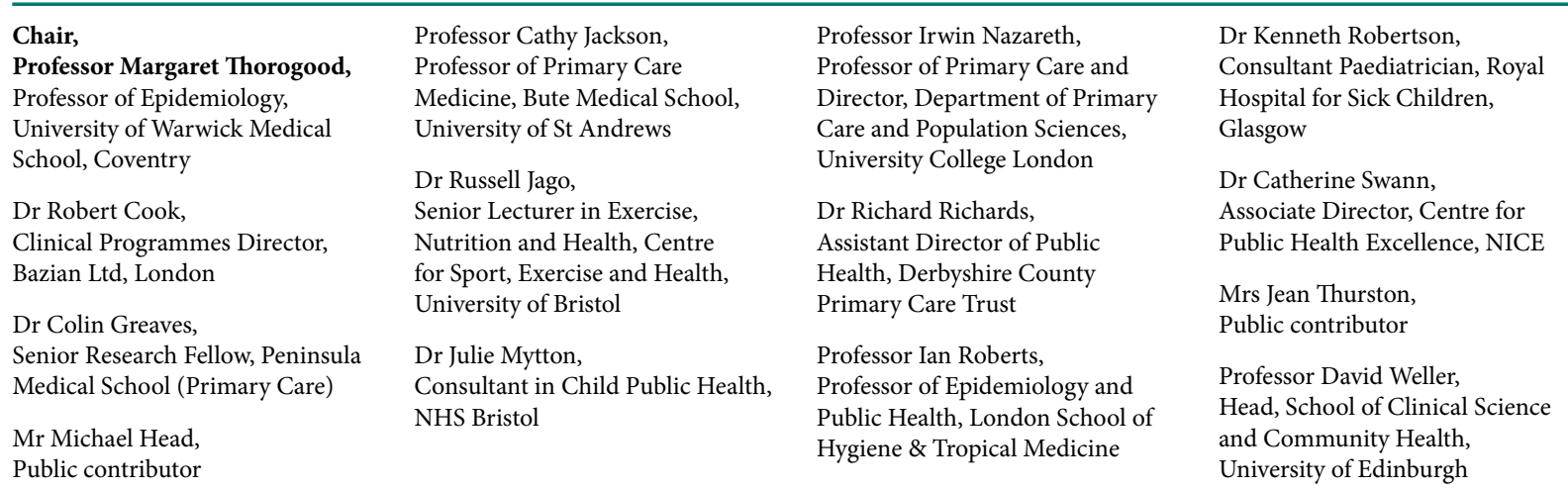

Observers

Ms Christine McGuire,

Research \& Development,

Department of Health
Dr Kay Pattison,

Senior NIHR Programme

Manager, Department of Health
Professor Tom Walley, CBE,

Director, NIHR HTA

programme, Professor of Clinical

Pharmacology, University of

Liverpool 


\section{External Devices and Physical Therapies Panel}

Members

\section{Chair,}

Dr John Pounsford,

Consultant Physician North Bristol

NHS Trust

Deputy Chair,

Professor E Andrea Nelson,

Reader in Wound Healing and

Director of Research, University

of Leeds

Professor Bipin Bhakta,

Charterhouse Professor in

Rehabilitation Medicine,

University of Leeds

Mrs Penny Calder,

Public contributor
Dr Dawn Carnes,

Senior Research Fellow, Barts and the London School of Medicine and Dentistry

Dr Emma Clark,

Clinician Scientist Fellow \& Cons.

Rheumatologist, University of Bristol

Mrs Anthea De Barton-Watson,

Public contributor

Professor Nadine Foster, Professor of Musculoskeletal Health in Primary Care Arthritis Research, Keele University
Dr Shaheen Hamdy,

Clinical Senior Lecturer and

Consultant Physician, University

of Manchester

Professor Christine Norton,

Professor of Clinical Nursing

Innovation, Bucks New University

and Imperial College Healthcare

NHS Trust

Dr Lorraine Pinnigton,

Associate Professor in

Rehabilitation, University of

Nottingham

Dr Kate Radford,

Senior Lecturer (Research),

University of Central Lancashire
Mr Jim Reece,

Public contributor

Professor Maria Stokes

Professor of Neuromusculoskeletal

Rehabilitation, University of

Southampton

Dr Pippa Tyrrell,

Senior Lecturer/Consultant

Salford Royal Foundation

Hospitals' Trust and University of Manchester

Dr Nefyn Williams,

Clinical Senior Lecturer, Cardiff University

\section{Observers}

\section{Dr Kay Pattison,}

Senior NIHR Programme

Manager, Department of Health
Dr Morven Roberts,

Clinical Trials Manager, Health

Services and Public Health

Services Board, Medical Research

Council
Professor Tom Walley, CBE,

Director, NIHR HTA

programme, Professor of Clinical

Pharmacology, University of

Liverpool
Dr Ursula Wells,

Principal Research Officer, Policy

Research Programme, Department of Health

\section{Interventional Procedures Panel}

Members

\section{Chair, \\ Professor Jonathan Michaels,} Professor of Vascular Surgery,

University of Sheffield

\section{Deputy Chair,}

Mr Michael Thomas,

Consultant Colorectal Surgeon, Bristol Royal Infirmary

Mrs Isabel Boyer,

Public contributor

Mr Sankaran Chandra Sekharan, Consultant Surgeon, Breast Surgery, Colchester Hospital University NHS Foundation Trust

Professor Nicholas Clarke,

Consultant Orthopaedic Surgeon,

Southampton University Hospitals NHS Trust

Ms Leonie Cooke Public contributor

\section{Mr Seumas Eckford,}

Consultant in Obstetrics \&

Gynaecology, North Devon

District Hospital

Professor Sam Eljamel,

Consultant Neurosurgeon,

Ninewells Hospital and Medical

School, Dundee

Dr Adele Fielding,

Senior Lecturer and Honorary

Consultant in Haematology,

University College London

Medical School

Dr Matthew Hatton,

Consultant in Clinical Oncology,

Sheffield Teaching Hospital

Foundation Trust

Dr John Holden,

General Practitioner, Garswood

Surgery, Wigan
Dr Fiona Lecky,

Senior Lecturer/Honorary

Consultant in Emergency

Medicine, University of

Manchester/Salford Royal

Hospitals NHS Foundation Trust

Dr Nadim Malik,

Consultant Cardiologist/Honorary

Lecturer, University of Manchester

Mr Hisham Mehanna,

Consultant \& Honorary Associate

Professor, University Hospitals

Coventry \& Warwickshire NHS Trust

Dr Jane Montgomery,

Consultant in Anaesthetics and

Critical Care, South Devon

Healthcare NHS Foundation Trust

Professor Yit Chiun Yang,

Consultant Ophthalmologist,

Royal Wolverhampton Hospitals

NHS Trust

\section{Observers}

Dr Kay Pattison,

Senior NIHR Programme

Manager, Department of Health
Dr Morven Roberts,

Clinical Trials Manager, Health

Services and Public Health

Services Board, Medical Research

Council
Professor Tom Walley, CBE

Director, NIHR HTA

programme, Professor of Clinical

Pharmacology, University of

Liverpool
Dr Ursula Wells,

Principal Research Officer, Policy

Research Programme, Department of Health 


\section{Pharmaceuticals Panel}

Members

\section{Chair,}

Professor Imti Choonara

Professor in Child Health,

University of Nottingham

\section{Deputy Chair,}

Dr Yoon K Loke,

Senior Lecturer in Clinical

Pharmacology, University of East

Anglia

Dr Martin Ashton-Key,

Medical Advisor, National

Commissioning Group, NHS

London

Dr Peter Elton,

Director of Public Health, Bury

Primary Care Trust

Dr Ben Goldacre,

Research Fellow, Epidemiology

London School of Hygiene and

Tropical Medicine
Dr James Gray,

Consultant Microbiologist,

Department of Microbiology,

Birmingham Children's Hospital

NHS Foundation Trust

Dr Jurjees Hasan,

Consultant in Medical Oncology,

The Christie, Manchester

Dr Carl Heneghan,

Deputy Director Centre for

Evidence-Based Medicine and

Clinical Lecturer, Department of

Primary Health Care, University of Oxford

\section{Dr Dyfrig Hughes,}

Reader in Pharmacoeconomics and Deputy Director, Centre for Economics and Policy in Health, IMSCaR, Bangor University
Dr Maria Kouimtzi,

Pharmacy and Informatics

Director, Global Clinical Solutions,

Wiley-Blackwell

Professor Femi Oyebode,

Consultant Psychiatrist and Head

of Department, University of

Birmingham

Dr Andrew Prentice,

Senior Lecturer and Consultant

Obstetrician and Gynaecologist,

The Rosie Hospital, University of

Cambridge

Ms Amanda Roberts,

Public contributor

Dr Gillian Shepherd,

Director, Health and Clinical

Excellence, Merck Serono Ltd
Mrs Katrina Simister

Assistant Director New Medicines,

National Prescribing Centre,

Liverpool

Professor Donald Singer,

Professor of Clinical

Pharmacology and Therapeutics,

Clinical Sciences Research

Institute, CSB, University of

Warwick Medical School

Mr David Symes,

Public contributor

Dr Arnold Zermansky,

General Practitioner, Senior

Research Fellow, Pharmacy

Practice and Medicines

Management Group, Leeds

University

\section{Observers}

\section{Dr Kay Pattison,}

Senior NIHR Programme

Manager, Department of Health

Mr Simon Reeve,

Head of Clinical and Cost-

Effectiveness, Medicines,

Pharmacy and Industry Group,

Department of Health
Dr Heike Weber,

Programme Manager, Medical

Research Council

Professor Tom Walley, CBE,

Director, NIHR HTA

programme, Professor of Clinical

Pharmacology, University of

Liverpool
Dr Ursula Wells,

Principal Research Officer, Policy Research Programme, Department of Health

\section{Psychological and Community Therapies Panel}

\section{Members}

\begin{tabular}{ll}
\hline Chair, & Mrs Val Carlill, \\
Professor Scott Weich, & Public contribut
\end{tabular}

Professor of Psychiatry, University

of Warwick, Coventry

\section{Deputy Chair,}

\section{Dr Howard Ring}

Consultant \& University Lecture in Psychiatry, University of

Cambridge

Professor Jane Barlow, Professor of Public Health in the Early Years, Health Sciences Research Institute, Warwick Medical Schoo

Dr Sabyasachi Bhaumik Consultant Psychiatrist, Leicestershire Partnership NHS Trust

\section{Public contributor}

Dr Steve Cunningham,

Consultant Respiratory

Paediatrician, Lothian Health

Board

Dr Anne Hesketh,

Senior Clinical Lecturer in Speech and Language Therapy, University of Manchester

Dr Peter Langdon,

Senior Clinical Lecturer, Schoo of Medicine, Health Policy and Practice, University of East Anglia

Dr Yann Lefeuvre,

GP Partner, Burrage Road Surgery, London
Dr Jeremy J Murphy,

Consultant Physician and

Cardiologist, County Durham and

Darlington Foundation Trust

Dr Richard Neal,

Clinical Senior Lecturer in General Practice, Cardiff University

Mr John Needham,

Public contributor

Ms Mary Nettle,

Mental Health User Consultant

Professor John Potter,

Professor of Ageing and Stroke Medicine, University of East

Anglia

Dr Greta Rait,

Senior Clinical Lecturer and

General Practitioner, University

College London
Dr Paul Ramchandani,

Senior Research Fellow/Cons.

Child Psychiatrist, University of Oxford

Dr Karen Roberts,

Nurse/Consultant, Dunston Hill Hospital, Tyne and Wear

Dr Karim Saad,

Consultant in Old Age Psychiatry, Coventry and Warwickshire Partnership Trust

Dr Lesley Stockton,

Lecturer, School of Health

Sciences, University of Liverpool

Dr Simon Wright,

GP Partner, Walkden Medical

Centre, Manchester

\section{Observers}

\section{Dr Kay Pattison,}

Senior NIHR Programme

Manager, Department of Health
Dr Morven Roberts,

Clinical Trials Manager, Health

Services and Public Health

Services Board, Medical Research

Council
Professor Tom Walley, CBE, Director, NIHR HTA programme, Professor of Clinical Pharmacology, University of Liverpool
Dr Ursula Wells,

Principal Research Officer, Policy Research Programme, Department of Health 


\section{Expert Advisory Network}

\section{Members}

Professor Douglas Altman, Professor of Statistics in Medicine, Centre for Statistics in Medicine, University of Oxford

Professor John Bond, Professor of Social Gerontology \& Health Services Research, University of Newcastle upon Tyne

Professor Andrew Bradbury, Professor of Vascular Surgery, Solihull Hospital, Birmingham

Mr Shaun Brogan,

Chief Executive, Ridgeway Primary Care Group, Aylesbury

Mrs Stella Burnside OBE, Chief Executive, Regulation and Improvement Authority, Belfast

Ms Tracy Bury,

Project Manager, World

Confederation of Physical Therapy, London

Professor Iain T Cameron,

Professor of Obstetrics and Gynaecology and Head of the School of Medicine, University of Southampton

Professor Bruce Campbell, Consultant Vascular \& General Surgeon, Royal Devon \& Exeter Hospital, Wonford

Dr Christine Clark Medical Writer and Consultant Pharmacist, Rossendale

Professor Collette Clifford, Professor of Nursing and Head of Research, The Medical School, University of Birmingham

Professor Barry Cookson, Director, Laboratory of Hospital Infection, Public Health Laboratory Service, London

Dr Carl Counsell,

Clinical Senior Lecturer in Neurology, University of Aberdeen

Professor Howard Cuckle, Professor of Reproductive Epidemiology, Department of Paediatrics, Obstetrics \& Gynaecology, University of Leeds

Professor Carol Dezateux,

Professor of Paediatric

Epidemiology, Institute of Child

Health, London

Mr John Dunning,

Consultant Cardiothoracic

Surgeon, Papworth Hospital NHS

Trust, Cambridge

Mr Jonothan Earnshaw,

Consultant Vascular Surgeon,

Gloucestershire Royal Hospital,

Gloucester
Professor Martin Eccles,

Professor of Clinical Effectiveness,

Centre for Health Services

Research, University of Newcastle upon Tyne

Professor Pam Enderby,

Dean of Faculty of Medicine,

Institute of General Practice

and Primary Care, University of

Sheffield

Professor Gene Feder,

Professor of Primary Care

Research \& Development, Centre

for Health Sciences, Barts and Th

London School of Medicine and

Dentistry

Mr Leonard R Fenwick,

Chief Executive, Freeman

Hospital, Newcastle upon Tyne

Mrs Gillian Fletcher,

Antenatal Teacher and Tutor and

President, National Childbirth

Trust, Henfield

Professor Jayne Franklyn,

Professor of Medicine, University of Birmingham

Mr Tam Fry,

Honorary Chairman, Child

Growth Foundation, London

Professor Fiona Gilbert,

Consultant Radiologist and NCRN

Member, University of Aberdeen

Professor Paul Gregg,

Professor of Orthopaedic Surgical

Science, South Tees Hospital NHS

Trust

Bec Hanley,

Co-director, TwoCan Associates,

West Sussex

Dr Maryann L Hardy,

Senior Lecturer, University of

Bradford

Mrs Sharon Hart,

Healthcare Management

Consultant, Reading

Professor Robert E Hawkins,

CRC Professor and Director of

Medical Oncology, Christie CRC

Research Centre, Christie Hospital

NHS Trust, Manchester

Professor Richard Hobbs,

Head of Department of Primary

Care \& General Practice,

University of Birmingham

Professor Alan Horwich,

Dean and Section Chairman,

The Institute of Cancer Research, London

Professor Allen Hutchinson, Director of Public Health and Deputy Dean of ScHARR,

University of Sheffield
Professor Peter Jones,

Professor of Psychiatry, University of Cambridge, Cambridge

Professor Stan Kaye,

Cancer Research UK Professor of Medical Oncology, Royal Marsden Hospital and Institute of Cancer Research, Surrey

Dr Duncan Keeley,

General Practitioner (Dr Burch \& Ptnrs), The Health Centre, Thame

Dr Donna Lamping,

Research Degrees Programme

Director and Reader in

Psychology, Health Services

Research Unit, London School of

Hygiene and Tropical Medicine,

London

Professor James Lindesay

Professor of Psychiatry for the

Elderly, University of Leicester

Professor Julian Little,

Professor of Human Genome

Epidemiology, University of

Ottawa

Professor Alistaire McGuire,

Professor of Health Economics,

London School of Economics

Professor Neill McIntosh

Edward Clark Professor of Child

Life and Health, University of Edinburgh

Professor Rajan Madhok,

Consultant in Public Health, South

Manchester Primary Care Trust

Professor Sir Alexander Markham Director, Molecular Medicine Unit, St James's University

Hospital, Leeds

Dr Peter Moore

Freelance Science Writer, Ashtead

Dr Andrew Mortimore,

Public Health Director,

Southampton City Primary Care

Trust

Dr Sue Moss,

Associate Director, Cancer

Screening Evaluation Unit,

Institute of Cancer Research,

Sutton

Professor Miranda Mugford, Professor of Health Economics and Group Co-ordinator, University of East Anglia

Professor Jim Neilson,

Head of School of Reproductive

\& Developmental Medicine

and Professor of Obstetrics

and Gynaecology, University of

Liverpool
Mrs Julietta Patnick,

Director, NHS Cancer Screening

Programmes, Sheffield

Professor Robert Peveler, Professor of Liaison Psychiatry, Royal South Hants Hospital,

Southampton

Professor Chris Price,

Director of Clinical Research,

Bayer Diagnostics Europe, Stoke Poges

Professor William Rosenberg,

Professor of Hepatology and

Consultant Physician, University of Southampton

Professor Peter Sandercock, Professor of Medical Neurology, Department of Clinical

Neurosciences, University of Edinburgh

Dr Philip Shackley,

Senior Lecturer in Health

Economics, Sheffield Vascular

Institute, University of Sheffield

Dr Eamonn Sheridan,

Consultant in Clinical Genetics, S James's University Hospital, Leeds

Dr Margaret Somerville,

Director of Public Health

Learning, Peninsula Medical

School, University of Plymouth

Professor Sarah Stewart-Brown, Professor of Public Health,

Division of Health in the Community, University of Warwick, Coventry

Dr Nick Summerton, GP Appraiser and Codirector, Research Network, Yorkshire Clinical Consultant, Primary Care and Public Health, University of Oxford

Professor Ala Szczepura, Professor of Health Service Research, Centre for Health Services Studies, University of Warwick, Coventry

Dr Ross Taylor,

Senior Lecturer, University of Aberdeen

Dr Richard Tiner,

Medical Director, Medical

Department, Association of the British Pharmaceutical Industry

Mrs Joan Webster,

Consumer Member, Southern

Derbyshire Community Health Council

Professor Martin Whittle,

Clinical Co-director, National Co-ordinating Centre for Women's and Children's Health, Lymington 



\section{Feedback}

The HTA programme and the authors would like to know your views about this report.

The Correspondence Page on the HTA website (www.hta.ac.uk) is a convenient way to publish your comments. If you prefer, you can send your comments to the address below, telling us whether you would like us to transfer them to the website.

We look forward to hearing from you.

NETSCC, Health Technology Assessment

Alpha House

University of Southampton Science Park

Southampton SO16 7NS, UK

Email: hta@hta.ac.uk

www.hta.ac.uk 